NIST Technical Note 1455-1

December 2007 Revision

\title{
Performance of Home Smoke Alarms Analysis of the Response of Several Available Technologies in Residential Fire Settings
}

\author{
Richard W. Bukowski \\ Richard D. Peacock \\ Jason D. Averill \\ Thomas G. Cleary \\ Nelson P. Bryner \\ William D. Walton \\ Paul A. Reneke \\ Erica D. Kuligowski
}





\section{Performance of Home Smoke Alarms Analysis of the Response of Several Available Technologies in Residential Fire Settings}

Richard W. Bukowski Richard D. Peacock Jason D. Averill Thomas G. Cleary Nelson P. Bryner William D. Walton Paul A. Reneke Erica D. Kuligowski

Fire Research Division Building and Fire Research Laboratory

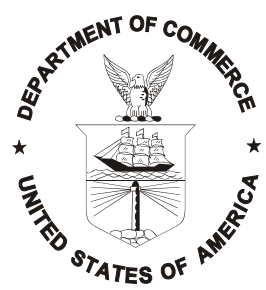

U.S. Department of Commerce Donald L. Evans, Secretary

Technology Administration Phillip J. Bond, Under Secretary for Technology 
Certain commercial entities, equipment, or materials may be identified in this document in order to describe an experimental procedure or concept adequately. Such identification is not intended to imply recommendation or endorsement by the National Institute of Standards and Technology, nor is it intended to imply that the entities, materials, or equipment are necessarily the best available for the purpose.

The policy of the National Institute of Standards and Technology is to use metric units of measurement in all its publications. In this document intended for the construction and building materials industries, however, in North America certain non-metric units are so widely used instead of metric units that it is more practical and less confusing to include certain measurement values in customary units only.

National Institute of Standards and Technology Technical Note 1455-1 Natl. Inst. Stand. Technol. Tech. Note 1455-1, 396 pages (December 2007) CODEN: NSPUE2

For sale by the Superintendent of Documents, U.S. Government Printing Office Internet: bookstore.gpo.gov — Phone: (202) 512-1800 — Fax: (202) 512-2250 Mail: Stop SSOP, Washington, DC 20402-0001 


\section{Bibliographic Information}

\section{Abstract}

This report presents the results of the project and provides details of the response of a range of residential smoke alarm technologies in a controlled laboratory test and in a series of real-scale tests conducted in two different residential structures. The data developed in this study include measurement of temperature and smoke obscuration in addition to gas concentrations for a range of fire scenarios and residences. The results are intended to provide both insight into siting and response characteristics of residential smoke alarms and a set of reference data for future enhancements to alarm technology based on fires from current materials and constructions.

Smoke alarms of either the ionization type or the photoelectric type consistently provide time for occupants to escape from most residential fires, although in some cases the escape time provided can be short. Consistent with prior findings, ionization type alarms provide somewhat better response to flaming fires than photoelectric alarms, and photoelectric alarms provide (often) considerably faster response to smoldering fires than ionization type alarms.

Escape times in this study were systematically shorter than those found in a similar study conducted in the 1970's. This is related to some combination of faster fire development times for today's products that provide the main fuel sources for fires, such as upholstered furniture and mattresses, different criteria for time to untenable conditions, and improved understanding of the speed and range of threats to tenability.

\section{Keywords}

detector sensitivity; fire tests; heat alarms; ionization alarms; photoelectric alarms; building fires; residential buildings; smoke alarms 


\section{Revision History}

Since its initial publication in 2004, NIST TN 1455 has been scrutinized by fire research professionals and several inconsistencies have been identified. Analysis of noted inconsistencies has led to the identification of a number of errors in data and computations that impact the alarm performance assessment. These errors did not impact the major conclusions of the study. A description of each error and the corrective action taken for NIST Technical Note 1455-1 is given below.

- Table 27 has been changed to reflect a correction of a rounding error and to add a footnote for the best-case scenario. (July 2004) ${ }^{1}$

- In the original report, the method NIST used to determine the detection time in the smoldering fire involving the 2-story house was in error. The results of the smoldering mattress test (test SDC 21) should not have been included in the analysis since the fire development did not allow enough alarms to respond by the time the test concluded. In addition, test SDC 14 was inadvertently excluded from the original analysis. Tables 11, $12,23,24,27,28,30$, and 32 were revised to correct these errors. (March 2006)

- Section 1.3.10 was corrected to define the format for the test data as comma-separated spreadsheet file. (November 2007).

- Table 11 was corrected to note that test SDC09 was conducted with the bedroom door closed. (November 2007)

- Appendix A Calculation of Times to Untenable Conditions. From test SDC30 to SDC41, the carbon monoxide (GASC_1) and carbon dioxide (GASC_3) data columns were switched for both ISO FED and NIST FED calculations. The corrective action was to switch the data columns to the proper position and recalculate the gas tenability values. Since tenability times for these tests are based on smoke obscuration (which occurred prior to reaching the gas tenability limit), no change in the report text resulted from this correction. (November 2007)

- Appendix A Calculated Alarm Times. From test SDC01 though SDC15, two sets of dual photo/ion alarms were not functioning properly and did not transmit alarms to the data acquisition system. They were subsequently coded as present but never reaching alarm. This had the effect of assignment of the end of test as a surrogate alarm time for each of the non-functioning alarms. The corrective action taken was the removal of all

\footnotetext{
${ }^{1}$ Dates in parentheses indicate when the report was revised to correct the noted error.
} 
non-functioning dual photo/ion alarms from the Appendix A Calculated Alarm Times spreadsheets. Tables 13, 15, 21, 23, 24, 27, and 28 were revised. (November 2007)

- Gas analyzer concentrations. NIST Report of Test 4016, October 2001 and NIST Report of Test 4017, May 2002. Several carbon monoxide and carbon dioxide gas analyzers exhibited uncompensated baseline drifts that significantly impacted the gas species tenability limit calculations. The largest errors where observed in the first manufactured home test series (SDC01 -SDC15). The corrective action taken was to apply a baseline correction to all gas analyzers such that at the start of a test, carbon monoxide analyzers read zero, carbon dioxide read between 0.04 to 0.06 volume percent, and oxygen analyzers read 20.95 volume percent. After baseline adjustments, the times to untenable conditions in Appendix A were re-calculated. Tables 14, 27, and 28 were revised based on the re-calculated tenability times. (November 2007)

- $\quad$ Excessive gas analyzer noise - The following carbon monoxide gas analyzer data were excessively noisy indicating sampling or instrumentation malfunction:

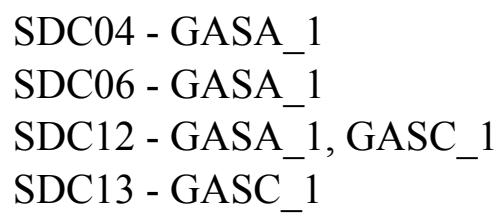

The corrective action taken was that all of the excessively noisy gas data were not used in tenability calculations, thus the assessment of tenable conditions at these sampling locations is incomplete. Fortunately, there was always a gas sampling point closer to the fire that would be the likely location where the gas tenability limit would be reached first. A single spurious data point was filtered out from SDC04 - GASC_1. Since tenability limits for these tests are based on smoke obscuration (which occurred prior to reaching the gas tenability limit), no change in the report text resulted from this correction.

(November 2007)

- $\quad$ Smoke optical density meter drift - NIST Report of Test 4016, October 2001 and NIST Report of Test 4017, May 2002. The following smoke meter data were removed because of output signal drift that made the optical density measurement unreliable.

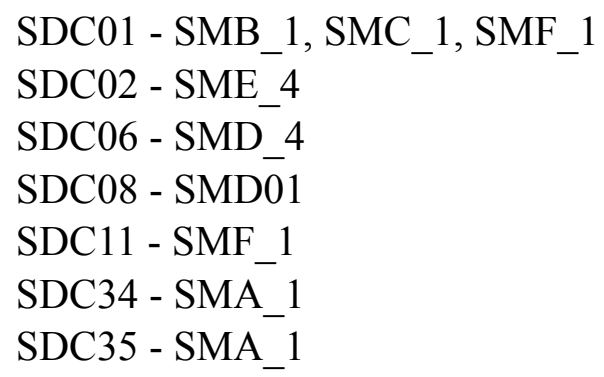




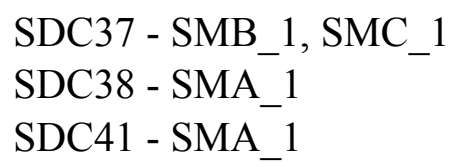

Only smoke meters SME_4 and SMD_4 affect the time to untenable conditions.

The corrective action taken was to re-calculate Appendix A Calculation of Times to Untenable Conditions. Tables 21, 27 and 28 were revised based on the re-calculated tenability times. (November 2007)

- The following smoke alarms were not functioning:

$$
\begin{aligned}
& \text { SDC09 - Pho1_B } \\
& \text { SDC11 - Pho1_D } \\
& \text { SDC36 - Ion1_C } \\
& \text { SDC37 - Ion_1C } \\
& \text { SDC38 - Ion_1C } \\
& \text { SDC39 - Ion_1C } \\
& \text { SDC41 - Ion1_D }
\end{aligned}
$$

This had the effect of assignment of the end of test as a surrogate alarm time for each of the non-functioning alarms. The corrective action taken was to remove these alarms from Appendix A Calculated Alarm Times. Tables 21, 23, 27, and 28 were revised based on the re-calculated tenability times. (November 2007)

- $\quad$ Appendix A Calculated Alarm Times. For tests SDC30, SDC31, SDC34, SDC35, and SDC41 location (E) was mislabeled as a bedroom. It should have been labeled as the living room. The corrective action taken was the proper label was assigned. Average time to alarm, and available egress times were updated and the values reported in tables 23 and 27 were corrected. (November 2007) 


\section{Contents}

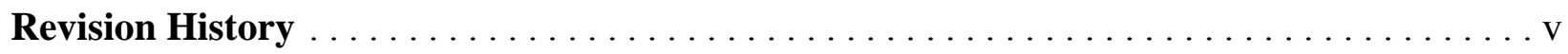

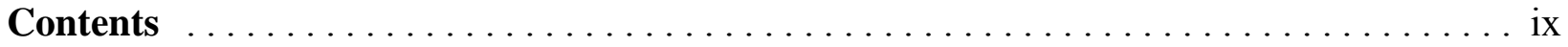

Figures and Tables $\ldots \ldots \ldots \ldots \ldots \ldots \ldots \ldots \ldots \ldots \ldots \ldots \ldots \ldots \ldots \ldots \ldots \ldots \ldots$

Executive Summary $\ldots \ldots \ldots \ldots \ldots \ldots \ldots \ldots \ldots \ldots \ldots \ldots \ldots \ldots \ldots \ldots$ xiii

Acknowledgments $\ldots \ldots \ldots \ldots \ldots \ldots \ldots \ldots \ldots \ldots \ldots \ldots \ldots \ldots \ldots \ldots \ldots \ldots \ldots$

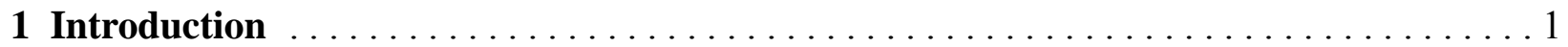

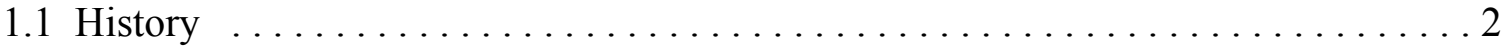

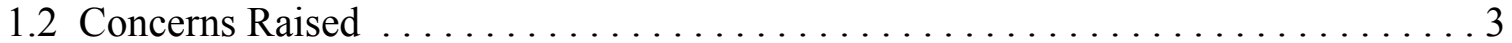

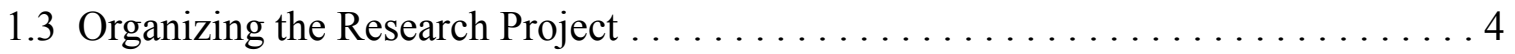

1.3.1 Evaluate the Performance of Current Smoke Alarm Technology . . . . . . . 4

1.3.2 Test Conditions Representative of Current Fatal Residential Fires . . . . . . 5

1.3.3 Evaluate the Efficacy of Current Requirements for Number and Location of

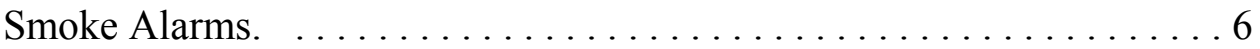

1.3.4 Develop Standard Nuisance Alarm Sources to Be Included in the Test

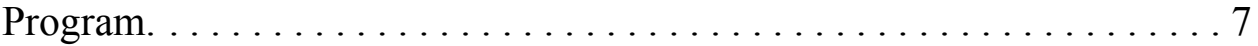

1.3.5 Examine Other Fire Detection Technologies in Combination with Smoke

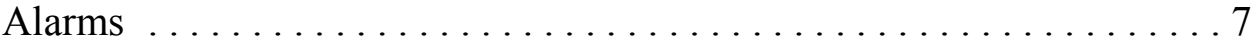

1.3.6 Obtain Data on the Potential for Improvements in Performance by New

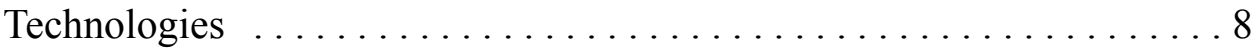

1.3.7 Include Fuel Items That Incorporate Materials and Constructions Representative of Current Residential Furnishings . . . . . . . . 8

1.3.8 Fully Characterize Test Detectors and Alarms in a Consistent Manner to Facilitate Comparisons $\ldots \ldots \ldots \ldots \ldots \ldots$

1.3.9 Utilize Fire Models to Extend the Applicability of the Test Arrangements and Maximize the Test Instrumentation $\ldots \ldots \ldots \ldots \ldots \ldots$

1.3.10 Make All of the Data Collected as Widely Accessible as Possible . . . . . 8

1.3.11 Provide Opportunities to Enhance Public Fire Safety Education . . . . . . 9

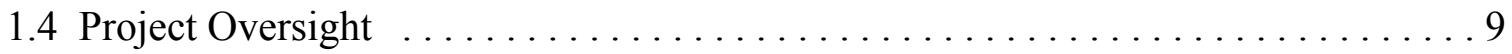

2 Residential Fire Alarms, Sensor Response and Calibration in the FE/DE . . . . . . 11

2.1 Residential Alarms Included in the Study $\ldots \ldots \ldots \ldots \ldots \ldots \ldots \ldots \ldots \ldots$ 


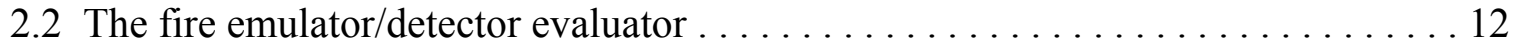

2.3 Smoke Aerosols . . ..................................... 14

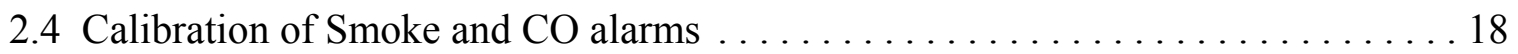

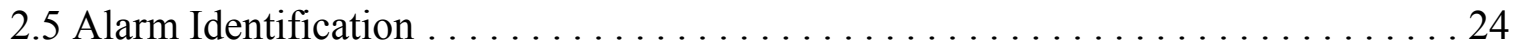

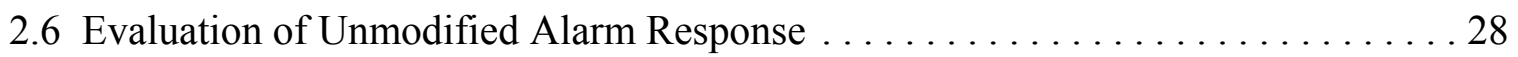

2.7 Effect of Sensor Board Location on Response . . . . . . . . . . . . . . . 47

2.8 Thresholds for Modified Alarms . . . . . . . . . . . . . . . . . . . . . 62

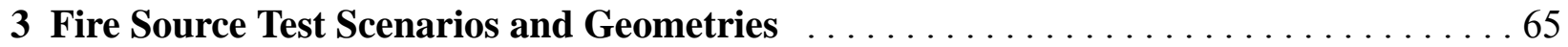

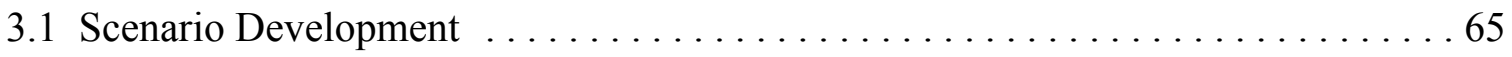

3.2 Material Selection .................................... 67

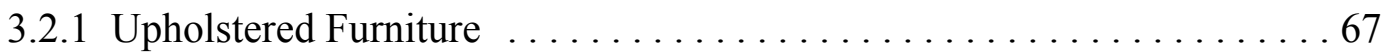

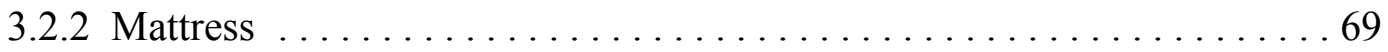

3.2.3 Cooking Materials ..............................69 69

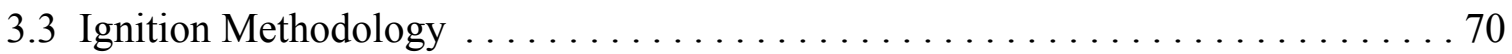

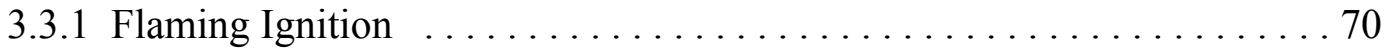

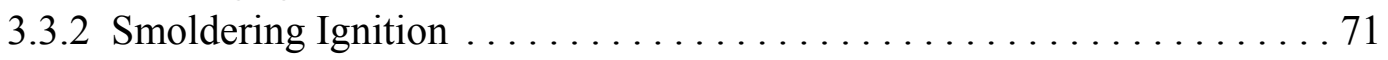

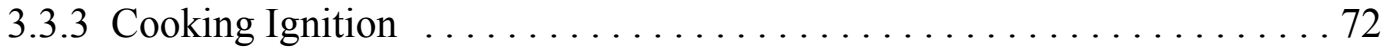

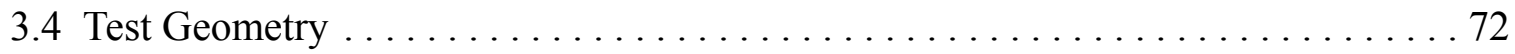

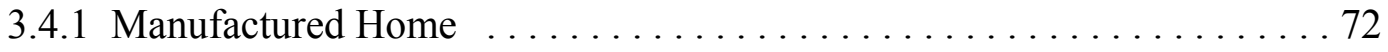

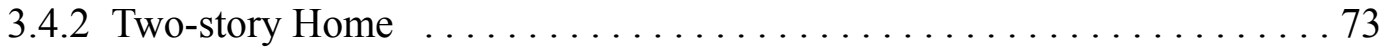

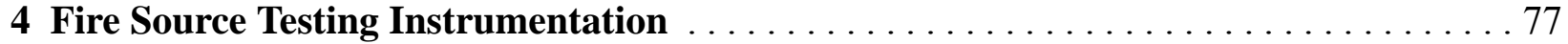

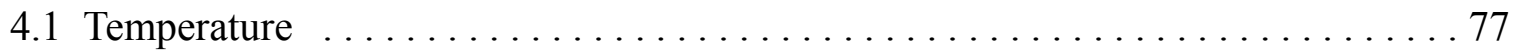

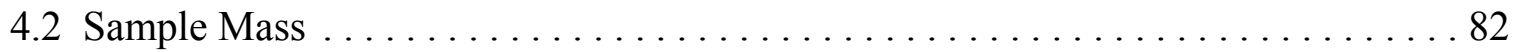

4.3 Primary Gases $-\mathrm{CO}, \mathrm{CO}_{2}$, and $\mathrm{O}_{2} \ldots \ldots \ldots \ldots \ldots \ldots \ldots \ldots \ldots \ldots \ldots \ldots$

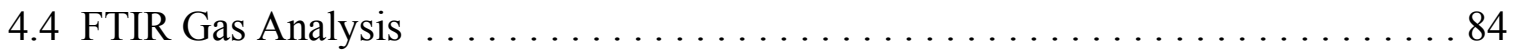

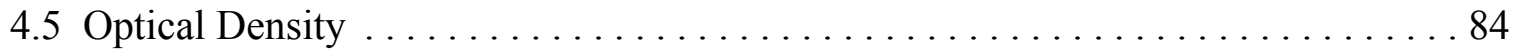

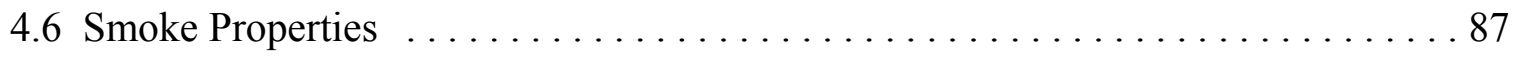

4.7 Smoke and CO Alarm Response $\ldots \ldots \ldots \ldots \ldots \ldots \ldots \ldots \ldots \ldots \ldots \ldots . \ldots \ldots \ldots$

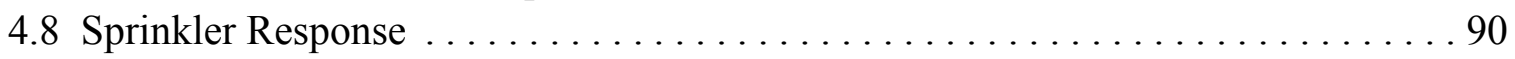

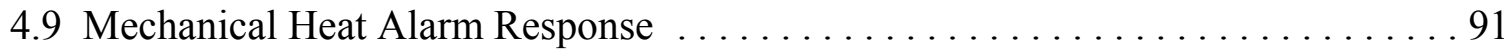

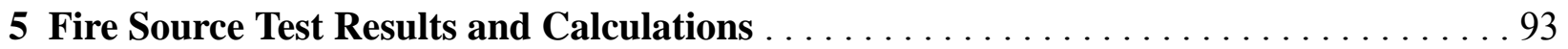

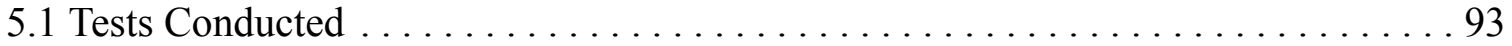

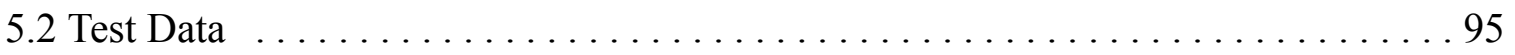

5.3 Calculation of Alarm Times . . . . . . . . . . . . . . . . . . . . . . 104

5.4 Calculation of Time to Untenable Conditions . . . . . . . . . . . . . . . 119

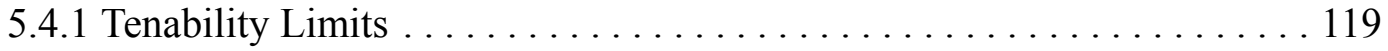

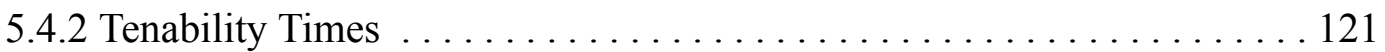

5.5 Assessment of Overall Alarm Performance . . . . . . . . . . . . . . . 122

5.6 Aerosol Concentration and Size Measurements ..................... 124 


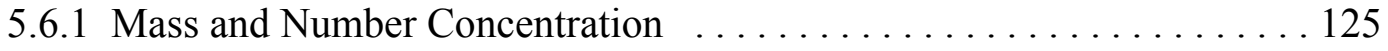

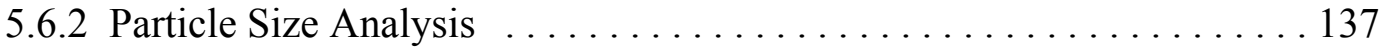

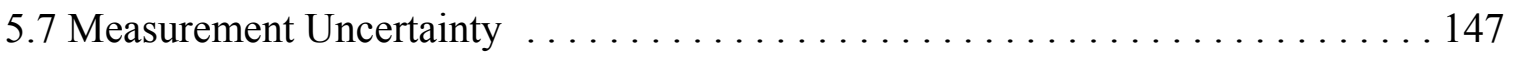

6 Residential Smoke Alarm Nuisance Source Testing . . . . . . . . . . . . . . . . . . 149

6.1 Nuisance Scenario Tests . . . . . . . . . . . . . . . . . . . . . . . 150

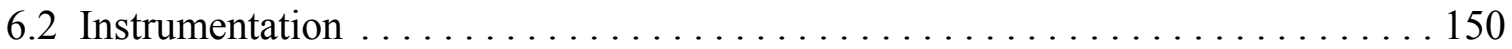

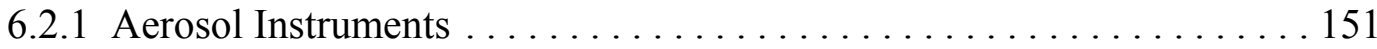

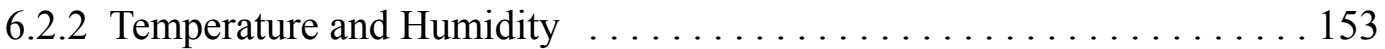

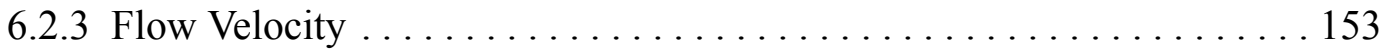

6.2.4 Analog Output Photoelectric, Ionization and Sensors ............. 154

6.3 Results . . . . . . . . . . . . . . . . . . . . . . . . . . . . 155

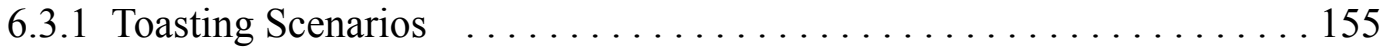

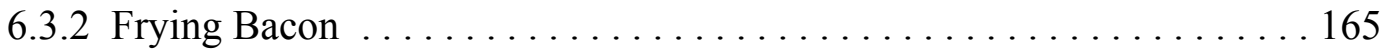

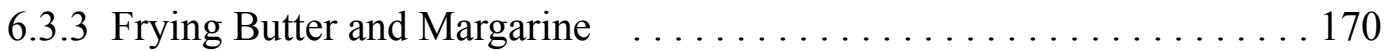

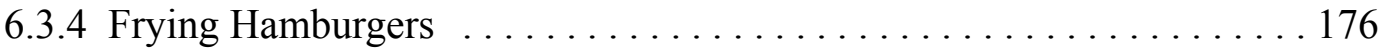

6.3.5 Deep-frying Tortillas and French-fried Potatoes ............. 180

6.3.6 Broiled and Baked/Broiled Pizza . . . . . . . . . . . . . . . . . . . . . 183

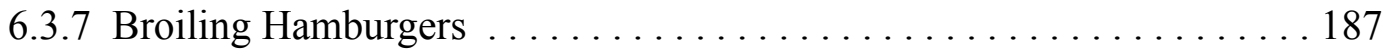

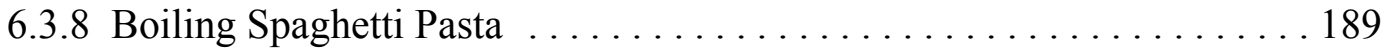

6.3.9 Candle Burning .................................. 193

6.3.10 Cigarette Smoking .............................. 194

6.4 Controlled Incipient Fire Sources . . . . . . . . . . . . . . . . . . . . . . . . . . . 194

6.4.1 Cotton Smolder ................................... 195

6.4.2 Wood Smolder ................................... 199

6.4 .3 Polyurethane Foam Smolder ........................... 201

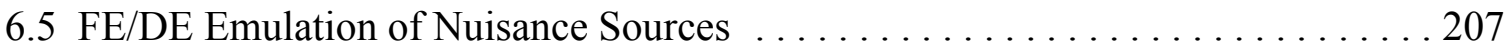

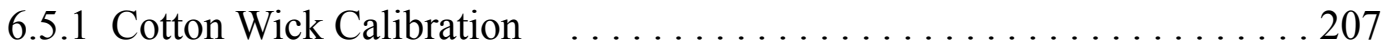

6.5.2 Cotton Smolder Smoke Fire Scenario ..................... 211

6.5.3 Wood Smolder Smoke Fire Scenario . . . . . . . . . . . . . . . . . . . . 211

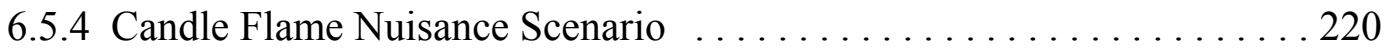

6.5.5 Heated Margarine or Butter Nuisance Scenario .............. 221

6.5.6 Toasting Bread Nuisance Scenario $\ldots \ldots \ldots \ldots \ldots \ldots \ldots \ldots . \ldots . \ldots . \ldots 21$

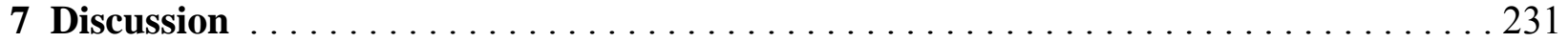

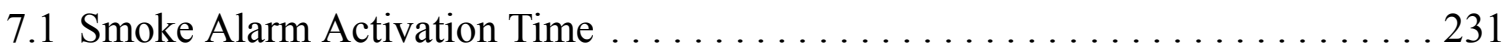

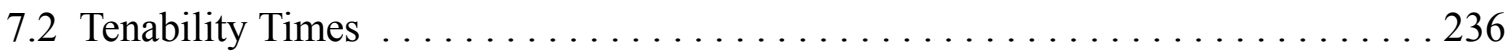

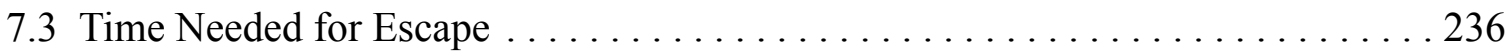

7.3.1 Movement Speed ............................... 237

7.3.2 Premovement Activities ........................... 237

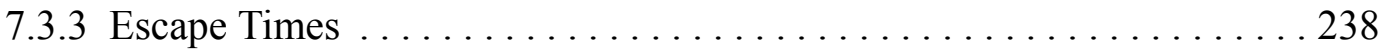

7.4 Smoke Alarm Performance .................................... 240 


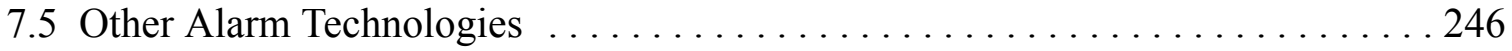

7.5.1 Carbon Monoxide Alarms . . . . . . . . . . . . . . . . . . . . 247

7.5 .2 Heat Alarms . . . . . . . . . . . . . . . . . . 247

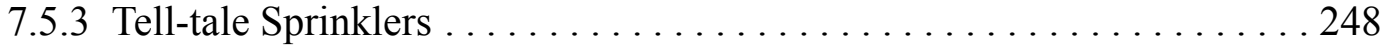

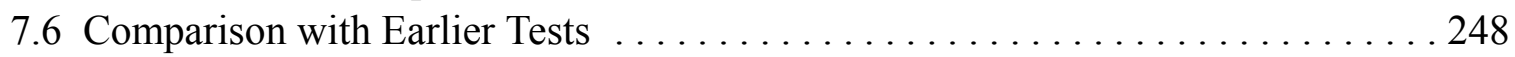

7.7 Nuisance Alarms . . . . . . . . . . . . . . . . . . . . . 251

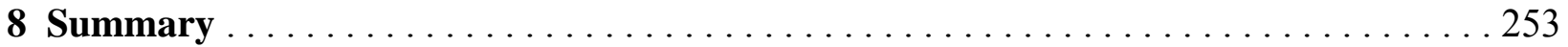

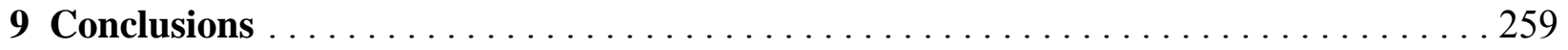

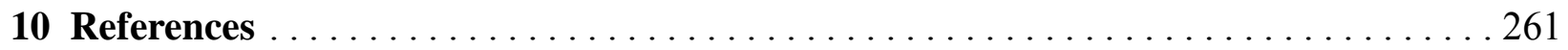

Appendix A: Alarm Activation and Time to Untenable Conditions During Tests of Residential Smoke Alarms Included in the Study $\ldots \ldots \ldots \ldots \ldots \ldots \ldots \ldots \ldots \ldots \ldots \ldots$

Appendix B: FTIR Gas Measurement in Home Smoke Alarm Tests . . . . . . . . . . . B-1 


\section{Figures and Tables}

\section{Figures}

Figure 1. Sample sensor test board used during tests of residential smoke alarms $\ldots \ldots \ldots 11$

Figure 2. Schematic of the FE/DE (all units in $\mathrm{cm}$ ) $\ldots \ldots \ldots \ldots \ldots \ldots \ldots \ldots \ldots \ldots$

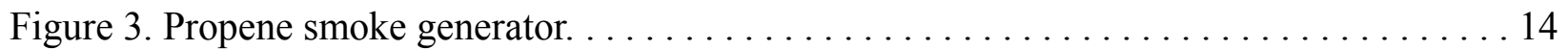

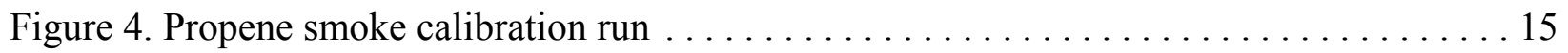

Figure 5. Smolder source, staged wick ignition device $\ldots \ldots \ldots \ldots \ldots \ldots \ldots \ldots \ldots$

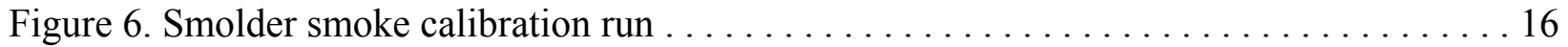

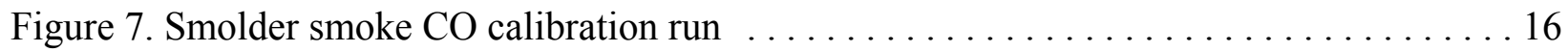

Figure 8. UL grey smoke (smolder smoke) sensitivity limits and FE/DE data . . . . . . . 17

Figure 9. UL black smoke sensitivity limits and corresponding FE/DE data . . . . . . . . 17

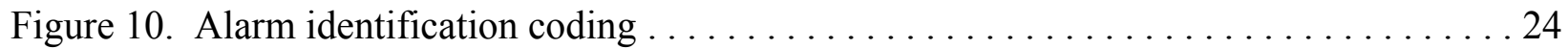

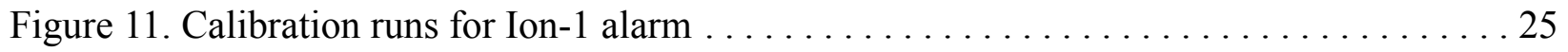

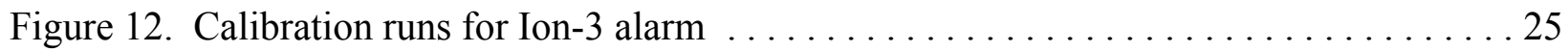

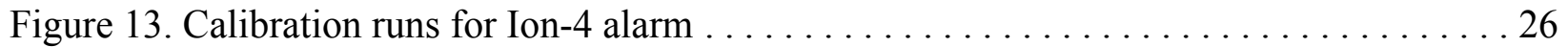

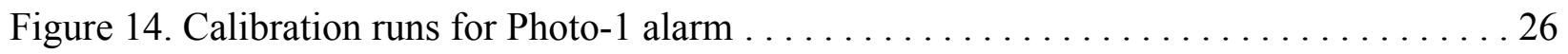

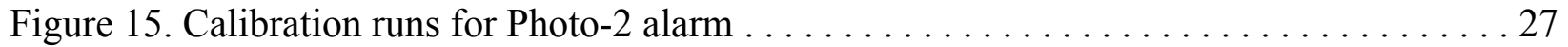

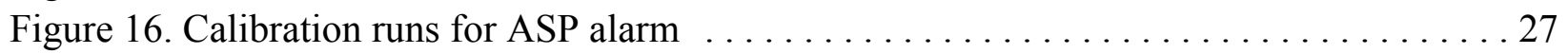

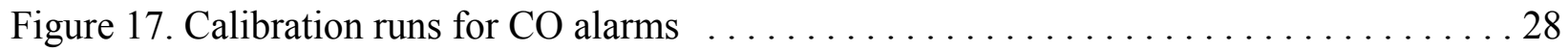

Figure 18. Photo-1, eight wicks -12 s delay, Disp 1 and Disp 7 alarms . . . . . . . . 30

Figure 19. Photo-1, 16 wicks -12 s delay, Disp 1 and Disp 7 alarms . . . . . . . . 30

Figure 20. Photo-1, eight wicks -12 s delay, Disp 1 and Disp 7 alarms $\ldots \ldots \ldots \ldots 1$

Figure 21. Photo-1, eight wicks -30 s delay, Disp 4 and Disp 10 alarms . . . . . . . . 31

Figure 22. Photo-1, eight wicks - 12 s delay, Disp 37 and Disp 40 alarms . . . . . . . . 32

Figure 23. Photo-1, eight wicks, 12 s delay, Disp 46 and Disp 55 alarms . . . . . . . 32

Figure 24. Photo-1, eight wicks - 12 s delay, Disp 52 and Disp 58 alarms . . . . . . 33

Figure 25. Photo-1, eight wicks - 12 s delay, Disp 61 and Disp 64 alarms . . . . . . . 33

Figure 26. Photo-1, eight wicks - 12 s delay, Disp 10 and Disp 55 alarms . . . . . . . 34

Figure 27. Photo-1, 16 wicks -12 s delay, Disp 10 and Disp 55 alarms . . . . . . . . 34

Figure 28. Ion-1, three wicks - 30 s delay, Disp 11 and Disp 38 alarms . . . . . . . . . 36

Figure 29. Ion-1, four wicks - $30 \mathrm{~s}$ delay, Disp 11 and Disp 38 alarms . . . . . . . . . . 36

Figure 30. Ion-1, four wicks - $30 \mathrm{~s}$ delay, Disp 2 and Disp 5 alarms $\ldots \ldots \ldots \ldots \ldots$

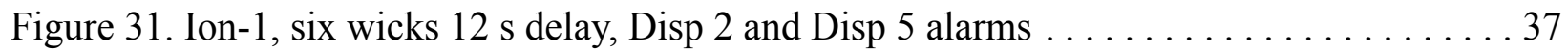

Figure 32. Ion-1, four wicks 30 s delay, Disp 8 and Disp 44 alarms . . . . . . . . . . . 38

Figure 33. Ion-1, six wicks 12 s delay, Disp 8 and Disp 44 alarms . . . . . . . . . . 38

Figure 34. Ion-1, six wicks 12 s delay, Disp 41 and Disp 51 alarms . . . . . . . . . . . . 39

Figure 35. Ion-1, three wicks 12 s delay, Disp 53 and Disp 44 alarms . . . . . . . . . . . 39

Figure 36. Ion-1, six wicks 12 s delay, Disp 60 and Disp 63 alarms . . . . . . . . . . 40 
Figure 37. Ion-1, six wicks 12 s delay, Disp 57 and Disp 60 alarms . . . . . . . . . . 40

Figure 38. Ion-3, six wicks - 12 s delay, Disp 39 and Disp 48 alarms . . . . . . . . . . 41

Figure 39. Ion-3, four wicks - 30 s delay, Disp 39 and Disp 48 alarms . . . . . . . . . 41

Figure 40. Ion-3, three wicks 30 s delay, Disp 39 and Disp 48 alarms . . . . . . . . . . . 42

Figure 41. Ion-3, three wicks $30 \mathrm{~s}$ delay, Disp 39 and Disp 48 alarms . . . . . . . . . 42

Figure 42. Ion-3, three wicks $30 \mathrm{~s}$ delay, Disp 3 and Disp 6 alarms $\ldots \ldots \ldots \ldots \ldots$

Figure 43. Ion-3, three wicks $30 \mathrm{~s}$ delay, Disp 9 and Disp 12 alarms . . . . . . . . . 43

Figure 44. Ion-3, three wicks $30 \mathrm{~s}$ delay, Disp 30 and Disp 45 alarms . . . . . . . . . . 44

Figure 45. Ion-3, three wicks 30 s delay, Disp 54 and Disp 56 alarms . . . . . . . . . . . 44

Figure 46. Ion-3, three wicks $30 \mathrm{~s}$ delay, Disp 59 and Disp 62 alarms . . . . . . . . . . 45

Figure 47. Ion-3, three wicks $30 \mathrm{~s}$ delay, Disp 53 and Disp 50 alarms . . . . . . . . . . 45

Figure 48. UL grey smoke (smolder smoke) sensitivity limits and FE/DE data . . . . . . . 46

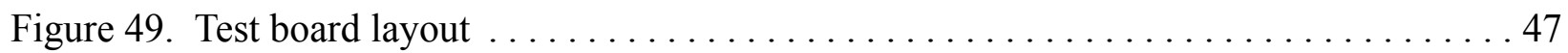

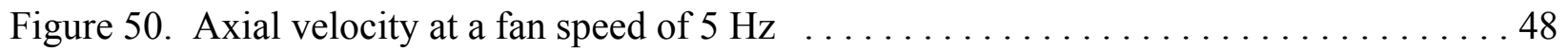

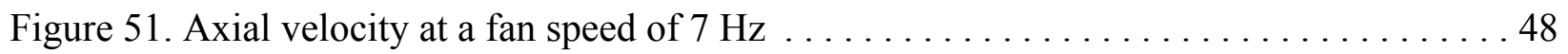

Figure 52 . Axial velocity at a fan speed of $12 \mathrm{~Hz} \ldots \ldots \ldots \ldots \ldots \ldots \ldots \ldots$

Figure 53. Ion-1 response, fan speed $5 \mathrm{~Hz}$ (see table 4$) \ldots \ldots \ldots \ldots \ldots \ldots \ldots \ldots \ldots \ldots$

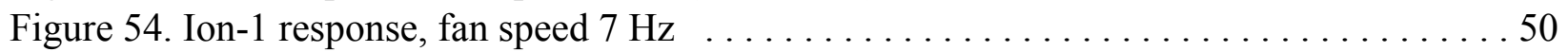

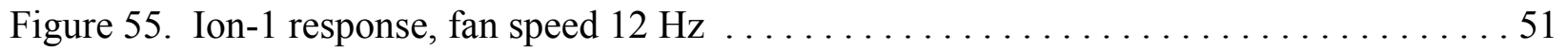

Figure 56. Ion-1 response, fan speed $5 \mathrm{~Hz}$ - repeat test $\ldots \ldots \ldots \ldots \ldots \ldots \ldots \ldots \ldots \ldots$

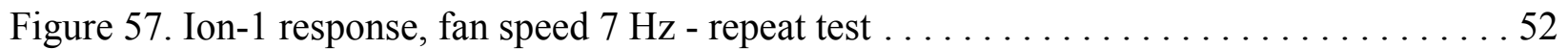

Figure 58 . Ion-1 response, fan speed $12 \mathrm{~Hz}$ - repeat test . . . . . . . . . . . . . . 52

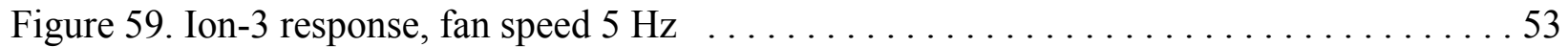

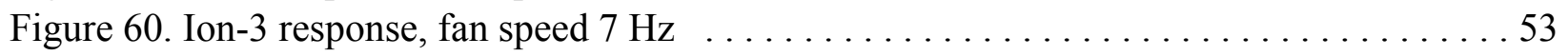

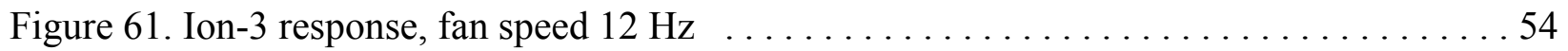

Figure 62 . Ion-3 response, fan speed $5 \mathrm{~Hz}$ - repeat test $\ldots \ldots \ldots \ldots \ldots \ldots \ldots \ldots \ldots$

Figure 63 . Ion-3 response, fan speed $7 \mathrm{~Hz}$ - repeat test $\ldots \ldots \ldots \ldots \ldots \ldots \ldots \ldots$

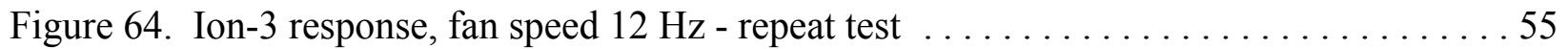

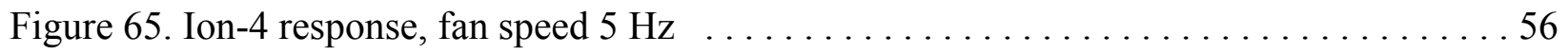

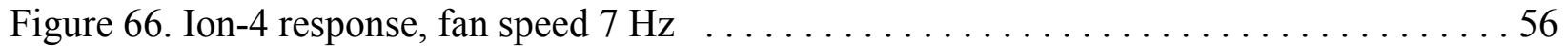

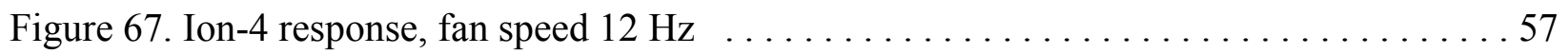

Figure 68 . Ion-4 response, fan speed $5 \mathrm{~Hz}$ - repeat test $\ldots \ldots \ldots \ldots \ldots \ldots \ldots \ldots \ldots$

Figure 69. Ion-4 response, fan speed $7 \mathrm{~Hz}$ - repeat test $\ldots \ldots \ldots \ldots \ldots \ldots \ldots \ldots$

Figure 70. Ion-4 response, fan speed $12 \mathrm{~Hz}$ - repeat test $\ldots \ldots \ldots \ldots \ldots \ldots \ldots$

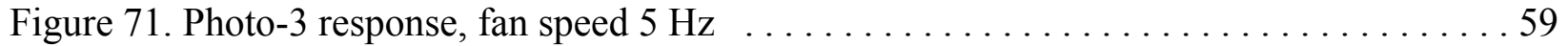

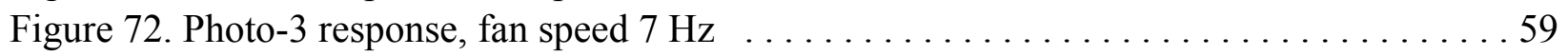

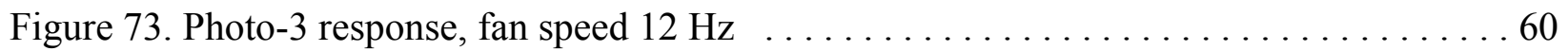

Figure 74. Photo-3 response, fan speed $5 \mathrm{~Hz}-$ repeat test $\ldots \ldots \ldots \ldots \ldots \ldots \ldots \ldots$

Figure 75. Photo-3 response, fan speed $7 \mathrm{~Hz}$ - repeat test $\ldots \ldots \ldots \ldots \ldots \ldots \ldots \ldots$

Figure 76. Photo-3 response, fan speed $7 \mathrm{~Hz}$ - repeat test . . . . . . . . . . . . . 61

Figure 77. Measurements of upholstered chair used for smoldering experiments . . . . . . 67

Figure 78. Upholstered chair used for smoldering experiments $\ldots \ldots \ldots \ldots \ldots \ldots \ldots$ 
Figure 79. Measurements of upholstered chair used for flaming experiments $\ldots \ldots \ldots 68$

Figure 80 . Upholstered chair used for flaming experiments $\ldots \ldots \ldots \ldots \ldots \ldots \ldots$

Figure 81. Mattress used for smoldering and flaming experiments . . . . . . . . . . 69

Figure 82. Corn oil used for kitchen cooking materials fire experiments . . . . . . . . . . . . 69

Figure 83. "Electric match" used for flaming ignitions $\ldots \ldots \ldots \ldots \ldots \ldots \ldots \ldots \ldots \ldots$

Figure 84. Location of "electric match" ignition source for flaming upholstered chair

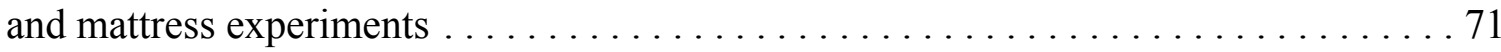

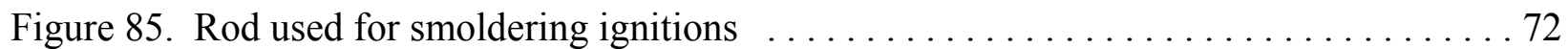

Figure 86 . Heating ignition source with cooking oil $\ldots \ldots \ldots \ldots \ldots \ldots \ldots \ldots \ldots$

Figure 87. Layout of manufactured home used for test of smoke alarm performance . . . . . 74

Figure 88. Layout of first floor of two-story home used for tests of smoke alarm performance 75

Figure 89. Layout of second floor of two-story home used for tests of smoke alarm

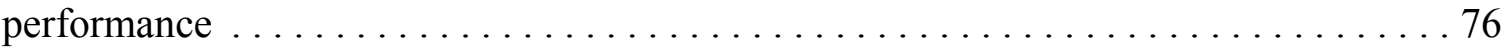

Figure 90. Sample instrumentation showing relative locations of temperature, primary gas analysis, smoke obscuration, and smoke alarm response measurements $\ldots \ldots \ldots 77$

Figure 91. Instrumentation in manufactured home during tests of smoke alarm performance . . 79 Figure 92. Instrumentation in first floor of two-story home during tests of smoke alarm

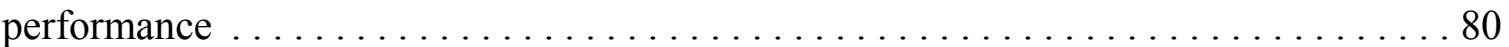

Figure 93. Instrumentation in second floor of two-story home during tests of smoke alarm

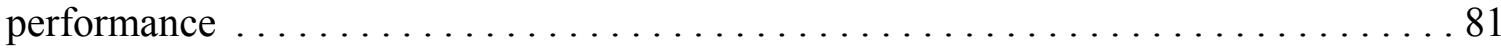

Figure 94. Example of mass loss measurement showing upholstered chair on load cell prior to one of the flaming ignition tests and load cell apparatus mounted beneath manufactured

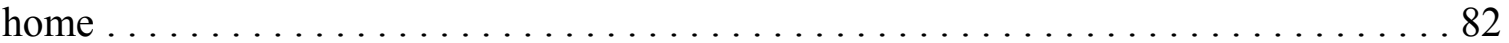

Figure 95. Optical density instrument designs . . . . . . . . . . . . . . . . . 84

Figure 96. Smoke properties measurement showing collection lines (foreground) and smoke

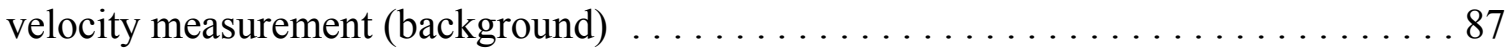

Figure 97. Calibration of residential ionization chamber $\ldots \ldots \ldots \ldots \ldots \ldots \ldots \ldots$

Figure 98. Measurement station showing several alarms arranged for testing . . . . . . . . 89 Figure 99. Measurement station showing unmodified smoke alarms arranged for testing . . . 91 Figure 100. Mass loss of burning mattress during flaming ignition test of a mattress, test SDC05 (Note that the mass loss is averaged to minimize noise in the data. Thus, peak mass loss and initiation of suppression do not exactly coincide) $\ldots \ldots \ldots \ldots \ldots \ldots$

Figure 101. Gas temperatures from ceiling to floor in remote bedroom during a flaming ignition

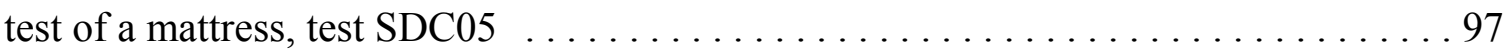

Figure 102. Gas temperatures from ceiling to floor in main bedroom (ignition location) during a

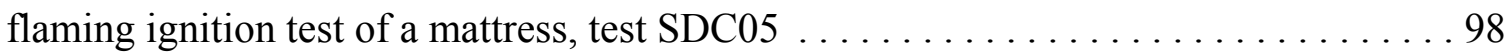

Figure 103. Gas temperatures from ceiling to floor in hallway outside remote bedroom in a flaming ignition test of a mattress, test SDC05 . . . . . . . . . . . . 99

Figure 104. Gas temperatures from ceiling to floor in hallway outside main bedroom in a

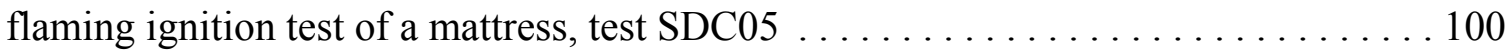

Figure 105. Gas temperatures from ceiling to floor in living area during a flaming ignition test of

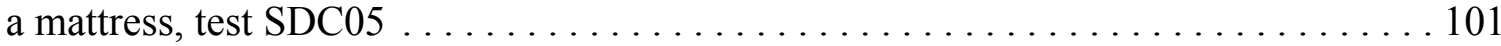


Figure 106. Gas temperatures from ceiling to floor in front door hallway during a flaming ignition test of a mattress, test SDC05 ....................... 102

Figure 107. Gas temperature in closed bedroom during a flaming ignition test of a mattress,

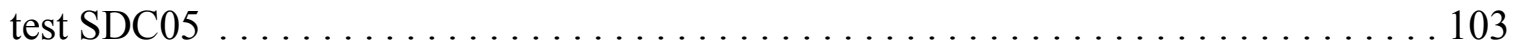

Figure 108. Smoke obscuration at $20 \mathrm{~mm}$ and $900 \mathrm{~mm}$ below ceiling in remote bedroom during flaming ignition test of a mattress, test SDC05 .................. 105

Figure 109. Smoke obscuration at $20 \mathrm{~mm}$ and $900 \mathrm{~mm}$ below ceiling in main bedroom (ignition location) during flaming ignition test of a mattress, test SDC05 .......... 106

Figure 110. Smoke obscuration at $20 \mathrm{~mm}$ and $900 \mathrm{~mm}$ below ceiling in hallway outside remote bedroom during flaming ignition test of a mattress, test SDC05 ........... 107

Figure 111. Smoke obscuration at $20 \mathrm{~mm}$ and $900 \mathrm{~mm}$ below ceiling in hallway outside main bedroom during flaming ignition test of a mattress, test SDC05 .......... 108

Figure 112. Smoke obscuration at $20 \mathrm{~mm}$ and $900 \mathrm{~mm}$ below ceiling in living area during flaming ignition test of a mattress, test SDC05 .................... 109

Figure 113. Smoke obscuration at $20 \mathrm{~mm}$ and $900 \mathrm{~mm}$ below ceiling in front door hallway during flaming ignition test of a mattress, test SDC05 ............... 110

Figure 114. Smoke obscuration at $1520 \mathrm{~mm}$ below ceiling in closed bedroom during flaming ignition test of a mattress, test SDC05 . . . . . . . . . . . . . . . . 111

Figure 115. Carbon monoxide concentration $900 \mathrm{~mm}$ below ceiling at four locations during a flaming ignition test of a mattress, test SDC05 . . . . . . . . . . . . . . 112

Figure 116. Carbon dioxide concentration $900 \mathrm{~mm}$ below ceiling at four locations during a flaming ignition test of a mattress, test SDC05 .................. 113

Figure 117. Oxygen concentration $900 \mathrm{~mm}$ below ceiling at four locations during a flaming

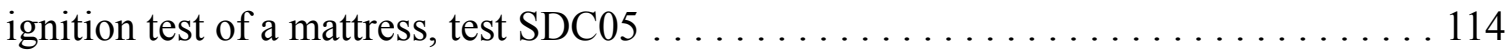

Figure 118. Measured output for several analog-modified smoke alarms in hallway outside main bedroom during a flaming ignition test of a mattress, test SDC05 $\ldots \ldots \ldots \ldots \ldots 115$

Figure 119. Measured output for several analog-modified carbon monoxide alarms in hallway outside main bedroom during a flaming ignition test of a mattress, test SDC05 . . 116

Figure 120. Measured output for a heat alarm in hallway outside main bedroom during a flaming

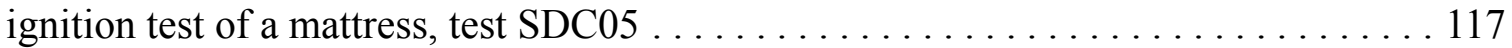

Figure 121. Measured output for a tell-tale residential sprinkler in hallway outside main bedroom during a flaming ignition test of a mattress, test SDC05 $\ldots \ldots \ldots \ldots 118$

Figure 122. Smoke properties for smoldering chair scenario SDC01 . . . . . . . . . . 127

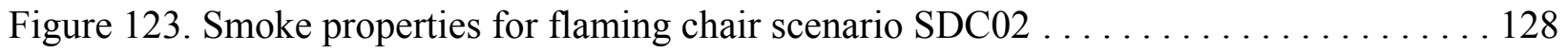

Figure 124. Smoke properties for smoldering mattress scenario SDC06 . . . . . . . . . . 129

Figure 125. Smoke properties for flaming mattress scenario SDC07 . . . . . . . . . . . 129

Figure 126. Smoke properties for smoldering mattress scenario SDC08 $\ldots \ldots \ldots \ldots \ldots 130$

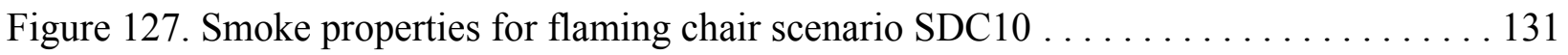

Figure 128. Smoke properties for smoldering chair scenario SDC11 . . . . . . . . . . 133

Figure 129. Smoke properties for cooking oil fire scenario SDC12 ............... 134

Figure 130. Smoke properties for cooking oil fire scenario SDC13 . .............. 134

Figure 131. Smoke properties for smoldering mattress with burn room door closed SDC14 . 135 


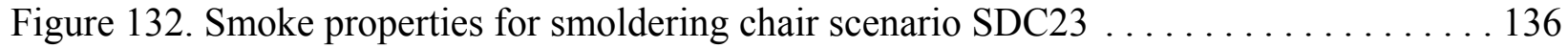

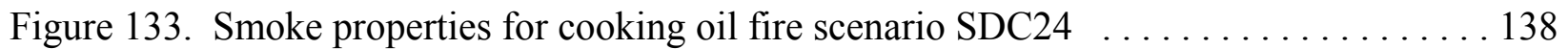

Figure 134. Smoke properties for flaming chair scenario SDC25 . . . . . . . . . . . . . 139

Figure 135. Smoke properties for flaming chair scenario SDC26 . . . . . . . . . . . . . . 139

Figure 136. Smoke properties for smoldering chair scenario with air conditioning SDC27 . . 140

Figure 137. Cascade impactor results for flaming chair scenario SDC02 . . . . . . . . 143

Figure 138. Cascade impactor results for flaming chair scenario SDC10 . . . . . . . . . 143

Figure 139. Cascade impactor results for cooking oil fire scenarios SDC12 and SDC13 . . . 144

Figure 140. Cascade impactor results for smoldering chair fire scenarios SDC23 and SDC27 145

Figure 141. Schematic of the manufactured home for nuisance testing $\ldots \ldots \ldots \ldots 151$

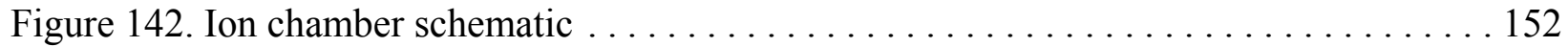

Figure 143. Response of the ion chamber and MIC to cotton smolder smoke $\ldots \ldots \ldots \ldots 152$

Figure 144. Thermocouple and sampling line placement $\ldots \ldots \ldots \ldots \ldots \ldots \ldots \ldots$

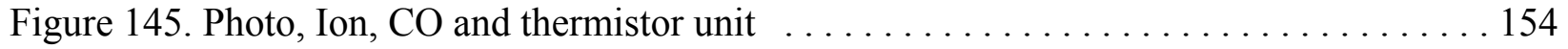

Figure 146. Velocity and speed components for toasting bread nuisance source

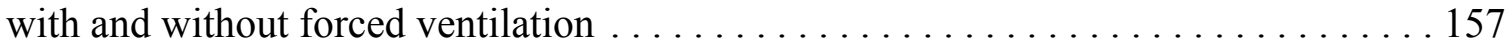

Figure 147. Toasting bread scenario - toaster on at $\mathrm{t}=0$, off at $240 \mathrm{~s}$. No forced flow $\ldots \ldots 158$

Figure 148. Toasting bread scenario - toaster on at $\mathrm{t}=0$, off at $255 \mathrm{~s}$.

No forced flow, remote bedroom window open $\ldots \ldots \ldots \ldots \ldots \ldots \ldots \ldots \ldots \ldots \ldots$

Figure 149. Toasting bread scenario - toaster on at $\mathrm{t}=0$, off at $255 \mathrm{~s}$. Room fan turned on . . . 161

Figure 150. Toasting bread scenario - toaster on at $\mathrm{t}=0$, off at $270 \mathrm{~s}$. Room fan turned on . . 162

Figure 151. Toasting frozen bagel scenario - toaster on at $\mathrm{t}=0$, off at $300 \mathrm{~s}$.

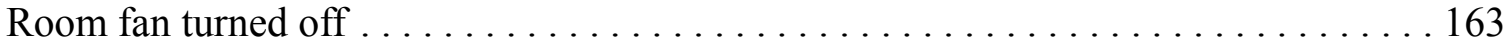

Figure 152. Toasting frozen bagel scenario - toaster on at $\mathrm{t}=0$, off at $300 \mathrm{~s}$.

Room fan turned on . . . . . . . . . . . . . . . . . . . . . . . 164

Figure 153. Frying bacon scenario - cast-iron pan, LPG hot plate, no forced flow, burner lit at

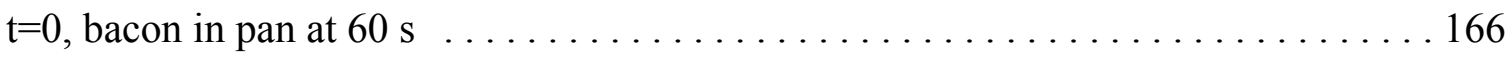

Figure 154. Frying bacon scenario - cast-iron pan, LPG hot plate, no forced flow, burner lit at

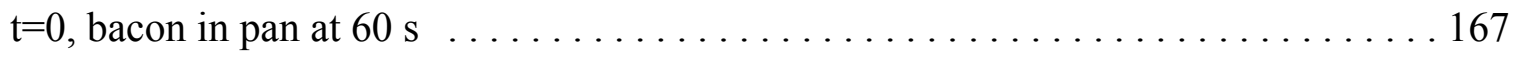

Figure 155. Frying bacon scenario - aluminum pan, electric range, no forced flow, bacon in pan

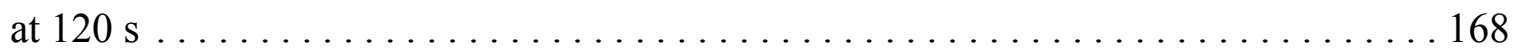

Figure 156. Frying bacon scenario - aluminum pan, electric range, floor fan on, bacon in pan at

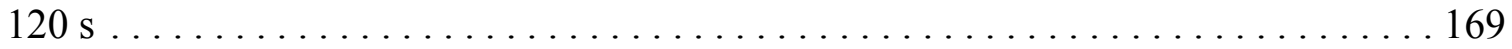

Figure 157. Frying butter scenario - aluminum pan, electric range, floor fan off, butter in pan at

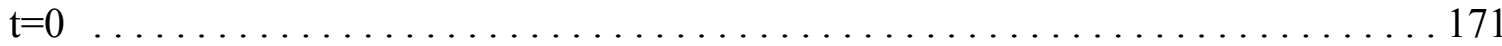

Figure 158. . Frying butter scenario - aluminum pan, electric range, floor fan off, butter in pan at

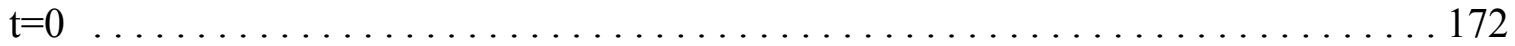

Figure 159. Frying butter scenario - cast iron pan, electric range, floor fan off, butter in pan at

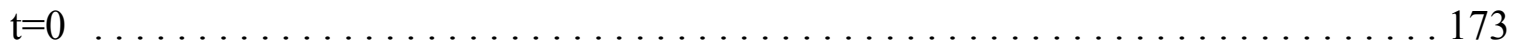

Figure 160. Frying butter scenario - cast iron pan, electric range, floor fan on, butter in pan at

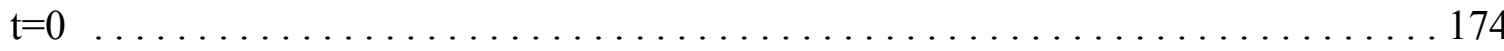


Figure 161. Frying margarine scenario - cast iron pan, electric range, floor fan off, margarine in

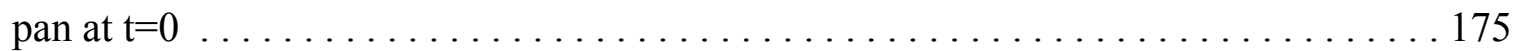

Figure 162. Frying hamburgers - three hamburgers in non-stick frying pan, floor fan off.

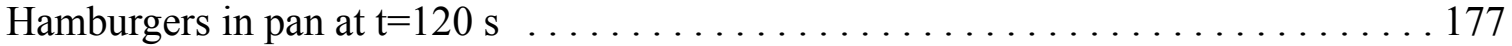

Figure 163. Frying hamburgers - three hamburgers in non-stick frying pan, floor fan on, living room window open. Hamburgers in pan at $\mathrm{t}=160 \mathrm{~s} \ldots \ldots \ldots \ldots \ldots \ldots \ldots$

Figure 164. Frying hamburgers - three hamburgers in non-stick frying pan, floor fan on.

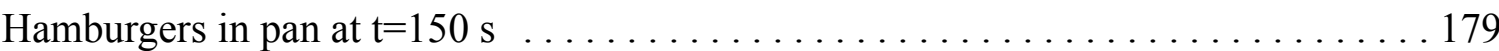

Figure 165. Deep-frying Tortillas scenario - steel wok, LPG hotplate, floor fan off, first tortillas

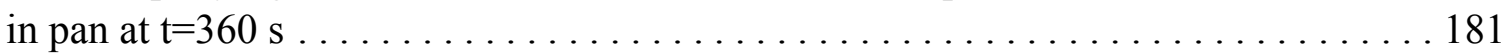

Figure 166. Deep-frying french fries scenario - steel wok, LPG hotplate, floor fan off, first

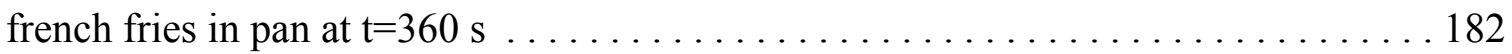

Figure 167. Broiling Pizza - one frozen pizza in oven set on broil, floor fan off.

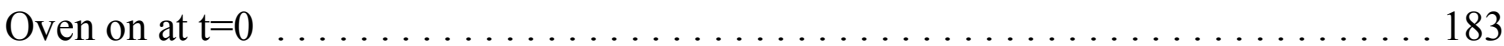

Figure 168. Bake/ broil pizza - one frozen pizza in oven set on bake and pre-heated to $350{ }^{\circ} \mathrm{F}$, then set to broil $630 \mathrm{~s}$ later. Floor fan was off. Pizza in at $\mathrm{t}=0 \ldots \ldots \ldots \ldots \ldots$

Figure 169. Bake/ broil pizza - one frozen pizza in oven set on bake and pre-heated to $350{ }^{\circ} \mathrm{F}$, then set to broil $630 \mathrm{~s}$ later. Floor fan was on. Pizza in at $\mathrm{t}=0 \ldots \ldots \ldots \ldots \ldots$

Figure 170. Broiling hamburgers - four hamburgers in oven set on broil, floor fan off. Oven on

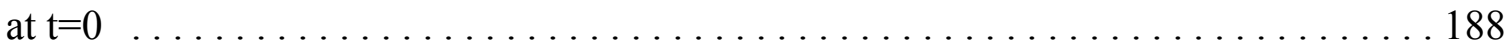

Figure 171. Broiling hamburgers - four hamburgers in oven set on broil, floor fan on. Oven on

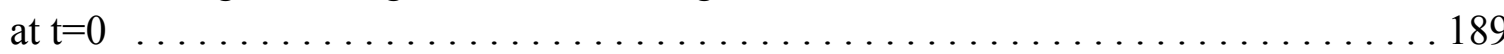

Figure 172. Boiling spaghetti pasta $-1 / 3$ package $(150 \mathrm{~g})$, floor fan off. Pasta placed in pot at

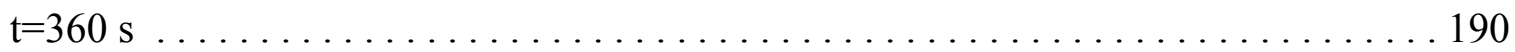

Figure173. Boiling spaghetti pasta $-2 / 3$ package $(300 \mathrm{~g})$, floor fan off. Pasta placed in pot at

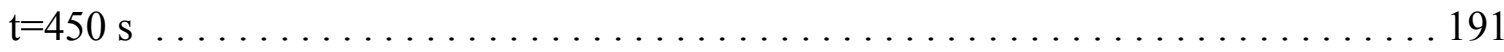

Figure 174. Boiling spaghetti pasta - 1/2 package (225 g), lid on, floor fan off. Pasta placed in

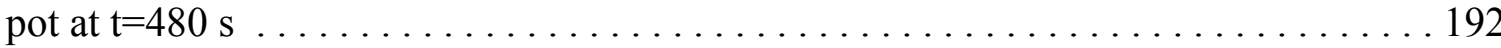

Figure 175. Candles burning - four tea candles burning on electric range top area. Floor fan was off. Candles lit at $\mathrm{t}=0 \ldots \ldots \ldots \ldots$

Figure 176. Cigarette smoking - two smokers smoking one cigarette each in kitchen area.

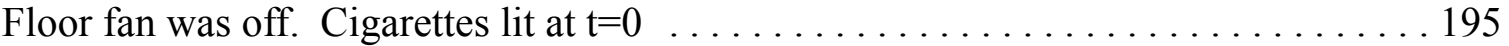

Figure 177. Cigarette smoking - two smokers smoking one cigarette each in kitchen area.

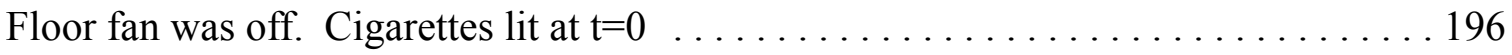

Figure 178. Smoldering cotton wicks - eight sets of four wicks were ignited with a $12 \mathrm{~s}$ delay using the staged wick ignition device, located on the living room floor at the chair burn location. Fan was off. Ignition sequence started at $\mathrm{t}=30 \mathrm{~s} \ldots \ldots \ldots \ldots \ldots \ldots$

Figure 179. Smoldering cotton wicks -eight sets of four wicks were ignited with a $12 \mathrm{~s}$ delay using the staged wick ignition device, located on the living room floor at the chair burn location. Fan was on. Ignition sequence started at $\mathrm{t}=30 \mathrm{~s} \ldots \ldots \ldots \ldots \ldots$ 
Figure 180. Smoldering wood blocks - eight beech wood blocks on an electric hotplate, located on the living room floor at the chair burn location. Fan was off. Power to hotplate on at

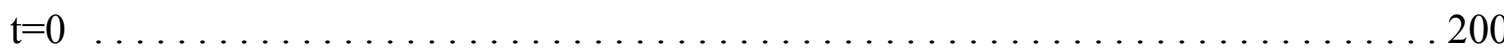

Figure 181. Smoldering wood blocks - eight beech wood blocks on an electric hotplate, located on the living room floor at the chair burn location. Fan was on. Power to hotplate on at $\mathrm{t}=0$

Figure 182. Smoldering foam - A polyurethane foam block, located on the living room floor at the chair burn location and ignited with an electrically powered nichrome wire heater imbedded in the block. Fan was off. Power to igniter on at $\mathrm{t}=0$. Sample flamed at $798 \mathrm{~s}$

Figure 183. Smoldering foam - a polyurethane foam block, located on the living room floor at the chair burn location and ignited with an electrically powered nichrome wire heater imbedded in the block. Fan was off. Power to igniter on at $\mathrm{t}=0$. Sample stopped

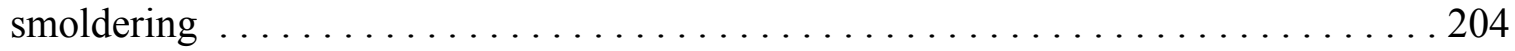

Figure 184. Smoldering foam - a polyurethane foam block, located on the living room floor at the chair burn location and ignited with an electrically powered nichrome wire heater imbedded in the block. Fan was on. Power to igniter on at $\mathrm{t}=0 \ldots \ldots \ldots \ldots \ldots 205$

Figure 185. Smoldering foam - a polyurethane foam block, located on the living room floor at the chair burn location and ignited with an electrically powered nichrome wire heater imbedded in the block. Fan was on. Power to igniter on at $\mathrm{t}=0 \ldots \ldots \ldots \ldots \ldots$

Figure 186. Photoelectric sensor response to cotton smolder smoke calibration test.

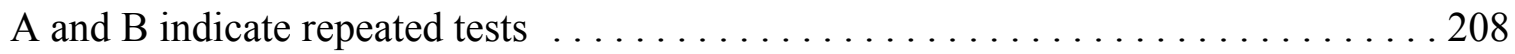

Figure 187. Ionization sensor response to cotton smolder smoke calibration test.

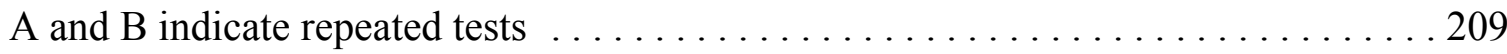

Figure 188. Electrochemical cell $\mathrm{CO}$ sensor response to cotton smolder smoke

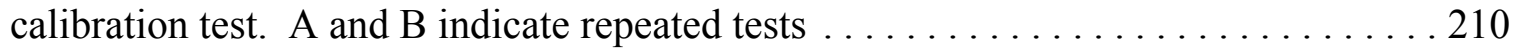

Figure 189. Smoldering cotton fire scenario, fan speed $7 \mathrm{~Hz} \ldots \ldots \ldots \ldots \ldots \ldots \ldots 212$

Figure 190. Smoldering cotton fire scenario, fan speed $7 \mathrm{~Hz} \ldots \ldots \ldots \ldots \ldots \ldots \ldots 213$

Figure 191. Smoldering cotton fire scenario, fan speed $12 \mathrm{~Hz} \ldots \ldots \ldots \ldots \ldots \ldots \ldots \ldots$

Figure 192. Smoldering cotton fire scenario, fan speed $12 \mathrm{~Hz} \ldots \ldots \ldots \ldots \ldots \ldots \ldots \ldots$

Figure 193. Smoldering wood fire scenario, fan speed $7 \mathrm{~Hz} \ldots \ldots \ldots \ldots \ldots \ldots$

Figure 194. Smoldering wood fire scenario, fan speed $7 \mathrm{~Hz} \ldots \ldots \ldots \ldots \ldots \ldots \ldots \ldots$

Figure 195. Smoldering wood fire scenario, fan speed $12 \mathrm{~Hz} \ldots \ldots \ldots \ldots \ldots \ldots \ldots$

Figure 196. Smoldering wood fire scenario, fan speed $12 \mathrm{~Hz} \ldots \ldots \ldots \ldots \ldots \ldots \ldots$

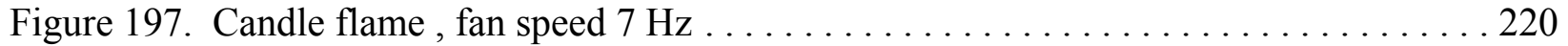

Figure 198. Margarine heated in a pan, fan speed $7 \mathrm{~Hz} \ldots \ldots \ldots \ldots \ldots \ldots \ldots \ldots \ldots$

Figure 199. Margarine heated in a pan, fan speed $7 \mathrm{~Hz} \ldots \ldots \ldots \ldots \ldots \ldots \ldots \ldots$

Figure 200. Butter heated in a pan, fan speed $7 \mathrm{~Hz} \ldots \ldots \ldots \ldots \ldots \ldots \ldots \ldots \ldots$

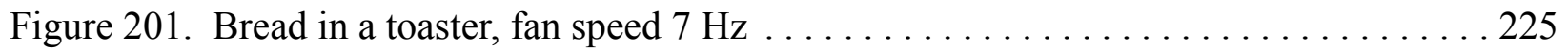

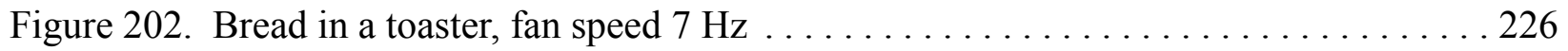

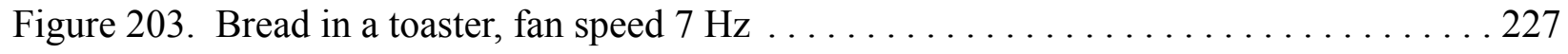

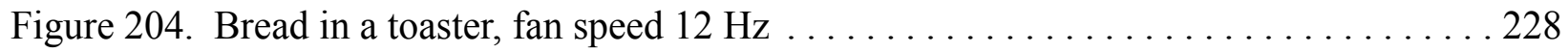




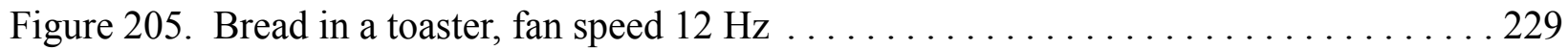

Figure 206. Average available egress time for several different alarm and fire types with alarms

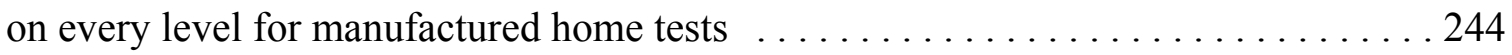

Figure 207. Average available egress time for several different alarm and fire types with alarms

on every level + bedrooms for manufactured home tests $\ldots \ldots \ldots \ldots \ldots \ldots 244$

Figure 208. Average available egress time for several different alarm and fire types with alarms

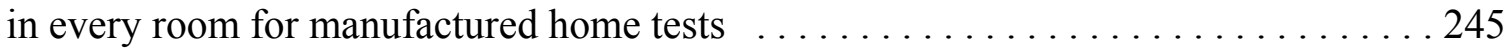

Figure 209. Test structure, sample interior room, and instrumentation in original 1975 Indiana

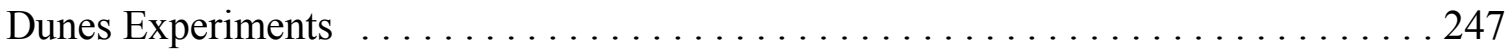

\section{Tables}

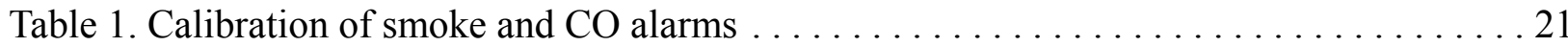

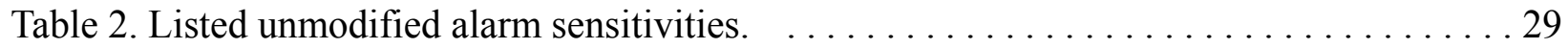

Table 3. MIC output and obscuration during tests of two ionization alarms $\ldots \ldots \ldots \ldots . \ldots 46$

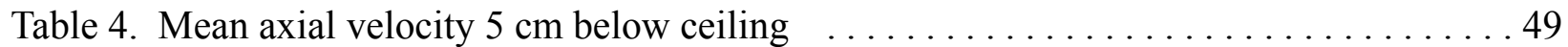

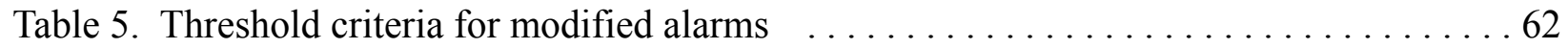

Table 6. Top fire scenarios ranked by frequency of occurrence, $1992-1996 \ldots \ldots \ldots \ldots 66$

Table 7. Locations for temperature measurement in tests of smoke alarm response . . . . . . . 78

Table 8. Locations for primary gas measurement in tests of smoke alarm response $\ldots \ldots .83$

Table 9. Locations for primary optical density measurements in tests of smoke alarm

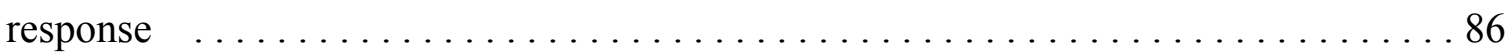

Table 10. Alarm and sprinkler locations in tests of smoke and $\mathrm{CO}$ alarm response ........ 90

Table 11. Test conditions for tests conducted in a manufactured home . . . . . . . . . . 94

Table 12. Test Conditions for tests conducted in a two-story home $\ldots \ldots \ldots \ldots \ldots \ldots$

Table 13. Alarm Times for Several Smoke Alarms in Hallway Outside Main Bedroom During Flaming Ignition Test of a Mattress, Test SDC05 . . . . . . . . . . . . . . 119

Table 14. Calculated tenability for a flaming ignition test of a mattress, test SDC05 . . . . 122

Table 15. Activation times for several smoke alarms during a flaming ignition test of a mattress, test SDC05 ...................................... 123

Table 16. Activation times for several alternative technologies during a flaming ignition test of a

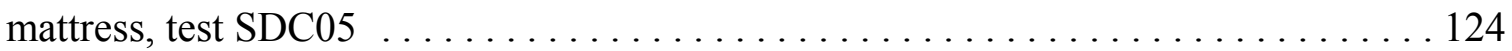

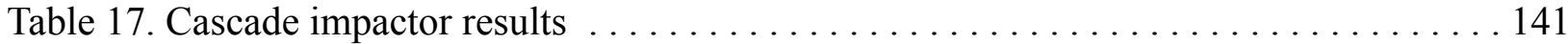

Table 18. Estimated particle size from smoldering chair scenario $\ldots \ldots \ldots \ldots \ldots \ldots \ldots \ldots$

Table 19. Estimated particle size from flaming sources $\ldots \ldots \ldots \ldots \ldots \ldots \ldots \ldots \ldots . \ldots \ldots$

Table 20. Estimated particle size from cooking oil fire scenario $\ldots \ldots \ldots \ldots \ldots \ldots \ldots 147$

Table 21. Variability for tenability time and alarm time during tests of residential smoke alarms (expressed as the ratio of standard uncertainty for replicate tests and the average value for

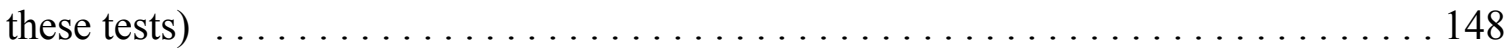

Table 22. Alarm sensitivity for photoelectric and ionization sensors $\ldots \ldots \ldots \ldots \ldots \ldots \ldots 5$ 
Table. 23. Average time to alarm (in seconds) for several smoke alarms and fire scenarios in a manufactured home ................................... 234

Table 24. Average time to alarm (in seconds) for several smoke alarms and fire scenarios in a

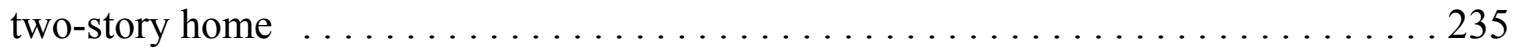

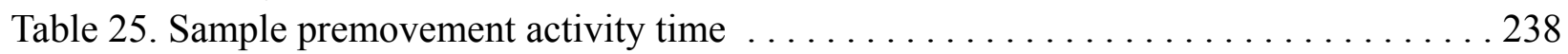

Table 26. Estimates of required escape times for best and worst case scenarios $\ldots \ldots \ldots \ldots 240$

Table. 27. Available egress time (in seconds) for several different alarm technologies and fire scenarios in a manufactured home . . . . . . . . . . . . . . . . . . . 242

Table 28. Available egress time (in seconds) for several different alarm technologies and fire

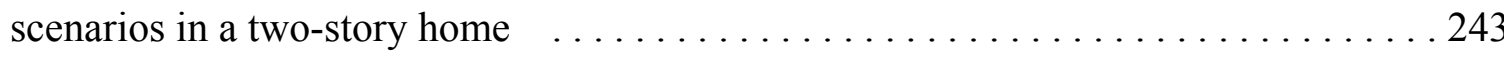

Table. 29. Activation time (in seconds) for several different fire detection technologies and fire scenarios in a manufactured home. .......................... 246

Table 30. Comparison of alarm times and times to untenable conditions for 1975 and current

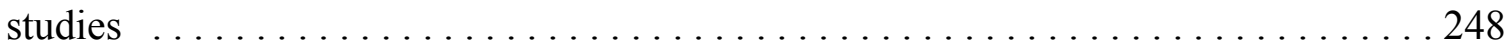

Table 31. Comparison of tenability criteria used in the 1975 and current studies . . . . . . 248

Table 32. Comparison of fire growth rates in the 1975 and current studies $\ldots \ldots \ldots \ldots \ldots 248$ 


\section{Executive Summary}

According to estimates by the National Fire Protection Association and the U. S. Fire Administration, U. S. home usage of smoke alarms rose from less than $10 \%$ in 1975 to at least $95 \%$ in 2000 , while the number of home fire deaths was cut nearly in half. Thus the home smoke alarm is credited as the greatest success story in fire safety in the last part of the $20^{\text {th }}$ century, because it alone represented a highly effective fire safety technology with leverage on most of the fire death problem that went from only token usage to nearly universal usage in a remarkably short time. Other highly effective fire safety technologies either affect a smaller share of the fire death problem (e.g., child-resistant lighter, cigarette-resistant mattress or upholstered furniture) or have yet to see more than token usage (e.g., home fire sprinkler, reduced ignition-strength cigarette).

A seminal component that underpinned this success was the existence of a comprehensive, independent set of tests conducted in 1975-76 that clearly demonstrated the potential of smoke alarms to save lives. These were the so-called Indiana Dunes tests sponsored by The National Institute of Standards and Technology, NIST, (then the National Bureau of Standards) and conducted by Illinois Institute of Technology Research Institute and Underwriters Laboratories.

In the past few years questions were being raised about the efficacy of some smoke alarm technologies, whether the numbers and locations in homes still represented the optimum configuration, and if multi-sensor designs (as are becoming popular for commercial fire alarm systems) might perform better or produce fewer nuisance alarms. Some simply thought that it was time to reexamine the technology in light of its importance to public safety. To this end a consortium of four federal agencies and Underwriters Laboratories (with in-kind support from several others) funded this project.

The present work followed a design similar to that used in the Indiana Dunes tests. Tests were conducted in actual homes with representative sizes and floorplans, utilized actual furnishings and household items for fire sources, and tested actual smoke alarms currently sold in retail stores. Smoke alarm performance was quantified in terms of the escape time provided by groups of alarms installed in accordance with typical code provisions. While some of the smoke alarms were modified by their manufacturers to provide continuous, analog output from their sensors (which allowed the analysis of the performance of multi-sensors and decision algorithms) these were compared to identical models purchased from retail stores and included in all tests. Additionally a separate study of nuisance alarm sources was conducted because this was identified as an important issue in a prior study by the U.S. Consumer Product Safety Commission. 
The fire emulator/detector evaluator (FE/DE) is a single-pass "wind tunnel" at NIST designed to reproduce all relevant conditions needed to assess the performance of spot-type particulate, thermal and gas sensor detectors or combination detectors. Specifically, the FE/DE allows for the control of the flow velocity, air temperature, gas species, and aerosol concentrations at a test section wherein detectors and sensors are exposed to these environmental conditions. Included in this report are complete data from tests conducted in the $\mathrm{FE} / \mathrm{DE}$ apparatus and calibrations for all of the alarms and detectors used in this study. Calibration in the FE/DE of the alarms used in the current study showed that the sensitivity of the alarms was consistent with manufacturer ratings and, on average, of equivalent sensitivity to those used in the 1975 study. The average sensitivity measured for all alarms tested was $5.1 \% / \mathrm{m} \pm 1 \% / \mathrm{m}(1.5 \% / \mathrm{ft} \pm 0.4 \% / \mathrm{ft})$. In the 1975 study, the average of all alarms tested was $6.3 \% / \mathrm{m} \pm 2 \% / \mathrm{m}(1.9 \% / \mathrm{ft} \pm 0.7 \% / \mathrm{ft})$. While the average for the 1975 tests is higher, the uncertainty in the data overlaps.

An analysis of residential fire statistics was conducted for this study to identify the important fire scenarios that were included in the study. Flaming and smoldering upholstered furniture and mattresses account for the top four most deadly fire scenarios. Flaming cooking materials are involved more than five times more frequently than any other material. These scenarios included the top five ranked by number of deaths, and among the top ten ranked by frequency of occurrence.

Fire produces heat, smoke, and toxic gases, all of which can threaten human life. In these experiments environmental parameters that have an impact on human response were measured. Parameters include temperature rise, toxic gas production, and smoke obscuration. Aspects of these that can be measured directly include the gas temperature, mass loss of the burning item, species concentrations, including $\mathrm{CO}, \mathrm{CO}_{2}, \mathrm{O}_{2}$, and other gases. Two different geometries of residential structures were used for the tests. The manufactured home geometry represented an array of residential layouts. The arrangement of the manufactured home was sufficiently generic as to represent an apartment, condominium, or small ranch house, in addition to a manufactured home, although it did include a sloped ceiling throughout the structure. The primary partitioning of the $84.7 \mathrm{~m}^{2}\left(911 \mathrm{ft}^{2}\right)$ home consisted of three bedrooms, one full bathroom, one kitchen/dining area, one living room, and two hallways. The $139 \mathrm{~m}^{2}\left(1495 \mathrm{ft}^{2}\right)$ two-story home was a brickclad 3 bedroom home. The first floor consisted of a foyer, den, family room, kitchen, dining room, bathroom, and stairwell to the upstairs. The home did not have a basement. The 2-car garage was accessible from the first floor den. The second floor consisted of a stairwell to the downstairs, hallway, 2 bathrooms, and two smaller bedrooms and one master bedroom. A total of 36 tests were conducted in the two homes; 27 in the manufactured home, and 8 in the 2-story home.

The experimental design provided data on the performance of alarms in various installation arrangements. Groups of alarms were located in the room of fire origin, at least one bedroom, and in a central location on every level. Thus, by considering the alarm times of devices in various locations against tenability times for any test it was possible to determine the escape 
time provided by various installation arrangements including every room, every level with or without every bedroom, and single detectors. Since both ionization and photoelectric types were included at each location the performance by sensor type was also quantified.

Smoke alarms of either type installed on every level generally provided the necessary escape time for different fire types and locations. Adding smoke alarms in bedrooms increased the escape time provided, especially for smoldering fires. These tests quantified an increased escape time for fires starting in the bedroom.

The results obtained were similar to those of the earlier work. Both common residential smoke alarm technologies (ionization and photoelectric) provided positive escape times in most fire scenarios with the ionization type reacting earlier to flaming fires and the photoelectric type reacting earlier to smoldering fires. The main difference from the earlier work is that the amount of escape time provided is consistently less. For example, average times to untenable conditions for flaming tests was $3 \mathrm{~min}$ compared to $17 \mathrm{~min}$ in the prior work. While some of this difference may be attributed to the tenability criteria used in the current study, it is also clear that fire growth in the current tests are significantly faster than in the earlier tests. For flaming fires, time to alarm activation and measurement of elevated temperatures in the room of fire origin support this faster fire growth observation. However, the smoldering fire scenarios are very difficult to reproduce experimentally and tenability times in the present study have an uncertainty (based upon one standard deviation) which overlaps the uncertainty from the 1975 study. Therefore, caution should be exhibited in drawing conclusions based upon comparisons of smoldering tenability times between the two studies. It is important to note that while both the 1975 study and the current study attempted to use a representative sample of available and important furnishings, each study included only a small fraction of those available in the marketplace. Still, this study is consistent with other recent studies of furniture and mattresses, even though there may be significant differences in the burning behavior between items of furniture.

Activation times for other fire detection technologies were also collected. As expected, $\mathrm{CO}$ alarms respond best to fires which produce considerable quantities of carbon monoxide during the combustion process, i.e., smoldering fire scenarios and the closed-door flaming mattress (which smoldered after the room of fire origin became oxygen limited). Tell-tell sprinklers and heat alarms responded to the flaming fire scenarios as well as to the smoldering fire scenarios after a transition to flaming combustion. Activation times of these devices support the current practice of use only in conjunction with smoke alarms.

Nuisance alarms in residential settings from typical cooking activities, smoking or candle flames are affected by the properties of the aerosol produced and its concentration, the location of an alarm relative to the source, and the air flow that transports smoke to an alarm. This is not surprising, as the same observations have been made in the fire tests here and other studies. This study provides a detailed set of data that can be used to address several issues involving nuisance alarms and reinforces current suggested practices. Clearly, the advice that alarms not be 
installed close to cooking appliances if at all possible is valid. These results show that homeowners who are able to move the location of an alarm that frequently experiences nuisance alarms would do well to maximize its distance from cooking appliances while keeping it in the area to be protected. It was observed that ionization alarms had a propensity to alarm when exposed to nuisance aerosols produced in the early stages of some cooking activities, prior to noticeable smoke production. This phenomenon could be particularly vexing to homeowners who experience such nuisance alarms. Carbon monoxide electrochemical cells at all alarm locations gathered data on the level of carbon monoxide produced during the tests and transported to the alarms. These data in conjunction with the complete fire test series data could be used to verify combined smoke/CO alarm algorithms.

The FE/DE nuisance source tests captured salient features of some of the manufactured home tests. More work needs to be done to produce a set of tests and the performance criteria that covers a significant range of residential nuisance sources, and would assure a benefit in terms of nuisance alarm reduction. This work is part of ongoing research with the FE/DE at NIST. The nuisance alarm study also represents a wealth of data valuable in establishing (amazingly for the first time) test methods and criteria for what a smoke alarm should not respond to. Testing labs are examining NIST's unique FE/DE apparatus to replace the current, product specific test apparatus that do not allow multi-sensor devices to be evaluated.

The present work has produced an extensive database of documented data on typical residential fires. All data collected in the tests are available in electronic form (http://smokealarm.nist.gov) and are already being used by the smoke alarm industry to improve their products, by the product approval agencies to improve testing procedures, and by researchers to validate and improve fire models.

The present work has considerable potential for communicating important fire safety messages to the public. The material has been made freely available and NIST plans to produce examples and is willing to work with others wishing to do the same. 


\section{Acknowledgments}

The authors gratefully acknowledge the efforts of the Fire Metrology Group and others, including D. Stroup, W. Twilley, J. Lee, G. Roadarmel, J. McElroy, M. Selepak, D. Weinert, M. Donnelly, M. Nyden, C. Davis, and L. DeLauter. G. Forney developed the web conversion for the test data. In addition, an in-kind contribution from the National Research Council-Canada measured toxicological species with FTIR within the manufactured home. The measurements were taken by Dr. J. Su and Dr. J. Kanabus-Kaminska.

The steering committee for the project included representatives from government and other organizations interested in the research. Their expertise provided direction throughout the project and insightful review of this report. Members of the committee included S. Coulberson, Ceners for Disease Control and Prevention; J. Hall, National Fire Protection Association; A. Lee, U.S. Consumer Product Safety Commission (CPSC); R. McCarthy, U.S. Fire Administration (USFA); J. Milke, University of Maryland; M. Nealy, CPSC; J. Ottoson, USFA; I. Papier and P. Patty of Underwriters Laboratories Inc.; J. Su, National Research Council Canada; and E. Taylor, U.S. Department of Housing and Urban Development, Healthy Homes Initiative.

The home smoke alarm project was sponsored by the U.S. Consumer Product Safety Commission, Centers for Disease Control and Prevention, U.S. Fire Administration, and the U.S. Department of Housing and Urban Development, Healthy Homes Initiative, Underwriters Laboratories. The National Fire Protection Association (In-kind contribution), and National Research Council Canada, (In-kind contribution). 
xxviii 


\title{
Performance of Home Smoke Alarms Analysis of the Response of Several Available Technologies in Residential Fires ${ }^{2}$
}

\author{
Richard W. Bukowski, Richard D. Peacock, Jason D. Averill, Thomas G. Cleary, \\ Nelson P. Bryner, William D. Walton, Paul A. Reneke, and Erica D. Kuligowski
}

Fire Research Division

Building and Fire Research Laboratory

\section{Introduction}

Smoke alarms are often the primary life safety strategy for occupants in the event of an unwanted residential fire. As the number of annual residential fire deaths (about 3000) far outpaces the number of commercial and industrial fire deaths (just over 100 in all non-residential structure fires) it is crucial to understand the level of safety provided by smoke alarms in the residential environment. In 1975, the Illinois Institute of Technology Research Institute (IITRI) and Underwriters Laboratories, Inc (UL), with funding from the National Bureau of Standards (NBS, now the National Institute of Standards and Technology (NIST)), evaluated the performance of commercially available smoke alarms in 76 residential fire experiments representative of the major fatal fire scenarios of the time, in actual homes scheduled for demolition. The purpose of the project, known as the Indiana Dunes Tests, was to evaluate the "requirements for fire alarms to protect residential occupancies." [1] The tests became the basis for the siting and response characteristics of residential smoke alarms, worldwide.

Since 1975, however, the materials and construction of contents that represent the major combustible items in a typical residential structure have changed and the response characteristics of current smoke alarms have also changed as the technology matured. Thus, a systematic evaluation of current residential smoke alarm requirements is again considered necessary. The United States Consumer Product Safety Commission (CPSC), the United States Fire Administration (USFA), the Centers for Disease Control and Prevention (CDC), the United States Department of Housing and Urban Development (HUD), Underwriters Laboratories, Inc. (UL), the National Fire Protection Association (NFPA), and the National Research Council

\footnotetext{
${ }^{2}$ Certain commercial entities, equipment, or materials may be identified in this report in order to describe an experimental procedure or concept adequately. Such identification is not intended to imply recommendation or endorsement by the National Institute of Standards and Technology, nor is it intended to imply that the entities, materials, or equipment are necessarily the best available for the purpose.
} 
Canada (NRCC), in conjunction with NIST's Building and Fire Research Laboratory (BFRL), have joined forces to evaluate the current state of residential smoke alarm requirements.

The overall purpose of the project is to determine how different types of fire alarms can respond to threatening residential fire settings in order to permit egress of typical occupant sets. The plan was to conduct real-scale tests of current smoke alarms and related technologies in actual homes with actual contents as fuels.

This report presents the results of the project and provides details of the response of a range of residential smoke alarm technologies in a controlled laboratory test and in a series of real-scale tests conducted in two different residential structures. The results are intended to provide both insight into siting and response characteristics of residential smoke alarms and a set of reference data for future enhancements to alarm technology based on fires from current materials and constructions.

\subsection{History}

As early as 1961 the potential of early warning of fire to reduce fatalities was recognized [2], however the costs associated with commercial detectors and alarm systems was prohibitive and such systems were found in fewer than $1 \%$ of U.S. dwellings. In 1965 the first self-contained (so-called single-station) smoke alarms were developed but it was not until the battery-powered smoke alarm was marketed in 1969 that homeowners began to take notice.

Initial sales were slow due largely to costs near $\$ 100$ but interest by large, consumer products companies brought more efficient production and marketing to bear and costs declined. By 1975 prices had dropped below $\$ 39.95$ (which General Electric cited as a level that brought impulse buyers) and sales really began to accelerate.

At about this time the National Bureau of Standards (now NIST) was promoting building code requirements for the installation of smoke alarms in new and existing dwelling units. Research to develop minimum product performance standards was being conducted in cooperation with Underwriters Laboratories (UL) and installation requirements were proposed to the National Fire Protection Association (NFPA) Standard 74, Household Fire Warning Equipment.

Initially, the installation scheme was based on the McGuire and Ruscoe recommendations [2] but it was recognized that testing was needed to verify the adequacy of the number, sensitivity, and location of devices in typical homes. This led to the conduct of the so-called Indiana Dunes Tests [1] that pioneered the every-level smoke alarm requirement now found in nearly every building code in the world. 
In the decade 1975-1985 smoke alarm sales were estimated in the 14 million per year range and (state and local) building codes were commonly amended to require smoke alarms at least in new residential construction. Many jurisdictions reported precipitous drops in residential fire fatalities, in many cases reaching zero [3]. This success inspired other jurisdictions to require smoke detectors in existing dwellings and programs to distribute free smoke alarms to the disadvantaged.

According to estimates by the National Fire Protection Association and the U. S. Fire Administration, U. S. home usage of smoke alarms rose from less than $10 \%$ in 1975 to at least $95 \%$ in 2000, while the number of home fire deaths was cut nearly in half. Thus the home smoke alarm is credited as the greatest success story in fire safety in the last part of the $20^{\text {th }}$ century, because it alone represented a highly effective fire safety technology with leverage on most of the fire death problem that went from only token usage to nearly universal usage in a remarkably short time. Other highly effective fire safety technologies either affect a smaller share of the fire death problem (e.g., child-resistant lighter, cigarette-resistant mattress or upholstered furniture) or have yet to see more than token usage (e.g., home fire sprinkler, reduced ignition-strength cigarette).

\subsection{Concerns Raised}

In the early 1990s the U.S. Consumer Product Safety Commission (CPSC), in conjunction with the US Fire Administration, NFPA, NIST, and the Centers for Disease Control and Prevention undertook an extensive study called the National Smoke Detector Project. The objective was to examine who did and did not have detectors, how many were working (many were now more than 10 years old), and, if not working, why. Also included were consumer awareness issues, availability in economically disadvantaged households, and rates of testing and battery replacement [4].

While the results of the study were generally good there were areas for concern. Surveys of representative homes showed that most had working smoke detectors (since renamed smoke alarms) correctly installed. While a third of the smoke detectors did not work on initial test, half of these were made operational by restoring power (mostly installing a fresh battery). Homeowner interviews revealed that most of these were intentionally disconnected due to nuisance alarms, mostly from cooking. While there were some failures identified there were no large or systematic problems identified with detector designs or manufacturing practices that cast any doubt on their long term reliability.

In this period, reports surfaced that some privately funded testing had shown delayed response from smoke alarms using ionization-type sensors to smoldering fires. While detailed reports were never published in the open literature, these persistent reports were the cause of some concern. Given the significant public benefits resulting from smoke alarms in homes CPSC, 
NIST, and others felt that it was time to make a new investment in research that would address these concerns directly and would examine ways to improve the protection provided while reducing nuisance alarms that were leading to disabling of the alarms.

\subsection{Organizing the Research Project}

Under the leadership of CPSC staff and with technical support from NIST, a series of public meetings were held to garner support for a new research program. Funding was provided by CPSC, USFA, HUD (Healthy Homes Initiative), CDC, and Underwriters Laboratories with additional support from NFPA, National Research Council of Canada, University of Maryland, and the US smoke alarm manufacturers to make this ambitious program possible. The level of resources allowed a broad program of research objectives to be identified. These were:

1. Evaluate the performance of current smoke-alarm technology.

2. Test conditions representative of current fatal residential fires.

3. Evaluate the efficacy of current requirements for number and location of smoke alarms.

4. Develop standard nuisance alarm sources to be included in the test program.

5. Examine other fire detection technologies in combination with smoke alarms (example: residential sprinkler and heat detectors).

6. Obtain data on the potential for improvements in performance by new technologies.

7. Include fuel items that incorporate materials and constructions representative of current residential furnishings.

8. Fully characterize test detectors and alarms in a consistent manner to facilitate comparisons.

9. Utilize fire models to extend the applicability of the test arrangements and maximize the test instrumentation.

10. Make all of the data collected as widely accessible as possible.

11. Provide opportunities to enhance public fire safety education.

The considerations made to address each of these objectives are discussed in the following sections.

\subsubsection{Evaluate the Performance of Current Smoke Alarm Technology}

Current technology includes two operating principles - the ionization-type sensor and the photoelectric (scattering) sensor. The major manufacturers produce both types representing unique designs that exhibit individual performance characteristics. Thus, the project included examples of current products containing each of the sensor designs so that the results would be representative of the full range of product performance expected. 
In addition, representative electrical and mechanical heat detectors, and residential sprinklers were included in the test program to provide reference data on activation times. Also included were current residential carbon monoxide (CO) alarms employing two of the three sensor types found in commercial products (metal oxide and electrochemical cells). The biometric sensors were not included because they could not be modified for continuous, analog output. The CO alarms evaluated are specifically marketed as not being suitable as fire detectors, but rather are for detecting high levels of $\mathrm{CO}$ from malfunctioning or improper use of combustion appliances. However, they were included because they are increasingly being required in addition to smoke alarms and because they may be useful in improving performance and reducing nuisance alarms in conjunction with smoke alarms.

All prior studies had been done with ordinary alarm devices that operate on an alarm threshold basis - produce an alarm signal when some pre-determined alarm level is exceeded. This limits the ability to evaluate the change in performance from variations above or below the set threshold, and does not allow determination of performance of multiple sensors or of alarm algorithms. Thus it was decided to include devices that were modified (by their manufacturers) to produce a continuous, analog output signal from their sensor.

While greatly increasing the usefulness of the data such modification by the manufacturer raises the issue of whether the performance is representative of an unmodified commercial product. To address this the project employed two approaches:

- First, there were numerous unmodified devices of the identical model as the analog device that were purchased by NIST from local retail sources. These served as a comparison to the analog devices when characterized in NIST's fire emulator / detector evaluator (FE/DE) apparatus [5]. The FE/DE is a single-pass "wind tunnel" designed to reproduce all relevant conditions needed to assess the performance of spot-type particulate, thermal and gas sensor detectors or combination detectors.

- Second, these unmodified devices were used in every test in the room of fire origin.

\subsubsection{Test Conditions Representative of Current Fatal Residential Fires}

To ensure that the room sizes and arrangements, materials of construction, and ventilation conditions are representative of actual dwellings, all tests were conducted in real dwelling units. First, a manufactured home was procured and delivered to the NIST site for use in both fire tests and nuisance alarm tests. The floor plan selected was a three bedroom, two bath arrangement with a master suite at one end and the other bedrooms at the other end. This size and arrangement represents not only manufactured homes but also apartments and condominiums of about $100 \mathrm{~m}^{2}$ (or about $1000 \mathrm{ft}^{2}$ ). 
A second test site, obtained through the Federal Emergency Management Agency (FEMA), was a three bedroom, two-story, brick home in Kinston, NC. The home was in an area impacted by a hurricane where the homes were purchased to be demolished and the owners relocated. While most homes in the area had flood damage, this one was on a higher elevation lot and had not been damaged. Floor plan drawings of both homes are provided in section 3.4.

Of key importance to the representativeness of the study was the selection of the test scenarios. These include the room of fire origin, ignition source and first item ignited, and the ventilation conditions that affect the fire development and combustion chemistry. Here we began with an NFPA analysis of the USFA's National Fire Incident Reporting System (NFIRS) data on fatal residential fires.

In addition to the scenarios themselves, the test matrix considered the need to perform sufficient replicates to allow estimates of experimental uncertainty and repeatability.

Finally the desire to run tests in a wide range of geometries (room sizes, materials, arrangements, and ventilation conditions) is limited by the costs of full-scale tests and available resources. Here the availability of computer fire models can provide a means to assess the potential impact of other geometries on smoke alarm performance. NIST's computational fluid dynamics (CFD) model, FDS, was used in advance of the tests to assist in planning and instrumentation and to provide quantification of modeling uncertainty. CFD models are now available to extrapolate the test results to other geometries of interest.

\subsubsection{Evaluate the Efficacy of Current Requirements for Number and Location of Smoke Alarms.}

Current U.S. model building codes contain requirements for smoke alarms in residential occupancies. These codes typically require smoke alarms on every level (story) outside sleeping areas and in all sleeping areas (bedrooms). Bedroom alarms are generally required only in newly constructed units. This requirement added in the mid-1990s based on the need for audibility with bedroom doors closed. Proposals to extend bedroom alarms to existing construction have lacked justification on life safety grounds compared to the significant expense.

The design of the experiments was meant to provide data on the performance of alarms in various installation arrangements. Groups of alarms were located in the room of fire origin, at least one bedroom, and in a central location on every level. Thus, by considering the alarm times of devices in various locations against tenability times for any test it was possible to determine the escape time provided by various installation arrangements including every room, every level with or without every bedroom, and single detectors. Since both ionization and photoelectric types were included at each location the performance by sensor type is also quantified. 
The inclusion of analog sensors allowed the evaluation of performance changes resulting from variation in alarm threshold settings. The analysis in this report is based on typical alarm threshold settings. Data are also available to allow comparison at other threshold values.

\subsubsection{Develop Standard Nuisance Alarm Sources to Be Included in the Test Program.}

Smoke alarms are susceptible to alarming when exposed to non-fire aerosols. In residential settings, this typically involves cooking activities or transient, high humidity conditions (i.e., "shower steam"). The Smoke Detector Operability Survey [6] conducted by the U.S. Consumer Product Safety Commission reported that about one half of the 1012 respondents indicated they experienced nuisance alarms, with $80 \%$ of those attributed to cooking activities, and an additional $6 \%$ citing steam from bathrooms. Dust, and tobacco smoke are also mentioned sources. The survey also reported that of the alarms with missing or disconnected batteries, or disconnected AC power, more than one third of respondents indicated that power was removed due to nuisance alarms.

The objective of this research task was to develop a basis for standard residential nuisance source testing. The approach taken was to define a set of nuisance scenarios, replicate the events that cause nuisance alarms, and quantify the important variables that cause nuisance alarms. Translating the results to a set of nuisance source conditions reproducible in a suitable test-bed (i.e., a test room or the fire emulator/detector evaluator) would allow for more comprehensive detector performance testing. Preliminary tests were performed in the FE/DE with this in mind. Programming realistic and reproducible fire and nuisance conditions in the FE/DE is an ongoing research project at NIST.

\subsubsection{Examine Other Fire Detection Technologies in Combination with Smoke Alarms}

Prior studies have evaluated alternate detection technologies such as heat detectors [1] and metal oxide gas sensing [7] compared to and in combination with particulate smoke sensing. In the present study residential carbon monoxide alarms were included to evaluate their performance individually and in combination with smoke and heat sensing. By including these data, the use of multi-criteria algorithms to combine the signals from different sensors can be examined as opposed to the simple AND and OR logic of the past. Similar work has been performed by others $[8,9]$ for a broader range of sensor types but many of these are not commercially feasible, especially for the price-sensitive residential market.

Simple combinations of smoke sensors with $\mathrm{CO}$ and thermal signals were evaluated for performance against fires and nuisance sources. More complex algorithms were evaluated under a grant to University of Maryland and will be reported separately.

Residential fire sprinklers have increasingly been required in new, multiple dwelling units (apartments, condominiums, and some townhomes) and under the relevant standards include smoke alarms in the same number and locations as without sprinklers. While these sprinklers have been extensively evaluated and have an outstanding record of saving lives and property [10], there are no research programs that provide data on how the two systems work together. 
Thus typical residential sprinklers were included in these tests to provide comparative data on activation times but the sprinklers were not connected to water as this would have interfered with the data on smoke alarm performance.

\subsubsection{Obtain Data on the Potential for Improvements in Performance by New Technologies}

Simple alarm times are not sufficient to identify potential performance improvements; so analog sensor data were recorded for both the fire performance and nuisance alarm testing. Additional instrumentation was selected and located to supplement the analog sensor data and allow better quantification of results. Examples include thermocouple measurements of temperatures and temperature gradients, smoke and gas species concentrations in several locations, and convective flow velocities in the ceiling layer at the level of the sensors.

\subsubsection{Include Fuel Items That Incorporate Materials and Constructions Representative of Current Residential Furnishings}

Fuel (combustible) items were selected as generically appropriate to the room of origin and fire scenario identified from NFIRS analysis and typical of materials and constructions in common use today. Specifically, upholstered chairs were used for living room fires and mattresses for bedroom fires that were purchased from local, retail sources. No modifications were made and no accelerants were used to enhance or retard the burning behavior of the items. Cooking oil used in kitchen fires was also a normal commercial product that was not modified in any way.

\subsubsection{Fully Characterize Test Detectors and Alarms in a Consistent Manner to Facilitate Comparisons}

All detectors (smoke, heat, and CO) were characterized in NIST's FE/DE apparatus [5] under similar conditions using an appropriate source. This allows direct calibration of all devices in a comparable way and a means to verify the performance of sensors in combination or utilizing algorithms that may be suggested by the results of this study.

\subsubsection{Utilize Fire Models to Extend the Applicability of the Test Arrangements and Maximize the Test Instrumentation}

Modeling, using NIST's CFAST and FDS models, was performed prior to testing to evaluate the impacts of the selected fuels and fire scenarios on the instrumentation and test structures and to assist in identifying the most appropriate instrument locations.

\subsubsection{Make All of the Data Collected as Widely Accessible as Possible}

Clearly the best method of (global) dissemination of the test data was to employ the Internet. Thus, a public web site was established (http://smokealarm.nist.gov/) on which the detailed results of tests were published as NIST Report of Tests [11, 12]. This publication vehicle exists as a means to quickly disseminate test data that is factual in nature - no observations or conclusions can be included. Thus the web site contains floor plans of the test sites showing instrument locations, details of the fuels and test conditions, and test data in electronic form. For 
this we used the Fire Data Management System (FDMS) format [13] and comma-separated spreadsheet (.csv) files.

\subsubsection{Provide Opportunities to Enhance Public Fire Safety Education}

The information obtained in these tests will provide a basis for numerous public fire safety messages and will quantify the protection provided by residential smoke alarms. The project data will be used to show the reduction in protection resulting from one or more devices being nonfunctional as a motivation for testing and maintenance by the homeowner. Videos will be made available for use in public safety announcements and educational materials. A press day was held that resulted in stories carried on all the major news networks and on more than 75 local outlets from Maine to Hawaii.

\subsection{Project Oversight}

The project was initiated by NIST in October 2000 with a 24 month schedule for completion. A Project Steering Committee was established with representatives of each of the sponsoring organizations along with NFPA, the University of Maryland, and the National Research Council of Canada. In addition a larger mailing list of interested parties was organized by CPSC to whom copies of the quarterly reports were sent by email. Semi-annual meetings were held with the Steering committee and annual briefings were held for the larger group in order to keep communications open. However, in keeping with NIST policy, recommendations from either group were given every consideration but were not a priori considered binding on the NIST team. 


\section{Residential Fire Alarms, Sensor Response and Calibration in the FE/DE}

\subsection{Residential Alarms Included in the Study}

Figure 1 shows one of the fire alarm sensor test boards used during the tests. Two classes of alarms were used in the test series, unmodified off-the-shelf smoke alarms and modified smoke and $\mathrm{CO}$ alarms that produce a continuous voltage signal in response to the sensor environment (such as smoke or $\mathrm{CO}$ concentrations, ambient pressure, humidity, and temperature). The unmodified alarms consisted of a photoelectric model and two ionization alarms corresponding to the modified models provided by the manufacturers. These alarms were purchased from retail establishments for use in this test

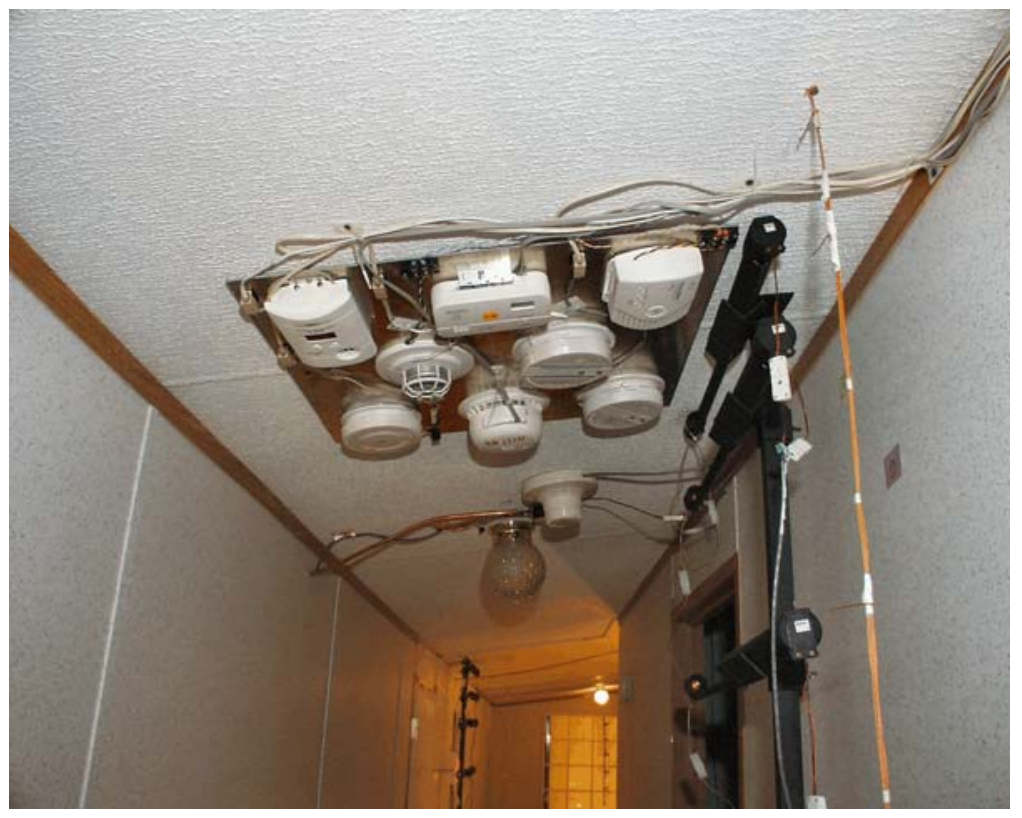

Figure 1. Sample sensor test board used during tests of residential smoke alarms series. The alarm response was verified by a single test in the FE/DE for at least 12 alarms of each type. The modified smoke and $\mathrm{CO}$ alarms were provided by their respective manufacturers. These represented three photoelectric type smoke alarms (one of which was of an aspirated design), three ionization type smoke alarms, and three $\mathrm{CO}$ alarms, two of which employed an electrochemical cell, and one which employed a tin oxide semiconductor (so-called Taguchi-type) sensor. Each alarm was assigned an arbitrary number for this report. A naming convention using the alarm type (i.e., Ion) and the arbitrary number is used throughout the report to identify individual alarms (i.e., Ion-1 identifies one particular model of the ionization alarms; additional identification details the location for each alarm in each test - see section 2.4). Calibration tests were performed on the modified smoke and $\mathrm{CO}$ alarms in order to develop voltage response curves for smoke and carbon monoxide gas exposures. The effects of local flow conditions and sensor test board location on the sensor response were examined in the FE/DE to quantify alarm response biasing due to a particular alarm's position on the test board in the test series. Details of these experiments are given below. 
Essentially all residential smoke alarms sold in the United States are listed by Underwriters Laboratories Inc. and meet the requirements of test standard UL 217 [14]. From tests performed on a particular model of detector, a sensitivity value is derived from an average of smoke levels reached at alarm for repeated tests. This sensitivity is recorded on the back of the smoke detector along with a lower and upper range that defines the allowed variation in sensitivity for the particular model. Alarms are periodically checked during the manufacturing process in accordance with UL 217 to make sure the model type still alarms within its sensitivity range.

During the formulation of the research plan for the Home Smoke Alarm Project it was decided that modified smoke and $\mathrm{CO}$ alarms that provide a continuous signal would be used. The purpose for this decision was two-fold. First, using the voltage response curve, any single sensitivity (alarm point) can be specified for a particular alarm model or type of alarm. Therefore any range of sensitivities can be examined. Second, continuous sensor output is useful for experimental algorithm development; it lends itself to signal processing feature extraction, and multi-sensor/multi-criteria algorithm development. The time investment in the modification of the smoke alarms, and their individual calibrations required that they be re-used throughout the test series. In almost all cases, calibrations were re-checked after a test series was complete for alarms used in that series.

\subsection{The fire emulator/detector evaluator}

The fire emulator/detector evaluator (FE/DE) is a single-pass "wind tunnel" at NIST designed to reproduce all relevant conditions needed to assess the performance of spot-type particulate, thermal and gas sensor detectors or combination detectors. Specifically, the FE/DE allows for the control of the flow velocity, air temperature, gas species, and aerosol concentrations at a test section wherein detectors and sensors are exposed to these environmental conditions [5]. A schematic of the FE/DE is shown in figure 2 .

Room air is drawn into the opening, and exhausted to a ventilation hood at the end of the duct. The air velocity at the test section is controlled over a range of flows between $0.02 \mathrm{~m} / \mathrm{s}(4 \mathrm{ft} / \mathrm{min})$ to over $2 \mathrm{~m} / \mathrm{s}$ ( $400 \mathrm{ft} / \mathrm{min})$ by means of the computer-controlled axial blower. Air is first propelled through the annular finned heating elements, then travels along the duct to the test section. The flow is conditioned before it reaches the test section by passing through a $0.1 \mathrm{~m}$ (4 in) long aluminum honeycomb with $5 \mathrm{~mm}$ rectangular openings. The goal is to provide a carefully controlled flow profile indicative of what would be experienced by a detector in a ceiling jet flow. A rectangular baffle plate covering $1 / 2$ of the duct cross-section may be placed in the center of the duct $0.3 \mathrm{~m}(1 \mathrm{ft})$ upstream from the test section to produce velocity fluctuations similar to those observed in room flows. Additional information on flows during room tests is discussed in section 6 . 


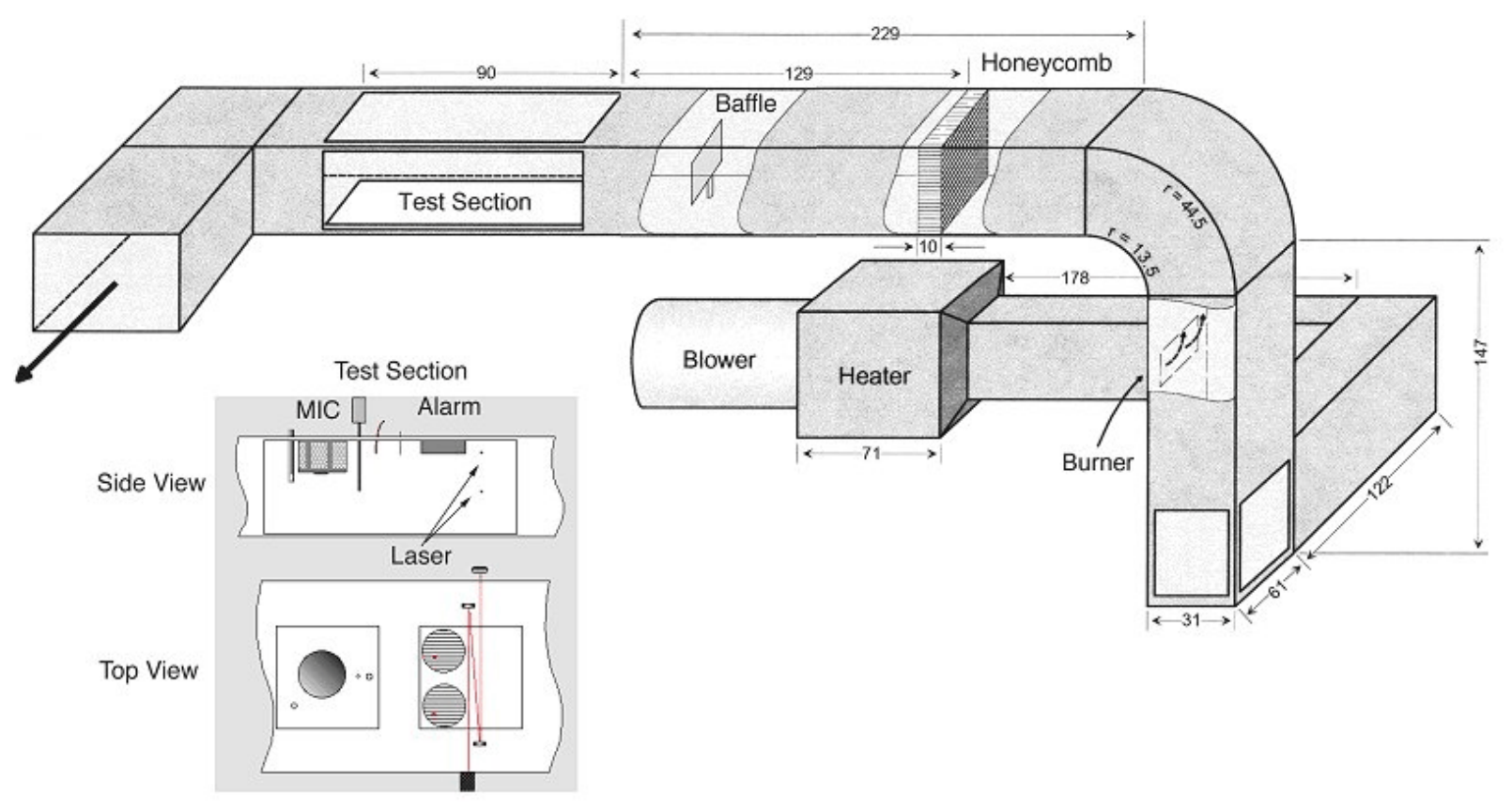

Figure 2. Schematic of the FE/DE (all units in $\mathrm{cm}$ )

Heat may be added to the flow by a series of 9 annular finned heating elements. Each element is rated at $5 \mathrm{~kW}$ for a total maximum heat input of $45 \mathrm{~kW}$. Power to the heating elements is controlled by a feedback controller that receives set-point values from an input file and compares them to the air temperature upon exiting the heaters. A rate of temperature rise in airflow of $0.5{ }^{\circ} \mathrm{C} / \mathrm{s}$ is achievable at the test section, up to maximum of about $80{ }^{\circ} \mathrm{C}$. For the testing described in this report, this feature was not used; any temperature rise is attributed to the smoke source heat output. Air temperature at the test section is measured with a chromel-alumel thermocouple located $5 \mathrm{~cm}$ below the duct ceiling.

$\mathrm{CO}, \mathrm{CO}_{2}$, or other gas blends may be metered into the flow via electronic mass flow controllers. $\mathrm{CO}$, and $\mathrm{CO}_{2}$ gas concentrations at the test section are monitored by a dual gas non-dispersive infrared (NDIR) analyzer (Siemens Ultramat 22P CO/CO 2 analyzer). Various types of smokes and non-combustion aerosols may be introduced into the flow, including flaming soot, smolder smokes, dust, nebulized liquid mists, and cooking aerosols. Laser light transmission measurements across the duct at the test section are used to calculate the light extinction coefficient, optical density, or obscuration of the aerosol. Extinction coefficient or optical density is the typical "concentration" measurement of smoke. In this report though, obscuration expressed in terms of (percent per foot) will be used often as it is the industry standard measurement unit. A linearly polarized helium-neon laser at $632.8 \mathrm{~nm}$ wavelength coupled with a stabilizer utilizing a liquid crystal polarizer is the light source. This system maintains nearly constant laser intensity. The main beam is split and introduced at two heights: the center of the duct, and $5 \mathrm{~cm}$ below the top of the duct. Each light beam is reflected off two mirrors inside the 
duct and directed at a photodetector placed on the opposite side of where the beam enters the duct. The total light transmission path length inside the duct is $1.52 \mathrm{~m} \pm 0.01 \mathrm{~m}$. The photodetector output voltage is linear with respect to the transmitted light intensity. The standard relative uncertainty due to random fluctuations in the output is $0.06 \%$ of the measured light transmittance (light intensity divided by smoke-free initial light intensity). A measuring ionization chamber (MIC) is located in the test section to provide a reference chamber current measurement appropriate for characterizing ionization detectors. The chamber voltage potential was set at $18 \mathrm{~V}$, the flow rate was set at $30 \mathrm{~L} / \mathrm{min}$ and the chamber current was measured with a picoammeter that provides a proportional analog voltage. The picoammeter voltage output was calibrated with a precision current source $\left(99.7746 \mathrm{pA}\right.$ at $\left.20^{\circ} \mathrm{C}\right)$ constructed from a weston standard cell and a $10 \mathrm{G} \Omega$ resistor. The clean air chamber output was typically $95 \mathrm{pA} \pm 5 \mathrm{pA}$ with a standard relative measurement uncertainty of $2 \mathrm{pA}$. Relative humidity was recorded with a capacitance-type humidity sensor (Oakton model WD-35612-10) with a stated measurement uncertainty of $2 \%$. Barometric pressure in the FE/DE laboratory was recorded by an electronic manometer (Druck model DPI 145) with the measurement uncertainty of $1 \mathrm{~Pa}$.

\subsection{Smoke Aerosols}

The modified smoke alarms were calibrated against two smoke sources: a propene flame soot generator, and smoldering cotton wick smoke. Initially, all detectors were tested against each smoke source twice. Repeat calibration checks were performed with the cotton smolder smoke source.

The propene smoke generator provides black soot typical of flaming hydrocarbon or plastics fire smoke, figure 3 . The propene smoke generator is directly attached to the $\mathrm{FE} / \mathrm{DE}$ duct at the vertical riser section. The concentration of smoke is varied by changing the fuel flow of the burner, and opening or closing dampers allowing more or less flow from the burner to enter the duct. For the calibration runs performed in this study, the

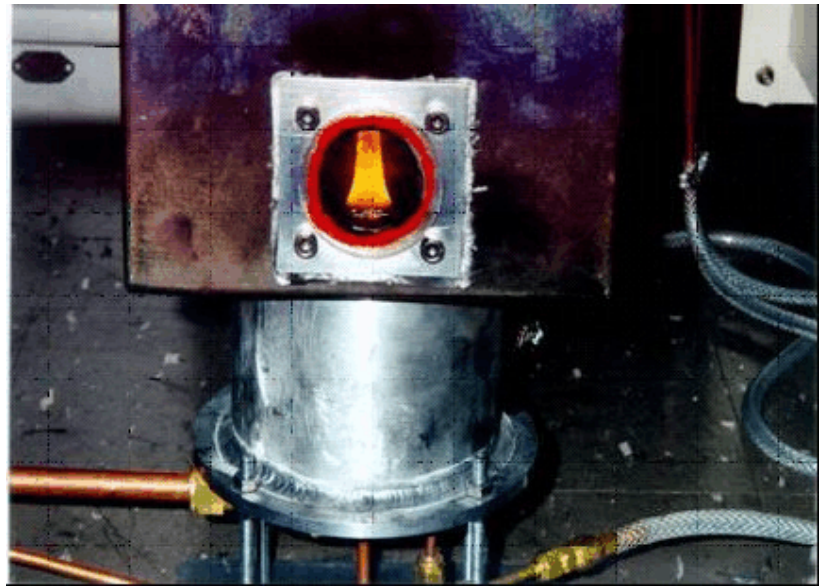

Figure 3. Propene smoke generator. 
burner fuel and air were

incremented every

$170 \mathrm{~s}$ in a series of 8 steps to

produce steps in the soot

concentration ending with a light

transmittance value of

approximately 0.7 . The flat sections

of the upper laser beam light

transmission curve (spanning

approximately $120 \mathrm{~s}$ ) are compared to the detector signal over the same time interval. Figure 4 shows the light transmittance and MIC current output as a function of time for one of the test runs. The curves consist of a series of stepped outputs which were averaged to determine values

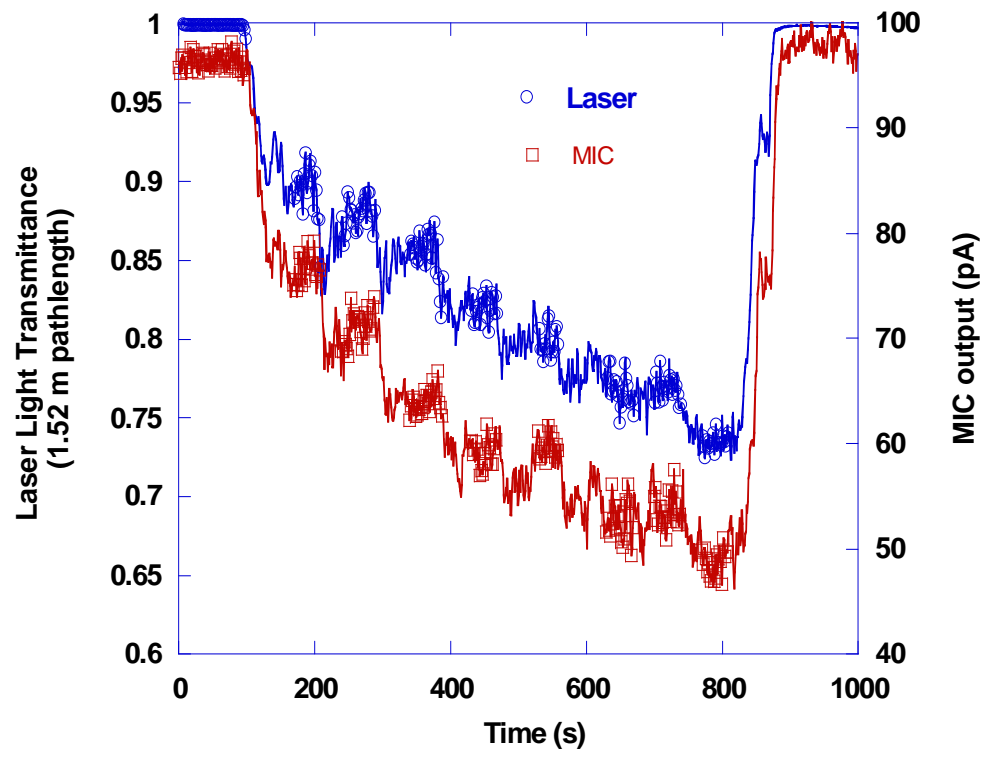

Figure 4. Propene smoke calibration run for the calibration curves.

Smoldering cotton smoke is generated by a staged-wick-ignition device (figure 5) that ignites wicks by applying power to electrical heating wires in a prescribed computercontrolled sequence to affect a specific rate of smoke build-up at the test section. Eight groups of up to four individual wicks can be ignited in sequence to provide the controlled rate-of-rise in smoke concentration at the test section. The cotton smolder source is similar to the cotton smolder test fire 3 in EN 54 part 9 [8] and the UL standard [14]. Power is applied at $200 \mathrm{~s}$ intervals to each of the four sets of four wick igniters followed by 2 sets of 8 wick igniters to yield six

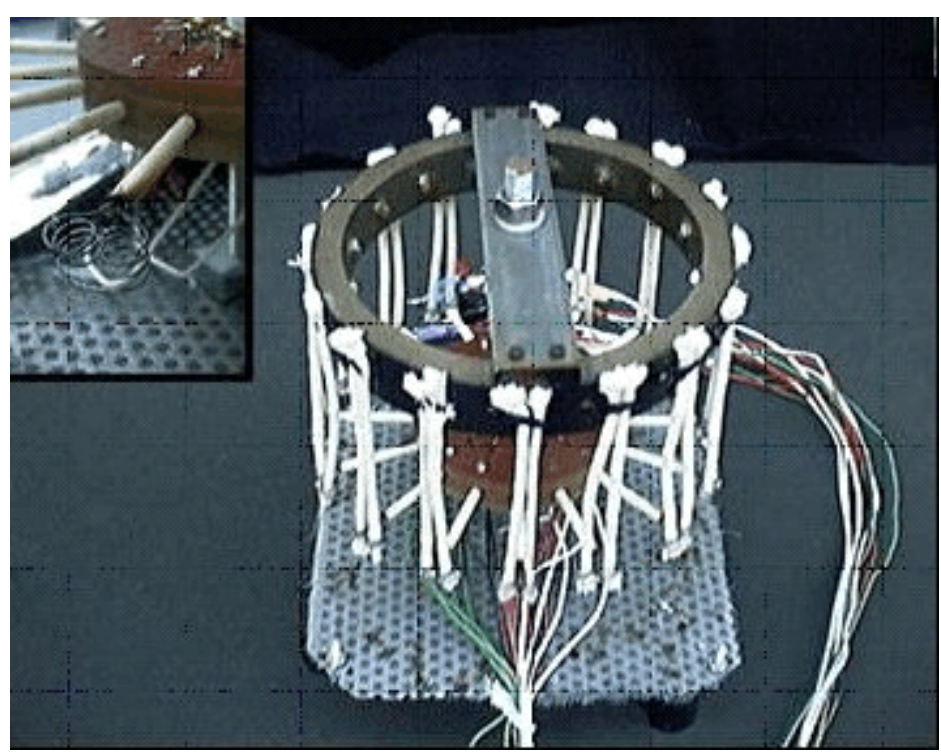

Figure 5. Smolder source, staged wick ignition device steps in the smolder smoke concentration. Figure 6 shows the light transmittance and MIC current output as a function of time for one of the test runs, with the steady values used for calibration curves highlighted. The smoldering cotton smoke source was used to produce increasing levels of $\mathrm{CO}$ for calibrating the $\mathrm{CO}$ alarms as well. Figure 7 shows the $\mathrm{CO}$ and $\mathrm{CO}_{2}$ volume fractions as a function of time for the same test. 
Examining the relationship between the test smokes used here and those used in the UL standard [14] is important in order to establish the validity of computed alarm times from the smoke sensor voltage values recorded in the test series. The smolder smoke source is used to specify an alarm's sensitivity, thus establishing the relationship between the UL cotton wick aerosol and the cotton wick aerosol produced here is of primary concern. Direct comparison of the physical properties and size distribution of the test smokes was not within the scope of this study. However, the UL standard allows for alternate aerosol generation equipment so long as the relationship between the MIC and the light transmittance values fall within specified sensitivity test limits. This relationship fixes the obscuration range at ionization alarm levels, but not necessarily the obscuration range at photoelectric alarm levels for alternate aerosols.

The relationship for cotton smolder smoke is shown in figure 8 . Along with the sensitivity test limits, results from 12 separate $\mathrm{FE} / \mathrm{DE}$ tests (spanning a 6 month test interval) are shown, where the mean values over the steady time periods are plotted along with the corresponding standard deviations. It is seen that the FE/DE results fall within the UL cotton smolder smoke test limits down to light transmittance values of about 0.9 , then deviate outside of those bounds for lesser light transmittance values. The observation that the FE/DE test results stay within the bounds down to a transmittance of 0.9 is significant because the sensitivity range for most residential ionization alarms falls within a transmittance range above 0.9 for the smolder smoke.

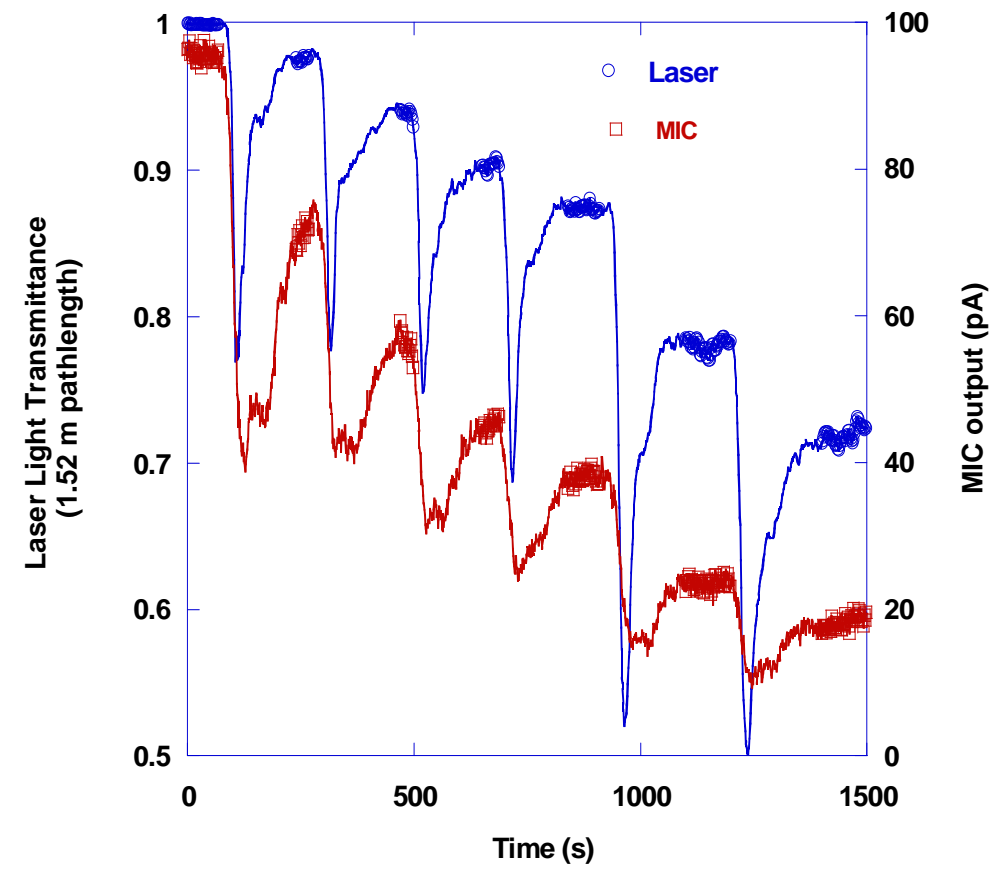

Figure 6. Smolder smoke calibration run

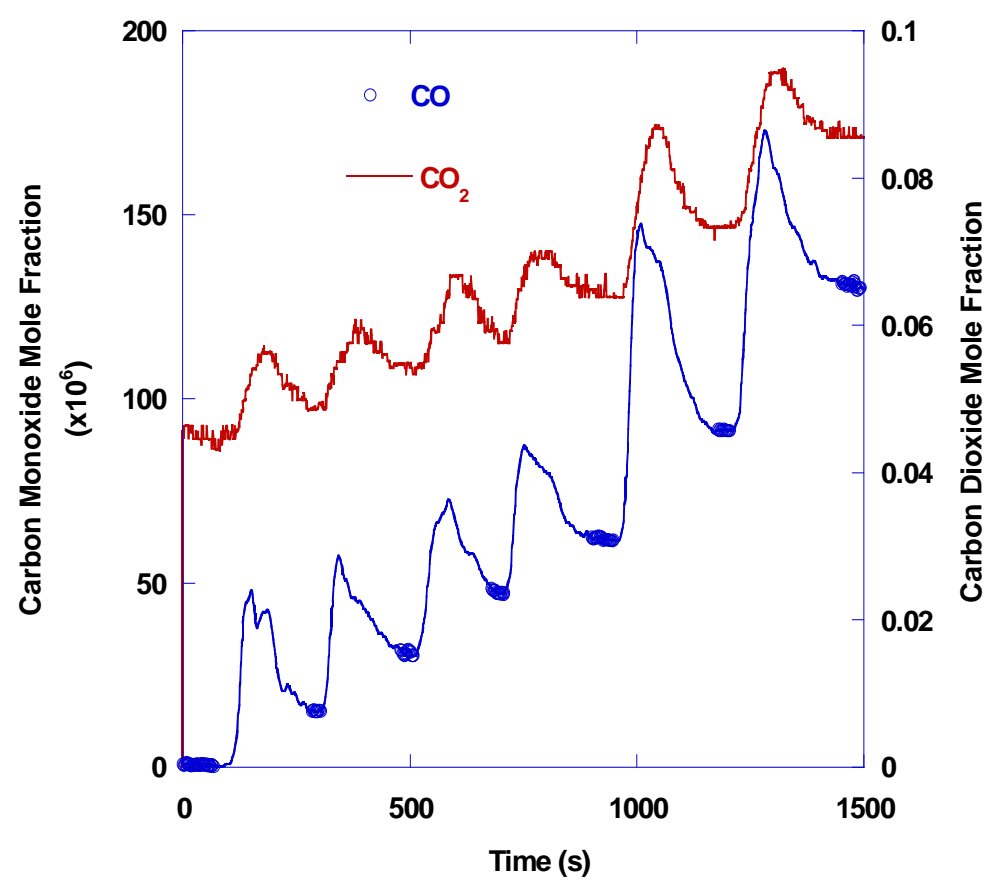

Figure 7. Smolder smoke CO calibration run 
Thus, within the range typical of ionization smoke alarms, the response is within the limits of the UL 217 test.

Residential photoelectric alarms typically alarm at transmittances greater than 0.84 in the UL test. While the $\mathrm{FE} / \mathrm{DE}$ smoke deviates outside the MIC limits at a transmittance of about 0.9 , this observation is not by itself a cause for concern.

The deviation between UL and FE/DE test smoke results at lower light transmittance values suggests a smoke size distribution difference between the two smolder smokes. Since the UL

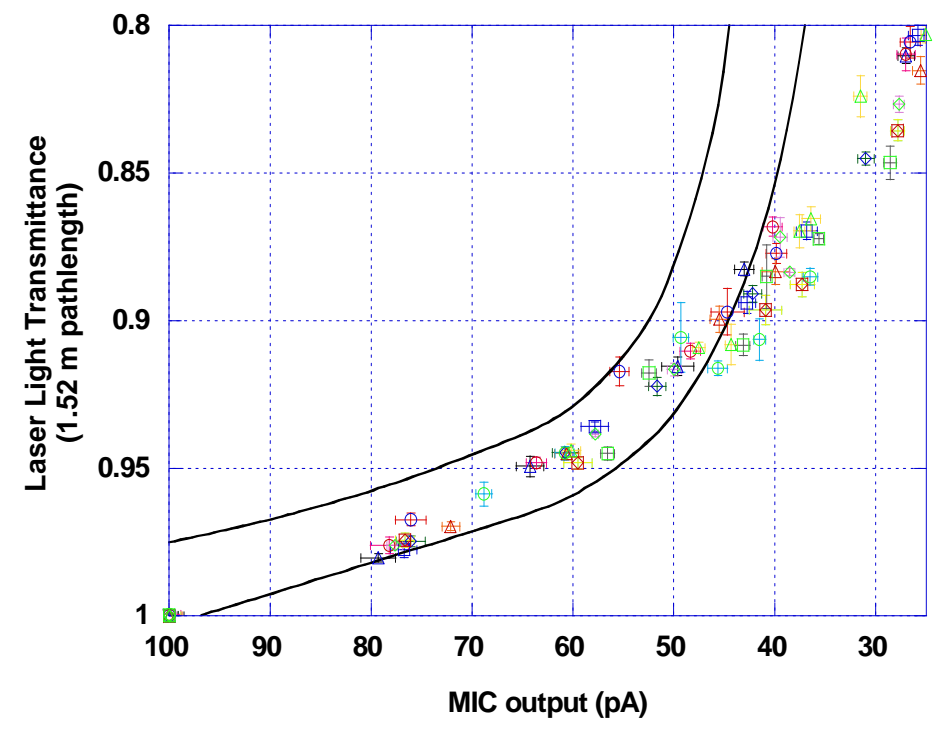

Figure 8. UL grey smoke (smolder smoke) sensitivity limits and FE/DE data smoke box is a re-circulating system, it can take between $12 \mathrm{~min}$ to $17 \mathrm{~min}$ to reach a light transmittance value of 0.8 . The FE/DE is an open, single-pass flow system and the smoke transport time for these calibration tests is on the order of $30 \mathrm{~s}$. Therefore, potentially more smoke aging (i.e. coagulation growth of the smolder aerosol) can take place in the smoke box relative to the FE/DE. Assuming that fresh smolder smokes are identical in the smoke box and the $\mathrm{FE} / \mathrm{DE}$, additional coagulation growth in the smoke box will tend to increase light extinction while decreasing the MIC output for a fixed amount of smoke. This could explain MIC difference between the two sources at lower transmittances. The difference between relative levels of obscuration and light scattering of fresh and aged smolder smokes should be less pronounced. There are two reasons for this supposition. First, coagulation growth tends to incorporate the smallest

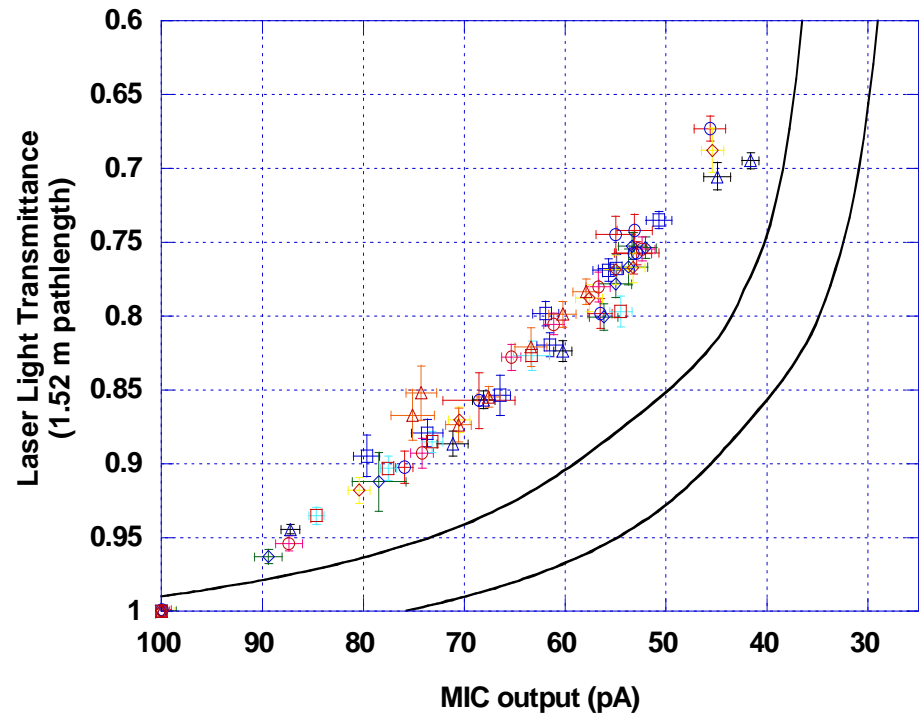

Figure 9. UL black smoke sensitivity limits and corresponding FE/DE data particles into bigger particles. The smaller particles scatter little light, and contribute almost nothing to obscuration, thus they are not missed. Second, the growth of particles that scatter significant amounts of light will tend to 
increase the scattered light at the same rate the obscuration is increased since for the light colored smolder smoke scattering dominates the light extinction.

The relationship for black smoke is shown in figure 9. Along with the sensitivity test limits, results from 9 separate FE/DE tests are shown as well. It is seen that for the most part, the FE/DE results fall outside the UL black smoke test limits yielding lower transmittance values over the MIC output range, although the trend is the same. Although certainly related to the characteristics of the smoke, the reasons for the difference has not been studied.

\subsection{Calibration of Smoke and CO alarms}

Calibration details for each type of detector are described below, including alarm power requirements, data acquisition details, and observations on sensor range limits. While the data collection conditions were similar between the laboratory calibrations runs and field tests for the analog alarms, slight differences between alarm power, signal transmission noise environment, and amplifier characteristics could affect initial (clean air) sensor voltage readings, not to mention background environmental effects. It is important to account for these differences in some cases by shifting field test baselines.

The FE/DE uses a custom routine programmed with National Instrument's Labview software running on a Pentium PC computer. Analog data acquisition is performed with two AT-MIO/AI E series multifunction I/O boards. The modified alarm analog signals were digitized on a ATMIO-16E-10 board configured for 8 differential inputs, with a voltage range of $0 \mathrm{~V}$ to $10 \mathrm{~V}$. It has 12 bit resolution, and an offset error of $\pm 0.5 \mathrm{mV}$ and a gain error of $\pm 0.01 \%$ of reading. The input impedance of the programmable gain amplifier is $100 \mathrm{G} \Omega$ in parallel with $50 \mathrm{pF}$ with input and offset bias currents of $\pm 200 \mathrm{pA}$ and $100 \mathrm{pA}$ respectively. All floating signal sources (i.e. battery powered alarms) had their ground reference (negative terminal) tied to the board analog input ground.

For smoke alarms, typically two flaming soot and two smoldering smoke calibration runs were performed for each detector prior to field test use, followed by a single smoldering smoke calibration run after each use in a field test series. CO alarms were subjected to a single smoldering smoke calibration run prior to field tests with the smoldering smoke calibration rerun after a field test series. The calibration data for each detector is available [15]. The data fields for the smoke alarms consist of test time, air temperature, laser light transmittance from the upper laser beam, relative humidity in the duct, barometric pressure in the laboratory, MIC output, and alarm voltage signal. The continuous time data is segmented into time blocks with nominally steady smoke production to produce the steady values for calibration. Over these steady periods, the mean values of alarm voltage, MIC output, and laser light transmittance were computed and recorded along with the standard deviation. From the mean laser light output, the 
extinction $\left(\mathrm{m}^{-1}\right), k=\ln \left(I / I_{0}\right) / L_{m}$, where $I$ is the light intensity, $I_{0}$ is the initial light intensity, and $L_{m}$ is the path length in meters. Obscuration $(\% / \mathrm{ft})$, $o b s=\left(1-\left(I / I_{0}\right)^{1 / L_{f}}\right) \times 100$, where $L_{f}$ is the path length in feet, were computed along with their standard deviation. The data fields for the ionization alarms include a dimensionless variable, $Y$, computed from the alarm voltage signal whose functional form was derived from ionization chamber theory $[16,17]$. $Y$ is defined as:

$$
Y=\left(\frac{\Delta V}{V_{O}}\right)\left(\frac{2-\Delta V / V_{O}}{1-\Delta V / V_{O}}\right)
$$

Since the ion chamber current is proportional to the ion alarm analog voltage $(V)$, a simple substitution is made. $V_{0}$ is the initial "clean air" chamber voltage and $\Delta V$ is $\left(V_{0}-V\right)$. The benefit of converting the alarm output to the dimensionless variable $Y$ is that it is a linear function of obscuration. Best fit lines through obscuration versus $Y$ provides the calibration equation. These best fit lines are presented along with the calibration data, beginning in figure 11. During the recalibration of detectors, the picoammeter used to measure the MIC chamber current ceased functioning and subsequently, only light extinction data are reported for re-calibration runs.

The data collected for the $\mathrm{CO}$ alarms include: $\mathrm{CO}$ and $\mathrm{CO}_{2}$ analyzer voltage output, and the $\mathrm{CO}$ alarm voltage, (the MIC output is not included). The conversion factor between voltage and volume fraction for the $\mathrm{CO}$ analyzer is 93.0 (volume fraction $\times 10^{6}$ )/ $\mathrm{V}$, with a stated measurement uncertainty of $2.5 \times 10^{-6}$ volume fraction. The conversion factor for the $\mathrm{CO}_{2}$ analyzer is $7.48 \times 10^{-3}$ (volume fraction) $/ \mathrm{V}$, with a stated measurement uncertainty of $4.0 \times 10^{-5}$ volume fraction.

Ionization smoke alarm Ion-1: The analog output was provided by the alarm's application specific integrated circuit (ASIC) as a voltage signal proportional to the ion chamber current flow. $9.00 \mathrm{~V}$ was provided by a variable voltage DC power supply. Clean air voltage level was on the order of $6 \mathrm{~V}$ and the value decreased with increasing smoke level.

Ionization smoke alarm Ion-3: Similar to Ion-1, the analog voltage was provided by the alarm's application specific integrated circuit (ASIC) as a voltage signal proportional to the ion chamber current flow. $9.00 \mathrm{~V}$ was provided by a variable voltage DC power supply. Clean air voltage level was on the order of $4 \mathrm{~V}$ and the value decreased with increasing smoke level.

Ionization smoke alarm Ion-4: This alarm provided a digital signal that was transmitted to a multiplexing box that converted the digital signal to an analog voltage. The multiplexing box also provided the power to the detector. The clean air voltage level was nominally $0 \mathrm{~V}$, with an increasing output in the presence of smoke. In order to compute $Y$ values, a cutoff voltage of $2 \mathrm{~V}$ was specified and the voltage signal was transformed by the equation: 


$$
\text { New Signal }=2-\text { Old Signal }
$$

With this transformation, new signal decreases with increasing smoke and equation (1) is used to compute $Y$. The $2 \mathrm{~V}$ cutoff voltage was chosen as an approximate upper limit for the output of the alarm.

Photoelectric smoke alarm Photo-1: This alarm was modified by adding a sample and hold IC and an amplifier to monitor and amplify the photo-detector signal. It needed a dual power supply of $\pm 9 \mathrm{~V}$, which was provided by a series/parallel (6x2) arrangement of 1.5 volt AA size alkaline batteries. The nominal clean air voltage level was $0.2 \mathrm{~V}$.

Photoelectric smoke alarm Photo-3: Identical to Ion-4, this alarm provided a digital signal that was transmitted to a multiplexing box that converted the digital signal to an analog voltage. The multiplexing box also provided the power to the detector. The clean air voltage level was nominally $0 \mathrm{~V}$. The voltage output increased in the presence of smoke.

Photoelectric smoke alarm ASP-1: This alarm is a commercial design and already has the provision for an amplified analog voltage proportional to the photodetector signal. It has an integrated fan that aspirates room air through a special filter medium and into the sensing chamber periodically at a fixed time interval. While it has no smoke entry lag characteristics, its response was affected by the aspiration time interval (normally $30 \mathrm{~s}$ ). It was powered at $12.0 \mathrm{~V}$ by a variable voltage DC power supply.

Carbon monoxide alarm CO-1: This alarm contained a semiconductor tin oxide CO sensor and provided a digital signal that was transmitted to a multiplexing box that converted the digital signal to an analog voltage. The multiplexing box also provided the power to the detector. As a consequence of its programming, this alarm did not produce any voltage change until the $\mathrm{CO}$ volume fraction was greater than $50 \times 10^{-6}$ (as indicated on the unit's LED display). Calibrations for this alarm start at 50 (volume fraction $\left.\times 10^{6}\right)$.

Carbon monoxide alarm CO-2: This alarm contained a $\mathrm{CO}$ electrochemical cell, and provided an analog voltage proportional to the concentration of $\mathrm{CO}$. These alarms were powered by a fresh alkaline 9 volt battery. The nominal clean air voltage level was $0.45 \mathrm{~V}$.

Carbon monoxide alarm CO-3: This alarm contained a $\mathrm{CO}$ electrochemical cell, and provided an analog voltage proportional to the concentration of $\mathrm{CO} .9 .00 \mathrm{~V}$ was provided by a variable voltage DC power supply. Clean air voltage level was on the order of $0 \mathrm{~V}$.

Representative calibration plots are show in figures 11 to 17 for each alarm type. A single smoldering test was used to specify a specific alarm's calibration used for particular test series. 
The test series are identified as S1, S2 and S3 for the first manufactured homes series (tests 1 to 15), the two-story home (tests 20 to 28), and the second manufactured home series (tests 30 to 42) respectively. The alarm calibrations are listed in table 1.

Table 1. Calibration of smoke and $\mathrm{CO}$ alarms

\begin{tabular}{|c|c|c|c|c|}
\hline Detector & Test Series $^{\mathrm{a}}$ & $\mathrm{m}_{0}^{\mathrm{b}}$ & $\mathrm{m}_{1}^{\mathrm{b}}$ & $\mathrm{R}^{\mathrm{c}}$ \\
\hline \multirow{2}{*}{ Aspirated-1- - -3 } & s1 & -1.629 & 2.971 & 0.988 \\
\hline & s3 & -1.392 & 2.064 & 0.999 \\
\hline \multirow[t]{3}{*}{ Aspirated-1- - -5 } & s1 & -1.512 & 2.568 & 0.996 \\
\hline & $\mathrm{s} 2$ & -1.349 & 2 & 0.999 \\
\hline & s3 & -1.672 & 2.144 & 0.985 \\
\hline \multirow{2}{*}{ Aspirated-1- - -6 } & s1 & -1.276 & 1.846 & 0.996 \\
\hline & $\mathrm{s} 2$ & -1.714 & 2.324 & 0.996 \\
\hline \multirow{3}{*}{ Aspirated-1- - -7 } & s1 & -1.37 & 2.625 & 0.991 \\
\hline & $\mathrm{s} 2$ & -1.598 & 3.021 & 0.994 \\
\hline & s3 & -2.338 & 3.994 & 0.981 \\
\hline Aspirated-1- - -12 & $\mathrm{s} 1, \mathrm{~s} 2$ & -1.727 & 2.998 & 0.999 \\
\hline CO-1- - -1 & s1 & -44.67 & 239.1 & 0.999 \\
\hline CO-1- - -2 & $\mathrm{s} 2$ & -0.733 & 109.1 & 0.98 \\
\hline $\mathrm{CO}-1---3$ & $\mathrm{~s} 2$ & -9.807 & 153.7 & 0.993 \\
\hline CO-1- - -4 & s1 & -4.379 & 129.8 & 0.999 \\
\hline $\mathrm{CO}-1---5$ & s1 & -43.62 & 233.2 & 0.97 \\
\hline \multirow[t]{2}{*}{ CO-1- - -6 } & s1 & -19.05 & 100.7 & 0.991 \\
\hline & s3 & 1.61 & 114.1 & 0.986 \\
\hline CO-1- - -8 & s1 & -33.82 & 193 & 0.975 \\
\hline \multirow[t]{2}{*}{ CO-1- - -9 } & s1 & -22.58 & 180.9 & 0.997 \\
\hline & s3 & 24.76 & 153.4 & 0.958 \\
\hline CO-1- - -10 & $\mathrm{s} 2$ & -10.32 & 132.5 & 0.988 \\
\hline CO-1- - -14 & s3 & 56.17 & 110 & 0.979 \\
\hline CO-1- - -16 & $\begin{array}{l}\mathrm{s} 2, \mathrm{~s} 3 \\
\end{array}$ & 27.5 & 132.2 & 0.969 \\
\hline CO-1- - -17 & $\mathrm{s} 2, \mathrm{~s} 3$ & 34.38 & 140 & 0.944 \\
\hline CO-2- - -1 & s1 & -45.93 & 109.4 & 0.995 \\
\hline $\mathrm{CO}-2---2$ & s1 & -45.07 & 92.91 & 0.993 \\
\hline $\mathrm{CO}-2---3$ & s3 & -59.45 & 117.6 & 0.999 \\
\hline $\mathrm{CO}-2---5$ & s1 & -48.8 & 113.7 & 0.999 \\
\hline \multirow{2}{*}{ CO-2- - -6 } & $\mathrm{s} 2$ & -57.92 & 115.2 & 0.999 \\
\hline & s3 & -74.84 & 179.5 & 0.998 \\
\hline \multirow[t]{2}{*}{ CO-2- - -8 } & $\mathrm{s} 2$ & -52.18 & 119.9 & 0.999 \\
\hline & s3 & -56.24 & 144.3 & 0.999 \\
\hline \multirow[t]{2}{*}{ CO-2- - -9 } & $\mathrm{s} 2$ & -58.96 & 137.9 & 0.998 \\
\hline & s3 & -63.73 & 128.8 & 0.991 \\
\hline CO-2- - -11 & s1 & -50.37 & 104.25 & 0.997 \\
\hline \multirow[t]{2}{*}{ CO-2- - - 12} & $\mathrm{~s} 2$ & -45.4 & 93.62 & 0.997 \\
\hline & s3 & -48.92 & 98.93 & 0.997 \\
\hline $\mathrm{CO}-2---13$ & s2 & -44.05 & 103.4 & 0.999 \\
\hline CO-3- - -1 & $\mathrm{s} 2$ & -6.924 & 166.1 & 0.999 \\
\hline
\end{tabular}




\begin{tabular}{|c|c|c|c|c|}
\hline Detector & Test Series ${ }^{\mathrm{a}}$ & $\mathrm{m}_{0}^{\mathrm{b}}$ & $\mathrm{m}_{1}^{\mathrm{b}}$ & $\mathrm{R}^{\mathrm{c}}$ \\
\hline CO-3- - - 2 & s2 & -2.237 & 165.7 & 0.999 \\
\hline \multirow[t]{2}{*}{ CO-3- - -3 } & s2 & -5.251 & 161.6 & 0.996 \\
\hline & s3 & -2.551 & 165.4 & 0.997 \\
\hline CO-3- - -4 & s1 & -6.457 & 163.4 & 0.998 \\
\hline CO-3- - -5 & s1 & -6.232 & 170.8 & 0.999 \\
\hline \multirow[t]{2}{*}{ CO-3- - -6 } & s1 & -0.882 & 172.9 & 0.99 \\
\hline & s3 & -1.96 & 173.8 & 0.999 \\
\hline \multirow[t]{2}{*}{ CO-3- - -7 } & $\mathrm{s} 2$ & -2.464 & 171.2 & 0.999 \\
\hline & s3 & -2.58 & 168.8 & 0.995 \\
\hline CO-3- - -8 & s1 & -2.542 & 165.5 & 0.998 \\
\hline $\mathrm{CO}-3---10$ & s1 & -2.954 & 165.7 & 0.998 \\
\hline $\mathrm{CO}-3---11$ & s3 & -4.059 & 163.1 & 0.998 \\
\hline Ion-1- - -1 & s1 & & 2.151 & 0.998 \\
\hline Ion-1- - -2 & s3 & & 2.07 & 0.997 \\
\hline Ion-1- - -3 & $\mathrm{s} 2$ & & 1.789 & 0.995 \\
\hline Ion-1- - -4 & s1 & & 1.66 & 0.991 \\
\hline \multirow[t]{2}{*}{ Ion-1- - -5 } & s2 & & 2.164 & 0.998 \\
\hline & s3 & & 1.827 & 0.994 \\
\hline Ion-1- - -6 & $\mathrm{s} 1$ & & 2.27 & 0.997 \\
\hline Ion-1- - -7 & $\mathrm{s} 1$ & & 1.685 & 0.987 \\
\hline Ion-1- - -8 & s3 & & 1.823 & 0.997 \\
\hline \multirow[t]{2}{*}{ Ion-1- - -9 } & s2 & & 1.93 & 0.999 \\
\hline & s3 & & 1.863 & 0.992 \\
\hline Ion-1- - -10 & s1 & & 1.919 & 0.998 \\
\hline \multirow[t]{2}{*}{ Ion-1- - -11 } & s1 & & 2.093 & 0.997 \\
\hline & s3 & & 2.095 & 0.999 \\
\hline Ion-3- - -1 & s3 & & 1.247 & 0.985 \\
\hline Ion-3- - -2 & s3 & & 1.197 & 0.988 \\
\hline Ion-3- - -3 & s1 & & 1.215 & 0.949 \\
\hline Ion-3- - -4 & s2 & & 1.558 & 0.998 \\
\hline Ion-3- - -6 & s1 & & 1.421 & 0.997 \\
\hline \multirow[t]{2}{*}{ Ion-3- - -7 } & s2 & & 1.001 & 0.998 \\
\hline & s3 & & 1.007 & 0.999 \\
\hline Ion-3- - -9 & s1 & & 1.144 & 0.998 \\
\hline Ion-3- - -10 & s1 & & 0.984 & 0.999 \\
\hline \multirow[t]{2}{*}{ Ion-3- - -11 } & s2 & & 1.184 & 0.987 \\
\hline & s3 & & 1.299 & 0.989 \\
\hline Ion-3- - -13 & s1 & & 1.147 & 0.995 \\
\hline Ion-3- - -17 & s2 & & 1.066 & 0.988 \\
\hline \multirow[t]{2}{*}{ Ion-4- - - $1^{\mathrm{d}}$} & s1 & & 1.462 & 0.996 \\
\hline & s3 & & 1.506 & 0.998 \\
\hline Ion-4- - - $2^{\mathrm{d}}$ & $\mathrm{s} 2$ & & 2.374 & 0.99 \\
\hline Ion-4- - - $3^{\mathrm{d}}$ & s1 & & 1.463 & 0.992 \\
\hline Ion-4- - - $4^{\mathrm{d}}$ & s3 & & 2.926 & 0.997 \\
\hline Ion-4- - - $5^{\mathrm{d}}$ & s1 & & 1.806 & 0.953 \\
\hline \multirow[t]{2}{*}{ Ion-4- - - $6^{\mathrm{d}}$} & s1 & & 3.353 & 0.984 \\
\hline & s3 & & 2.624 & 0.985 \\
\hline Ion-4- - - $8^{\mathrm{d}}$ & s1 & & 2.562 & 0.997 \\
\hline Ion-4- - - $9^{\mathrm{d}}$ & $\mathrm{s} 2$ & & 1.35 & 0.996 \\
\hline
\end{tabular}




\begin{tabular}{|c|c|c|c|c|}
\hline Detector & Test Series ${ }^{\mathrm{a}}$ & $\mathrm{m}_{0}{ }^{\mathrm{b}}$ & $\mathrm{m}_{1}^{\mathrm{b}}$ & $\mathrm{R}^{\mathrm{c}}$ \\
\hline & s3 & & 1.222 & 0.998 \\
\hline \multirow[t]{2}{*}{ Ion-4- - - $10^{\mathrm{d}}$} & s1 & & 2.642 & 0.993 \\
\hline & s3 & & 2.498 & 0.984 \\
\hline Ion-4- - -11 d & $\mathrm{s} 2$ & & 1.832 & 0.996 \\
\hline Ion-4- - -12 & $\mathrm{s} 2$ & & 3.202 & 0.985 \\
\hline \multirow[t]{3}{*}{ Photo-1- - -1 } & s1 & -0.801 & 5.779 & 0.997 \\
\hline & $\mathrm{s} 2$ & -2.351 & 7.755 & 0.997 \\
\hline & s3 & -2.274 & 8.117 & 0.997 \\
\hline \multirow[t]{3}{*}{ Photo-1- - - -2 } & s1 & -2.185 & 5.786 & 0.997 \\
\hline & $\mathrm{s} 2$ & -3.761 & 8.383 & 0.994 \\
\hline & s3 & -2.758 & 8.589 & 0.987 \\
\hline \multirow[t]{2}{*}{ Photo-1- - -3 } & s1 & -1.498 & 6.717 & 0.997 \\
\hline & $\mathrm{s} 2, \mathrm{~s} 3$ & -1.566 & 7.855 & 0.998 \\
\hline \multirow[t]{3}{*}{ Photo-1- - -4 } & s1 & -1.523 & 8.569 & 0.995 \\
\hline & $\mathrm{s} 2$ & -0.0972 & 0.452 & 0.999 \\
\hline & s3 & -2.569 & 14.15 & 0.996 \\
\hline Photo-1- - -5 & s1 & -1.963 & 6.882 & 0.996 \\
\hline \multirow[t]{3}{*}{ Photo-1- - -6 } & s1 & -1.56 & 9.248 & 0.997 \\
\hline & $\mathrm{s} 2$ & -2.195 & 11.82 & 0.997 \\
\hline & s3 & -2.154 & 12.67 & 0.997 \\
\hline \multirow[t]{2}{*}{ Photo-3- - - $1^{\mathrm{d}}$} & $\mathrm{s} 2$ & -1.724 & 11.03 & 0.999 \\
\hline & s3 & -2.415 & 12.87 & 0.999 \\
\hline Photo-3- - - $2^{\mathrm{d}}$ & s2 & -1.701 & 9.473 & 0.998 \\
\hline Photo-3- - $-3^{\mathrm{d}}$ & s1 & -1.734 & 10.83 & 0.999 \\
\hline \multirow[t]{2}{*}{ Photo-3- - - $4^{\mathrm{d}}$} & s1 & -0.875 & 8.524 & 0.995 \\
\hline & s3 & -2.408 & 11.2 & 0.998 \\
\hline Photo-3- - - $5^{\mathrm{d}}$ & s1 & -2.096 & 11.563 & 0.999 \\
\hline \multirow[t]{2}{*}{ Photo-3- - - $6^{\mathrm{d}}$} & s1 & -1.421 & 9 & 0.999 \\
\hline & s3 & -1.825 & 12.032 & 0.997 \\
\hline Photo-3- - - $8^{\mathrm{d}}$ & s1 & -1.411 & 10.574 & $\overline{0.996}$ \\
\hline \multirow[t]{2}{*}{ Photo-3- - - $9^{\mathrm{d}}$} & s1 & -1.289 & 9.323 & 0.999 \\
\hline & s3 & -1.532 & 10.124 & 0.998 \\
\hline
\end{tabular}

a For the purposes of these calibrations, the test series is identified as $\mathrm{s} 1$ for tests $1-15, \mathrm{~s} 2$ for tests $20-28$, and s3 for tests 30-41.

b $O b s_{i o n}=m_{l} Y($ in $\% / \mathrm{ft})$, where $Y$ is defined in eq. (1)

$O b s_{\text {photo }}=m_{0}+m_{1} V($ in $\% / \mathrm{ft})$, where $V$ is in volts

$X_{C O}=m_{0}+m_{1} V$ (in volume fraction $\times 10^{6}$ ), where $V$ is in volts

c $\quad \mathrm{R}$ is the square root of the coefficient of determination for the regression equation defined in note $\mathrm{b}$.

d Alarms Photo-3 and Ion-4 are components of a single dual alarm.

Due to differences in power supply levels, data acquisition hardware, signal noise pickup, and other environmental effects, initial "clean air" voltage signals measured in the field tests usually deviated slightly from those observed in laboratory tests. In applying the calibration equations to test series data, differences in initial "clean air" values were handled in a consistent manner. First, an average initial value was obtained from the twenty recorded values preceding the start of the test (time $=0$ ), typically $20 \mathrm{~s}$ of data. Second, adjustments were made using this initial 
voltage. In the case of photoelectric and $\mathrm{CO}$ sensors, the difference between the calibration "clean air" value $\left(-m_{0} / m_{l}\right)$ and the average initial value was added to the data to adjust for any offset. In the case of the ionization sensors, equation 1 was applied to the test data and using the initial "clean air" average as $V_{0}$.

\subsection{Alarm Identification}

Throughout the report, each alarm is identified with a specific code that identifies the model of the alarm, the location the alarm was used in a test, and the specific sample of the alarm used (see figure 10). The code has the format 'device type' - 'model' - 'alarm board location' - 'alarm board position' - 'alarm sample' where 'device type' specifies the technology of the device, 'model' is a number which identifies a specific model of the type, 'alarm board location' is a letter code that specifies where in the structure the alarm was located for a specific test, 'alarm board position' is a number that specifies the location of the alarm on an alarm board in a particular test, and 'alarm sample' is a number which identifies a specific sample of an alarm. For example, Ion-1-A-3-8 describes a specific model of an ionization alarm (Ion-1) located on alarm sensor board ' $A$ ' at position 3 (-A-3) and it is identified as the eighth sample of that particular model alarm. Since the calibrations were not dependent upon a position in a test and these test locations were randomly varied throughout the test series to minimize systematic errors due to specific alarm placement, the 'alarm board location' and 'alarm board position' are left blank in table 1. Appendix A includes full identification for every alarm in each of the tests.

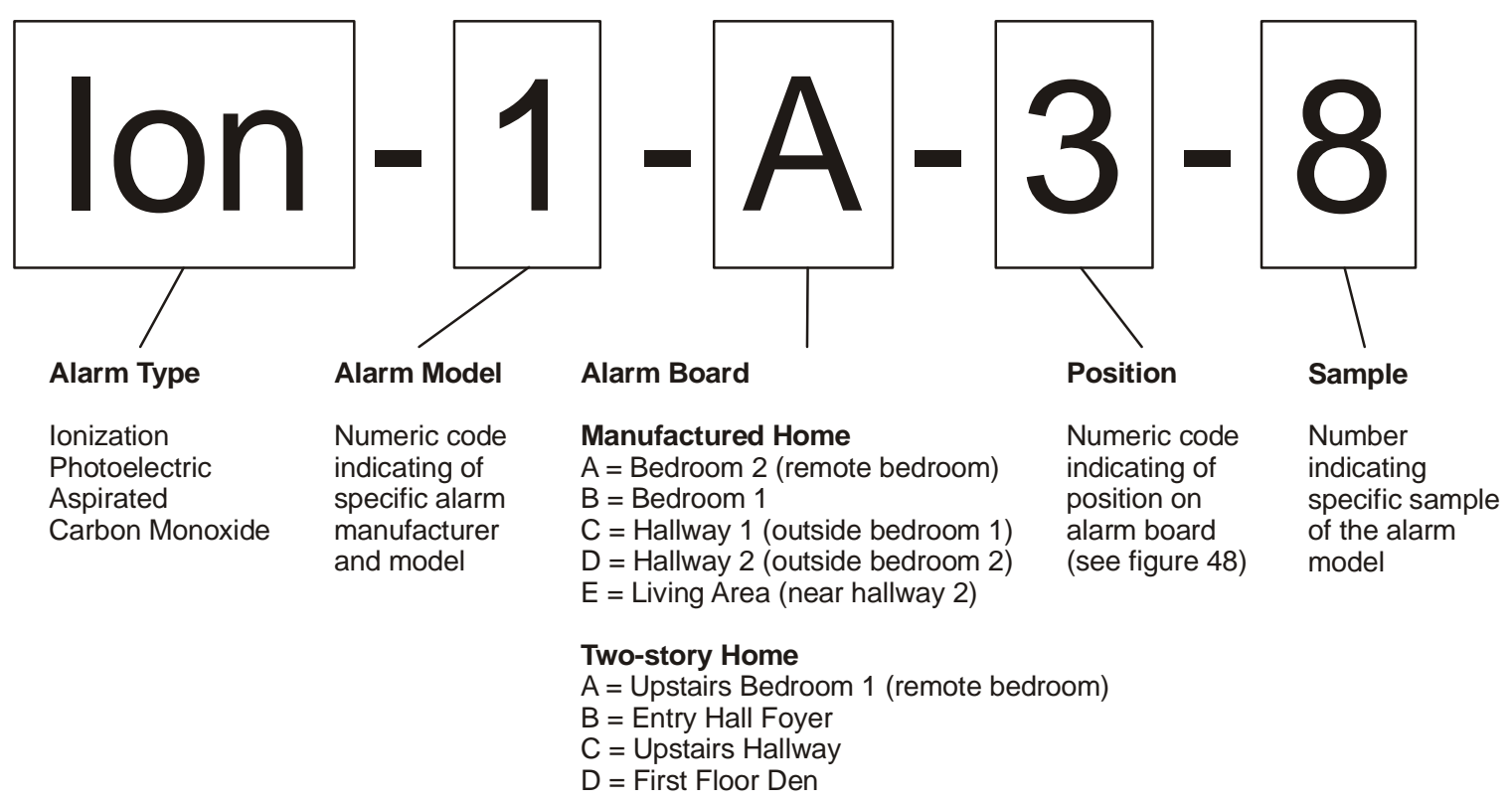

Figure 10. Alarm identification coding 


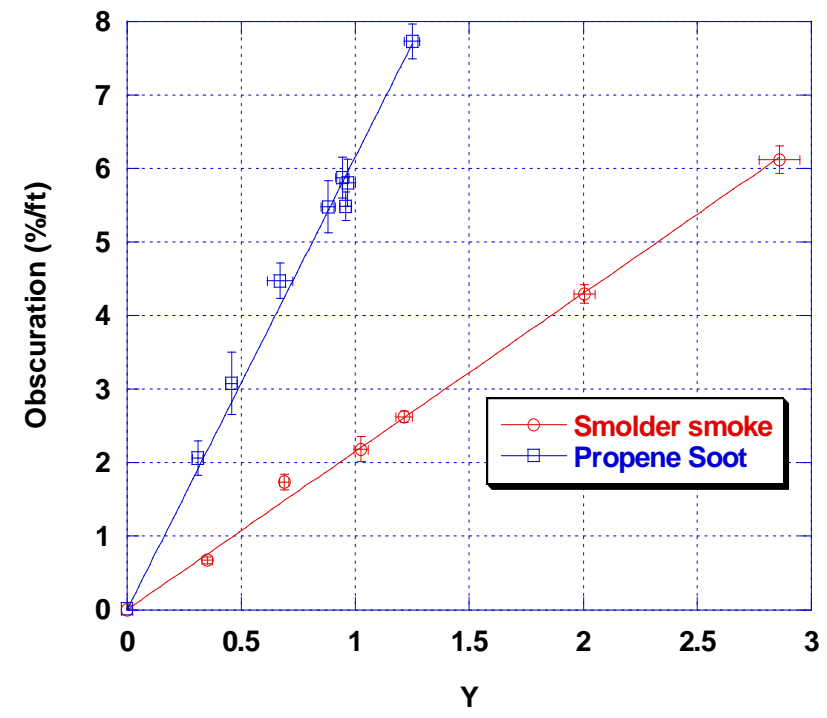

Figure 11. Calibration runs for Ion-1 alarm

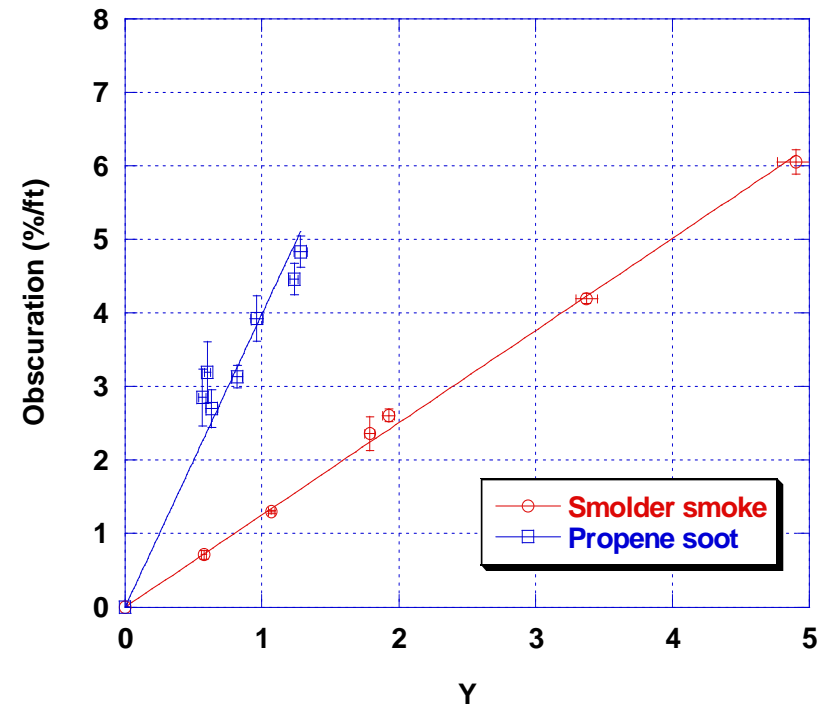

Figure 12. Calibration runs for Ion-3 alarm 


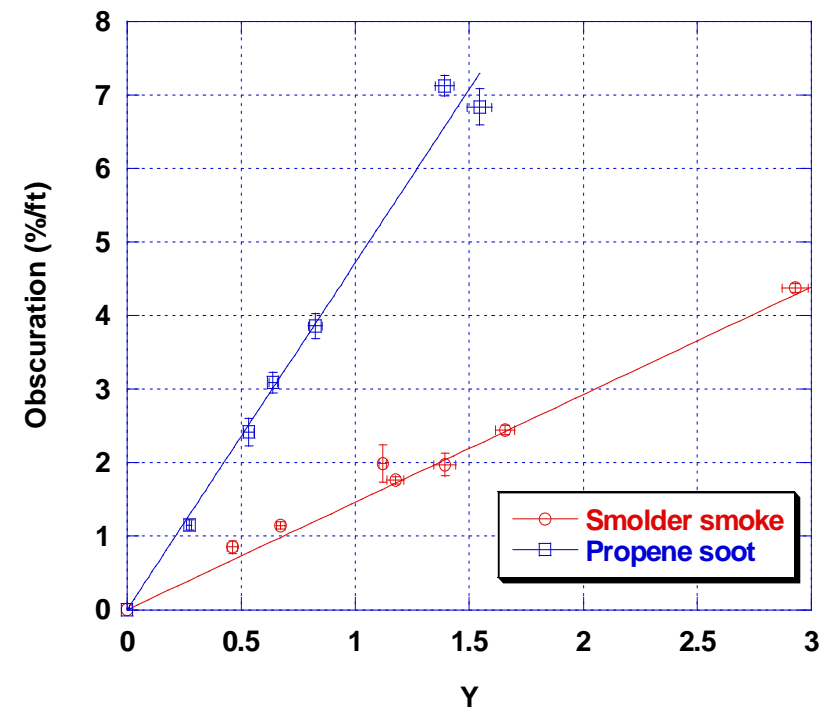

Figure 13. Calibration runs for Ion-4 alarm

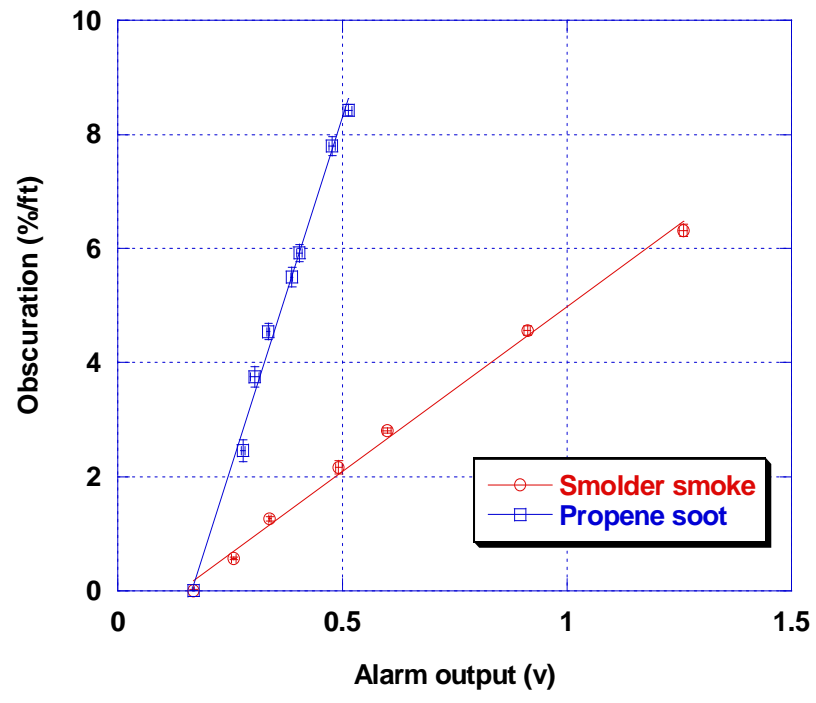

Figure 14. Calibration runs for Photo-1 alarm 


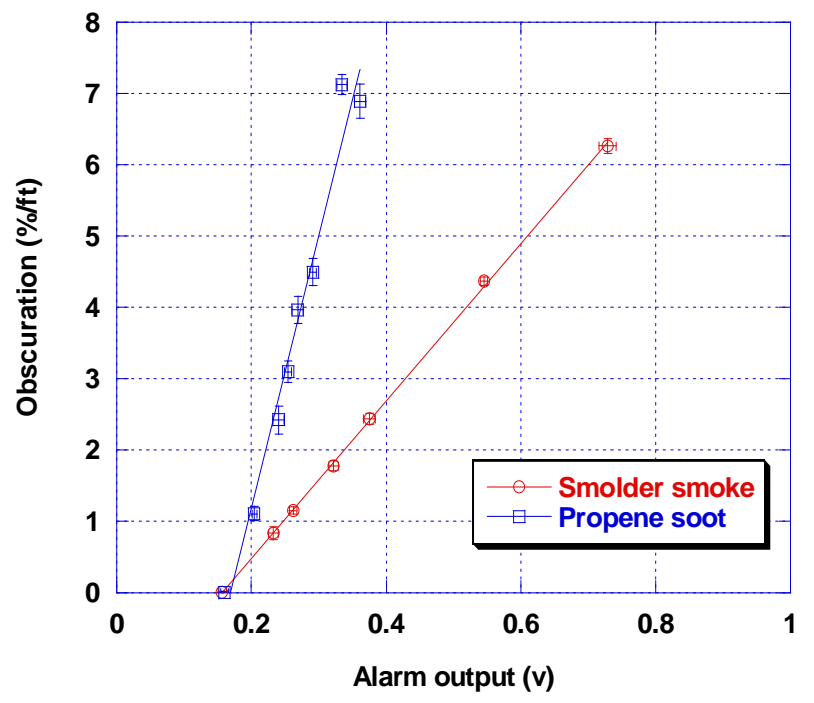

Figure 15. Calibration runs for Photo-2 alarm

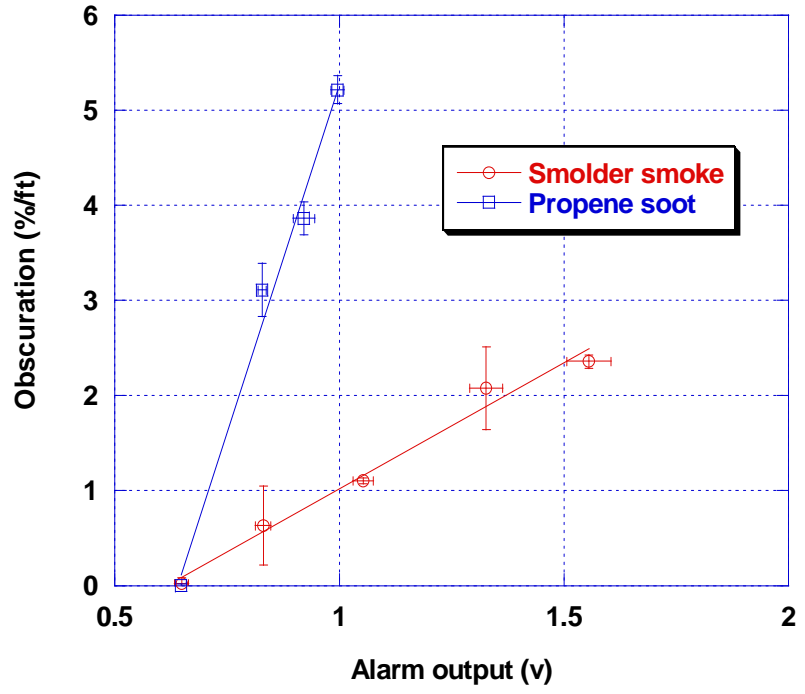

Figure 16. Calibration runs for ASP alarm 


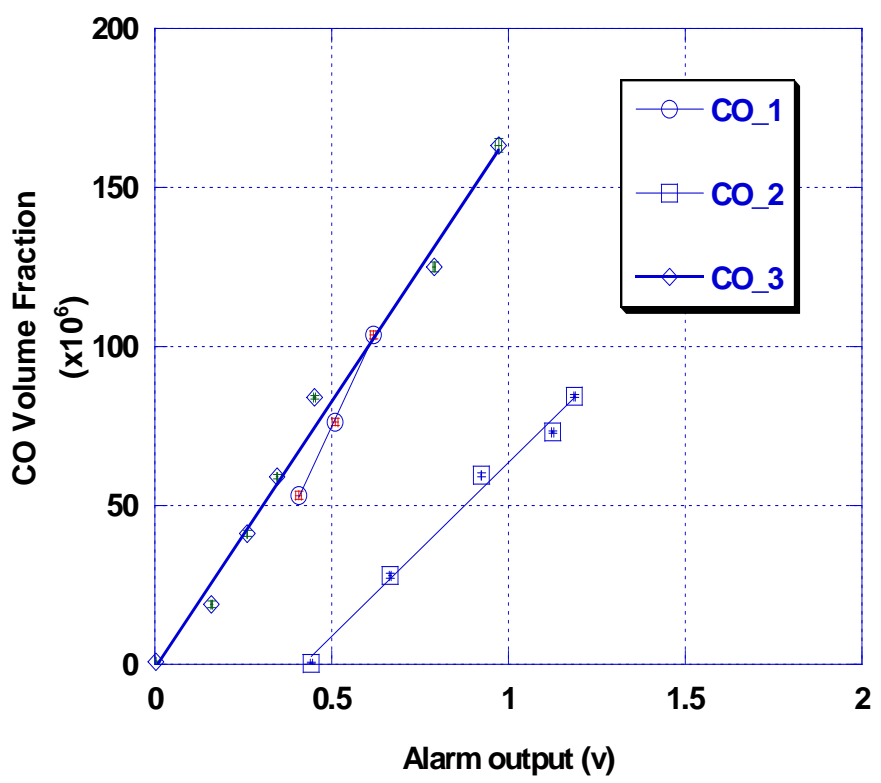

Figure 17. Calibration runs for $\mathrm{CO}$ alarms

\subsection{Evaluation of Unmodified Alarm Response}

Unmodified smoke alarms of the same models selected for modified alarms Ion-1, Ion-3 and Photo-1 were used in the test series at locations were thermal damage might potentially occur, and to supplement the analog output alarm results. Each of these alarms were powered with a previously unused $9 \mathrm{~V}$ alkaline battery and the alarm state was determined by monitoring the interconnect voltage signal. This signal changes from nominally $0 \mathrm{~V}$ to approximately $7 \mathrm{~V}$ to $9 \mathrm{~V}$ when the alarm threshold is met, with a $3 \mathrm{~s}$ delay in the response. No adjustment was made in the data for this delay.

In order to verify the manufacturer-specified alarm response, a number of alarms were tested after they had been used in fire tests. Cotton wick smoke, generated by the staged wick ignition device, was used to produce an increasing level of smoke in the FE/DE (the ignition protocol was different than the one used in the calibration of modified alarms.) After $30 \mathrm{~s}$ delay from the start of data logging, groups of wicks were ignited in series. The delay time between the application of power to the ignition coils was either $12 \mathrm{~s}$ or $30 \mathrm{~s}$, with between one and four wicks ignited at each step. The FE/DE fan speed was set to $12 \mathrm{~Hz}$ which produced a mean axial velocity of $0.16 \mathrm{~m} / \mathrm{s}$ at the center of the duct test section, and $50 \mathrm{~mm}(2 \mathrm{in})$ below the ceiling. Alarms pairs were placed in the FE/DE test section side by side and the interconnect voltage signal was recorded for each alarm. The nominal alarm sensitivities recorded on the alarms and the production range is given in table 2 along with the corresponding transmittance values over a $1.52 \mathrm{~m}(5.0 \mathrm{ft})$ path length. 
Table 2. Listed unmodified alarm sensitivities.

\begin{tabular}{|l|l|l|l|l|}
\hline Alarm Model & $\begin{array}{l}\text { Sensitivity } \\
\% / \mathrm{m}(\% / \mathrm{ft})\end{array}$ & $\begin{array}{l}\text { Sensitivity } \\
\text { Range } \\
\% / \mathrm{m}(\% / \mathrm{ft})\end{array}$ & Transmittance & $\begin{array}{l}\text { Transmittance } \\
\text { Range (hi/low) }\end{array}$ \\
\hline Ion-1 & $4.13(1.26)$ & $\pm 1.2( \pm 0.38)$ & 0.939 & $0.957 / 0.921$ \\
\hline Ion-3 & $4.23(1.29)$ & $\pm 1.7( \pm 0.51)$ & 0.937 & $0.962 / 0.913$ \\
\hline Photo-1 & $6.76(2.06)$ & $\pm 4.3( \pm 1.3)$ & 0.901 & $0.963 / 0.843$ \\
\hline
\end{tabular}

The following graphs show the upper laser transmittance and the alarm interconnect voltage for

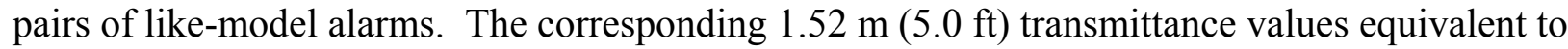
the model's listed sensitivity range are shown as dashed horizontal lines. At the test flow velocity, the smoke entry lag time should be short, thus detectors should alarm somewhere within the upper and lower transmittance boundaries.

Figures $18-27$ show the results for 12 Photo-1 photoelectric type alarms. Typically, 8 wicks ignited in pairs of two every 12 seconds was sufficient to produce enough smoke to set off the alarms. Disposable alarms Disp-10 and Disp-46 appeared to be less sensitive than the majority of the photoelectric alarms. Disp-10 alarmed when a group of 16 wicks was used (figure 27). The light transmittance at alarm was typically at the middle sensitivity value. Thus, the unmodified alarms can be expected to provide alarms consistent with the manufacturer's specification. Similar and consistent alarm criteria will be chosen for the measurements from the modified alarms. 


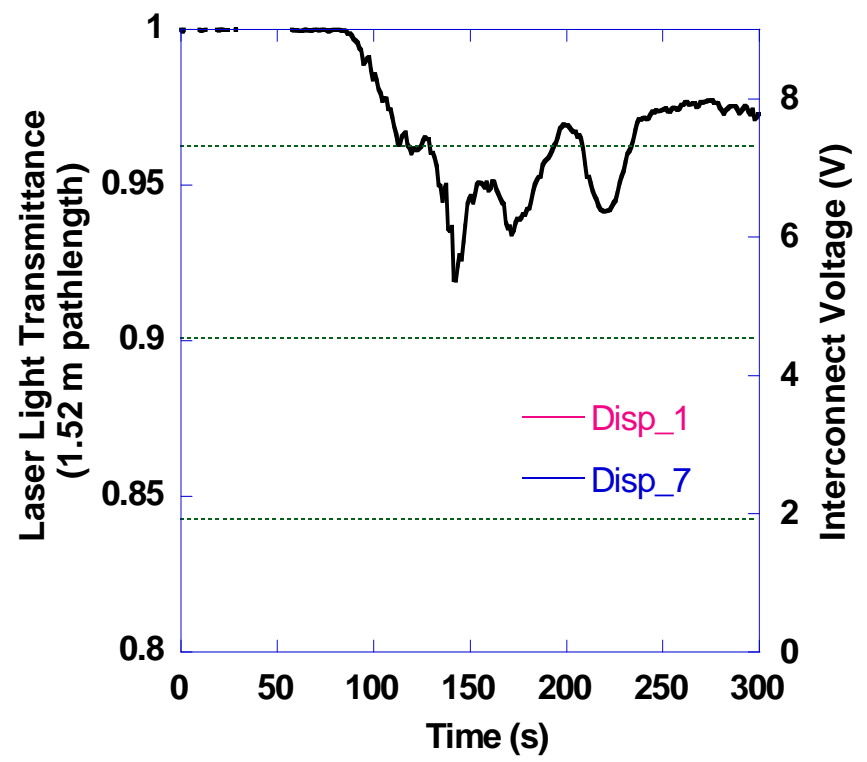

Figure 18. Photo-1, eight wicks - $12 \mathrm{~s}$ delay, Disp 1 and Disp 7 alarms

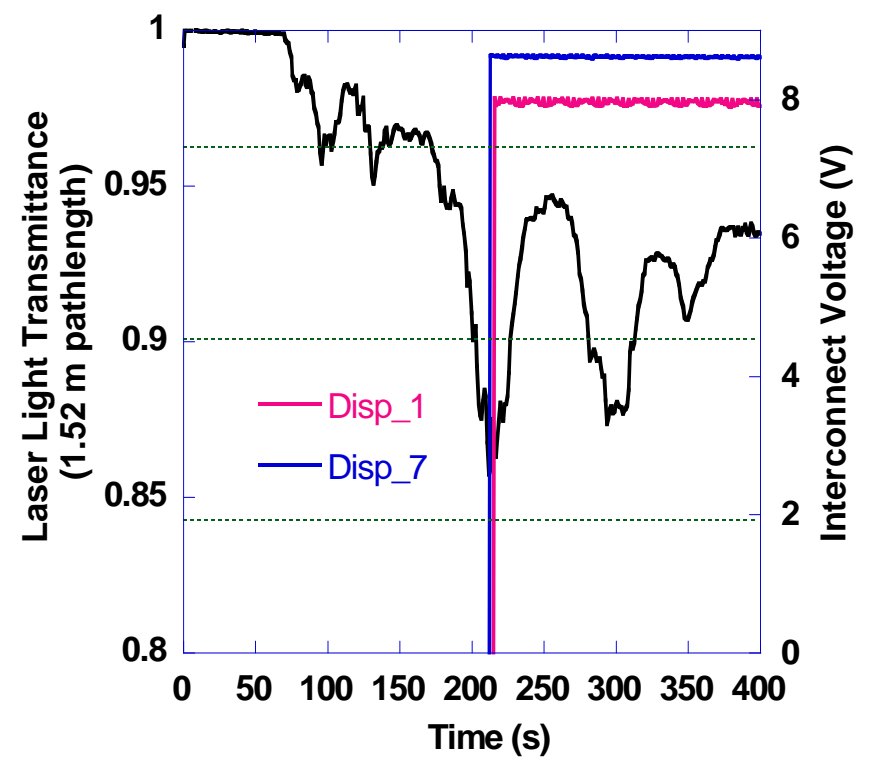

Figure 19. Photo-1, 16 wicks - 12 s delay, Disp 1 and Disp 7 alarms 


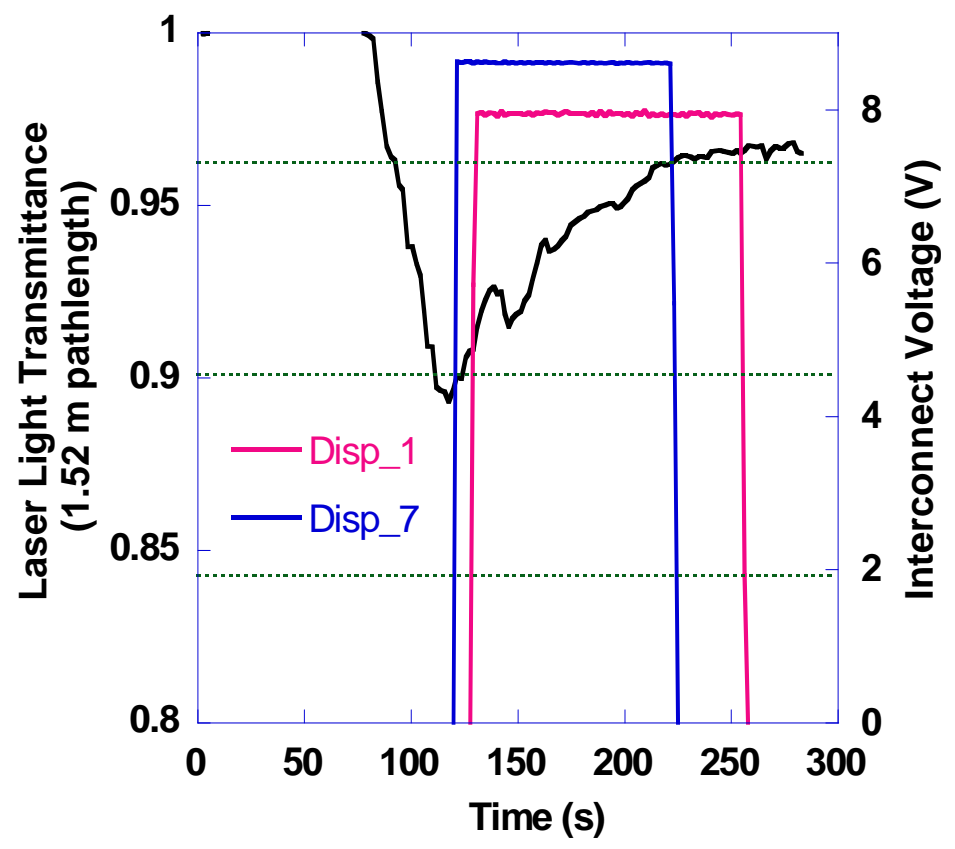

Figure 20. Photo-1, eight wicks - 12 s delay, Disp 1 and Disp 7 alarms

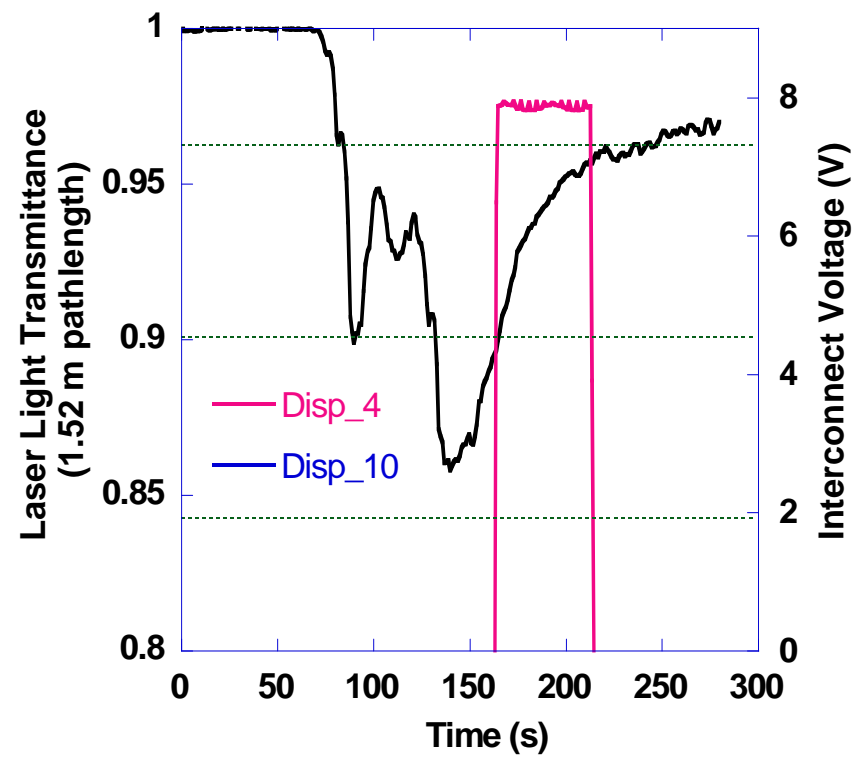

Figure 21. Photo-1, eight wicks - 30 s delay, Disp 4 and Disp 10 alarms 


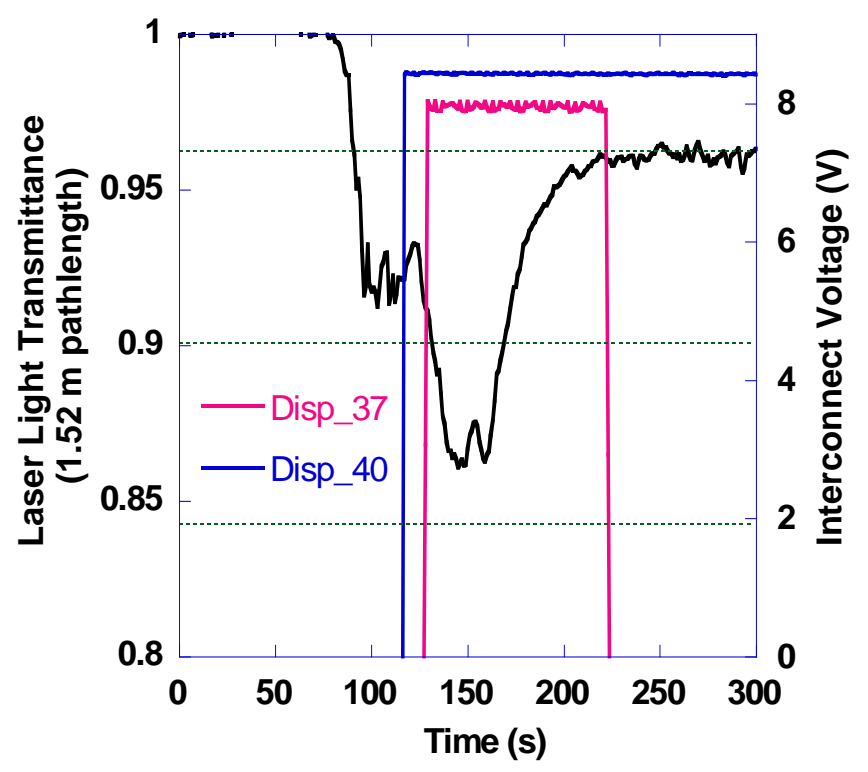

Figure 22. Photo-1, eight wicks - 12 s delay,

Disp 37 and Disp 40 alarms

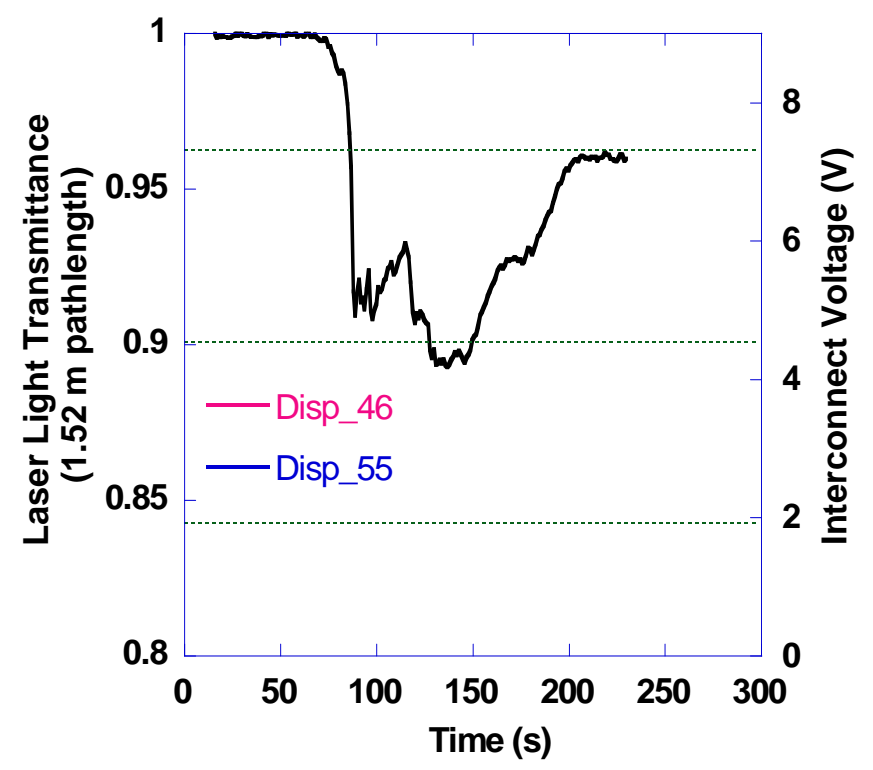

Figure 23. Photo-1, eight wicks, $12 \mathrm{~s}$ delay,

Disp 46 and Disp 55 alarms 


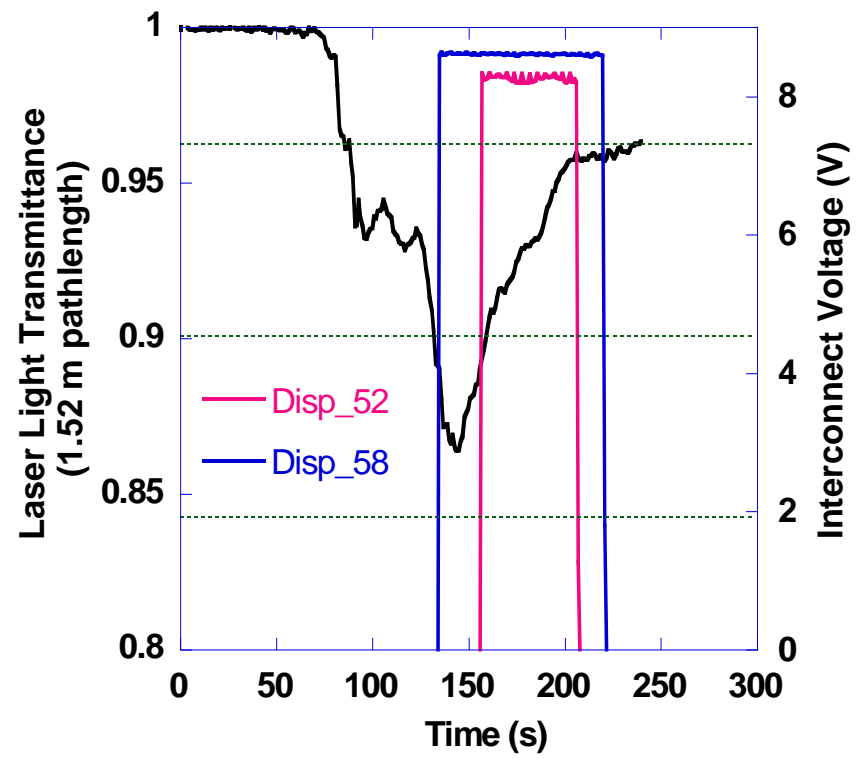

Figure 24. Photo-1, eight wicks - 12 s delay, Disp 52 and Disp 58 alarms

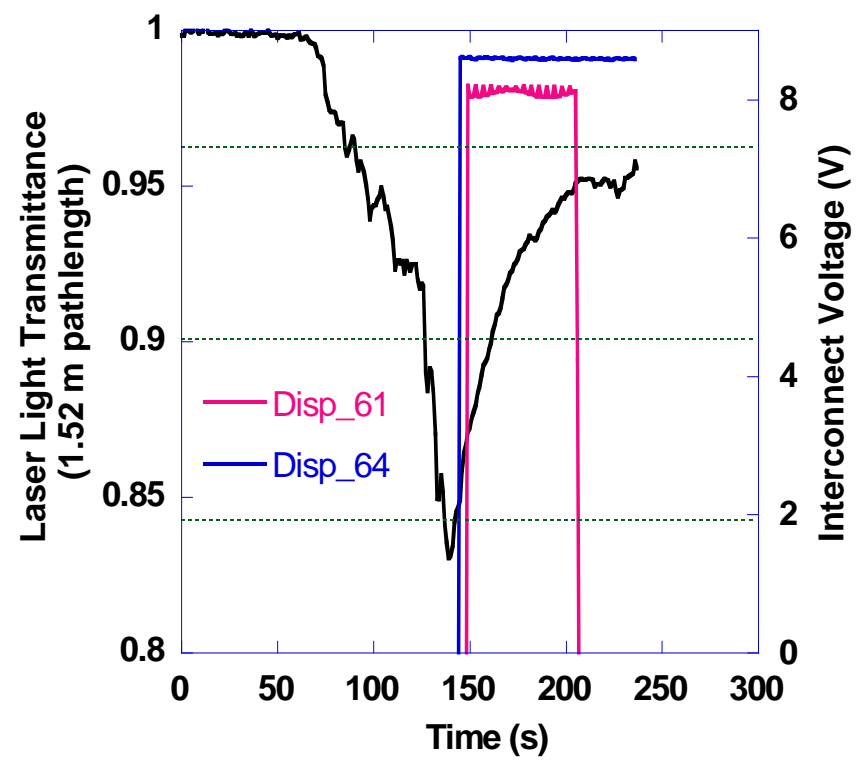

Figure 25. Photo-1, eight wicks - 12 s delay, Disp 61 and Disp 64 alarms 


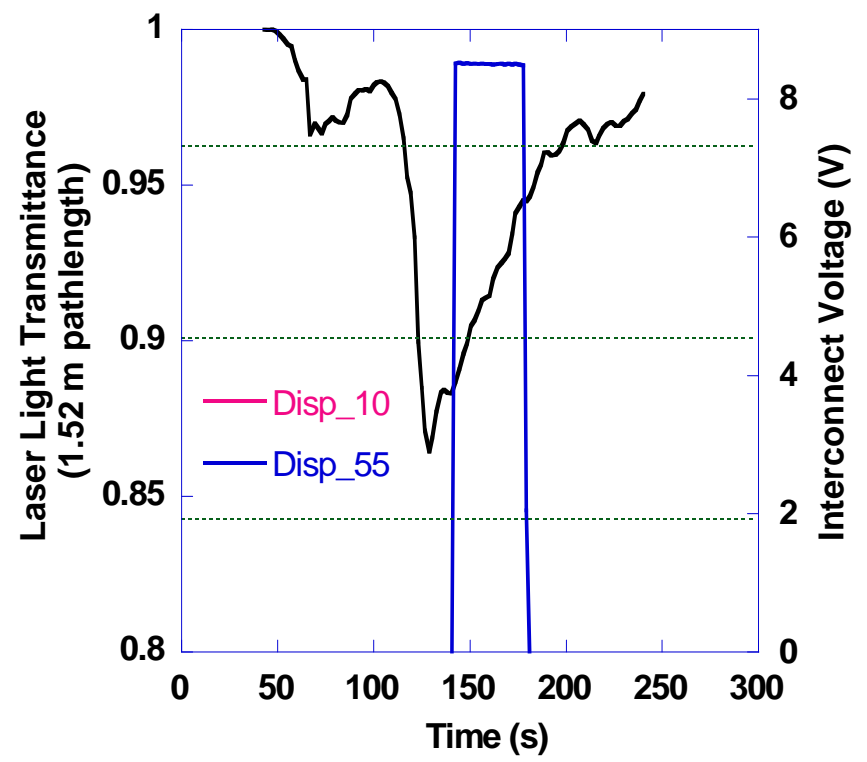

Figure 26. Photo-1, eight wicks - 12 s delay, Disp 10 and Disp 55 alarms

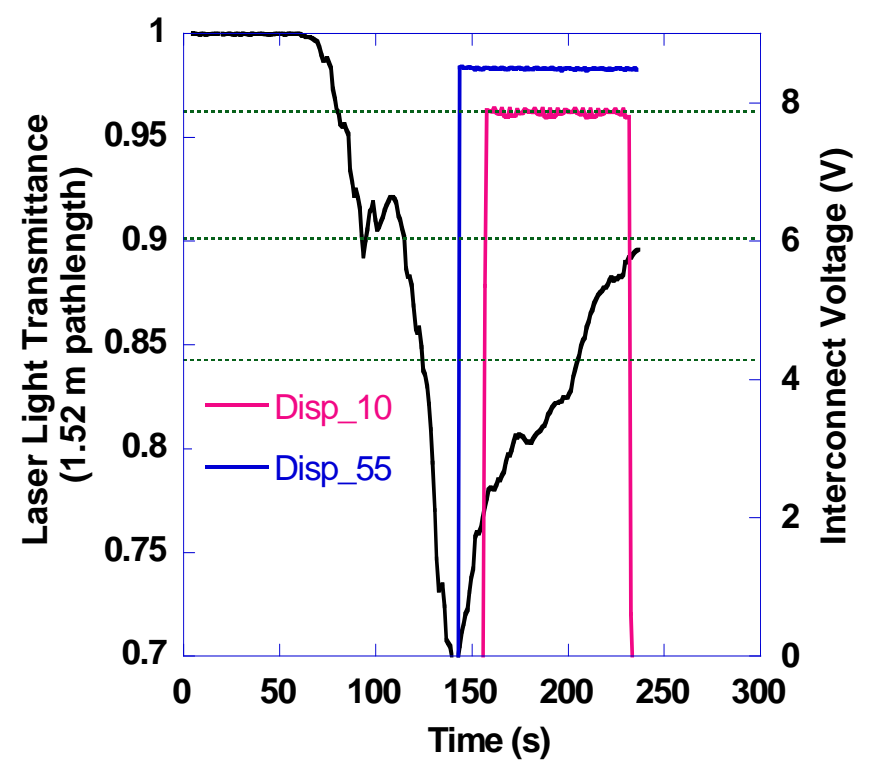

Figure 27. Photo-1, 16 wicks - 12 s delay,

Disp 10 and Disp 55 alarms 
Figures $28-37$ show the results for 12 Ion-1 ionization type alarms. Typically, 4-6 wicks ignited separately every $30 \mathrm{~s}$ was sufficient to produce alarms. The light transmittance at alarm was typically at the upper sensitivity value. On one occasion, the alarms were set off at transmittance levels above the upper sensitivity value.

Figures $38-47$ show the results for 12 Ion-3 ionization type alarms. Typically, 3 wicks ignited separately every $30 \mathrm{~s}$ was sufficient to produce alarms. The light transmittance at alarm was typically above the upper sensitivity value for this alarm.

For the most part, these tests confirm that each model of unmodified alarm yielded consistent sensitivity results with cotton wick smoke generated in the FE/DE. The two ionization alarms with the same nominal sensitivity showed a demonstrable difference in the actual sensitivities for the alarms tested here. Moreover, the tendency for the ionization alarms to appear more sensitive than the nominal sensitivity listed on the alarms raises some questions related to the differences between smoke generation techniques, smoke properties, and measurement techniques of the UL smoke box and the FE/DE.

Recall that the steady smoke generated in the calibration tests falls within the allowed bounds of light transmittance versus MIC output up to a transmittance value of approximately 0.9 (figure 44). The MIC picoammeter was not functioning during the verification tests, so several cotton smolder tests were performed after it was repaired to assure consistent results with earlier tests (figure 8). These results, shown in figure 48, follow the lower sensitivity limit quite closely until a transmittance value of 0.94 , then the FE/DE results deviate outside the UL limits for grey smoke. Additional tests were conducted to better quantify differences between the two ionization alarms relative to their response to the FE/DE smolder smoke. Separate tests using one, two, three, and four wicks were conducted. An Ion_1 and an Ion-3 alarm were placed in the test section, and the wicks were allowed to burn for several hundred seconds in order to achieve steady obscuration and MIC output values. The mean obscuration and MIC output along with the alarm state were obtained during the steady burning period. Table 3 shows the results for four tests. 


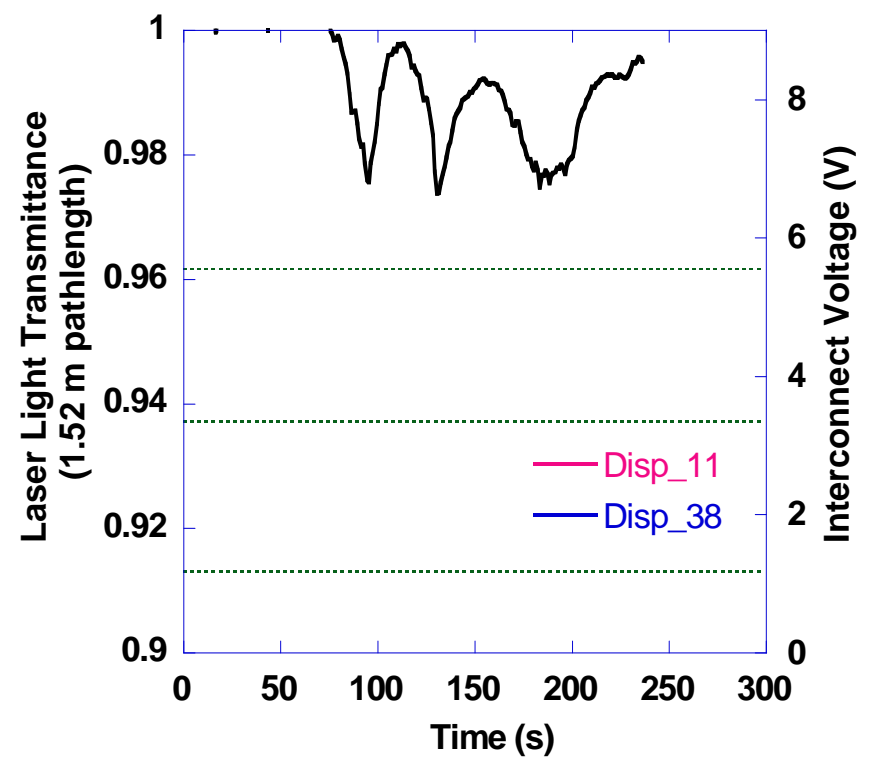

Figure 28. Ion-1, three wicks - 30 s delay,

Disp 11 and Disp 38 alarms

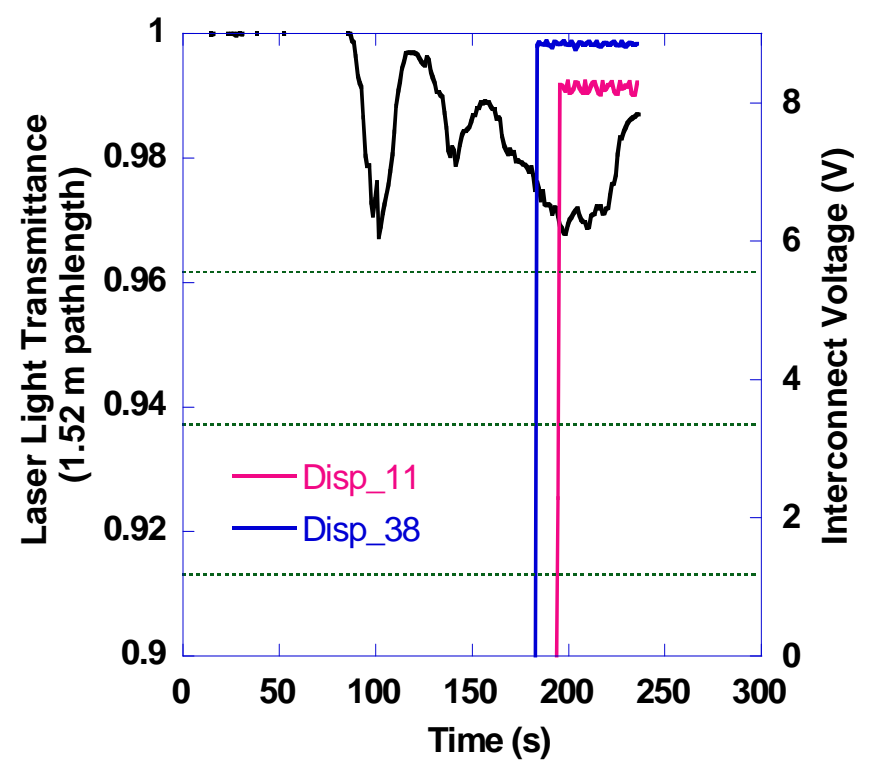

Figure 29. Ion-1, four wicks - $30 \mathrm{~s}$ delay,

Disp 11 and Disp 38 alarms 


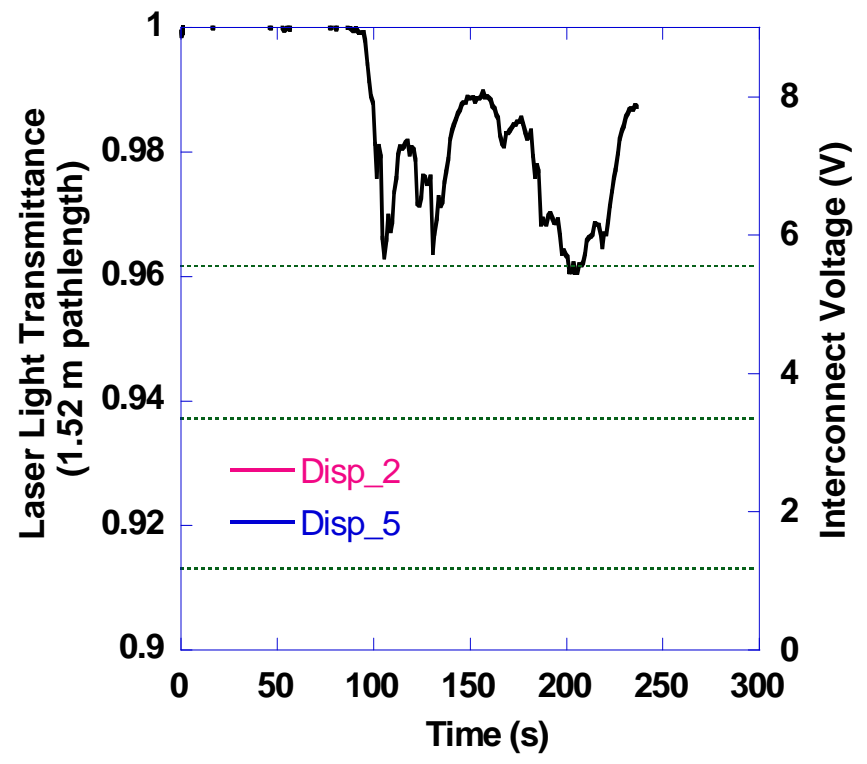

Figure 30. Ion-1, four wicks - 30 s delay, Disp 2 and Disp 5 alarms

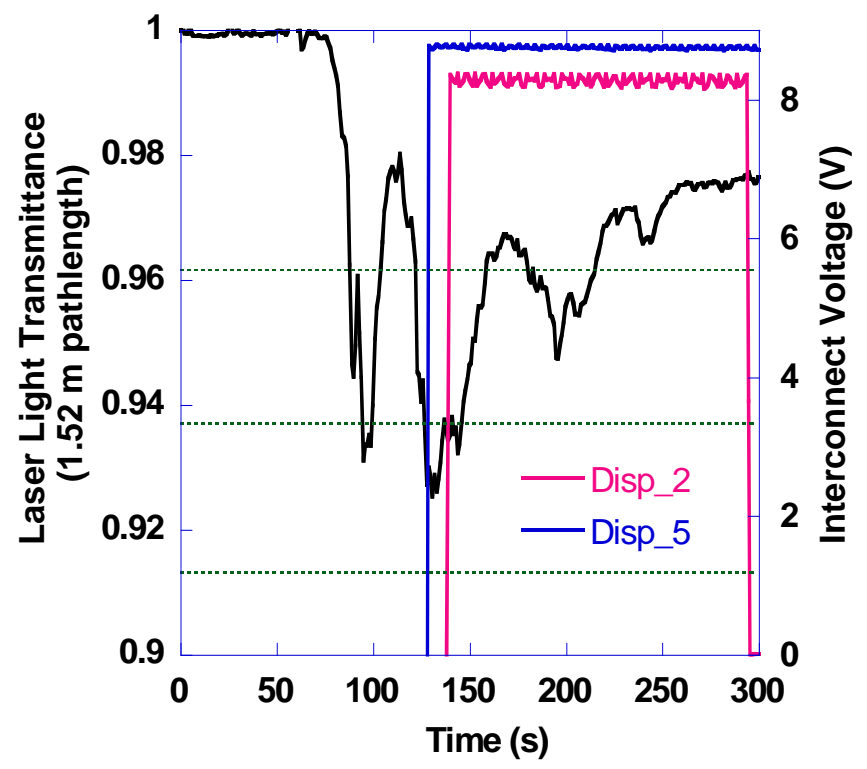

Figure 31. Ion-1, six wicks 12 s delay, Disp 2 and Disp 5 alarms 


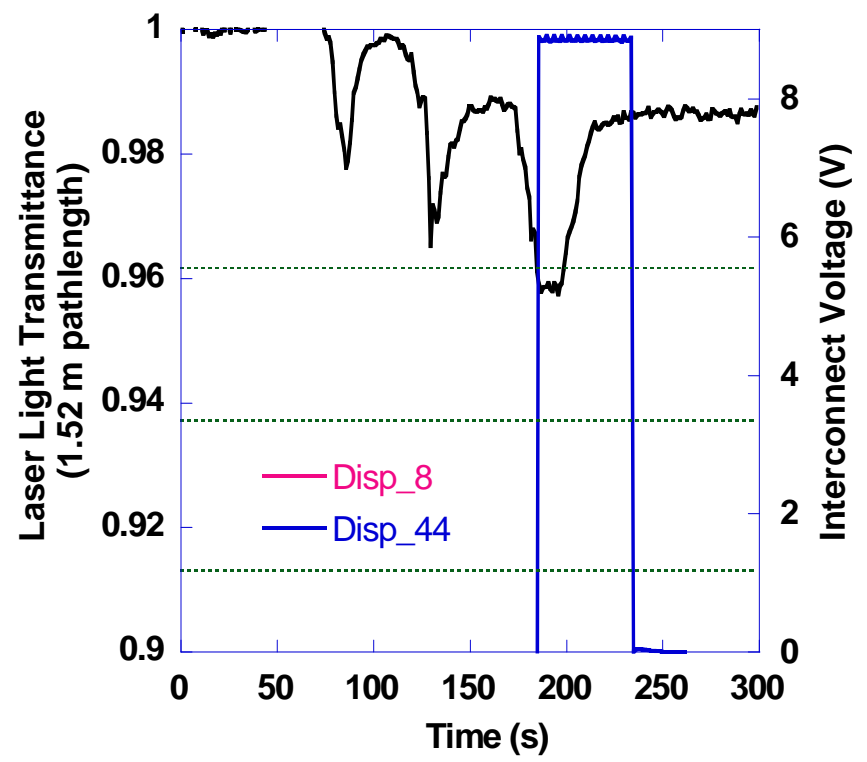

Figure 32. Ion-1, four wicks 30 s delay,

Disp 8 and Disp 44 alarms

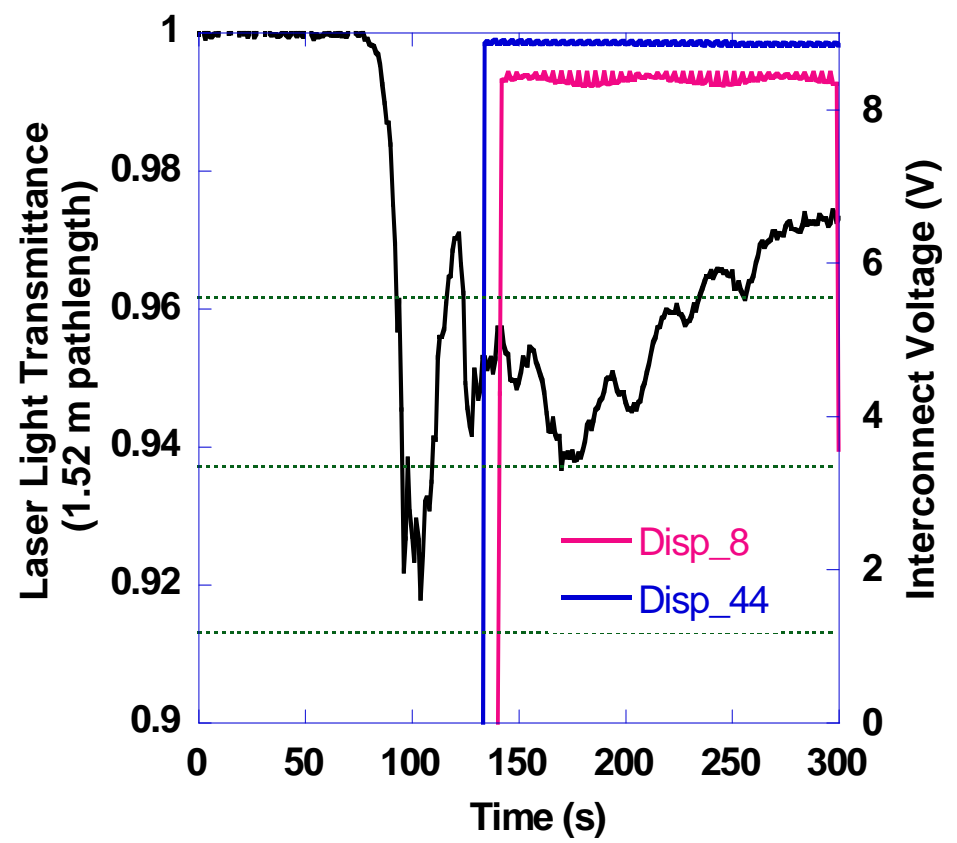

Figure 33. Ion-1, six wicks 12 s delay,

Disp 8 and Disp 44 alarms 


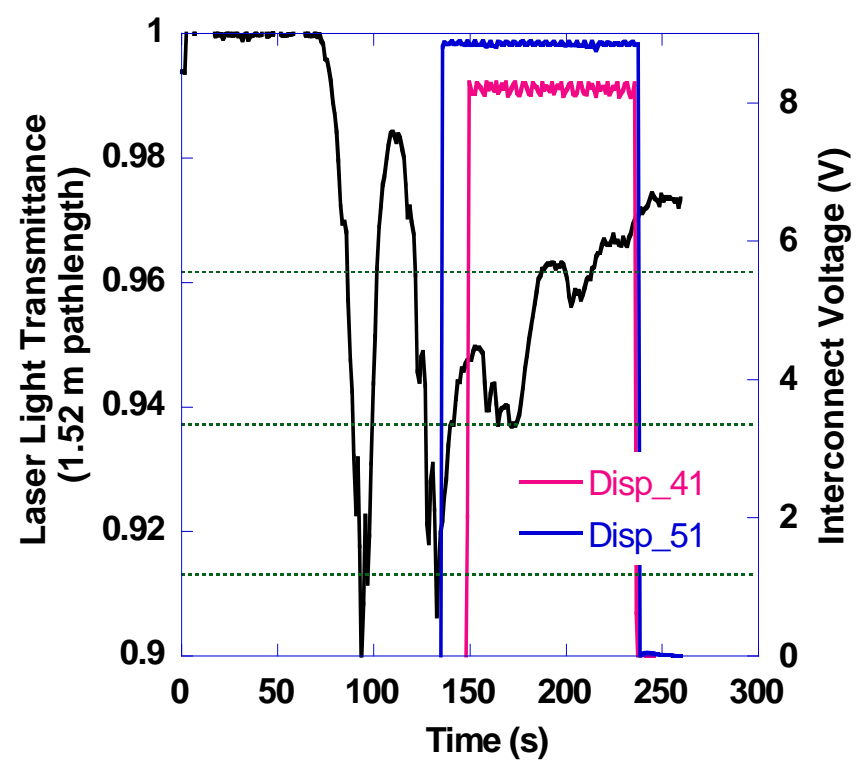

Figure 34. Ion-1, six wicks 12 s delay,

Disp 41 and Disp 51 alarms

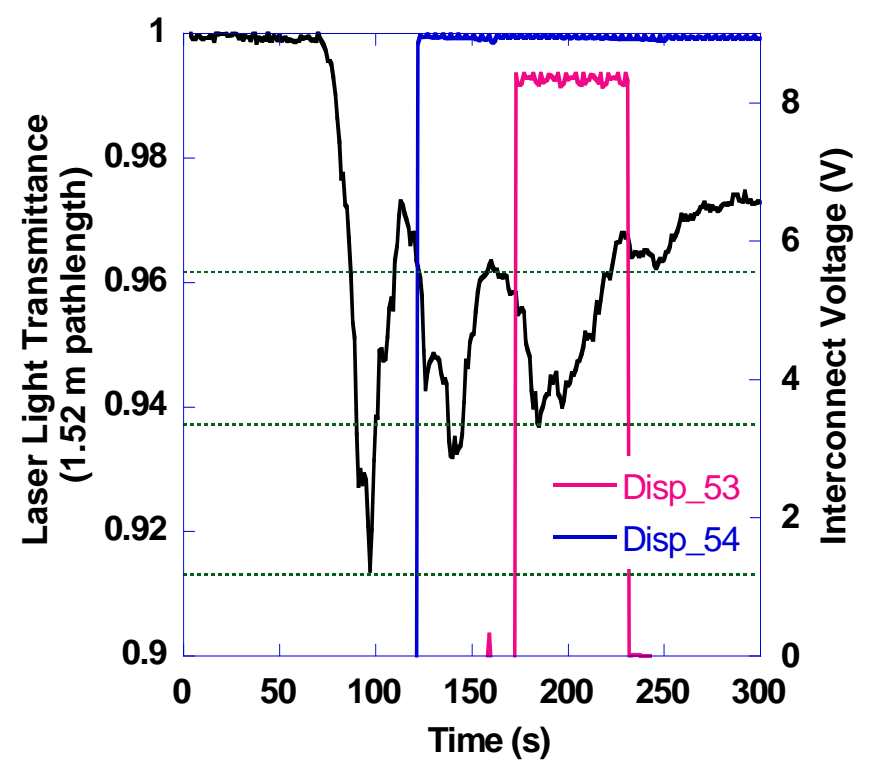

Figure 35. Ion-1, three wicks 12 s delay,

Disp 53 and Disp 44 alarms 


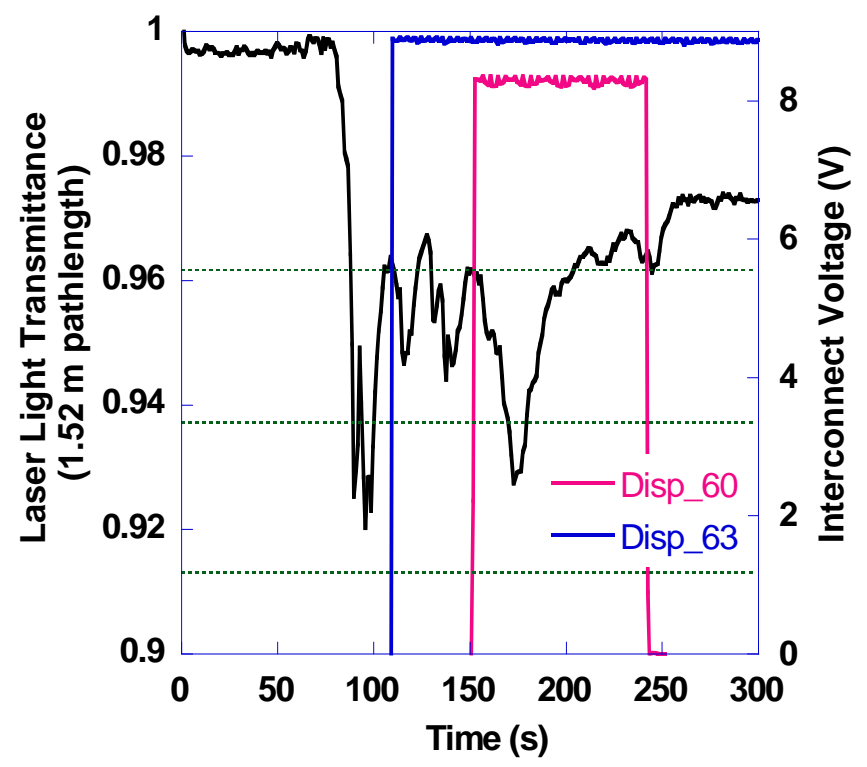

Figure 36. Ion-1, six wicks 12 s delay,

Disp 60 and Disp 63 alarms

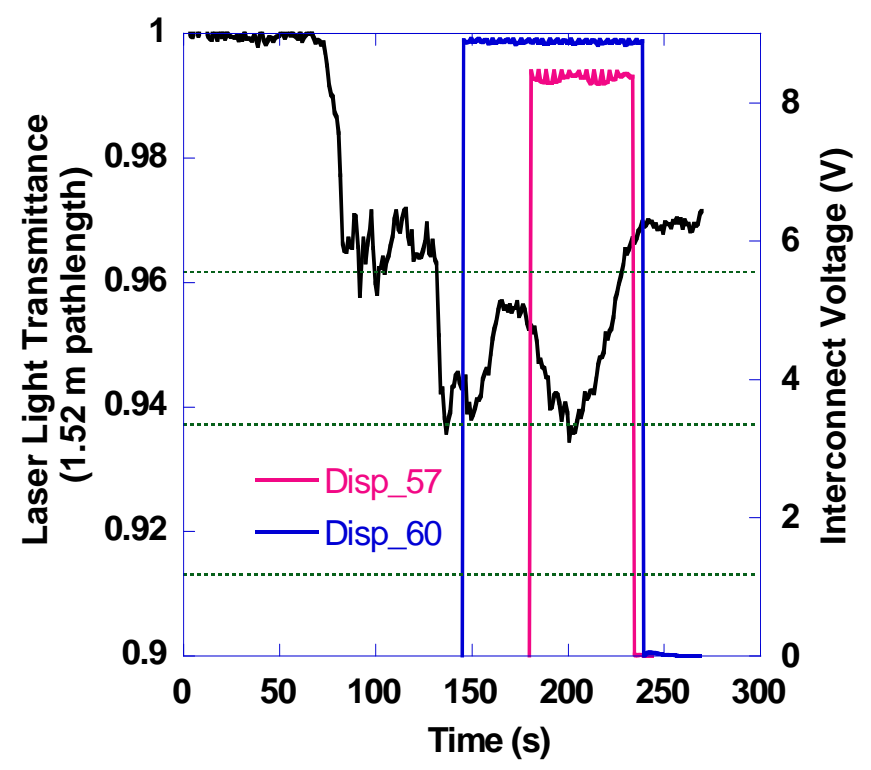

Figure 37. Ion-1, six wicks 12 s delay,

Disp 57 and Disp 60 alarms 


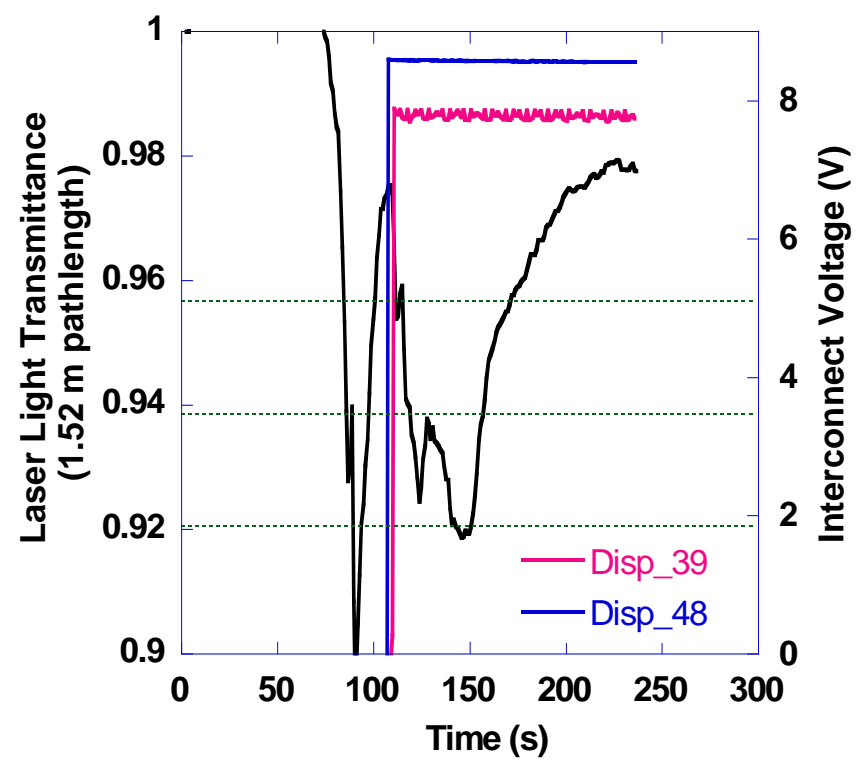

Figure 38. Ion-3, six wicks - 12 s delay, Disp 39 and Disp 48 alarms

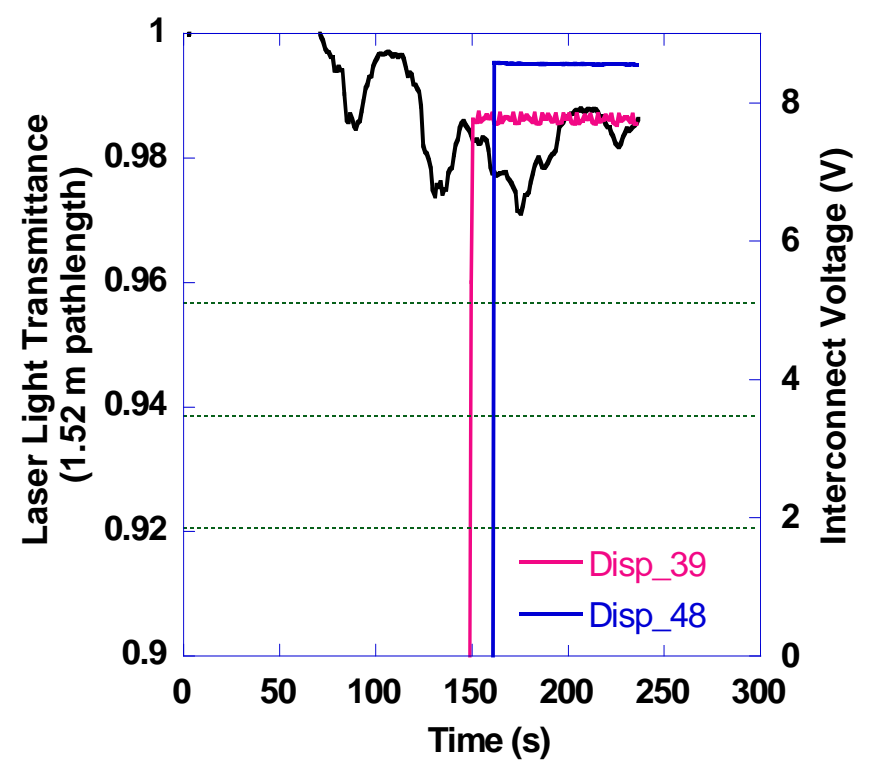

Figure 39. Ion-3, four wicks - 30 s delay, Disp 39 and Disp 48 alarms 


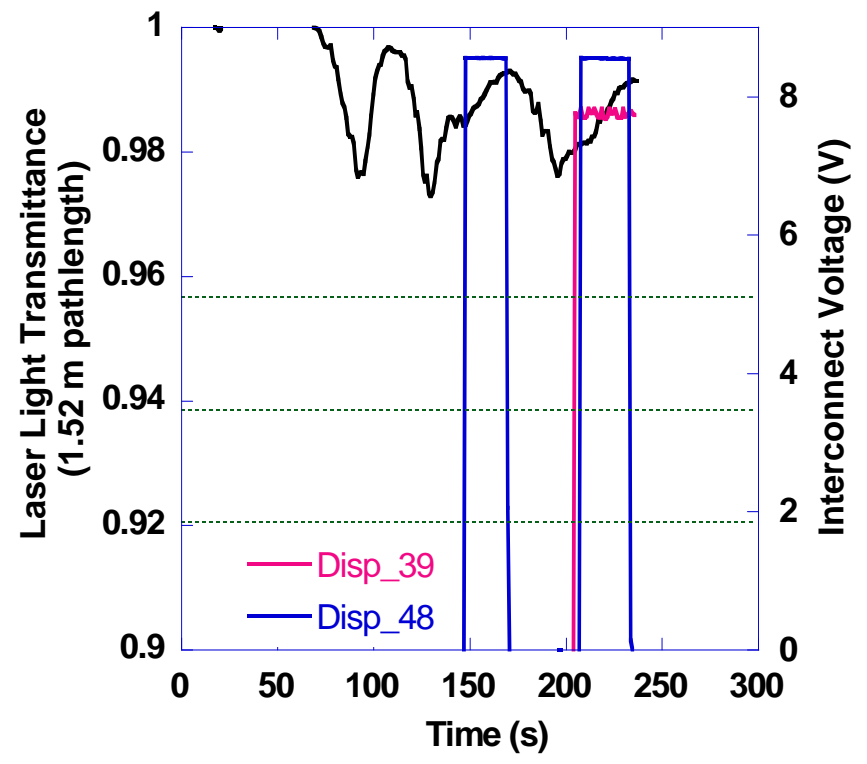

Figure 40. Ion-3, three wicks 30 s delay, Disp 39 and Disp 48 alarms

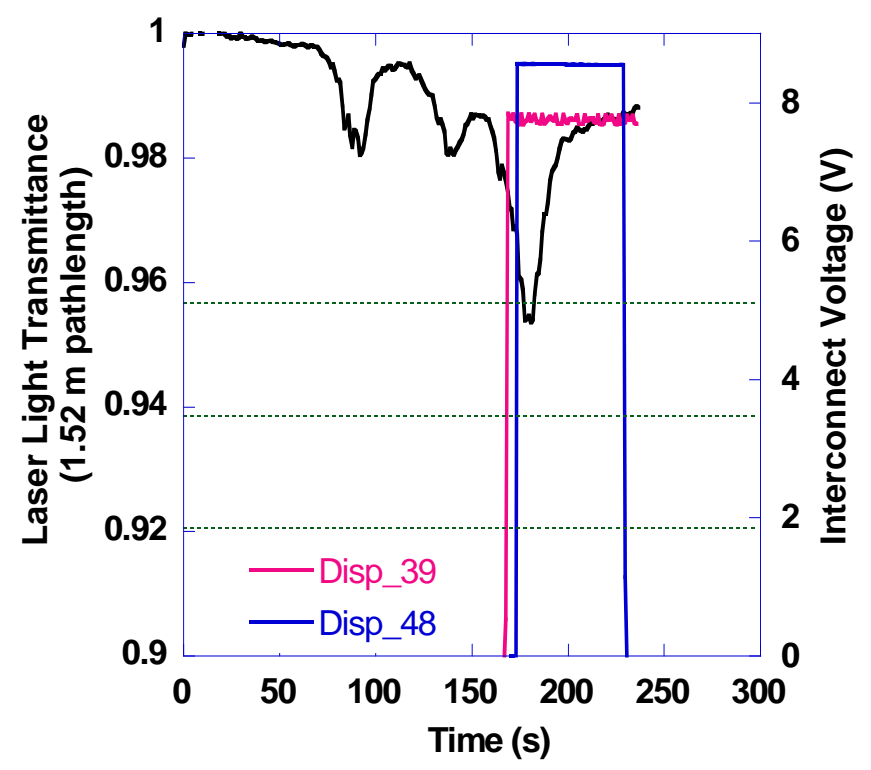

Figure 41. Ion-3, three wicks $30 \mathrm{~s}$ delay, Disp 39 and Disp 48 alarms 


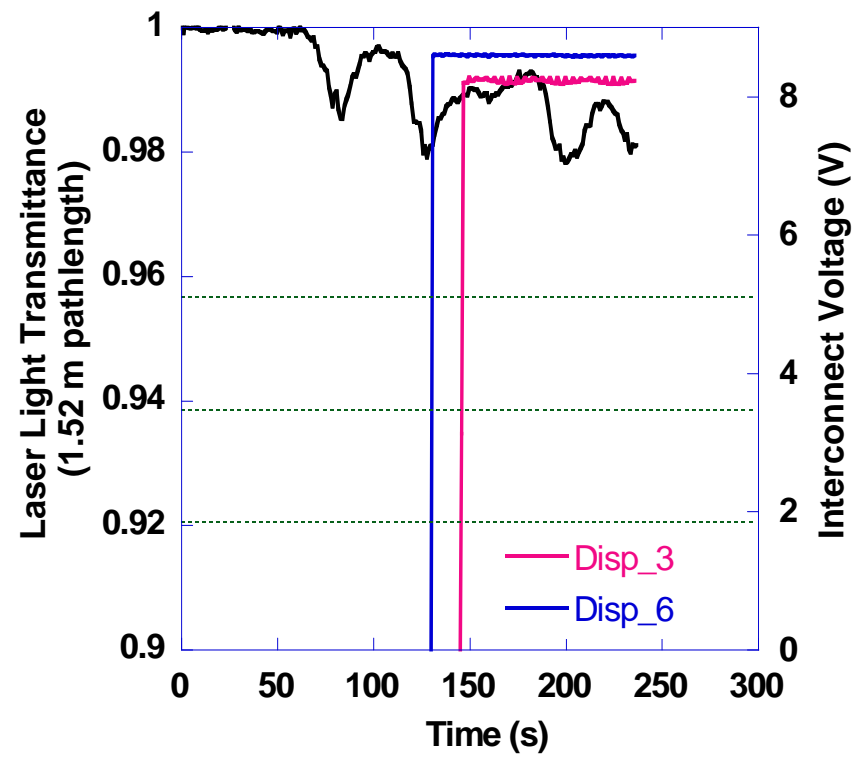

Figure 42. Ion-3, three wicks 30 s delay, Disp 3 and Disp 6 alarms

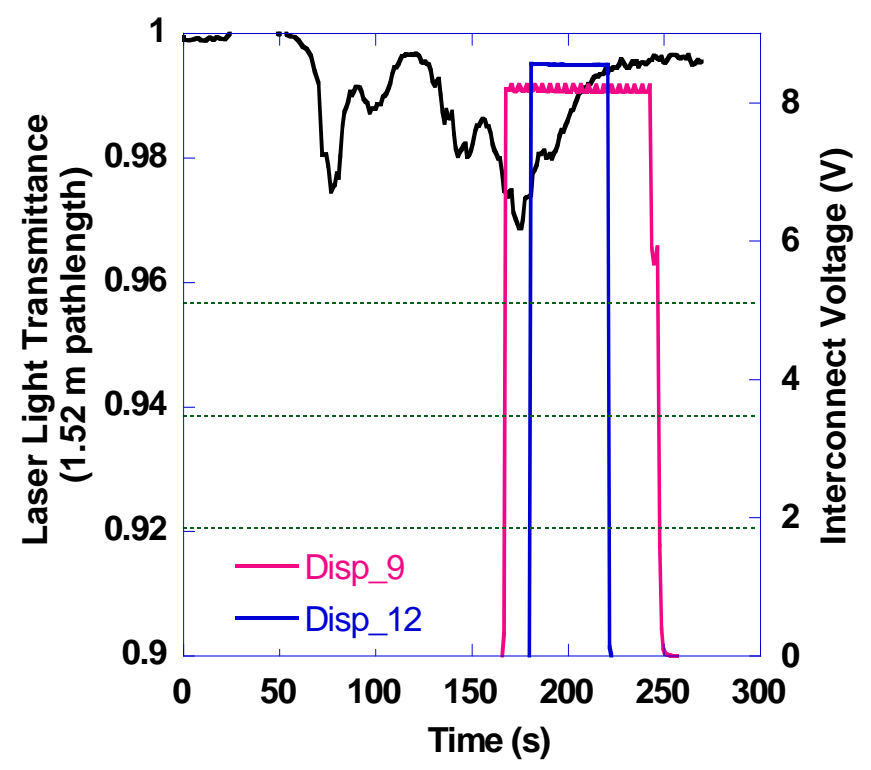

Figure 43. Ion-3, three wicks 30 s delay,

Disp 9 and Disp 12 alarms 


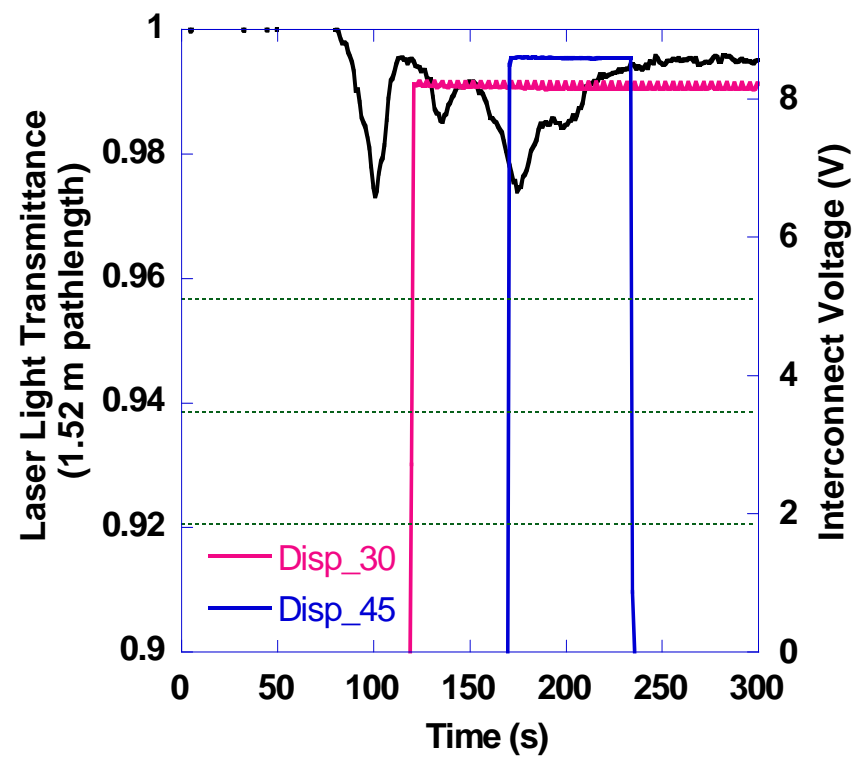

Figure 44. Ion-3, three wicks 30 s delay, Disp 30 and Disp 45 alarms

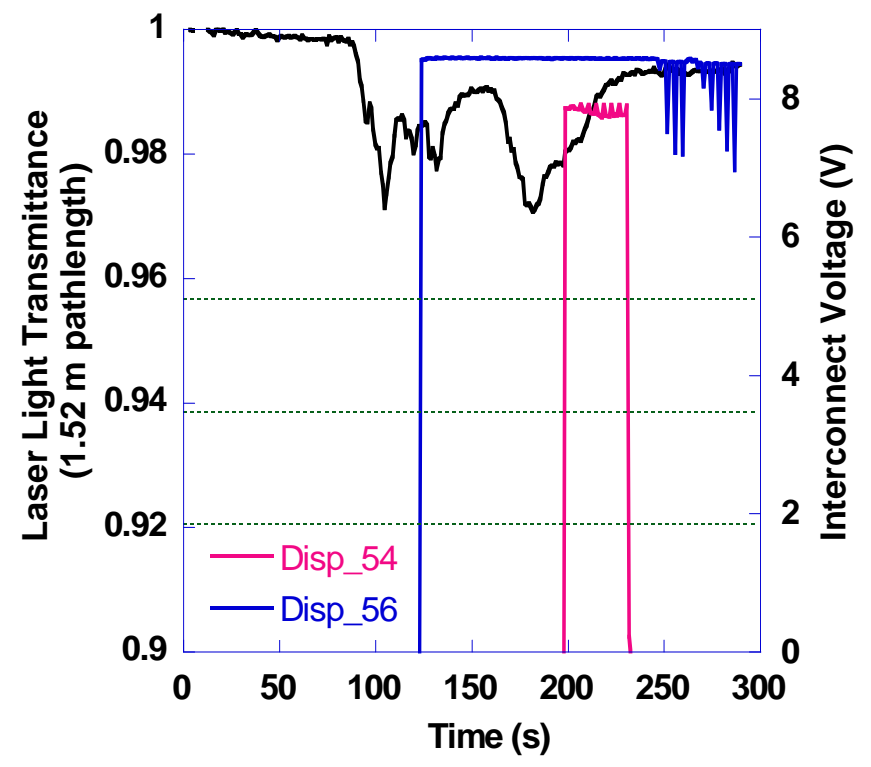

Figure 45. Ion-3, three wicks 30 s delay, Disp 54 and Disp 56 alarms 


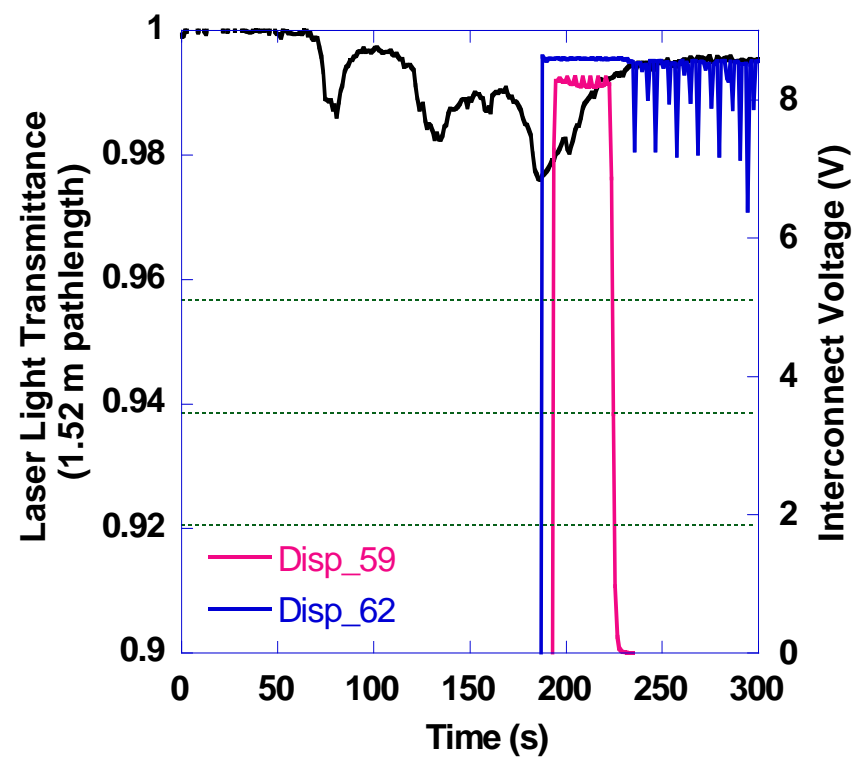

Figure 46. Ion-3, three wicks 30 s delay, Disp 59 and Disp 62 alarms

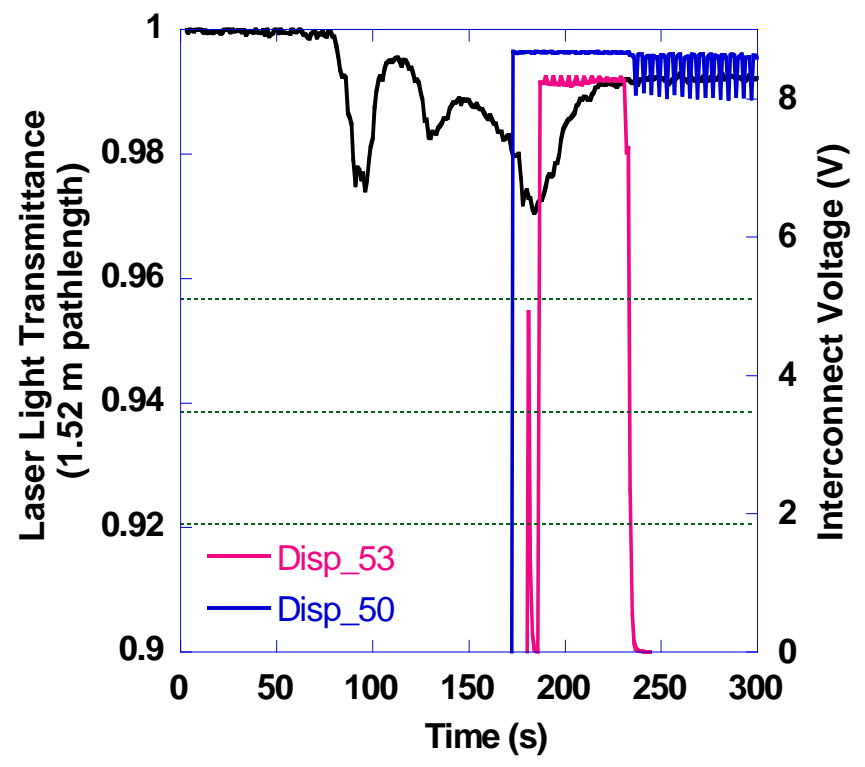

Figure 47. Ion-3, three wicks 30 s delay, Disp 53 and Disp 50 alarms 


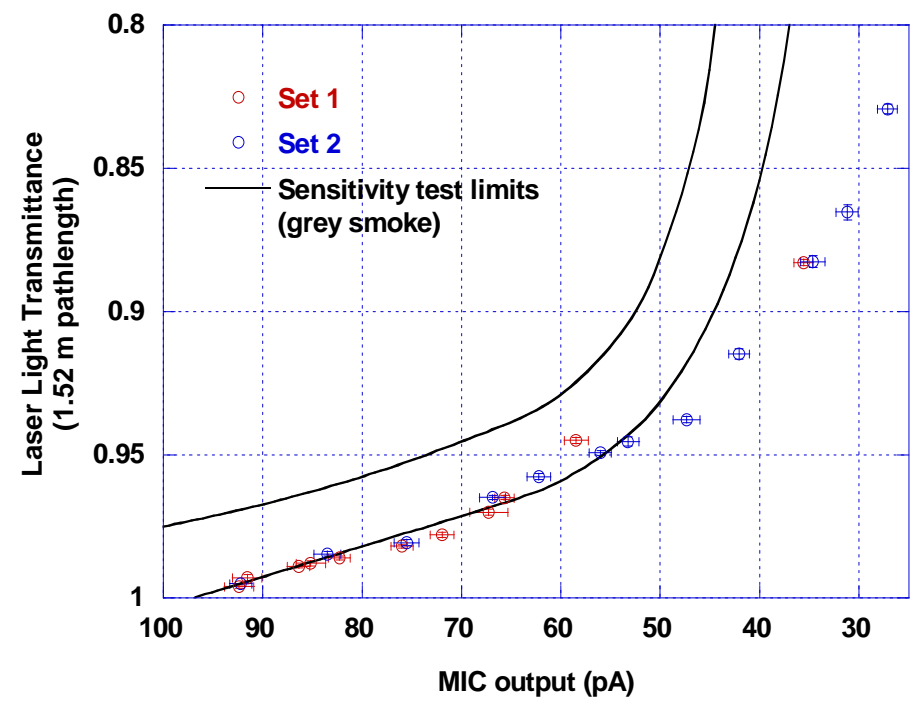

Figure 48. UL grey smoke (smolder smoke) sensitivity limits and FE/DE data

Table 3. MIC output and obscuration during tests of two ionization alarms

\begin{tabular}{|l|l|l|l|l|}
\hline $\begin{array}{l}\text { Number of } \\
\text { wicks }\end{array}$ & Alarm state & Transmittance & MIC output & $\begin{array}{l}\text { Obscuration } \\
(\% / \mathrm{m} / \% / \mathrm{ft})\end{array}$ \\
\hline 1 & $\begin{array}{l}\text { Ion-1 - off } \\
\text { Ion-3 - off }\end{array}$ & 0.985 & 85.2 & $1.0 / 0.31$ \\
\hline 2 & $\begin{array}{l}\text { Ion-1 - off } \\
\text { Ion-3 - on }\end{array}$ & 0.981 & 77.1 & $1.3 / 0.39$ \\
\hline 3 & $\begin{array}{l}\text { Ion- 1- off } \\
\text { Ion-3 - on }\end{array}$ & 0.965 & 68.3 & $2.4 / 0.72$ \\
\hline 4 & $\begin{array}{l}\text { Ion-1 - on } \\
\text { Ion-3 - on }\end{array}$ & 0.958 & 63.5 & $2.9 / 0.87$ \\
\hline
\end{tabular}

From the table above, an obscuration ranging from $1.3 \% / \mathrm{m}$ to $2.9 \% / \mathrm{m}(0.39 \% / \mathrm{ft}$ to $0.87 \% / \mathrm{ft})$ was necessary for the devices to trip an alarm state. To insure the device would be expected to alarm (to insure both devices trip an alarm state would require an obscuration of $2.9 \% / \mathrm{m}$ or $0.87 \% / \mathrm{ft})$, an approximate minimum value of $2.8 \% / \mathrm{m} \pm 1.6 \% / \mathrm{m}(0.85 \% / \mathrm{ft} \pm 0.5 \% / \mathrm{ft})$ was used as representative of this range. This would correspond to MIC output values of $80.6 \mathrm{pA}$, $59.5 \mathrm{pA}$, and $50.9 \mathrm{pA}$ for high, nominal and low sensitivity. 


\subsection{Effect of Sensor Board Location on Response}

The test board layout was a compromise between placing multiple alarms at approximately the same physical location, while limiting the local flow effects the crowding of alarms will have and the biasing of sensor response due to position. The layout of the test board is shown in figure 49. Since the primary purpose of this study was the performance of smoke alarms, preference was given to the positioning of the smoke alarms and positions 1-5 were reserved for smoke alarms, while positions $6-8$ were reserved for $\mathrm{CO}$ alarms. The arrow indicates the board orientation in the expected resultant flow during the fire tests. It is obvious from the layout that positions 4 and 5 may experience a flow blocking effect.

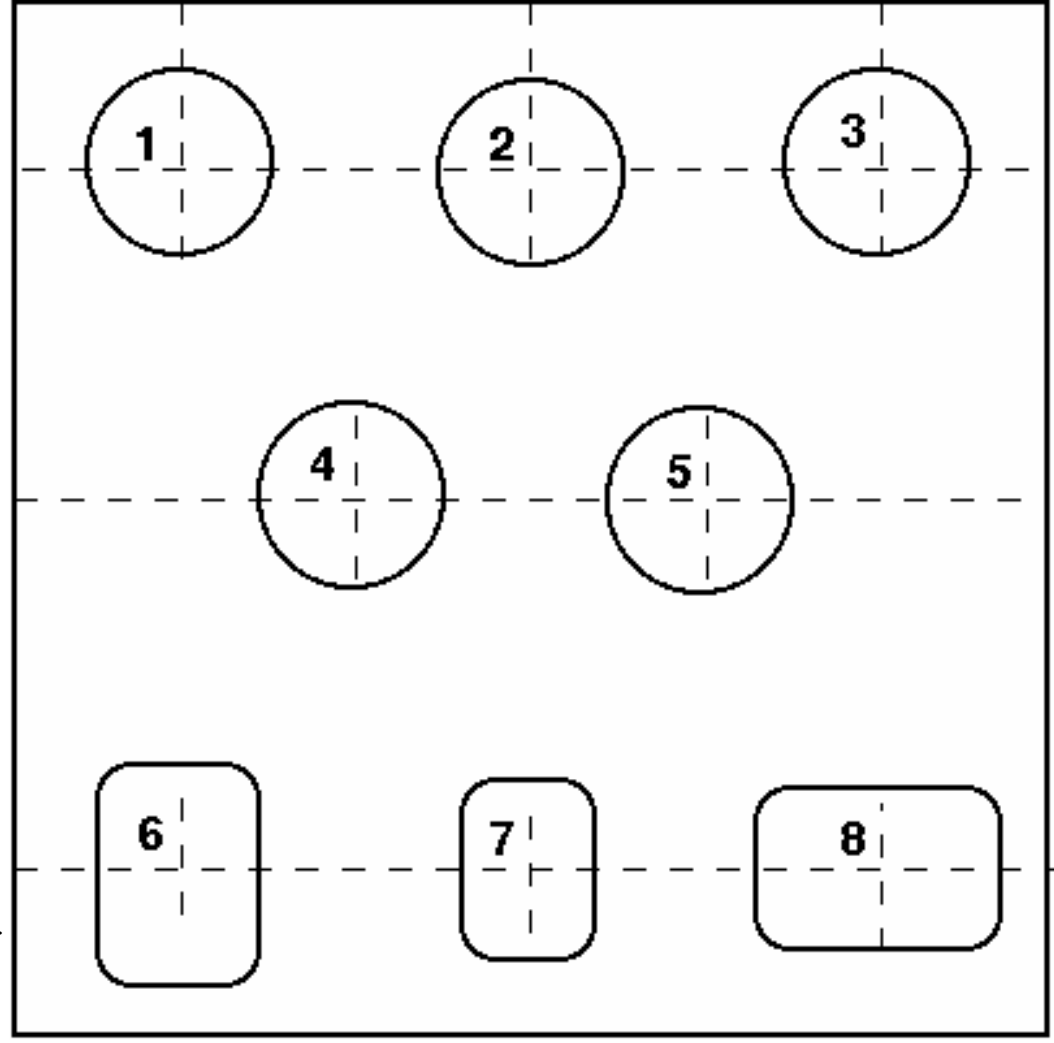

A series of tests were carried out in the FE/DE to quantify the Figure 49. Test board layout effects of the test board position of a smoke sensor on its response for 6 flow conditions. The test board was mounted on the ceiling of the FE/DE duct at the test section approximately $20 \mathrm{~cm}$ from the laser beam. Duplicate tests were performed on two different board configurations at 3 separate FE/DE fan speeds with and without a baffle placed upstream of the test section. The baffle is a $10 \mathrm{~cm}$ high by $20 \mathrm{~cm}$ long steel plate oriented across duct flow. Its function is to promote velocity fluctuations that are similar to those observed in room flows in section 6 . Table 4 shows the mean axial velocity at a height $5 \mathrm{~cm}$ below the ceiling and standard deviation for each configuration. Figures $50-52$ show the results for the three fan speeds with and without the baffle present. 


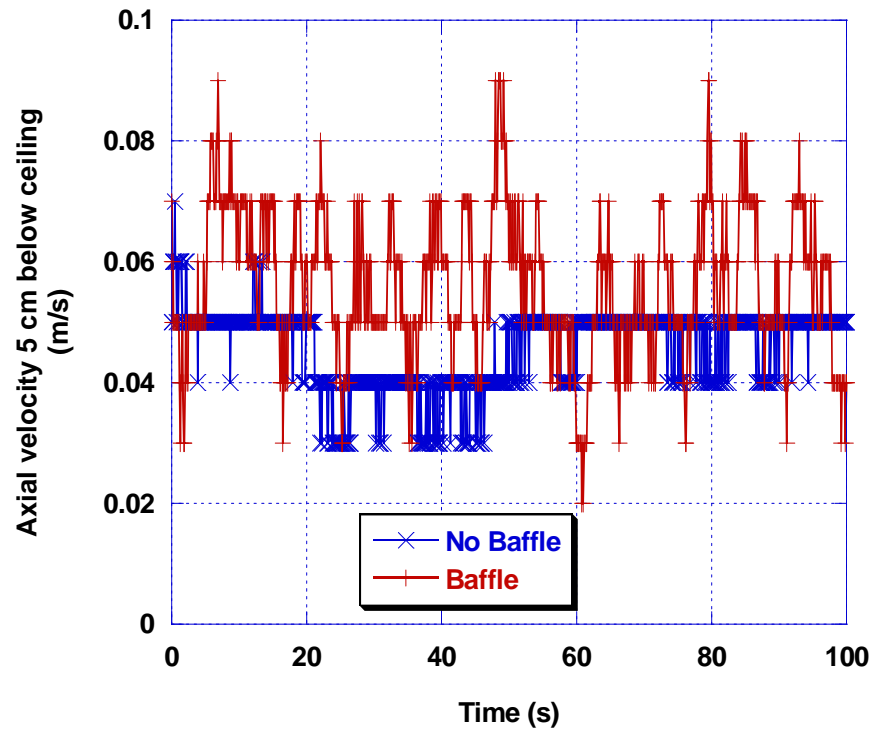

Figure 50. Axial velocity at a fan speed of $5 \mathrm{~Hz}$

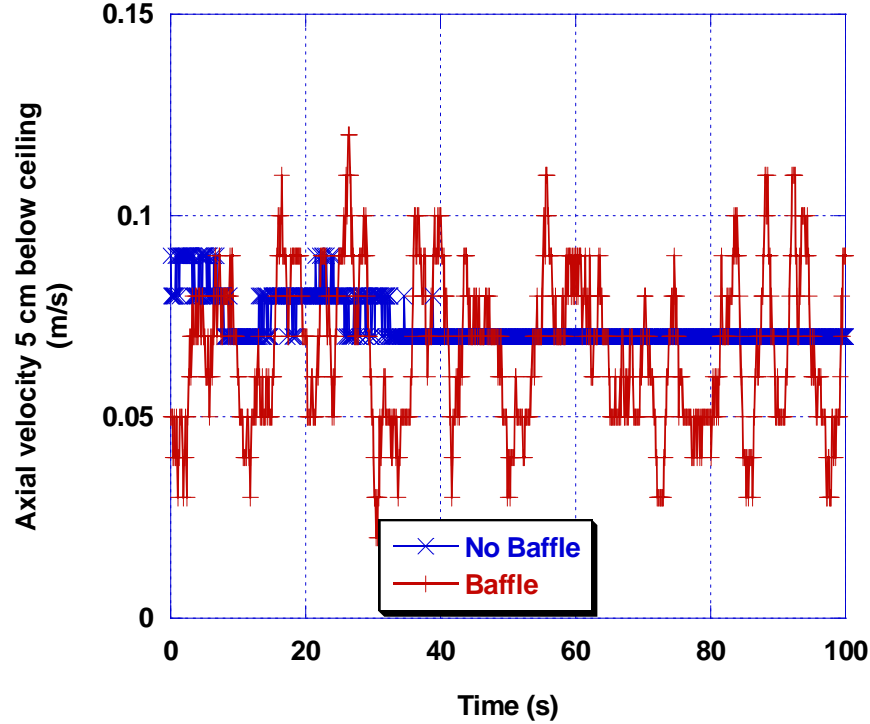

Figure 51. Axial velocity at a fan speed of $7 \mathrm{~Hz}$ 


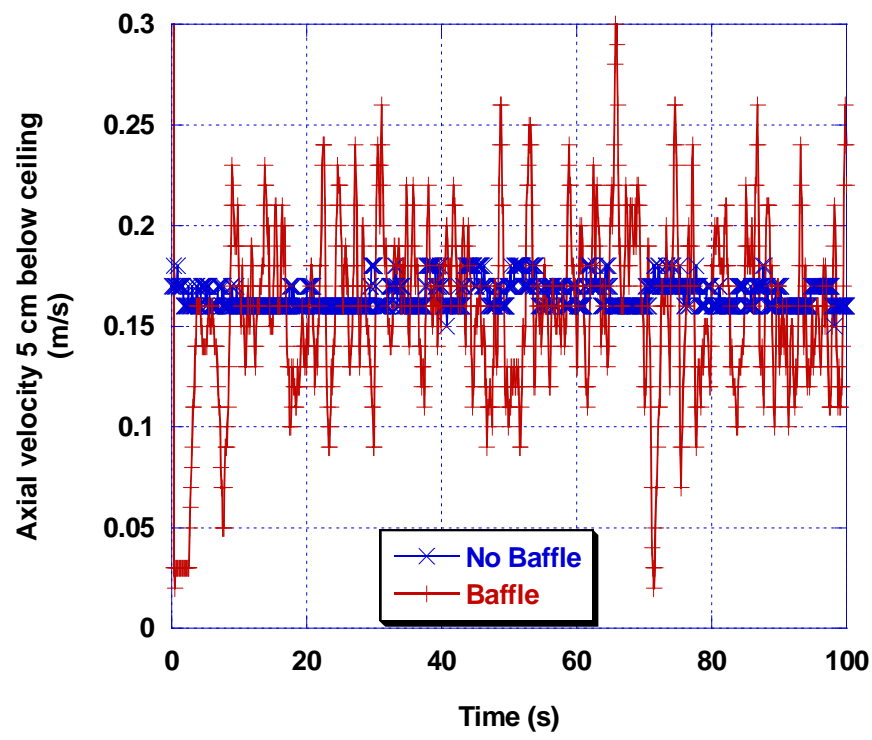

Figure 52. Axial velocity at a fan speed of $12 \mathrm{~Hz}$

Table 4. Mean axial velocity $5 \mathrm{~cm}$ below ceiling

\begin{tabular}{|l|l|l|l|}
\hline Fan speed & & $\begin{array}{l}\text { With baffle } \\
(\mathrm{m} / \mathrm{s})\end{array}$ & $\begin{array}{l}\text { Without baffle } \\
(\mathrm{m} / \mathrm{s})\end{array}$ \\
\hline $5 \mathrm{~Hz}$ & Velocity & 0.057 & 0.047 \\
& Standard deviation & 0.01 & 0.004 \\
\hline $7 \mathrm{~Hz}$ & Velocity & 0.073 & 0.073 \\
& Standard deviation & 0.021 & 0.005 \\
\hline $12 \mathrm{~Hz}$ & Velocity & 0.159 & 0.162 \\
& Standard deviation & 0.043 & 0.01 \\
\hline
\end{tabular}

Alarm positions for board 1 are: position 1 - Ion-3- - -6, position 2 - Ion-1- - -1, position 3 Photo-1 - - -3, position 4 - (Ion-4- - -8 and Photo-3- - -8; these sensors are co-located in the same alarm housing), and position 5 empty. Alarm positions for board 2 are: position 1 is empty, position 2 - Ion-3- - -13, position 3 - (Ion-4- - -3 and Photo-3- - -3), position 4 - Ion3- - -4, and position 5 - Photo-1 - - -6. Thus, a Photo-1 type detector was in a front location on board 1 and a rear location on board 2, while Ion-4 and Photo-3 were in a rear location on board 1 and in a front location on board 2. Ion-1 was located in the front on board 1 and in a rear location on board 2; however, since location 1 is empty on board 2, the full effects of blockages are not expected. Ion-3 remained in a front location on each board. Smoke was generated with the same staged wick ignition device described earlier, using eight pairs of wicks with a $12 \mathrm{~s}$ ignition delay between pairs. Figures 53-76 show the results for each alarm. 


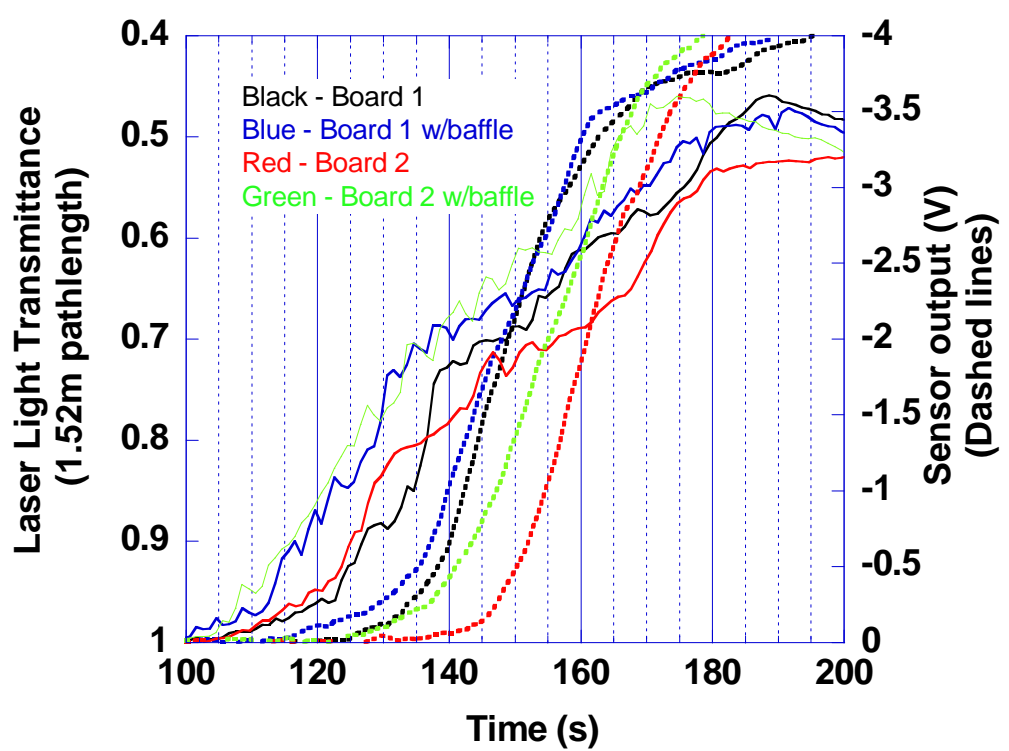

Figure 53. Ion-1 response, fan speed $5 \mathrm{~Hz}$ (see table 4)

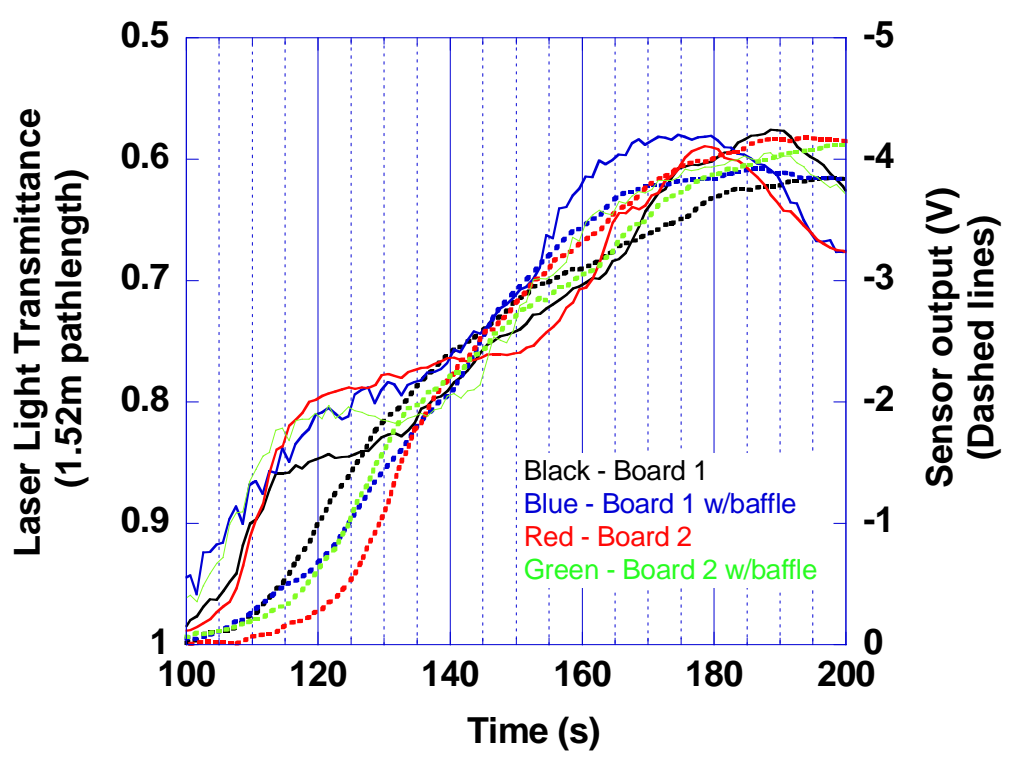

Figure 54. Ion-1 response, fan speed $7 \mathrm{~Hz}$ 


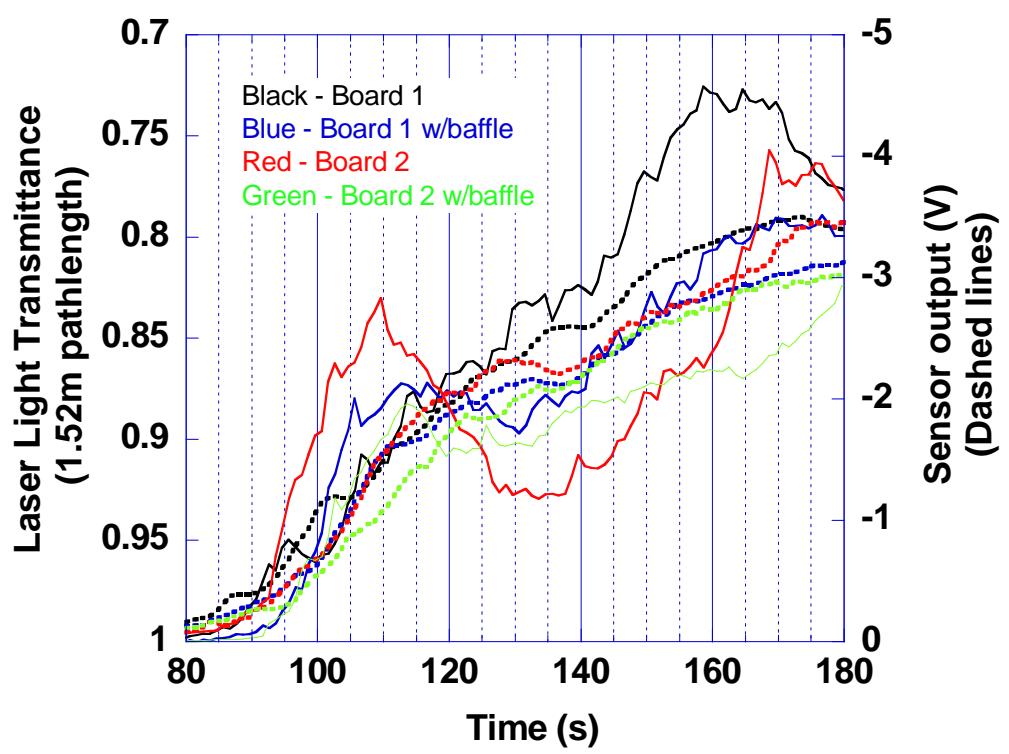

Figure 55. Ion-1 response, fan speed $12 \mathrm{~Hz}$

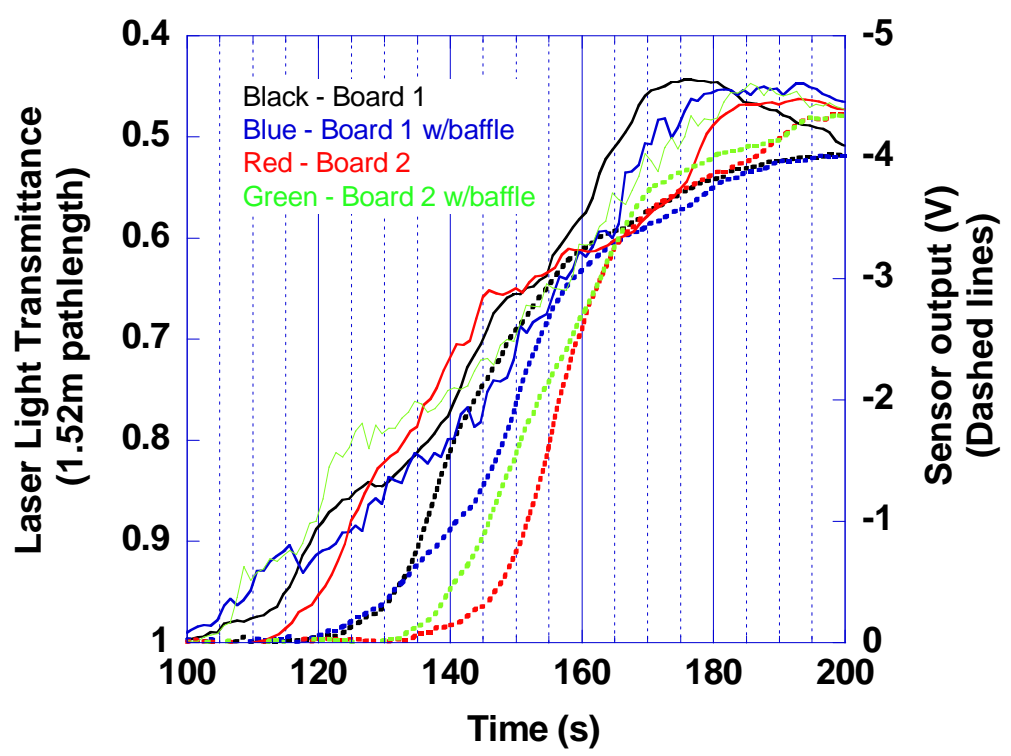

Figure 56. Ion-1 response, fan speed $5 \mathrm{~Hz}$ - repeat test 


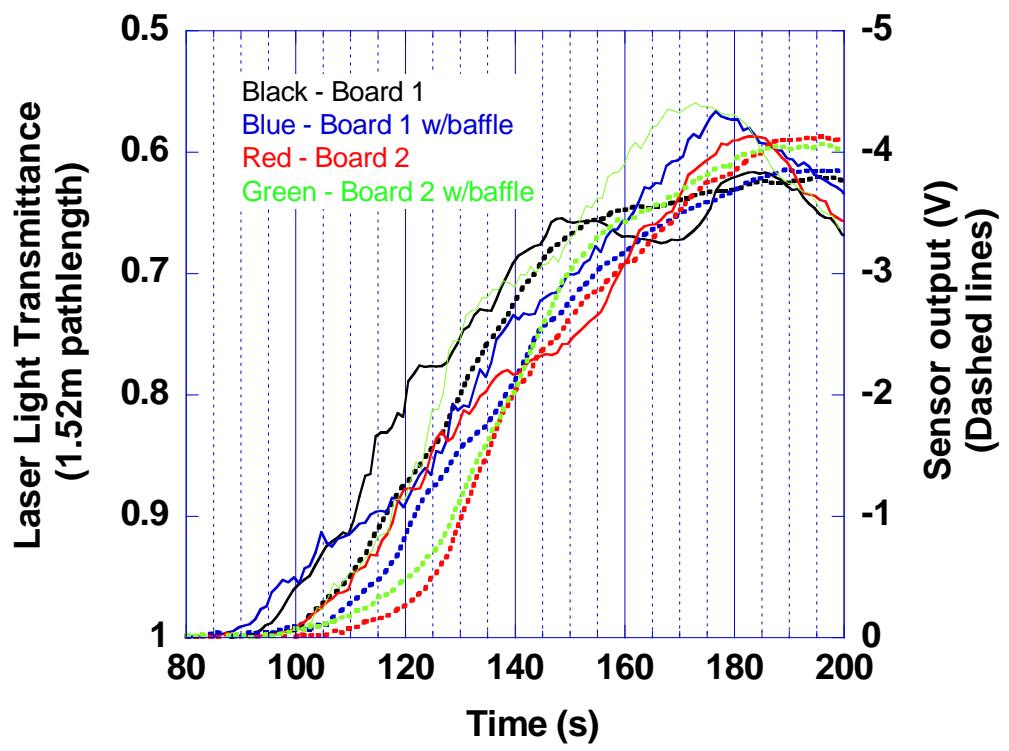

Figure 57. Ion-1 response, fan speed $7 \mathrm{~Hz}$ - repeat test

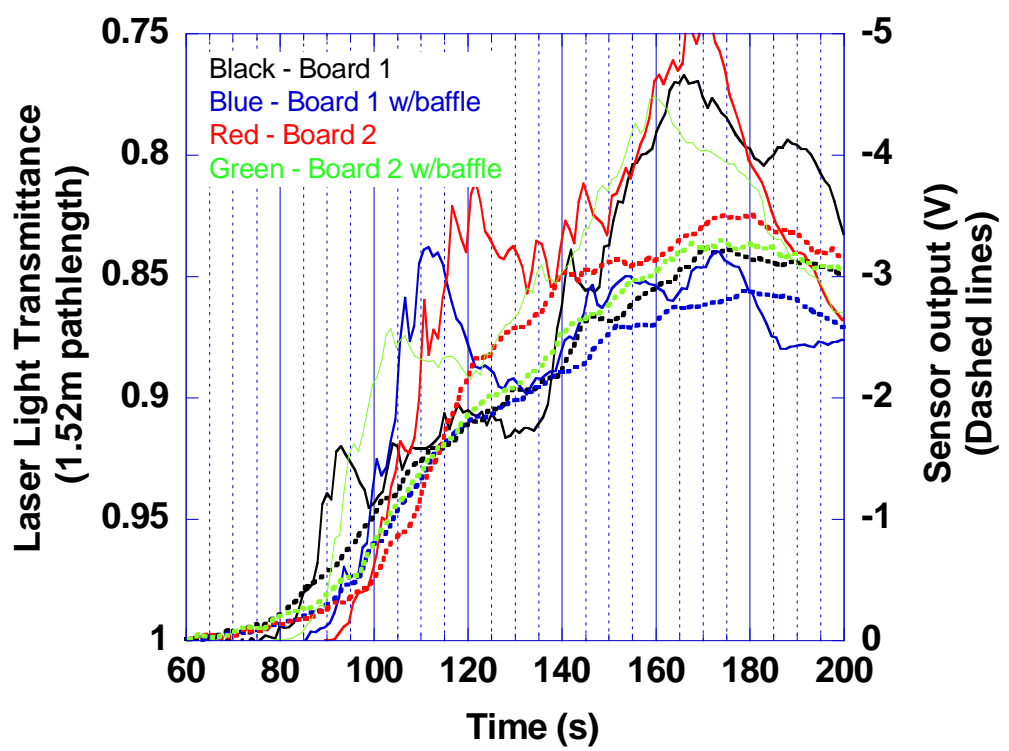

Figure 58. Ion-1 response, fan speed $12 \mathrm{~Hz}$ - repeat test 


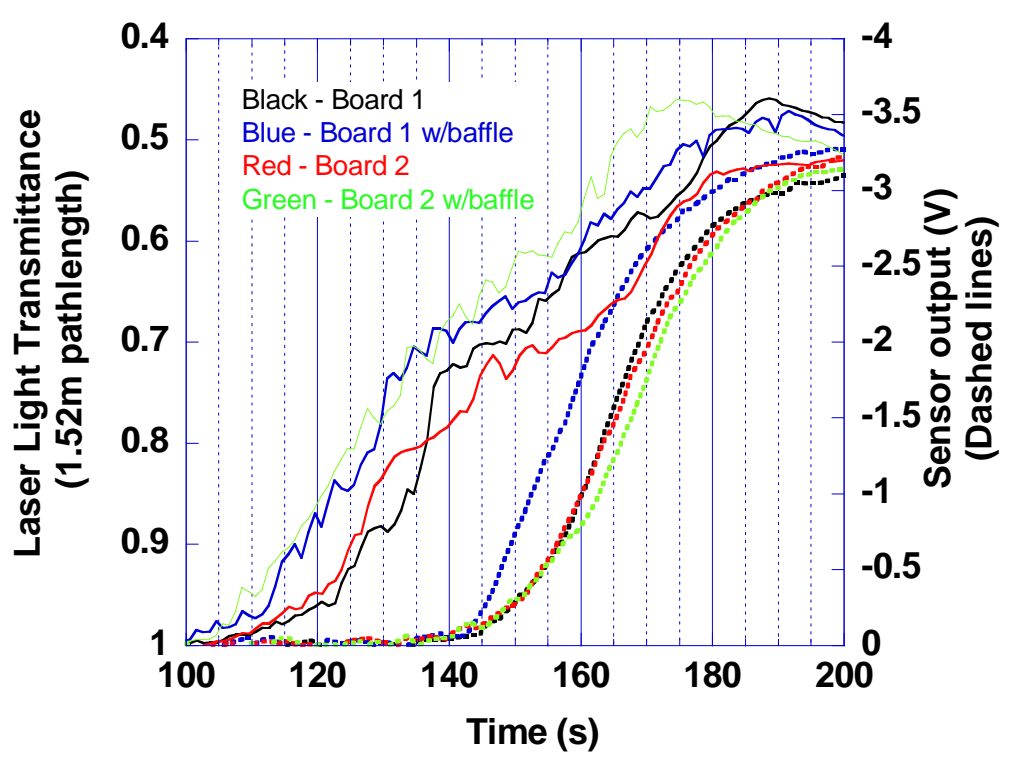

Figure 59. Ion-3 response, fan speed $5 \mathrm{~Hz}$

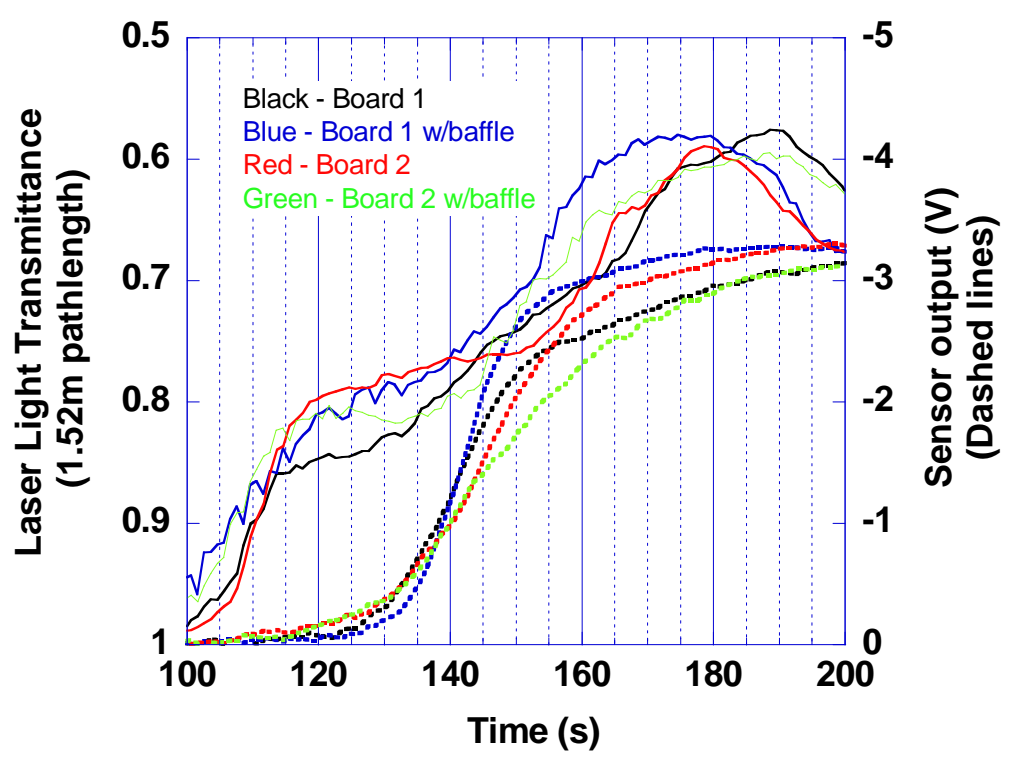

Figure 60. Ion-3 response, fan speed $7 \mathrm{~Hz}$ 


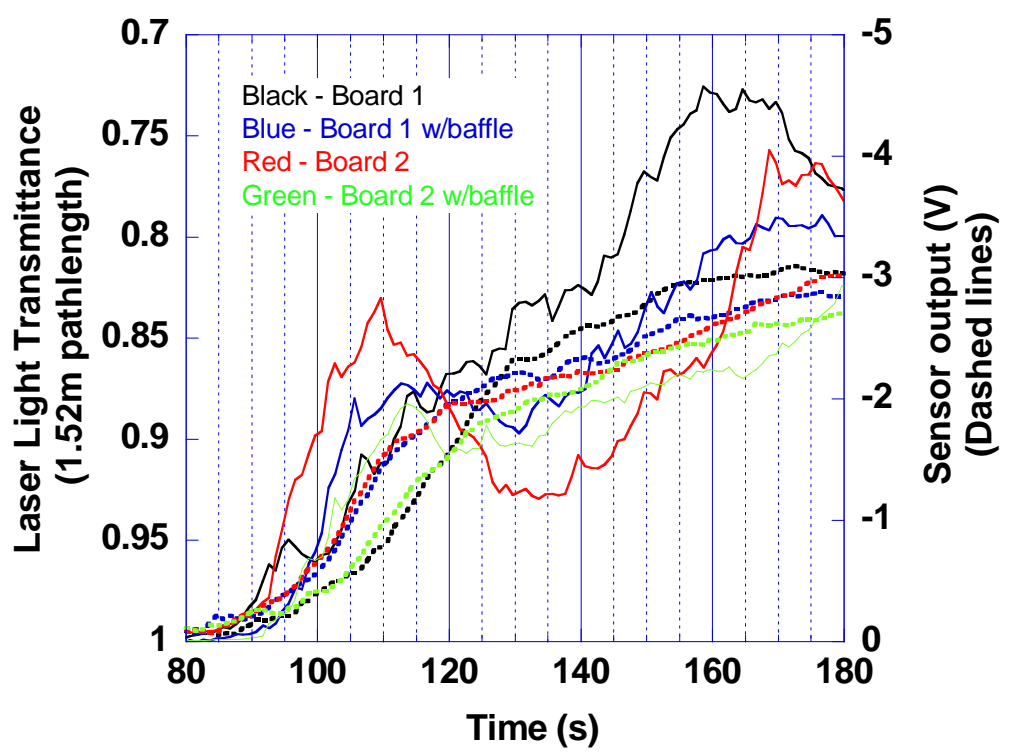

Figure 61. Ion-3 response, fan speed $12 \mathrm{~Hz}$

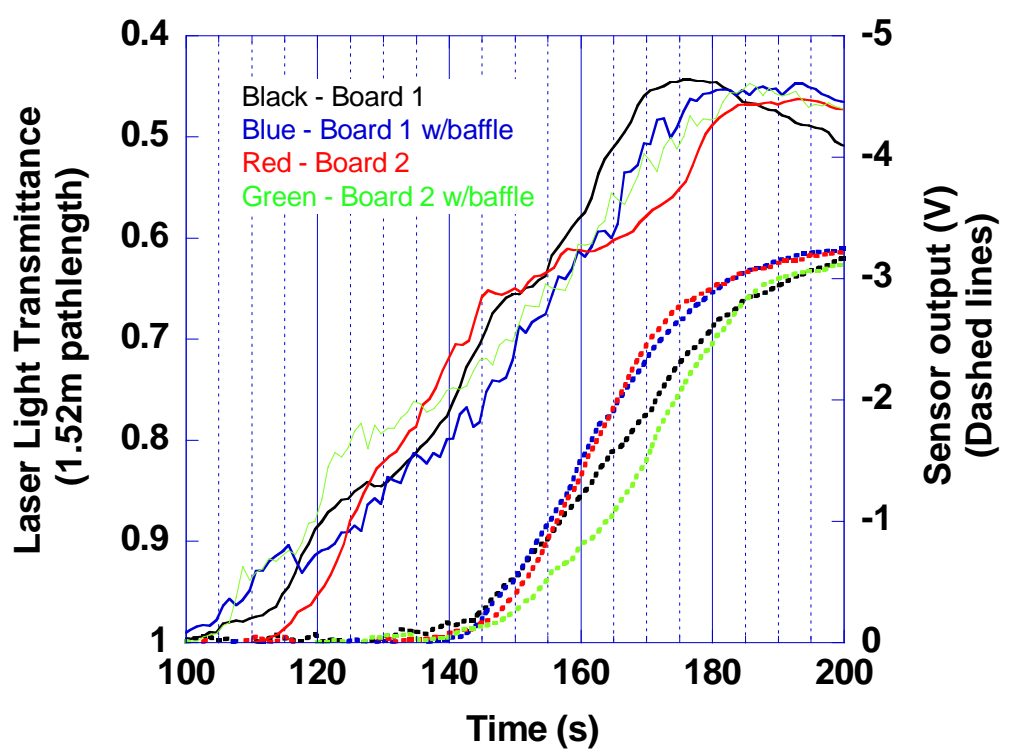

Figure 62. Ion-3 response, fan speed $5 \mathrm{~Hz}$ - repeat test 


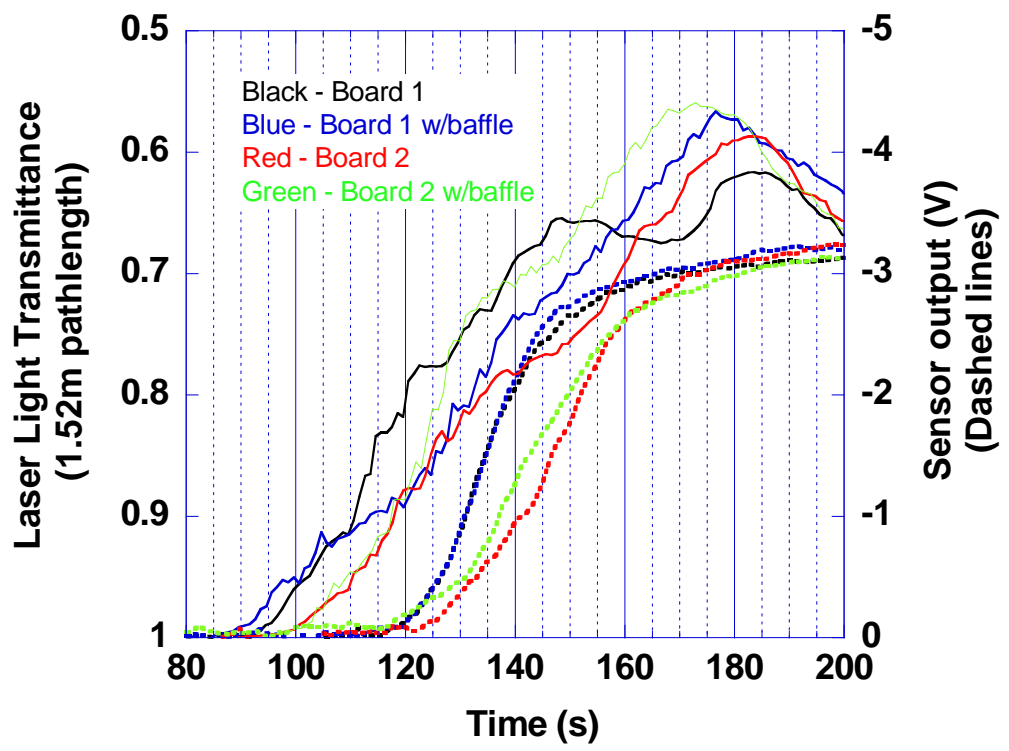

Figure 63. Ion-3 response, fan speed $7 \mathrm{~Hz}$ - repeat test

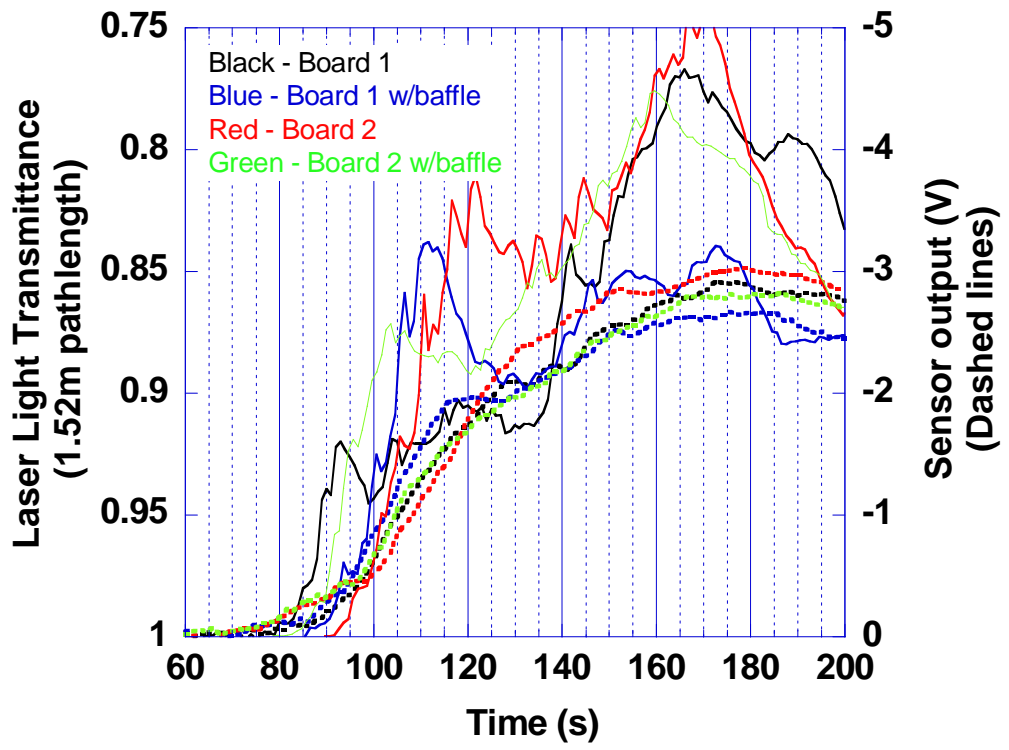

Figure 64. Ion-3 response, fan speed $12 \mathrm{~Hz}$ - repeat test 


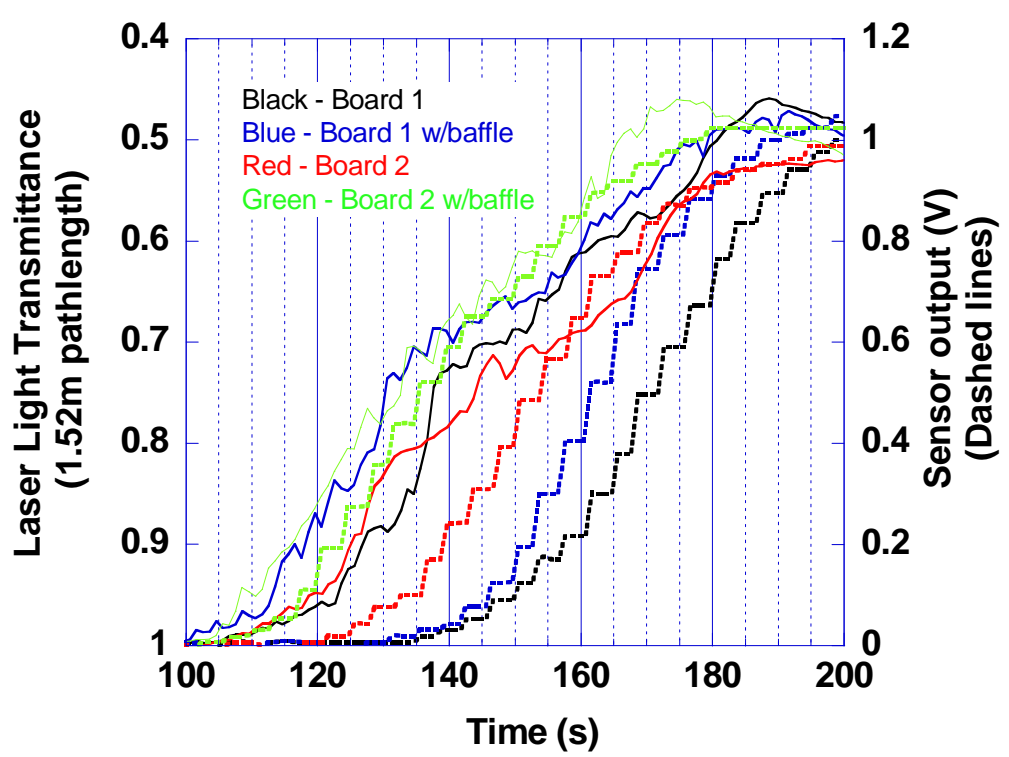

Figure 65. Ion-4 response, fan speed $5 \mathrm{~Hz}$

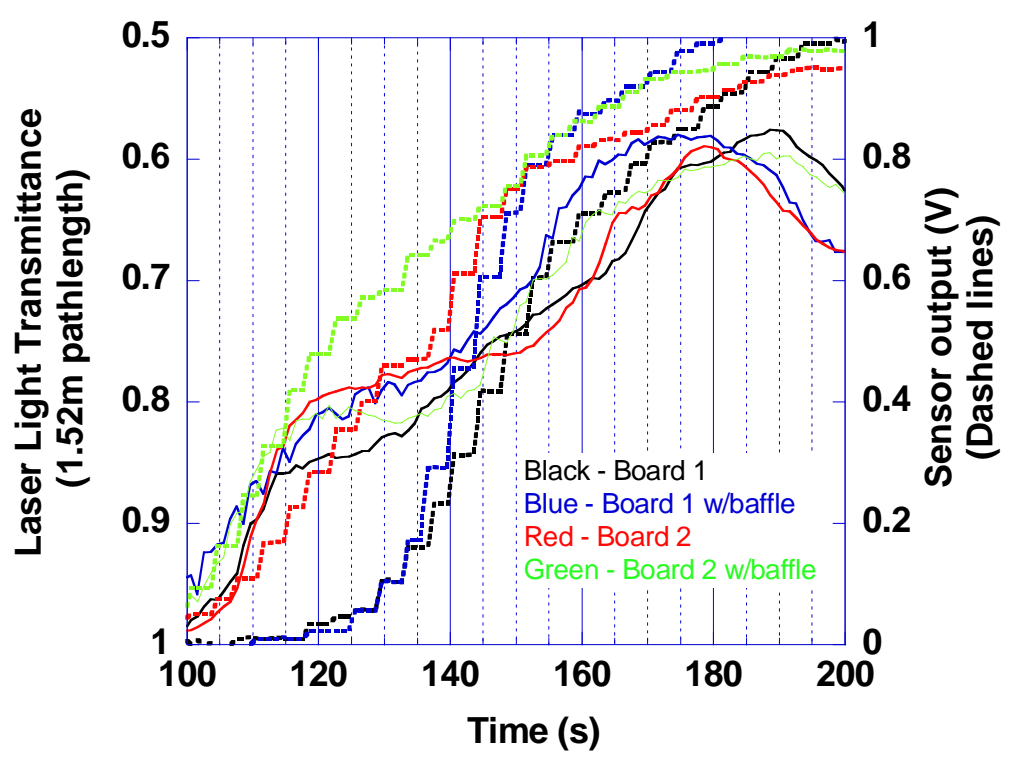

Figure 66. Ion-4 response, fan speed $7 \mathrm{~Hz}$ 


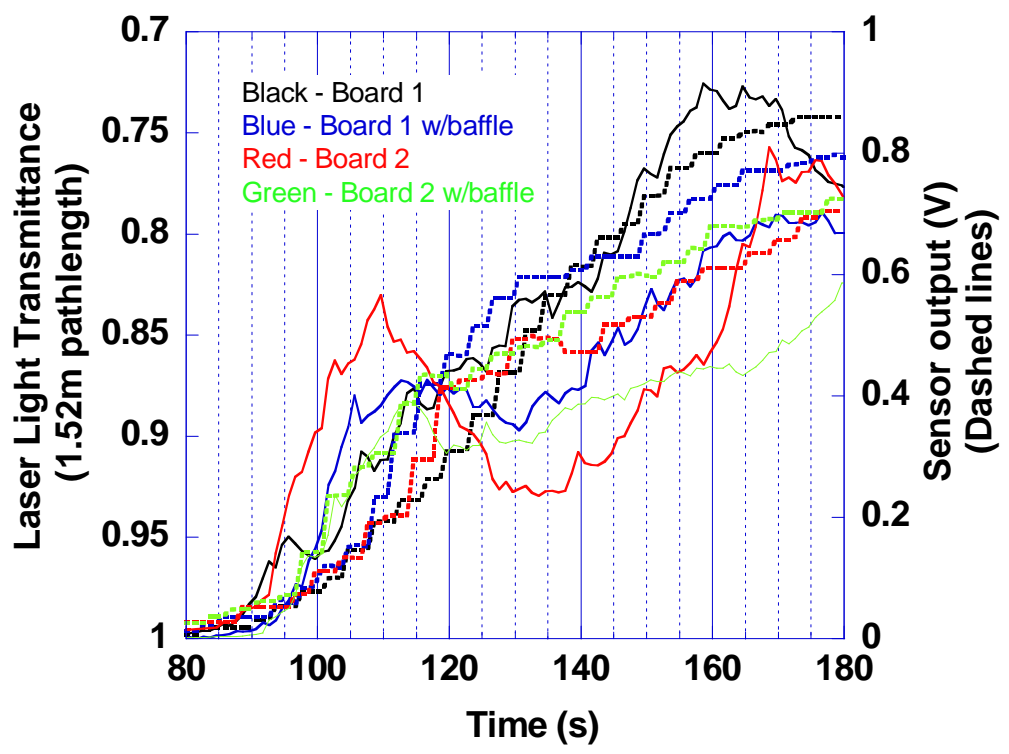

Figure 67. Ion-4 response, fan speed $12 \mathrm{~Hz}$

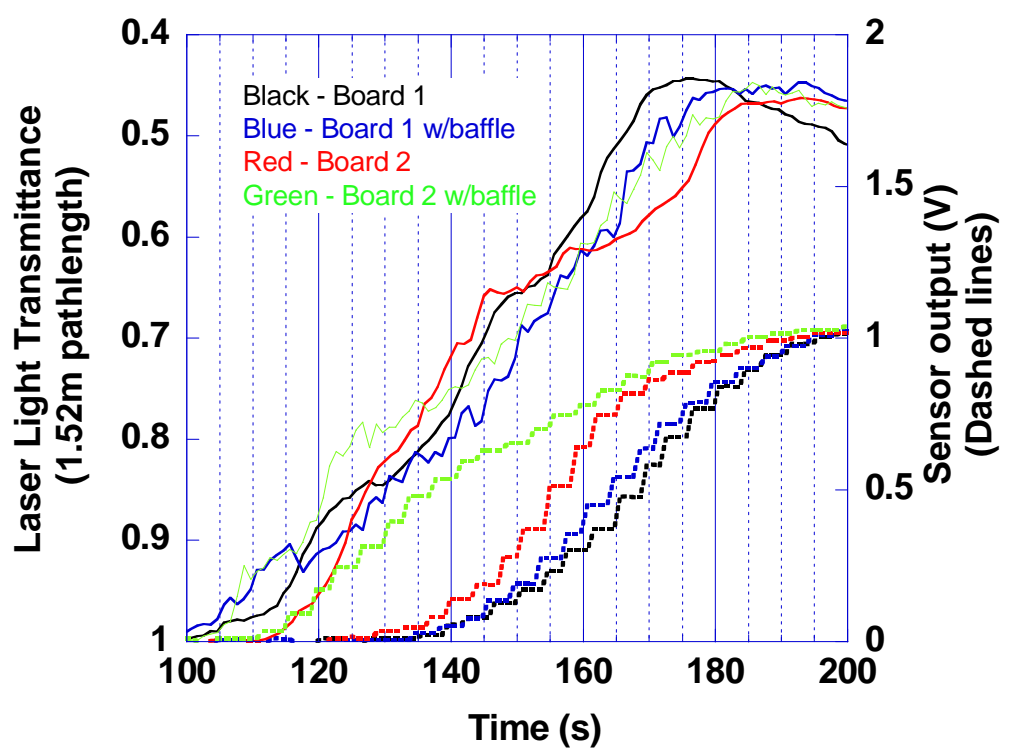

Figure 68. Ion-4 response, fan speed $5 \mathrm{~Hz}$ - repeat test 


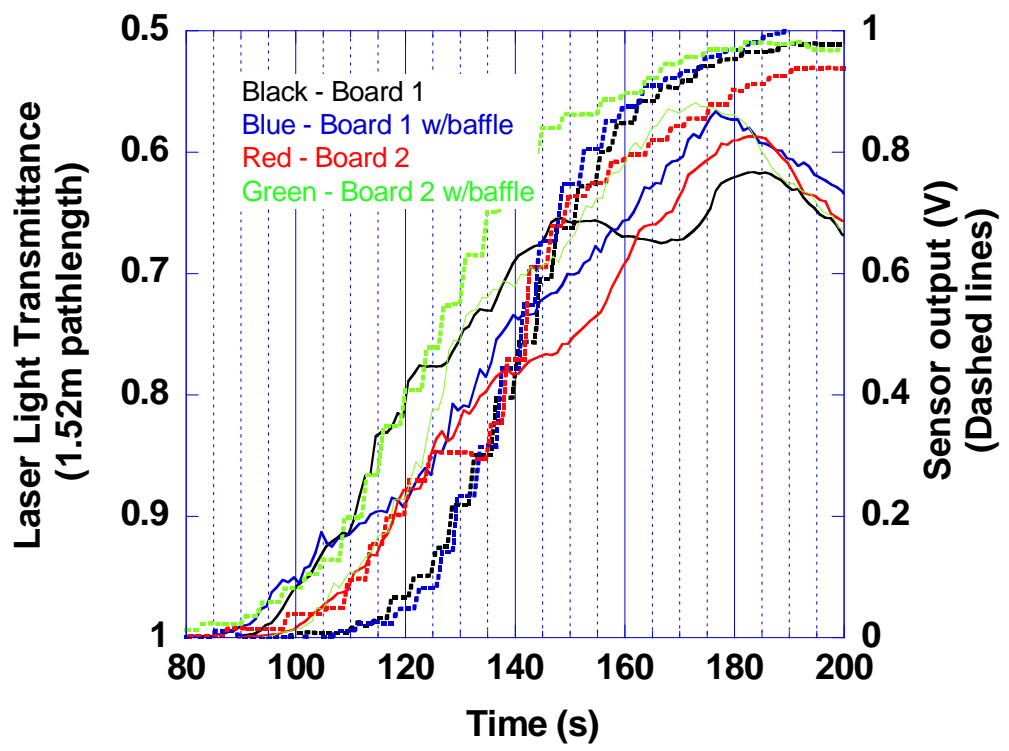

Figure 69. Ion-4 response, fan speed $7 \mathrm{~Hz}$ - repeat test

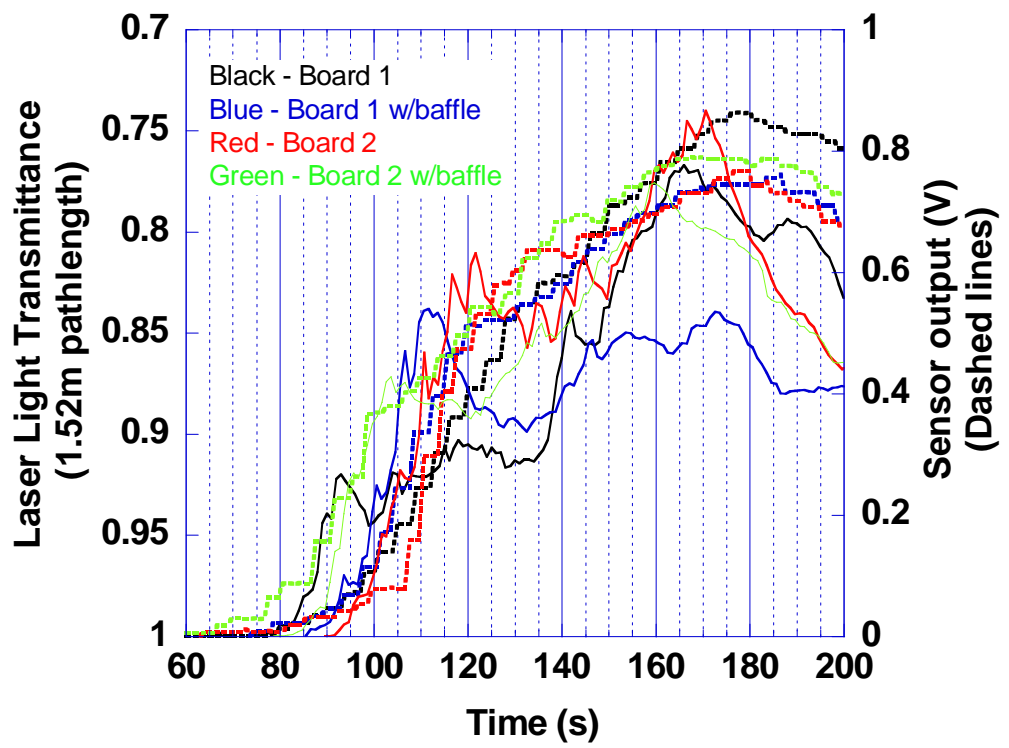

Figure 70. Ion-4 response, fan speed $12 \mathrm{~Hz}$ - repeat test 


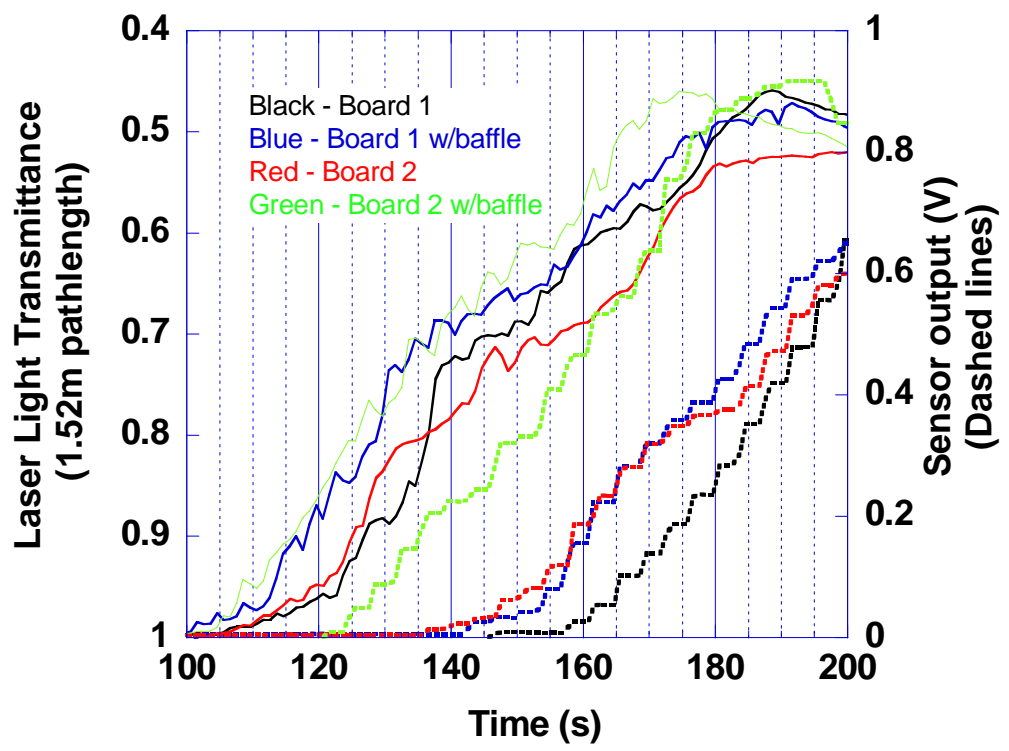

Figure 71. Photo-3 response, fan speed $5 \mathrm{~Hz}$

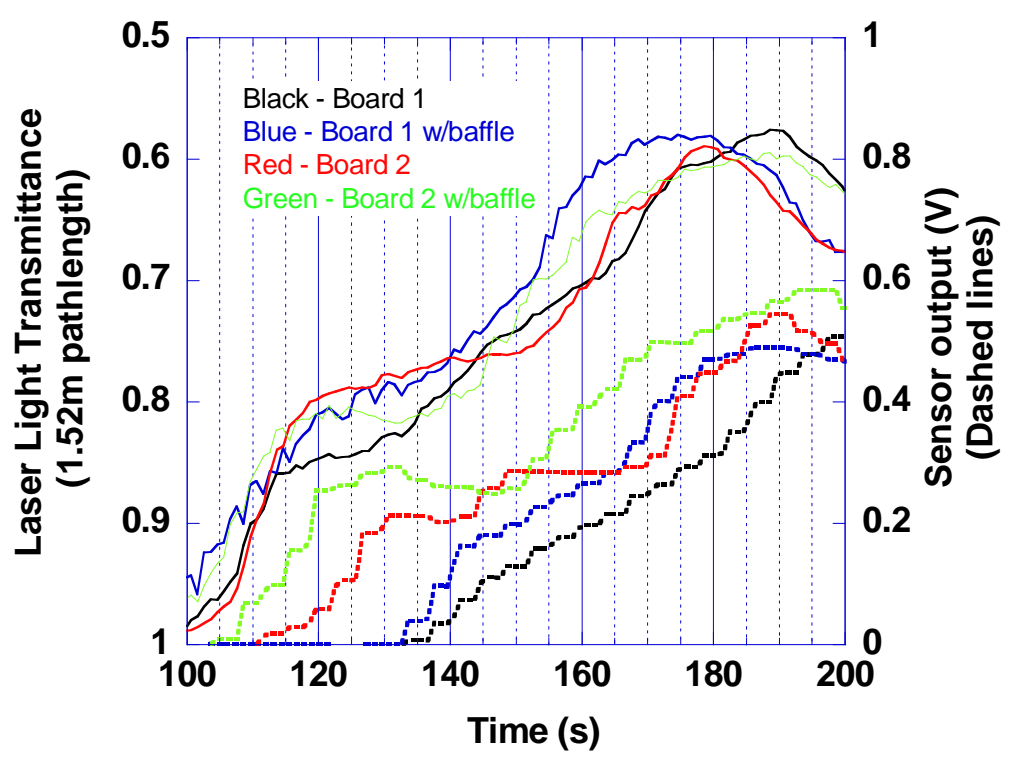

Figure 72. Photo-3 response, fan speed $7 \mathrm{~Hz}$ 


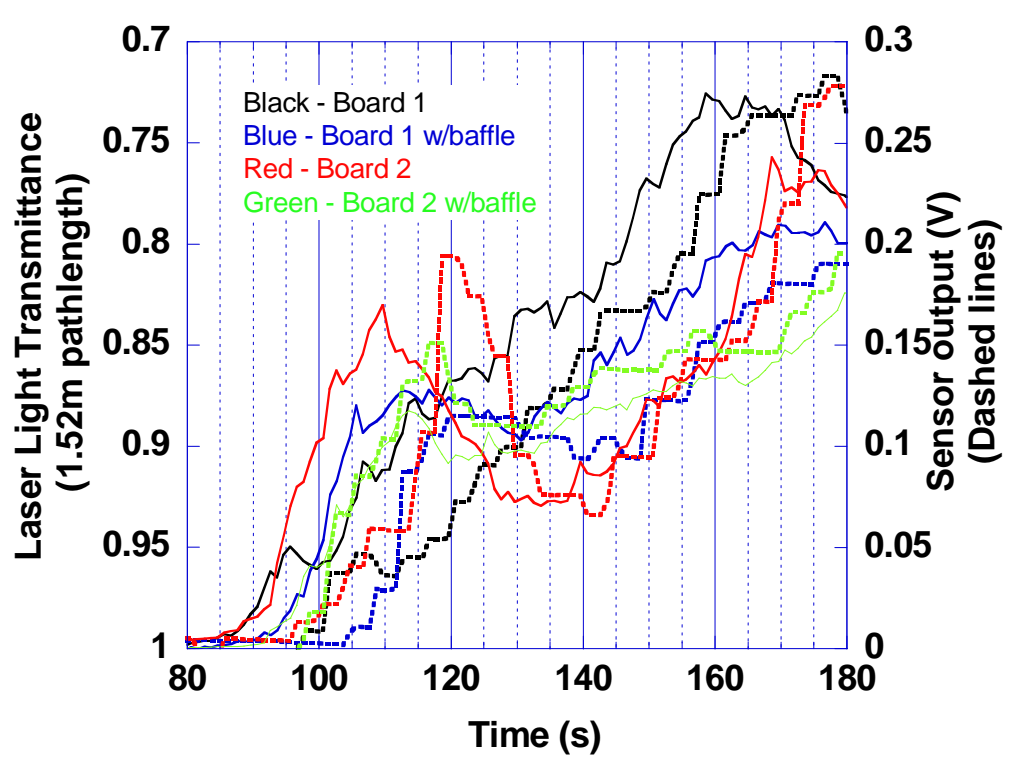

Figure 73. Photo-3 response, fan speed $12 \mathrm{~Hz}$

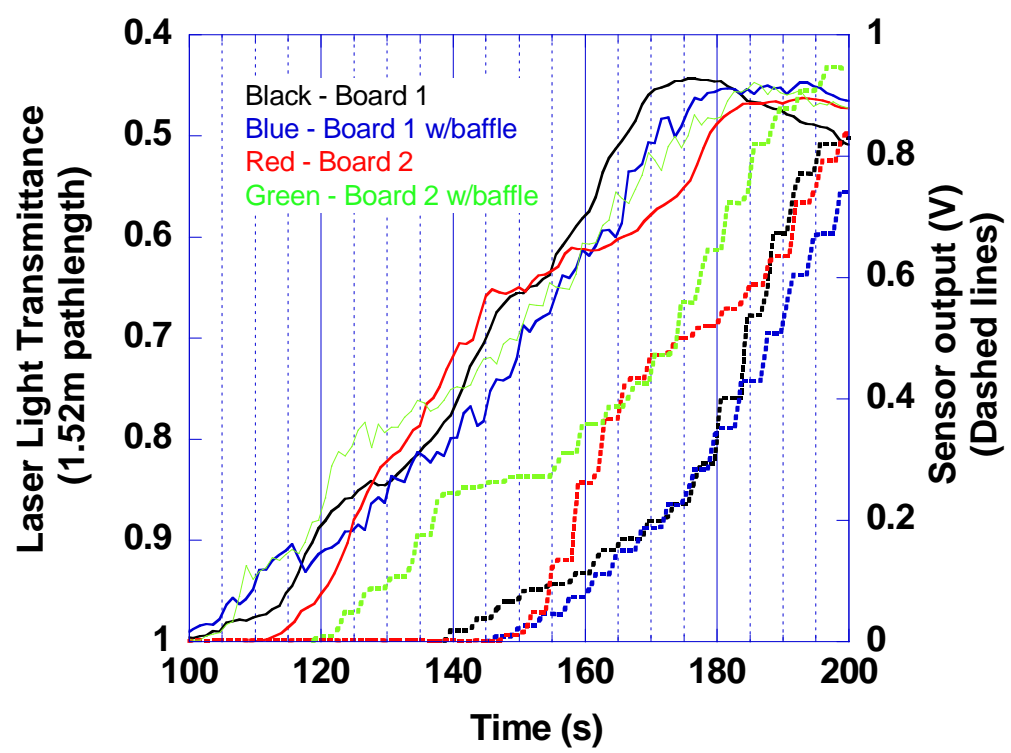

Figure 74. Photo-3 response, fan speed $5 \mathrm{~Hz}$ - repeat test 


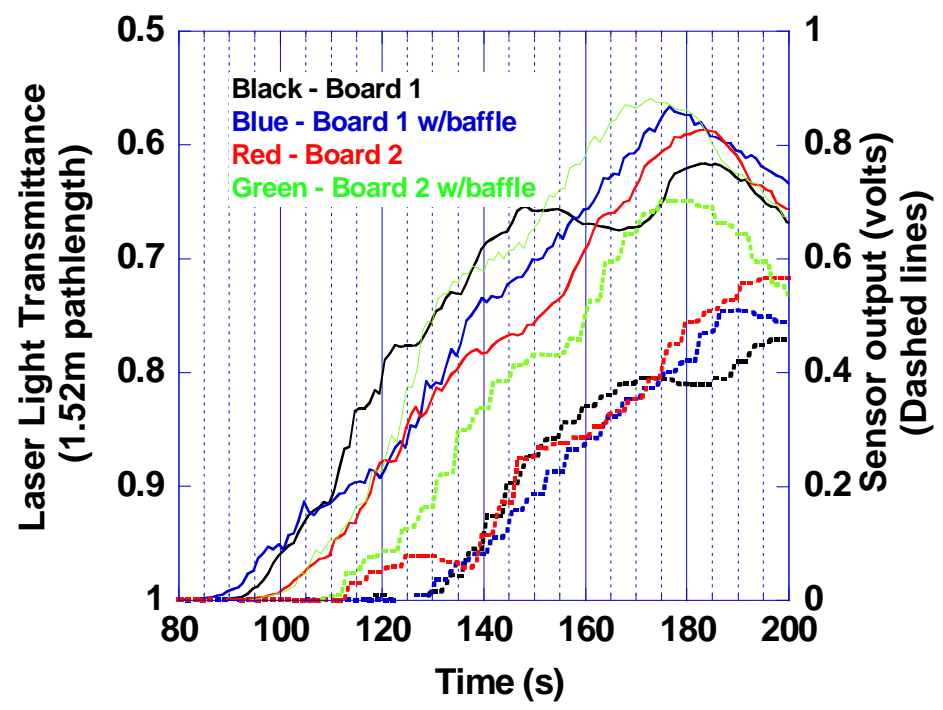

Figure 75. Photo-3 response, fan speed $7 \mathrm{~Hz}$ - repeat test

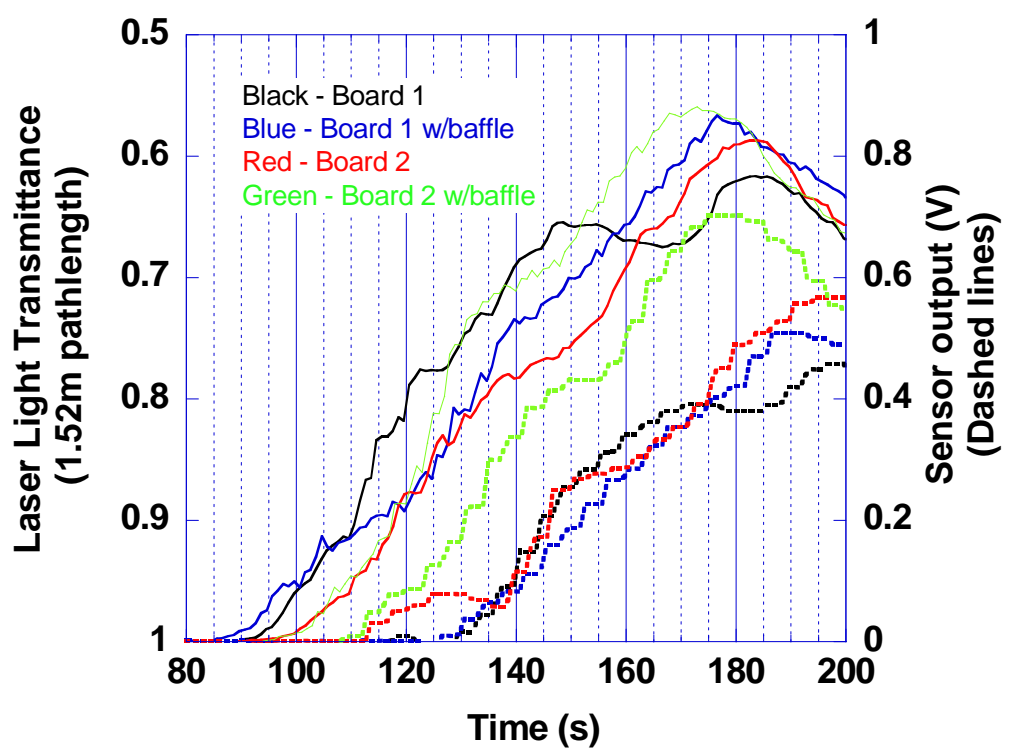

Figure 76. Photo-3 response, fan speed $7 \mathrm{~Hz}$ - repeat test 
A photo-1 alarm malfunctioned and the results for that alarm type are not included. The results show at low flow $(5 \mathrm{~Hz})$ there is an additional response delay on the order of $10 \mathrm{~s}$ at alarm concentrations for alarms placed in positions 4 and 5 versus the front three positions due to the additional transport time and blockage effects. At $7 \mathrm{~Hz}$, the effect is less pronounced, on the order of a 5 s delay. At $12 \mathrm{~Hz}$ there appears to be no effect. In general, the baffle lessens the effect of position on the alarm response at the low flows. It is concluded that at all but the low flows there is little or no appreciable bias affecting the alarm response and the board position. It is important to note that this simultaneous data were only available for the ionization alarms since only one photoelectric alarm was included in the data. The effects of background flows are discussed further in section 6 from testing of nuisance sources in the manufactured home.

\subsection{Thresholds for Modified Alarms}

Since the modified CO and smoke alarms do not produce an alarm signal, appropriate thresholds need to be specified to perform the calculations of time to alarm presented in the Appendix A. Selection of alarm thresholds was driven by the desire to consistently apply the same reasonable criterion for each sensor type. Moreover, high and low ranges were specified for photoelectric and ionization alarms characteristic of allowed production ranges. For $\mathrm{CO}$ alarms, high and low ranges were specified to indicate sensitivity of a fixed threshold.

Table 5 gives the alarm

Table 5. Threshold criteria for modified alarms thresholds used in the fire test series analysis. The justification for the $\mathrm{CO}$ alarm threshold and range is based on levels that should be above potential

\begin{tabular}{|l|c|c|c|}
\cline { 2 - 4 } \multicolumn{1}{c|}{} & Low & Mid & High \\
\hline Ionization $(\% / \mathrm{m} / \% / \mathrm{ft})$ & $2.6 / 0.8$ & $4.3 / 1.3$ & $5.9 / 1.8$ \\
\hline Photoelectric $(\% / \mathrm{m} / \% / \mathrm{ft})$ & $3.3 / 1.0$ & $6.6 / 2.0$ & $9.8 / 3.0$ \\
\hline $\mathrm{CO}($ volume fraction / ppm) & $2.5 \times 10^{-5} / 25$ & $5.0 \times 10^{-5} / 50$ & $1.0 \times 10^{-4} / 100$ \\
\hline
\end{tabular}
house $\mathrm{CO}$ concentration levels. The photoelectric alarm threshold and range are indicative of typical nominal values and falls within the observed alarm threshold for the unmodified photoelectric alarms tested in the FE/DE. The ionization alarm threshold and range are indicative of typical nominal values, however, the observed alarm threshold for one unmodified ionization alarm fell outside the range. It was decided to use the nominal threshold value of $4.3 \% / \mathrm{m}(1.3 \% / \mathrm{ft})$ based on two observations. First, the cotton smoke source appears to meet the UL criterion for typical ionization alarm values, and second, it is a more conservative value than the apparent alarm thresholds for the unmodified ionization alarms. Furthermore, the average relative time difference between an alarm sensitivity settings of $4.3 \% / \mathrm{m}$ and $3.2 \% / \mathrm{m}$ (that is, 100 times the \{time to alarm at $4.3 \% / \mathrm{m}$ - time to alarm at $3.2 \% / \mathrm{m}$ \} divided by the time to alarm at $4.3 \% / \mathrm{m}$ 
for all (modified) ionization alarms in all tests was computed to be $2.9 \%$, indicating that the time to reach alarm is not particularly sensitive to threshold settings near the chosen $4.3 \% / \mathrm{m}$ setting used for these fire tests. 


\section{Fire Source Test Scenarios and Geometries}

In order to evaluate the performance of residential smoke alarms in-situ, a test series was designed to encompass multiple fire scenarios, residential geometries, and alarm technologies. This section describes the design of the test series.

\subsection{Scenario Development}

Of key importance to the representativeness of the study was the selection of the test scenarios. These include the room of fire origin, ignition source and first item ignited, and the ventilation conditions that affect the fire development and combustion chemistry. Here we began with an NFPA analysis of USFA's NFIRS data on fatal residential fires conducted for CPSC [18]. The statistics were categorized according to:

- $\quad$ fire location (living room, bedroom, kitchen, and other),

- $\quad$ fire type (smoldering, flaming, and fast flaming),

- $\quad$ first item ignited.

The data were divided into the most frequent fires, and fires resulting in the greatest number of deaths. Relatively hazardous fires may be fires that occur more frequently than others, or ones that result in a high number of deaths. Of particular interest are fires that occur relatively frequently, and when they do, they result in a disproportionately high death rate. Table 6 shows the top ten scenarios for frequency of occurrence and number of deaths. Flaming and smoldering upholstered furniture and mattresses account for the top four most deadly fire scenarios. Flaming cooking materials are involved more than five times more frequently than any other material. The scenarios chosen for testing included the top five ranked by number of deaths, and among the top ten ranked by frequency of occurrence:

- $\quad$ smoldering upholstered furniture in the living room,

- flaming upholstered furniture in the living room,

- smoldering mattress in the bedroom,

- $\quad$ flaming mattress in the bedroom, and

- cooking materials in the kitchen.

These chosen scenarios are consistent with typical scenarios included for the performance-based design option in NFPA 101 [19] and available guides for performance-based design [20]. 
Table 6. Top fire scenarios ranked by frequency of occurrence, $1992-1996$

\begin{tabular}{|c|c|c|c|}
\hline Location & Fire Type & First Item Ignited & Frequency \\
\hline \multicolumn{4}{|c|}{ Ranked by Frequency of Occurrence } \\
\hline Kitchen & Flaming & Cooking Materials & 82905 \\
\hline Bedroom & Flaming & Mattress & 15914 \\
\hline Kitchen & Flaming & Wire / Cable & 7499 \\
\hline Bedroom & Smoldering & Mattress & 6437 \\
\hline Kitchen & Fast Flaming & Cooking & 5234 \\
\hline Bedroom & Flaming & Wire / Cable & 4551 \\
\hline Kitchen & Flaming & Interior Wall Covering & 4271 \\
\hline Living Room & Smoldering & Upholstered Furniture & 4060 \\
\hline Living Room & Flaming & Upholstered Furniture & 3715 \\
\hline Living Room & Flaming & Wire / Cable & 3481 \\
\hline \multicolumn{4}{|c|}{ Ranked by Number of Deaths } \\
\hline Living Room & Smoldering & Upholstered Furniture & 372 \\
\hline Bedroom & Smoldering & Mattress & 251 \\
\hline Bedroom & Flaming & Mattress & 249 \\
\hline Living Room & Flaming & Upholstered Furniture & 160 \\
\hline Kitchen & Flaming & Cooking Materials & 142 \\
\hline Kitchen & Flaming & Clothing & 79 \\
\hline Living Room & Flaming & Wire / Cable & 61 \\
\hline Living Room & Flaming & Interior Wall Covering & 52 \\
\hline Bedroom & Flaming & Clothing & 51 \\
\hline Kitchen & Flaming & Structural Member / Framing & 50 \\
\hline
\end{tabular}

Source: 1992 - 1996 NFIRS Data [18] 


\subsection{Material Selection}

\subsubsection{Upholstered Furniture}

Once the fire scenarios were determined, the materials were procured. The primary goal of each material was to provide a realistic residential fire signature. The burning behavior of individual items, however, can vary dramatically depending upon the specific materials, geometry, and construction used to fabricate the items. Therefore, two different pieces of upholstered living room furniture were selected in order to take advantage of different combustion characteristics of the fuels. Figures 77 and 78 show the upholstered chair selected for the smoldering experiments. The smoldering chair weighed $34 \mathrm{~kg}$. Extended propagation (greater than one hour) of the smoldering front necessitates a large quantity of foam for the seat cushion. The seat cushion consisted of $79 \%$ urethane foam and $21 \%$ synthetic fiber. The cushioning in the rest of the chair consists of $68 \%$ urethane foam, $29 \%$ felted textile fibers of unknown kind, $2 \%$ resin treated synthetic fiber, and $1 \%$ olefin foam. The frame of the chair was constructed of wood.
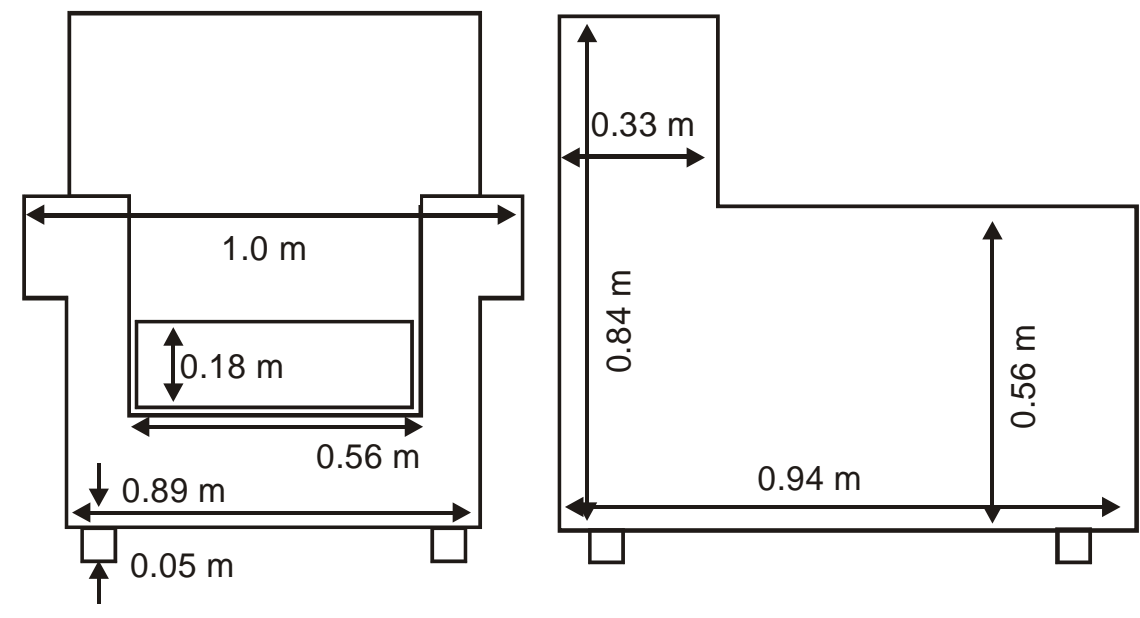

Figure 77. Measurements of upholstered chair used for smoldering experiments

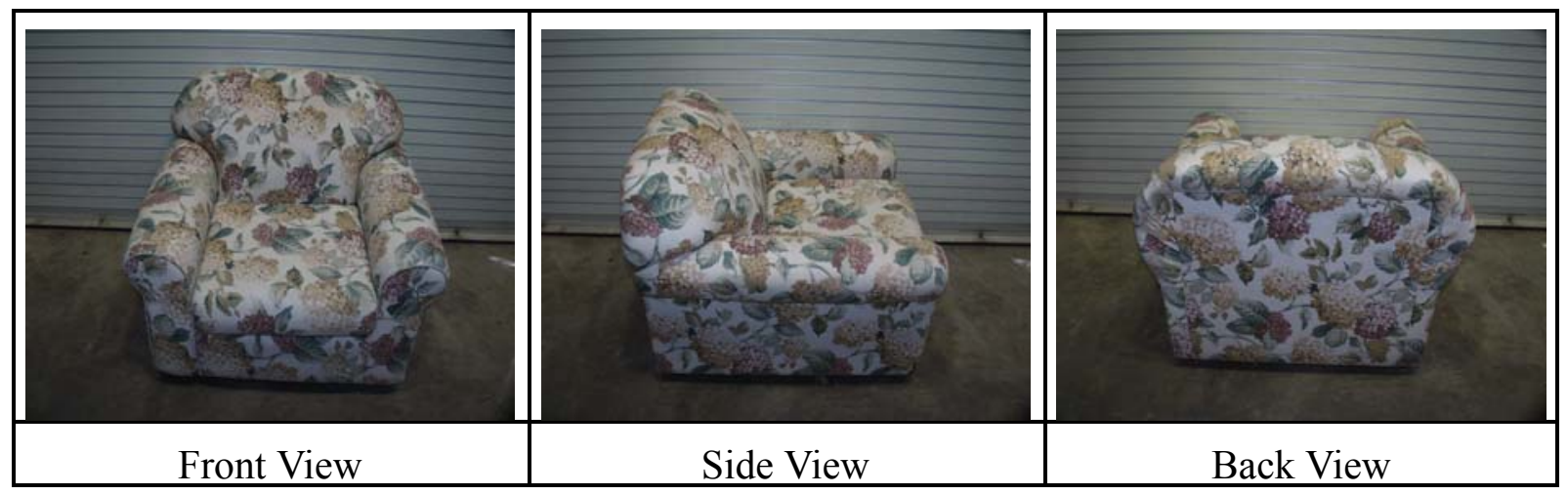

Figure 78. Upholstered chair used for smoldering experiments 
The flaming chair was chosen with a lower foam content than the smoldering chair to reduce the peak heat release rate of the chair. A high peak heat release rate may have resulted in unintentional damage to the test structures. Thus, the total quantity of foam in the flaming chair was less than the total quantity of foam in the smoldering chair. The quantity of wood in the framing was not a consideration because the fires were always extinguished prior to the framing becoming substantially involved. Figures 79 and 80 show the chair selected for the flaming living room experiments.

The flaming chair weighed $28 \mathrm{~kg}$. The seat cushion consisted of $79 \%$ polyurethane foam and $21 \%$ polyester fiber. The remaining cushioning consisted of $28 \%$ polyurethane foam and $72 \%$ felted textile fiber of unknown composition. A separate back pillow consisted of $100 \%$ polyester fiber. The frame was constructed of wood.
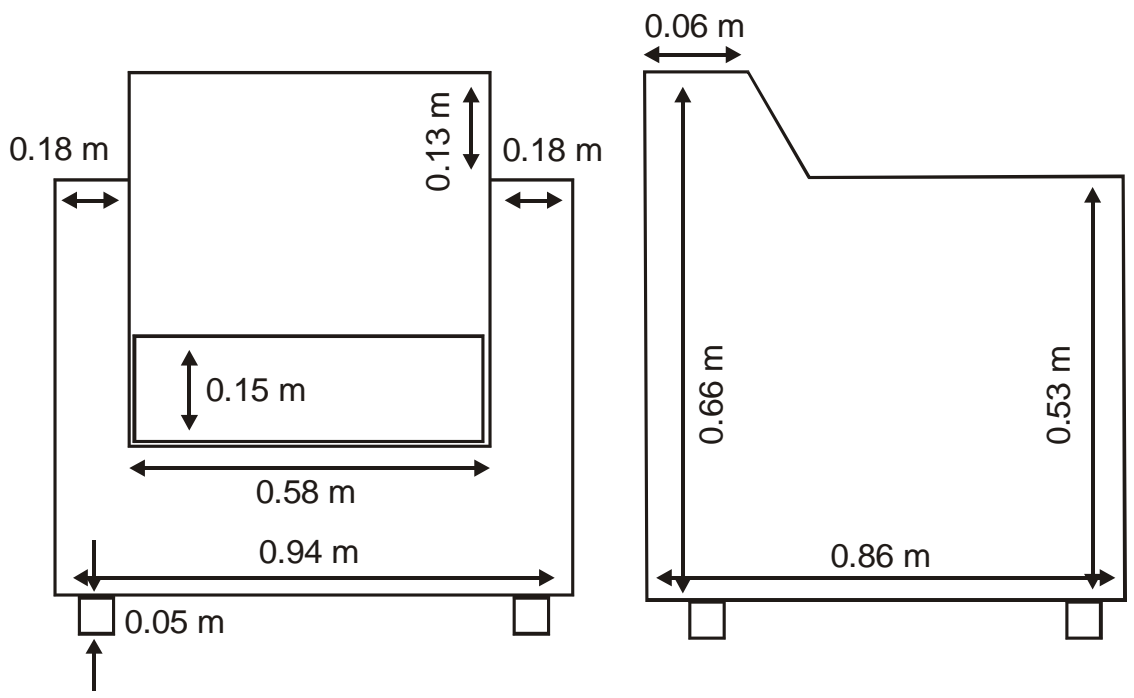

Figure 79. Measurements of upholstered chair used for flaming experiments

Both smoldering and

flaming chairs were obtained from a furniture rental retailer in order to reproduce the flaming or smoldering characteristics of lived-in furniture.

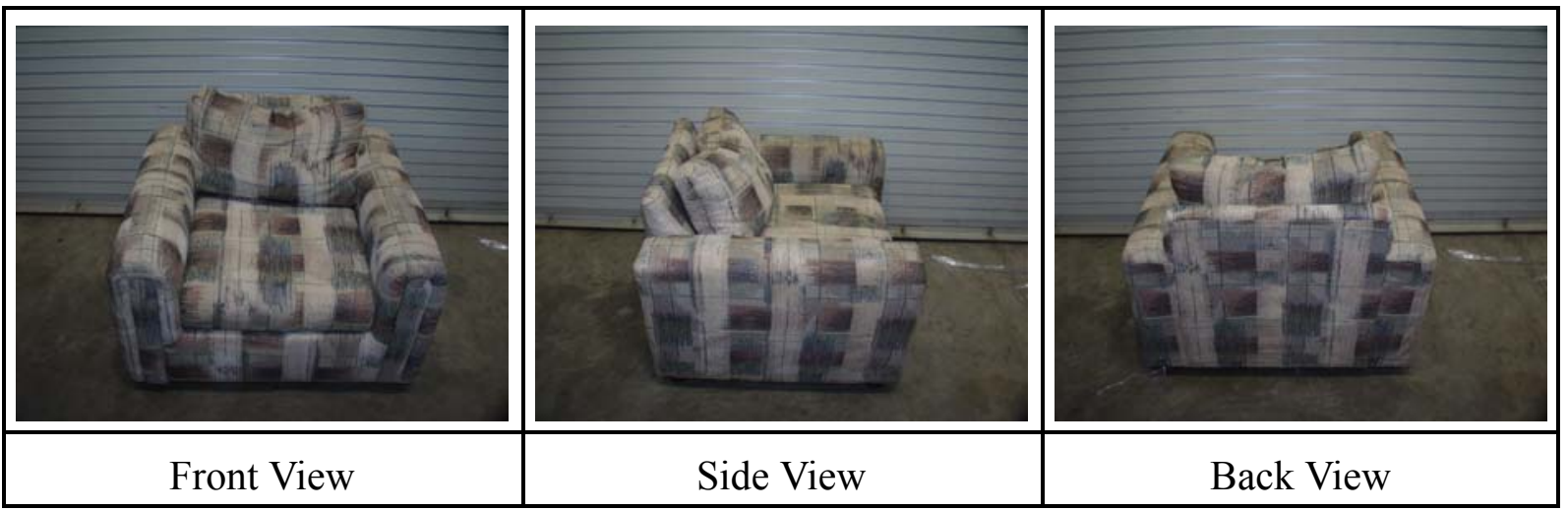

Figure 80. Upholstered chair used for flaming experiments 


\subsubsection{Mattress}

For the smoldering and flaming bedroom scenarios, a single mattress style was obtained. The twin size mattress had a "pillowtop" layer of foam. While the "pillow-top" mattresses are popular due to the extra cushioning it provides, the layers of foam also provide a route for the smoldering front to spread. The stuffing material consisted of $66 \%$ resin treated shredded padding of

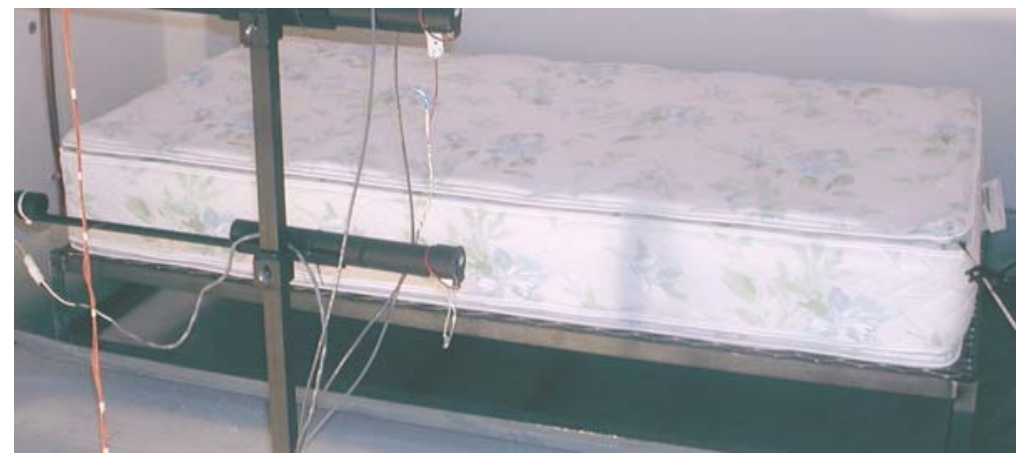

Figure 81. Mattress used for smoldering and flaming experiments

unknown composition and $34 \%$ polyurethane foam. The mattress rigidity was provided by a metal innerspring unit. Figure 81 shows a picture of the mattress. The mattress mass was $19 \mathrm{~kg}$.

\subsubsection{Cooking Materials}

The most frequently occurring fire in the United States is the cooking materials fire. $500 \mathrm{~mL}$ of $100 \%$ corn oil was heated on a gas range until the fuel ignited and was consumed in the fire. Corn oil was selected because it is commonly used and a repeatable fuel source.

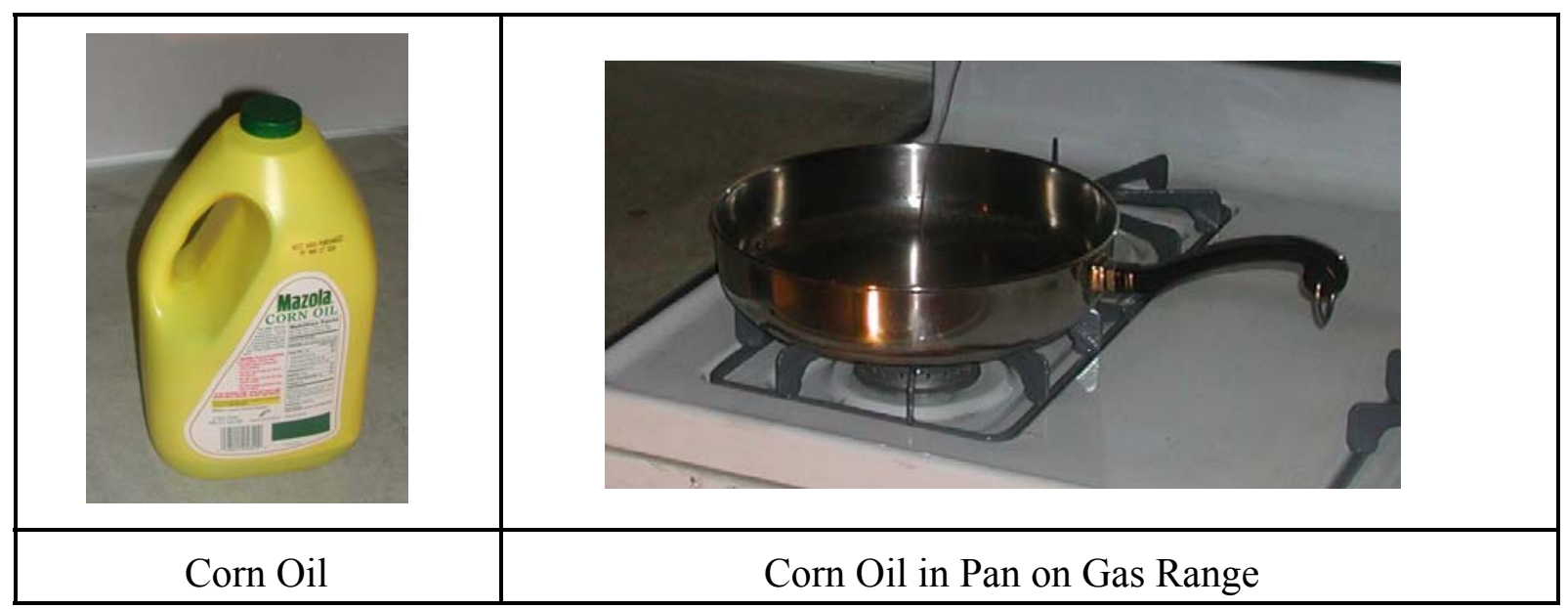

Figure 82. Corn oil used for kitchen cooking materials fire experiments 


\subsection{Ignition Methodology}

There were three primary ignition sources: flaming, smoldering, and cooking. The flaming ignition source required a moderate flame source which would quickly ignite the material. The smoldering source necessitated a slow, steady introduction of heat to the material in order to initiate a self-propagating smolder front. The cooking material ignition source was easier to define with a gas cooking range heating source. The size, timing, and location of each source were design to give repeatable results, to the extent possible.

\subsubsection{Flaming Ignition}

One of the goals of the experiments was to quantify the performance of differing smoke detector technologies, which suggests that a slower fire growth rate will act to more clearly differentiate smoke detector alarm times. Thus, a relatively small ignition source, an electric match, was chosen. An electric match (see figure 83) is a cardboard match book with the cover flipped back, and with a small gauge nichrome wire loop run through the sulfur ends of the matches. The other end of the wire was attached to a switched power source. When the switch was closed, the wire heated quickly, igniting the matchbook within $2 \mathrm{~s}$. Since the match book would burn out quickly, a folded piece of paper surrounding the match book assembly extended the flaming for approximately $20 \mathrm{~s}$ to ensure ignition of the surrounding material.

The geometric placement of the electric match

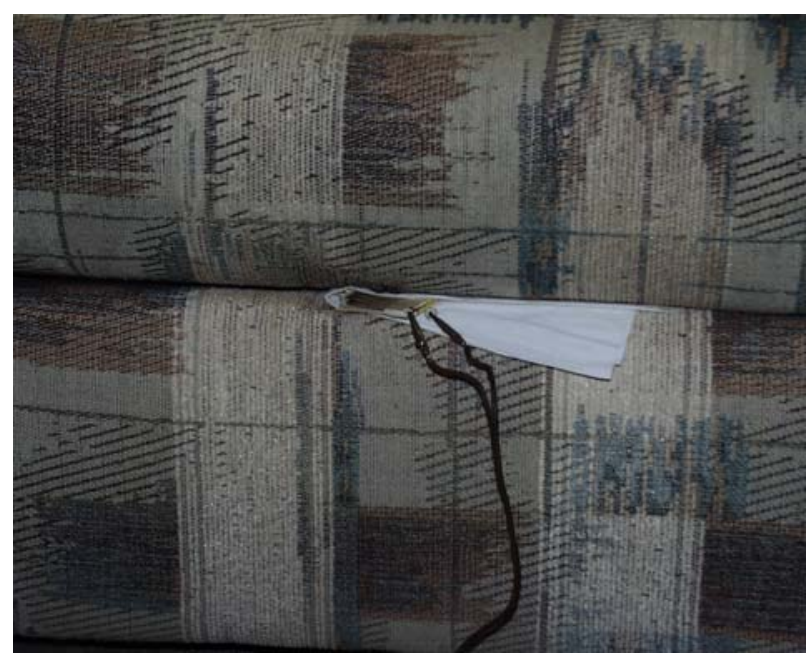

Figure 83. "Electric match" used for flaming ignitions

assembly was selected in order to minimize the fire growth rate. Beyond the characteristics of the fuel, the flame spread rate is largely determined by the available surface area and the orientation of the surface. In a horizontal orientation, the area of material involved in the combustion process grows in a circular manner, away from the origin. Thus, eliminating any direction for the flames to propagate reduces the surface area available to the fire. Locating the origin at the edge of a horizontal surface reduces by half the available surface area. Flames travel more rapidly up vertical surfaces than across horizontal surfaces due to buoyant convection of the heated gases.

In order to reduce the fire growth rate, the origin was located away from large vertical surfaces (the arms and back of the chair and the sides of the mattress) since flames travel more rapidly 
vertically than horizontally. The electric match was located near an edge or corner to minimize the available surface area. The location of the electric match was in the front of the chair between the cushion and the frame. This minimized the horizontal surface area and maximized the distance to the arms and back as shown in figure 84. A similar rationale was used to select the ignition point for the mattress, also shown in figure 84 .

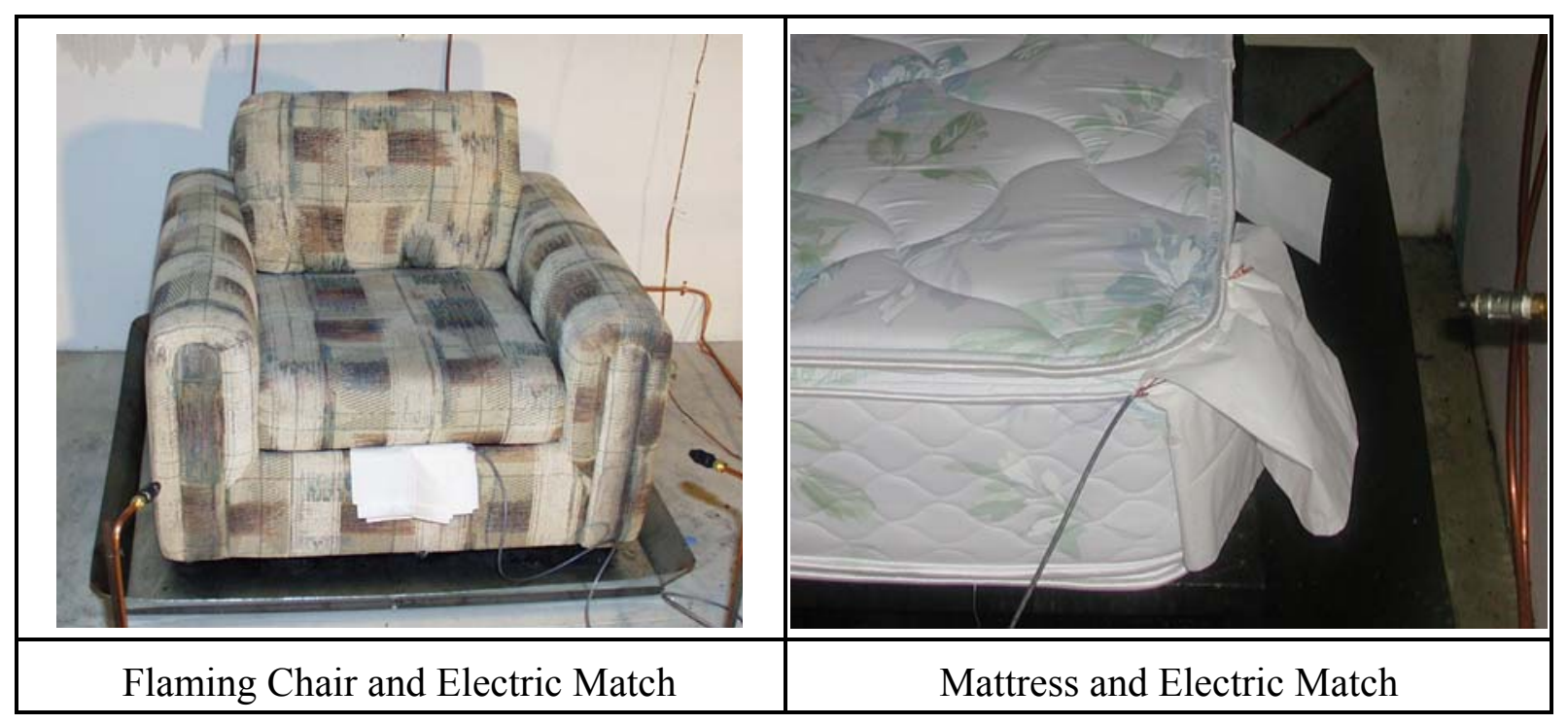

Figure 84. Location of "electric match" ignition source for flaming upholstered chair and mattress experiments

\subsubsection{Smoldering Ignition}

Sustained smoldering in the urethane foam was difficult to reliably reproduce. If the rate of heating was too high, the foam would either melt and move away from the smoldering region, or runaway reactions would transition the material to flaming combustion. If the rate of heating was too low, the heat input would not sustain the pyrolysis of the material and the smoldering front would extinguish. $30 \mathrm{~min}$ to120 min of smoldering could be achieved in these experiments, if sufficient urethane foam was available, using the techniques described here. 
Figure 85 shows the smoldering rod fabricated for theses tests. The rod material was ceramic through which nichrome wire was enclosed. The total length of wire was about $0.76 \mathrm{~m}$ (30 in) A loop was formed by the wire at the end of the rod. The diameter of the loop was approximately $0.13 \mathrm{~m}$ (5 in). The wires were connected to a variac to control the voltage (and thus the current applied) to the loop.

Smoldering was initiated with a low current that was slowly increased as necessary to insure sufficient heat to sustain extended smoldering of

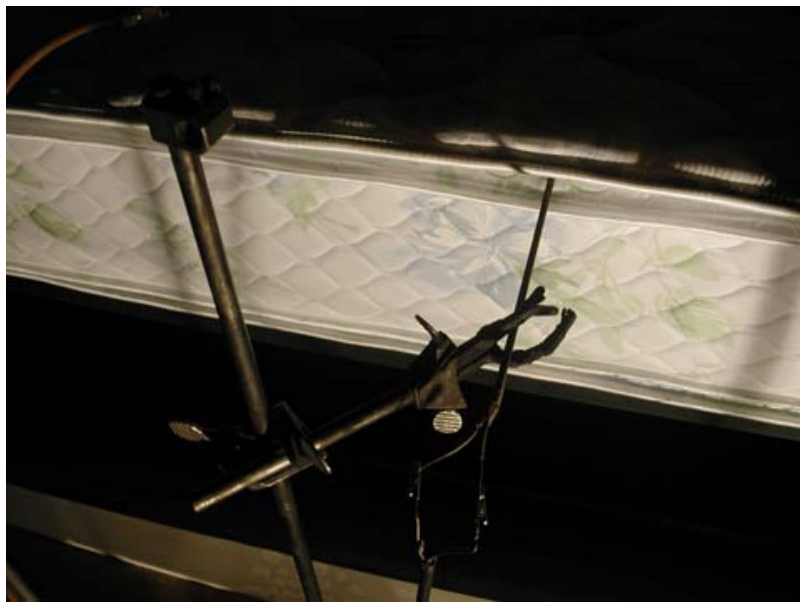
the polyurethane foam. Typical heat output was approximately $0.2 \mathrm{~kW}$ A thermocouple,

Figure 85. Rod used for smoldering ignitions attached to the top surface of the seat cushion or mattress above the wire loop was monitored during the test to insure continued smoldering. The test was terminated once flaming occurred.

\subsubsection{Cooking Ignition}

The cooking oil fire scenario produced both extended pyrolyzing and extended flaming combustion. The $500 \mathrm{~mL}$ of corn oil was poured into a $0.3 \mathrm{~m}$ (12 in) diameter aluminum saute pan. A propane burner provided approximately $1.5 \mathrm{~kW}$ to the underside of the pan, typical of cooking applications. The corn oil pyrolyzed for a period of time, after which a transition to flaming occurred, consuming the remainder of the corn oil. Figure 86 shows the heating ignition source.

\subsection{Test Geometry}

\subsubsection{Manufactured Home}

The manufactured home geometry represented an array of residential layouts. The arrangement of the manufactured home was sufficiently generic as to

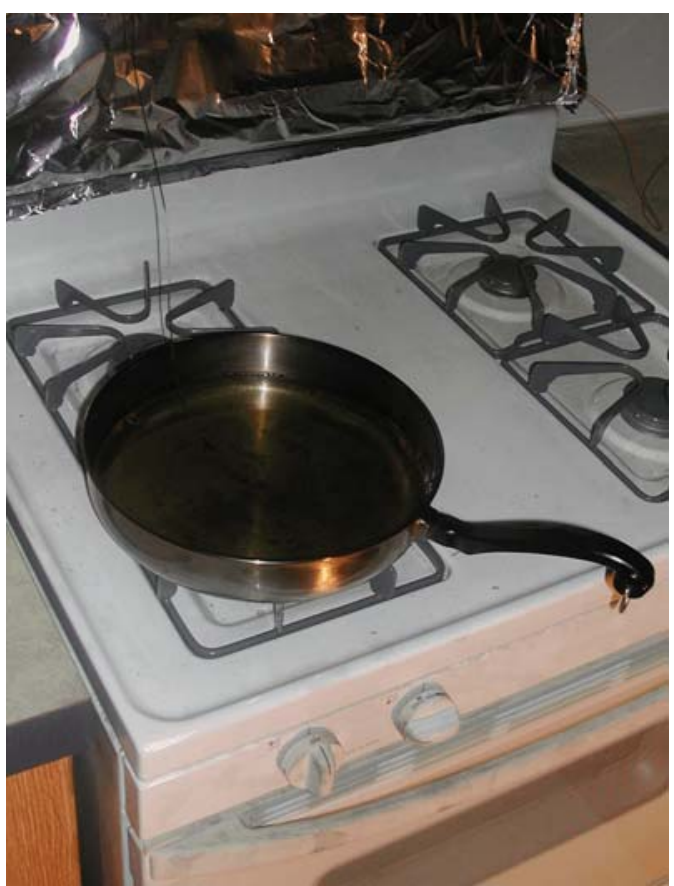

Figure 86. Heating ignition source with cooking oil represent an apartment, condominium, or small ranch house, in addition to a manufactured home. The primary partitioning of the $85 \mathrm{~m}^{2}\left(910 \mathrm{ft}^{2}\right)$ home consisted of three bedrooms, one full bathroom, one kitchen/dining area, one living room, and two hallways. Each bedroom also 
contained a small closet. Figure 87 shows the general layout of the manufactured home. For testing, the doors to the bedroom 3 and the bathroom were always closed. Conditions in the middle bedroom were monitored during the tests to assess the tenability of the environment behind the closed door. In the bathroom, a partially open window $\left(0.06 \mathrm{~m}^{2}\right)$ minimized the pressure differential to the outside during tests. Additionally, both doors leading outside the manufactured home were closed during testing. The ceiling of the manufactured home was peaked on the long axis, reaching a height of $2.4 \mathrm{~m}(8 \mathrm{ft})$. The outside walls of the manufactured home were approximately $2.1 \mathrm{~m}(7 \mathrm{ft})$ in height. The slope of the ceiling was approximately $8.4^{\circ}$. Smoke detector arrays were mounted parallel to the ceiling.

\subsubsection{Two-story Home}

The two-story home in Kinston, N.C. was a brick-clad 3 bedroom home, $139 \mathrm{~m}^{2}\left(1490 \mathrm{ft}^{2}\right)$ in size. The first floor consisted of a foyer, den, family room, kitchen, dining room, bathroom, and stairwell to the upstairs. The home did not have a basement. The 2 car garage was accessible from the first floor den. The second floor consisted of a stairwell to the downstairs, hallway, 2 bathrooms, and two smaller bedrooms and one master bedroom. Figures 88 and 89 show the layout of the two-story home. 


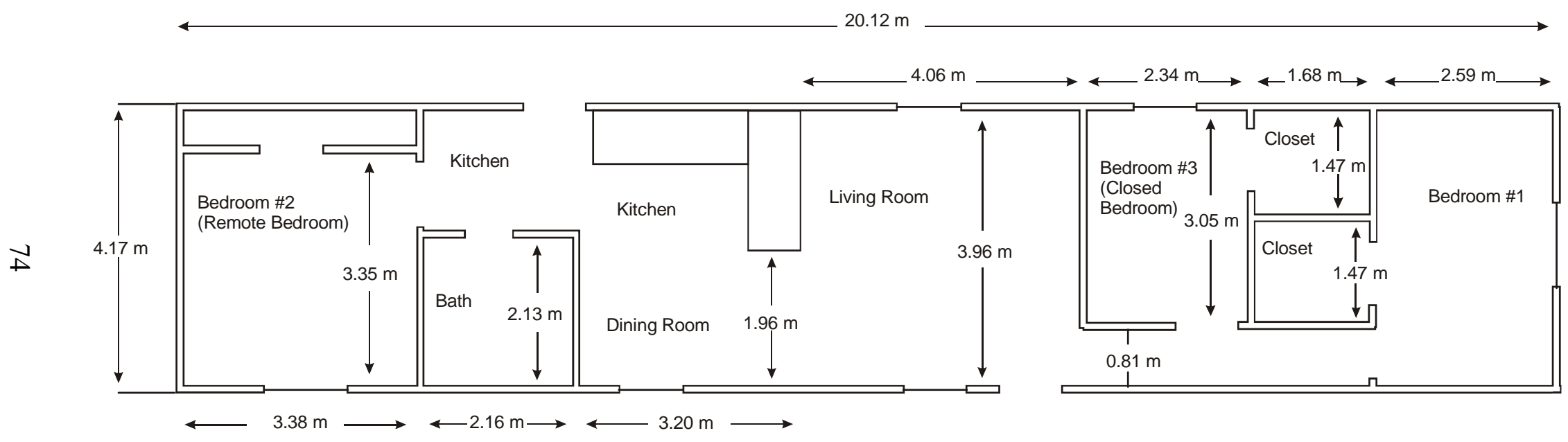

Figure 87. Layout of manufactured home used for test of smoke alarm performance 


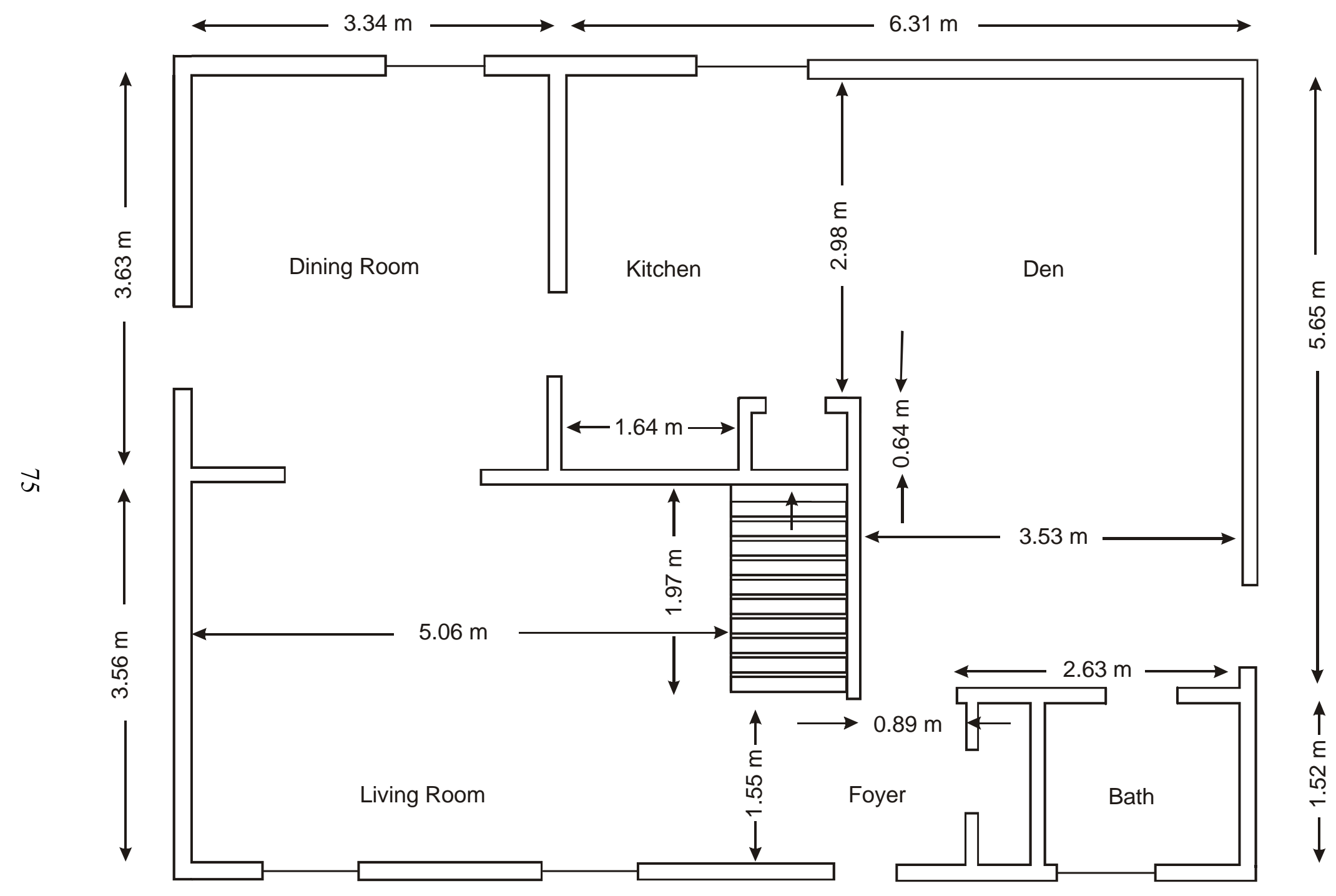

Figure 88. Layout of first floor of two-story home used for tests of smoke alarm performance 


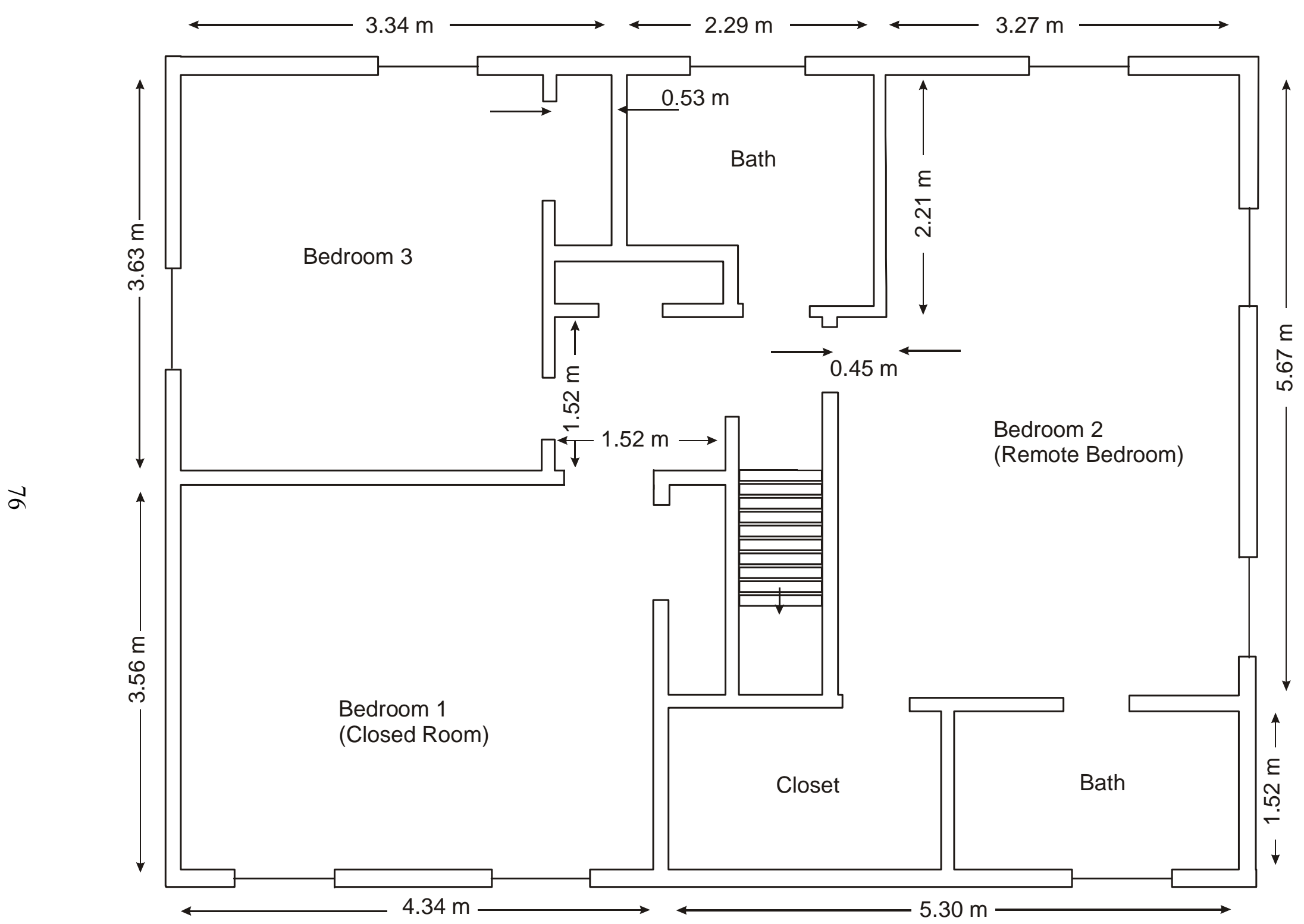

Figure 89. Layout of second floor of two-story home used for tests of smoke alarm performance 


\section{Fire Source Testing Instrumentation}

Fire produces heat, smoke, and toxic gases, all of which can threaten human life. In these experiments environmental parameters that have an impact on human response were measured. Parameters included temperature rise, toxic gas production, and smoke obscuration. Aspects of these that can be measured directly include the gas temperature, mass loss of the burning item, species concentrations, including $\mathrm{CO}, \mathrm{CO}_{2}, \mathrm{O}_{2}$, and other gases. The instrumentation and methods used to measure each of these quantities are discussed below. Readers interested in a general overview of large-scale fire testing data collection and analyses are referred to Peacock and Babrauskas [21].

\subsection{Temperature}

Temperature measurements were accomplished using small diameter, bare-bead type $\mathrm{K}$ thermocouples. Thermocouple trees that consist of thermocouples spaced evenly from floor to ceiling provide a measure of vertical temperature stratification (an example is shown in figure 90). Bare-bead trees were arranged in the room of origin, in at least one remote bedroom, and along the egress path to provide vertical temperature distributions as a function of time. The barebead thermocouples were constructed from $0.25 \mathrm{~mm}$ diameter wire. Table 7, below, summarizes the location of

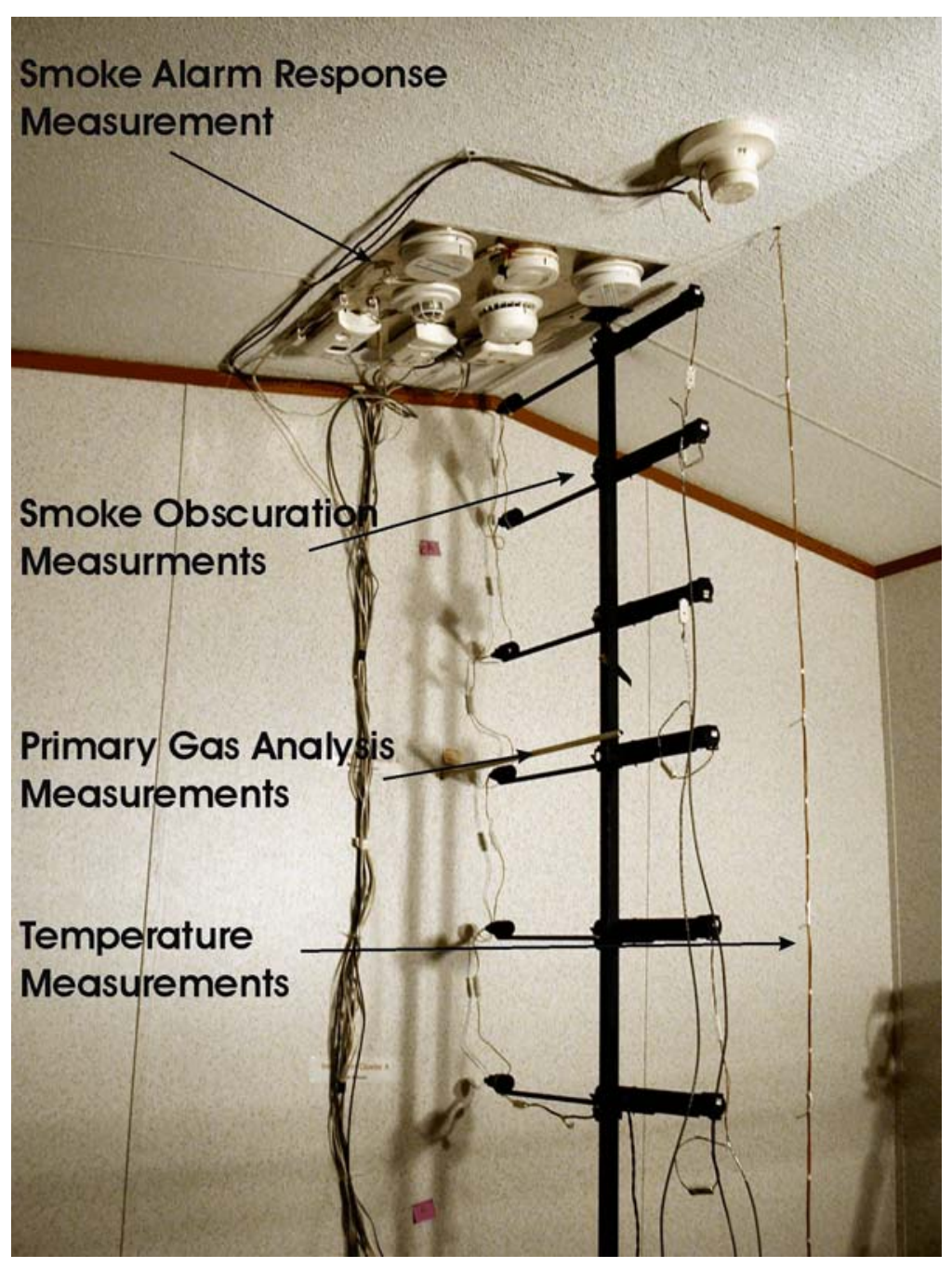

Figure 90. Sample instrumentation showing relative locations of temperature, primary gas analysis, smoke obscuration, and smoke alarm response measurements 
temperature measurement in the different test structures. Figures 91 to 93 show the instrumentation in the manufactured home and two-story home, respectively.

For a series of well-controlled gas burner tests [22], the standard uncertainty for peak gas temperature was found to be $\pm 16{ }^{\circ} \mathrm{C}$. These are expressed as the standard deviation of the peak values for 12 replicate tests. While random error in the current experiments is expected to be comparable to these values, additional uncertainty due to variation in the ignition and fire growth of the fire sources from this test series can be expected. Replicate tests were conducted for this report to better quantify uncertainty.

Table 7. Locations for temperature measurement in tests of smoke alarm response

\begin{tabular}{|c|c|c|}
\hline Structure & Location & $\begin{array}{l}\text { Vertical Positions } \\
\text { (mm from ceiling) }\end{array}$ \\
\hline \multirow{7}{*}{$\begin{array}{l}\text { Manufactured } \\
\text { Home }\end{array}$} & Bedroom 2 (remote bedroom) & $20,300,610,900,1220,1520,1820$ \\
\hline & $\begin{array}{l}\text { Bedroom } 1 \text { (room of fire origin for } \\
\text { mattress tests) }\end{array}$ & $20,300,610,900,1220,1520,1820$ \\
\hline & Hallway 1 (outside bedroom 2) & $20,300,610,900,1220,1520,1820$ \\
\hline & Hallway 2 (outside bedrooms 1 and 3) & $20,300,610,900,1220,1520,1820$ \\
\hline & $\begin{array}{l}\text { Living area (room of fire origin for } \\
\text { upholstered chair and cooking oil tests) }\end{array}$ & $20,300,610,900,1220,1520,1820$ \\
\hline & Hallway 2 (near living room) & $20,300,610,900,1220,1520,1820$ \\
\hline & Bedroom 3 (closed bedroom) & 1520 \\
\hline \multirow{7}{*}{$\begin{array}{l}\text { Two-Story } \\
\text { House }\end{array}$} & Bedroom 2 (remote bedroom) & $20,300,610,900,1220$ \\
\hline & Second floor hallway & $20,300,610,900,1220$ \\
\hline & $\begin{array}{l}\text { Bedroom } 3 \text { (room of fire origin for } \\
\text { mattress tests) }\end{array}$ & $20,300,610,900,1220$ \\
\hline & First floor foyer & $20,300,610,900,1220$ \\
\hline & First floor den & $20,300,610,900,1220$ \\
\hline & $\begin{array}{l}\text { Living room (room of fire origin for } \\
\text { upholstered chair tests) }\end{array}$ & $20,300,610,900,1220$ \\
\hline & $\begin{array}{l}\text { Kitchen (room of fire origin for cooling } \\
\text { oil tests) }\end{array}$ & $20,300,610,900,1220$ \\
\hline
\end{tabular}




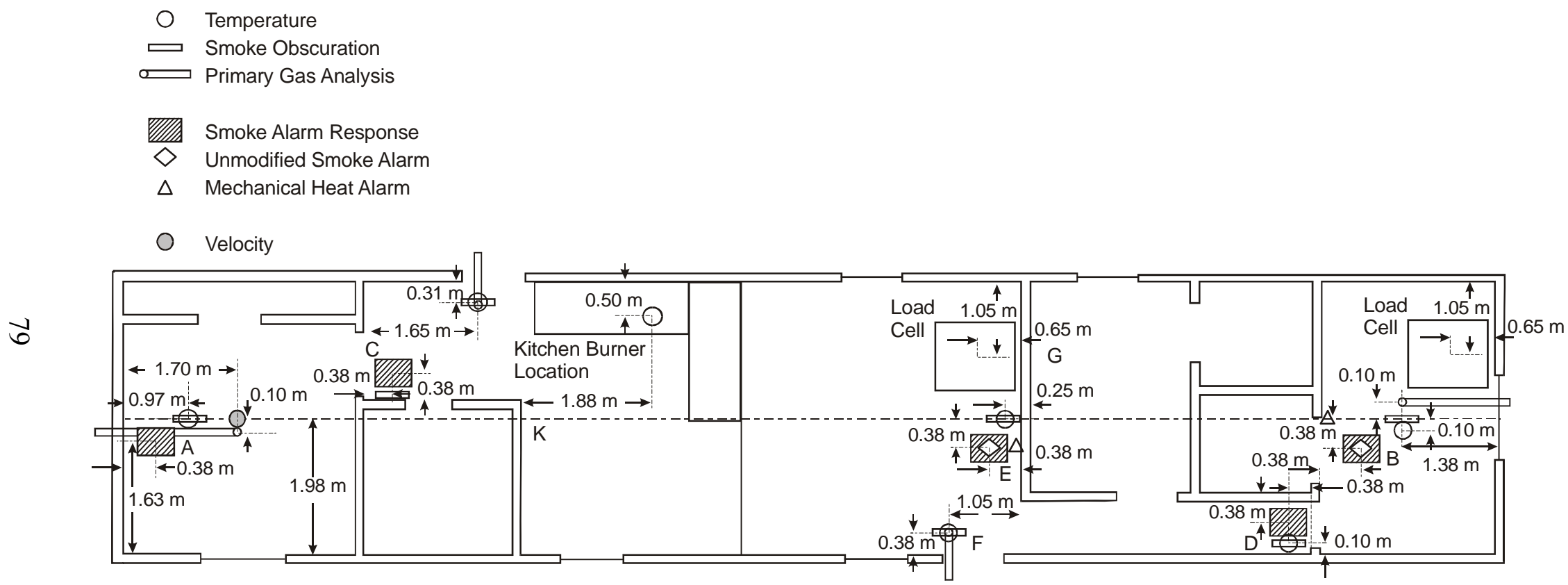

Figure 91. Instrumentation in manufactured home during tests of smoke alarm performance 


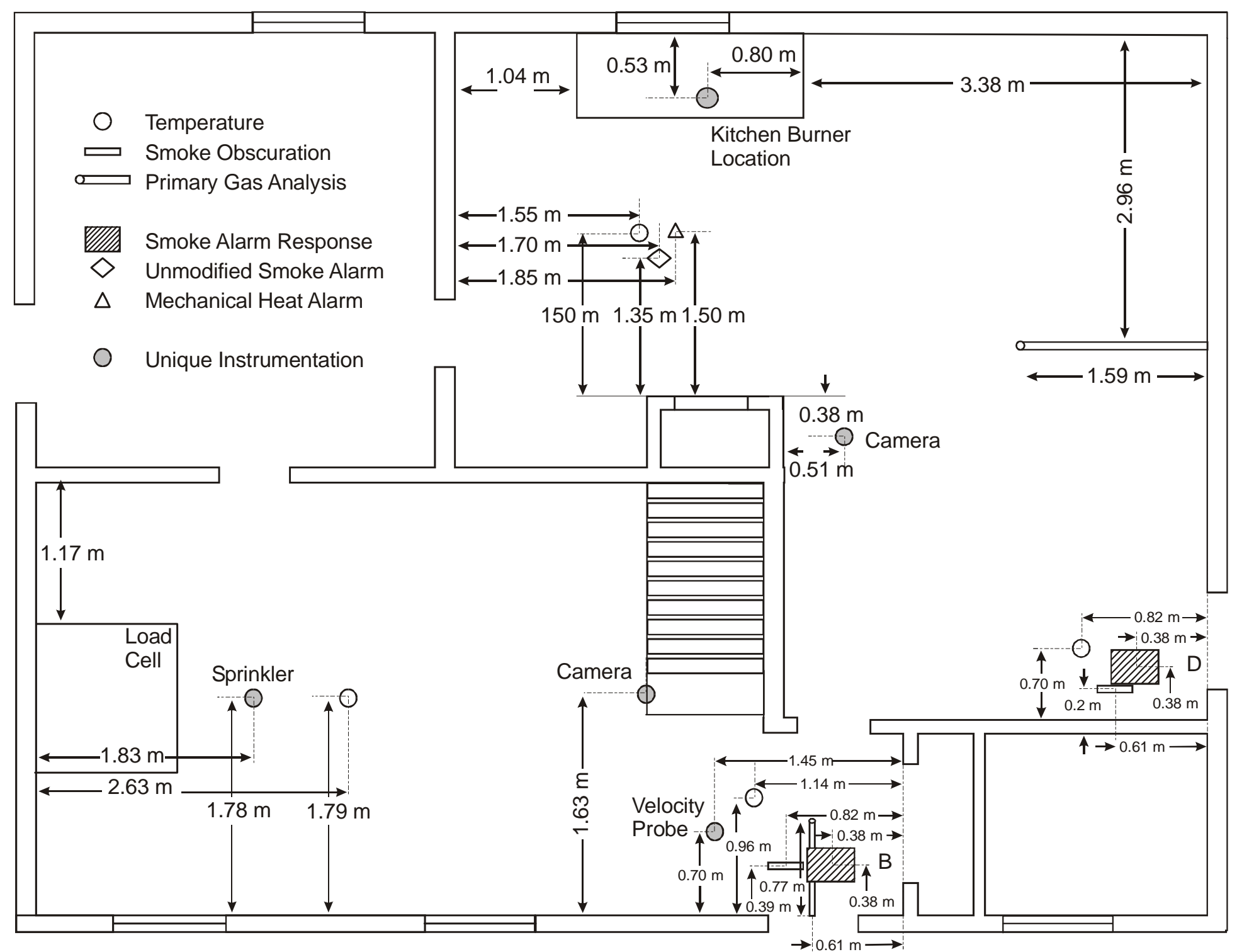

Figure 92. Instrumentation in first floor of two-story home during tests of smoke alarm performance 


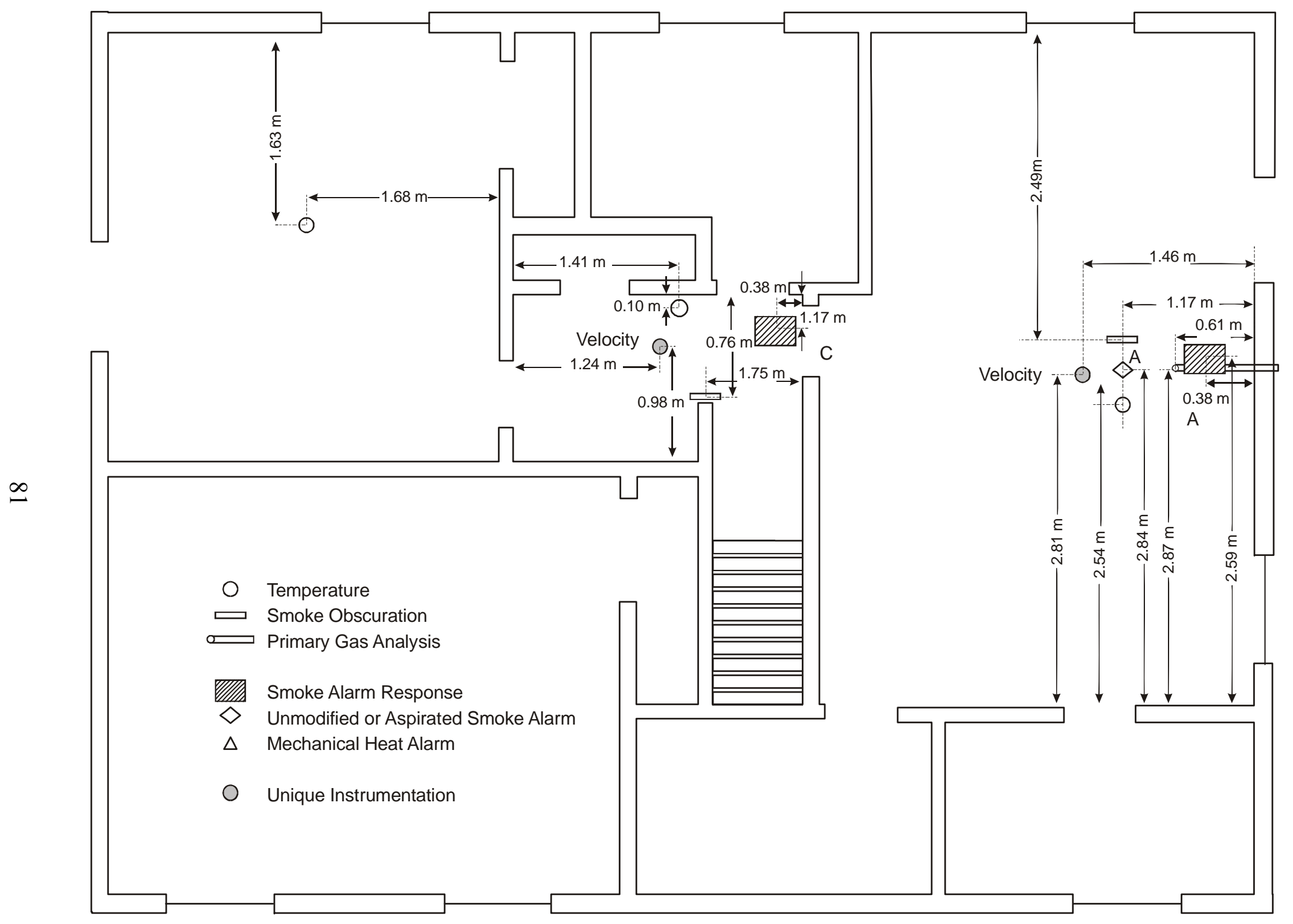

Figure 93. Instrumentation in second floor of two-story home during tests of smoke alarm performance 


\subsection{Sample Mass}

Mass loss from the object of fire origin was recorded using a floor-mounted load cell apparatus. Figure 94 shows the apparatus for one of the flaming ignition tests. As the object of fire origin is consumed by the fire, the mass decreases. The mass was measured over time, yielding the instantaneous measure of specimen mass, as well as the total mass consumed by the fire. The mass loss rate is simply the rate of change of the measured mass loss. It is determined from the slope of a straight line fit through seven adjacent points to minimize noise in the instantaneous measurements. Mass loss rate is directly correlated with the generation of combustion products (toxic species, smoke, etc.), as well as heat release rate.

Standard uncertainty for the mass loss measurements is expressed as a percentage of the full-scale resolution of the load cells. For the load cell used for these tests, this is approximately $\pm 2 \mathrm{~g}$. While random error in the current experiments is expected to be comparable to these values, additional uncertainty due to variation in the ignition and fire growth of the fire sources from this test series can be expected. Replicate tests were conducted for this report to better quantify uncertainty.
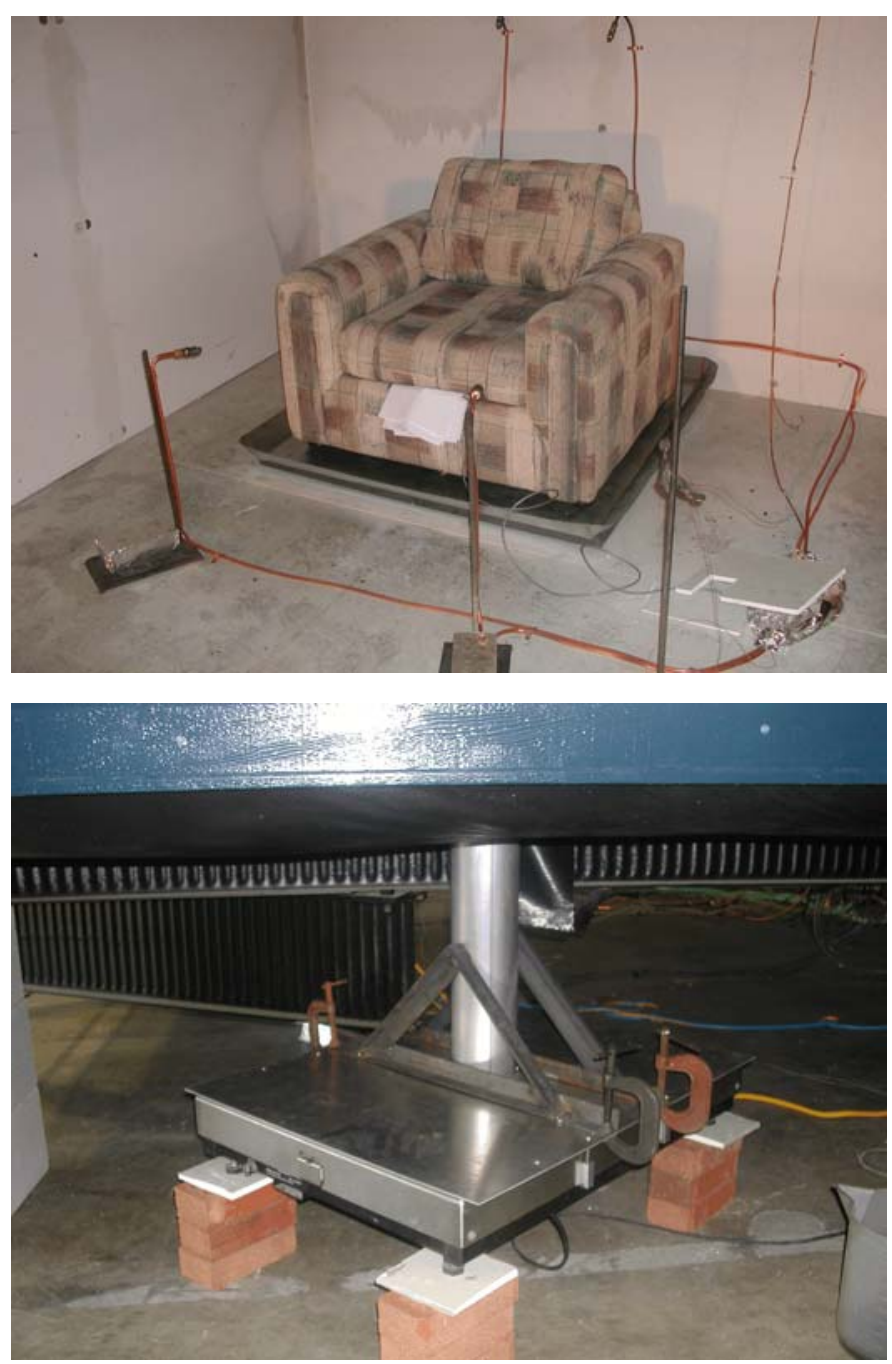

Figure 94. Example of mass loss measurement showing upholstered chair on load cell prior to one of the flaming ignition tests and load cell apparatus mounted beneath manufactured home 


\subsection{Primary Gases - $\mathrm{CO}, \mathrm{CO}_{2}$, and $\mathrm{O}_{2}$}

Measurement of $\mathrm{CO}, \mathrm{CO}_{2}$, and $\mathrm{O}_{2}$ concentrations, along with temperature and smoke obscuration, provide an assessment of the tenability of a fire environment and allow determination of available egress time (discussed in section 5.4). $\mathrm{CO}$ and $\mathrm{CO}_{2}$ measurements were performed with nondispersive infrared (NDIR) analysis. NDIR works on the principle that a gas species will absorb infrared light at a known wavelength. These instruments are carefully designed to avoid absorption bands that are near those of strong interfering bands such as those of water. $\mathrm{O}_{2}$ measurements were made using paramagnetic analyzers. In these tests, primary gas concentration measurements were made in the room of fire origin, in at least one remote bedroom and at two points along the egress path. An example of the measurement point at one of the locations is shown in figure 90. For all experiments and locations, the NDIR gas analysis sampling point was located at an elevation of $1.5 \mathrm{~m}(5 \mathrm{ft})$ from floor level. Table 8 , below, summarizes the location of gas analysis in the two test geometries.

Table 8. Locations for primary gas measurement in tests of smoke alarm response

\begin{tabular}{|l|l|l|}
\hline Structure & Location & $\begin{array}{l}\text { Vertical Positions } \\
(\mathrm{mm} \text { from ceiling) }\end{array}$ \\
\hline \multirow{4}{*}{$\begin{array}{l}\text { Manufactured } \\
\text { Home }\end{array}$} & Bedroom 2 (remote bedroom) & 900 \\
\cline { 2 - 3 } & Bedroom 1 (only for mattress tests) & 900 \\
\cline { 2 - 3 } & Hallway 1 (outside bedroom 2) & 900 \\
\cline { 2 - 3 } & $\begin{array}{l}\text { Living area (only for upholstered chair and cooking } \\
\text { oil tests) }\end{array}$ & 900 \\
\cline { 2 - 3 } & Hallway 2 (near living room) & 900 \\
\hline \multirow{2}{*}{$\begin{array}{l}\text { Two-Story } \\
\text { House }\end{array}$} & Bedroom 2 (remote bedroom) & 900 \\
\cline { 2 - 3 } & First floor foyer & 900 \\
\hline
\end{tabular}

Upstream of the analyzers, the gas stream was filtered to remove particulates and also passed through ice water and dry ice traps to remove water from the samples. Each analyzer was calibrated with a known concentration of gas prior to each test to insure correct operation and provide appropriate small corrections to manufacturer-supplied calibration curves. Typically, this correction was less than $1 \%$ of the measured value. Additionally, delay times to account for line delays in the instrumentation for each measurement location was determined by introducing a gas pulse at the measurement point and noting the time required for the analyzer to respond. 
With different line lengths, vacuum pumps, and filters in each analyzer system, these delays can be expected to be different for each analyzer. With multiple replicates, this delay ranged from $9 \mathrm{~s} \pm 1 \mathrm{~s}$ to $34 \mathrm{~s} \pm 3 \mathrm{~s}$. Test data were shifted to account for these delay times.

For a series of well-controlled gas burner tests [22], the standard uncertainty for oxygen concentration, carbon dioxide concentration, and carbon monoxide concentration was found to be $\pm 0.6 \%$ volume fraction, $\pm 0.4 \%$ volume fraction, and $\pm 0.06 \%$ volume fraction, respectively. These are expressed as the standard deviation of the peak values for 12 replicate tests. While random error in the current experiments is expected to be comparable to these values, additional uncertainty due to variation in the ignition and fire growth of the fire sources from this test series can be expected. Replicate tests were conducted for this report to better quantify uncertainty.

\subsection{FTIR Gas Analysis}

In the manufactured home tests, the secondary gas measurement was performed by representatives from National Research Council of Canada using FTIR (Fourier Transform Infrared) techniques. FTIR gas analysis provides measures of additional gases which may effect tenability, such as $\mathrm{HCN}$, $\mathrm{HCl}, \mathrm{NO}$, or $\mathrm{NO}_{2}$, in addition to providing a second measure of $\mathrm{CO}$ and $\mathrm{CO}_{2}$. Data on secondary gases are included as Appendix B.

\subsection{Optical Density}

Optical density was measured using laserbased light extinction measurement trees. A laser beam's signal strength is measured over time. As smoke passes through the laser beam, the smoke absorbs and reflects a fraction of the light, reducing the light level at an in-line receiver. Figure 95 shows the two designs of smoke obscuration instruments used in the tests. The light source was a low-cost laser pointer consisting of Class II laser diode with a stated wavelength range of $630 \mathrm{~nm}$ to $680 \mathrm{~nm}$ and a maximum power output of less than a 1

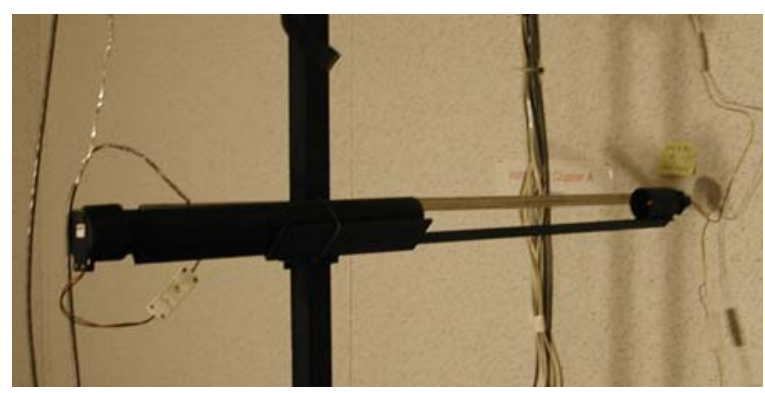

(a)

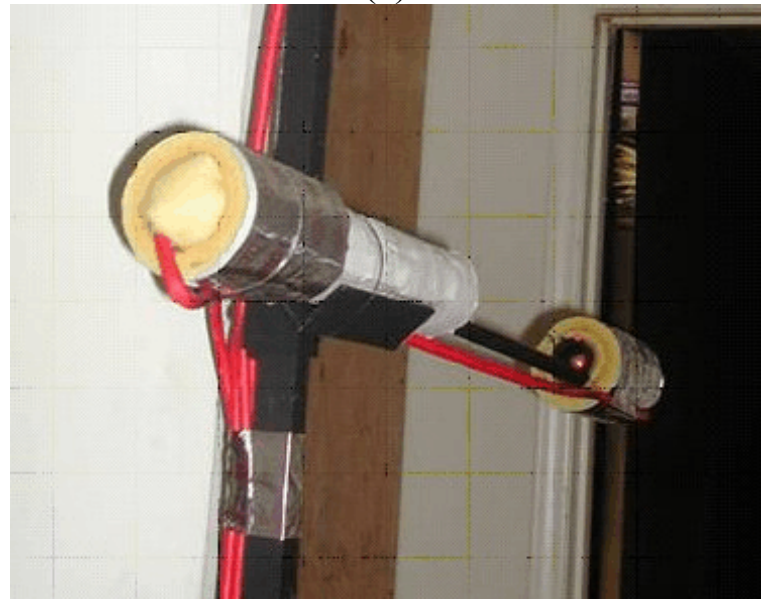

(b)

Figure 95. Optical density instrument designs 
$\mathrm{mW}$, coupled to a plastic lens. The laser was powered by a regulated power supply set at a constant voltage of $4.50 \mathrm{~V}$. The light receiver was a silicon photovoltaic cell (i.e., solar cell) where the open-circuit voltage was monitored by an analog voltage input channel of the data acquisition system. The "clean air" voltage was typically between $0.15 \mathrm{~V}$ and $0.3 \mathrm{~V}$ depending on the smoke meter, and its alignment before a test. Prior to each test, the smoke meters were powered up, allowed to reach a steady state, and calibrated with neutral density filters covering a range in optical densities. Neutral density filters typically have an uncertainty of $2 \%$ of the stated value. The data were fitted to calibration curves, which were then used to compute the optical densities from the voltages gathered during the subsequent test. The relative uncertainty of the field-calibrated smoke meters was typically $10 \%$ of the reading.

Optical density was measured near smoke alarms, in the room of origin, in at least one remote bedroom, and along the egress path. Smoke obscuration coupled with irritant effects may impair the ability of occupants to egress in fires [23]. Figure 90 shows an example set of smoke obscuration instruments in one of the bedrooms of the manufactured home. Measurements of smoke obscuration from floor to ceiling were made in the room of fire origin, at least one remote bedroom, and at locations along the egress path. Table 9, below, summarizes the location of optical density measurements in the two test geometries.

As testing progressed, the design of the optical density instruments evolved. Three different designs were used in the three test series. In test 1-15, the design shown in figure 95a was used. Although the simplest design, the output of the in-line receiver changed at high temperature. Since the primary focus of these tests was early detection, the design provided reasonable results for all but the hottest measurement locations. For tests 20-41, the design was modified to include high temperature insulation around both the laser and receiver ends of the instrument (figure 95b). In addition, for tests 30-41, more insulation was added along with a glass window on the receiver end to clearly define the path length. 
Table 9. Locations for primary optical density measurements in tests of smoke alarm response

\begin{tabular}{|l|l|l|}
\hline \multirow{4}{*}{ Structure } & Location & $\begin{array}{l}\text { Vertical Positions } \\
(\mathrm{mm} \text { from ceiling) }\end{array}$ \\
\hline \multirow{4}{*}{$\begin{array}{l}\text { Manufactured } \\
\text { Home }\end{array}$} & Bedroom 2 (remote bedroom) & 20,900 \\
\cline { 2 - 3 } & Bedroom 1 (room of fire origin for mattress tests) & 20,900 \\
\cline { 2 - 3 } & Hallway 1 (outside bedroom 2) & 20,900 \\
\cline { 2 - 3 } & Hallway 2 (outside bedrooms 1 and 3) & 20,900 \\
\cline { 2 - 3 } & $\begin{array}{l}\text { Living area (room of fire origin for upholstered chair } \\
\text { and cooking oil tests) }\end{array}$ & 20,900 \\
\cline { 2 - 3 } & Hallway 2 (near living room) & 20,900 \\
\cline { 2 - 3 } & Bedroom 3 (closed bedroom) & 1020 \\
\hline \multirow{4}{*}{$\begin{array}{l}\text { Two-Story } \\
\text { House }\end{array}$} & Bedroom 2 (remote bedroom) & 20,900 \\
\cline { 2 - 3 } & Second floor hallway & 20,900 \\
\cline { 2 - 3 } & First floor foyer & 20,900 \\
\cline { 2 - 3 } & First floor den & \\
\hline
\end{tabular}

The main function of the field test smoke meters was to provide obscuration data for assessing tenability limits. An optical density of $0.25 \mathrm{~m}^{-1}(1.5 \mathrm{~m}$ from the floor) was used in the analysis as the tenability criterion for smoke obscuration. It is important to note that heat transfer from hot gases transported to the smoke meters causes their voltage output to drift, giving a false indication of smoke and a larger apparent optical density than what would be due to the smoke in the optical path. The time after a significant amount of heat transfer occurred represents a period of smoke meter operation where the smoke obscuration results would not be reliable.

Smoldering fire tests typically did not produce significantly elevated temperatures, thus the uncertainty in a smoke meter obscuration measurement during smoldering phases is essentially the ambient temperature calibration uncertainty. Laboratory tests on un-insulated and insulated smoke meters indicated that a temperature change approximately $20^{\circ} \mathrm{C}$ to $40{ }^{\circ} \mathrm{C}$ over $100 \mathrm{~s}$ to $200 \mathrm{~s}$, would start to affect the reliability of the computed smoke obscuration by the end of the heating period (the insulated meters performed better.) Therefore, caution must be used when analyzing these smoke meter results anytime the ambient temperature has changed. During the flaming phase of these tests, the optical density tenability limits were reached prior to significant smoke meter heating. 


\subsection{Smoke Properties}

In addition to optical density, other smoke properties were measured in selected tests in one or more locations. Figure 96 shows the instrumentation in the two-story home. The aerosol mass concentration was recorded with a tapered-element oscillating microbalance (TEOM model 1100, Rupprecht and Pastashnick Co., Inc) designed for diesel soot monitoring. This is a widely used instrument for continuous recording of mass concentration. It consists of a filter attached to a tube oscillating in an inverted pendulum fashion. Particle-laden flow is drawn through the filter and down the tube. Aerosol particles that deposit on the filter change the

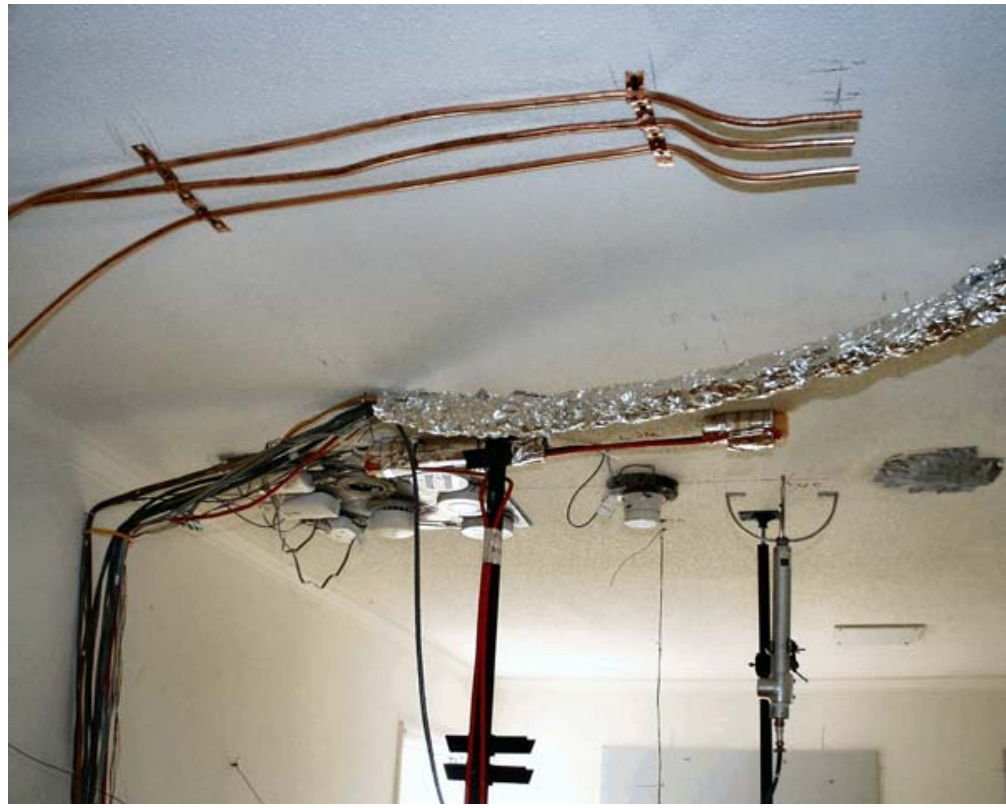

Figure 96. Smoke properties measurement showing collection lines (foreground) and smoke velocity measurement (background) frequency of oscillation, which is sensed and converted into a change in mass. The flow rate is fixed at $3.00 \mathrm{~L} / \mathrm{min}$, hence the aerosol mass concentration can be computed. The air entering the instrument is heated to approximately $45^{\circ} \mathrm{C}$. The range of the instrument was between $0.2 \mathrm{mg} / \mathrm{m}^{3}$ to about $500 \mathrm{mg} / \mathrm{m}^{3}$ with an absolute uncertainty if $0.1 \mathrm{mg} / \mathrm{m}^{3}$. An attempt was made to sample from two different locations during some of the manufactured home tests by switching between the sampling locations every $30 \mathrm{~s}$ with a two-way valve. The TEOM had problems recovering after flow switching, and this technique was abandon.

The number concentration was recorded with a condensation particle counter (model 3022A, Thermo-systems Inc.) This instrument counts all particles in the range of $10 \mathrm{~nm}$ to over $3 \mu \mathrm{m}$ by growing them to a single size via condensation of alcohol vapor and then counting them individually via single-particle light scattering, or by calibrated photometry. The instrument can record up to $1 \times 10^{7}$ particles $/ \mathrm{cm}^{3}$. This upper range was extended to $8 \times 10^{8}$ particles $/ \mathrm{cm}^{3}$ by employing a 80:1 diluter. The stated relative uncertainty is $10 \%$ of reading up to $5 \times 10^{5}$ particles $/ \mathrm{cm}^{3}$. As with the TEOM, an attempt was made to sample from two different locations in the two-story home tests. By switching the flow between two locations every $60 \mathrm{~s}$ it was possible to record the number concentration at $60 \mathrm{~s}$ intervals every $120 \mathrm{~s}$ for each location. 
Finally, a size distribution measurement of the smoke was made using a cascade impactor. A micro-orifice, uniform-deposit impactor (MOUDI) was used. It is a 12-stage impactor that measures the sized distribution from $18 \mu \mathrm{m}$ down to $0.03 \mu \mathrm{m}$. The MOUDI requires 28.3 SLM flow. It provides a time-averaged size distribution over its collection time period. Aerosols over a narrow aerodynamic size are collected on pre-weighed filters by impaction at each stage. After the last stage, a final filter collects all particles that were not impacted. The standard uncertainty of mass collected on any single stage is $0.01 \mathrm{mg}$. Typically, a sum of over $2 \mathrm{mg}$ of material was collected on all stages. The mass fraction of aerosol on each stage is computed and was used to estimate the mass median aerodynamic particle diameter and the width of the size distribution.

Ionization chambers were constructed to emulate the response of a measuring ionization chamber (MIC) to provide an estimate of the first moment of the size distribution. The design of these chambers is described in Section 6. In addition to the ionization chambers, individual modified residential ionization alarms were located at the sampling position for the chambers. Figure 97 shows the response of a modified alarm exposed to cotton smolder smoke generated in the FE/DE as compared to the Y value computed from a MIC located inside the FE/DE. The modified alarm was located inside a plenum and drew air from the FE/DE duct at the test section location. This comparison was used to compute $\mathrm{Y}$ values from the voltage data from all four modified alarms. In order to compute a $\mathrm{Y}$ value from the voltage, the voltage signal must be transformed into a variable that ranges from 0 to 1 over a smoke concentration of 0 to infinity (where the MIC goes from $100 \mathrm{pA}$ to $0 \mathrm{pA}$, and the normalized change in current goes from 0 to 1). The data in figure 97 were fitted via non-linear least squares regression to the following equation:

$Y=X(2-X) /(1-X)$ where the voltage was transformed into $X$, the normalized current change, by $X=\left(V_{\max }-V_{0}\right) /\left(V-V_{0}\right)$; $V_{0}$ is the initial clean air voltage and $V_{\max }$ is a maximum value obtained via the regression.

The regression curve is shown in figure 97. A best-fit value for $\mathrm{V}_{\max }$ was $3.139 \mathrm{~V}$ with a standard error of $0.013 \mathrm{~V}$. A correlation coefficient of 0.9975 indicates a good fit with the equation. Above values of $\mathrm{Y}=2.3$, the equation was extrapolated. Propagating the

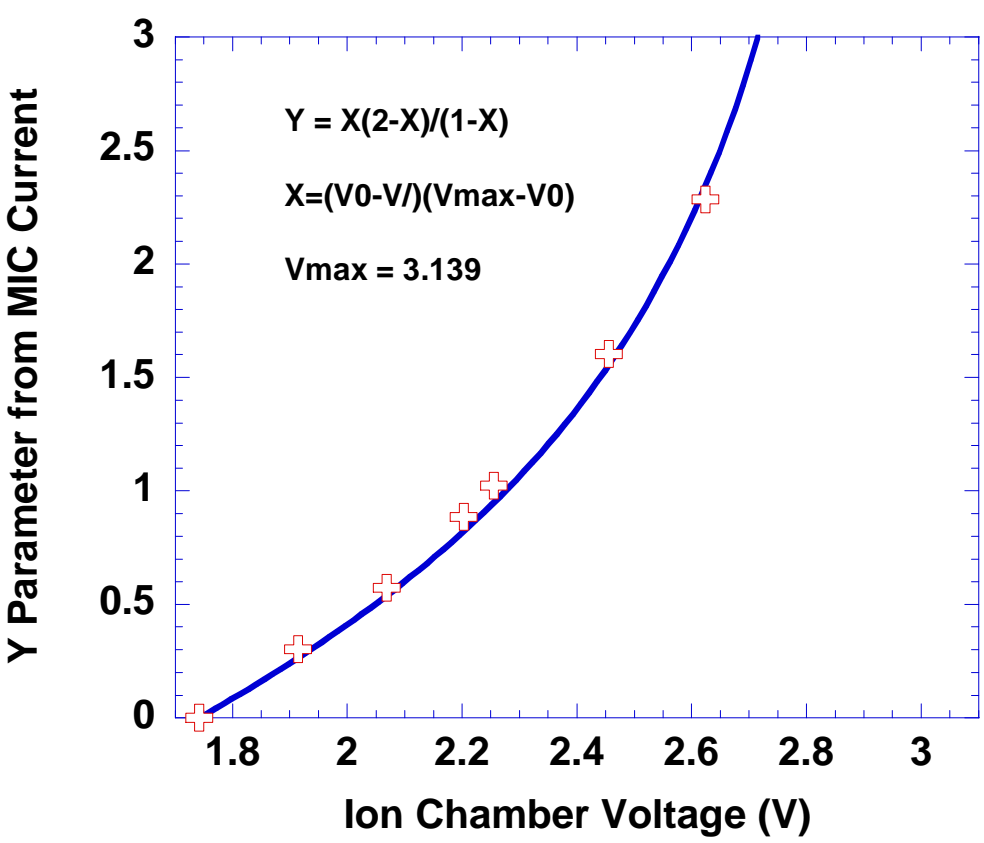

Figure 97. Calibration of residential ionization chamber 
uncertainty in X when computing Y yields a combined standard uncertainty in computed $\mathrm{Y}$ of 0.022 .

All sample lines were $9.5 \mathrm{~mm}$ (3/8 in) OD copper tubing. The main sampling location in the manufactured home tests was $25 \mathrm{~mm}$ below the ceiling in the remote bedroom, $1.60 \mathrm{~m}$ from the window and $1.65 \mathrm{~m}$ from the wall opposing the door. Air was flowed through the ionization chamber, and a fraction of the flow leaving the chamber was sent to the TEOM. Additionally, a sampling location was located $25 \mathrm{~mm}$ below the ceiling in the hallway $1.70 \mathrm{~m}$ from the back bedroom door, and $0.50 \mathrm{~m}$ from the exterior wall. The air sample from this location went to an ionization chamber.

The main sampling location in the two-story home was $25 \mathrm{~mm}$ below the ceiling in the upstairs remote bedroom $1.57 \mathrm{~m}$ from the back wall and $1.60 \mathrm{~m}$ from the garage side wall. The secondary sampling location was $25 \mathrm{~mm}$ below the ceiling in the den $2.44 \mathrm{~m}$ from the back wall and $1.60 \mathrm{~m}$ from the garage sidewall.

\subsection{Smoke and CO Alarm Response}

Figure 98 shows one of the alarm arrays. Alarm response was measured by direct recording of the voltage signal from ionization smoke alarms, photoelectric smoke alarms, and CO alarms arranged for analog output, instead of the more common alarm threshold. By recording analog output, the performance of the alarms at any desired threshold setting as well as the potential use of algorithms that reduce nuisance alarms can be evaluated. The analog signal was compared to unmodified alarms purchased in local, retail outlets, in the laboratory to verify that the

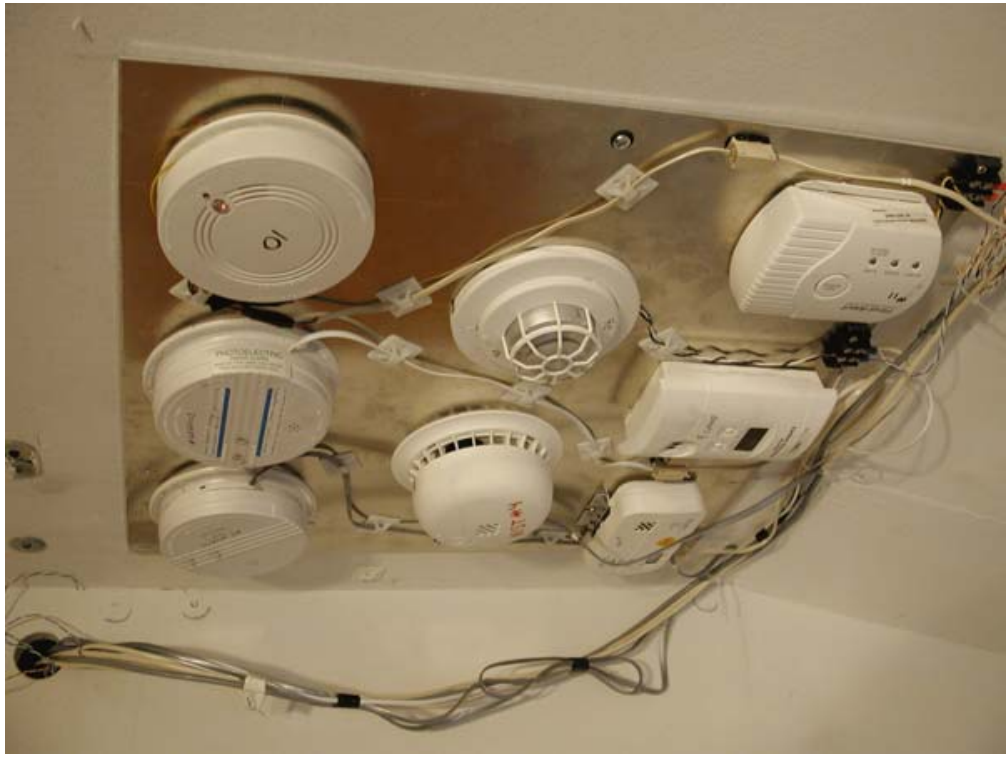

Figure 98. Measurement station showing several alarms arranged for testing modifications did not affect the alarm performance (see section 2.8 for details). Alarms were located in typical, code-required locations, as well as in the room of origin, in order to determine the effectiveness of alternative siting rules. In the room of fire origin, three unmodified alarms were used to avoid destruction 
of the limited supply of analog-modified alarms (figure 99). For these alarms, the interconnect signal was monitored to determine the time of alarm. Table 10 summarizes the alarm locations for the two test structures.

Table 10. Alarm and sprinkler locations in tests of smoke and $\mathrm{CO}$ alarm response

\begin{tabular}{|c|c|}
\hline Structure & Location \\
\hline \multirow{6}{*}{$\begin{array}{l}\text { Manufactured } \\
\text { Home }\end{array}$} & Smoke and CO Alarms - Bedroom 2 (remote bedroom) \\
\hline & $\begin{array}{l}\text { Smoke and CO Alarms and Tell-tale Sprinkler - Bedroom } 1 \text { (room of } \\
\text { fire origin for mattress tests) }\end{array}$ \\
\hline & Smoke and CO Alarms - Hallway 1 (outside bedroom 2) \\
\hline & Smoke and CO Alarms - Hallway 2 (outside bedrooms 1 and 3 ) \\
\hline & $\begin{array}{l}\text { Smoke and CO Alarms and Tell-tale Sprinkler - Living area (room of } \\
\text { fire origin for upholstered chair and cooking oil tests) }\end{array}$ \\
\hline & Smoke and CO Alarms - Hallway 2 (near living room) \\
\hline \multirow{5}{*}{ Two-Story House } & Smoke and CO Alarms - Bedroom 2 (remote bedroom) \\
\hline & Smoke and CO Alarms - Second floor hallway \\
\hline & Smoke and CO Alarms - First floor foyer \\
\hline & Tell-tale Sprinkler - Living Room \\
\hline & Smoke and CO Alarms - First floor den \\
\hline
\end{tabular}

\subsection{Sprinkler Response}

Residential sprinklers have made significant market penetration in multi-family housing. Many current codes include independent requirements for smoke alarms and sprinklers. This is not necessarily the most effective arrangement but limited data exist that demonstrate the relative performance of the combination. Sprinkler response was measured using an air pressurized (so called telltale) sprinkler. A small quantity of water at the sprinkler head provides an appropriate thermal sink. Upon activation, the pressure in the sprinkler line drops to ambient and the activation time is recorded as the time of this pressure drop. For the test installation, pipe lengths were sufficiently short so that measurement delay from sprinkler activation to noted response was less than $2 \mathrm{~s}$. Typical residential sprinklers (listed for installation in a sloped ceiling with up 
to a $26.6^{\circ}$ pitch for the

manufactured home) were

installed in the room of fire origin

in accordance with the requirements

of NFPA 13D, Standard for the

Installation of Sprinkler Systems

in One- and Two-Family

Dwellings and Manufactured

Homes. The sprinklers had a listed

activation temperature of $68^{\circ} \mathrm{C}$

$\left(155^{\circ} \mathrm{F}\right)$ with a $\mathrm{K}$-factor of 5.5

and were appropriate for spacing

up to $6 \mathrm{~m}(20 \mathrm{ft})$. Table 10

summarizes the alarm locations

for the two test structures.

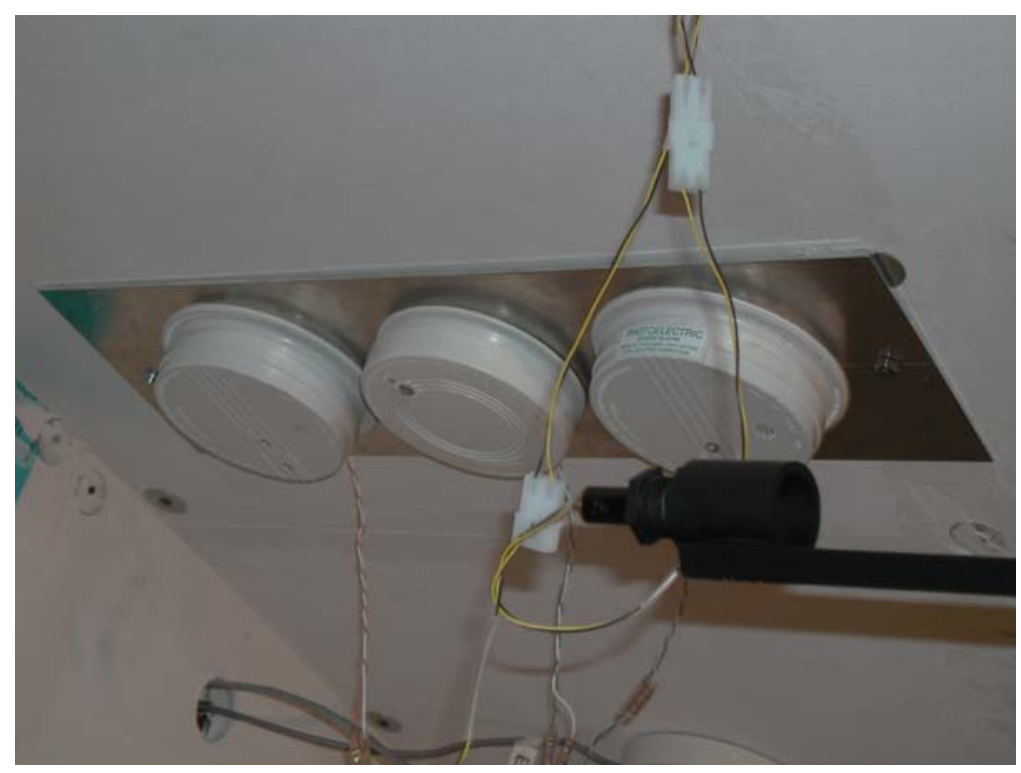

Figure 99. Measurement station showing unmodified

\subsection{Mechanical Heat Alarm}

smoke alarms arranged for testing

\section{Response}

Mechanical heat alarms were located in the room of origin in accordance with the requirements of NFPA 72, the National Fire Alarm Code. The heat alarms were listed for $57^{\circ} \mathrm{C}\left(135^{\circ} \mathrm{F}\right)$ activation at a spacing up to $21 \mathrm{~m}(70 \mathrm{ft})$. Heat alarm response can be compared to the performance of other smoke and heat alarms. Activation was recorded by the acoustic signal emitted by the device. 


\section{Fire Source Test Results and Calculations}

In this section, experimental test results are presented which characterize the environment in typical residential fire scenarios. Data presented include the time varying concentrations of $\mathrm{CO}$, $\mathrm{CO}_{2}$, and $\mathrm{O}_{2}$, gas velocities, smoke obscuration, and temperature at multiple locations in the structure. Additional measurement of smoke particle size and concentration are discussed later in the report.

\subsection{Tests Conducted}

Real-scale tests of current smoke, heat, and carbon monoxide alarms in actual homes with appropriate contents as fuels provide a base of data to evaluate the performance of modern residential alarm technologies. Fire scenarios (including ignition source, first item ignited, and room of fire origin) were selected based upon a statistical analysis of available fire loss data. Selected fires include a mattress fire in a bedroom, upholstered chair fire in a living area, and a cooking oil fire in a kitchen. In most tests, a single item fire was used. In one test, additional items in the room of fire origin were allowed to ignite to more fully characterize fire growth in the structure. Table 11 summarizes the 27 tests conducted in the manufactured home. Similarly, table 12 shows the 9 tests conducted in the two-story home. Multiple replicates of most scenarios were included to quantify repeatability and measurement uncertainty in the experiments.

Inclusion of all of the test data would overwhelm this report, with several thousand readings per instrument, 150 instruments per test, and 36 tests. All of the data from the tests conducted are available in two companion reports, one for the tests conducted in the manufactured home [24], and one for the tests conducted in the two-story home [25]. For each of the tests, these two reports include a summary of the test conditions and ignition source. Graphs of all test data along with spreadsheets of the data are included.

For this report, detailed presentation and analysis will be included for one of the tests. For this test, all of the measured data characterizing the environment (temperature, gas concentrations, smoke obscuration, and specimen mass loss) will be presented. For one of the alarm locations, data from all of the alarms (multiple photoelectric, ionization, aspirated photoelectric, and heat alarms) will be presented. Details of calculations to determine individual alarm times and to estimate time to untenable conditions will be discussed.

For the remainder of the tests, the results of identical analyses will be summarized. 
Table 11. Test conditions for tests conducted in a manufactured home

\begin{tabular}{|c|c|c|c|c|}
\hline Test & Ignition & Fuel Package & Fire Location & Comments \\
\hline SDC01 & Smoldering & Chair & Living Area & \\
\hline SDC02 & Flaming & Chair & Living Area & \\
\hline $\mathrm{SDC} 03$ & Smoldering & Mattress & Bedroom & Ignition failure \\
\hline SDC04 & Smoldering & Mattress & Bedroom & \\
\hline SDC05 & Flaming & Mattress & Bedroom & \\
\hline SDC06 & Smoldering & Mattress & Bedroom & \\
\hline SDC07 & Flaming & Mattress & Bedroom & \\
\hline SDC08 & Smoldering & Mattress & Bedroom & \\
\hline SDC09 & Flaming & Mattress & Bedroom & Bedroom door closed \\
\hline SDC10 & Flaming & Chair & Living Area & \\
\hline $\mathrm{SDC} 11$ & Smoldering & Chair & Living Area & \\
\hline $\mathrm{SDC} 12$ & Heating & Cooking Oil & Kitchen Area & \\
\hline SDC13 & Heating & Cooking Oil & Kitchen Area & \\
\hline SDC14 & Smoldering & Mattress & Bedroom & Bedroom door closed \\
\hline SDC15 & Flaming & Chair & Living Area & \\
\hline SDC30 & Smoldering & Chair & Living Area & Ignition failure \\
\hline SDC31 & Smoldering & Chair & Living Area & \\
\hline SDC32 & Flaming & Chair & Living Area & Ignition failure \\
\hline SDC33 & Flaming & Chair & Living Area & \\
\hline $\mathrm{SDC} 34$ & Smoldering & Chair & Living Area & \\
\hline SDC35 & Flaming & Chair & Living Area & \\
\hline SDC36 & Flaming & Mattress & Bedroom & Bedroom door closed \\
\hline SDC37 & Smoldering & Mattress & Bedroom & \\
\hline $\mathrm{SDC} 38$ & Flaming & Mattress & Bedroom & \\
\hline SDC39 & Flaming & Mattress & Bedroom & \\
\hline SDC40 & Smoldering & Mattress & Bedroom & \\
\hline SDC41 & Heating & Cooking Oil & Kitchen Area & \\
\hline
\end{tabular}


Table 12. Test Conditions for tests conducted in a two-story home

\begin{tabular}{|l|l|l|l|l|}
\hline Test & Ignition & Fuel Package & Fire Location & Comments \\
\hline SDC20 & Flaming & Mattress & Bedroom & Bedroom door closed \\
\hline SDC21 & Smoldering & Mattress & Bedroom & Alarms not reached \\
\hline SDC22 & Flaming & Mattress & Bedroom & Ignition failure \\
\hline SDC23 & Smoldering & Chair & Living Room & \\
\hline SDC24 & Heating & Cooking Oil & Kitchen & \\
\hline SDC25 & Flaming & Chair & Living Room & \\
\hline SDC26 & Flaming & Chair & Living Room & \\
\hline SDC27 & Smoldering & Chair & Living Room & $\begin{array}{l}\text { Air-conditioning } \\
\text { upstairs }\end{array}$ \\
\hline SDC28 & Flaming & Fully furnished room & Living Room & \\
\hline
\end{tabular}

\subsection{Test Data}

As an example of the analysis details, test SDC05 was chosen. This test was a flaming mattress test in a bedroom of the manufactured home. It was chosen as a single representative test, but does include all of the relevant test data. In addition for this test, all of the alarm types responded to the fire. Figures 100 through 121 present the data from test SDC05.

Mass loss of the burning mattress is shown in figure 100. Mass loss grows from shortly after ignition to a peak of approximately $150 \mathrm{~g}$ at the end of the test. Like all of the tests in the project, this test was terminated once untenable conditions had been reached in the path of egress from the home. A manually-operated water spray from copper tubing directed at the burning chair (see figure 94) at the end of the test quickly suppressed the fire resulting in a sharp drop in mass loss at the end of the test as the water collected in the pan supporting the mattress. The initiation of this manual suppression is noted in the figure as "Initiation of Suppression."

Figures 101 to 107 show measured profiles of gas temperature from ceiling to floor in several locations throughout the home. In the ignition location (in a bedroom at one end of the manufactured home, noted as "Main Bedroom" for this report), temperatures reached $120{ }^{\circ} \mathrm{C}$ near the ceiling (20 mm from the ceiling) and $98{ }^{\circ} \mathrm{C}$ near face level $(900 \mathrm{~mm}$ below ceiling or $1.5 \mathrm{~m}$ from the floor) at the end of the test. Further from the fire, peak temperatures were naturally lower, ranging from $87{ }^{\circ} \mathrm{C}$ just outside the main bedroom down to $42{ }^{\circ} \mathrm{C}$ in the bedroom farthest from the fire. In the closed bedroom, temperatures remain near ambient. 


\section{Mass Loss}

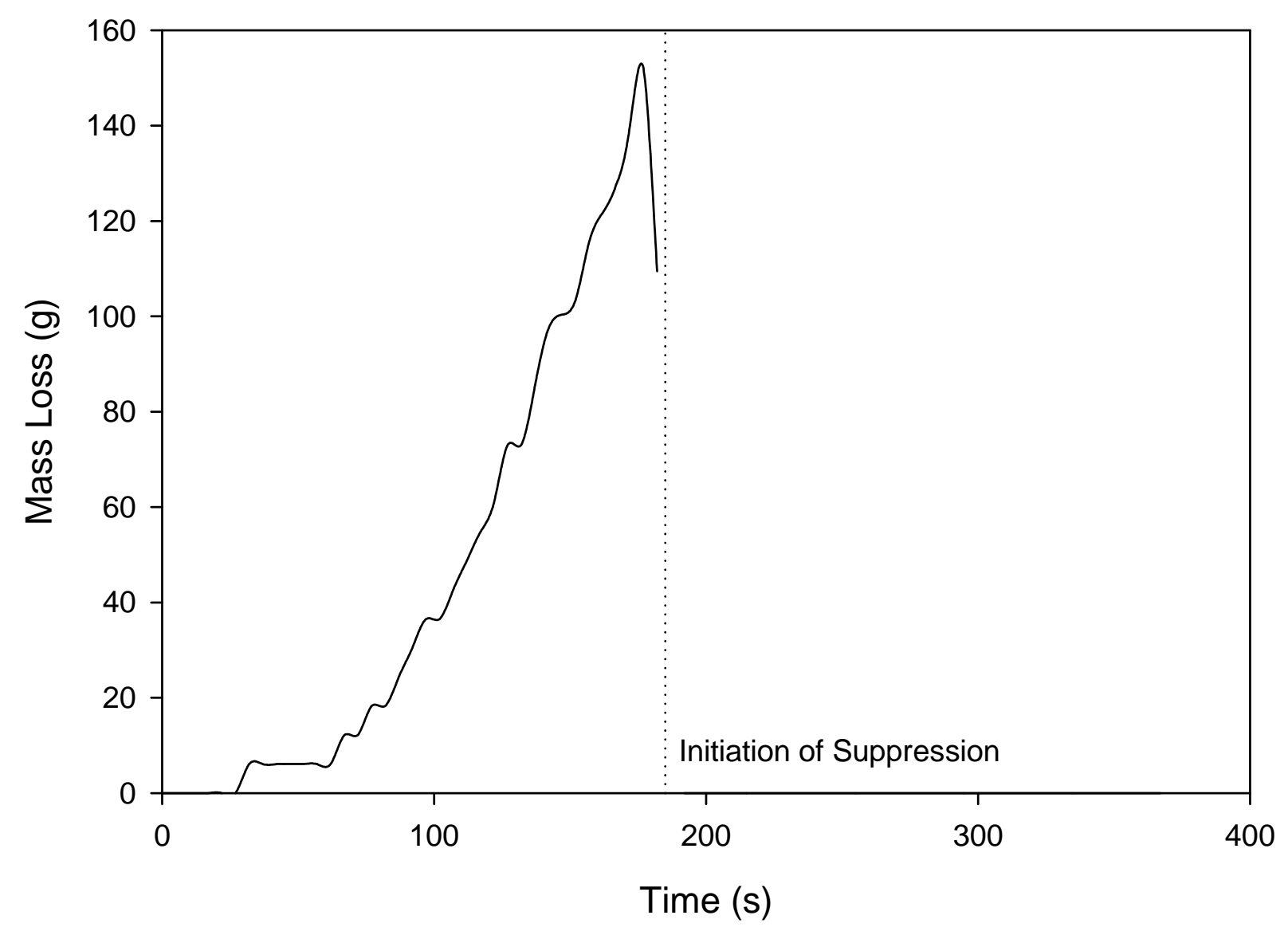

Figure 100. Mass loss of burning mattress during flaming ignition test of a mattress, test SDC05 (Note that the mass loss is averaged to minimize noise in the data. Thus, peak mass loss and initiation of suppression do not exactly coincide) 


\section{Remote Bedroom}

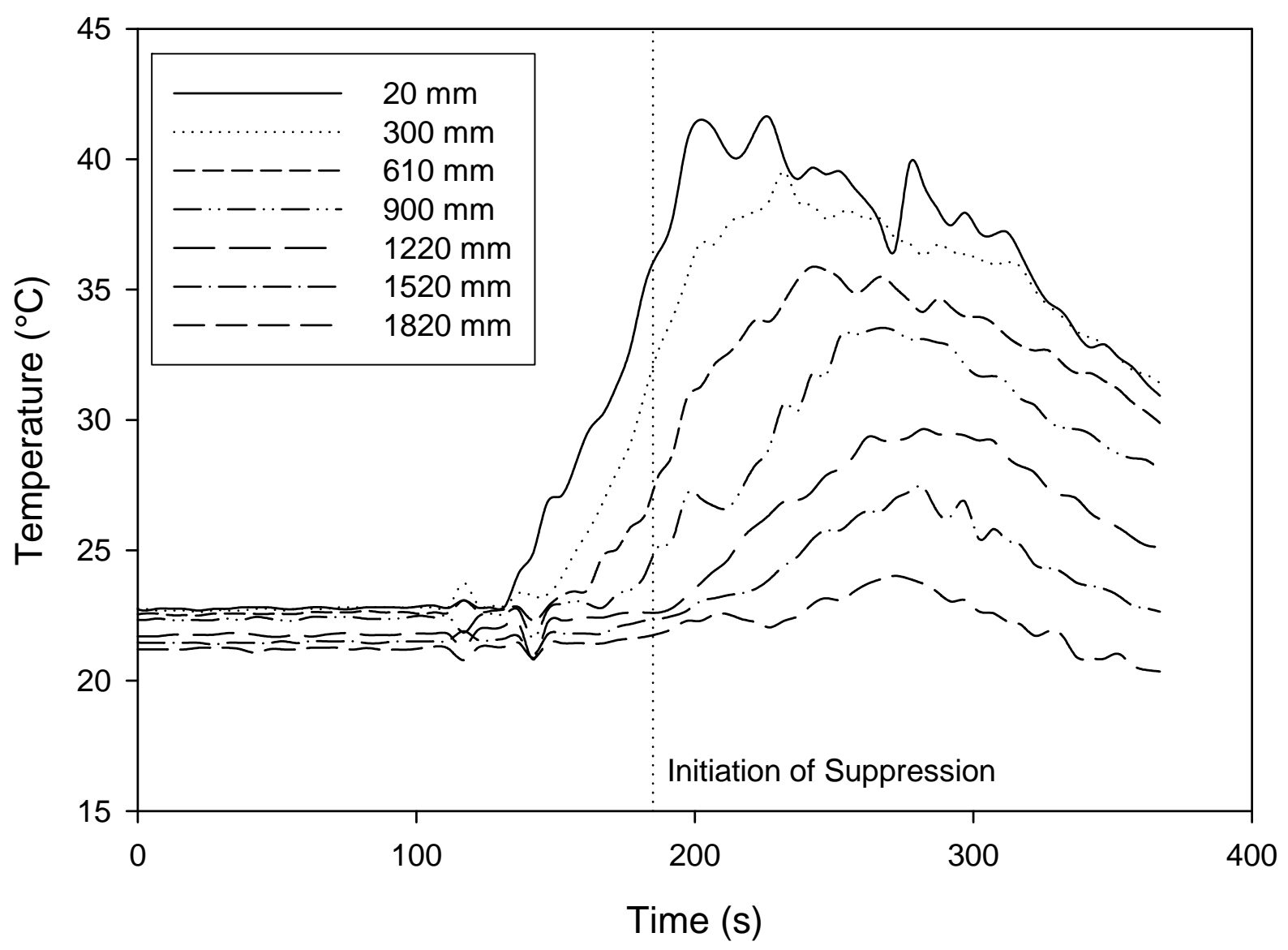

Figure 101. Gas temperatures from ceiling to floor in remote bedroom during a flaming ignition test of a mattress, test SDC05 


\section{Main Bedroom}

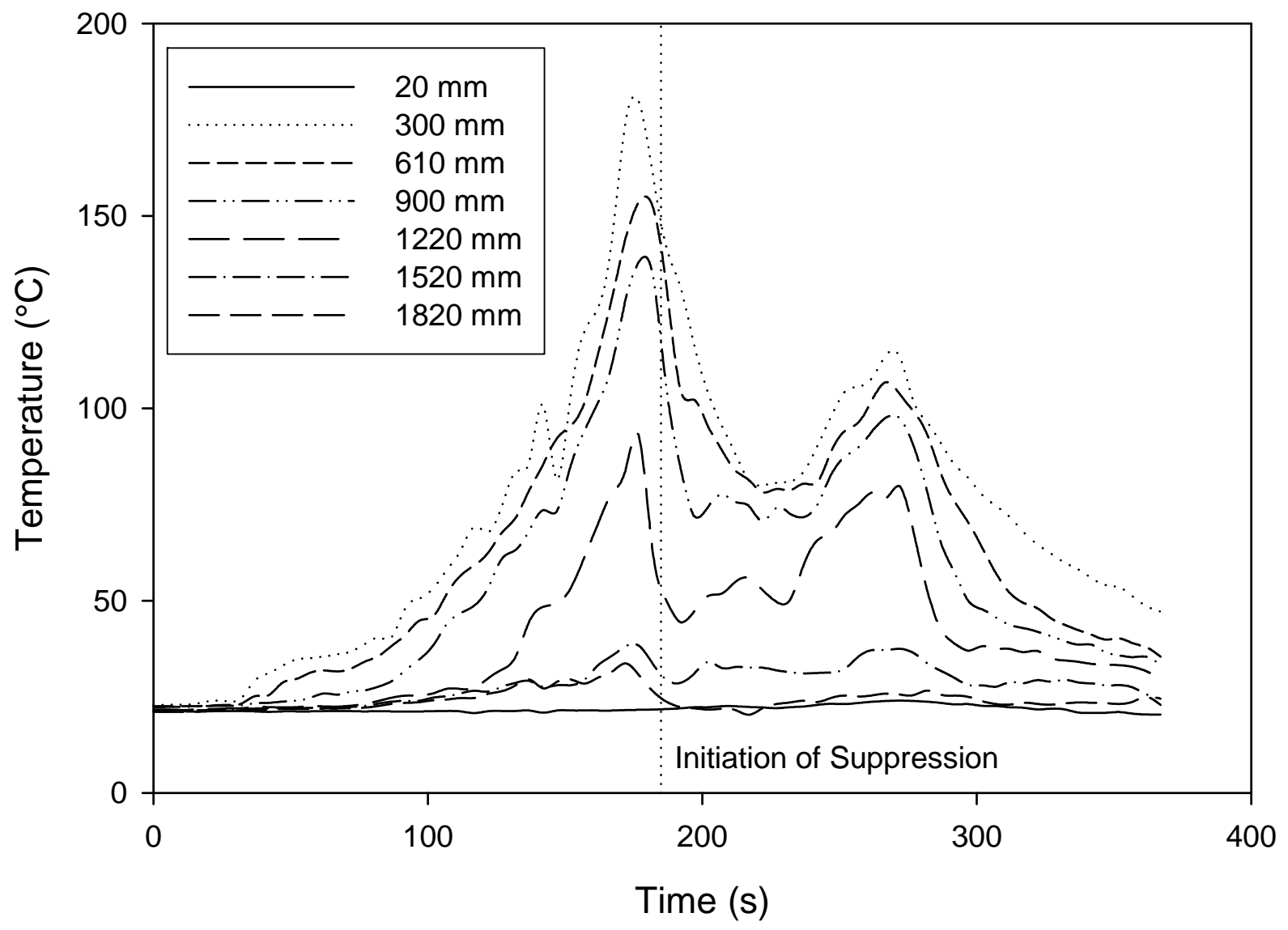

Figure 102. Gas temperatures from ceiling to floor in main bedroom (ignition location) during a flaming ignition test of a mattress, test SDC05 


\section{Hallway Outside Remote Bedroom}

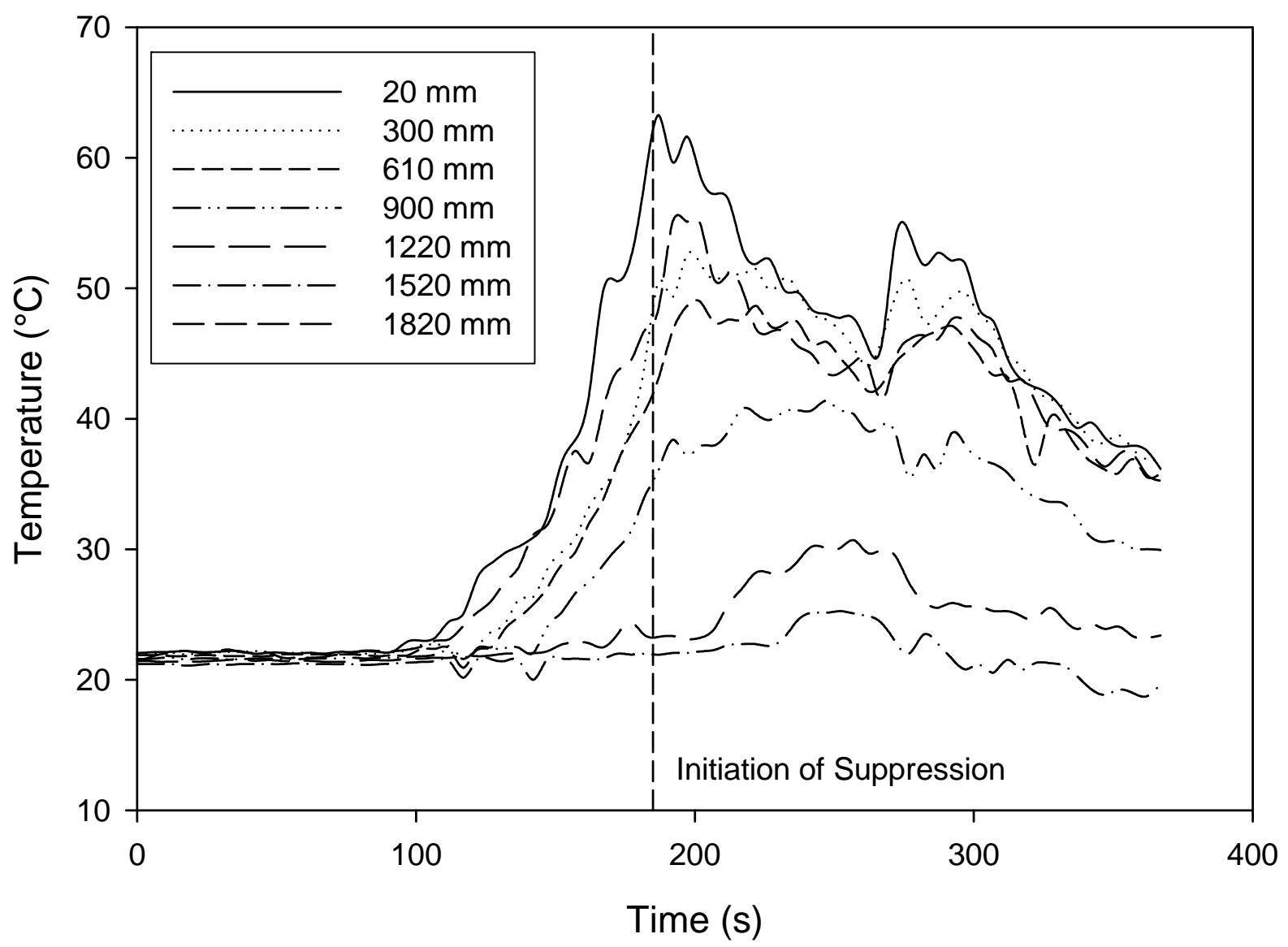

Figure 103. Gas temperatures from ceiling to floor in hallway outside remote bedroom in a flaming ignition test of a mattress, test SDC05 
Hallway Outside Main Bedroom

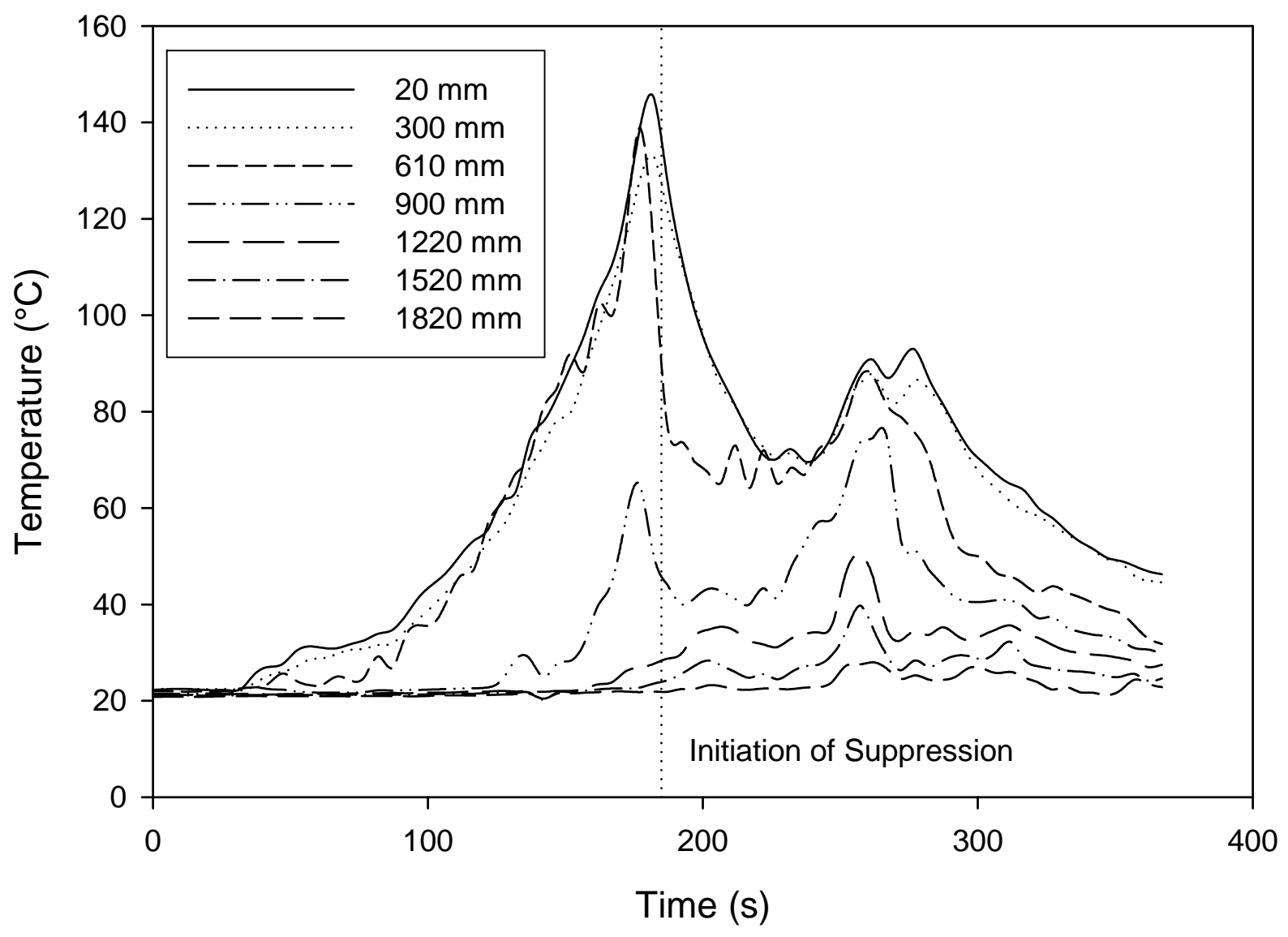

Figure 104. Gas temperatures from ceiling to floor in hallway outside main bedroom in a flaming ignition test of a mattress, test SDC05 


\section{Living Room}

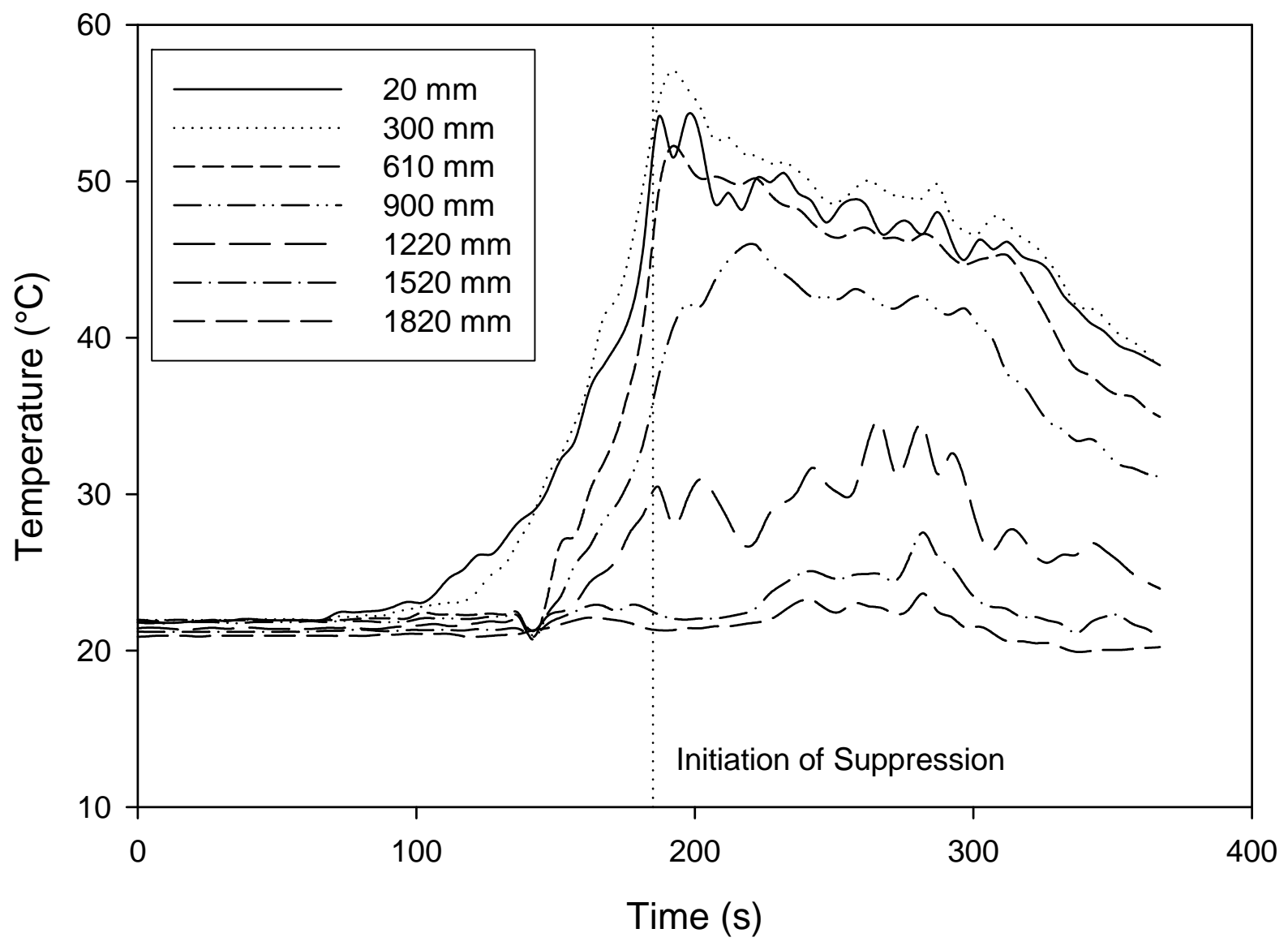

Figure 105. Gas temperatures from ceiling to floor in living area during a flaming ignition test of a mattress, test SDC05 


\section{Front Door Hallway}

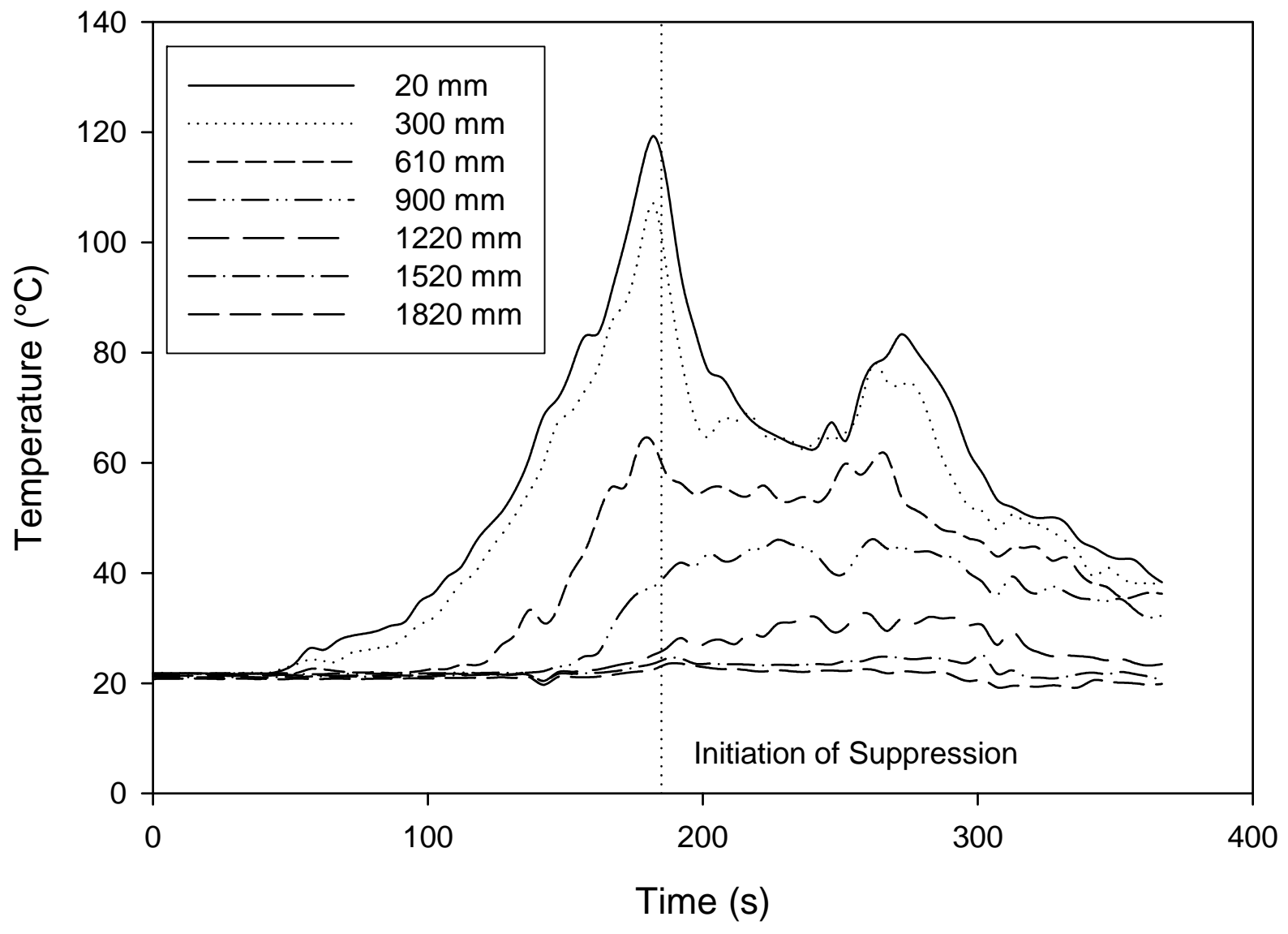

Figure 106. Gas temperatures from ceiling to floor in front door hallway during a flaming ignition test of a mattress, test SDC05 


\section{Closed Bedroom}

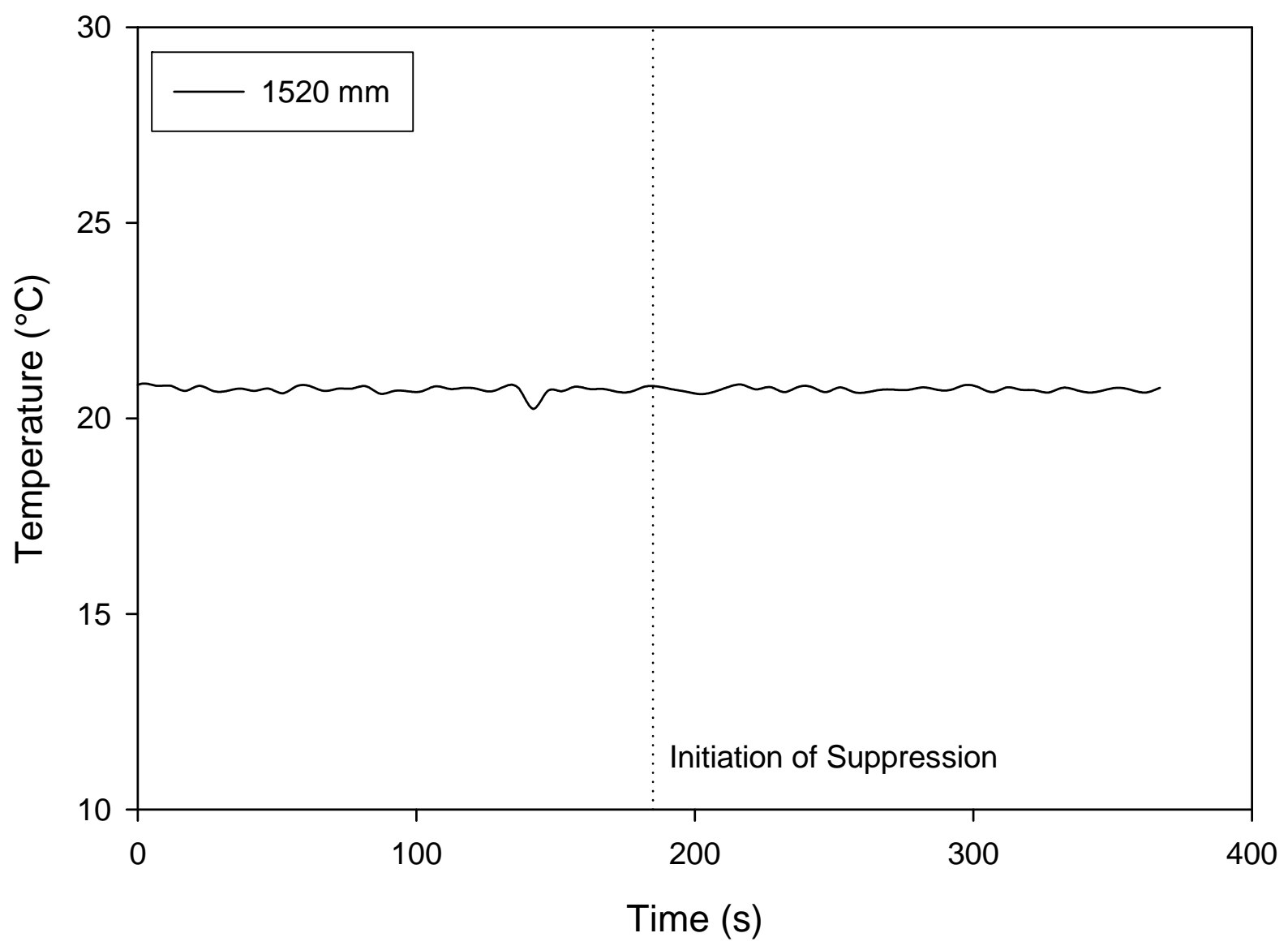

Figure 107. Gas temperature in closed bedroom during a flaming ignition test of a mattress, test SDC05 
Figures 108 through 114 show smoke obscuration near the ceiling ( $20 \mathrm{~mm}$ below the ceiling) and near face height ( $900 \mathrm{~mm}$ from ceiling or $1.5 \mathrm{~m}$ from floor) at several locations throughout the home. Like the gas temperatures, peak values are highest near the fire (an optical density of nearly $5.0 \mathrm{~m}^{-1}$ near the ceiling in the main bedroom) and peak values less than $2.0 \mathrm{~m}^{-1}$ further removed from the fire. In the main bedroom, the smoke meter near the ceiling shows an initial peak beginning about $50 \mathrm{~s}$ not evident in other locations. Examination of the video for this test shows little reason for this initial peak so it may indicate a malfunction in the instrument.

Values at the $1.5 \mathrm{~m}$ level are naturally lower, typically below $1.0 \mathrm{~m}^{-1}$. In the closed bedroom, an increase in obscuration is noticeable, but still near ambient levels throughout the test.

Figure 115 shows carbon monoxide concentration $900 \mathrm{~mm}$ below ceiling level ( $1.5 \mathrm{~m}$ from the floor) at several locations throughout the home. Peak values range from a high of $0.099 \%$ volume fraction near the fire down to $0.043 \%$ volume fraction remote from the fire - both shortly after initiation of suppression. Just prior to suppression, these values are $0.049 \%$ volume fraction and $0.017 \%$ volume fraction, respectively. Similarly, values from carbon dioxide (figure 116) range from $2.3 \%$ volume fraction near the fire down to $0.74 \%$ volume fraction remote from the fire overall and $2.0 \%$ volume fraction down to $0.25 \%$ volume fraction prior to suppression. Oxygen concentration (figure 117) lowers quickly in the main bedroom fire room to a low of about $19 \%$ volume fraction. By the end of the test, concentrations at all measurement points are similar at just under $20 \%$ volume fraction.

A total of 43 fire alarms were monitored in each test. Figures 118 to 121 show the measured output for the alarms at one measurement location (just outside the main bedroom). Many of the smoke alarms (figure 118) respond within about $40 \mathrm{~s}$ after ignition. In this test, all but one of the alarms responded to the fire. One alarm, Ion-4 (see figure 10 for details of the alarm identification codes), showed little response by the end of the test. The carbon monoxide alarms (figure 119) begin to respond within about $50 \mathrm{~s}$, with significant output after $100 \mathrm{~s}$. One of the alarms, CO-1, was inadvertently disconnected during this test. The heat alarm at $127 \mathrm{~s}$ (figure 120) and tell-tale sprinkler at $147 \mathrm{~s}$ (figure 121) respond within $20 \mathrm{~s}$ of one another. One of the photoelectric alarms shows little response until late in the test.

\subsection{Calculation of Alarm Times}

For each of the analog alarms, voltage readings were converted to engineering units typical for the alarm - obscuration for smoke alarms (from table 1) and concentration for carbon monoxide alarms (from a linear calibration with reference gases). Calibrations of each individual alarm was determined in the FE/DE (see section 2) and are shown in table 13. Typical alarm points for each type of alarm were used to determine a time of alarm for each alarm at each measurement location: 


\section{Remote Bedroom}

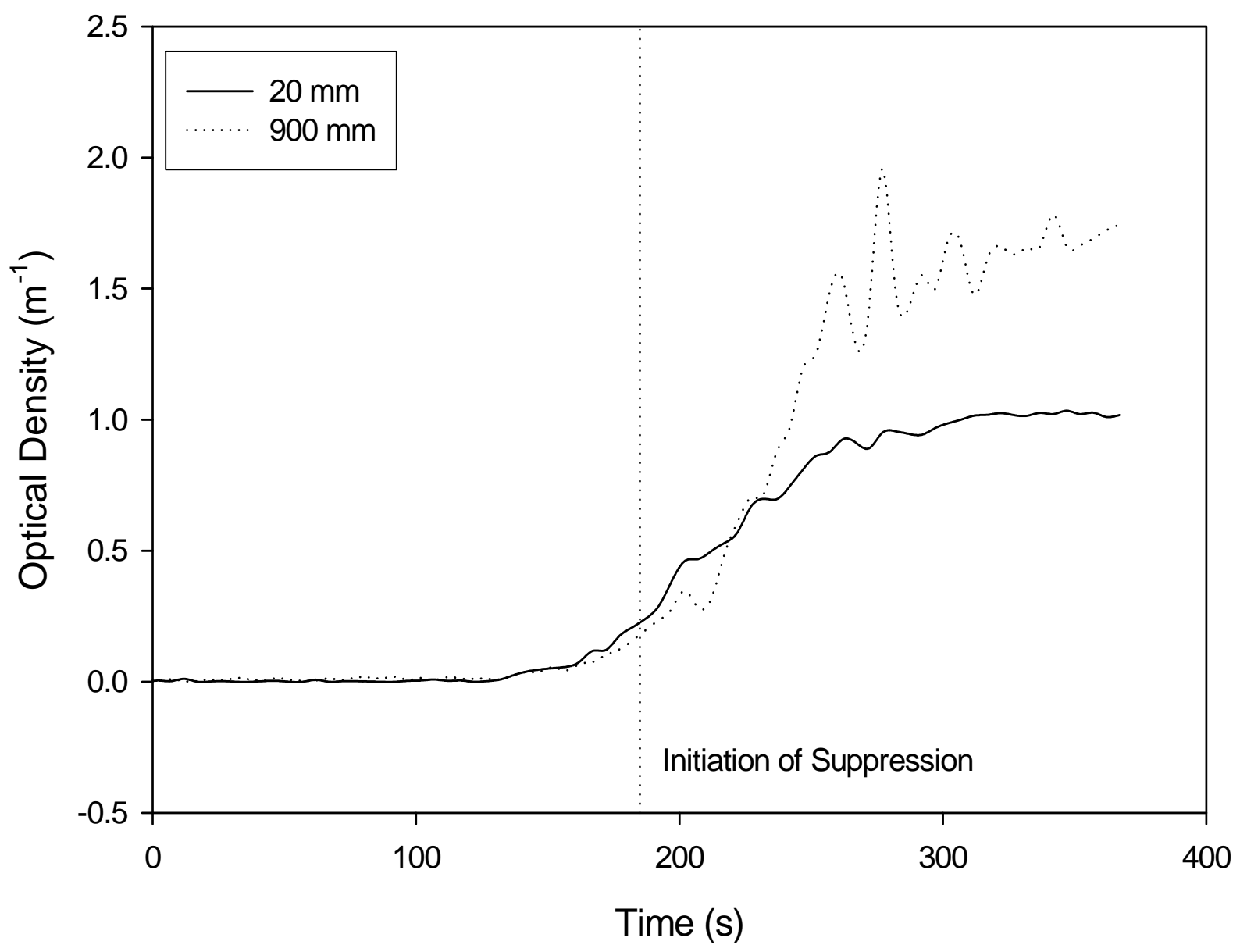

Figure 108. Smoke obscuration at $20 \mathrm{~mm}$ and $900 \mathrm{~mm}$ below ceiling in remote bedroom during flaming ignition test of a mattress, test SDC05 


\section{Main Bedroom}

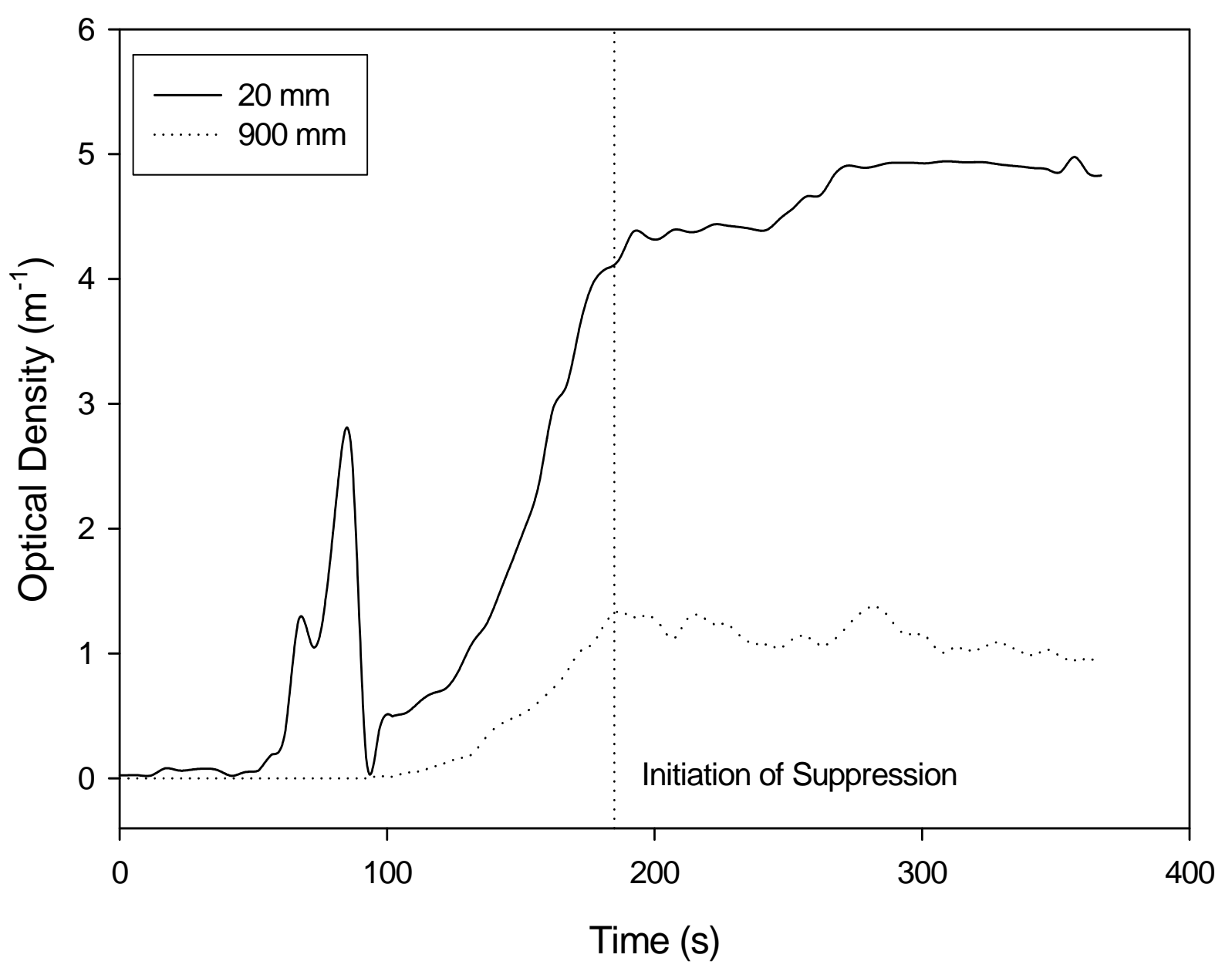

Figure 109. Smoke obscuration at $20 \mathrm{~mm}$ and $900 \mathrm{~mm}$ below ceiling in main bedroom (ignition location) during flaming ignition test of a mattress, test SDC05 


\section{Hallway Outside Remote Bedroom}

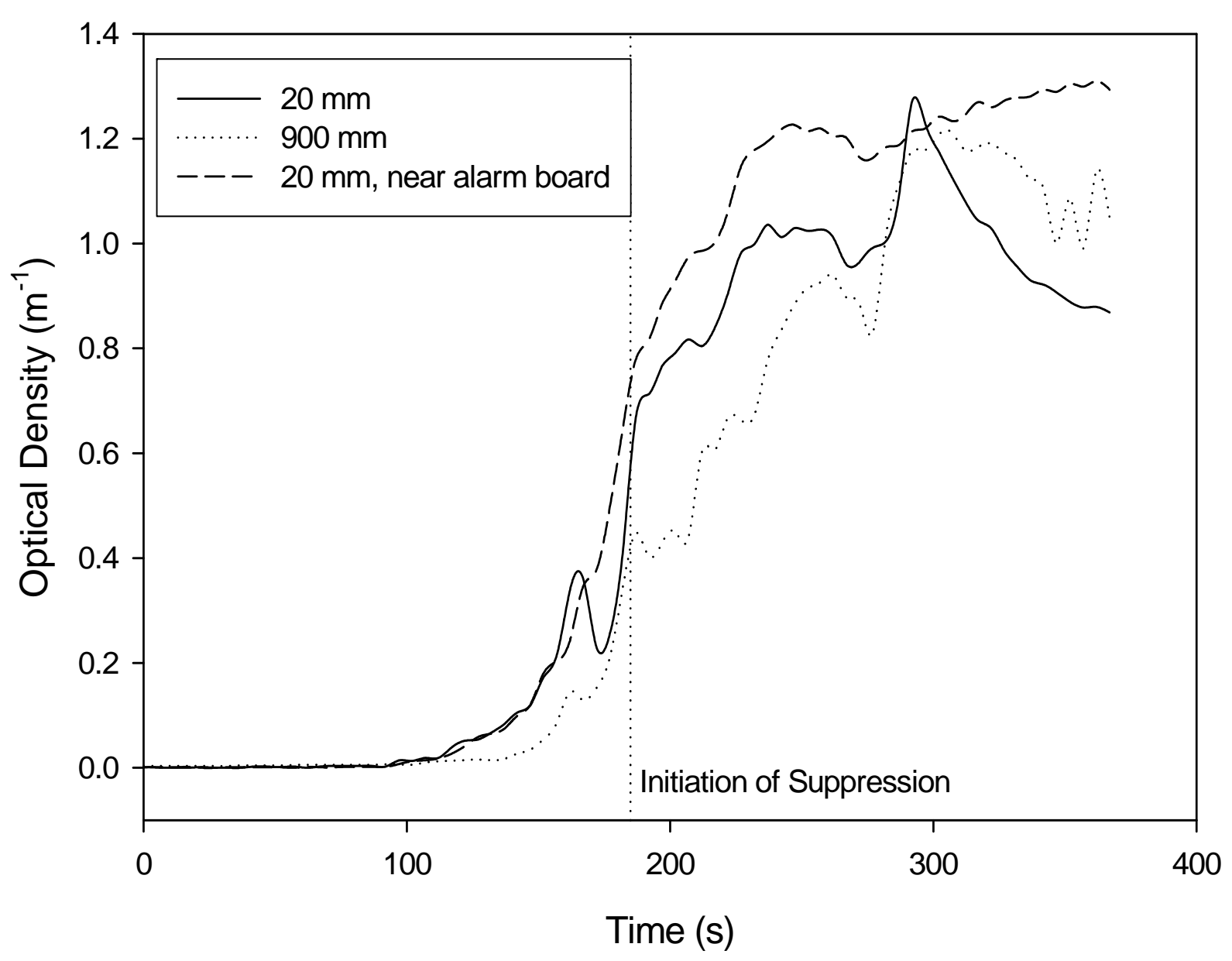

Figure 110. Smoke obscuration at $20 \mathrm{~mm}$ and $900 \mathrm{~mm}$ below ceiling in hallway outside remote bedroom during flaming ignition test of a mattress, test SDC05 


\section{Hallway Outside Main Bedroom}

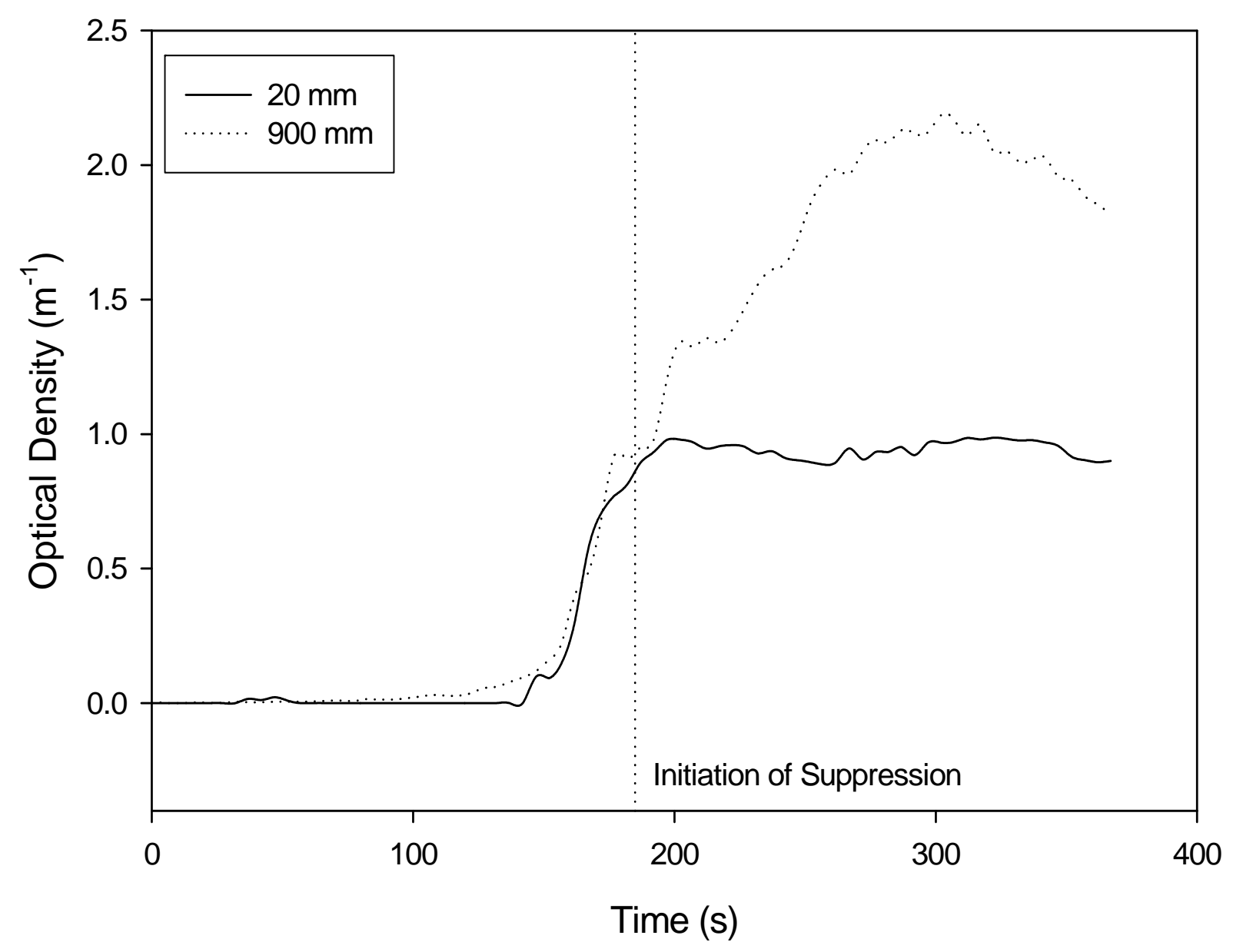

Figure 111. Smoke obscuration at $20 \mathrm{~mm}$ and $900 \mathrm{~mm}$ below ceiling in hallway outside main bedroom during flaming ignition test of a mattress, test SDC05 


\section{Living Room}

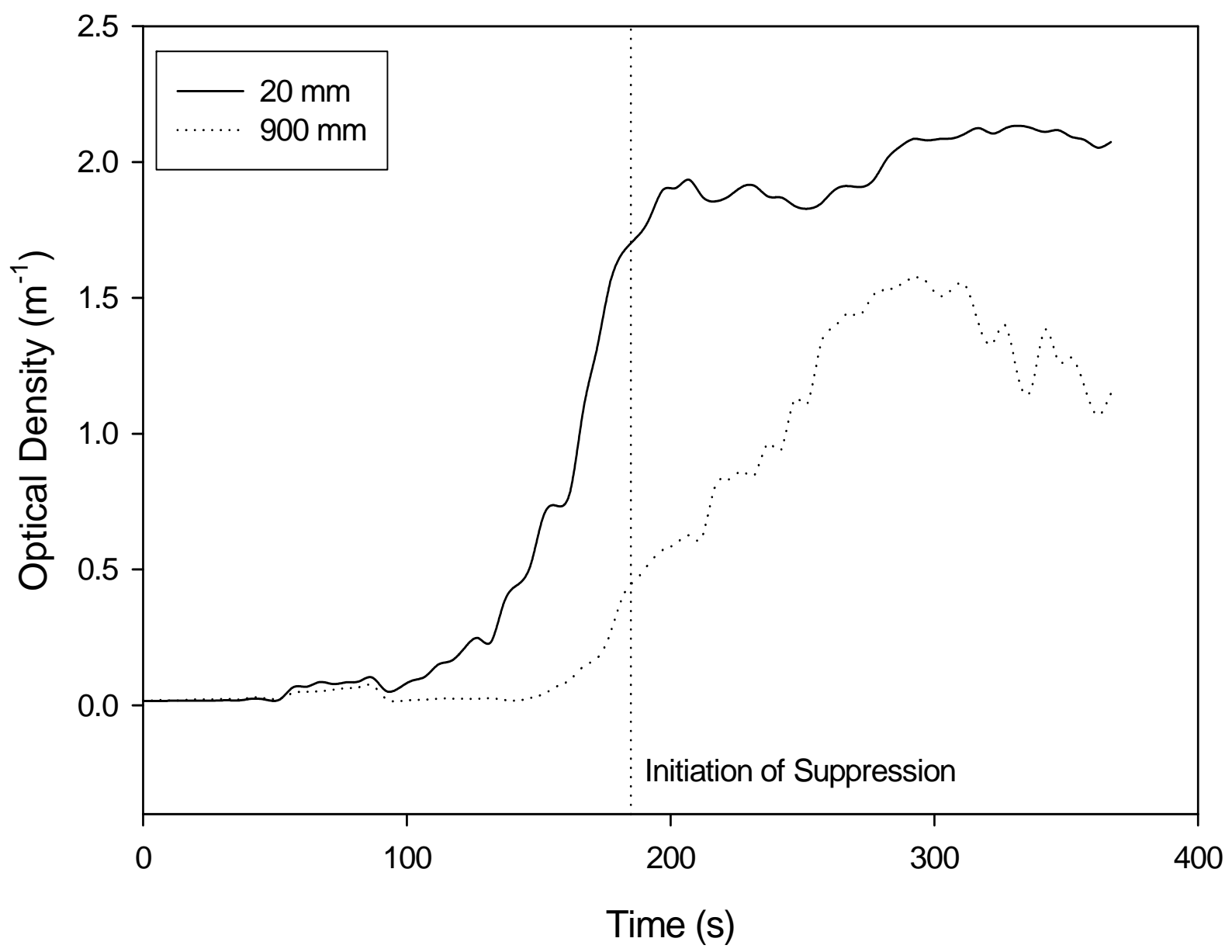

Figure 112. Smoke obscuration at $20 \mathrm{~mm}$ and $900 \mathrm{~mm}$ below ceiling in living area during flaming ignition test of a mattress, test SDC05 


\section{Front Door Hallway}

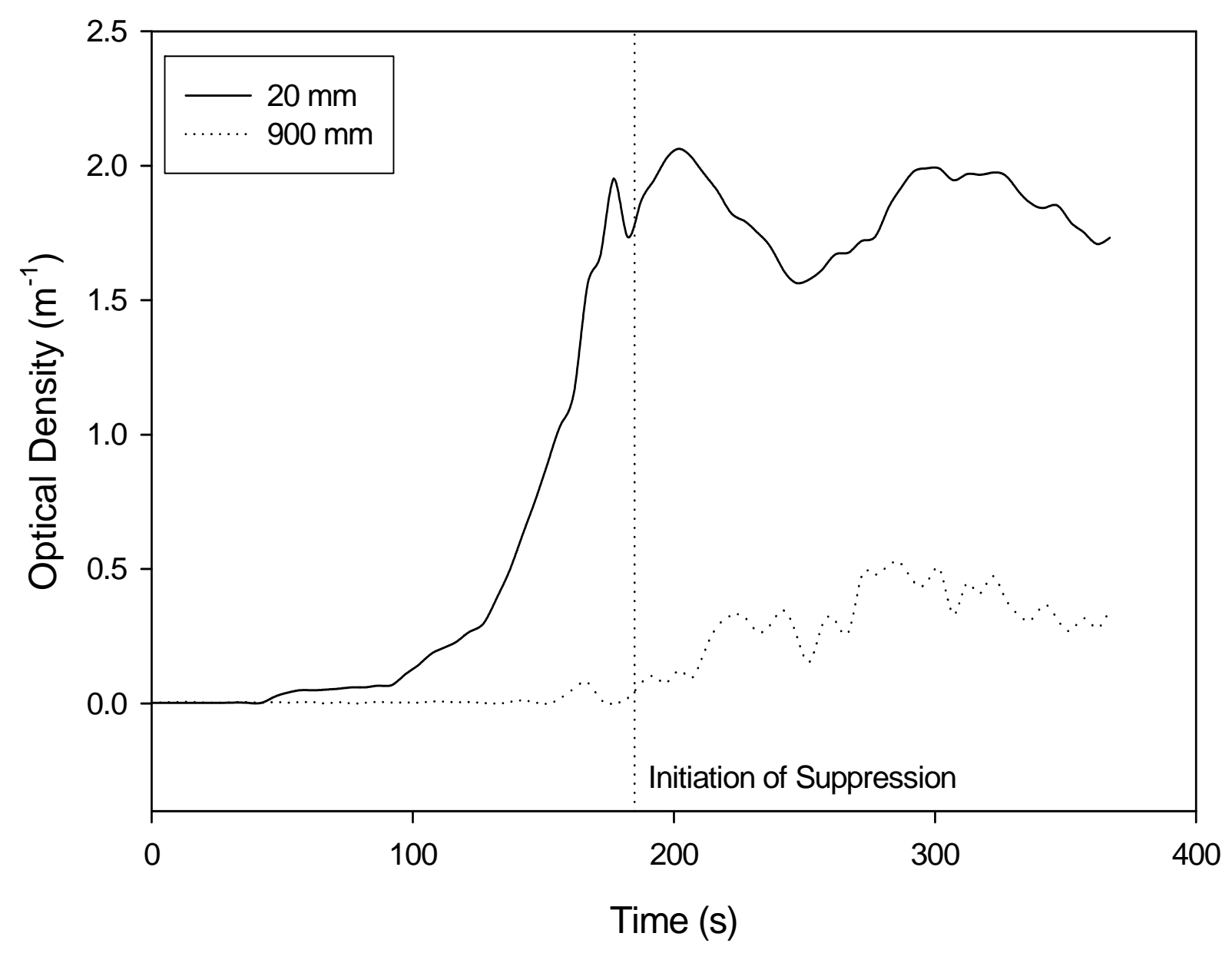

Figure 113. Smoke obscuration at $20 \mathrm{~mm}$ and $900 \mathrm{~mm}$ below ceiling in front door hallway during flaming ignition test of a mattress, test SDC05 


\section{Closed Bedroom}

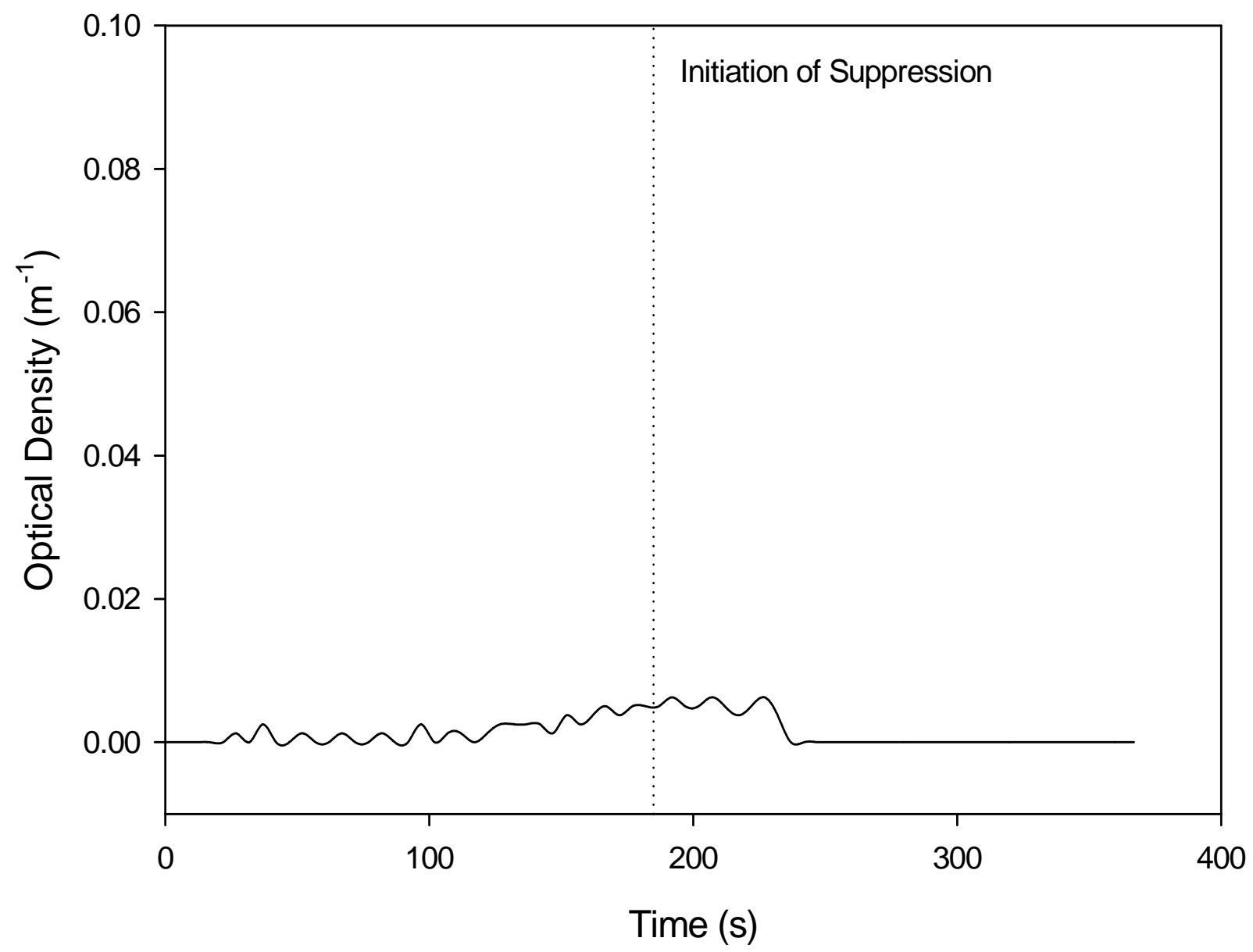

Figure 114. Smoke obscuration at $1520 \mathrm{~mm}$ below ceiling in closed bedroom during flaming ignition test of a mattress, test SDC05 


\section{Carbon Monoxide}

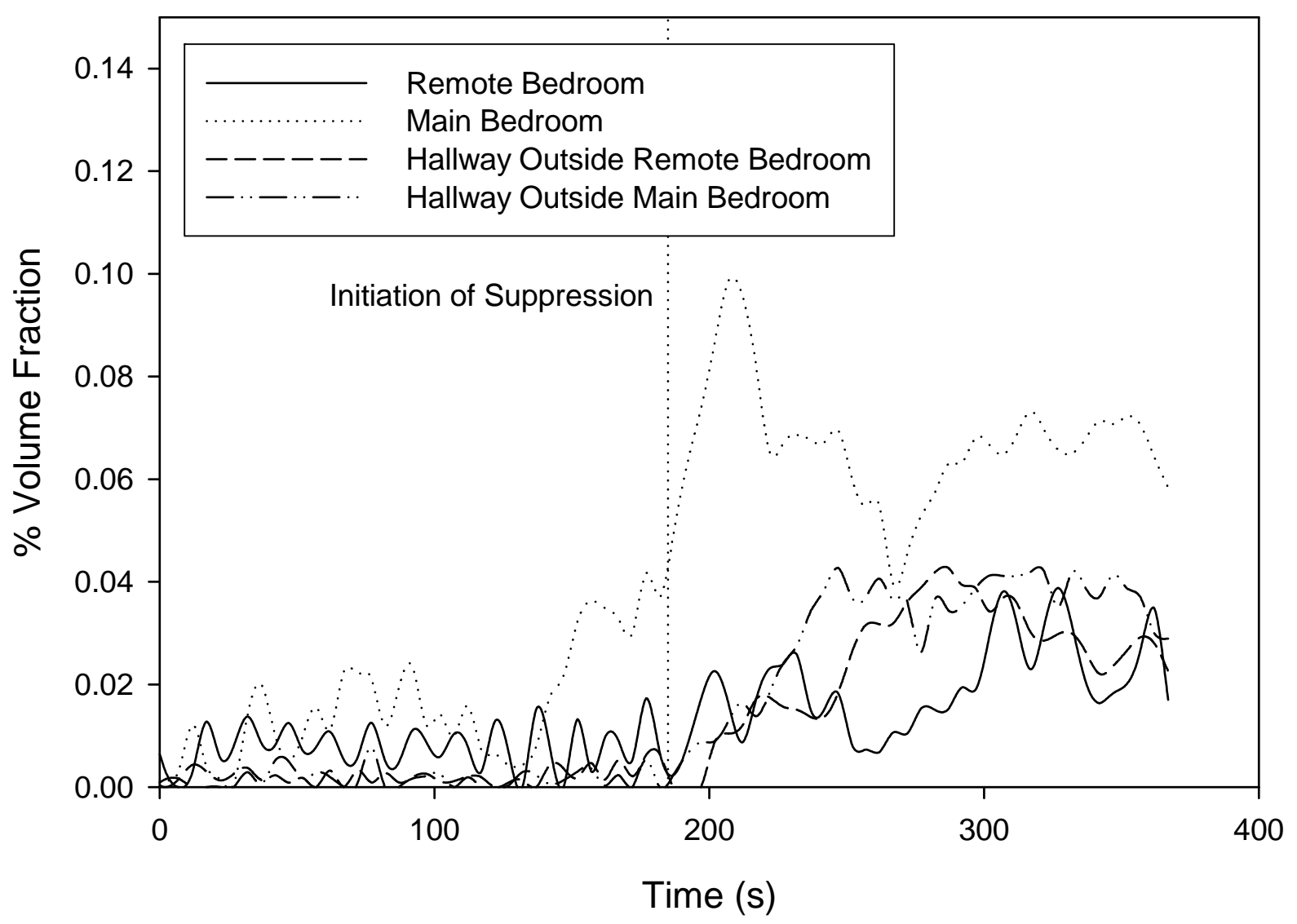

Figure 115. Carbon monoxide concentration $900 \mathrm{~mm}$ below ceiling at four locations during a flaming ignition test of a mattress, test SDC05 


\section{Carbon Dioxide}

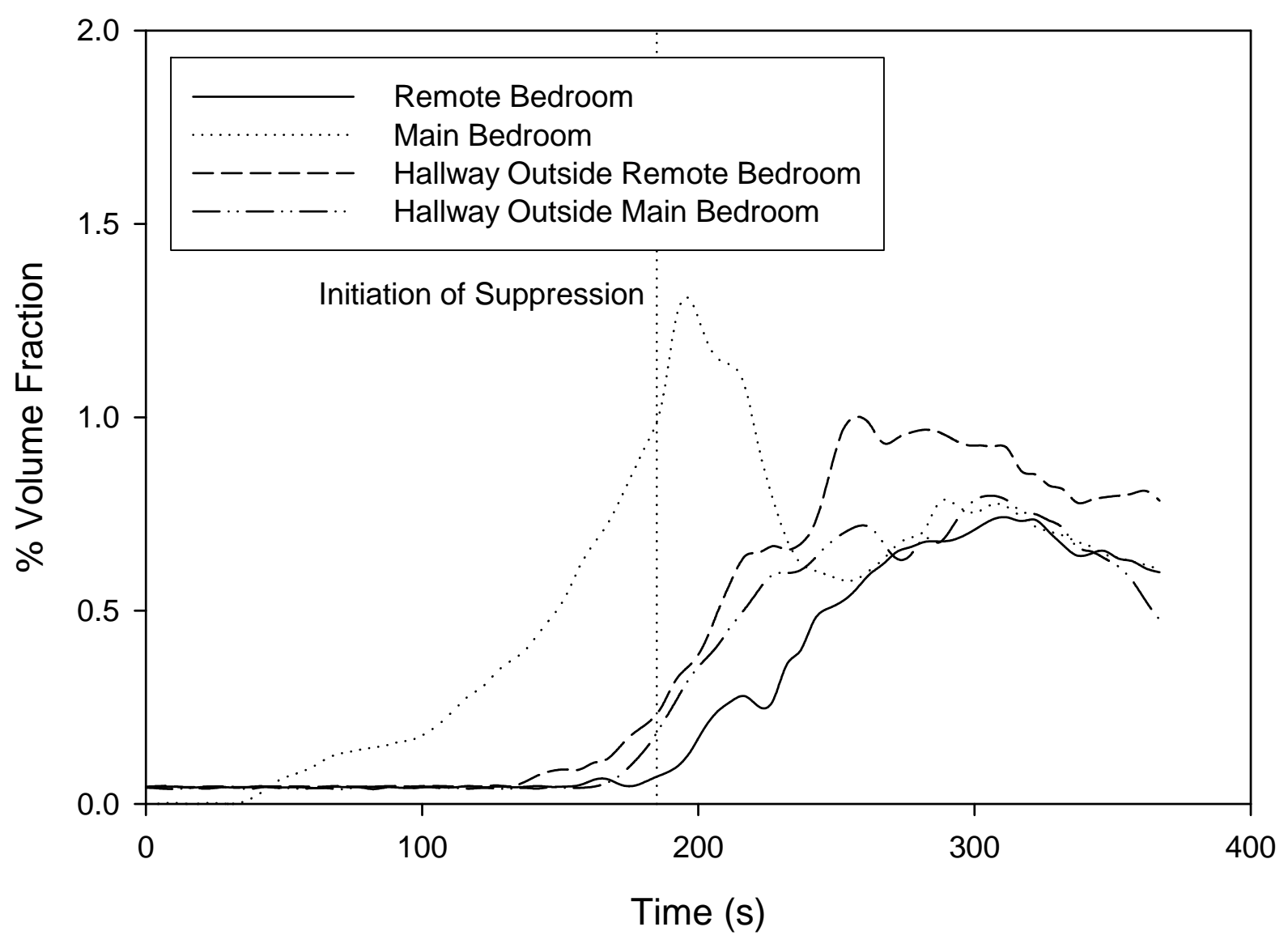

Figure 116. Carbon dioxide concentration $900 \mathrm{~mm}$ below ceiling at four locations during a flaming ignition test of a mattress, test SDC05 


\section{Oxygen}

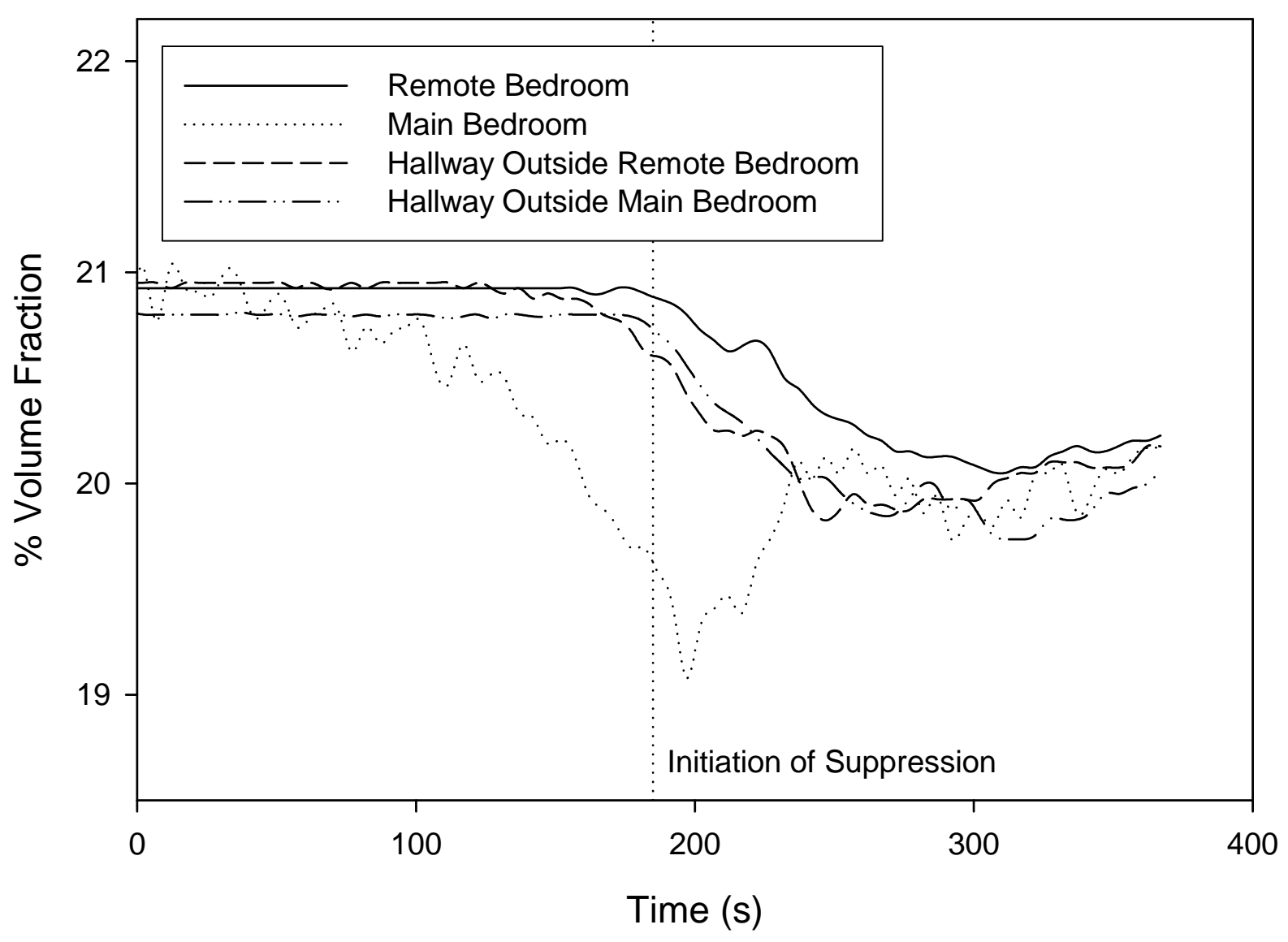

Figure 117. Oxygen concentration $900 \mathrm{~mm}$ below ceiling at four locations during a flaming ignition test of a mattress, test SDC05 


\section{Smoke Alarm Output}

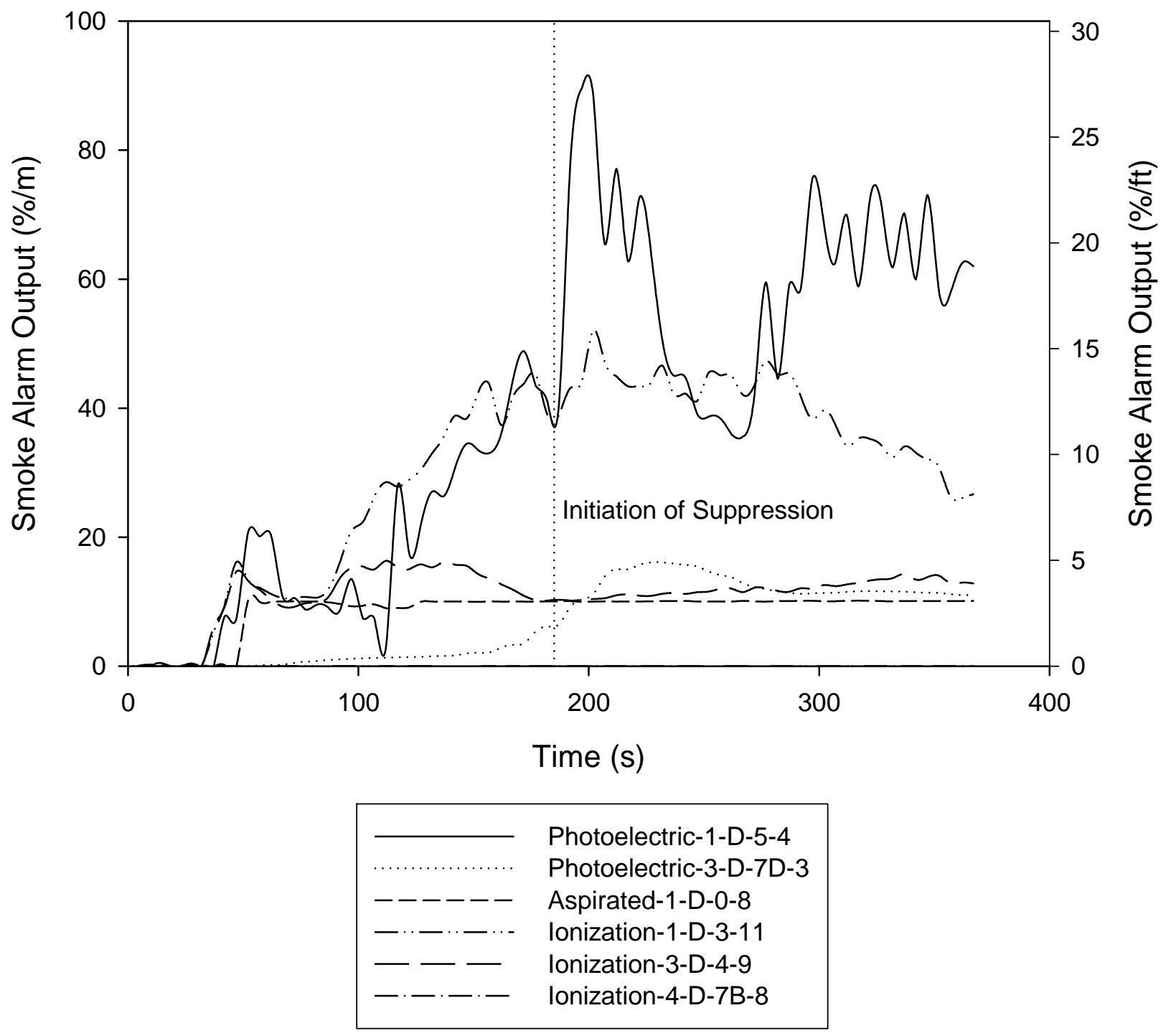

Figure 118. Measured output for several analog-modified smoke alarms in hallway outside main bedroom during a flaming ignition test of a mattress, test SDC05 


\section{CO Alarm Output}

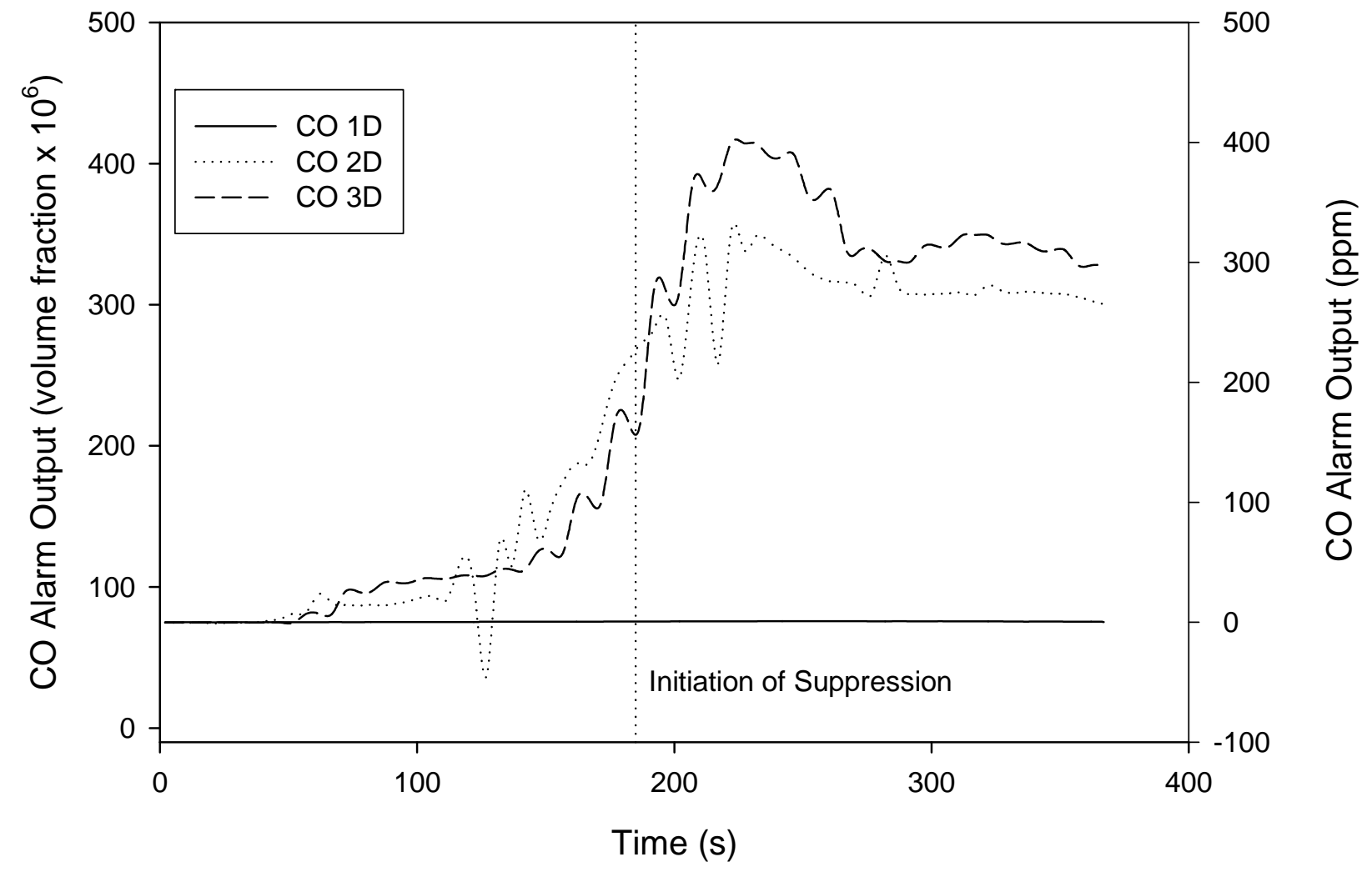

Figure 119. Measured output for several analog-modified carbon monoxide alarms in hallway outside main bedroom during a flaming ignition test of a mattress, test SDC05 


\section{Heat Alarm}

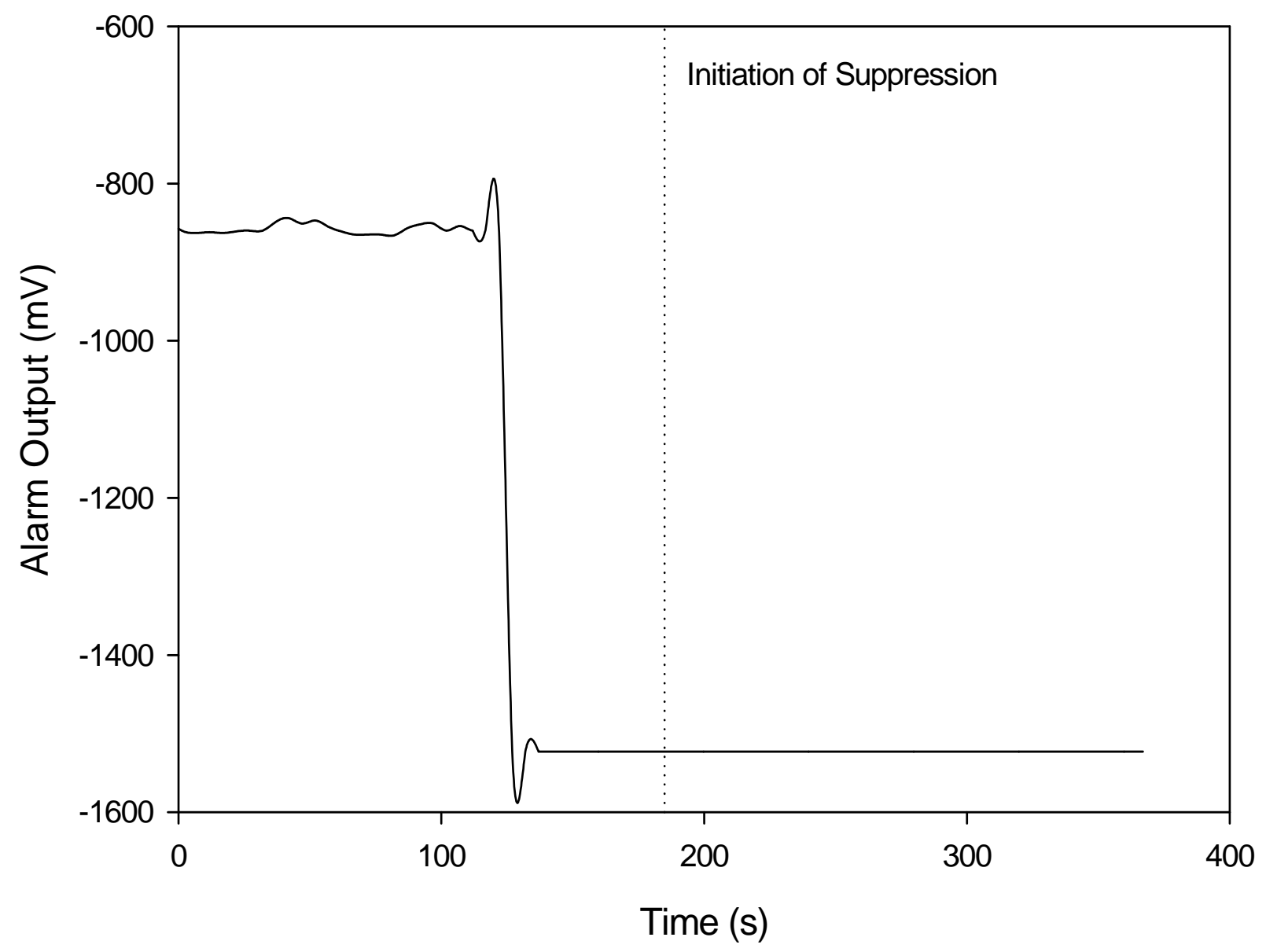

Figure 120. Measured output for a heat alarm in hallway outside main bedroom during a flaming ignition test of a mattress, test SDC05 


\section{Sprinkler Response}

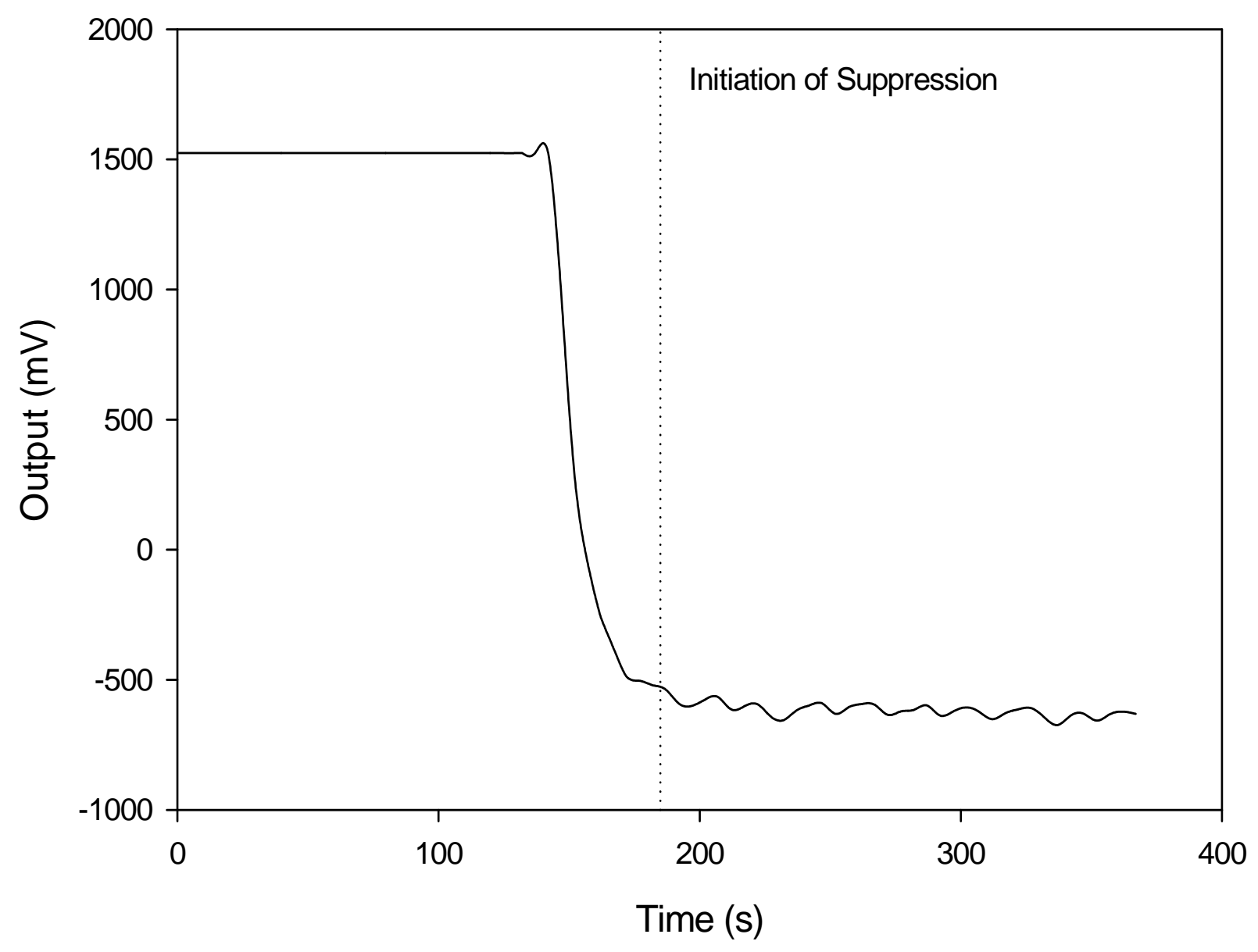

Figure 121. Measured output for a tell-tale residential sprinkler in hallway outside main bedroom during a flaming ignition test of a mattress, test SDC05 
- $\quad$ carbon monoxide alarms $-50 \times 10^{-6}$ volume fraction (50 ppm)

- $\quad$ ionization alarms $-4.3 \% / \mathrm{m}(1.3 \% / \mathrm{ft})$

- $\quad$ photoelectric alarms $-6.6 \% / \mathrm{m}(2 \% / \mathrm{ft})$

Section 2.8 discusses determination of these alarm points. For some alarms, a single alarm time was obvious from the output (for example, see figure 120). For the one location in the hallway outside the main bedroom, alarm times are shown in table 13. For this location, all alarms reached typical alarm points in times ranging from a first alarm at $37 \mathrm{~s}$ to one shortly after initiation of manual suppression at $192 \mathrm{~s}$. For many alarms, the choice of alarm point had little effect on the resulting alarm times.

Table 13. Alarm Times for Several Smoke Alarms in Hallway Outside Main Bedroom During Flaming Ignition Test of a Mattress, Test SDC05

\begin{tabular}{|l|l|c|c|c|c|c|}
\cline { 2 - 7 } \multicolumn{1}{c|}{} & \multicolumn{2}{c|}{ ID } & \multicolumn{2}{c|}{ Calibrations (from table 1) } & \multicolumn{3}{c|}{ Alarm Times (s) } \\
\cline { 2 - 7 } & & $\mathrm{m}_{0}$ & $\mathrm{~m}_{1}$ & low & mid & high \\
\hline Smoke Alarm & ION-1-D-3-7 & 0 & 2.09 & 37 & 37 & 42 \\
\hline Smoke Alarm & ION-3-D-4-13 & 0 & 1.14 & 37 & 37 & 37 \\
\hline Smoke Alarm & PHOTO-1-D-5-4 & -1.96 & 6.88 & 42 & 42 & 52 \\
\hline Smoke Alarm & ASPIRATED-1-D-0-8 & -1.27 & 1.84 & 52 & 52 & 52 \\
\hline CO Alarm & CO-1-D-1-5 & -43.6 & 233.3 & n.a. ${ }^{\text {b }}$ & n.a. & n.a. \\
\hline CO Alarm & CO-2-D-2-2 & -45.1 & 93.0 & 142 & 142 & 172 \\
\hline CO Alarm & CO-3-D-3-5 & -3.44 & 165.5 & 87 & 147 & 177 \\
\hline
\end{tabular}

a - Alarms Ion- 4 and Photo-3 are components of a dual mode alarm, but were not functioning for this test $\mathrm{b}-$ n.a. $=$ no activation indicated

\subsection{Calculation of Time to Untenable Conditions}

\subsubsection{Tenability Limits}

The time available for occupant egress is simply the amount of time from the first indication of a fire (in this case, the alarm time) to the time at which conditions become untenable. This topic has been the subject of current work by an international committee of experts working as ISO Technical Committee (TC) 92, subcommittee (SC) 3. This group has published a technical standard, ISO TS 13571, that recommends limits of human tolerance to fire products [26]. 
These limits are also consistent with the recommendations in the SFPE Handbook of Fire Protection Engineering. Additional discussion of ISO TS 13571 is included in section 7.2

Heat exposure: The current version of ISO TS 13571 includes equations for calculating incapacitation from skin exposure to radiant heating, from exposure to convected heat resulting form elevated gas temperatures, from exposure to the incapacitating effects of asphyxiant gases, and from exposure to irritant gases.

For these tests, heat exposure is determined as an effect of only elevated gas temperatures. At early times of fire development typical of the tests included in this project, the effects of radiant heating would be negligible. Thus, the Fractional Effective Dose (FED) for heat exposure is given from ISO TS 13571 as:

$$
F E D_{H E A T}=\sum_{t_{1}}^{t_{2}} \frac{T^{3.4}}{5 \times 10^{7}} \Delta t
$$

where $T$ is in ${ }^{\circ} \mathrm{C}$ and $\Delta t$ is in min.

Exposure to toxic gases: The FED equation for the incapacitating effects of asphyxiant gases, derived from the current version of ISO TS 13571 is:

$$
F E D_{\text {GASES }}=\sum_{t_{1}}^{t_{2}} \frac{C O}{35000} \Delta t+\sum_{t_{1}}^{t_{2}} \frac{e^{(H C N / 43)}}{220} \Delta t
$$

where $C O$ and $H C N$ are in volume fraction $\times 10^{6}(\mathrm{ppm})$ and $\Delta t$ is in min. $\mathrm{CO}$ and $\mathrm{HCN}$ are the average values over the time increment $\Delta t$. The person "receives" incremental doses of smoke until an incapacitating value of FED is reached.

To determine a time to incapacitation, an FED value of 0.3 , indicating incapacitation of the susceptible population was used [26]. This limit was used for both heat and gas tenability. While this is a conservative limit, it is reasonably consistent with the 1975 study. Section 7.6 compares tenability limits in the two studies.

ISO TS 13571 also includes an equation for incapacitation from irritant gases. The primary gas analysis available for this report included only $\mathrm{CO}, \mathrm{CO}_{2}$, and $\mathrm{O}_{2}$; data on irritant gases were not available.

Smoke obscuration: For smoke obscuration, an optical density of $0.25 \mathrm{~m}^{-1}$ was used as a tenability criterion, a value typically used by the smoke alarm industry. For all calculations, temperature, smoke obscuration, and gas concentration values $900 \mathrm{~mm}$ below the ceiling $(1.5 \mathrm{~m}$ from the floor) were used. This height was intended to be representative of the mouth and nose height for a typical adult during egress from the home. 


\subsubsection{Tenability Times}

Chapter 8 of the National Fire Alarm Code [27] entitled Fire Warning Equipment for Dwelling Units states that the primary function of a dwelling fire warning system is to, “... provide a reliable means to notify the occupants of a dwelling unit of the presence of a threatening fire and the need to escape to a place of safety before such escape might be impeded by untenable conditions in the normal path of egress." The chapter further explains that, "Fire warning systems for dwelling units are capable of protecting about half of the occupants in potentially fatal fires. Victims are often intimate with the fire, ..., such that they cannot escape even when warned early enough that escape should be possible." This is consistent with the earlier research of McGuire and Ruscoe [2]. When escape is not practical, other strategies such as protection-inplace, assisted escape or rescue may be necessary.

Similarly, the Life Safety Code [28] states in 4-1.1, "The goal of this code is to provide an environment for the occupants that is reasonably safe from fire and similar emergencies by the following means: (1) Protection of occupants not intimate with the initial fire development. (2) Improvement of the survivability of occupants intimate with the initial fire development."

The limitations applicable to the protection of people intimate with the fire are common and recognize the associated difficulties and costs of such protection. Based on this position in the Codes, the performance criteria applied to the fire alarms in this study were based on the egress time provided between alarm and the time that selected tenability criteria were exceeded at any point in the primary egress path in the home but not including the instruments located at the fire source. These instruments were used to quantify conditions produced by the fire but were not counted towards escape as they represent the conditions to which a person intimate with the fire would experience.

Table 14 shows calculated tenability values for test SDC05. The time to untenable conditions and value for each calculated tenability calculation is shown. To judge available safe egress time, the minimum time to untenable conditions for measurement locations outside the room of fire origin was used. For this test, only the smoke obscuration reached untenable conditions in times ranging from $137 \mathrm{~s}$ to $217 \mathrm{~s}$.

In the room of fire origin, the main bedroom, the convected heat criteria was close to untenable with an FED value of 0.273 - near the tenability criterion of FED $=0.3$. Results of tenability calculations for all of the tests is included as Appendix A. 
Table 14. Calculated tenability for a flaming ignition test of a mattress, test SDC05

\begin{tabular}{|c|c|c|}
\hline Measurement Location & $\begin{array}{c}\text { Time from Ignition } \\
\text { to Untenable } \\
\text { Conditions (s) }\end{array}$ & $\begin{array}{l}\text { Final Tenability } \\
\text { Value at End of Test }\end{array}$ \\
\hline \multicolumn{3}{|c|}{ ISO Gas FED = 0.3} \\
\hline Remote Bedroom (A) & n.r. ${ }^{\mathrm{a}}$ & 0.012 \\
\hline Burn Room Bedroom (BEK) & & 0.07 \\
\hline Hallway Outside Remote Bedroom (C) & & 0.021 \\
\hline Front Door Hallway (F) & & 0.028 \\
\hline \multicolumn{3}{|c|}{ ISO Convected Heat $=0.3$} \\
\hline Master Bedroom (A) & & 0.009 \\
\hline Burn Room Bedroom (BEK) & & 0.273 \\
\hline Hallway Outside Remote Bedroom (C) & & 0.016 \\
\hline Hallway Outside Main Bedroom (D) & & 0.013 \\
\hline Living Area (E) & & 0.02 \\
\hline Front Door Hallway $(\mathrm{F})$ & & 0.022 \\
\hline Closed Bedroom $(\mathrm{G})$ & & 0.004 \\
\hline \multicolumn{3}{|c|}{ Smoke Obscuration $=0.25 \mathrm{~m}^{-1}$} \\
\hline Remote Bedroom (A) & $197 *$ & 0.276 \\
\hline Burn Room Bedroom (BEK) & 137 & 0.329 \\
\hline Hallway Outside Remote Bedroom (C) & 182 & 0.341 \\
\hline Hallway Outside Main Bedroom (D) & 162 & 0.403 \\
\hline Living Area $(\mathrm{E})$ & 177 & 0.26 \\
\hline Front Door Hallway (F) & $217^{\mathrm{b}}$ & 0.291 \\
\hline Closed Bedroom $(\mathrm{G})$ & & 0.006 \\
\hline
\end{tabular}

a - tenability limit not reached by end of test

$b-$ occurs after initiation of suppression

\subsection{Assessment of Overall Alarm Performance}

With 43 separate alarms throughout the structure in each test, an assessment of overall performance of the alarms requires some grouping of the alarms. For existing homes (and all homes, prior to 1993) smoke alarms are required outside the sleeping rooms and on each additional story of the home. For this report, this arrangement will be referred to as "every level" and represents the minimum arrangement allowed by code. In 1993 the National Fire 
Alarm Code was revised to require smoke alarms in every bedroom for new construction in addition to the every level locations. We will refer to this arrangement as "every level + bedrooms." Finally, the greatest escape times would be guaranteed only if smoke alarms were required in every room - an arrangement that has never been required in any code. Such an "every room" result represents the maximum required performance that can be contrasted against "every level" as the minimum required performance as a function of number and location. Alarm times for heat alarms and residential sprinklers are based on the activation of devices in the room of fire origin.

Table 15 shows the overall smoke alarm performance for test SDC05 using typical alarm points as defined in section 5.3. For each alarm type, the time of first alarm activation is noted as a "First Alarm" time. The average of all alarms in a category is noted as "Average." The time to the last alarm activation is noted as "Last Alarm." Finally, the total number of alarms in a group which activated is shown. Table 16 shows activation times for several other technologies that may be used in conjunction with smoke alarms. Individual calculations for all of the alarms in all of the tests is included as Appendix A. A summary of these results is included in the discussion section, below.

Table 15. Activation times for several smoke alarms during a flaming ignition test of a mattress, test SDC05

\begin{tabular}{|c|c|c|c|c|c|c|}
\hline & & $\begin{array}{c}\text { First Alarm } \\
\text { (s) }\end{array}$ & $\begin{array}{c}\text { Average } \\
\text { (s) }\end{array}$ & $\begin{array}{l}\text { Last Alarm } \\
\text { (s) }\end{array}$ & Activated & $\begin{array}{c}\text { Total } \\
\text { Alarms } \\
\end{array}$ \\
\hline \multirow{4}{*}{$\begin{array}{l}\text { Every } \\
\text { Level }\end{array}$} & Photoelectric & 42 & 85 & 127 & 2 of 2 & \multirow{4}{*}{10} \\
\hline & Ionization & 37 & 77 & 117 & 4 of 4 & \\
\hline & Aspirated & 52 & 85 & 117 & 2 of 2 & \\
\hline & Dual & 107 & 135 & 162 & 2 of 2 & \\
\hline \multirow{4}{*}{$\begin{array}{c}\text { Every } \\
\text { Level }+ \\
\text { Bedrooms }\end{array}$} & Photoelectric & 42 & 95 & 167 & 4 of 4 & \multirow{4}{*}{19} \\
\hline & Ionization & 32 & 83 & 147 & 8 of 8 & \\
\hline & Aspirated & 52 & 112 & 167 & 3 of 3 & \\
\hline & Dual & 107 & 147 & 172 & 4 of 4 & \\
\hline \multirow{4}{*}{$\begin{array}{l}\text { Every } \\
\text { Room }\end{array}$} & Photoelectric & 42 & 99 & 167 & 5 of 5 & \multirow{4}{*}{25} \\
\hline & Ionization & 32 & 80 & 147 & 10 of 10 & \\
\hline & Aspirated & 52 & 106 & 167 & 4 of 4 & \\
\hline & Dual & 107 & 160 & 192 & 6 of 6 & \\
\hline
\end{tabular}

Note - Dual alarm Ion4/Pho3D was not functioning during this test 
Table 16. Activation times for several alternative technologies during a flaming ignition test of a mattress, test SDC05

\begin{tabular}{|c|l|c|c|c|c|}
\cline { 3 - 6 } \multicolumn{2}{c|}{} & $\begin{array}{c}\text { First Alarm } \\
(\mathrm{s})\end{array}$ & $\begin{array}{c}\text { Average } \\
(\mathrm{s})\end{array}$ & $\begin{array}{c}\text { Last Alarm } \\
(\mathrm{s})\end{array}$ & Activated \\
\hline Every Level & CO & 132 & 174 & 197 & 5 of 6 \\
\hline Every Level + Bedrooms & CO & 132 & 189 & 237 & 8 of 9 \\
\hline \multirow{3}{*}{ Every Room } & CO & 132 & 182 & 237 & 10 of 12 \\
\cline { 2 - 6 } & Heat & 140 & 140 & 140 & 1 of 1 \\
\cline { 2 - 6 } & $\begin{array}{l}\text { Tell-tale } \\
\text { Sprinkler }\end{array}$ & 147 & 147 & 147 & 1 of 1 \\
\hline
\end{tabular}

\subsection{Aerosol Concentration and Size Measurements}

The amount of smoke, its particle size distribution, and light scattering properties in the case of photoelectric sensors affect the response of smoke alarms. Mulholland and Lui studied the response of ionization and photoelectric alarms to nearly mono-disperse (single size) di-octyl pthalate aerosols over a size range from $0.05 \mu \mathrm{m}$ to $1.1 \mathrm{~mm}$ [29]. Theory and experimental results have established the relationship between the response of an ionization-type alarm and the product of the aerosol number concentration and the count mean diameter [16,30]. (The count mean diameter is defined as the sum over all sizes of the number of particles of a given size times the particle diameter divided by the total number of particles.) For light scattering photoelectric alarms, the size relationship is more complex for the range of particle sizes found in smoke. In addition, the light scattering properties of the material that forms the particles is important.

Surrogate measures of smoke concentration via light extinction techniques, such as the laser beam smoke extinction meters used in this study, may provide approximate measures of smoke mass concentration by application of Bouguer's Law if an appropriate specific extinction coefficient is know for the smoke causing the extinction. In the case of soot generated from flaming fires, an estimated mean value of $8.7 \mathrm{~m}^{2} / \mathrm{g}$ with an expanded uncertainty $(95 \%$ confidence interval) of $1.1 \mathrm{~m}^{2} / \mathrm{g}$ computed from a wide range of experiments has been reported [31]. In the case of non-soot smolder smokes, the specific extinction coefficient lies typically between $3 \mathrm{~m}^{2} / \mathrm{g}$ to $5 \mathrm{~m}^{2} / \mathrm{g}$.

The concentration and size distribution of smoke aerosols were measured during several selected tests conducted in the first manufactured home series and the two-story home series. 
Measurements of the aerosol number and mass concentration were recorded at fixed locations for selected tests. In addition, the continuous voltage output from modified residential ionization smoke alarms (some installed in plenums through which room air was drawn in and others located at the sampling location) were recorded. Response of these alarms was related to the response of a MIC reference chamber to establish an effective chamber constant for the modified alarms. The combination of these measures allows for an estimation of two mean particle sizes (the count mean diameter and the diameter of average mass) and potentially the width of the size distribution as a function of time. In a select number of fire tests, cascade impactor samples were collected and analyzed to estimate the mass median aerodynamic diameter and the geometric standard deviation for those samples.

\subsubsection{Mass and Number Concentration}

The number concentration, ion chamber, and mass concentration results for 15 tests are presented below. In addition to these results, estimates of the count mean diameter and diameter of average mass were computed at selected time periods.

SDC01 was a smoldering chair test in the manufactured home. Figure 122a shows the mass and number concentrations measured in the remote bedroom. Except for a slight increase at $1400 \mathrm{~s}$, the number concentration declined until $5200 \mathrm{~s}$ when it started to climb rapidly. Mass concentration started to climb at $5400 \mathrm{~s}$ and peaked around $7200 \mathrm{~s}$ when suppression action was taken.

Figure 122b shows the ionization chamber results in terms of the dimensionless " $Y$ " parameter. The two flow-through ionization chambers were Ion 1 and Ion 2. Ion 3 and Ion 4 were located next to the sampling locations at the ceiling. Ion 1 and Ion 3 were measuring the hallway location, and Ion 2 and Ion 4 were measuring the remote bedroom location. This was the arrangement for the tests conducted in the manufactured home. For the most part, the flowthrough and room ionization chambers sampling the same location responded similarly, with no significant response time difference. The difference between the two sampling locations reflects smoke transport time and differences in smoke concentration and size distribution.

In this particular test, there appears to be a discrepancy since the remote bedroom responded sooner and achieved a higher level than the hallway location. A possible explanation for this observation is that the back bedroom window was left open, and the bay doors to the test facility were also open during this test. Flow into the test building imposed a pressure gradient in the manufactured home that restricted flow down the hallway. Repeat tests where the window was closed and the building doors shut did not show this effect. 
SDC02 was a flaming chair test in the manufactured home. Figure 123a shows the number and mass concentrations for this test. Both started rising around $100 \mathrm{~s}$. Figure $123 \mathrm{~b}$ shows the ionization chamber results. The hallway chambers responded within $20 \mathrm{~s}$ of ignition, while the remote bedroom chambers responded $70 \mathrm{~s}$ after ignition. The difference between the response of the remote bedroom chambers and the mass and number concentration is attributed to differences in aerosol transport time to the instruments.

SDC06 was a smoldering mattress test in the manufactured home. Figure 124 shows the number concentration and the ionization chamber results. The mass concentration was not recorded during this test. The number concentration declined until about $6000 \mathrm{~s}$ when it shot up after the mattress flamed. The initial decrease in number concentration appears to be related to decrease in the ambient number concentration during the long period the house was closed up. Ionization chambers started to increase slightly around $2800 \mathrm{~s}$ then climbed rapidly between $5700 \mathrm{~s}$ and $5900 \mathrm{~s}$.

SDC07 was a flaming mattress test in the manufactured home. Figure 125 shows the number concentration and the ionization chamber results. Again, mass concentration was not recorded during this test. The hallway chambers increased rapidly around $50 \mathrm{~s}$ while the remote bedroom chambers increased nearly as rapidly, but delayed until about $130 \mathrm{~s}$. The number concentration started to increase very rapidly at $150 \mathrm{~s}$.

SDC08 was a smoldering mattress test in the manufactured home. Figure 126a shows the number and mass concentrations for this test. Number concentration was flat and no mass concentration was recorded until about $3750 \mathrm{~s}, 70 \mathrm{~s}$ after the mattress started to flame, when both increased rapidly. Figure $126 \mathrm{~b}$ shows the ionization chamber results. The chambers located in the hallway and target room started rising as early as $500 \mathrm{~s}$. The hallway ionization chambers increased rapidly around $3680 \mathrm{~s}$, while the target room chambers did the same around $3730 \mathrm{~s}$.

SDC10 was a flaming chair test in the manufactured home. Figure 127a shows the number and mass concentrations for this test. The number concentration started rising about $130 \mathrm{~s}$ after ignition with the mass concentration following $10 \mathrm{~s}$ later. Figure 127b shows the ionization chamber results. The hallway chambers started to increase sharply at $55 \mathrm{~s}$, while the remote bedroom chambers started to rise between $110 \mathrm{~s}$ and $120 \mathrm{~s}$. 

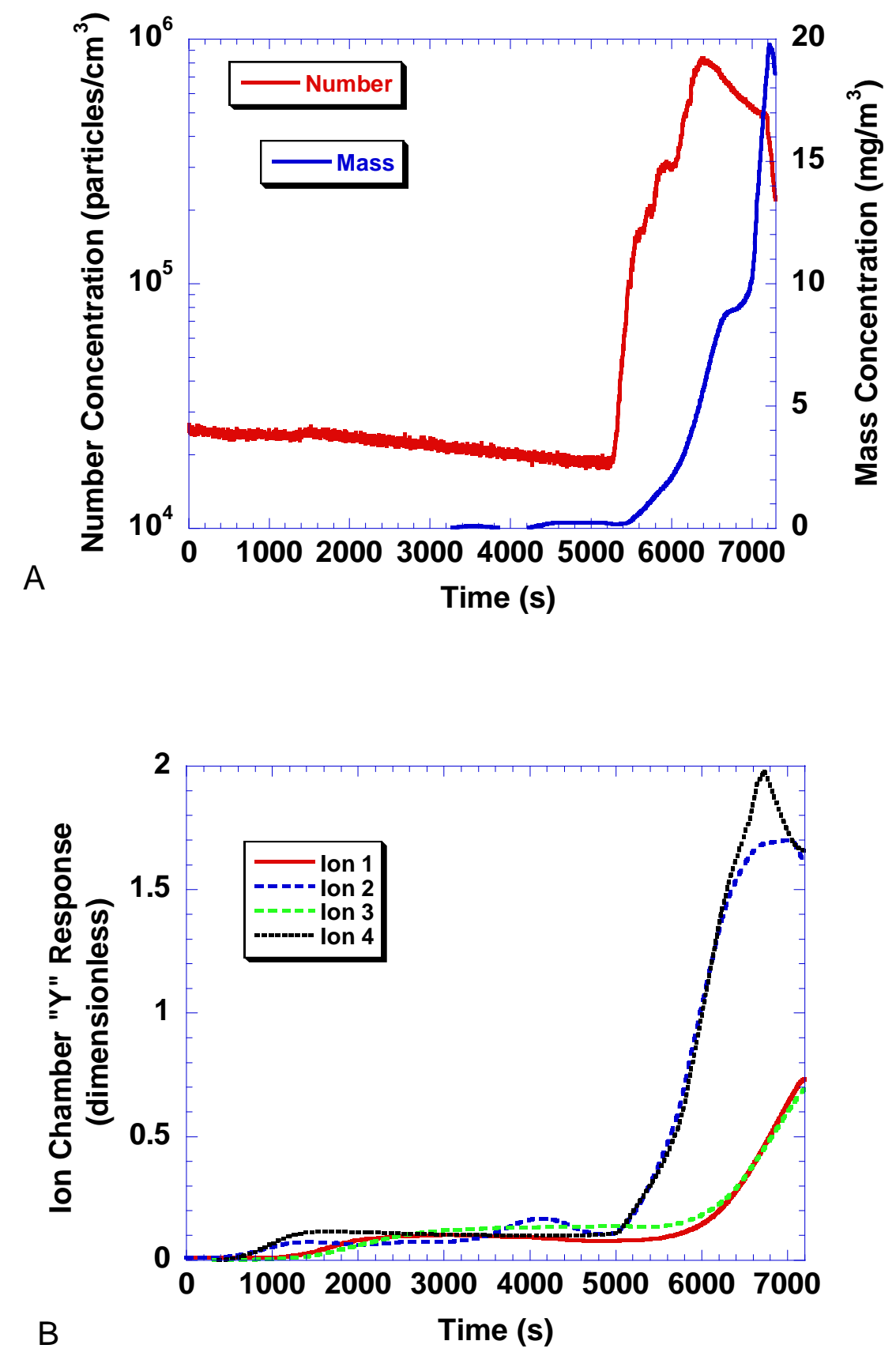

Figure 122. Smoke properties for smoldering chair scenario SDC01 

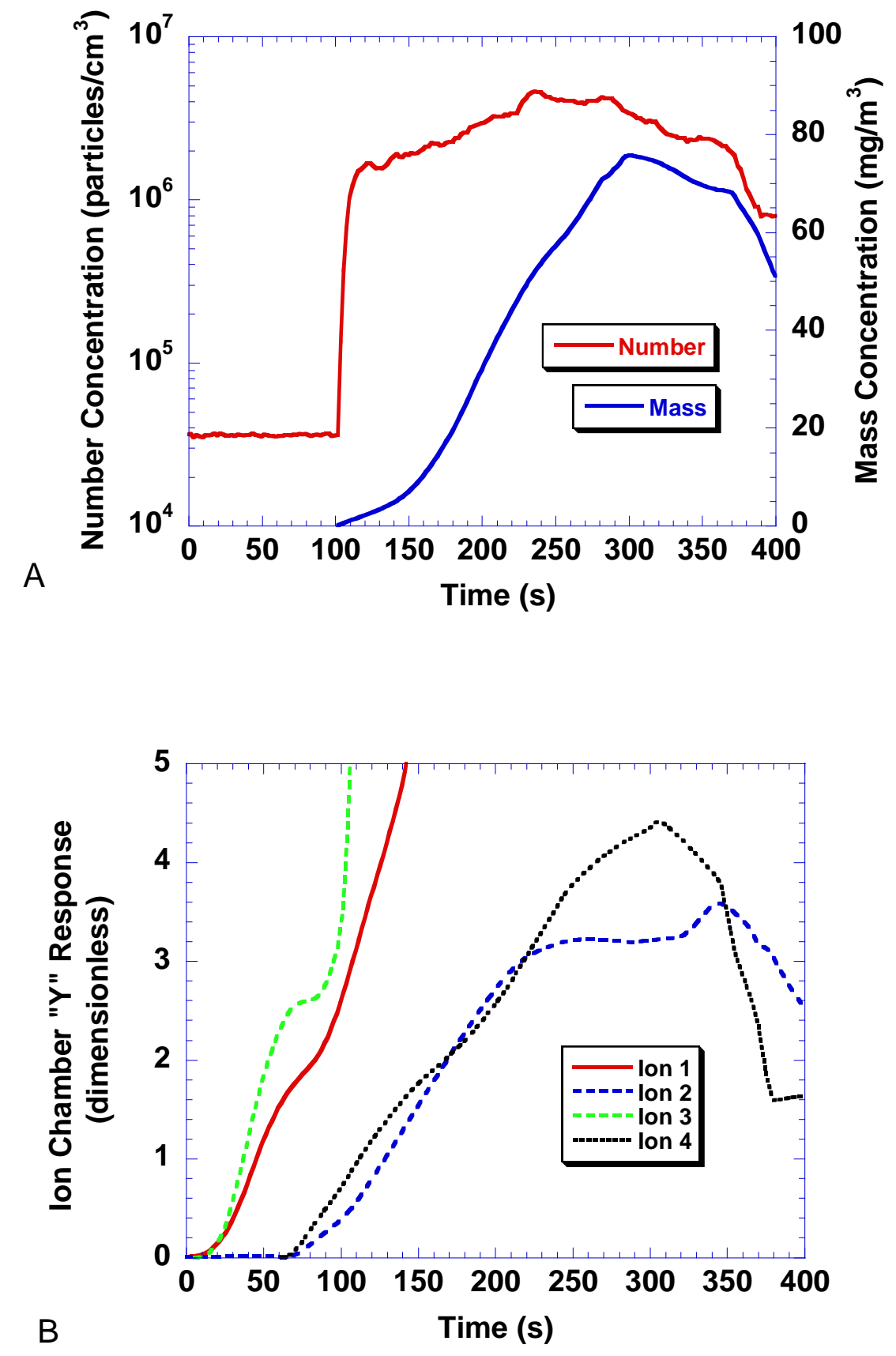

Figure 123. Smoke properties for flaming chair scenario SDC02 


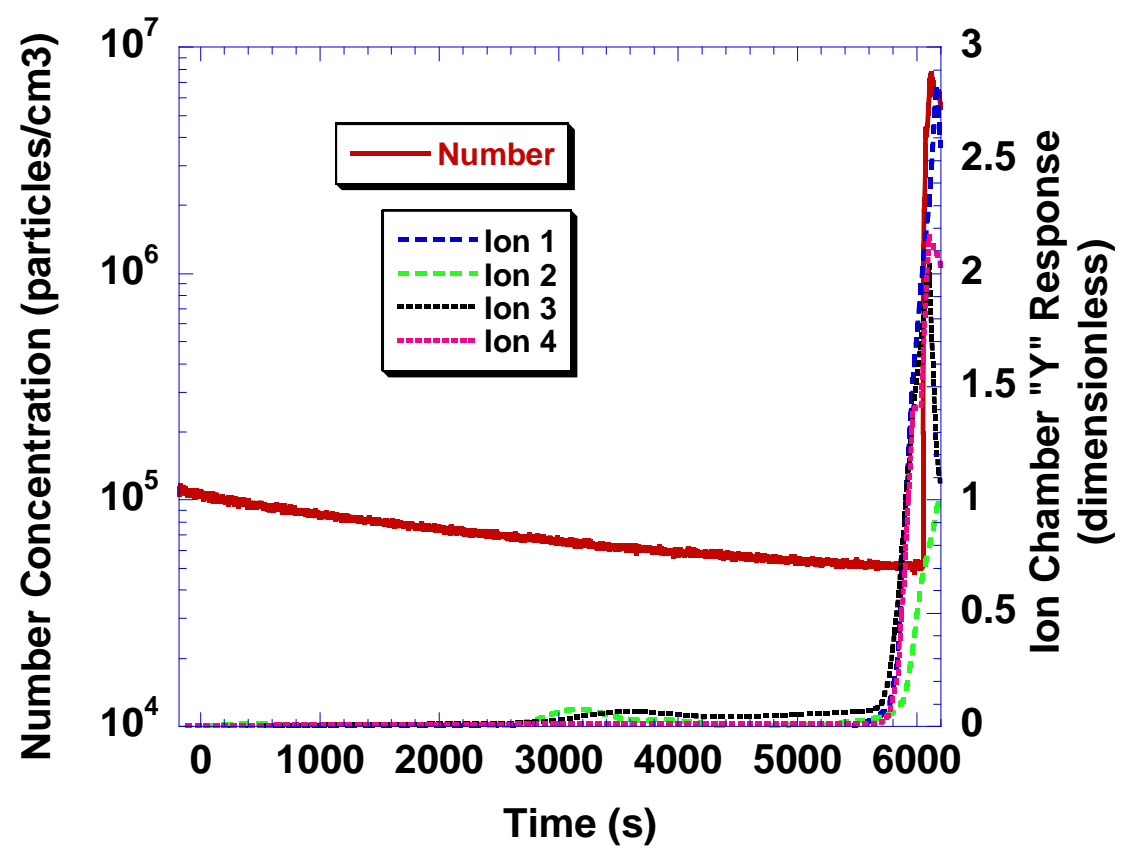

Figure 124. Smoke properties for smoldering mattress scenario SDC06

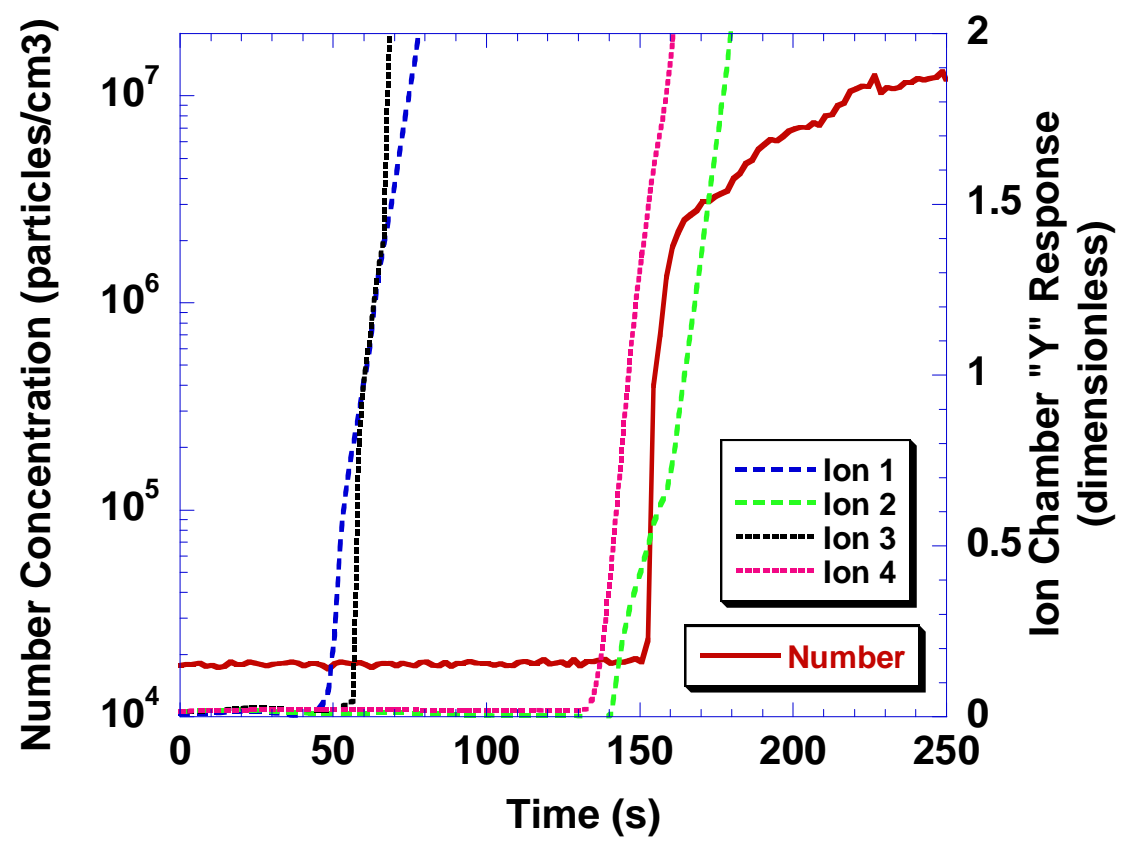

Figure 125. Smoke properties for flaming mattress scenario SDC07 

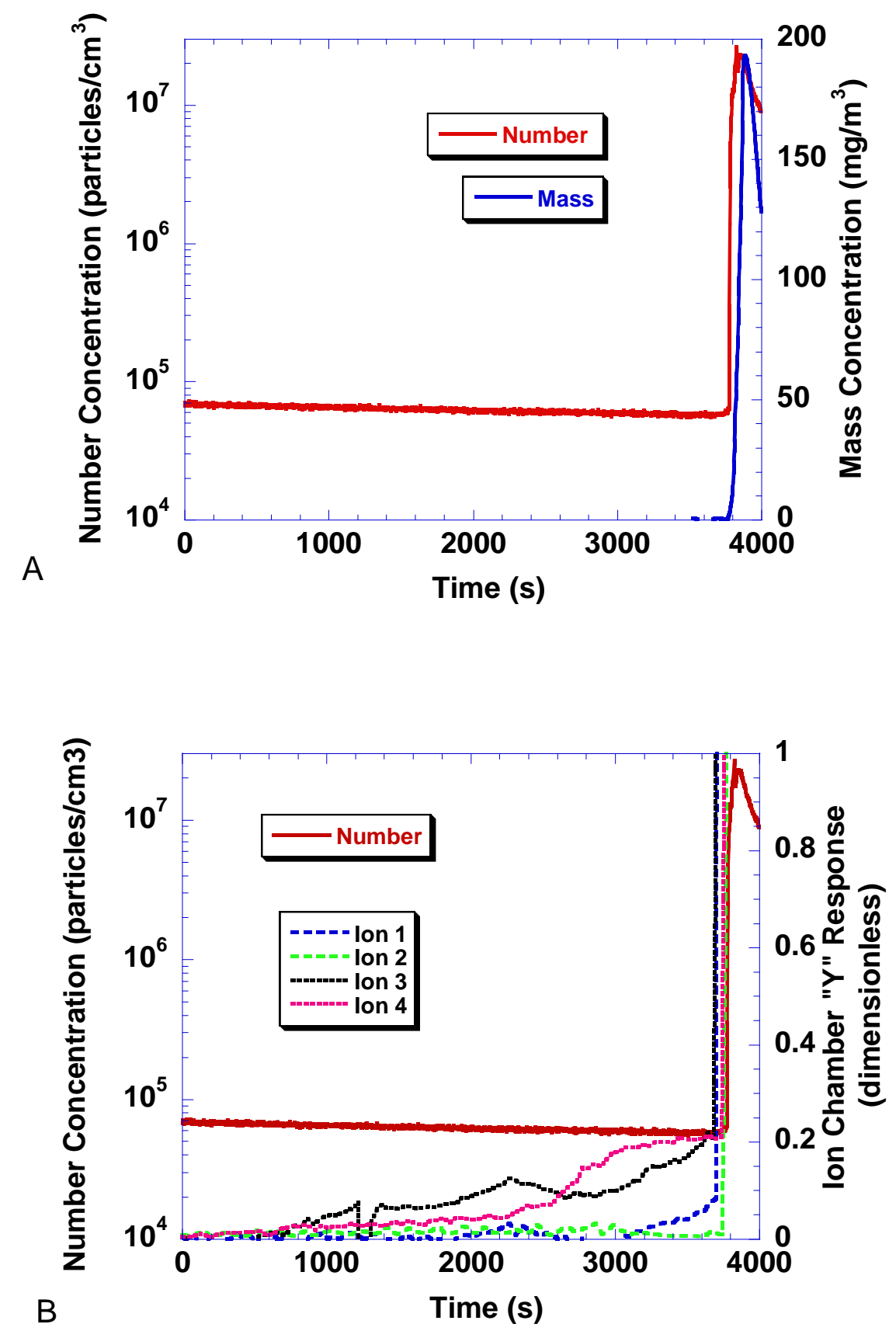

Figure 126. Smoke properties for smoldering mattress scenario SDC08 

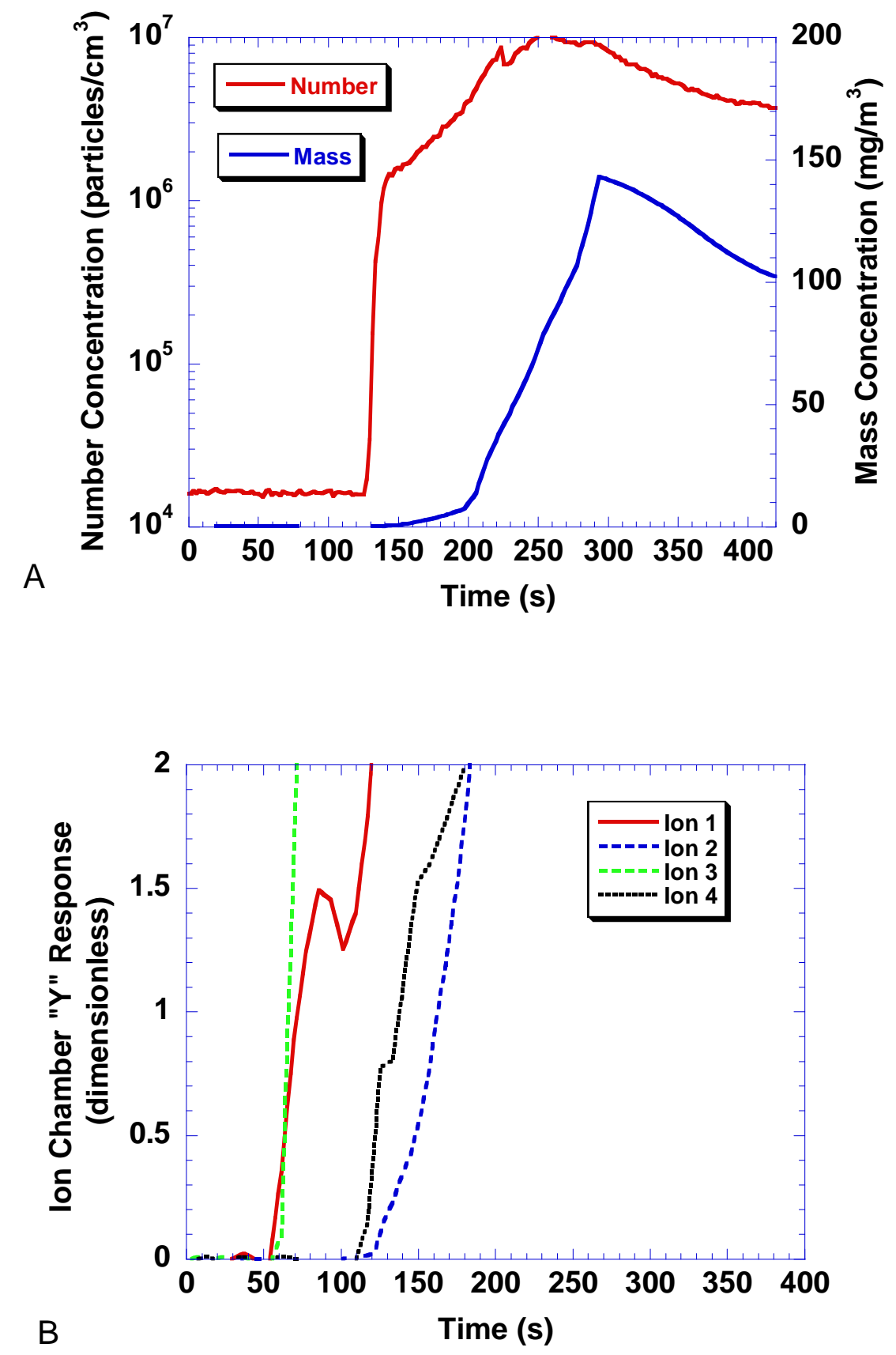

Figure 127. Smoke properties for flaming chair scenario SDC10 
SDC11 was a smoldering chair test in the manufactured home. Figure 128a shows the number and mass concentrations for this test. Only after transition to flaming at about $4200 \mathrm{~s}$ do both number and mass concentrations start to rise. Figure $128 \mathrm{~b}$ shows the ionization chamber results. The two chambers located inside the manufactured home started rising around $1000 \mathrm{~s}$, while the two flow-through chambers remained at nominally zero until after transition to flaming when all chambers increased rapidly. The environmental conditions that caused the room chambers to increase early in the test evidently were not transported to the flow-through chambers. It could be condensation or volatile particulates.

SDC12 was a cooking oil fire in the manufactured home. Figure 129 shows the number concentration and ionization chamber results. The number concentration started to increase at about $200 \mathrm{~s}$ due to the small particles produced by the gas burner. The number concentration increases at a moderate rate until transition to flaming at about $1400 \mathrm{~s}$. The hallway ionization chambers started to increase almost immediately after ignition of the burner, while the remote bedroom chambers did not start increasing until about $500 \mathrm{~s}$. All ionization chambers reached relatively high levels before ignition of the cooking oil.

SDC13 was a repeat of the cooking oil fire in the manufactured home. Figure 130 shows the number concentration and ionization chamber results. The results are similar to SDC12.

SDC14 was a smoldering mattress fire with the burn bedroom door closed in the manufactured home. Figure 131 shows the number concentration and ionization chamber results. Around $3400 \mathrm{~s}$ the mattress transitioned to flaming, and smoke was forced out the door cracks, which caused the hallway chambers to increase rapidly. The smoke drifted back to the remote bedroom and caused the bedroom ionization chambers to rise along with the number concentration. Around $4000 \mathrm{~s}$ the burn bedroom door was opened, the mattress burst into flames again, and consequentially, much more smoke was transported to the remote bedroom. Soon after, the exterior doors were opened and the fire extinguished.

SDC23 was a smoldering chair test in the two-story home. Figure 132a shows the number and mass concentrations recorded in the den. The mass concentration started to increase at $1000 \mathrm{~s}$ while the number concentration started to increase at $2000 \mathrm{~s}$. Around $4600 \mathrm{~s}$ the number and mass concentration started to increase rapidly. Figures $132 \mathrm{~b}$ and $132 \mathrm{c}$ show the number concentration and flow-through ionization chamber results for the den and remote bedroom locations respectively. The den ionization chamber started to rise at $600 \mathrm{~s}$ and reached a peak at $2000 \mathrm{~s}$. After $3800 \mathrm{~s}$ the data from the den ionization chamber was invalid. The remote bedroom ionization chamber started to rise at $1400 \mathrm{~s}$, while the number concentration started to increase at $1600 \mathrm{~s}$, leveled off to a plateau, then increased rapidly again at $4200 \mathrm{~s}$. The ionization chamber increased rapidly at $4600 \mathrm{~s}$. 

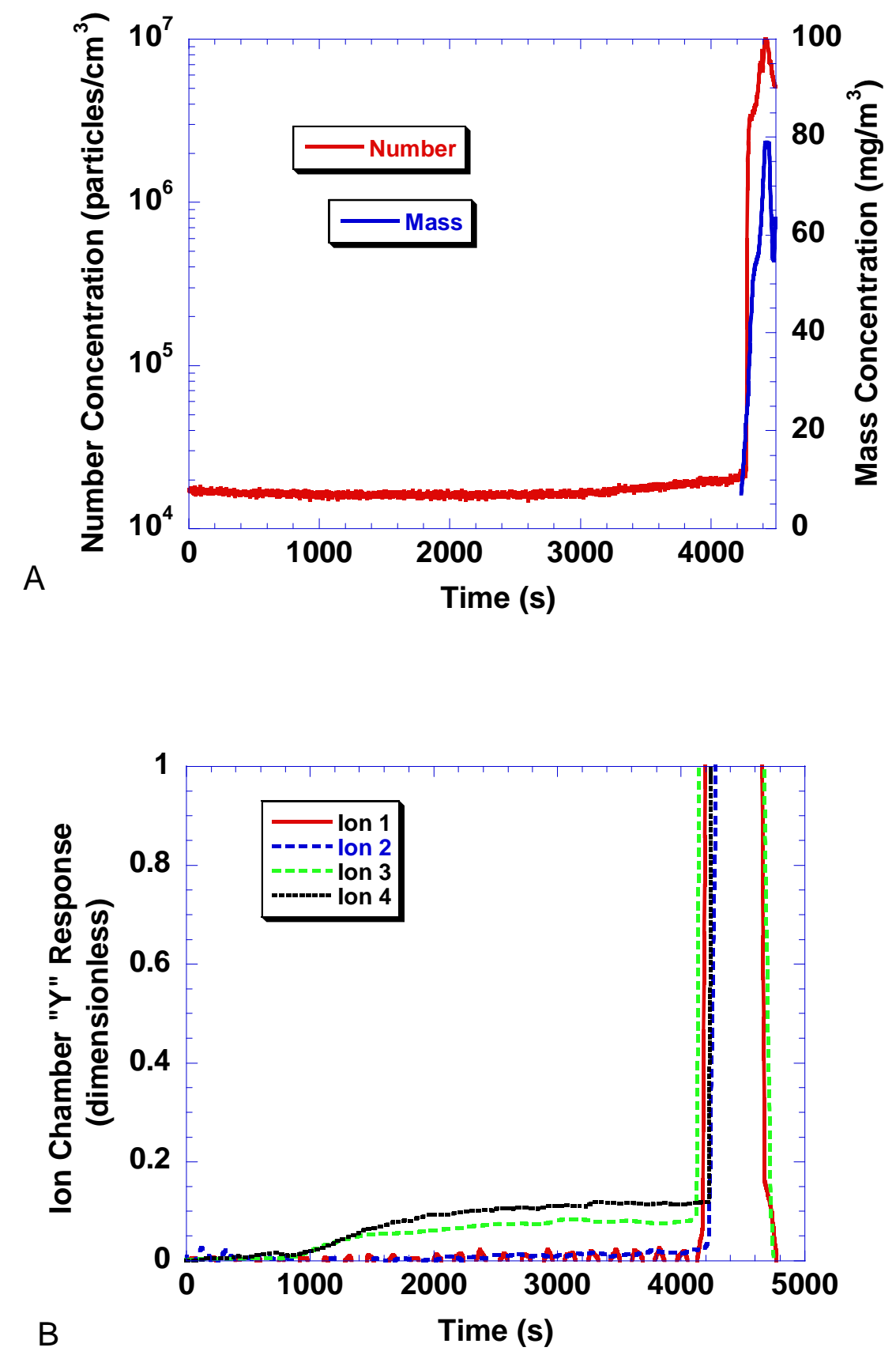

Figure 128. Smoke properties for smoldering chair scenario SDC11 


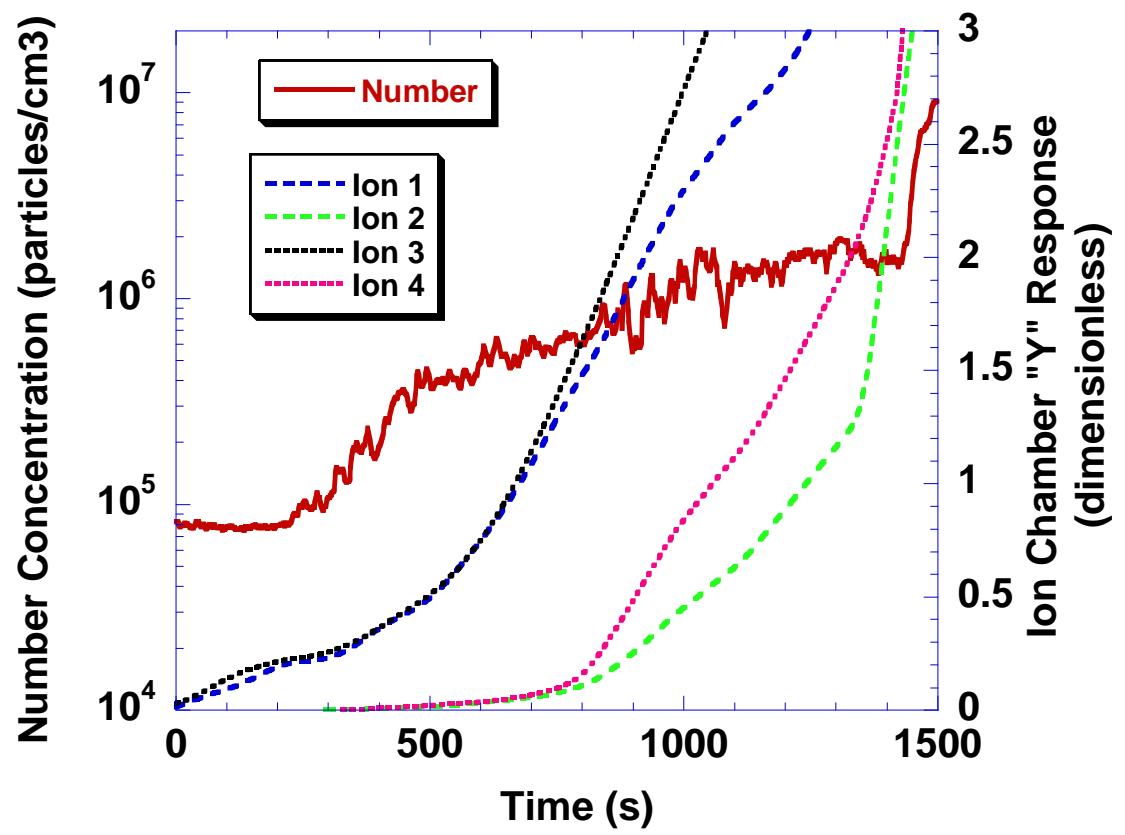

Figure 129. Smoke properties for cooking oil fire scenario SDC12

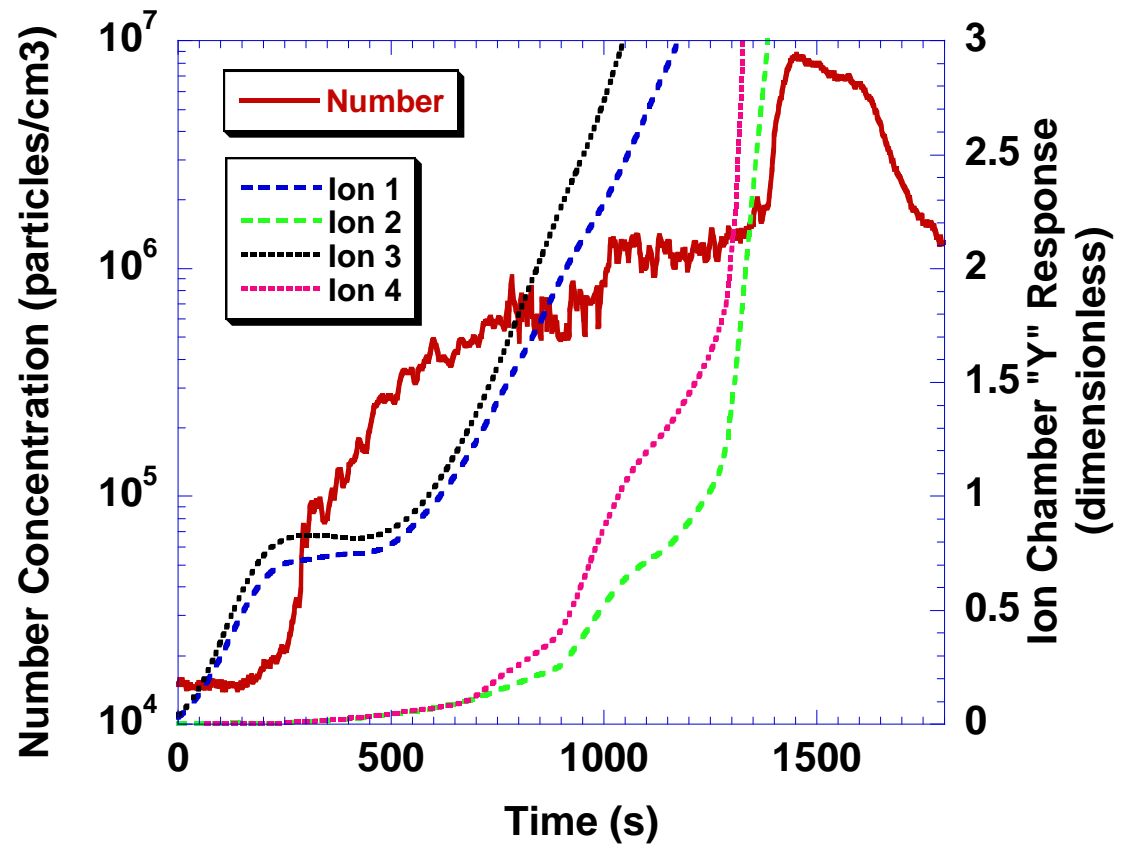

Figure 130. Smoke properties for cooking oil fire scenario SDC13 


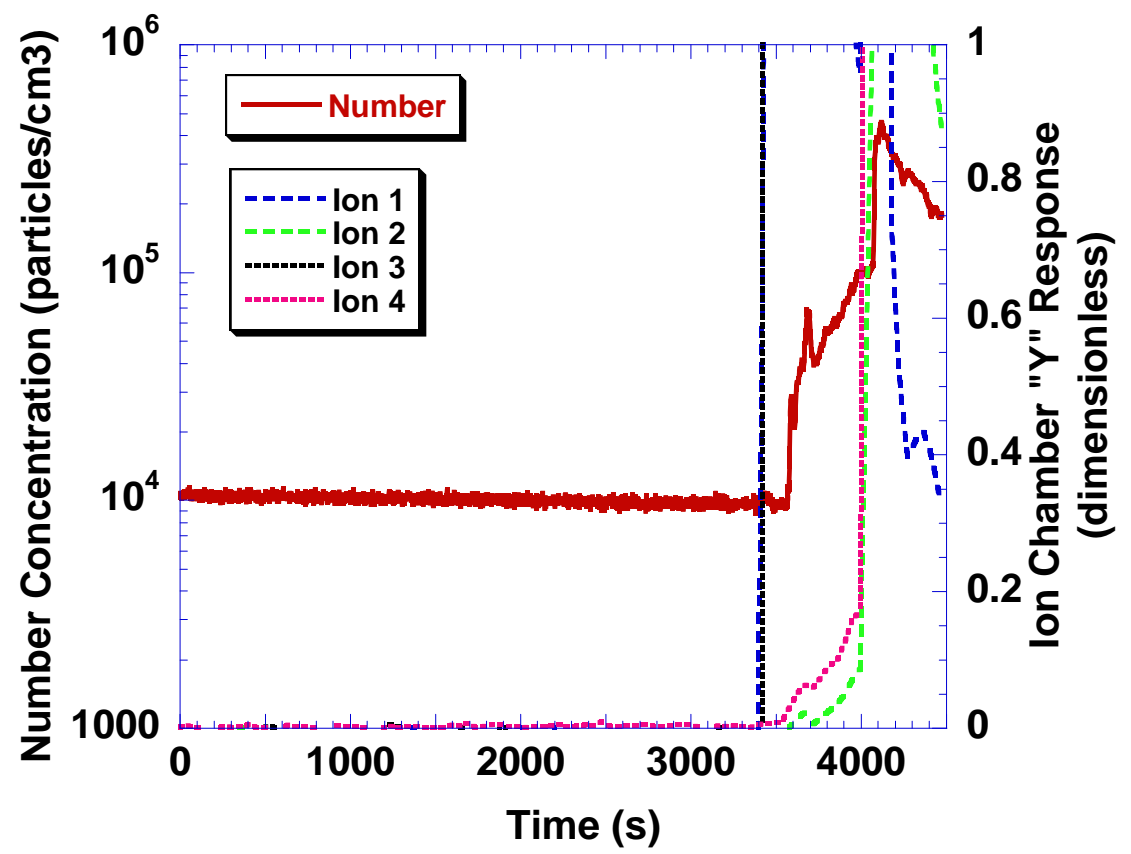

Figure 131. Smoke properties for smoldering mattress with burn room door closed SDC14 

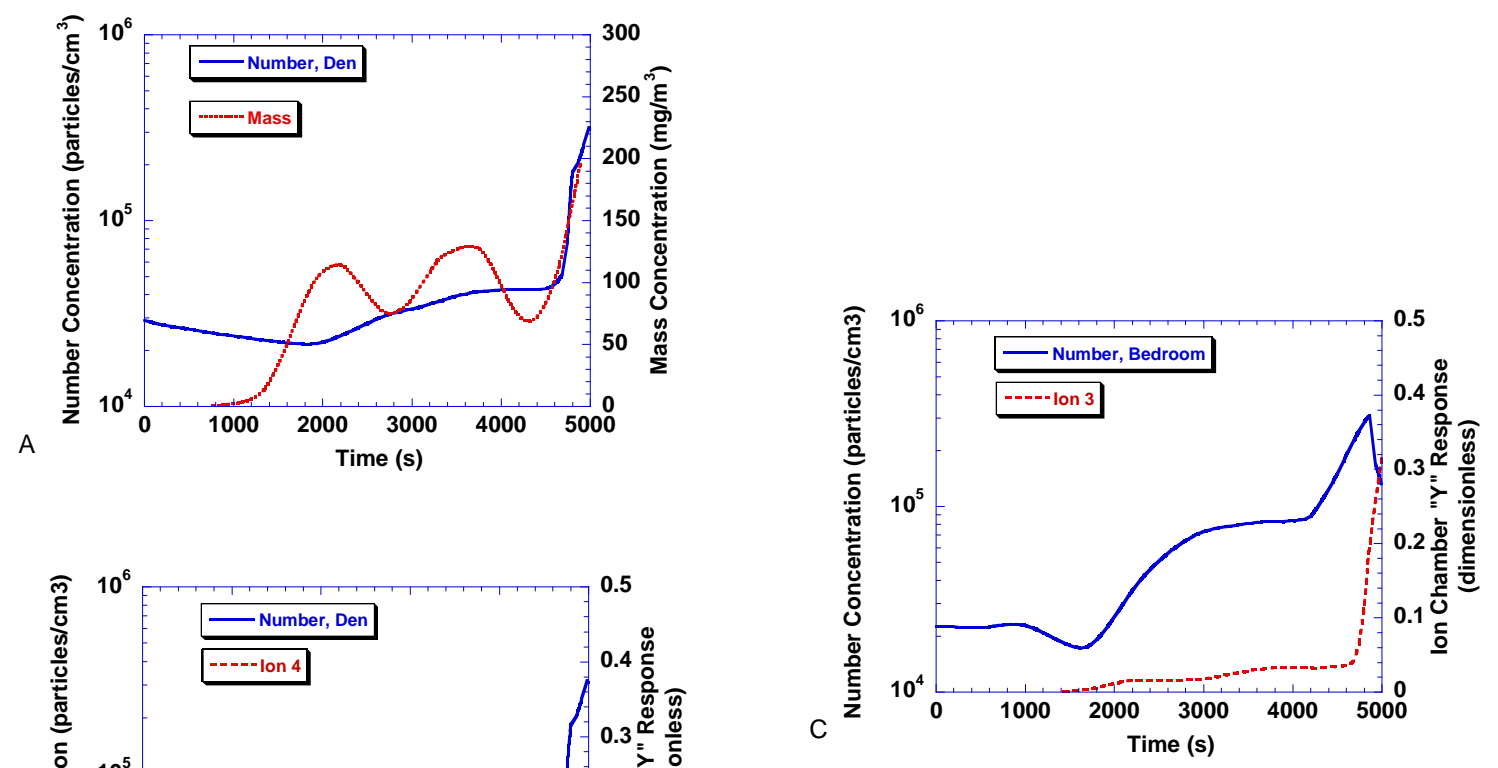

Figure 132. Smoke properties for smoldering chair scenario SDC23 
SDC24 was a cooking oil fire in the two-story home. Figure 133a shows the number and mass concentrations recorded in the den. The number concentration started to increase almost immediately following ignition of the gas burner. The mass concentration started to increase at $600 \mathrm{~s}$, and rose at an ever-increasing rate until the instrument upper range was reached at $1750 \mathrm{~s}$. Figure 133b shows the number concentration and ionization chamber results for the den. From $200 \mathrm{~s}$ to $1400 \mathrm{~s}$ the ion chamber signal increased steadily. From $1400 \mathrm{~s}$ to the peak at $1700 \mathrm{~s}$ the signal increased at a steeper rate. Figure 133c shows the number concentration and ionization chamber results for the remote bedroom. The number concentration increased steadily up to $1700 \mathrm{~s}$ when both it and the ionization chamber signal rose rapidly.

SDC25 was a flaming chair test in the two-story home. Figure 134 shows the mass concentration and ionization chamber results. The condensation particle counter was inoperable for this test and subsequent tests below. The den and remote bedroom ionization chamber signals started to increase rapidly around $110 \mathrm{~s}$ and $125 \mathrm{~s}$ respectively. The mass concentration in the den started to rise at $125 \mathrm{~s}$ and peaked around $275 \mathrm{~s}$.

SDC26 was a repeat of the flaming chair test above. Figure 135 shows the mass concentration and ionization chamber results. The results were similar to SDC25, but it appears that the early fire growth period was longer.

SDC27 was a smoldering chair test with an operating window-mounted air conditioning unit located in the remote bedroom. Figure 136 shows the mass concentration and ionization chamber results. Interestingly, the remote bedroom ionization chamber started to increase before the den ionization chamber, and reached a higher level. The mass concentration increased steadily from $1000 \mathrm{~s}$ to $3500 \mathrm{~s}$.

\subsubsection{Particle Size Analysis}

\subsubsection{Cascade Impactor Tests}

The size distribution results for each MOUDI cascade impactor run are presented below. It is customary to fit impactor data to a log-normal size distribution and report the mass median aerodynamic diameter (equivalent spherical particle with unit density) and geometric standard deviation.

Here, two techniques were used to fit the data: graphically on a log-probability chart, and by non-linear regression curve fitting of the data. On log-probability paper, a log-normal distribution is described by a straight line. Data points are fitted to a straight line that is weighted toward fitting the points around the $50 \%$ probability. The geometric standard deviation was estimated by taking the ratio of the diameter at $50 \%$ to the diameter at $16.1 \%$. Results for the MOUDI tests are presented in table 17. The sampling time was identified along 

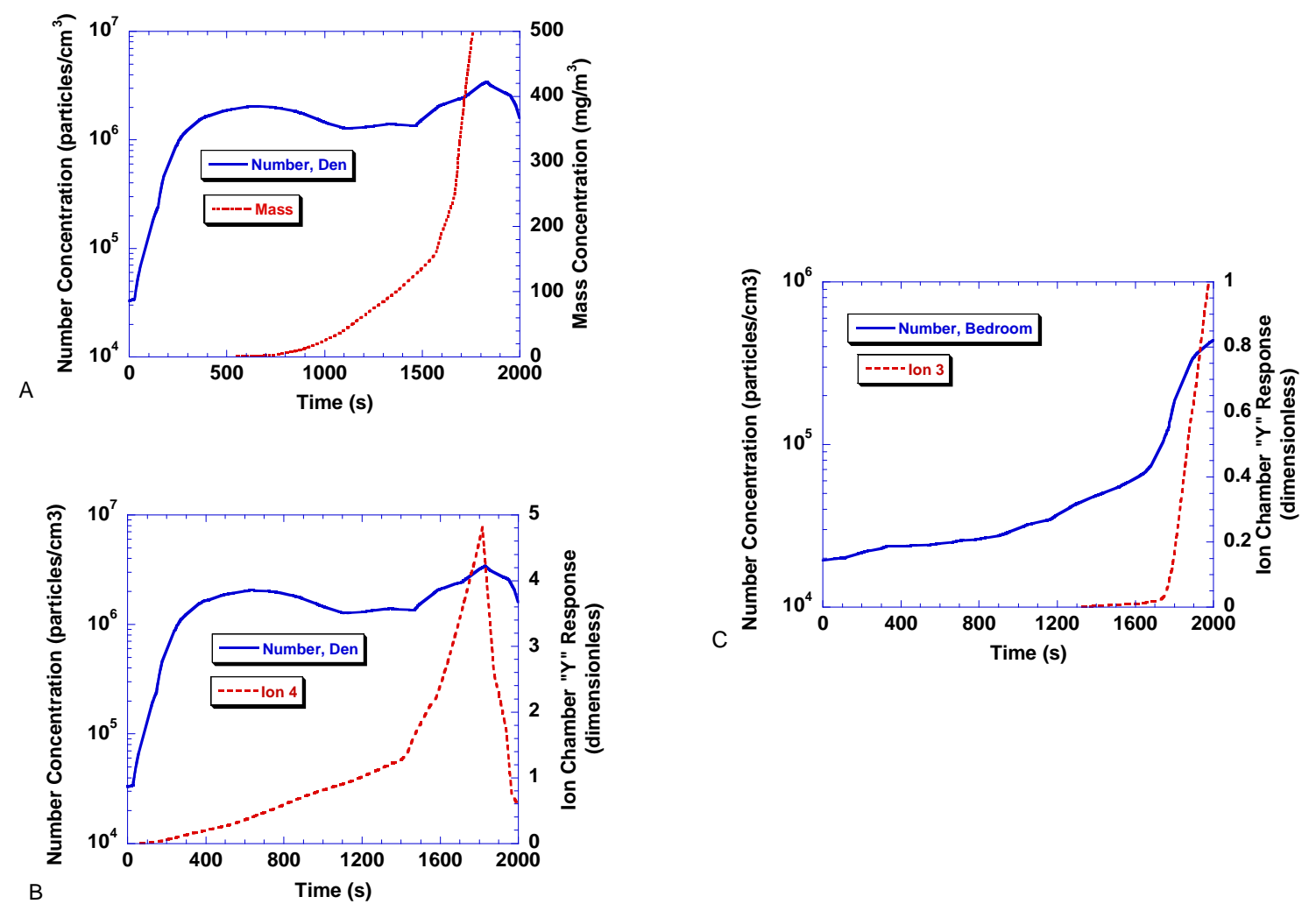

Figure 133. Smoke properties for cooking oil fire scenario SDC24 


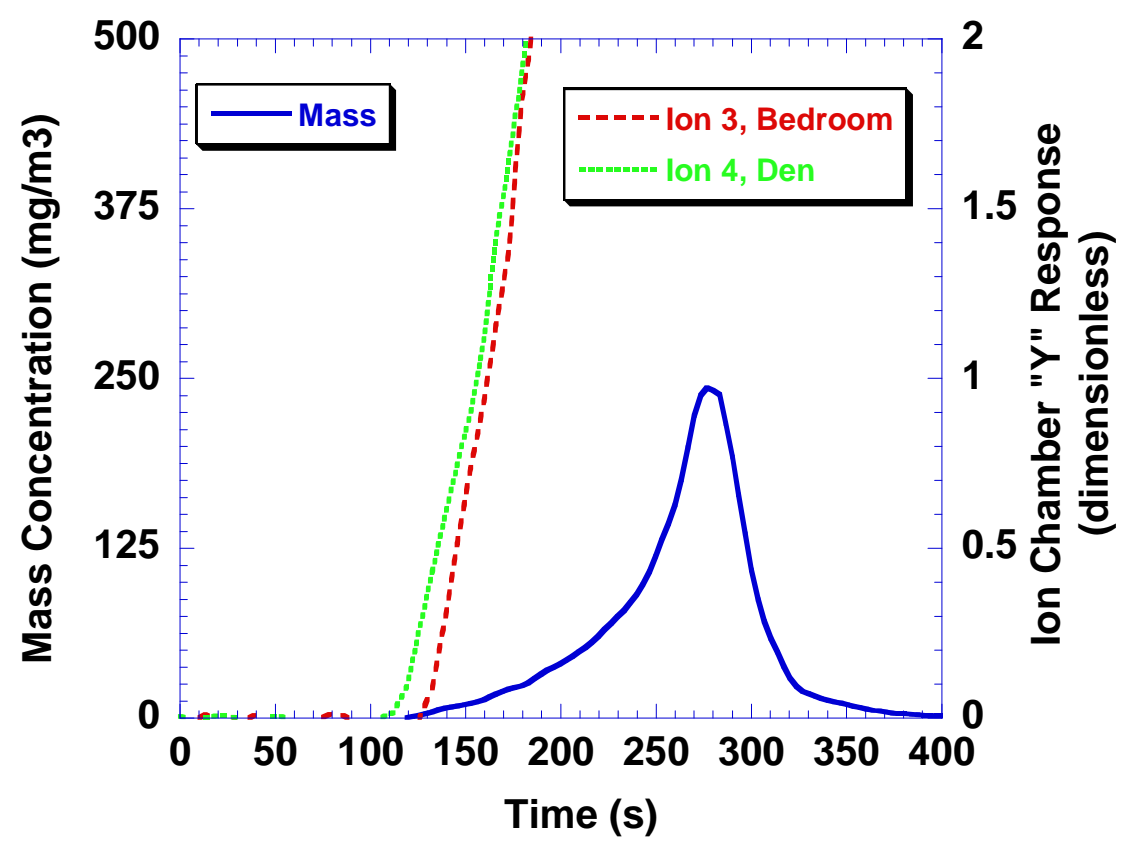

Figure 134. Smoke properties for flaming chair scenario SDC25

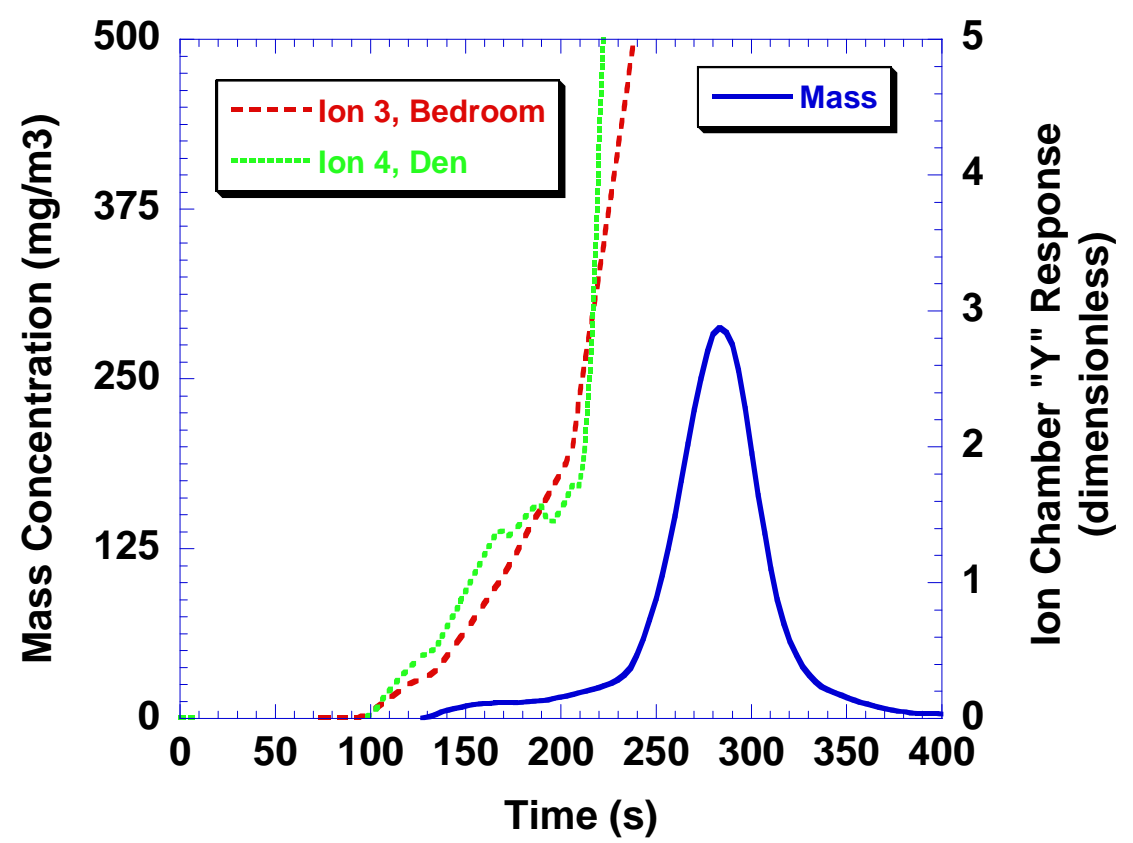

Figure 135. Smoke properties for flaming chair scenario SDC26 


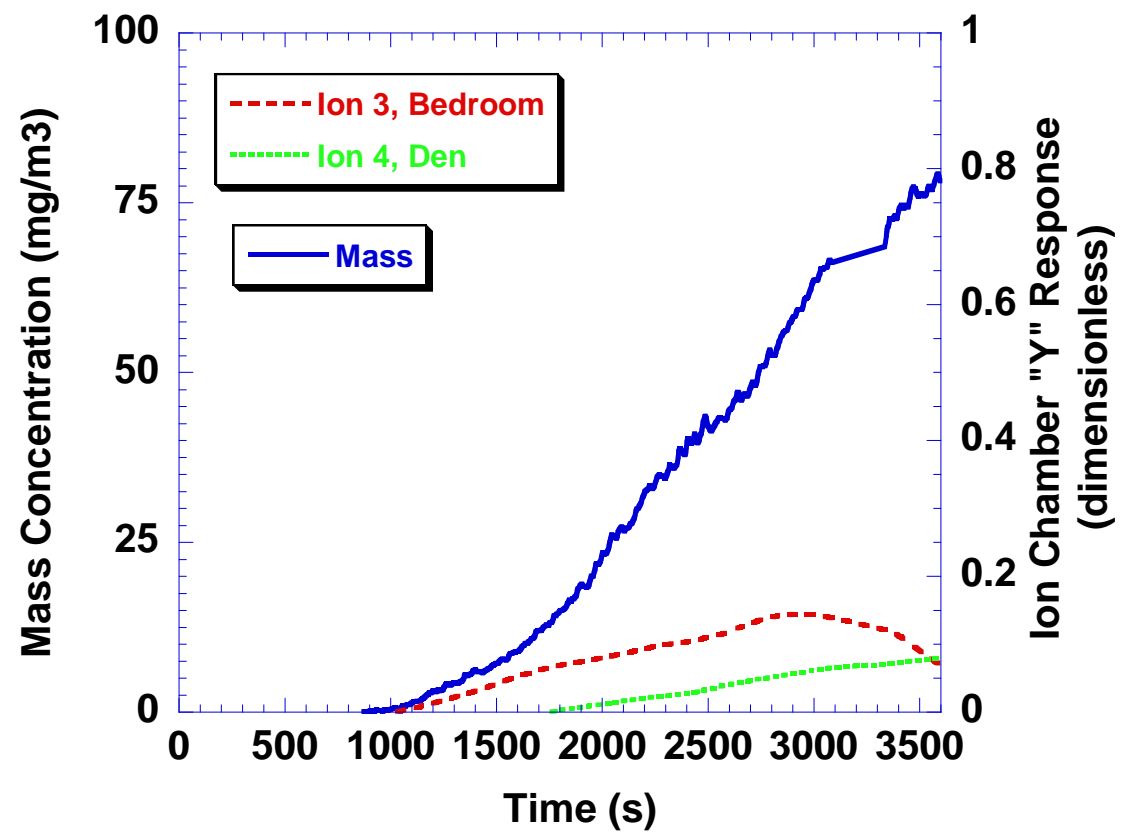

Figure 136. Smoke properties for smoldering chair scenario with air conditioning SDC27 
Table 17. Cascade impactor results

\begin{tabular}{|c|c|c|c|c|c|c|c|}
\hline Test & $\begin{array}{c}\text { Sampling } \\
\text { start / } \\
\text { duration (s) }\end{array}$ & $\begin{array}{l}\text { L-P fit } \\
\text { MMAD } \\
(\mu \mathrm{m})\end{array}$ & $\begin{array}{c}\text { L-P fit } \\
\sigma_{g}\end{array}$ & $\begin{array}{l}\text { L-S fit } \\
\text { MMAD } \\
(\mu \mathrm{m})\end{array}$ & $\begin{array}{c}\text { L-S fit } \\
\sigma_{g}\end{array}$ & \multicolumn{2}{|c|}{$\begin{array}{c}\text { Mean Mass } \\
\text { concentration, } \\
\mathrm{U}_{\mathrm{C}}\left(\mathrm{mg} / \mathrm{m}^{3}\right)\end{array}$} \\
\hline $\begin{array}{l}\text { SDC02 } \\
\text { Flaming } \\
\text { Chair }\end{array}$ & $360 / 60$ & 0.32 & 4.0 & -- & -- & 78.3 & 1.7 \\
\hline $\begin{array}{l}\text { SDC10 } \\
\text { Flaming } \\
\text { Chair }\end{array}$ & $200 / 60$ & 0.33 & 4.1 & -- & -- & 87.1 & 1.7 \\
\hline $\begin{array}{l}\text { SDC12 } \\
\text { Cooking } \\
\text { Oil }\end{array}$ & $980 / 240$ & 1.0 & 2.5 & 1.09 & 2.59 & 41.3 & 0.4 \\
\hline $\begin{array}{l}\text { SDC13 } \\
\text { Cooking } \\
\text { Oil }\end{array}$ & $830 / 270$ & 1.0 & 2.7 & 0.93 & 2.86 & 16.8 & 0.4 \\
\hline $\begin{array}{l}\text { SDC23 } \\
\text { Smoldering } \\
\text { Chair }\end{array}$ & $3520 / 600$ & 2.3 & 1.7 & 2.68 & 1.78 & 11.5 & 0.2 \\
\hline $\begin{array}{l}\text { SDC27 } \\
\text { Smoldering } \\
\text { Chair }\end{array}$ & $1850 / 300$ & 2.2 & 1.7 & 2.46 & 1.67 & 15.0 & 0.4 \\
\hline
\end{tabular}

L-P fit - log probability fit

MMAD - mass median aerodynamic diameter

$\sigma_{\mathrm{g}}-$ geometric standard deviation

L-S - least squares

$\mathrm{U}_{\mathrm{C}}-$ combined standard uncertainty 
with the average mass concentration obtained from the sum of masses on all stages and the backup filter.

Figures 137 and 138 show the results for SDC02 and SDC10, repeats of the flaming chair scenario. The collection times were nominally the same and near the end of the test. The mass median aerodynamic diameters of $0.32 \mu \mathrm{m}$ and $0.33 \mu \mathrm{m}$ were within $5 \%$ and the geometric standard deviations were nominally the same. The data from these two runs were not fitted by non-linear regression due to poor fitting of the very broad data. The aerodynamic size distribution of soot agglomerates yields very broad distribution. Cleary [32] reported mass median diameters of soot from an acetylene diffusion flame ranging from $0.3 \mu \mathrm{m}$ to over $8 \mu \mathrm{m}$ depending on the fuel flow. The open agglomerate structure of soot produces a drag force greater than an equivalent spherical volume and consequently, an agglomerate cross-sectional diameter is much greater than its aerodynamic diameter.

Figure 139 shows the results for SDC12 and SDC13, repeats of the cooking oil fire scenario in the manufactured home. Sample collection was conducted prior to ignition of the hot oil. The aerosol was made up of condensed pyrolyzate from the hot oil. The mass median diameters obtained from the log-probability fit for the two tests were the same $(1.0 \mu \mathrm{m})$, while the nonlinear regression fits were within $17 \%$ of each other with a mean value of $1.01 \mu \mathrm{m}$. The various geometric standard deviations ranged from 2.59 to 3.0 .

Figure 140 shows the results for SDC23 and SDC27, the smoldering chair tests in the two-story home. SDC27 differed from SDC23 by the placement and operation of a window air conditioning unit in the remote bedroom. The two samples were collected at different time periods from the beginning of the tests. The mass median aerodynamic diameters estimated by the log-probability fits were within $5 \%$ of each other for the repeated tests, while the non-linear regression fits were within $9 \%$ of each other. The mass median aerodynamic diameters computed by the non-linear regression were higher than those estimated by the log-probability fits by $16 \%$ and $12 \%$ for SDC23 and SDC 27 respectively.

\subsubsection{Particle size from moment analysis}

Estimates of the count mean diameter and diameter of average mass were computed from the number concentration, flow-through ionization chamber results and mass concentration data for selected time periods of several tests. The ratio of the ionization chamber "Y" to the number concentration is proportional to the count mean diameter, while the cubed root of the quotient the mass concentration to the number concentration is proportional to the diameter of average mass. Details of this analysis are available [33]. The material density factors into the calculation and when unknown, it was assumed to be $1 \mathrm{~g} / \mathrm{cm}^{3}$. The results are presented in the tables below where each table was dedicated to a particular scenario. 


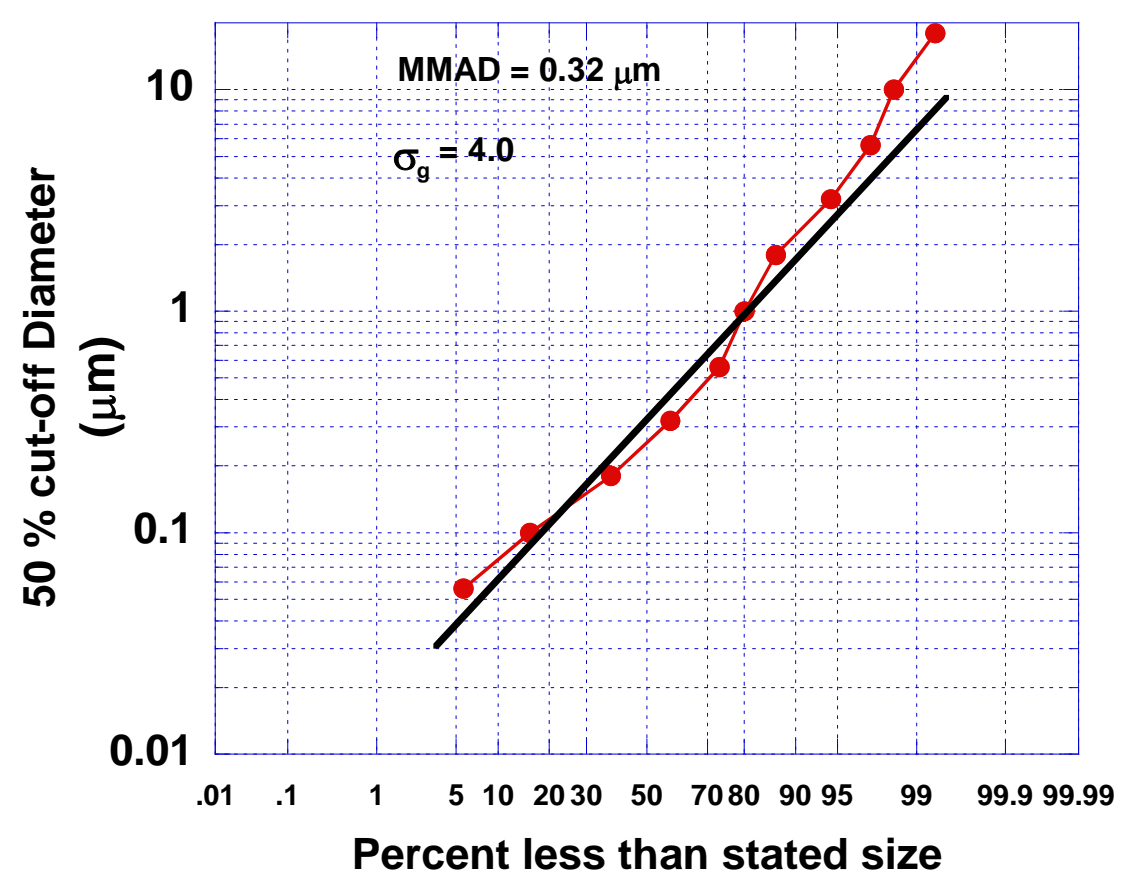

Figure 137. Cascade impactor results for flaming chair scenario SDC02

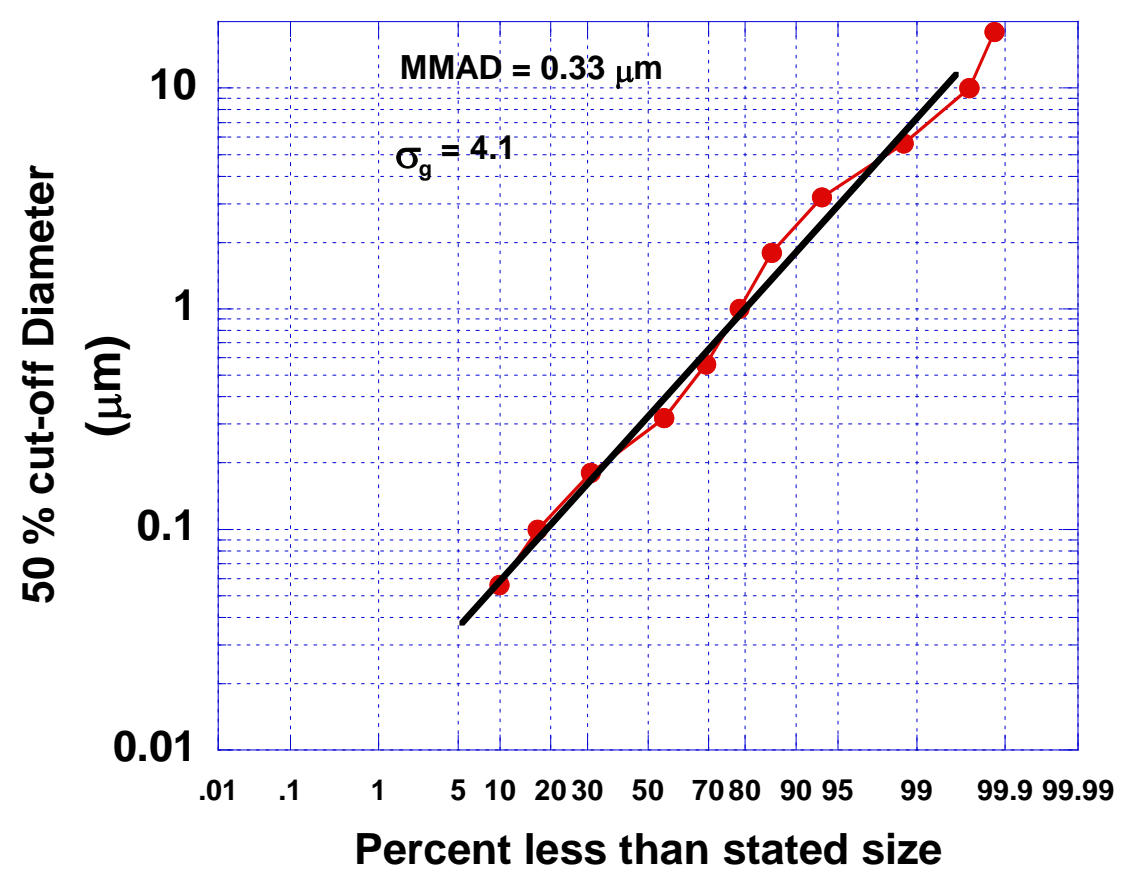

Figure 138. Cascade impactor results for flaming chair scenario SDC10 

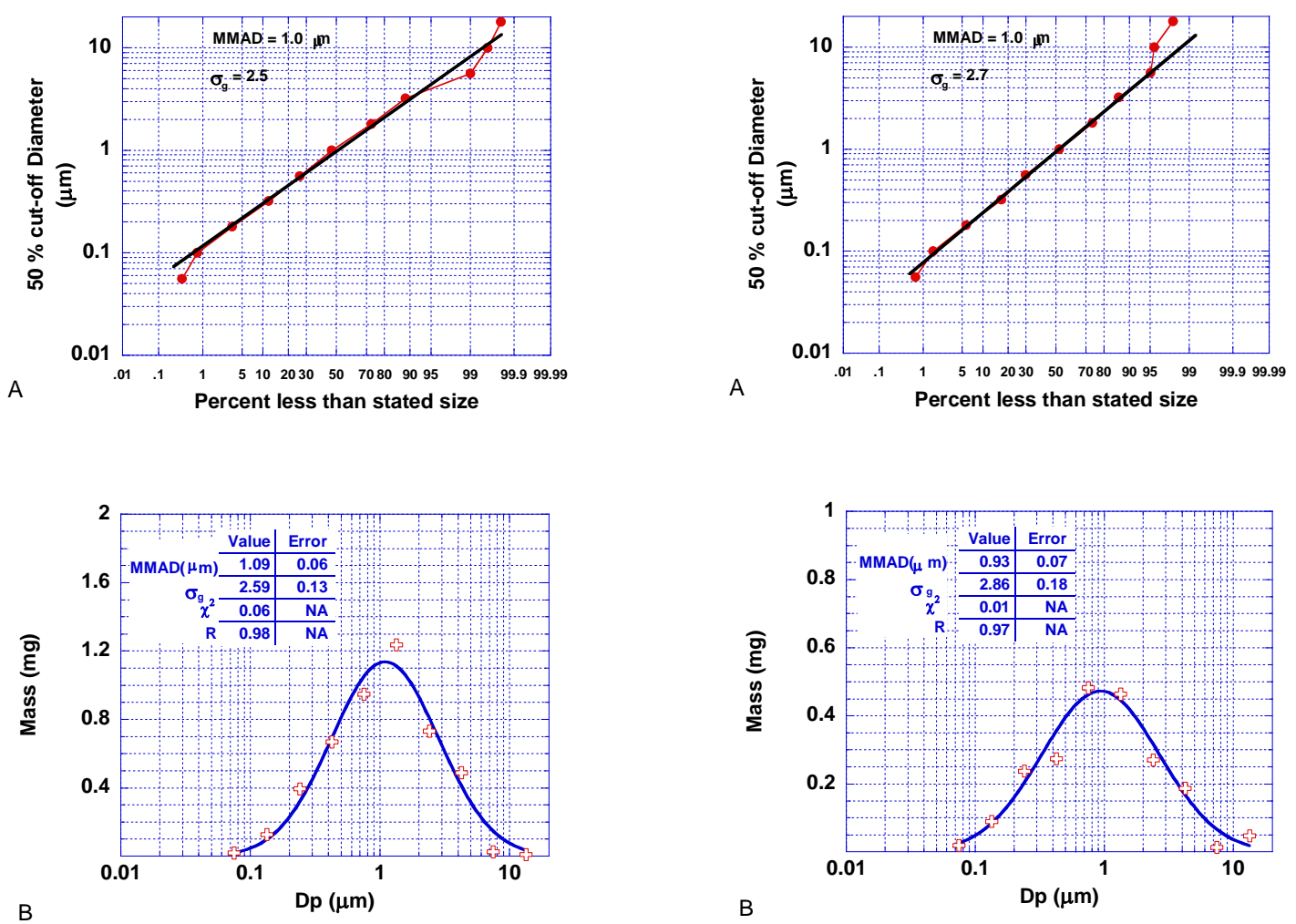

Test SDC12

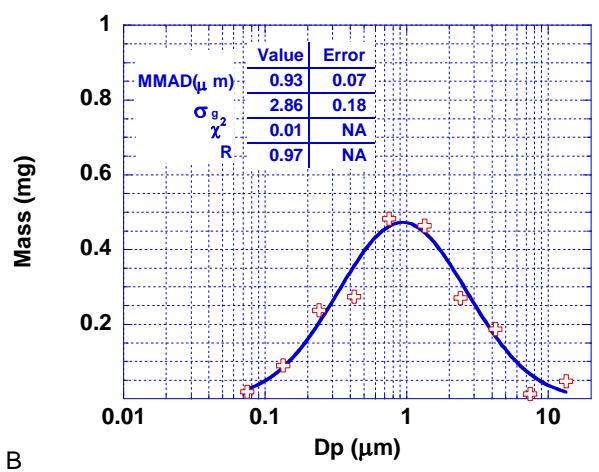

Test SDC13

Figure 139. Cascade impactor results for cooking oil fire scenarios SDC12 and SDC13 

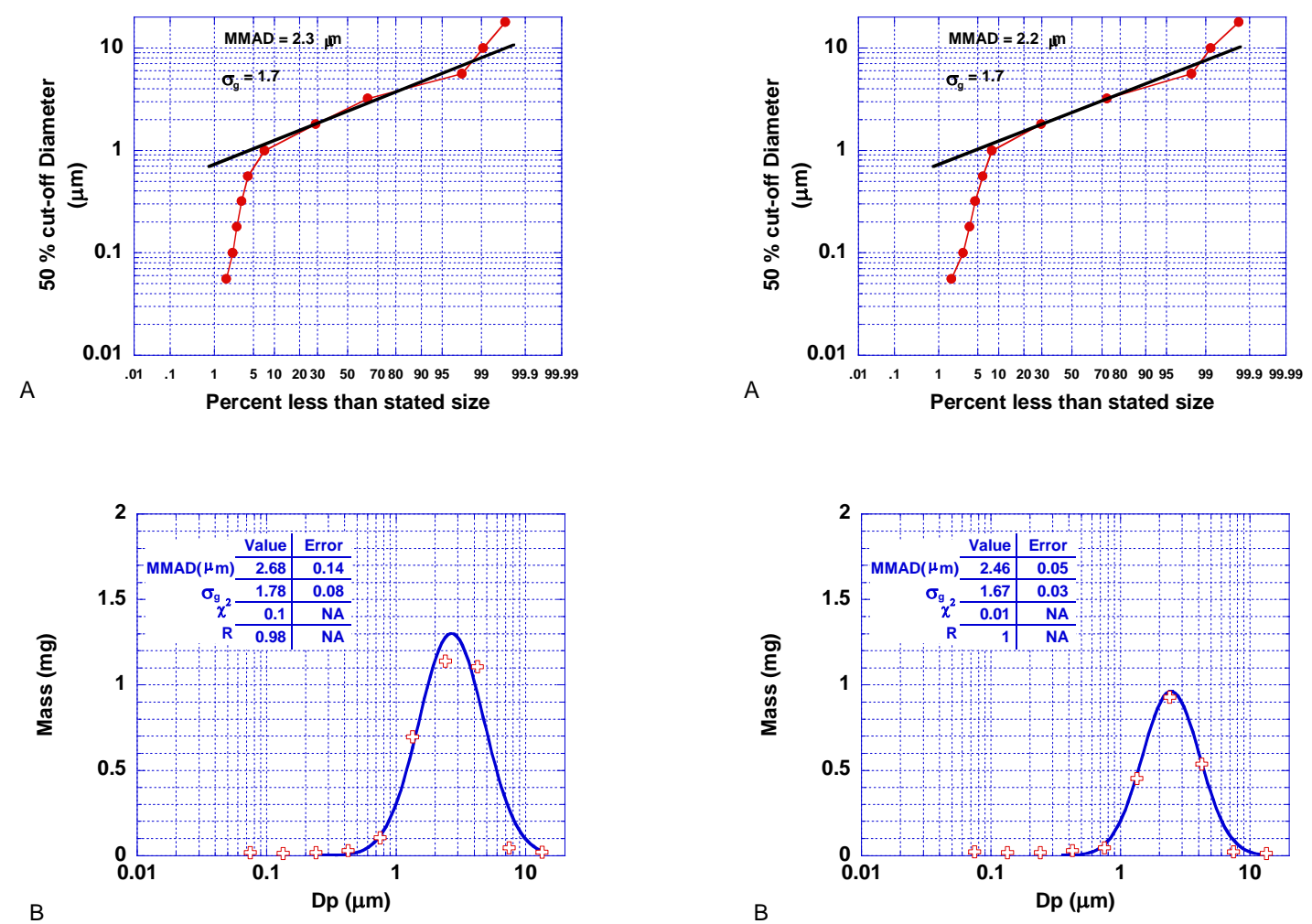

Test SDC23

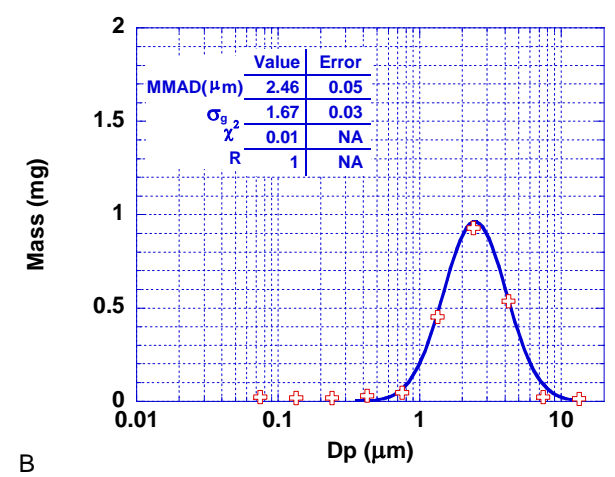

B

Test SDC27

Figure 140. Cascade impactor results for smoldering chair fire scenarios SDC23 and SDC27 
Table 18 presents the results for the smoldering chair scenario. Estimates were made for three tests during the smoldering period. For a fixed location, the observed trend was that both the count mean diameter (CMD) and diameter of average mass (Dam) increased as time increased. The exception to that trend was the estimate at $3000 \mathrm{~s}$ for SDC01, were the smoldering rate had leveled off for a long period. The diameter of average mass estimates from SDC01 were inconsistent with the count mean diameters. The most likely cause was a sampling bias where the room mass concentration was underestimated. Recall that during the manufactured home tests, the aerosol passed through the ionization chamber first, then it flowed to the TEOM. It was likely that a significant fraction of large particles were lost inside the ionization chamber and did not add to the mass concentration. The MOUDI size distribution measurement in the remote bedroom during SDC23 compares favorably with the den mean sizes estimated at $3000 \mathrm{~s}$.

Table 18. Estimated particle size from smoldering chair scenario

\begin{tabular}{|l|l|l|l|l|l|l|}
\hline Test & $\begin{array}{l}\text { Time } \\
(\mathrm{s})\end{array}$ & $\begin{array}{l}\text { Number } \\
\text { Conc. } \\
\left(\mathrm{cm}^{-3}\right)\end{array}$ & $\begin{array}{l}\text { "Y" } \\
\text { Ion } \\
\text { Chamber }\end{array}$ & $\begin{array}{l}\text { Mass } \\
\text { Conc. } \\
\left(\mathrm{mg} / \mathrm{m}^{3}\right)\end{array}$ & $\begin{array}{l}\text { CMD, Uc } \\
(\mu \mathrm{m})\end{array}$ & $\begin{array}{l}\text { Dam,Uc } \\
(\mu \mathrm{m})\end{array}$ \\
\hline SDC01 & 3000 & 21900 & 0.072 & - & $1.0,0.2$ & - \\
\hline & 6400 & $8.02 \times 10^{5}$ & 1.55 & 5.35 & $0.64,0.13$ & $\theta .23$ \\
\hline & 7000 & $5.21 \times 10^{5}$ & 1.55 & 10.5 & $0.93,0.19$ & $\theta .34$ \\
\hline SDC23 (den) & 3000 & 35600 & 0.096 & 85.3 & $0.82,0.16$ & $1.7,0.2$ \\
\hline (bedroom) & 3000 & 64600 & .017 & - & $0.08,0.02$ & - \\
\hline & 3520 & 81300 & 0.032 & - & $0.12,0.02$ & - \\
\hline SDC24 (den) & 850 & $1.83 \mathrm{e}^{6}$ & .658 & 9.46 & $0.11,0.02$ & $0.21,0.02$ \\
\hline & 1110 & 99000 & 0.913 & 44.7 & $0.22,0.04$ & $0.41,0.04$ \\
\hline & 1400 & 25600 & 1.27 & 123.5 & $0.39,0.08$ & $0.62,0.06$ \\
\hline
\end{tabular}

Table 19 presents the results for a flaming chair scenario and a smoldering mattress fire after it transitioned to flaming. The count mean diameter was computed using a chamber constant for flaming soot of $0.025 \mathrm{~cm}^{2}$ [262]. Again, the mass concentrations for the manufactured home test (SDC02) were suspected to be low. Table 20 presents the results for the cooking oil scenario. After an initial increase in particle size, the count mean diameter remained relatively constant prior to ignition of the oil.

The concentration and size distribution measurements provide a more fundamental characterization of the smoke levels compared to the light extinction measurements. The data gathered here provide detailed information that may be used to help interpret the smoke alarm responses observed in these tests, and to specify the smoke production rate and size characteristics for modeling exercises. 
Table 19. Estimated particle size from flaming sources

\begin{tabular}{|l|l|l|l|l|l|l|}
\hline Test & $\begin{array}{l}\text { Time } \\
(\mathrm{s})\end{array}$ & $\begin{array}{l}\text { Number } \\
\text { Conc. } \\
\left(\mathrm{cm}^{-3}\right)\end{array}$ & $\begin{array}{l}\text { "Y" } \\
\text { Ion } \\
\text { Chamber }\end{array}$ & $\begin{array}{l}\text { Mass } \\
\text { Conc. } \\
\left(\mathrm{mg} / \mathrm{m}^{3}\right)\end{array}$ & $\begin{array}{l}\text { CMD, Uc } \\
(\mu \mathrm{m})\end{array}$ & $\begin{array}{l}\text { Dam,Uc } \\
(\mu \mathrm{m})\end{array}$ \\
\hline SDC02 & 275 & $3.98 \times 10^{6}$ & 3.18 & 68.1 & $0.32,0.06$ & $\theta .26$ \\
\hline & 360 & $1.13 \times 10^{6}$ & 2.77 & 53.5 & $0.98,0.19$ & $\theta .3$ \\
\hline $\begin{array}{l}\text { SDC06 } \\
\text { (flaming) }\end{array}$ & 6150 & $6.51 \times 10^{6}$ & 2.88 & - & $0.18,0.04$ & - \\
\hline
\end{tabular}

Table 20. Estimated particle size from cooking oil fire scenario

\begin{tabular}{|l|l|l|l|l|l|l|}
\hline Test & $\begin{array}{l}\text { Time } \\
(\mathrm{s})\end{array}$ & $\begin{array}{l}\text { Number } \\
\text { Conc. } \\
\left(\mathrm{cm}^{-3}\right)\end{array}$ & $\begin{array}{l}\text { "Y" } \\
\text { Ion } \\
\text { Chamber }\end{array}$ & $\begin{array}{l}\text { Mass } \\
\text { Conc. } \\
\left(\mathrm{mg} / \mathrm{m}^{3}\right)\end{array}$ & $\begin{array}{l}\text { CMD, Uc } \\
(\mu \mathrm{m})\end{array}$ & $\begin{array}{l}\text { Dam,Uc } \\
(\mu \mathrm{m})\end{array}$ \\
\hline SDC12 & 1325 & $1.80 \times 10^{6}$ & 1.25 & - & $0.21,0.04$ & - \\
\hline & 1550 & $7.62 \times 10^{6}$ & 4.44 & - & $0.18,0.04$ & - \\
\hline SDC13 & 850 & $6.16 \times 10^{5}$ & 0.23 & - & $0.11,0.02$ & - \\
\hline & 1250 & $1.26 \times 10^{6}$ & 1.01 & - & $0.24,0.05$ & - \\
\hline & 1550 & $7.03 \times 10^{6}$ & 5.51 & - & $0.24,0.05$ & - \\
\hline
\end{tabular}

\subsection{Measurement Uncertainty}

Replicate tests were performed for the manufactured home tests to assess the uncertainty in alarm times and time to untenable conditions based on measurements of temperature, smoke obscuration, and toxic gas concentrations. Table 21 shows standard uncertainties expressed as a relative error (in this case calculated as the ratio of the standard uncertainty for replicate tests and the average value for the replicate tests). 
Table 21. Variability for tenability time and alarm time during tests of residential smoke alarms (expressed as the ratio of standard uncertainty for replicate tests and the average value for these tests)

\begin{tabular}{|l|c|c|c|c|c|}
\cline { 2 - 6 } \multicolumn{1}{c|}{} & \multirow{2}{*}{$\begin{array}{c}\text { Tenability } \\
\text { Time } \\
(\%)\end{array}$} & $\begin{array}{c}\text { Ionization } \\
\text { Alarm (\%) }\end{array}$ & $\begin{array}{c}\text { Photoelectric } \\
\text { Alarm (\%) }\end{array}$ & $\begin{array}{c}\text { Aspirated } \\
\text { Alarm (\%) }\end{array}$ & $\begin{array}{c}\text { CO Alarm } \\
(\%)\end{array}$ \\
\cline { 2 - 6 } & 10 & 24 & 28 & 32 & 21 \\
\hline Flaming & 32 & 30 & 51 & 39 & 19 \\
\hline Smoldering & 6 & 64 & 7 & 34 & 6 \\
\hline Cooking Oil & & & & & 4 \\
\hline
\end{tabular}




\section{Residential Smoke Alarm Nuisance Source Testing}

Smoke alarms are susceptible to alarming when exposed to non-fire aerosols. In residential settings, this typically involves cooking activities or transient, high humidity conditions (i.e., "shower steam"). The Smoke Detector Operability Survey: Report on Finding [6] conducted by the U.S. Consumer Products Safety Commission reported that about one half of the 1012 respondents indicated they experienced nuisance alarms, with $80 \%$ of those attributed to cooking activities, and an additional $6 \%$ citing steam from bathrooms. Dust, and tobacco smoke are also mentioned sources. The Survey also reported that of the alarms with missing or disconnected batteries, or disconnected $\mathrm{AC}$ power, more than one third of respondents indicated that power was removed due to nuisance alarms. The Smoke Detector Operability Survey: Engineering Laboratory Analysis found that there was a correlation between the occurrence of nuisance alarms and the sensitivity of the alarm, location of the alarm relative to the nuisance source, and the condition (cleanliness) of the alarm [34]. A field study of nuisance alarms in the Native American community reported that of 80 households with one or more ionization alarms 63 reported nuisance alarms [3]. The activities associated with these alarms and the percentage of detectors implicated in said nuisance alarms were: cooking (77\%), steam from bathroom (18 $\%$ ), cigarettes (6\%), and fireplace/wood stove (4\%), other, unknown and chirping (low battery alert) (12\%). The types of cooking activities identified with nuisance alarms included: frying, baking, boiling, and toaster/toaster oven. This study concluded that the type of sensor technology, and the location of an alarm play an important role in the frequency of nuisance alarms in residential settings.

The benefits of an alarm that is relatively insensitive to common nuisance sources while maintaining a high sensitivity to a wide range of fire scenarios are self-evident. In the last decade, a significant research effort has been expended to achieve more robust fire detection with a focus on new sensors (including gas sensing), signal processing techniques, and advanced algorithm development [35], [36], [37], [38]. The practical implementation of any new technologies on a broad basis depends on the participation of several key entities including: research organizations, standards bodies, testing organizations, and the commercial sector. The demonstration of improved fire detection, both enhanced sensitivity to fire and insensitivity to nuisance sources, through detector performance testing is a necessary objective. While standard fire tests are designed to quantify a given alarm's ability to detect fire conditions (e.g., UL 268 [39], and EN-54 [40] fire sensitivity tests), there are no consensus standards to test its ability to reject nuisance alarms. 
The objective of this research task was to develop a basis for standard residential nuisance source testing. The approach taken is to define a set of nuisance scenarios, replicate the events that cause nuisance alarms, and quantify the important variables that cause nuisance alarms. Translating the results to a set of nuisance source conditions reproducible in a suitable test-bed (i.e., a test room or the fire emulator/detector evaluator) would allow for more comprehensive detector performance testing. Preliminary tests were performed in the FE/DE with this in mind. Programming realistic and reproducible fire and nuisance conditions in the FE/DE is an ongoing research project at NIST.

\subsection{Nuisance Scenario Tests}

Nuisance scenario tests were performed in the manufactured home used for other tests in this study. The selections were based on what are commonly thought to be causes of residential nuisance alarms, and scenarios were designed to mimic normal activities (i.e. no intentional food burning, with the exception of toasted bread). No consideration was given to the probability of occurrence for any given scenario; the objective was to gather data on a number of scenarios. The bulk of the scenarios were related to cooking activities including: frying, deep-frying, baking, broiling, boiling, and toasting. In addition, cigarette smoking and candle burning were included. Neither a "shower steam" scenario, nor dust exposure test were performed in the manufactured home. Both have been investigated in the FE/DE in prior studies. Three smoldering fire scenarios (smoldering polyurethane foam, beech wood blocks and cotton wick) were examined for comparative purposes.

A schematic of the manufactured home is shown in figure 141. The dark shaded areas were closed off. During most tests, all external doors and windows were closed. Most scenarios were repeated with and without a floor fan blowing air from the master bedroom into the kitchen/living room area.

\subsection{Instrumentation}

Aerosol concentrations, temperature, humidity, flow velocity and analog output from photoelectric, ionization and carbon monoxide sensors were gathered. Most tests were videotaped. Figure 141 shows the approximate ceiling location of all the measurement positions. Details of the measurement are given below. Note, carbon monoxide data were not presented in the graphs, but are included in the data files, which in addition include all analog sensor outputs. The complete data set is available in electronic form [41]. 


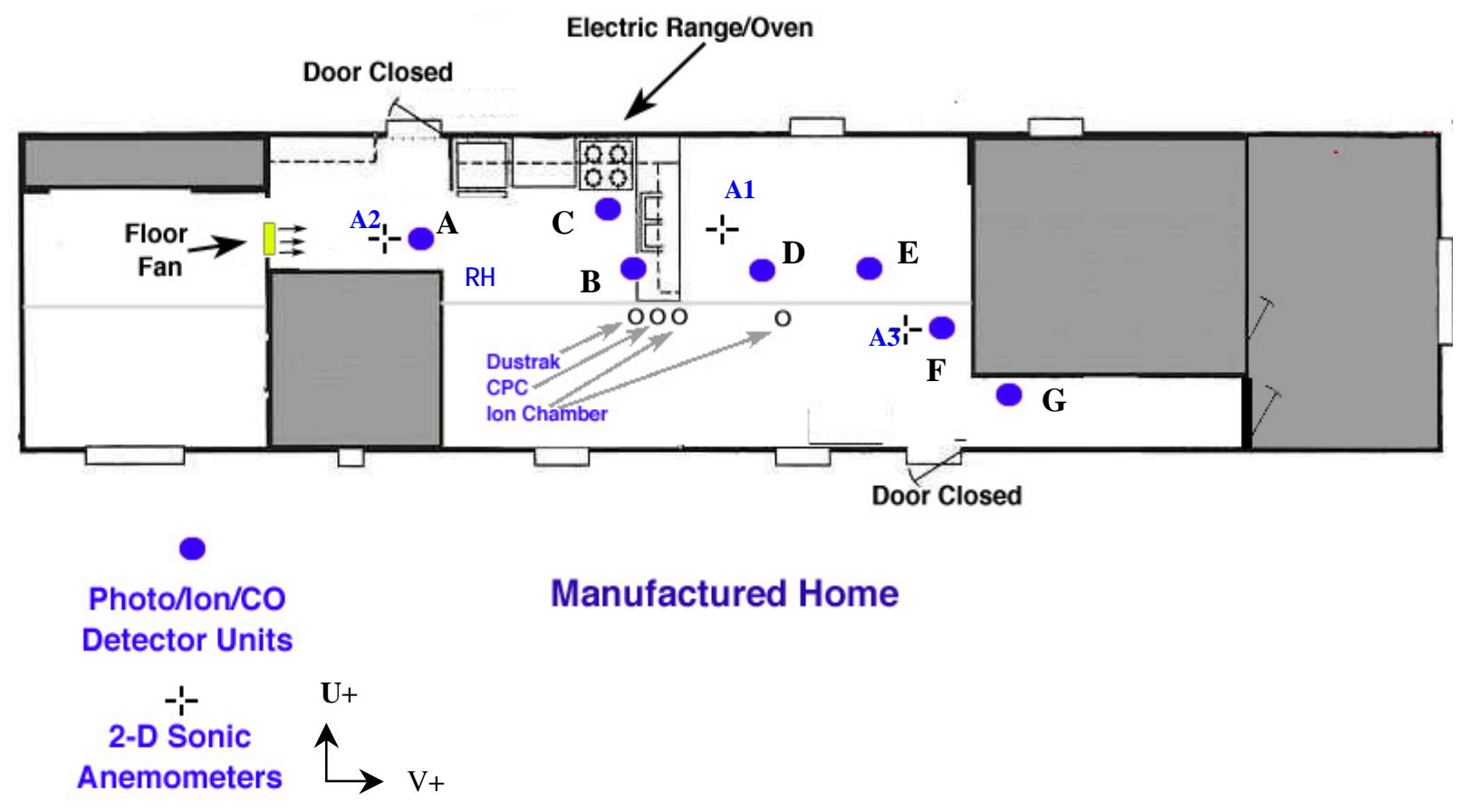

Figure 141. Schematic of the manufactured home for nuisance testing

\subsubsection{Aerosol Instruments}

Two portable aerosol instruments were used to gather aerosol number and mass concentrations during the tests. Number concentration was recorded with a TSI model 3007 portable condensation particle counter (CPC). This instrument is capable of counting particles greater than $10 \mathrm{~nm}$ in diameter up to concentrations of $5 \times 10^{5}$ particles $/ \mathrm{cm}^{3}$ with an uncertainty of $10 \%$ of the reading. The upper concentration range of the instrument is insufficient for many fire and nuisance conditions so the air sample was diluted with a fixed amount of clean air prior to entering the CPC. The air sample was drawn through a $1.5 \mathrm{~m}$ long, $6 \mathrm{~mm}$ ID PVC tube (Tygon brand, which is recognized to have low electrostatic particle loss characteristics.) to a laminar flow element that was attached to one branch of a "Y" tube fitting. The other branch of the fitting was attached to a HEPA filter, while the main branch was attached to the inlet of the CPC. The CPC internal pump draws air from the sampling tube and clean (particle-free) air through the filter into the unit. With this arrangement, approximately a 20 to 1 dilution of the air sample is achieved. The dilution ratio for each test was obtained by computing the ratio of undiluted to diluted background room aerosol. The uncertainty in the dilution ratio is estimated to be $2 \%$. A TSI model 8520 "Dustrak" portable aerosol mass monitor was used to gather the aerosol mass concentration. This device is a light scattering photometer that analyzes the light scattered at an angle of $90^{\circ}$ from particles flowing through the device. Its default calibration is set to the respirable fraction of standard ISO 12103-1 A1 test dust. It has a range from 
$0.001 \mathrm{mg} / \mathrm{m}^{3}$ to $150 \mathrm{mg} / \mathrm{m}^{3}$. The effective particle size measurement range is $0.1 \mu \mathrm{m}$ up to 10 $\mu \mathrm{m}$. The device can be calibrated for any aerosol with scattering properties different from the test dust provided the true mass concentration is determined. Here, the default calibration was used, so any given mass concentration measurement reported is relative and related measure to an equivalent mass of test dust. Since the device is not calibrated to each of the aerosols produced in the nuisance tests, the uncertainty in the measurement in not determined. However, the results are proportional to the mass concentration and correspond directly to the scattering signal strength of photoelectric detectors with an equivalent amount of aerosol in its sensing region. Both the CPC and Dustrak log data to internal memory along with a time stamp every second. Data were downloaded after each test.

Two flow-through ionization chamber devices were constructed from commercially available ionization

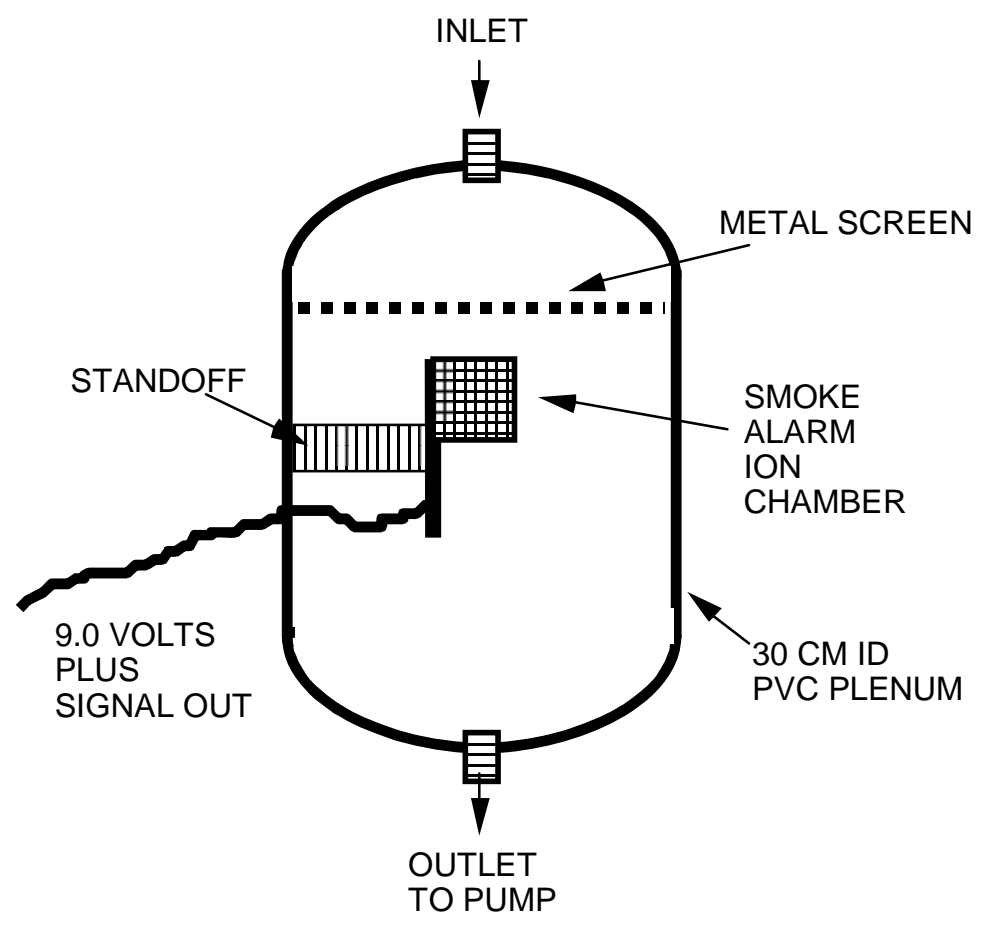

Figure 142. Ion chamber schematic alarms. Figure 142 is a schematic diagram of this device. To make the modification, the horn from the alarm circuit board was removed, and the voltage from the IC circuit pin that follows the chamber current was monitored. $17 \mathrm{~cm}^{3} / \mathrm{s}$ of room air was drawn through each device and each circuit was powered by $9.00 \mathrm{~V}$ from a regulated power supply. These devices function effectively as measuring ionization chambers (MIC), and their voltage output can be correlated to a MIC chamber current. Figure 143 shows the voltage output from the ion chamber devices and the MIC current for cotton smolder smoke generated in the FE/DE. The ion chamber device was located outside the FE/DE test section and the air sample was drawn into the devices by $1.5 \mathrm{~m}$ long, $10 \mathrm{~mm}$ ID copper tubing. The ion chamber voltage

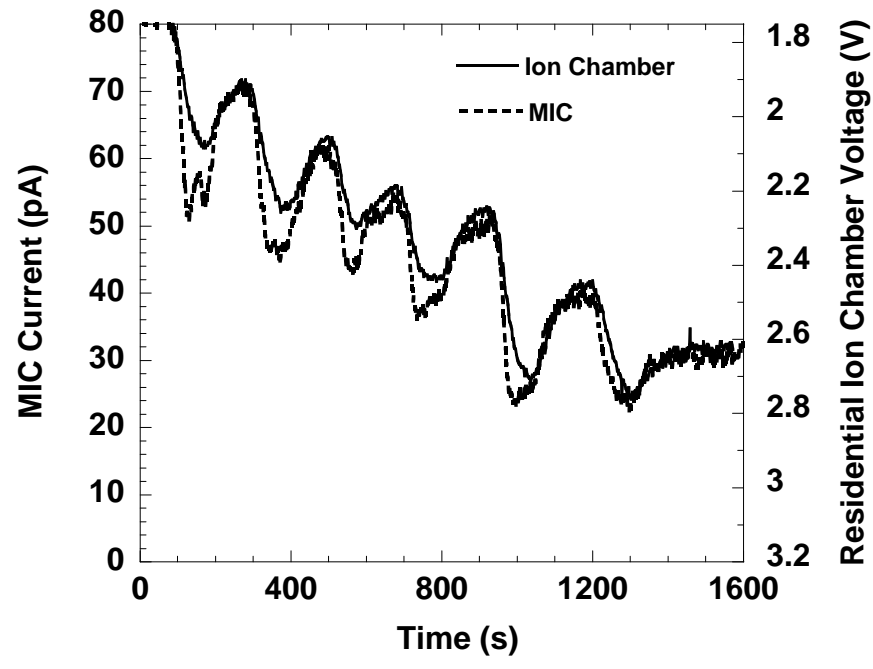

Figure 143. Response of the ion chamber and MIC to cotton smolder smoke 
lags the MIC current due to the time difference between the aerosol transport through the tubing and chamber filling, but the steady voltage value is proportional to the MIC current. The reported ion chamber values in the nuisance tests below differ by a constant value due to differing amplifier gains.

In the nuisance tests, the copper tubes used to transport the air sample to the devices were $2.5 \mathrm{~m}$ long. Chamber \#1 was located in the living room area, while chamber \#2 was located in the kitchen area next to the other aerosol sampling points (figure 140).

\subsubsection{Temperature and Humidity}

A type-K $0.5 \mathrm{~mm}$ stainless steel sheathed thermocouple monitored the ceiling air temperature. It was placed at the aerosol sampling location, $3 \mathrm{~cm}$ below the ceiling.

Figure 144 is a picture showing the thermocouple placement along with the CPC, Dustrak, and ion chamber 2 sampling lines.

An Oakton 35612 series thermohygrometer probe was used to monitor the relative humidity throughout the experiments. The probe was positioned $2.0 \mathrm{~m}$ off the floor at the position indicated by " $\mathrm{RH}$ " in figure 141. The stated measurement uncertainty for this device is $2.5 \%$ of the reading.

\subsubsection{Flow Velocity}

Three CATI sonic anemometers (Applied

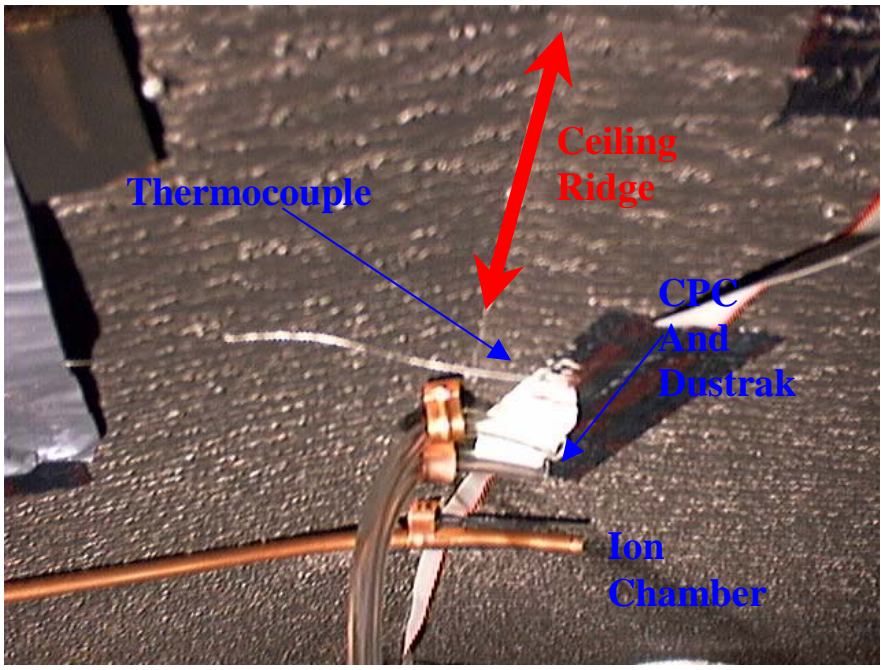

Figure 144. Thermocouple and sampling line placement

Technologies Inc.) were used to record the two-component air velocities perpendicular to the sloped ceiling at a depth of $5 \mathrm{~cm}$. Their locations are shown in figure 141 and identified as anemometer A1, A2, and A3. The component velocity orientation is also shown.

These devices use piezoelectric crystals to form ultrasonic transducers that can send and receive ultrasonic pulses. The forward and backward travel time of these pulses are used to compute the component velocity between two opposing transducers. The anemometers record the mean velocity over a $15 \mathrm{~cm}$ sonic path length (which equals the distance separating opposing transducers) at a frequency of up to $10 \mathrm{~Hz}$. The measurement resolution is $1 \mathrm{~cm} / \mathrm{s}$ with a stated uncertainty of $1 \mathrm{~cm} / \mathrm{s}$. 


\subsubsection{Analog Output Photoelectric, Ionization and Sensors}

Seven commercially available dual photo/ion smoke alarms were modified at NIST to provide continuous analog output (figure 145). The modifications made to the alarms included removing the horn, modifying the circuit board to suppress the alarm mode, and speeding up the IR light source to a flash rate of approximately every $2 \mathrm{~s}$. The photodetector was disconnected from the circuit board and monitored by a peak detection circuit. The photodetector signal was amplified with an op-amp circuit then sent over a multi-conductor ribbon cable to a single board computer (SBC) dedicated to each modified alarm. The signal was amplified by an instrument amplifier then, sent to the peak detector. The peak detector was reset every $2 \mathrm{~s}$ by a $1 \mathrm{~ms}$ digital signal pulse to the gate of a MOSFET attached across the peak circuit's holding capacitor. The voltage stored by the peak detector was sampled by the analog input circuit of the SBC every $2 \mathrm{~s}$, just prior to the reset. The SBC processed this data with a 3point median filter to suppress spurious signals. The voltage follower IC pin output that follows the chamber current was sampled to obtain the ionization signal. An electro-chemical CO cell removed from a commercially available $\mathrm{CO}$ alarm was fitted in the dual smoke alarm housing at the horn's previous location. The voltage drop across a resistor placed across the cells terminals was sampled. Both the Ion and $\mathrm{CO}$ voltages were sent to instrument amplifiers prior to being sampled by the analog input of the SBC. A $10 \mathrm{k} \Omega$ thermistor was located in the dual smoke alarm just sticking out of the detector housing at the LED light pipe hole.

The SBC logged the photo, ion, and CO sensor voltages every two seconds to non-volatile flash ram. The data were downloaded after each test. The voltages were expressed as 8 bit characters to minimize data storage. Each detector was calibrated in the FE/DE with the cotton wick smoke, and the sensor values are presented in engineering units of extinction $\left(\mathrm{m}^{-1}\right)$ and mole fraction of $\mathrm{CO}$. The locations of the sensor packages are indicated on figure 141 and represented in the results by the letters A - G. 
On several occasions, the SBC data were monitored via a data stream sent over a serial port to a laptop. This was observed to corrupt some of the sensor data saved to the flash ram, consequently, some data columns were left blank in the test data files. One photo sensor monitoring circuit did not function properly and was omitted from the test data files (Unit E).

\subsection{Results}

Selected results for each of the tests performed are shown in the figures below. Data from all of the tests are available [41]. The time to reach photoelectric and ionization alarm points was determined from ion and photoelectric sensor calibration test data, and estimated alarm sensitivities appropriate for the FE/DE cotton wick smoke. Estimated high, medium and low sensitivities for both photoelectric and ionization alarms are given in Table 22. These values cover the range expected for residential smoke alarms for each sensor type.

Table 22. Alarm sensitivity for photoelectric and ionization sensors

\begin{tabular}{|l|l|l|l|}
\hline Sensor & $\begin{array}{l}\text { High sensitivity } \\
\text { Extinction } \mathrm{m}^{-1}, \\
(\% / \mathrm{ft} \text { Obsc. })\end{array}$ & $\begin{array}{l}\text { Medium sensitivity } \\
\text { Extinction } \mathrm{m}^{-1}, \\
(\% / \mathrm{ft} \mathrm{Obsc.})\end{array}$ & $\begin{array}{l}\text { Low sensitivity } \\
\text { Extinction } \mathrm{m}^{-1}, \\
(\% / \mathrm{ft} \mathrm{Obsc.})\end{array}$ \\
\hline Photoelectric & $0.05,1.5$ & $0.083,2.5$ & $0.117,3.5$ \\
\hline Ionization & $0.016,0.5$ & $0.033,1.0$ & $0.050,1.5$ \\
\hline
\end{tabular}

\subsubsection{Toasting Scenarios}

Either two slices of white bread or frozen bagel halves were placed in a two-slice toaster, located on the counter to the left of the range. The toaster's automatic pop-up mechanism was disabled by holding the lever in the on position with a wrap of wire attached to the bottom of the toaster. Power was applied by plugging in an extension cord attached to the toaster. The cord extended out of the manufactured home through an access hole. At time $=0$ the power was applied to the toaster. The toaster was left on for up to $300 \mathrm{~s}$. This was sufficient to char ("burn") toast but not the frozen bagels. No flames were produced in these tests. This scenario is unique compared to all of the following nuisance scenarios in that it transitions from nuisance to an impending hazard as the bread chars since it could produce flames.

Three tests (two with bread, one with a frozen bagel) were conducted with no forced room airflow, while an additional three tests were conducted with the floor fan operating. Similar background conditions, (i.e., fan or no fan) ostensibly produced the same flow conditions at the 
measurement locations. In the repeated toasting bread test with no forced flow, the remote bedroom window was left open, which did affect the airflow slightly. Figure 146 shows the velocity components and speed. The reported speed is a time-smoothed representation computed from the component velocities. With no forced flow, background flow velocities were initially between $0 \mathrm{~m} / \mathrm{s}$ and $0.1 \mathrm{~m} / \mathrm{s}$. After the toaster is turned on, the plume from hot gases is evident with computed speeds up to $0.2 \mathrm{~m} / \mathrm{s}$. When the toaster power was removed, the flow at location A1 returned to its low background value; the return to background values was less pronounced at locations A2 and A3. With the floor fan turned on, speeds between $0.15 \mathrm{~m} / \mathrm{s}$ and $0.6 \mathrm{~m} / \mathrm{s}$ were recorded. The effects of the toaster heat plume were not clearly evident in these velocity measurements.

The results for each test are shown in a series of four graphs for each case. Figure 147A shows the computed time to reach various alarm thresholds for toasted bread with no forced flow.

Every threshold for all photoelectric and Ion sensors was reached in this test. Note that at alarm location $E$ is displayed in gray since no photoelectric alarm times are indicated; there was no functioning photoelectric alarm at this position. This notation is used in the figures that follow to indicate when no alarms were placed at a given position. In all cases the ion sensors reached the alarm thresholds before the photoelectric sensors. Generally, the closer the sensor was to the source the sooner the alarm threshold was reached. Figure 147B shows the temperature and relative humidity. There was approximately a $4{ }^{\circ} \mathrm{C}$ rise in temperature and a $7 \%$ drop in relative humidity during the test. Figure $147 \mathrm{C}$ shows the mass concentration and the number concentration readings. The earlier increase in the number concentration and the later increase in the mass concentration correspond to the earlier ionization alarm times followed later by photoelectric alarm times. The Dustrak surpassed its maximum range of $300 \mathrm{mg} / \mathrm{m}^{3}$ from about $240 \mathrm{~s}$ to $300 \mathrm{~s}$. 

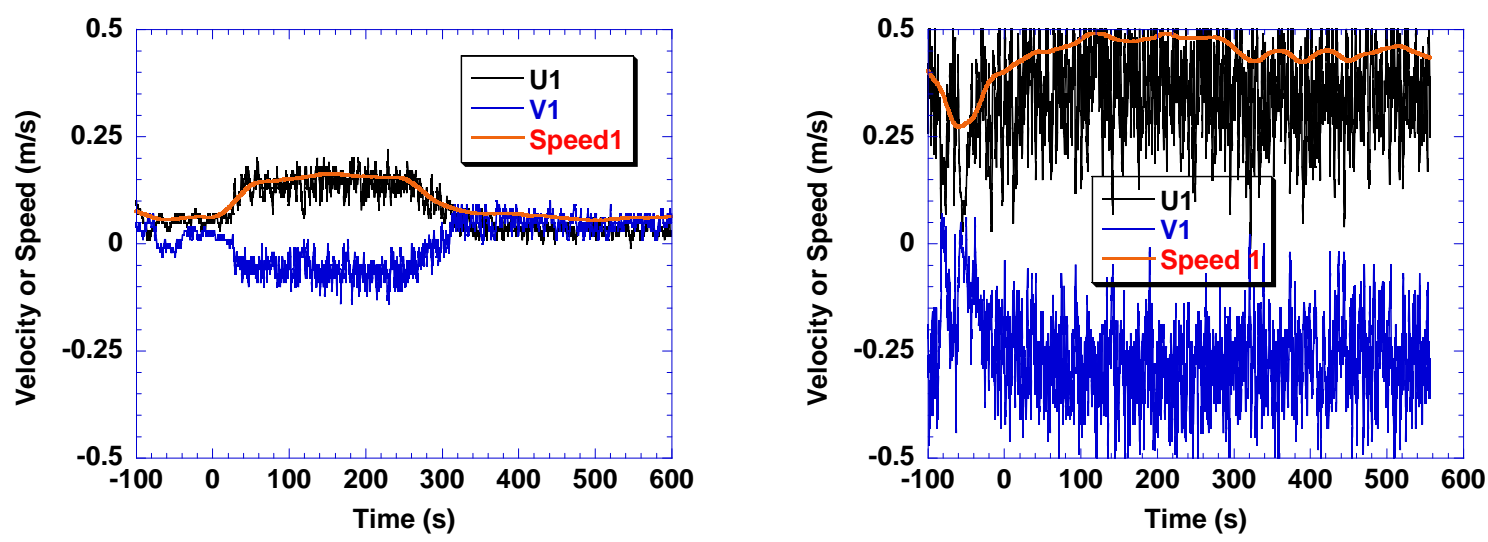

A (Bread, no forced airflow)

B (Bread, forced airflow)
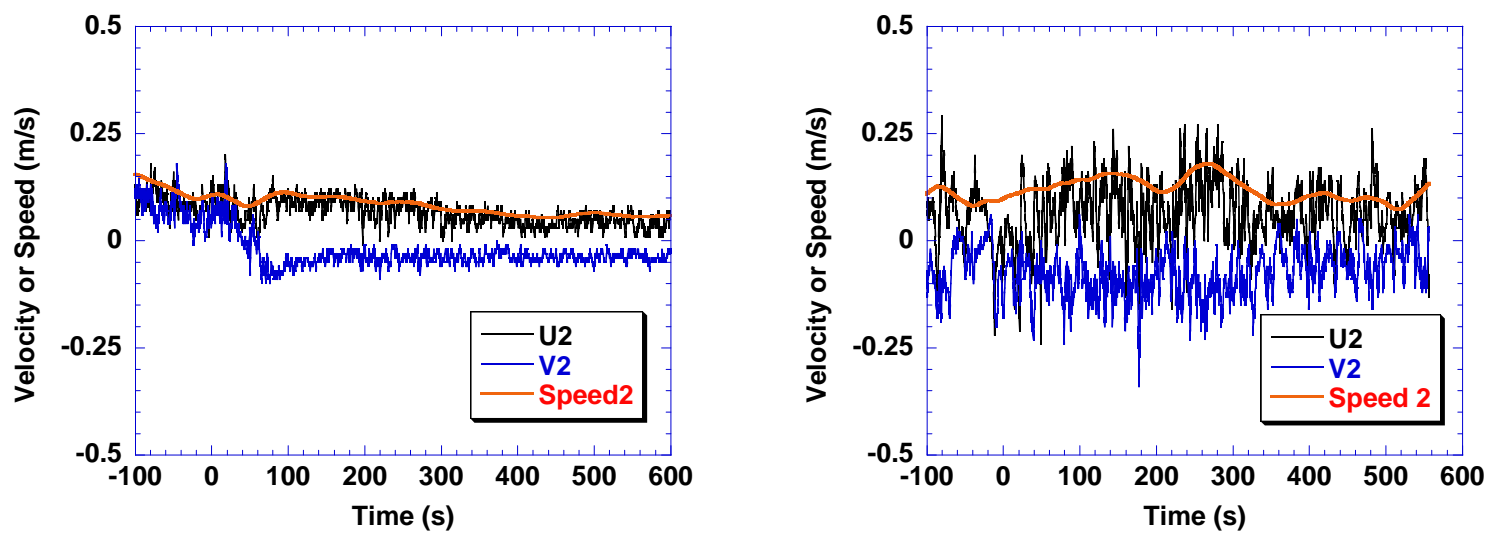

C (Bread, replicate, no forced airflow)

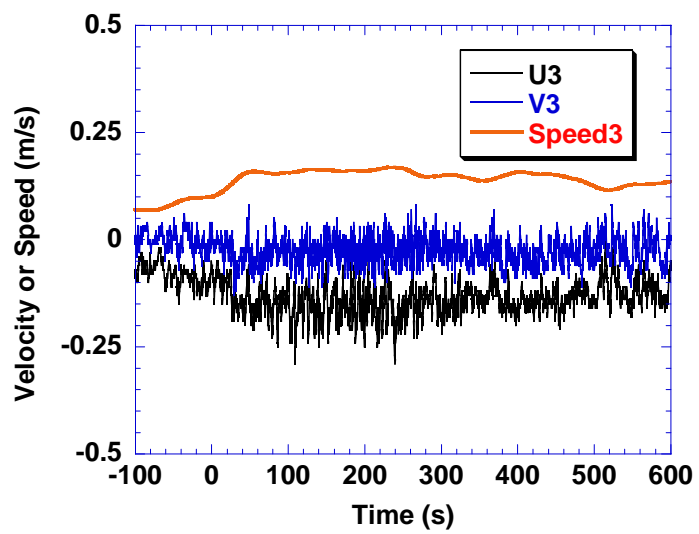

E (Bagel, no forced airflow)

D (Bread, replicate, forced airflow)

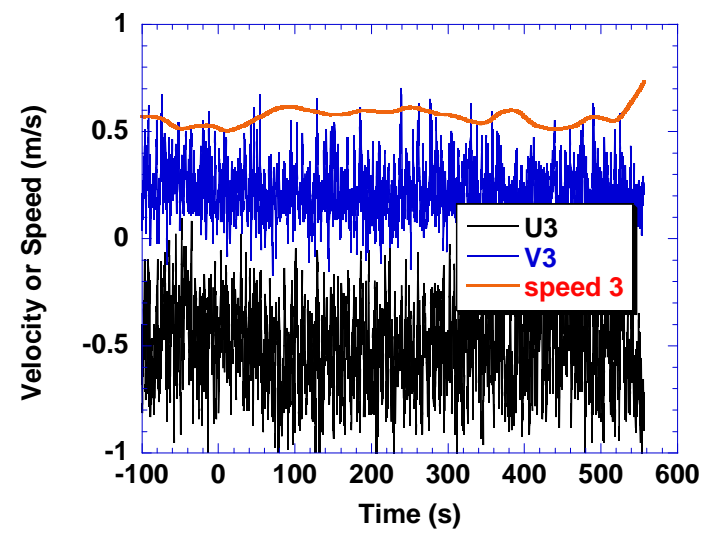

F (Bagel, forced airflow)

Figure 146. Velocity and speed components for toasting bread nuisance source with and without forced ventilation 

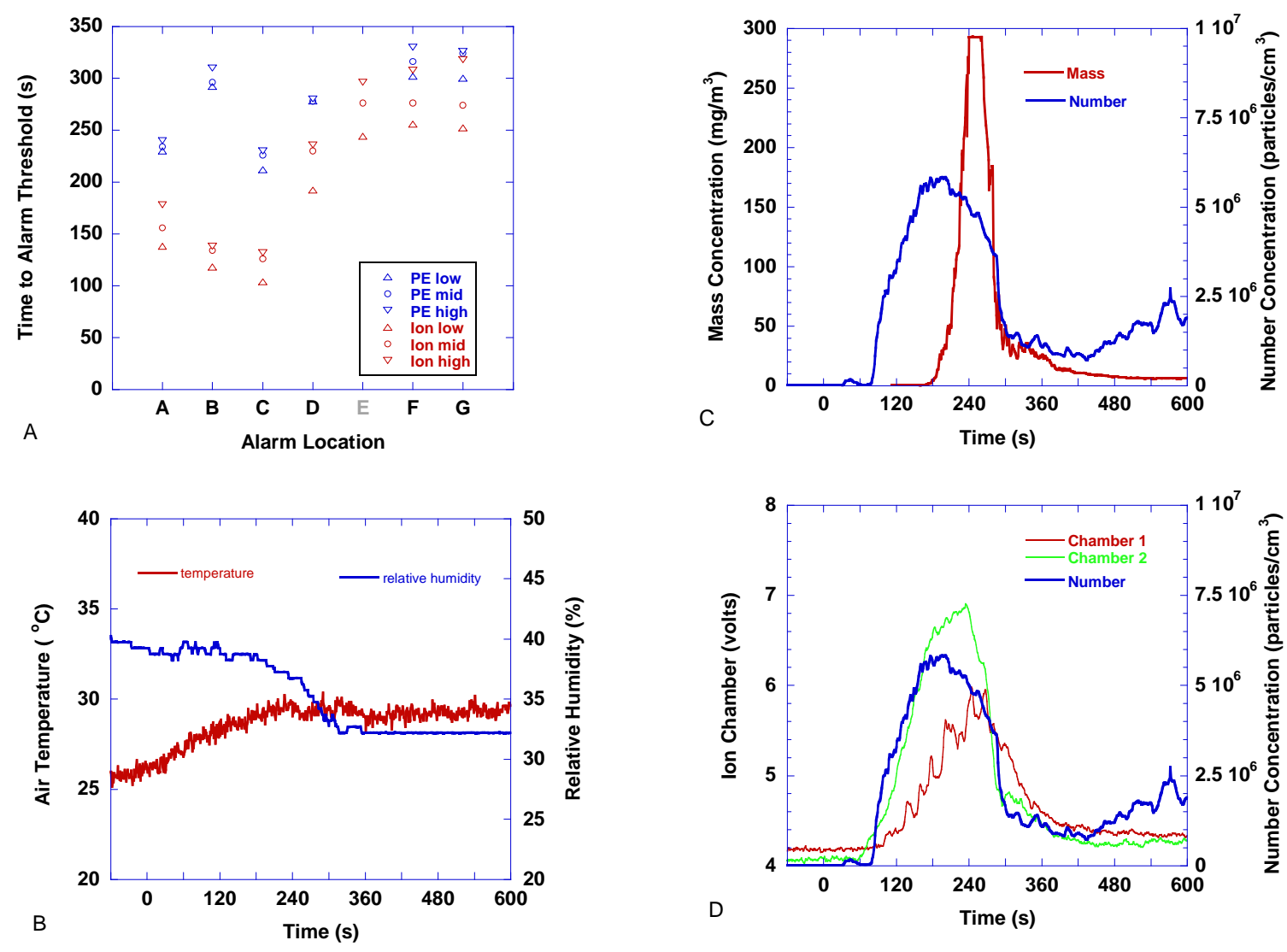

Figure 147. Toasting bread scenario - toaster on at $\mathrm{t}=0$, off at $240 \mathrm{~s}$. No forced flow

Figure 147D shows the number concentration and the voltage from the flow-through ion chambers. First, note that ion chamber 2 responds first and achieves a higher peak voltage due to the fact that it was located closer to the source. Second, note that the voltage signal from ion chamber 2 followed the number concentration curve which again is expected since they were at the same sampling location. The ion chamber voltage is related to the first moment of the particle size distribution, while the number concentration is essentially the zero ${ }^{\text {th }}$ moment of the size distribution. The ratio of these moments is equal to the count median diameter of the aerosol size distribution. Likewise, the ratio of the mass concentration ( $3^{\text {rd }}$ moment) to the number concentration is proportional to another particle diameter, the diameter of average mass. These characteristic particle sizes were computed from these moments for several smoke and nuisance source aerosols generated in the FE/DE [33]. These calculations were possible because the MIC was used to get the first moment since previous research had established its proportionality constant, and a direct mass measurement was taken to obtain the $3^{\text {rd }}$ moment. 
One can still observe trends in these particle size statistics by examining the number concentration, ion chamber output and the Dustrak mass concentration. In figure 147D, it is observed that the number concentration and the ion chamber begin to rise at about $60 \mathrm{~s}$ and the rate of rise for each is constant up to $150 \mathrm{~s}$. This implies that the count median diameter is constant while the number concentration increases. After $150 \mathrm{~s}$ the number concentration starts to level off then drop, while the ion chamber voltage continues to increase until it reaches a peak at $240 \mathrm{~s}$. This implies that the count median diameter starts to increase from $150 \mathrm{~s}$ to $240 \mathrm{~s}$. After $240 \mathrm{~s}$ the ion chamber voltage and the number concentration drop rapidly until reaching minimums at $420 \mathrm{~s}$. The ion chamber voltage remains relatively flat while the number concentration starts to rise suggesting a decrease in the count median diameter from that point on.

In figure $147 \mathrm{C}$, it is observed that the number concentration has reached its peak at $180 \mathrm{~s}$ before any significant amount of mass concentration was recorded. In fact, the mass concentration just begins to rise at $180 \mathrm{~s}$. The rapid increase in mass concentration along with the decreasing number concentration implies a significant increase in the diameter of average mass as the Dustrak reaches its peak. The steep drop in the mass concentration also implies a significant decrease in the diameter of average mass since the number concentration is decreasing at a much lower rate. At $420 \mathrm{~s}$ the mass concentration continued to fall while the number concentration started to rise implying continuing decrease in the diameter of average mass.

Figure 148 shows the results for a repeated test where the remote bedroom window was left open. The times to reach alarm thresholds are similar to the initial toasted bread test. Again, the trends were increasing ambient air temperature and decreasing relative humidity. The mass concentration, number concentration and ion chamber voltages show more fluctuation due to the open window disturbing the room flows, but they achieved maximum values similar to the previous test. Note that the number concentration started out higher than normal room air background $\left(1 \times 10^{6}\right.$ particles $\left./ \mathrm{cm}^{3}\right)$ because this test was run before the previous test aerosol concentration was cleared from the manufactured home. 

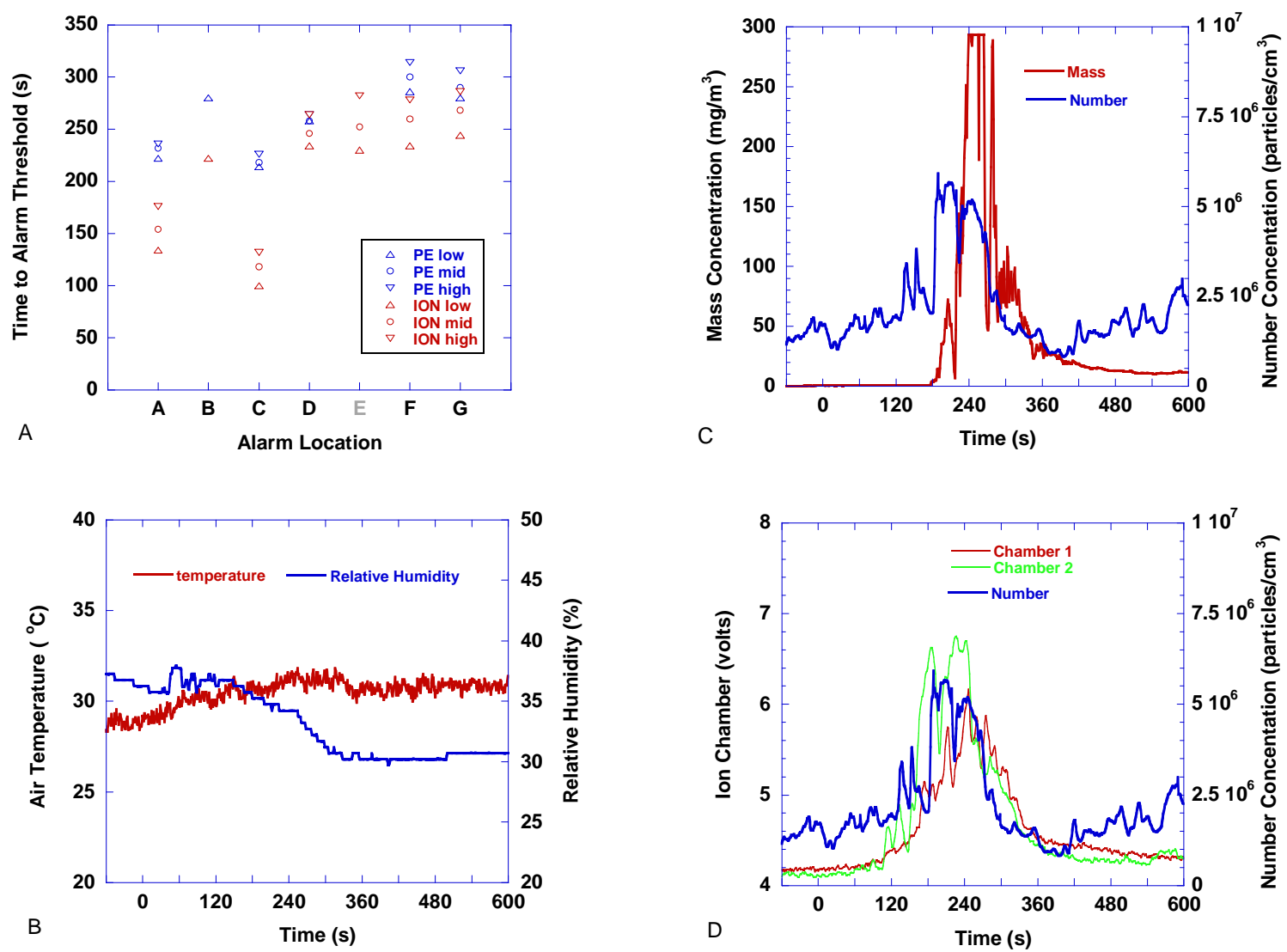

Figure 148. Toasting bread scenario - toaster on at $\mathrm{t}=0$, off at $255 \mathrm{~s}$.

No forced flow, remote bedroom window open

Figures 149 and 150 show the time to alarm results for the toasted bread cases with the floor fan on. Fewer alarm thresholds were reached compared to the cases without the fan flow, which was due primarily to the dilution effects of the forced flow. Consequently, the number and mass concentration and ion chamber voltage peaks were lower than the tests without the fan turned on. Another difference between the cases without and with the fan flow was the decay of the number concentration, mass concentration, and ion chamber voltage after the toaster was turned off, where after an initial drop, there was a more even linear decay in each of these values. The fan flow is promoting mixing throughout the entire volume of the open rooms. There was an observed difference between the two tests. 

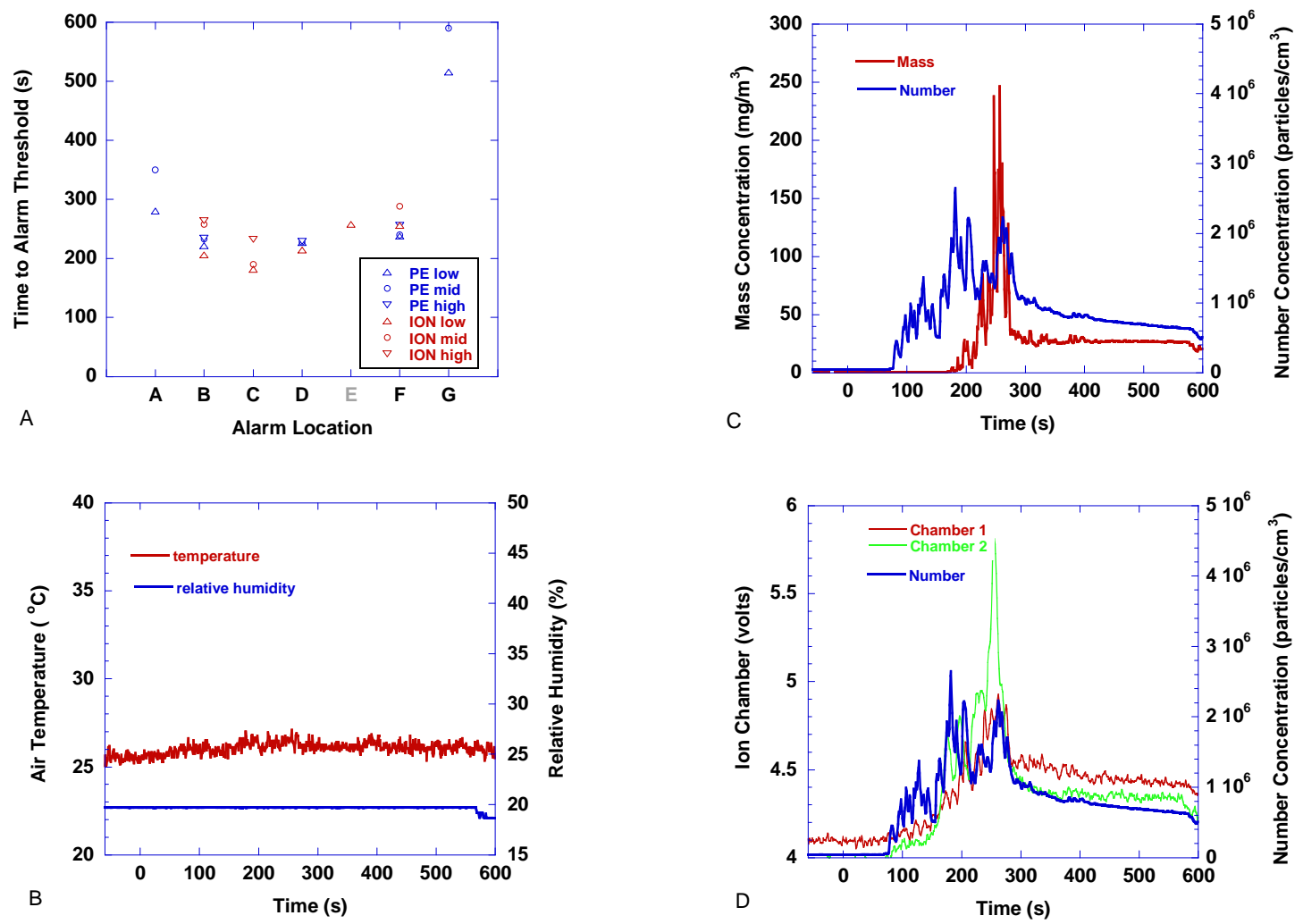

Figure 149. Toasting bread scenario - toaster on at $\mathrm{t}=0$, off at $255 \mathrm{~s}$. Room fan turned on 

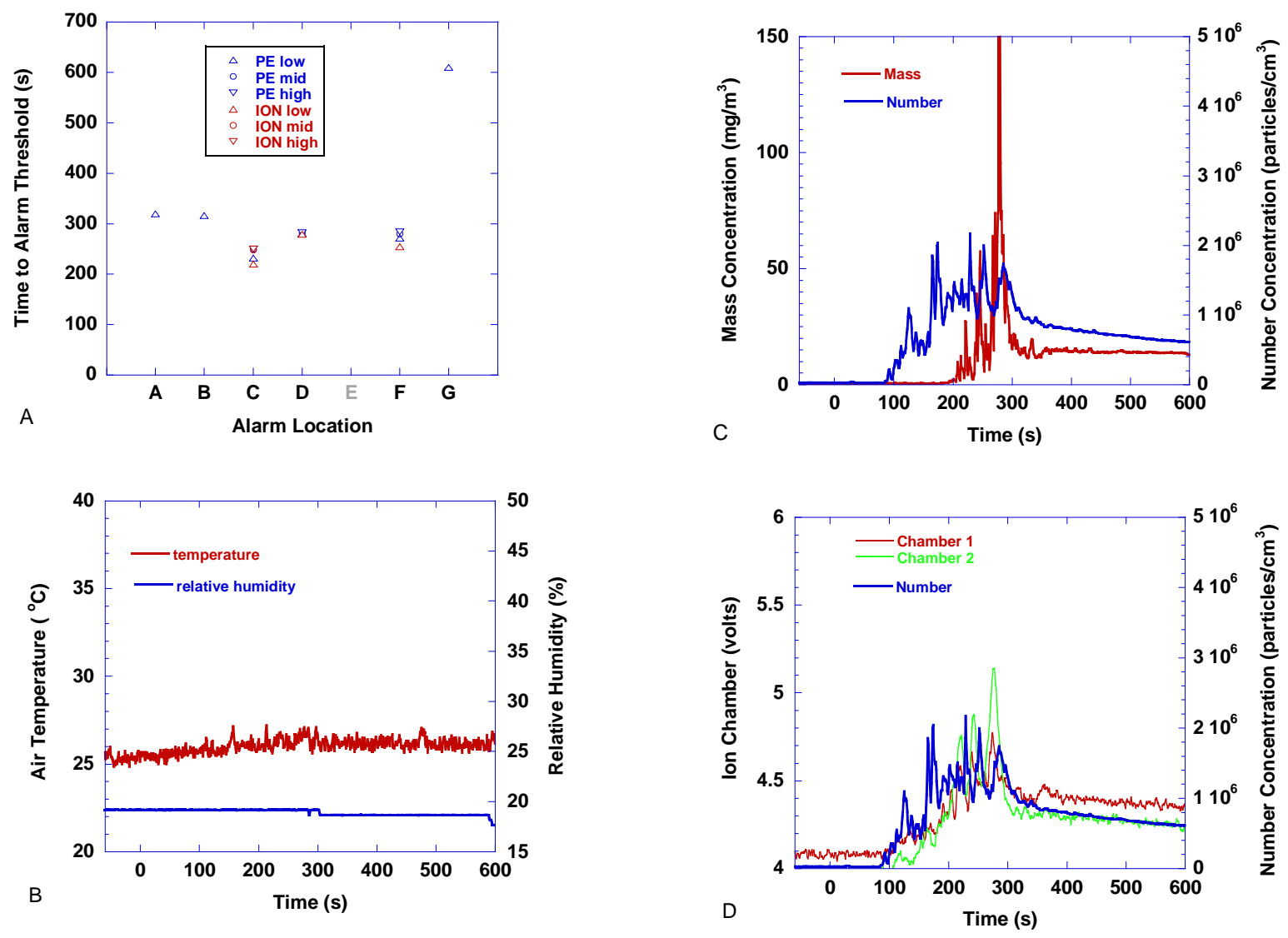

Figure 150. Toasting bread scenario - toaster on at $\mathrm{t}=0$, off at $270 \mathrm{~s}$. Room fan turned on

Figures 151 and 152 show the time to alarm results for the toasting frozen bagel tests. In these tests, only ionization alarm thresholds were achieved. The fan off, no forced flow test reached more threshold levels. The frozen bagels were toasted to a medium brown color and did not char significantly, thus the heavy gray smoke observed with the charring of bread was not present. The temperature and relative humidity show the same trend as the toasting bread scenarios. The peak number concentrations for these tests compare with the respective fan, no fan toasting bread scenarios, however, peak mass concentrations were an order of magnitude lower. 

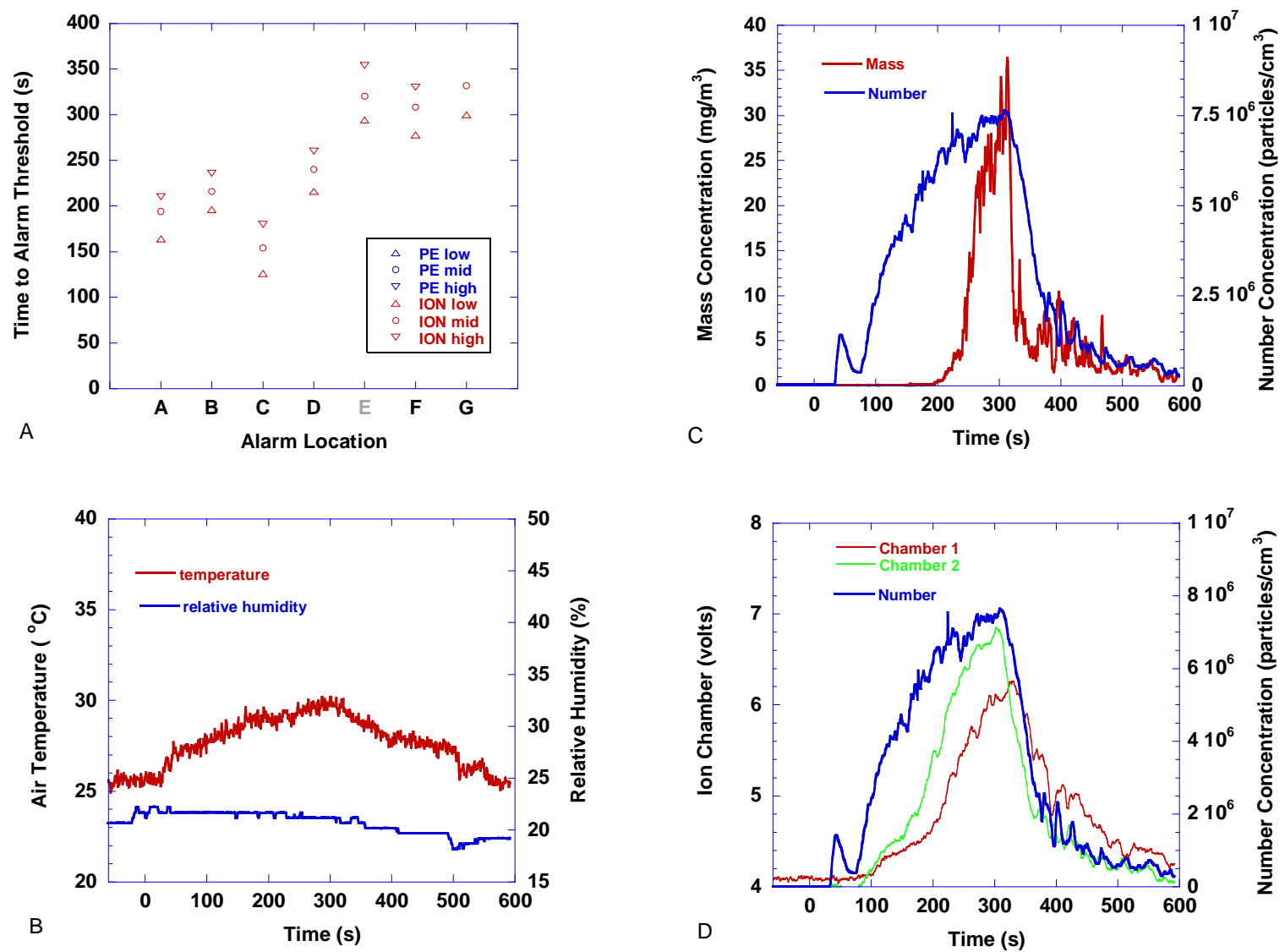

Figure 151. Toasting frozen bagel scenario toaster on at $\mathrm{t}=0$, off at $300 \mathrm{~s}$. Room fan turned off 

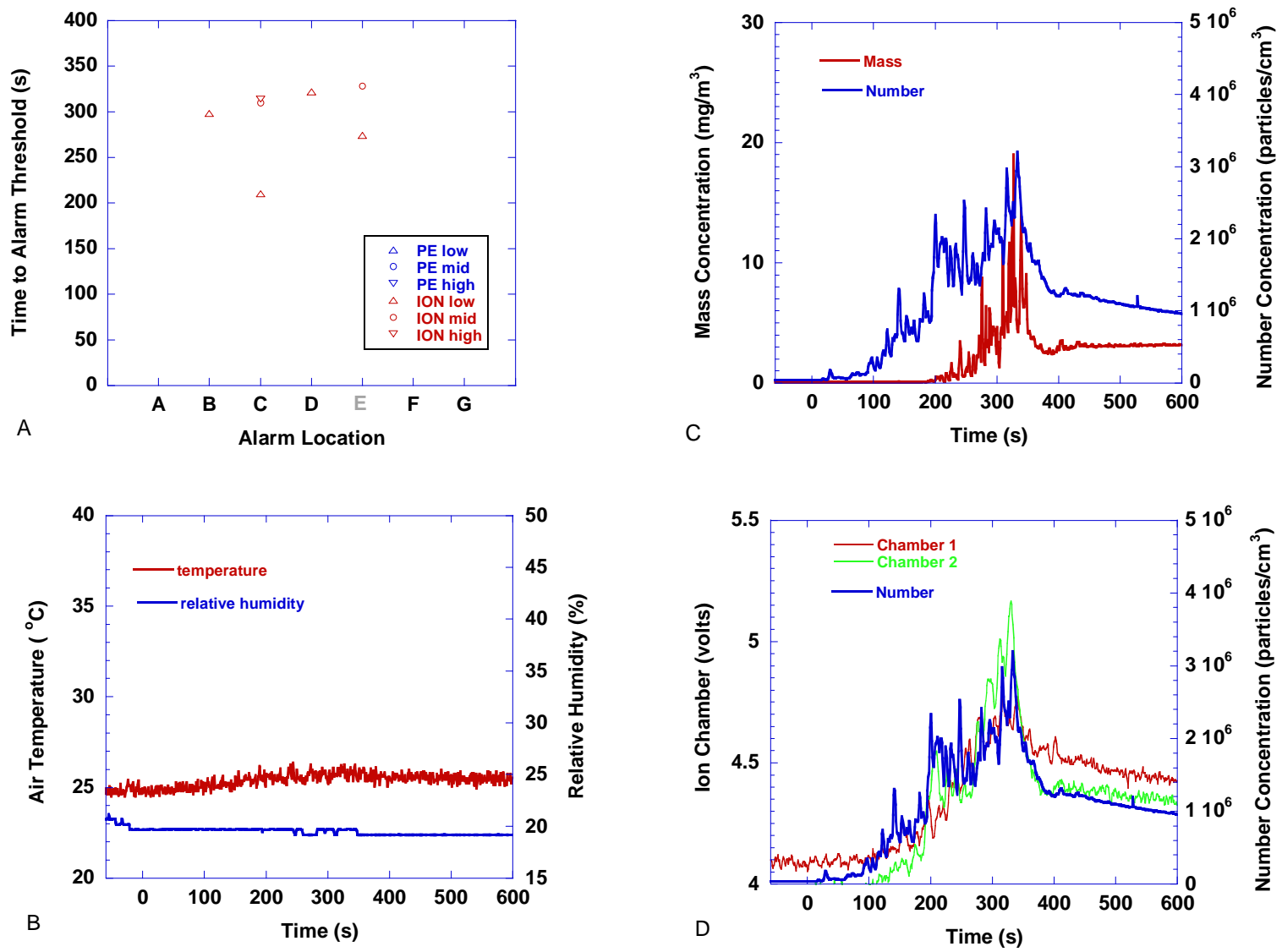

Figure 152. Toasting frozen bagel scenario toaster on at $\mathrm{t}=0$, off at $300 \mathrm{~s}$. Room fan turned on

Several generalizations describe the toasting scenarios:

- For the most part, a high concentration of small particles produced in the early stages of toasting contributed to the ion alarm events.

- With no forced flow, the particles were carried to the ceiling and were transported far from the source at sufficient concentration to cause ionization alarms.

- Forced flow diluted the aerosol concentration such that only ionization alarms closest to the source were susceptible to reaching their threshold. 
- $\quad$ Photoelectric alarm thresholds were met only after items started to char and produce visible smoke, i.e., the photoelectric alarms lag the ionization alarms due to the type of smoke aerosol produced.

- $\quad$ Forced flow reduced the number of photoelectric alarm thresholds met. However, at several locations, photoelectric alarm thresholds were met where ionization alarm thresholds were not.

\subsubsection{Frying Bacon}

Strips of bacon were fried in either a $300 \mathrm{~mm}$ (10 in) diameter cast-iron frying pan or $280 \mathrm{~mm}$ ( 9 in) aluminum non-stick frying pan heated by both a single-unit LPG hot plate (maximum output $4 \mathrm{~kW}$ ) or the electric range (large burner, nominally $1.5 \mathrm{~kW}$ maximum output). With the electric range, the aluminum non-stick pan was pre-heated on the high setting, then turned down to medium high after the bacon was added. Cooking time was between $5 \mathrm{~min}$ to $6 \mathrm{~min}$. With the LPG hot plate, LP gas was piped to the burner from a bottle located outside the manufactured home. After the burner was lit, the cast iron pan was pre-heated for $1 \mathrm{~min}$, then, the bacon was added. Cooking time was $10 \mathrm{~min}$. In all cases, approximately $230 \mathrm{~g}$ of bacon was cooked for a time sufficient to crisp the bacon, but not char it.

Figures 153 and 154 show results for bacon cooked in the cast iron pan with no forced air flow. (Note, there was no companion forced air flow test for this scenario.) Figures 153A and 154A show similar trends in that ionization alarm thresholds were reached first, followed by photoelectric alarm thresholds. For any fixed location, delays between ionization and photoelectric alarm ranged between several hundred seconds to 10 's of seconds. The temperature rise was about $7{ }^{\circ} \mathrm{C}$ during these tests. From figure 154B, the ignition of the hotplate was apparent from a temperature spike at time $=0$. The relative humidity initially increased, then decreased suggesting moisture driven off early the cooking process (as evidenced by condensed water vapor above the frying pan) was the primary cause (moisture from the combustion process also affects the relative humidity).

The number concentration rose to peak values of approximately $6 \times 10^{6}$ particles $/ \mathrm{cm}^{3}$ at the end of the cooking time, while the mass concentration peaked at about $50 \mathrm{mg} / \mathrm{m}^{3}$ at the end of each test. The ion chamber voltages rose steadily from about $180 \mathrm{~s}$ to the end of the cooking time (660 s). There was no evident rise in the number concentration from the LP gas combustion alone. 

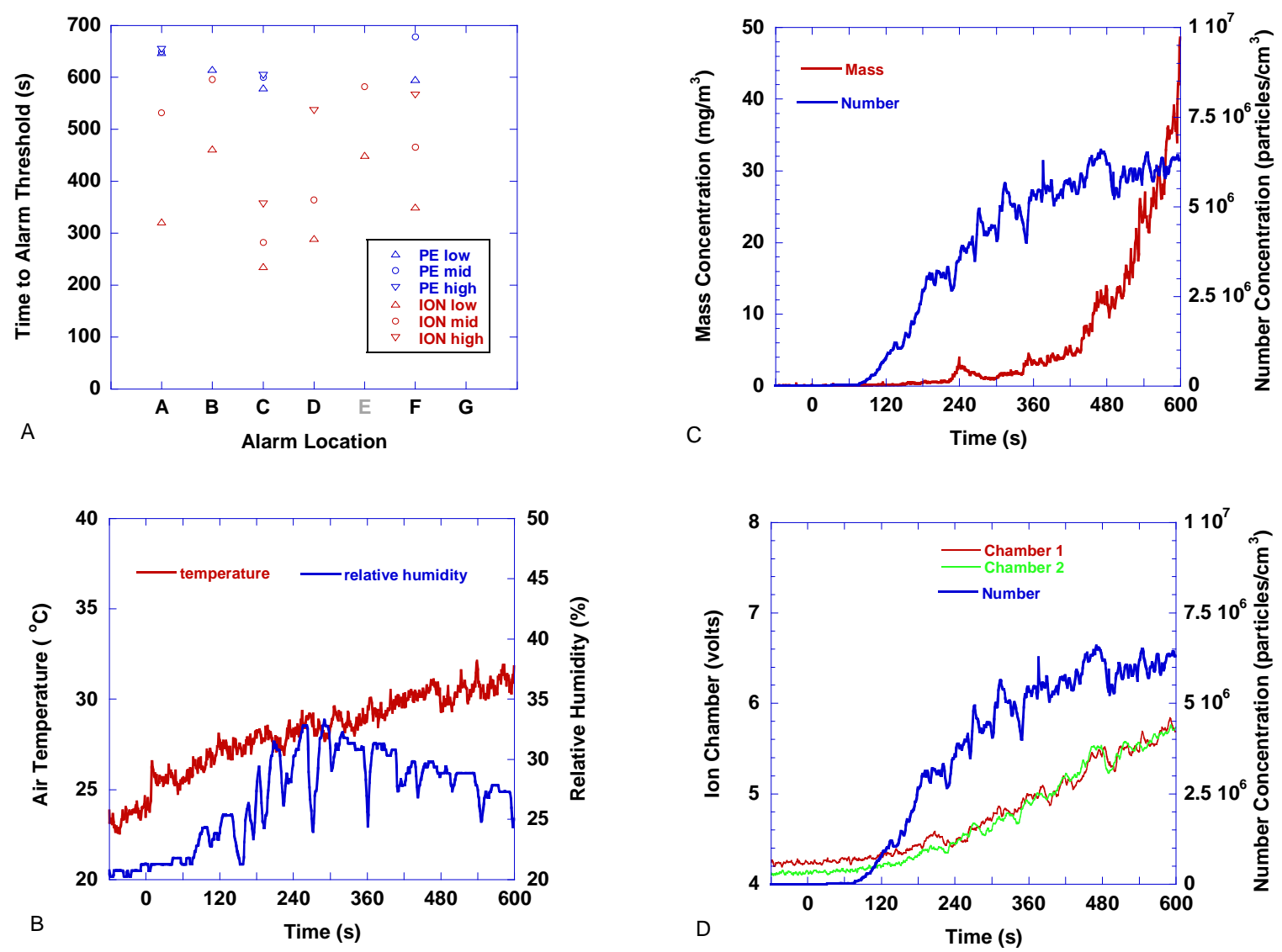

Figure 153. Frying bacon scenario - cast-iron pan, LPG hot plate, no forced flow, burner lit at $\mathrm{t}=0$, bacon in pan at $60 \mathrm{~s}$

Figure 155 shows the results for bacon cooked in the aluminum non-stick frying pan heated by the electric range with no forced air flow. Figure 155A shows the time to reach alarm thresholds. Usually, ionization thresholds were reached before photoelectric thresholds with the exception of the lower threshold at position G. Fewer thresholds were reached in this test compared to the cast iron frying tests above, even though the bacon was cooked to the same nominal doneness. The temperature rise was about $3{ }^{\circ} \mathrm{C}$ over the test time, and again the relative humidity increased to a peak, then decreased below the initial value by the end of the test.

There were two peaks in the number concentration attributed to the method of cooking (i.e. turning the bacon and moving it and pressing it with a spatula), on the order of $4 \times 10^{6}$ particles $/ \mathrm{cm}^{3}$. The mass concentration also experienced two peaks, the early one about $20 \mathrm{mg} / \mathrm{m}^{3}$ and the latter one about $80 \mathrm{mg} / \mathrm{m}^{3}$. The ion chamber voltages follow the number concentration 
trend and both experience two peak values. The magnitude of the second peak is greater than the first suggesting the mean particle size is larger, which is confirmed by the mass concentration peaks.
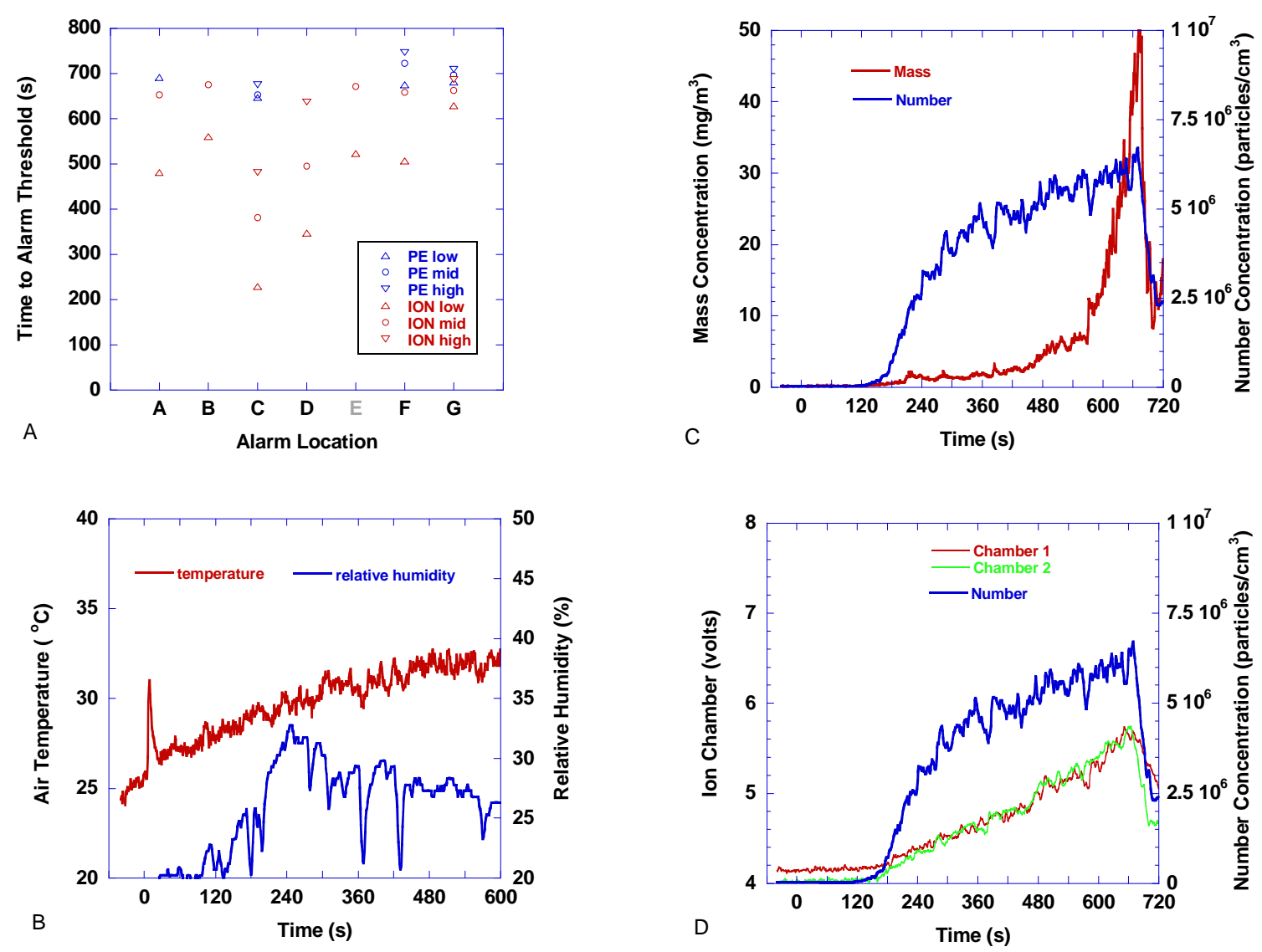

Figure 154. Frying bacon scenario - cast-iron pan, LPG hot plate, no forced flow, burner lit at $\mathrm{t}=0$, bacon in pan at $60 \mathrm{~s}$ 

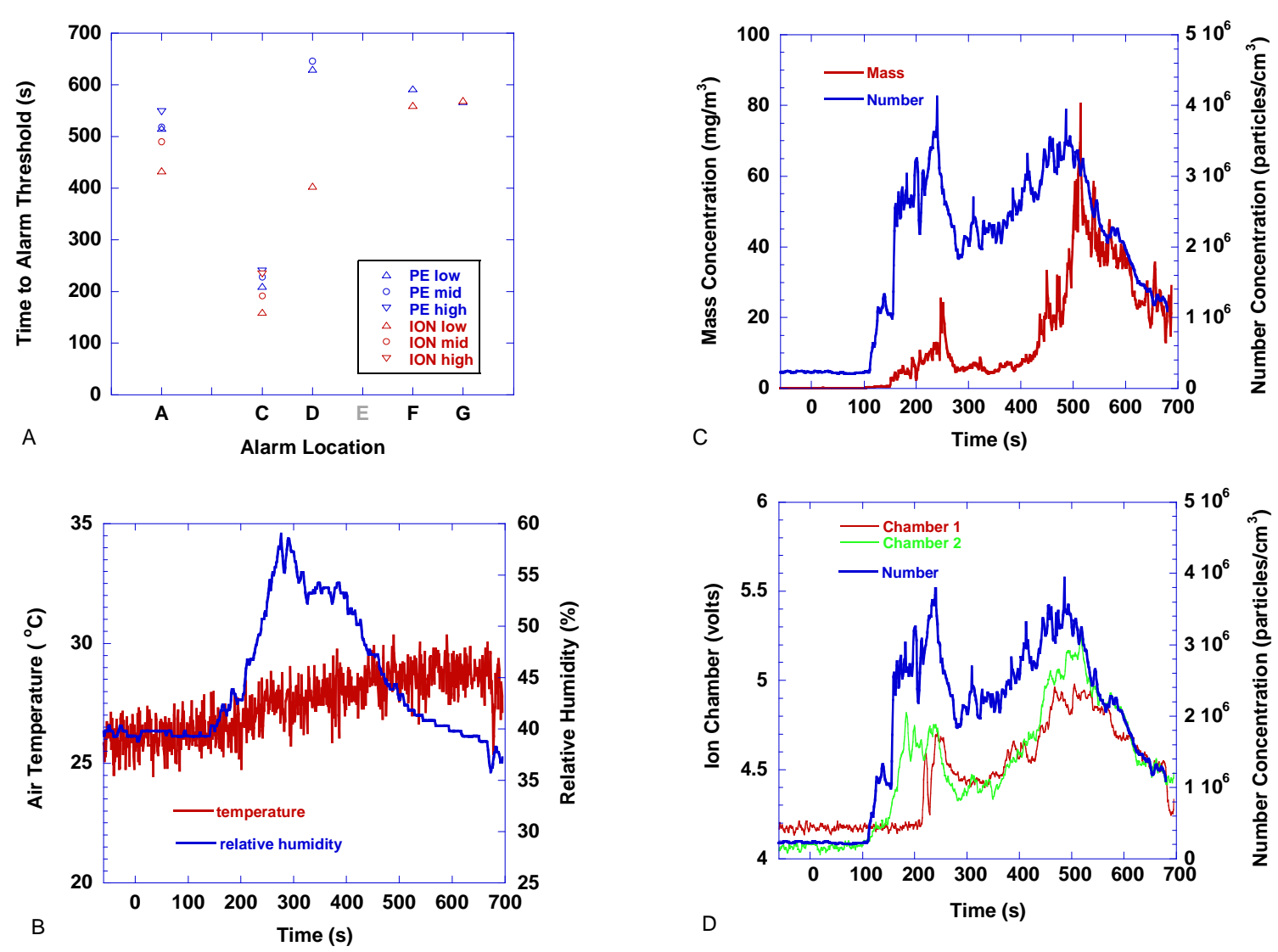

Figure 155. Frying bacon scenario - aluminum pan, electric range, no forced flow, bacon in pan at $120 \mathrm{~s}$ 
Figure 156 shows the results for bacon cooked in the aluminum non-stick frying pan heated by the electric range with forced air flow. During this test, only photoelectric alarm thresholds were met, and then at three locations. A slight decrease in relative humidity was observed, as was a slight increase in temperature. The number concentration peaked at about $1 \times 10^{6}$ particles $/ \mathrm{cm}^{3}$, while the mass concentration experienced a sharp peak to a value of $25 \mathrm{mg} / \mathrm{m}^{3}$. The ion chamber voltages follow the number concentration increase, but only reach values about $0.2 \mathrm{~V}$ above clean air background value.
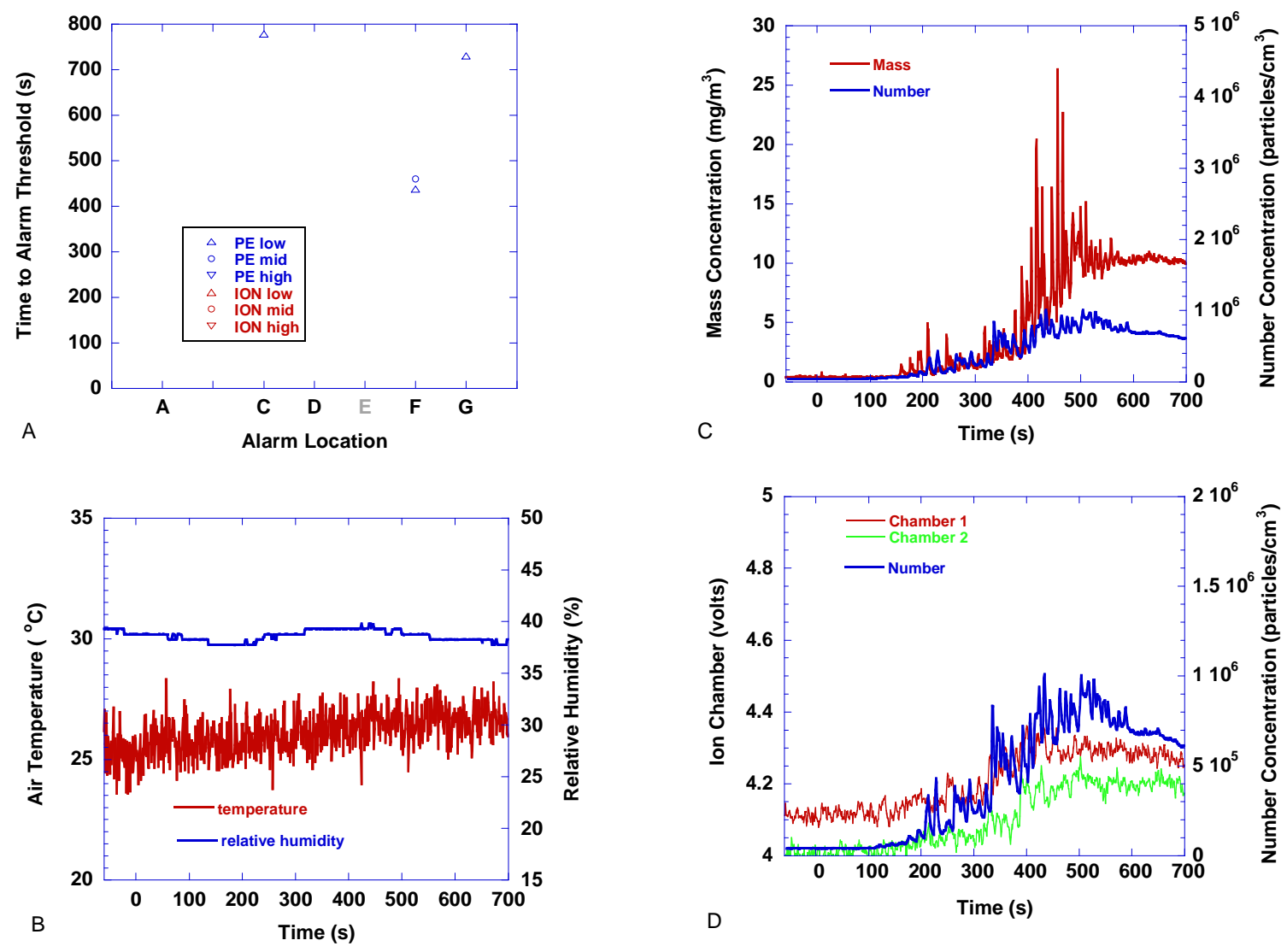

Figure 156. Frying bacon scenario - aluminum pan, electric range, floor fan on, bacon in pan at $120 \mathrm{~s}$

Several generalizations describe the frying bacon scenarios:

- With no forced air flow, ionization alarm thresholds were met before photoelectric alarm thresholds at most locations. 
- The combination of the type of pan and heat source affects the number of alarm thresholds met. The rendered byproducts from the bacon tended to stick to the cast iron pan and burn, producing more visible smoke during cooking.

- With forced airflow, all ionization alarms were suppressed, while three locations reached photoelectric alarm thresholds. The alarm closest to the source actually reached the lowest threshold after two alarms located farther from the source.

\subsubsection{Frying Butter and Margarine}

One tablespoon of butter or margarine was placed in either the aluminum or cast iron pan set on the large element burner. Power was set to high and the pan was left for $5 \mathrm{~min}$ to $6 \mathrm{~min}$, then power was turned off and the pan was removed from the burner and set aside.

Figures 157 and 158 show results for repeated tests of butter heated in the aluminum pan with no force air flow. Both photoelectric and ionization alarm thresholds were met at most locations. Usually the ionization thresholds were reached first. In both tests, the temperature rose about $4{ }^{\circ} \mathrm{C}$, while the relative humidity dropped from $20 \%$ to $15 \%$. The mass concentration peaked at about $360 \mathrm{~s}$ at values of $150 \mathrm{mg} / \mathrm{m}^{3}$ and $300 \mathrm{mg} / \mathrm{m}^{3}$. Number concentration maximums were on the order of $5 \times 10^{6}$ particle $/ \mathrm{cm}^{3}$. Ion chamber 2 voltage followed the number concentration curve, tracking peaks and valleys though advanced in time which is assumed to be due to a sample transport time difference. Ion Chamber 1 voltage on average is lower than chamber 2 voltage due to the fact that it is further from the source.

Figure 159 shows the results for butter heated in a cast iron pan with no forced air flow. Photoelectric alarms thresholds were met at all locations with working sensors (location E does not have a photoelectric sensor, and location $\mathrm{C}$ did not have a working sensor for this test) before ionization alarm thresholds were met. Temperature rise was about $6{ }^{\circ} \mathrm{C}$, and a slight decrease in relative humidity was observed. Mass concentration peaked at over $300 \mathrm{mg} / \mathrm{m}^{3}$, while the number concentration peaked at about $4 \times 10^{6}$ particle $/ \mathrm{cm}^{3}$. The ion chamber voltages follow the number concentration with chamber 2 peaking higher and slightly sooner than chamber 1 .

Figure 160 shows the results for butter heated in a cast iron pan with forced air flow. All locations with photoelectric sensors reached alarm thresholds, while only 2 locations reached ionization thresholds, and usually after the photoelectric thresholds were met. Temperature rise was about $2{ }^{\circ} \mathrm{C}$ and the relative humidity dropped a few percent during the test, then rebounded at the end. No CPC data was taken during this test. Mass concentration peaked above $35 \mathrm{mg} / \mathrm{m}^{3}$, while the ion chamber voltages rose between $0.3 \mathrm{~V}$ and $0.4 \mathrm{~V}$. 

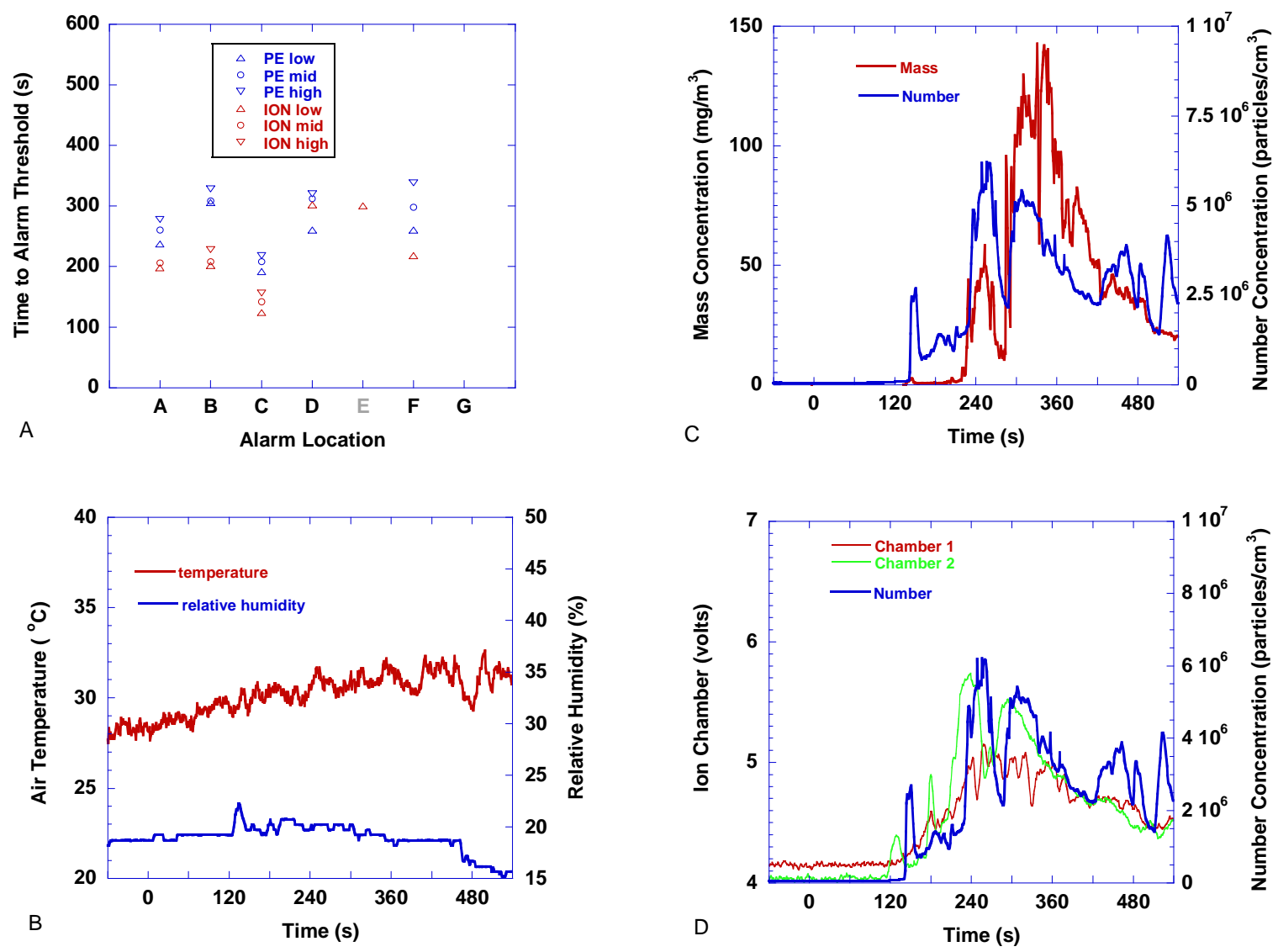

Figure 157. Frying butter scenario - aluminum pan, electric range, floor fan off, butter in pan at $\mathrm{t}=0$ 

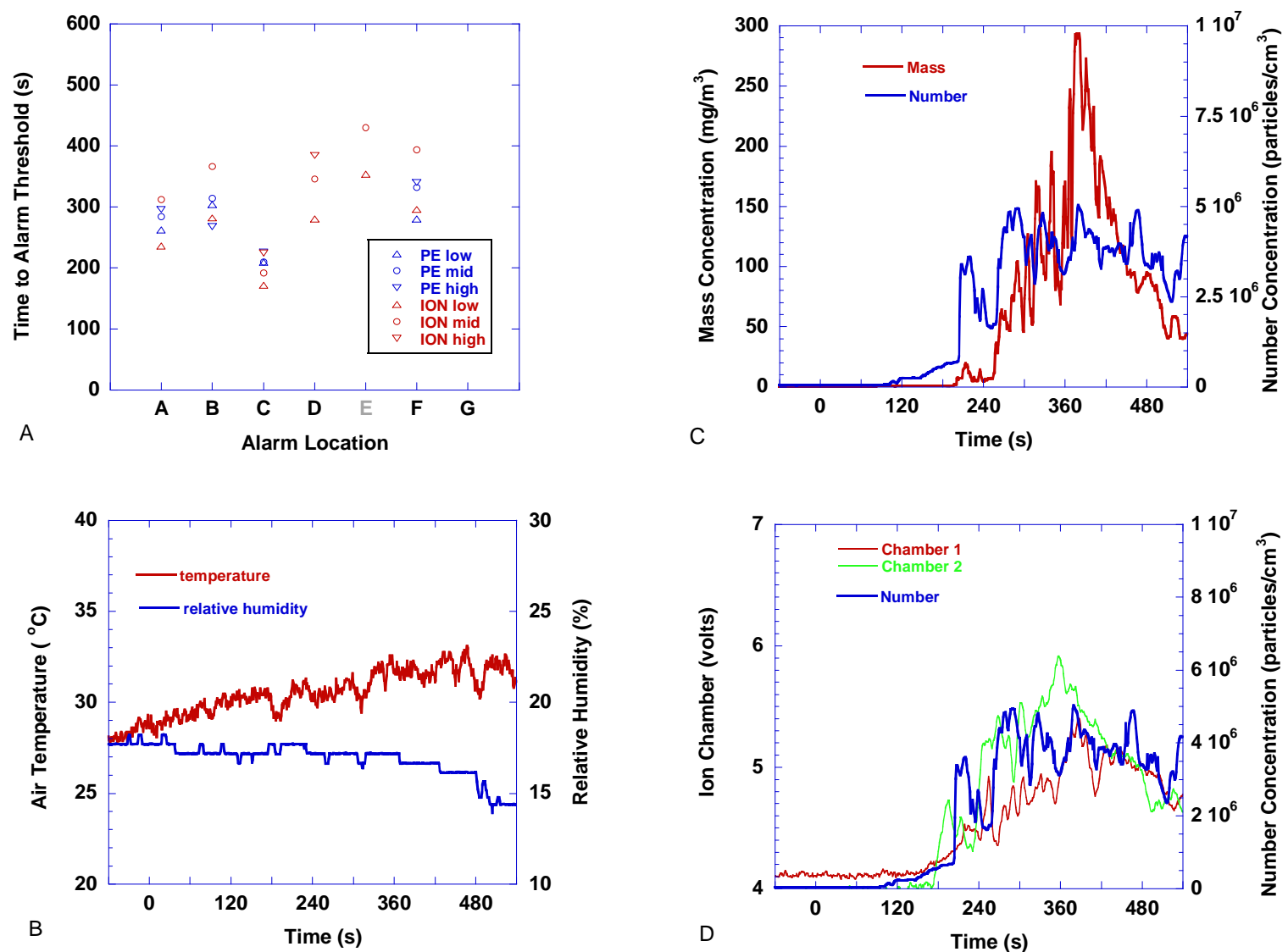

Figure 158. . Frying butter scenario - aluminum pan, electric range, floor fan off, butter in pan at $\mathrm{t}=0$ 

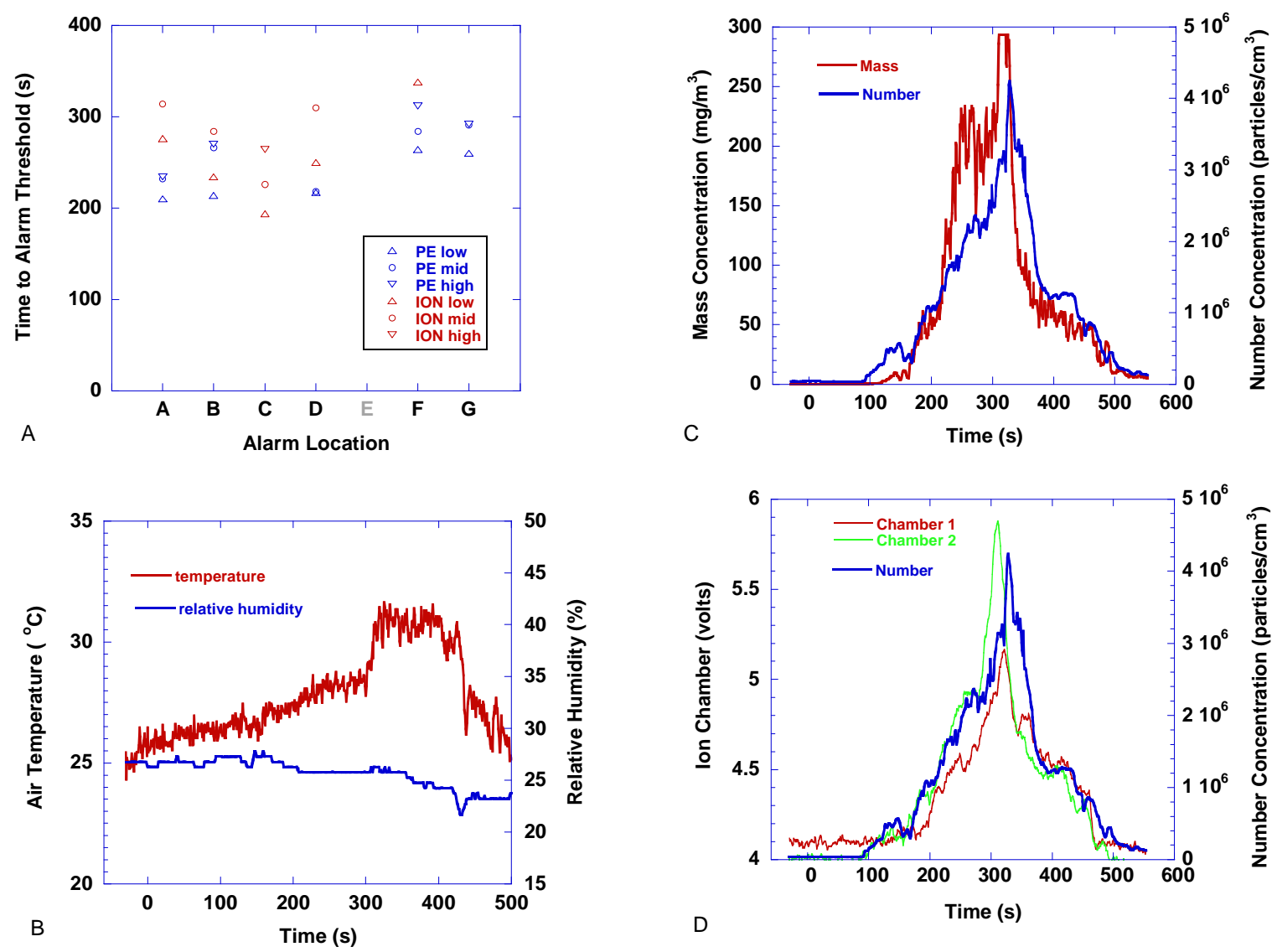

Figure 159. Frying butter scenario - cast iron pan, electric range, floor fan off, butter in pan at $\mathrm{t}=0$ 

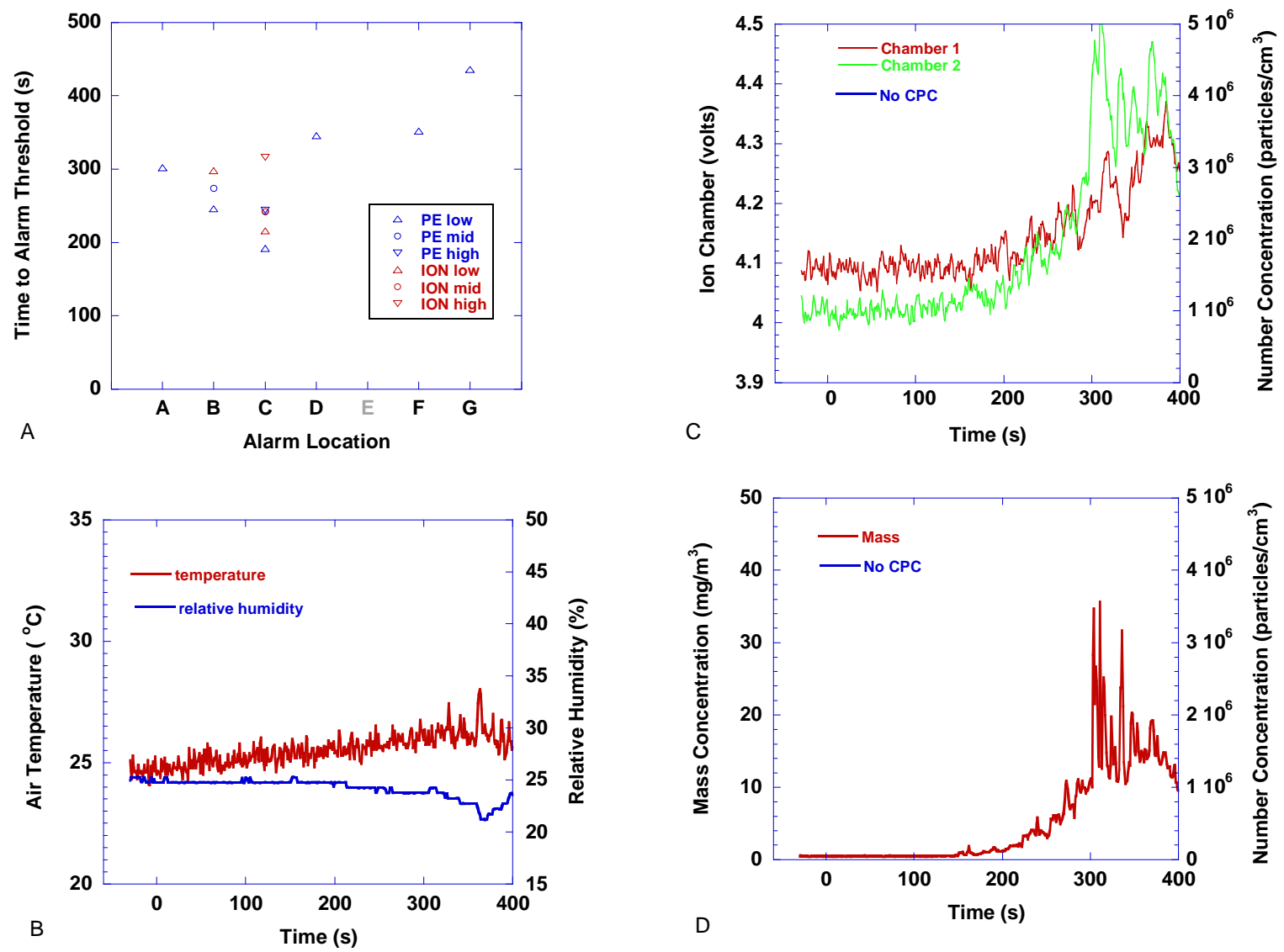

Figure 160. Frying butter scenario - cast iron pan, electric range, floor fan on, butter in pan at $\mathrm{t}=0$ 
Figure 161 shows the results for margarine heated in a cast iron pan with no forced air flow. Photoelectric alarm thresholds were met at all six locations that had photoelectric sensors, while ionization threshold was met only at the location closest to the source. There was little change in temperature or relative humidity during the test. The mass concentration experienced a sharp peak of $100 \mathrm{mg} / \mathrm{m}^{3} 310 \mathrm{~s}$ into the test. Four of the six alarm locations had already reached photoelectric alarm thresholds before that peak mass concentration was recorded. Number concentration peaked at a value of approximately $2.8 \times 10^{6}$ particles $/ \mathrm{cm}^{3}$. Ion chamber voltages rose between $0.5 \mathrm{~V}$ and $0.6 \mathrm{~V}$ for chambers 1 and 2 respectively.
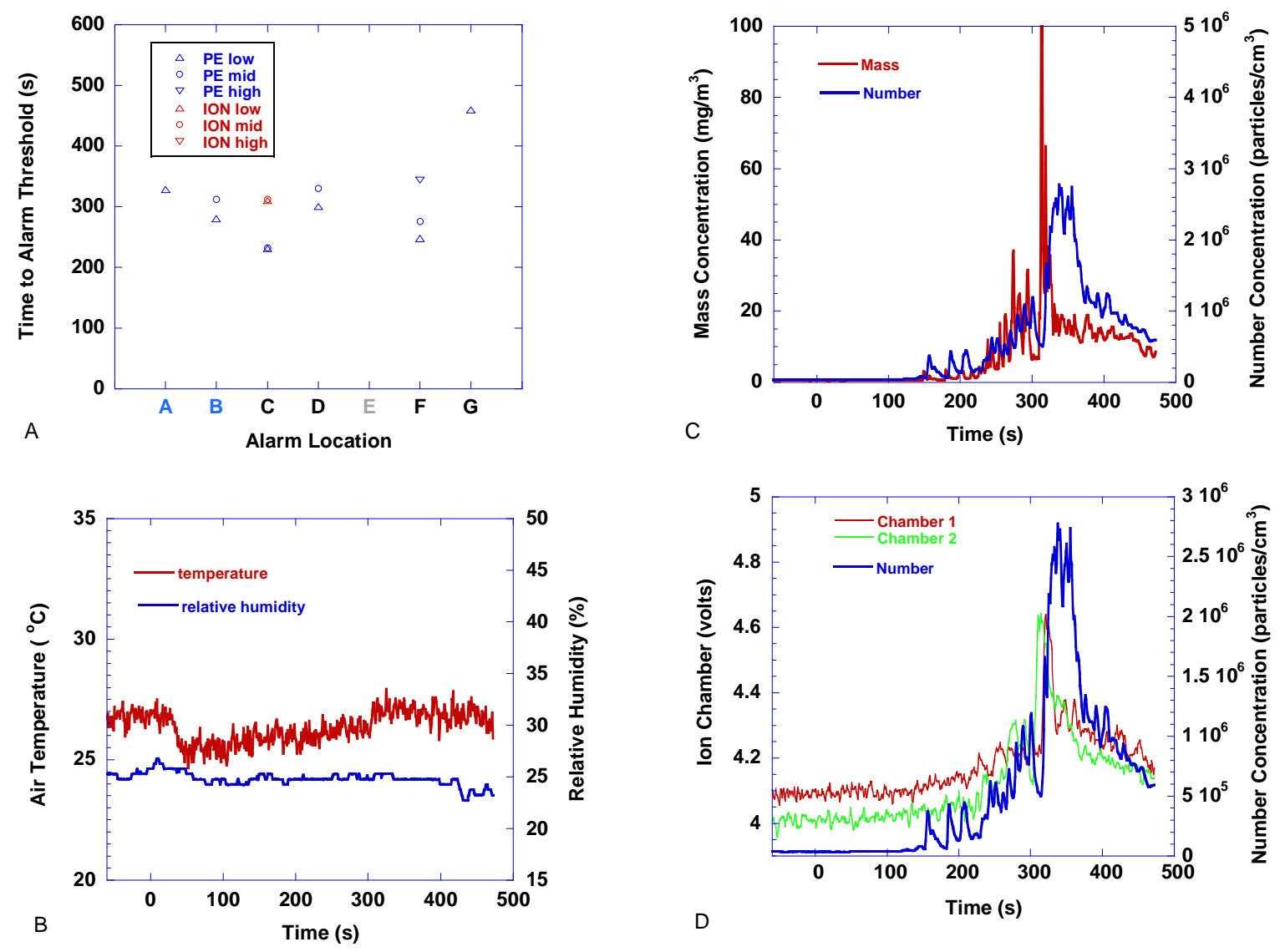

Figure 161. Frying margarine scenario - cast iron pan, electric range, floor fan off, margarine in pan at $\mathrm{t}=0$ 
The following generalizations describe butter or margarine heated in pans.

- Heating butter in the aluminum non-stick pan caused ionization alarm thresholds to be achieved first, while heating butter or margarine in the cast iron pan caused photoelectric alarm thresholds to be achieved first.

- Forced air flow reduced the number of locations that reached ionization alarm thresholds, but not the number of photoelectric alarm thresholds.

- Butter in the cast iron pan with no forced flow produced more alarm threshold conditions slightly earlier than margarine tested under the same conditions.

\subsubsection{Frying Hamburgers}

Three $110 \mathrm{~g}$ (quarter pound) frozen hamburgers ( $28 \%$ fat content) were cooked in the aluminum pan on the large electric burner. First, the pan was placed on the large electric burner and the power was set to high. After a preheat time (between $130 \mathrm{~s}$ to $160 \mathrm{~s}$ ) the power was lowered to a medium-high setting. The hamburgers were placed in the heated pan. The hamburgers were flipped after approximately $4 \mathrm{~min}$ and cooked for an additional $5 \mathrm{~min}$. The stove was turned off and the hamburgers were removed from the pan.

Figure 162 shows the results for test conditions with no forced flow. At about $250 \mathrm{~s}$, a threshold was met by the photoelectric sensor closest to the source. The rest of the locations reached alarm thresholds between $400 \mathrm{~s}$ and $600 \mathrm{~s}$. Some locations record photoelectric alarm thresholds first, while others record ionization alarm thresholds first. Temperature rose about $3{ }^{\circ} \mathrm{C}$ during the test while the relative humidity rose about $5 \%$. After initial peaks in the mass and number concentration, the mass concentration peaked at about $100 \mathrm{mg} / \mathrm{m}^{3}$, while the number concentration peaked at $4 \times 10^{6}$ particles $/ \mathrm{cm}^{3}$. Ion chamber voltage rise was $1.4 \mathrm{~V}$ and $1.1 \mathrm{~V}$ for chambers 2 and 1 respectively.

Figure 163 shows the results for test conditions with force air flow and the living room window open. All locations with a photoelectric sensor reach alarm thresholds for that sensor. Only two locations recorded ionization alarm thresholds. The temperature rise was about $2{ }^{\circ} \mathrm{C}$ during the test, while the relative humidity rose about $3 \%$. Mass concentration peaked at about $30 \mathrm{mg} / \mathrm{m}^{3}$, while the number concentration peaked at $2 \times 10^{6}$ particles $/ \mathrm{cm}^{3}$. The peak ion chamber voltage change was $0.4 \mathrm{~V}$ and $0.6 \mathrm{~V}$ for chamber 1 and 2 respectively.

Figure 164 shows the results for test conditions with forced air flow and the normal conditions of all exterior doors and windows closed. Five out of six locations reach photoelectric alarm thresholds, while none reach ionization alarm thresholds. The closer to the source the sooner the photoelectric thresholds were reached. Temperature rose about $2{ }^{\circ} \mathrm{C}$, while the relative humidity 
rose about $2 \%$. The mass concentration showed several short duration spikes, with the highest peak about $30 \mathrm{mg} / \mathrm{m}^{3}$. The number concentration peaked at about $1.6 \times 10^{6}$ particles $/ \mathrm{cm}^{3}$. Peak ion chamber voltage change was about $0.5 \mathrm{~V}$ for each chamber.
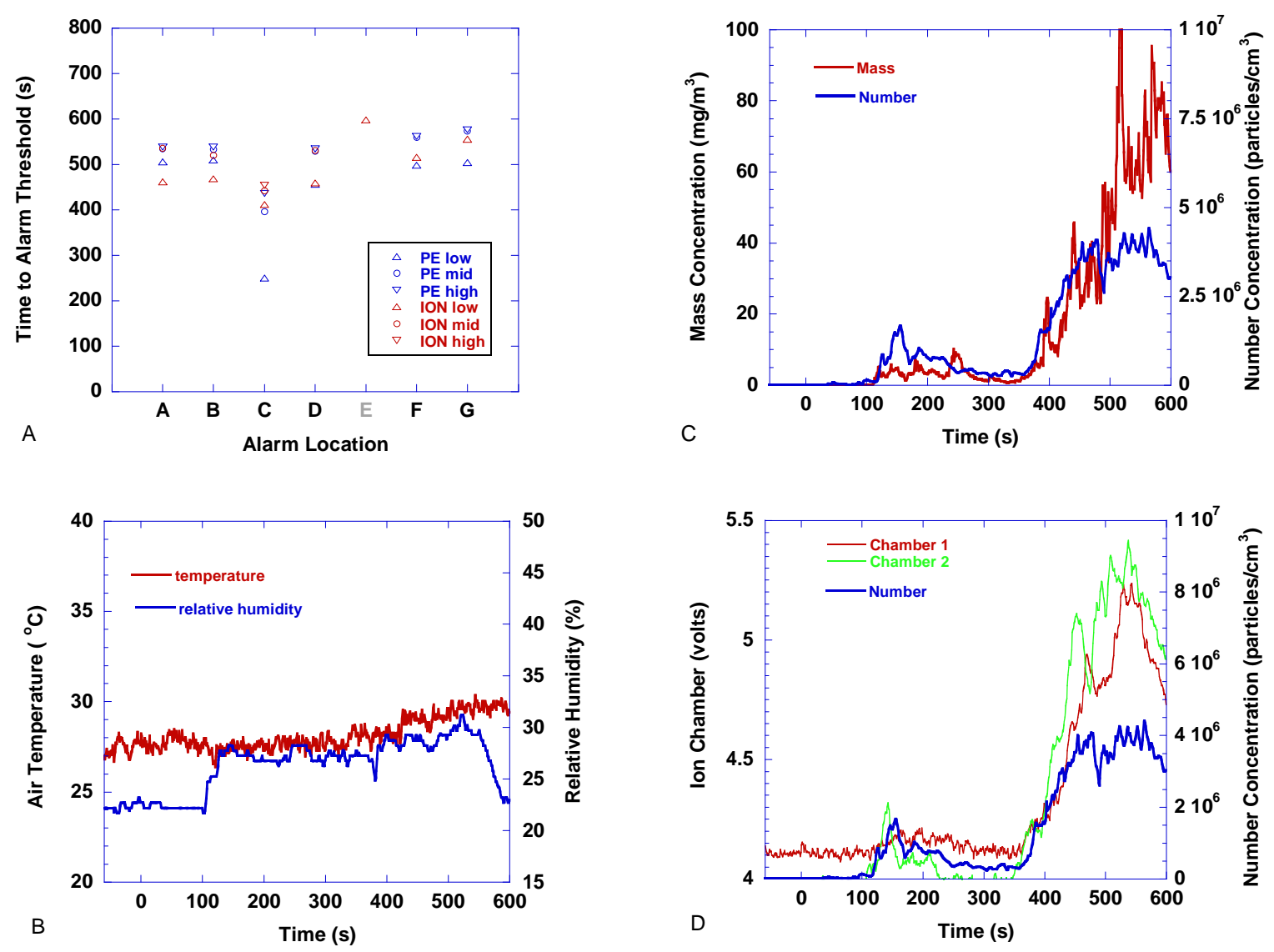

Figure 162. Frying hamburgers - three hamburgers in non-stick frying pan, floor fan off. Hamburgers in pan at $\mathrm{t}=120 \mathrm{~s}$ 

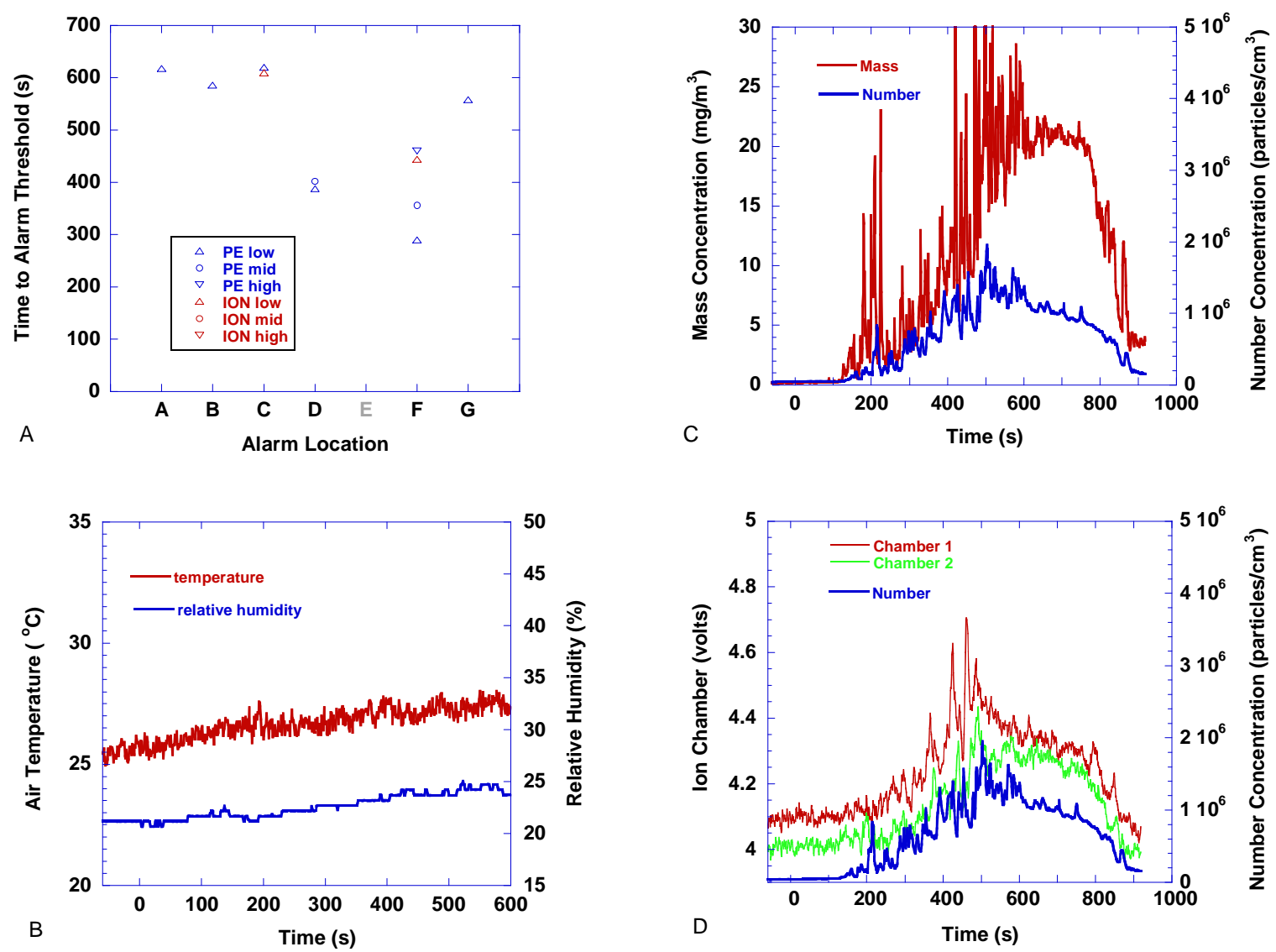

Figure 163. Frying hamburgers - three hamburgers in non-stick frying pan, floor fan on, living room window open. Hamburgers in pan at $\mathrm{t}=160 \mathrm{~s}$ 

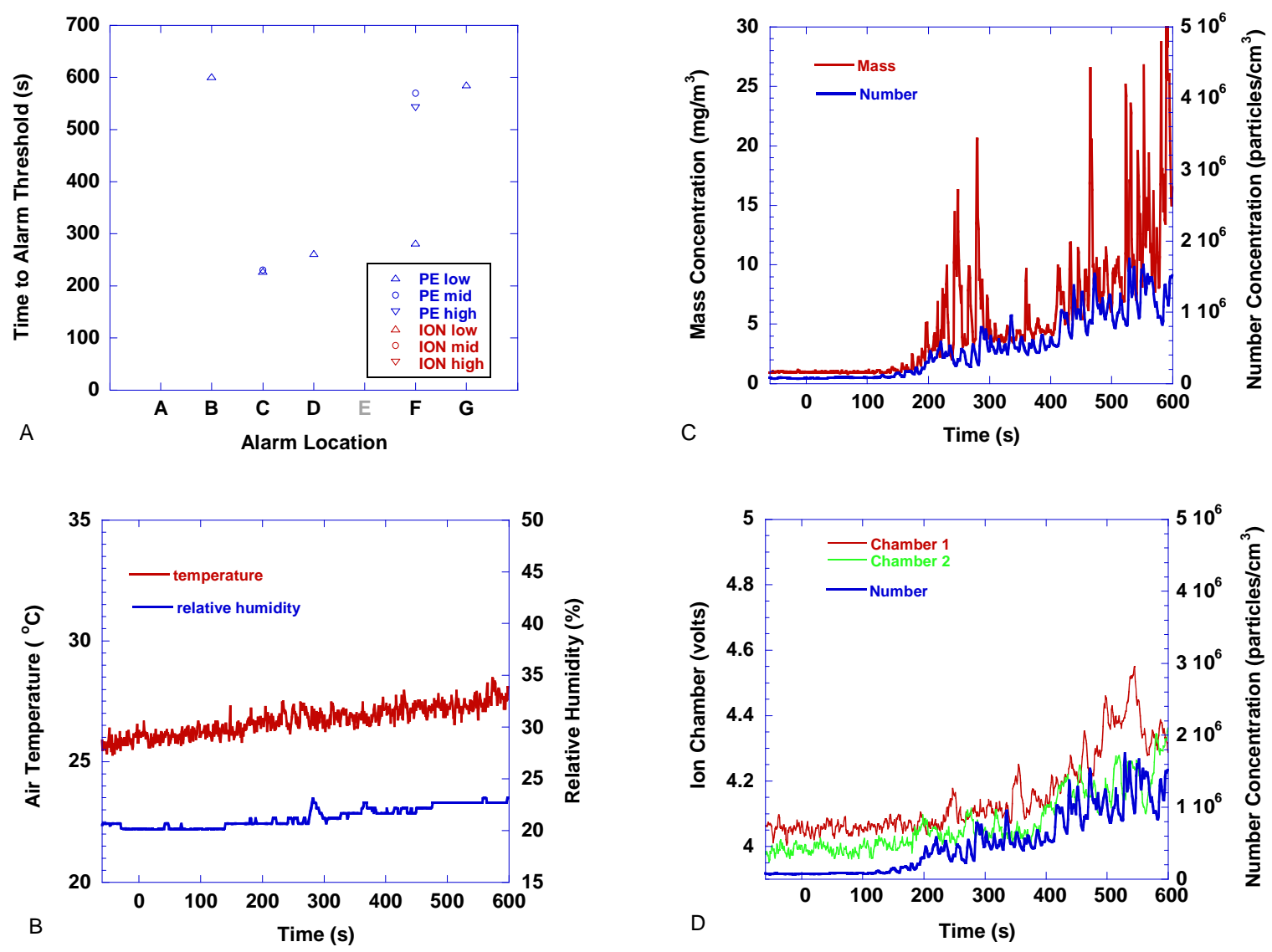

Figure 164. Frying hamburgers - three hamburgers in non-stick frying pan, floor fan on. Hamburgers in pan at $\mathrm{t}=150 \mathrm{~s}$ 
The following generalizations describe the frying hamburgers tests:

- With no forced flow, photoelectric and ionization alarm thresholds were recorded at all locations with no clear preference as to which type reached a threshold first at the specified locations.

- The forced flow tests yielded significantly less ionization alarm thresholds.

\subsubsection{Deep-frying Tortillas and French-fried Potatoes}

Using the LP hot plate, $0.15 \mathrm{~m}$ diameter (6 in) corn tortillas were deep-fried in a large flatbottom wok-style steel pan in corn oil approximately $50 \mathrm{~mm}$ in depth. The hot plate was set to the high setting. Six minutes after the burner was lit and the oil pre-heated, tortillas were added. A total of 10 tortillas were fried over a period of $10 \mathrm{~min}$. Using the cast iron pan, $30 \mathrm{~mm}$ of vegetable oil was pre-heated on the large electric burner element for eight minutes. $454 \mathrm{~g}(1 \mathrm{lb})$ of frozen "French-fried" potatoes were added to the pan and cooked for $5 \mathrm{~min}$ to $6 \mathrm{~min}$, removed and another $454 \mathrm{~g}$ of potatoes were added and cooked for an additional $5 \mathrm{~min}$ to $6 \mathrm{~min}$, then removed.

Figure 165 shows the results for tortilla frying test with no forced air flow. Three locations reached ionization alarm threshold levels. The temperature rose $9{ }^{\circ} \mathrm{C}$ over the test period, while relative humidity rise reached a maximum of about $8 \%$. The mass concentration did not rise above $2 \mathrm{mg} / \mathrm{m}^{3}$ during the test. The number concentration peaked at about $6.5 \times 10^{6}$ particles $/ \mathrm{cm}^{3}$. The ion chamber voltage rise follows the number concentration rise, with peak change in the voltages of $0.5 \mathrm{~V}$ and $0.6 \mathrm{~V}$ for chamber's 1 and 2 respectively.

Figure 166 shows the results for deep frying French fried potatoes with no forced air flow. Only one ionization alarm threshold was recorded at the location closest to the source. The temperature increased $5{ }^{\circ} \mathrm{C}$ during the test period. The relative humidity initially dropped $2 \%$ during the pre-heating stage, but showed a $10 \%$ rise after each batch of potatoes were added to the hot oil. As the water was driven off the potatoes, the relative humidity would drop. The mass concentration showed two spikes of $3 \mathrm{mg} / \mathrm{m}^{3}$ and $1 \mathrm{mg} / \mathrm{m}^{3}$ soon after the potatoes were dropped into the hot oil. The number concentration peaked at about $3.5 \times 10^{6}$ particles $/ \mathrm{cm}^{3}$. Ion chamber 2 voltage change peaked at $0.45 \mathrm{~V}$, while chamber 1 voltage change peaked at $0.3 \mathrm{~V}$.

The lack of sufficient aerosol mass concentration means it would be unlikely that these scenarios would produce any photoelectric alarms for any extended cooking time. Though, extended cooking times could produce aerosol levels sufficient to produce alarms in the more distant ionization alarms. Any spillage or spatter of hot oil on an electric heating element produces smoke and, some times, small short-lived flames, which would change the nature of the aerosol produced. Repeated spatter of the hot oil might produce enough smoke to reach the threshold for 
photoelectric alarms; however one could argue that such a situation is potentially hazardous and an alarm sounding would not be characterized as a nuisance alarm.
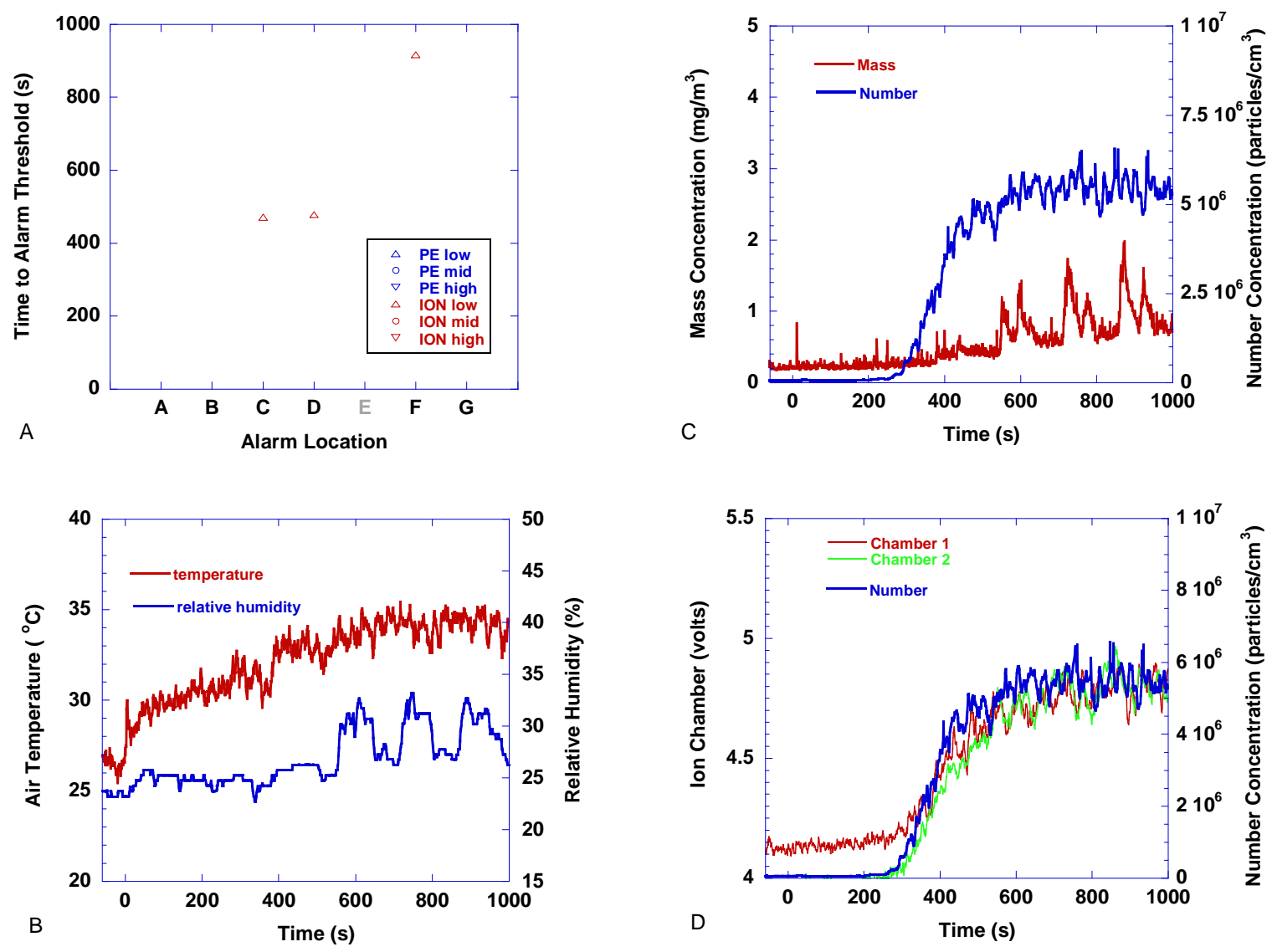

Figure 165. Deep-frying Tortillas scenario - steel wok, LPG hotplate, floor fan off, first tortillas in pan at $\mathrm{t}=360 \mathrm{~s}$ 

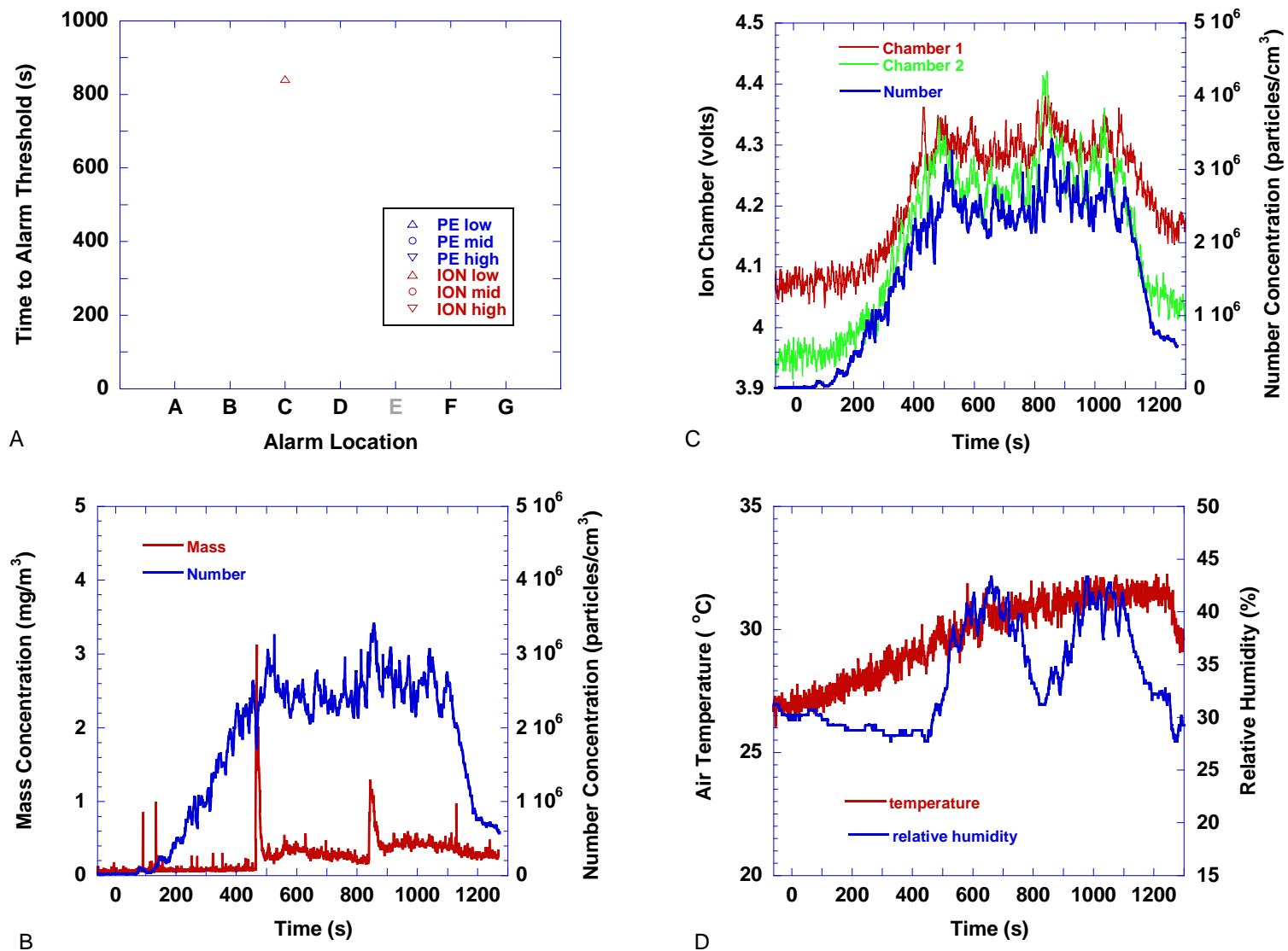

Figure 166. Deep-frying french fries scenario - steel wok, LPG hotplate, floor fan off, first french fries in pan at $\mathrm{t}=360 \mathrm{~s}$ 


\subsubsection{Broiled and Baked/Broiled Pizza}

For the broiled pizza scenario, a small (158 g) frozen plain pizza was placed on a large metal "broiler" pan that was covered with aluminum foil, and placed in the oven. The broiler was set to high and the door opened to the broiling position. The pizza cooked for approximately $13 \mathrm{~min}$ then was removed. There was no forced flow for this test.

Figure 167 shows the results for broiling frozen pizza. While five locations reached ionization alarm thresholds, and only two locations reached photoelectric alarm thresholds, the first threshold reached was for the photoelectric alarm closest to the source. This was reached almost $300 \mathrm{~s}$ before the lowest ionization threshold was reached at that location. Interestingly, there was nothing evident in the Dustrak or CPC readings at the time the first photoelectric threshold was met. Temperature rise was on the order of $5{ }^{\circ} \mathrm{C}$, though there was little change in the
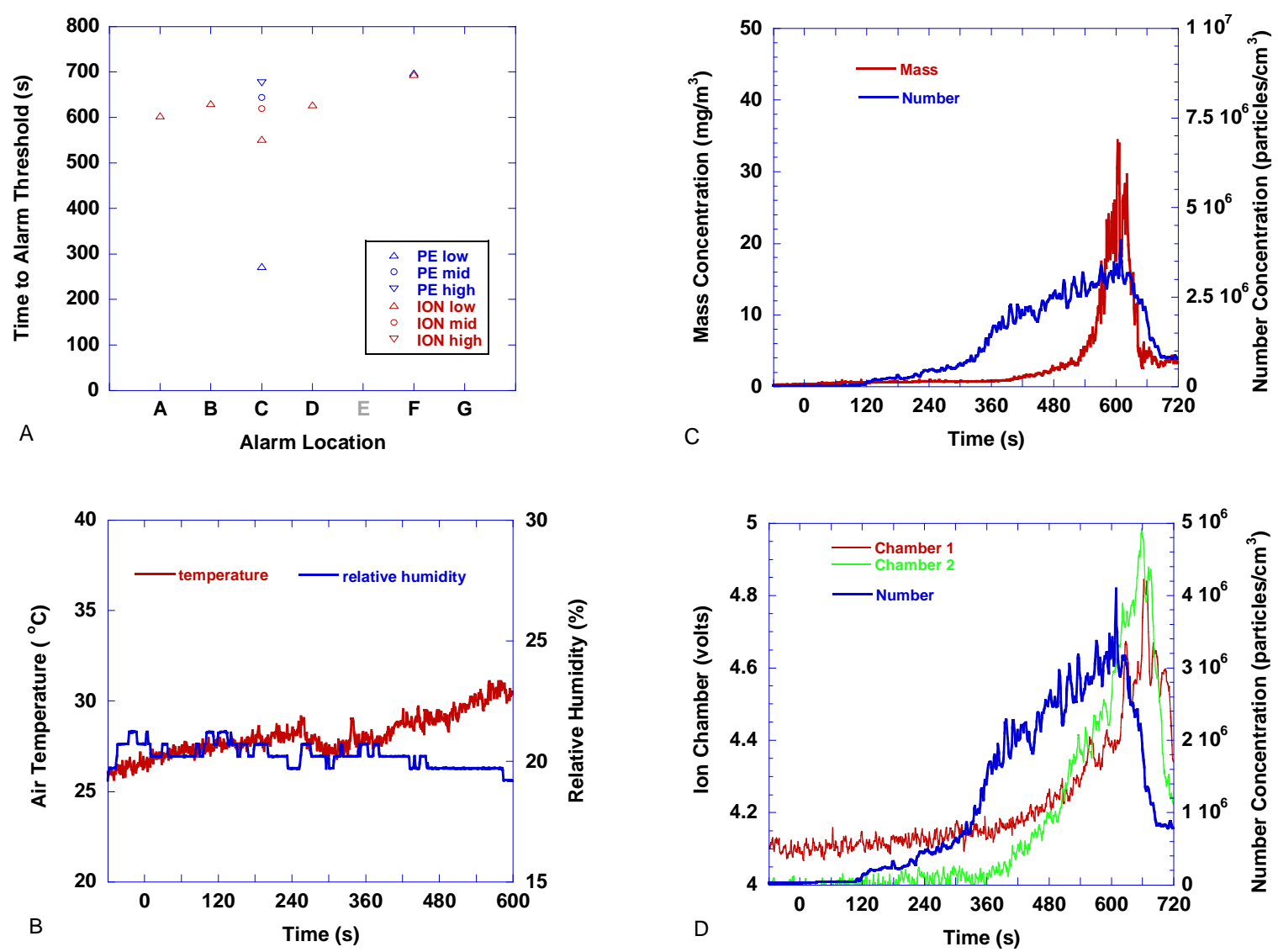

Figure 167. Broiling Pizza - one frozen pizza in oven set on broil, floor fan off. Oven on at $\mathrm{t}=0$ 
relative humidity. The number concentration starts to rise at $120 \mathrm{~s}$ and peaks at $4 \times 10^{6}$ particles $/ \mathrm{cm}^{3}$ just before $600 \mathrm{~s}$. The mass concentration starts to rise at $400 \mathrm{~s}$ and reaches a peak of $30 \mathrm{mg} / \mathrm{m}^{3}$ at $600 \mathrm{~s}$. The mass concentration increase is attributed to the browning of the cheese layer and small amounts of spatter hitting the broiler heating element. The ion chambers peaked at $600 \mathrm{~s}$ at voltage changes of approximately $0.7 \mathrm{~V}$ and $1.0 \mathrm{~V}$ for chamber 1 and 2 respectively. The voltage curve of chamber 2 does not follow the early rise in the number concentration suggesting that the aerosol produced early in the test was of a size too small to produce any significant ion chamber voltage change.

For the baked, then broiled pizza scenario, the oven was pre-heated to a setting of $176{ }^{\circ} \mathrm{C}$ $\left(350^{\circ} \mathrm{F}\right)$. At time $\mathrm{t}=0$ the oven door was opened and one small frozen pizza on the broiler pan was placed in the oven. The pizza was baked for $630 \mathrm{~s}$. During this time the oven door was opened once to visually check the pizza. After $630 \mathrm{~s}$, the oven door was opened to the broil position, the broiler was turned to high, and the pizza was broiled for an additional $10 \mathrm{~min}$. After broiling, the pizza was removed and the oven turned off. This scenario was repeated with the floor fan off and on. Typically, the pizzas were dark drown with some black, but deemed edible.

Figure 168 shows the results for the scenario with the floor fan off. All ionization alarm positions reached at least one threshold level, while only one position reached a photoelectric alarm threshold. The temperature showed two spikes in the baking phase, which were caused by the hot gases escaping the oven during the two periods the door was opened then closed. When the door was opened at the beginning of the broiling phase, the temperature jumped about $5{ }^{\circ} \mathrm{C}$, then continued to rise another $3{ }^{\circ} \mathrm{C}$ over the next $600 \mathrm{~s}$. The relative humidity dropped by about $5 \%$ during the broiling phase. Three spikes in the number concentration on the order of ( 2 to 3 $\mathrm{x} 10^{6}$ ) particles $/ \mathrm{cm}^{3}$ were observed in the number concentration at the three times the door was opened. By the end of the broiling phase, the number concentration had risen to $4 \times 10^{6}$ particles $/ \mathrm{cm}^{3}$. The mass concentration started to climb at about $900 \mathrm{~s}$ and peaked at $6 \mathrm{mg} / \mathrm{m}^{3}$ by the end of the broiling phase. Both ion chambers tracked the early number concentration spikes and reached a voltage change of about $1 \mathrm{~V}$ for each chamber.

Figure 169 shows the results for the scenario with the floor fan on. During this test, only one ionization alarm reached a threshold value. Spikes in the air temperature were observed at the times the oven door was opened. The temperature rose $6{ }^{\circ} \mathrm{C}$ during the test, while the relative humidity fell from $30 \%$ to $25 \%$. The number concentration also showed spikes when the oven door was opened, and it reached a peak of about $4 \times 10^{6}$ particles $/ \mathrm{cm}^{3}$ by the end of the test. The mass concentration started to rise at about $1000 \mathrm{~s}$ and peaked with a value of nearly $5 \mathrm{mg} / \mathrm{m}^{3}$ at $1225 \mathrm{~s}$. The ionization chambers followed the number concentration value and they reached peak values of about $0.5 \mathrm{~V}$ above their background levels. 

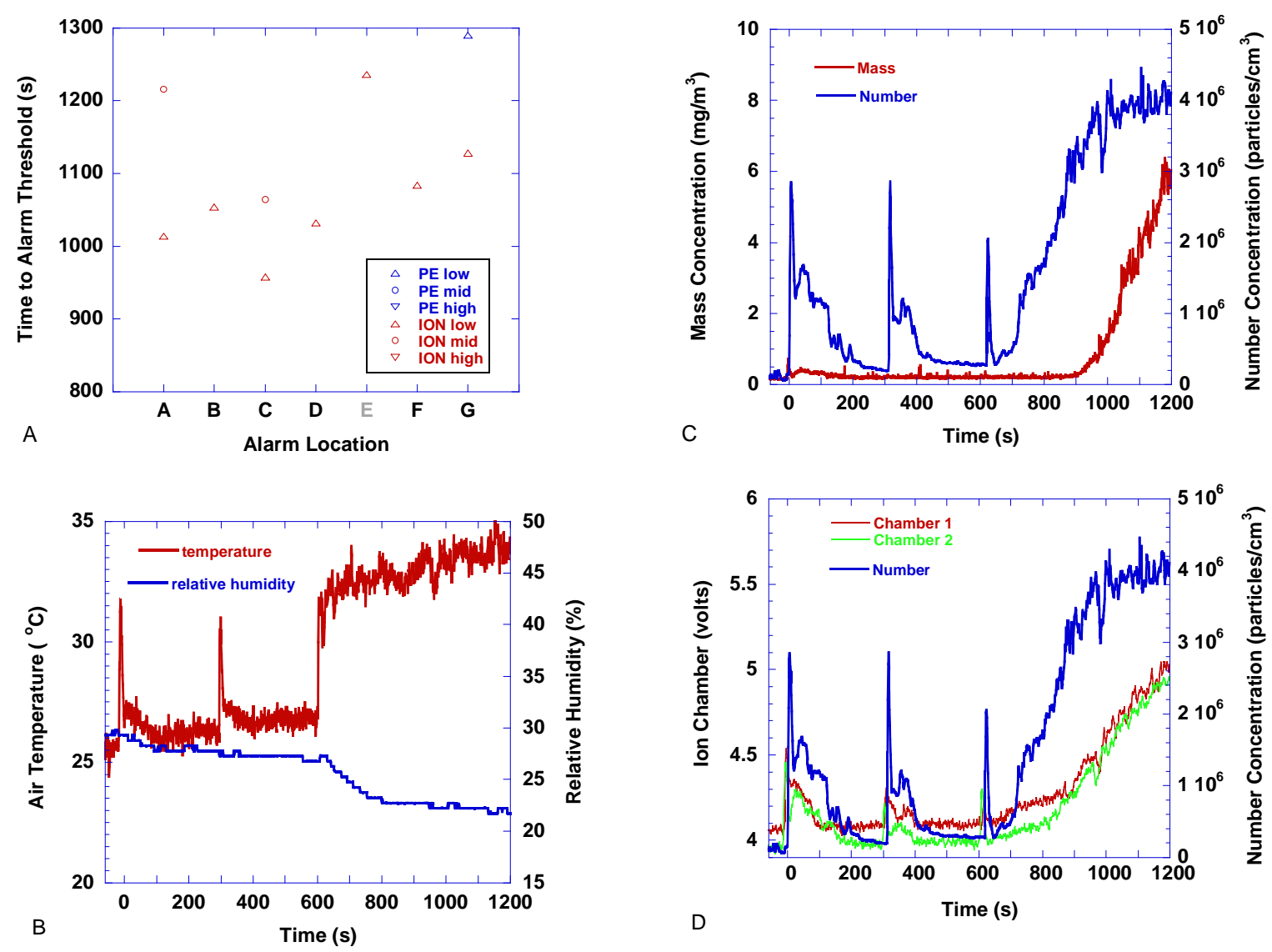

Figure 168. Bake/ broil pizza - one frozen pizza in oven set on bake and pre-heated to $350{ }^{\circ} \mathrm{F}$, then set to broil $630 \mathrm{~s}$ later. Floor fan was off. Pizza in at $\mathrm{t}=0$ 

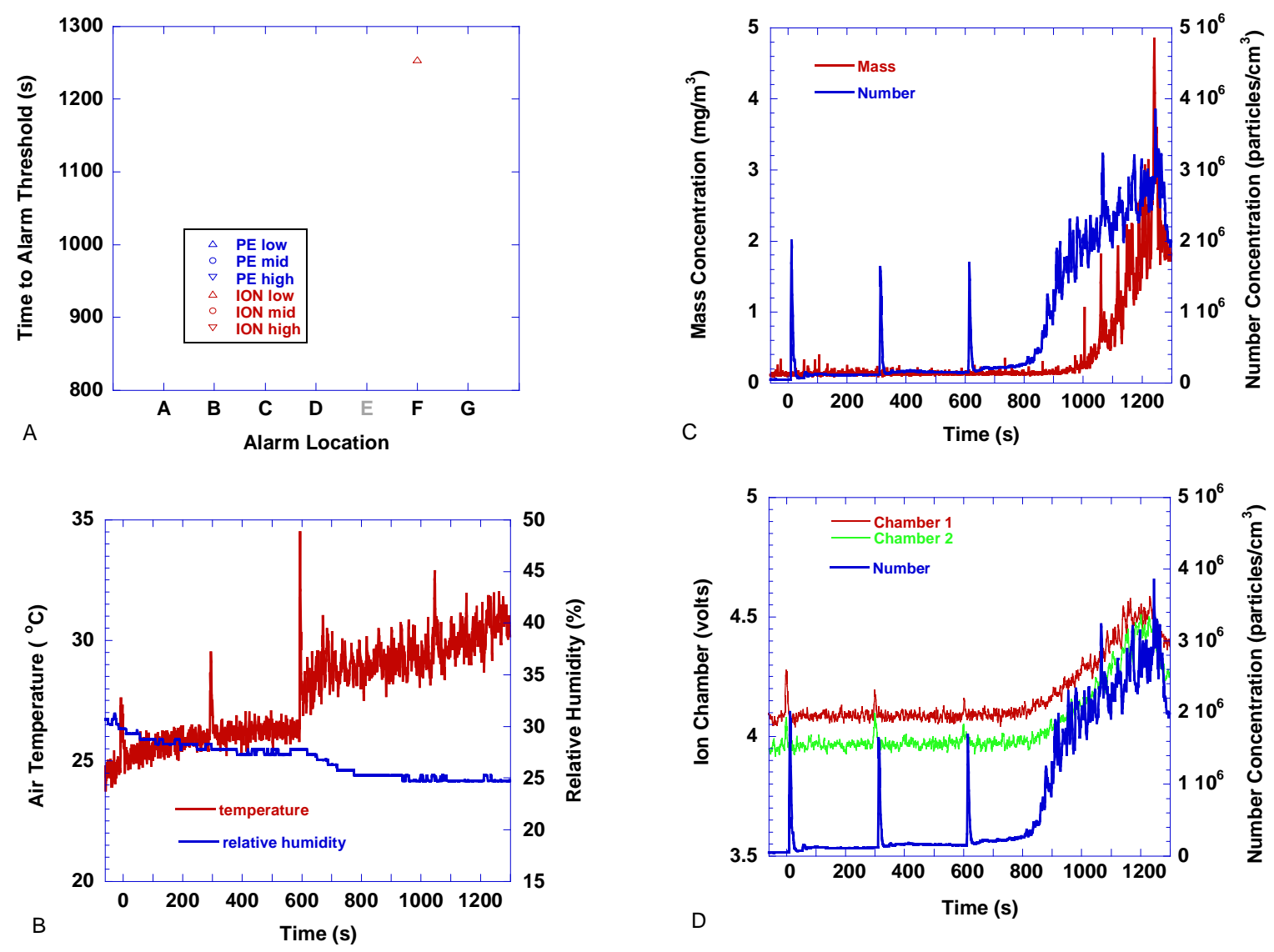

Figure 169. Bake/ broil pizza - one frozen pizza in oven set on bake and pre-heated to $350{ }^{\circ} \mathrm{F}$, then set to broil $630 \mathrm{~s}$ later. Floor fan was on. Pizza in at $\mathrm{t}=0$

The following generalizations characterize the broiling, and bake/broil scenarios:

- $\quad$ Every time the pre-heated oven was opened, a wave of heat and small particles spill out. Forced ventilation dilutes this source.

- Ionization alarm thresholds were reached more frequently than photoelectric alarms.

- Aerosol mass concentration is not significant until the cheese starts to brown on the pizzas.

- $\quad$ Forced flow significantly reduced the number of alarm thresholds reached. 


\subsubsection{Broiling Hamburgers}

Four $110 \mathrm{~g}$ (quarter pound) frozen hamburgers were placed on an aluminum foil covered broiler pan and placed in the oven. With the oven door open to the broil position, the oven was set to high broil. After $10 \mathrm{~min}$, the hamburgers were flipped, cooked for 5 to 6 min longer, then removed and the oven turned off.

Figure 170 shows the results for the case with the floor fan off. All ionization alarm locations reached alarm thresholds, while four out of six photoelectric alarms reached thresholds. Four ionization thresholds were reached before the hamburgers were flipped at $600 \mathrm{~s}$. The air temperature started to rise at $200 \mathrm{~s}$ and climbed steadily to about $6{ }^{\circ} \mathrm{C}$ above the ambient temperature. The relative humidity did not change appreciably. The number concentration started to rise at about $200 \mathrm{~s}$ and reached a peak of about $4 \times 10^{6}$ particles $/ \mathrm{cm}^{3}$ by the end of the test. The mass concentration started to rise at $500 \mathrm{~s}$ and reached a peak value of about $25 \mathrm{mg} / \mathrm{m}^{3}$ near the end of the test. The ionization chambers followed the number concentration and reached peaks about $1 \mathrm{~V}$ above the initial values.

Figure 171 shows the results for the case with the floor fan on. All of the ionization alarms reached thresholds, while five out of 6 photoelectric alarms reached thresholds. The air temperature climbed about $3{ }^{\circ} \mathrm{C}$ except for a short spike near the end of the test. The relative humidity remained at the initial value. The number concentration started to rise at $200 \mathrm{~s}$ and reached a peak at $800 \mathrm{~s}$ of nearly $5 \times 10^{6}$ particles $/ \mathrm{cm}^{3}$. The mass concentration started to rise at about $400 \mathrm{~s}$ and between $800 \mathrm{~s}$ and $1000 \mathrm{~s}$ reached spiked to about $15 \mathrm{mg} / \mathrm{m}^{3}$. Ionization chamber 2, which is closer to the source, started to rise at about $200 \mathrm{~s}$, while chamber 1 started to rise at about $300 \mathrm{~s}$. Chamber 2 voltage showed two peaks at $600 \mathrm{~s}$ and $800 \mathrm{~s}$ corresponding to peaks in the number concentration. By the end of the test, chamber 1 voltage had rise by about $0.9 \mathrm{~V}$ and chamber 2 had risen by about $1.1 \mathrm{~V}$.

The following generalizations characterize the broiling hamburger tests:

- More ionization alarm thresholds were reached than photoelectric thresholds.

- The forced airflow case saw more photoelectric alarm locations reaching thresholds than the no forced flow case.

- Number concentration in both tests started to increase $200 \mathrm{~s}$ before an appreciable rise in the mass concentration, suggesting smaller particles being produced in the early cooking stage. 

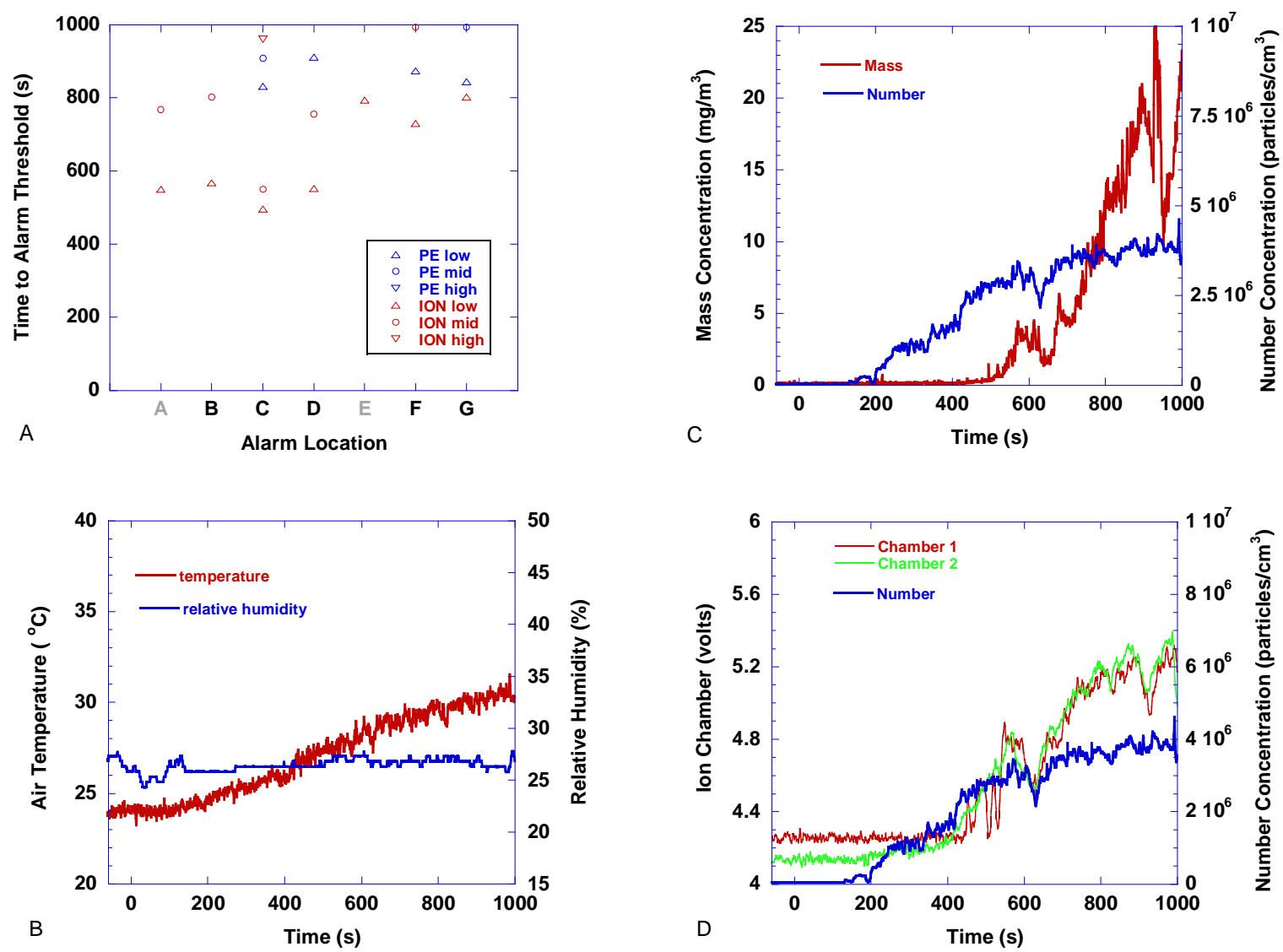

Figure 170. Broiling hamburgers - four hamburgers in oven set on broil, floor fan off. Oven on at $t=0$ 

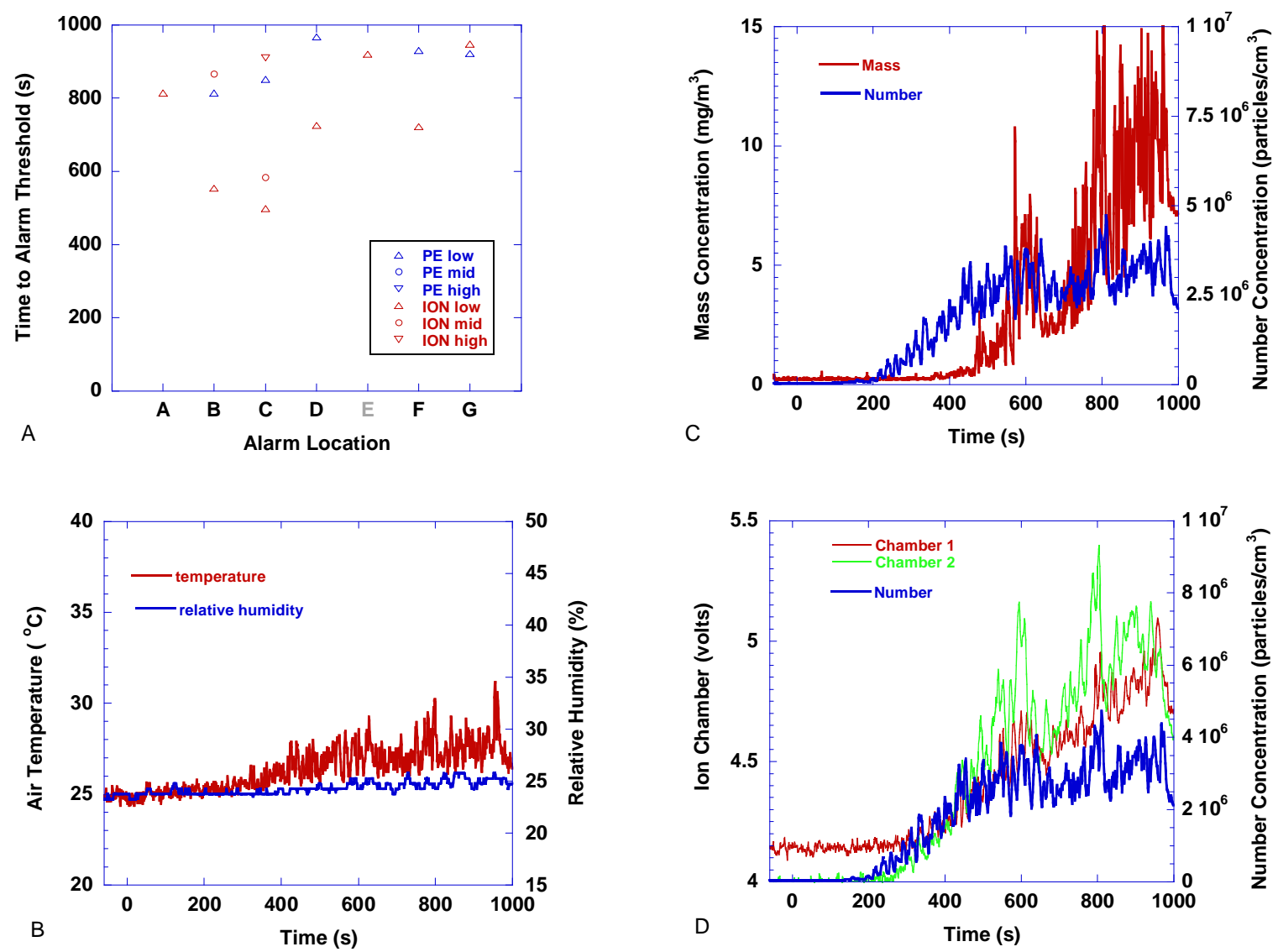

Figure 171. Broiling hamburgers - four hamburgers in oven set on broil, floor fan on. Oven on at $\mathrm{t}=0$

\subsubsection{Boiling Spaghetti Pasta}

In a "two quart" non-stick pot of boiling water, between $1 / 3$ and $2 / 3$ of a $454 \mathrm{~g}(1 \mathrm{lb})$ package of dry spaghetti pasta was cooked for $10 \mathrm{~min}$ to $13 \mathrm{~min}$. The water-filled pot was placed on the large electric burner element. The burner was set on high and, after the water began to boil, the pasta was added. In one variation, a tight-fitting lid was placed on the pot with the intention of causing the boiling water to spill over.

Figure 172 shows the results for the case with 1/3 package of spaghetti. A single ionization alarm threshold was reached at the location nearest to the source. The air temperature rose about $5{ }^{\circ} \mathrm{C}$ during the course of the test. The relative humidity rose from an initial value of $17 \%$ to 

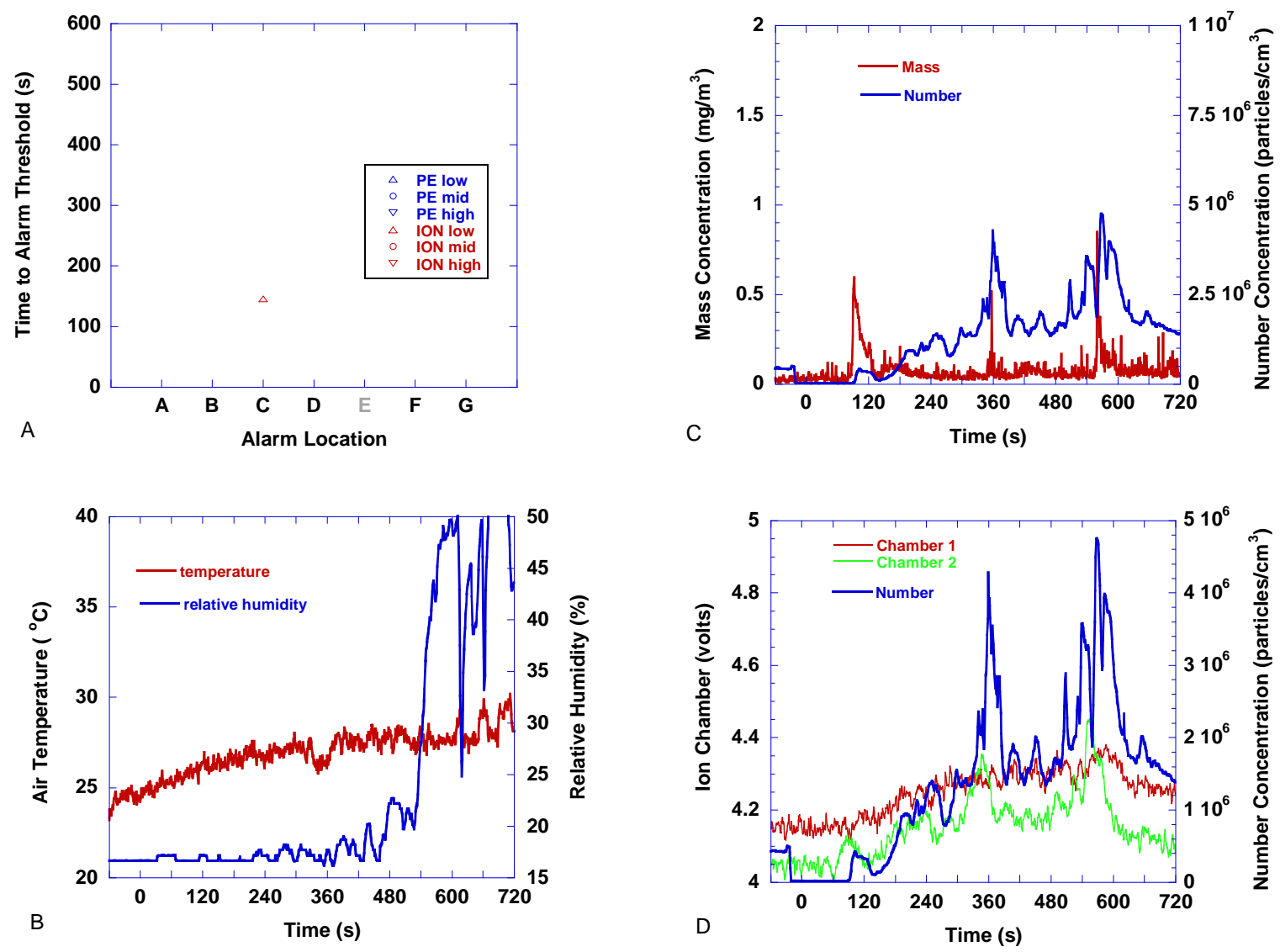

Figure 172. Boiling spaghetti pasta - 1/3 package (150 g), floor fan off.

Pasta placed in pot at $\mathrm{t}=360 \mathrm{~s}$

over $50 \%$ during the test. The number concentration started to climb at $120 \mathrm{~s}$ reaching a peak of $4 \times 10^{6}$ particles $/ \mathrm{cm}^{3}$ when the pasta was added, followed by another peak of $5 \times 10^{6}$ particles $/ \mathrm{cm}^{3}$ at $560 \mathrm{~s}$. The mass concentration shows a spike at $100 \mathrm{~s}$ possibly due to material on the burner element being driven off. The was a spike at $360 \mathrm{~s}$ when the pasta was added, and again a spike $560 \mathrm{~s}$; all spikes were below $1 \mathrm{mg} / \mathrm{m}^{3}$. Ionization chamber 2 followed the number concentration trend, peaking at $360 \mathrm{~s}$ and $560 \mathrm{~s}$, with a maximum value of $0.4 \mathrm{~V}$ above the initial value. Chamber 1 increased about $0.2 \mathrm{~V}$ above its initial value.

Figure 173 shows the results for the case with $2 / 3$ of a package of pasta. Only one ionization threshold was met at the location closest to the source, before the pasta was added. The temperature and relative humidity trends were the same as observed in the test above. The number concentration started to rise at about $180 \mathrm{~s}$, reaching a plateau at $360 \mathrm{~s}$ of $1.5 \times 10^{6}$ particles $/ \mathrm{cm}^{3}$, then spiking to a value of $3.7 \times 10^{6}$ partcles $/ \mathrm{cm}^{3}$ at $610 \mathrm{~s}$. The mass concentration 

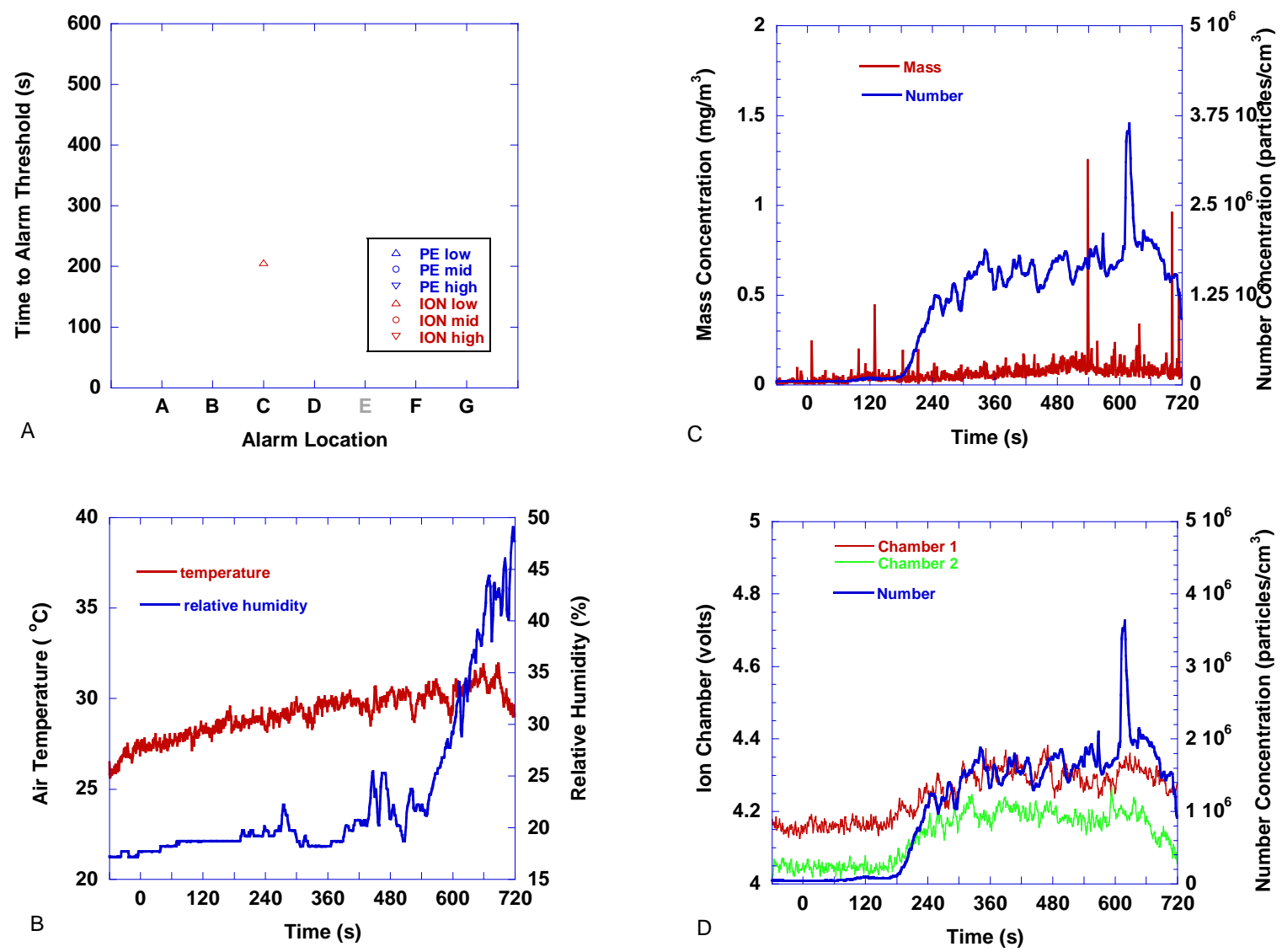

Figure173. Boiling spaghetti pasta - 2/3 package (300 g), floor fan off.

Pasta placed in pot at $\mathrm{t}=450 \mathrm{~s}$

showed only one spike greater than $1 \mathrm{mg} / \mathrm{m}^{3}$ at $540 \mathrm{~s}$. The ionization chambers followed the number concentration trend (except for the spike at $610 \mathrm{~s}$.) Chamber 1 and 2 increased approximately $0.1 \mathrm{~V}$ and $0.2 \mathrm{~V}$ above their initial values, respectively.

Figure 174 shows the results for the case with $1 / 2$ of a package of pasta cooked with the lid on. Only one ionization alarm reached a threshold, the alarm located closest to the source, $120 \mathrm{~s}$ after the pasta was added. The number concentration started to rise at $100 \mathrm{~s}$ climbing to a average value of $2.5 \times 10^{6}$ particles $/ \mathrm{cm}^{3}$ by $400 \mathrm{~s}$. At $625 \mathrm{~s}$ the number concentration spiked to nearly $5 \times 10^{6}$ particles $/ \mathrm{cm}^{3}$, then dropped to about $2 \times 10^{6}$ particles $/ \mathrm{cm}^{3}$. This spike is attributed to boil-over of the pot. The mass concentration, though elevated to about $0.5 \mathrm{mg} / \mathrm{m}^{3}$ initially, experienced only one peak above $1 \mathrm{mg} / \mathrm{m}^{3}$ at $530 \mathrm{~s}$. The lid was removed at $940 \mathrm{~s}$. Its removal did not appear to change room conditions. 

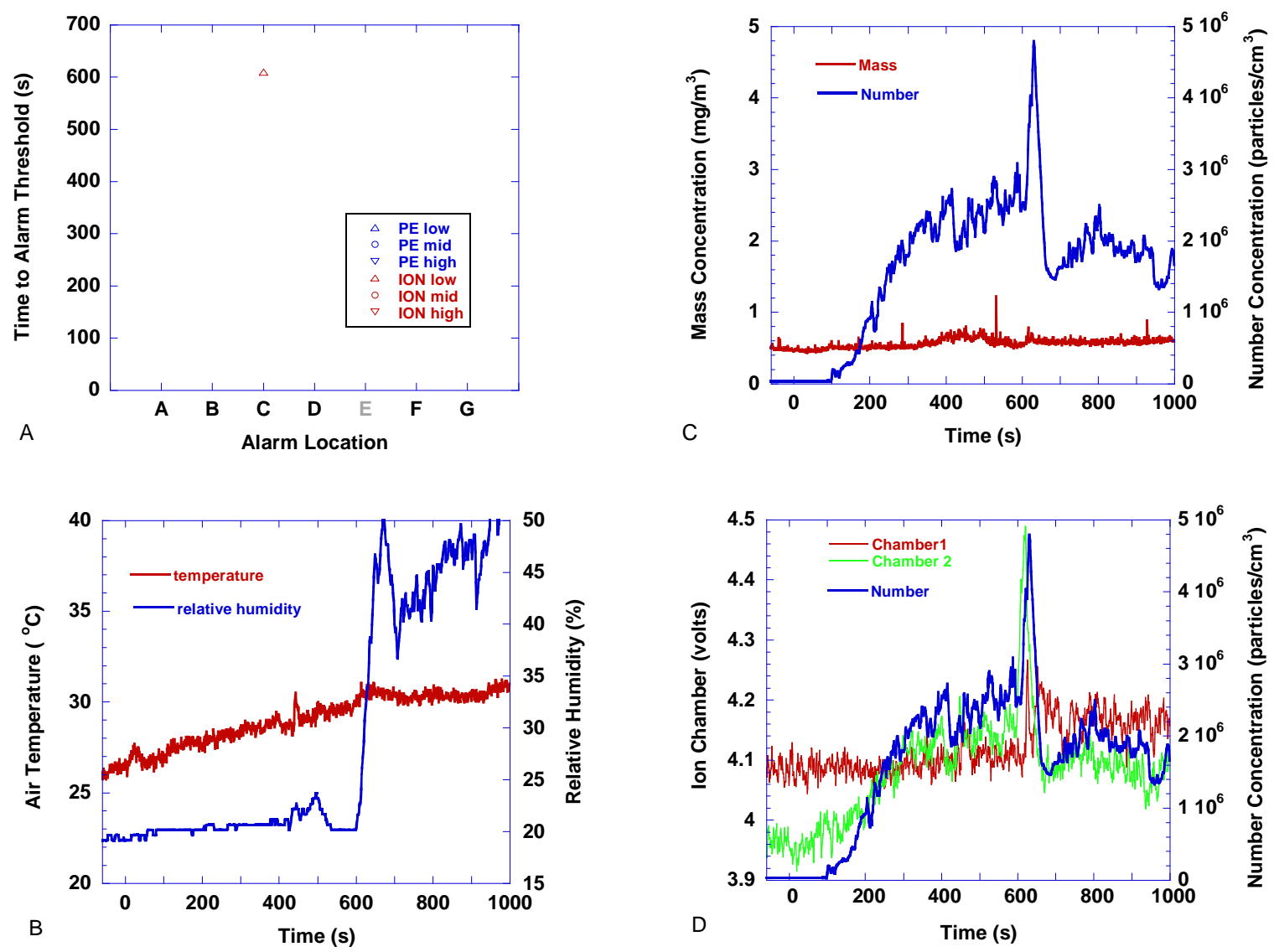

Figure 174. Boiling spaghetti pasta - 1/2 package (225 g), lid on, floor fan off.

Pasta placed in pot at $\mathrm{t}=480 \mathrm{~s}$

Since only one alarm threshold was reached in each test, and all attributed to the alarm closest to the source, this scenario was not particularly challenging. However, the aerosol measurements do suggest that the electric heating element on a high setting produces aerosols. It is assumed that the cleanliness of the element and the pan surface in contact with it affect this aerosol production. In general, the boiling of the pasta in and of itself did not account for much aerosol production unless boil-over of the pot occurs, in which case the pasta water splashing on the hot surfaces appears to cause more aerosol formation. 


\subsubsection{Candle Burning}

Four scented tea candles were placed on the counter top to the right of the stove and lit with a butane lighter. The candles burned for $15 \mathrm{~min}$, then they were extinguished. There was no forced flow during this test.

Figure 175 shows the results for this test. No alarm thresholds were reached during this test. Since there was no forced airflow, the candle flames were laminar, with no flickering. Air temperature rose about $2{ }^{\circ} \mathrm{C}$, and the relative humidity dropped by about $2 \%$ during the test. The number concentration spiked at about $50 \mathrm{~s}$ to $5 \times 10^{5}$ particles $/ \mathrm{cm}^{3}$, which is attributed to the butane lighter and the candle ignitions. Around $300 \mathrm{~s}$ the number concentration rose sharply for $1 \times 10^{5}$ particles $/ \mathrm{cm}^{3}$ to $6 \times 10^{5}$ particles $/ \mathrm{cm}^{3}$. This steep rise is most likely due to the ceiling jet
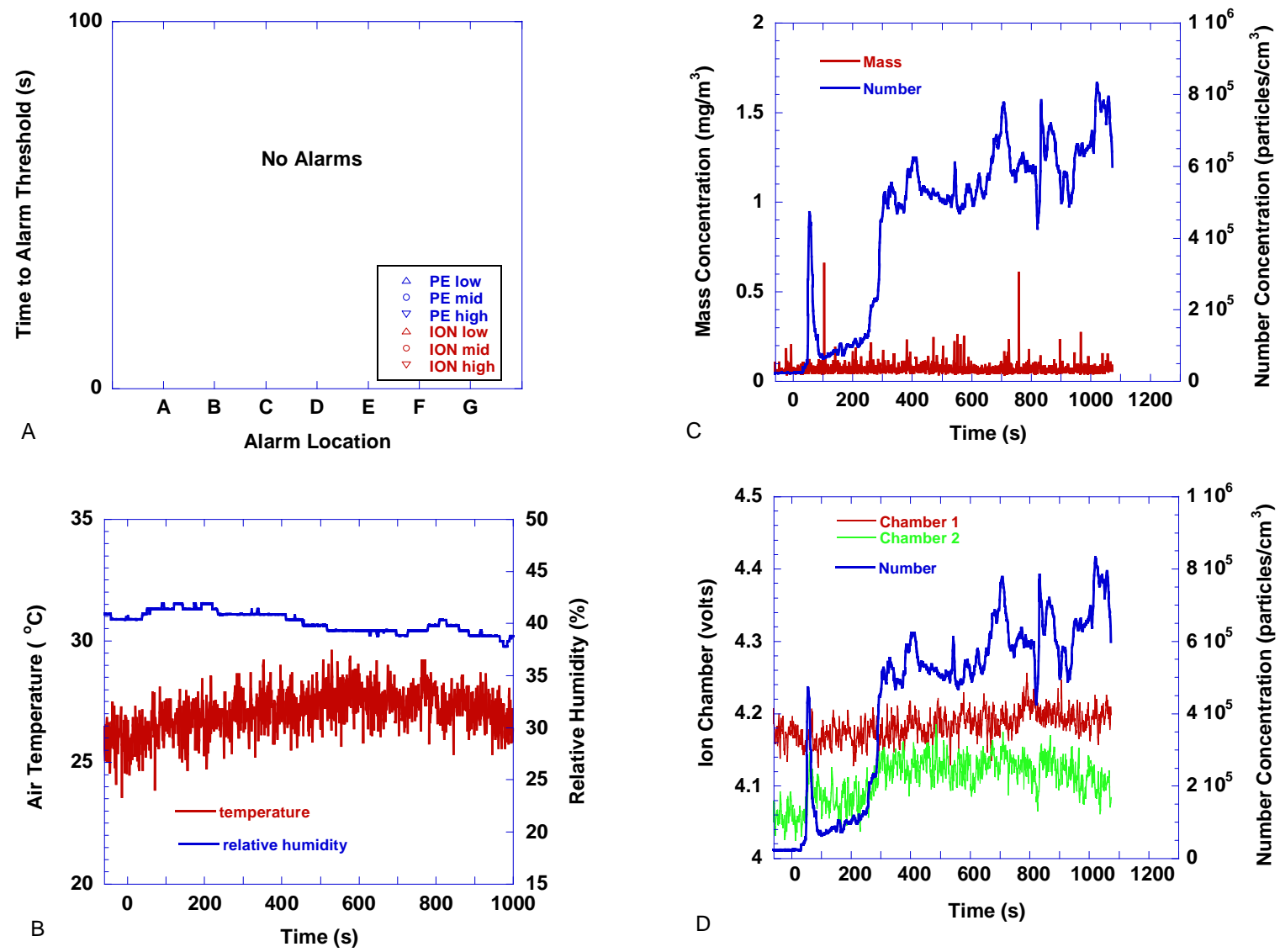

Figure 175. Candles burning - four tea candles burning on electric range top area.

Floor fan was off. Candles lit at $\mathrm{t}=0$ 
from the plume formed by the burning candles reaching the aerosol sampling location. The number concentration peaked at about $8 \times 10^{5}$ particles $/ \mathrm{cm}^{3}$ near the end of the test. No appreciable mass concentration was recorded; two spikes are attributed to local airborne dust or other background aerosol. Ionization chamber 2 voltage rose about $0.1 \mathrm{~V}$ above its initial value, while, chamber 1's voltage rose by a factor of 3 less.

Clearly, this scenario poses little threat for producing nuisance alarms. It is not clear if flickering candle flames or the presence of additives in the candles would produce enough aerosol to be a significant nuisance alarm threat.

\subsubsection{Cigarette Smoking}

Two persons seated in the kitchen area proceeded to smoke one cigarette each. In approximately $4 \mathrm{~min}$, the smokers finished their cigarettes and exited the manufactured home, closing the door behind them. In both tests, there was no forced air flow.

Figures 176 and 177 show the results for these two tests. In the first test, no alarm thresholds were reached, while in the second test, the ionization alarm located nearest to the smokers did reach two thresholds near the end of the smoking period. Little change in air temperature or relative humidity was observed during both tests. The maximum number concentration reached was $1.45 \times 10^{5}$ particles $/ \mathrm{cm}^{3}$ and $8.5 \times 10^{4}$ particles $/ \mathrm{cm}^{3}$ for the first and second test. Mass concentration peaks of $2.5 \mathrm{mg} / \mathrm{m}^{3}$ and $3 \mathrm{mg} / \mathrm{m}^{3}$ were recorded in the first and second tests. Neither ionization chamber voltage changed significantly from the background mean values during both tests.

It is surprising that the ionization alarms were recorded during the second test, while no indications of a significant level of aerosol was recorded by the particle counter nor ionization chamber 2. The mass concentrations during both tests appear to be approaching threshold levels for photoelectric alarms, suggesting repeated smoking, or more smokers could produce threshold level values.

\subsection{Controlled Incipient Fire Sources}

The following incipient fire sources were performed to provide comparative results and put the nuisance alarm tests into context with the FE/DE tests. The cotton smolder and pyrolyzing wood blocks tests, are variants of EN 54 fire sensitivity test sources. The cotton smolder source was the same as used for the FE/DE tests discussed in Chapter 2. Thus, these data are mainly useful to determine the differences in detector response due to the layout of the building and resulting flow patterns during the tests. In addition, the data show the detector responses at various locations throughout the manufactured home. 

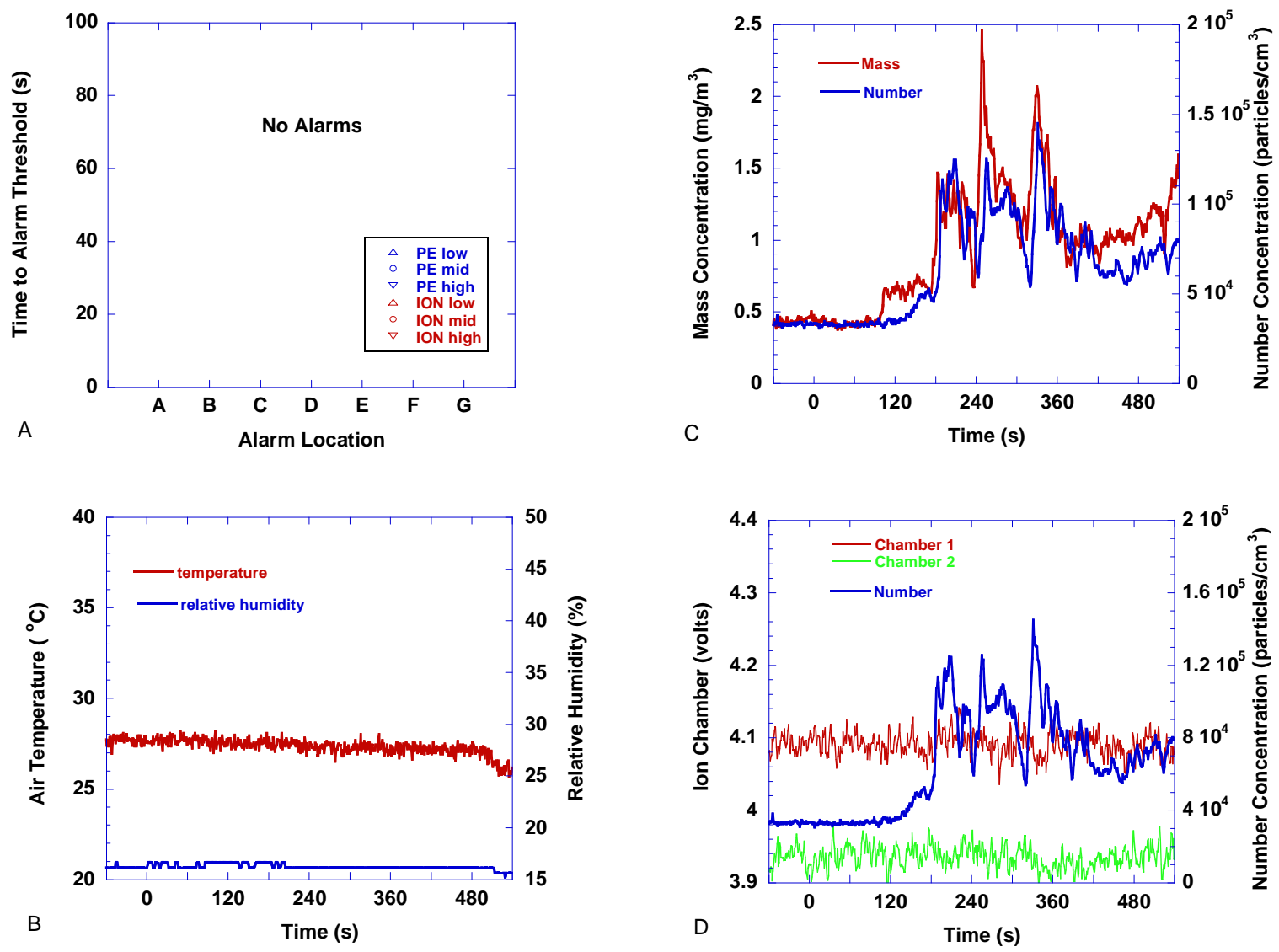

Figure 176. Cigarette smoking - two smokers smoking one cigarette each in kitchen area. Floor fan was off. Cigarettes lit at $\mathrm{t}=0$

\subsubsection{Cotton Smolder}

The staged wick ignition device was used to produce cotton smolder smoke. This is the same source used in the FE/DE to calibrate all of the modified analog output smoke alarms. 32 cotton wicks were placed in the staged wick ignition device, which was then set on the chair-burn load cell pan located in the living room area. After an initial $30 \mathrm{~s}$ delay, 8 sets of 4 wicks were ignited with a $12 \mathrm{~s}$ delay time between sets. The wicks smoldered for $30 \mathrm{~min}$ then the doors to the manufactured home were opened. The test was run with the floor fan off and repeated with the floor fan on. 

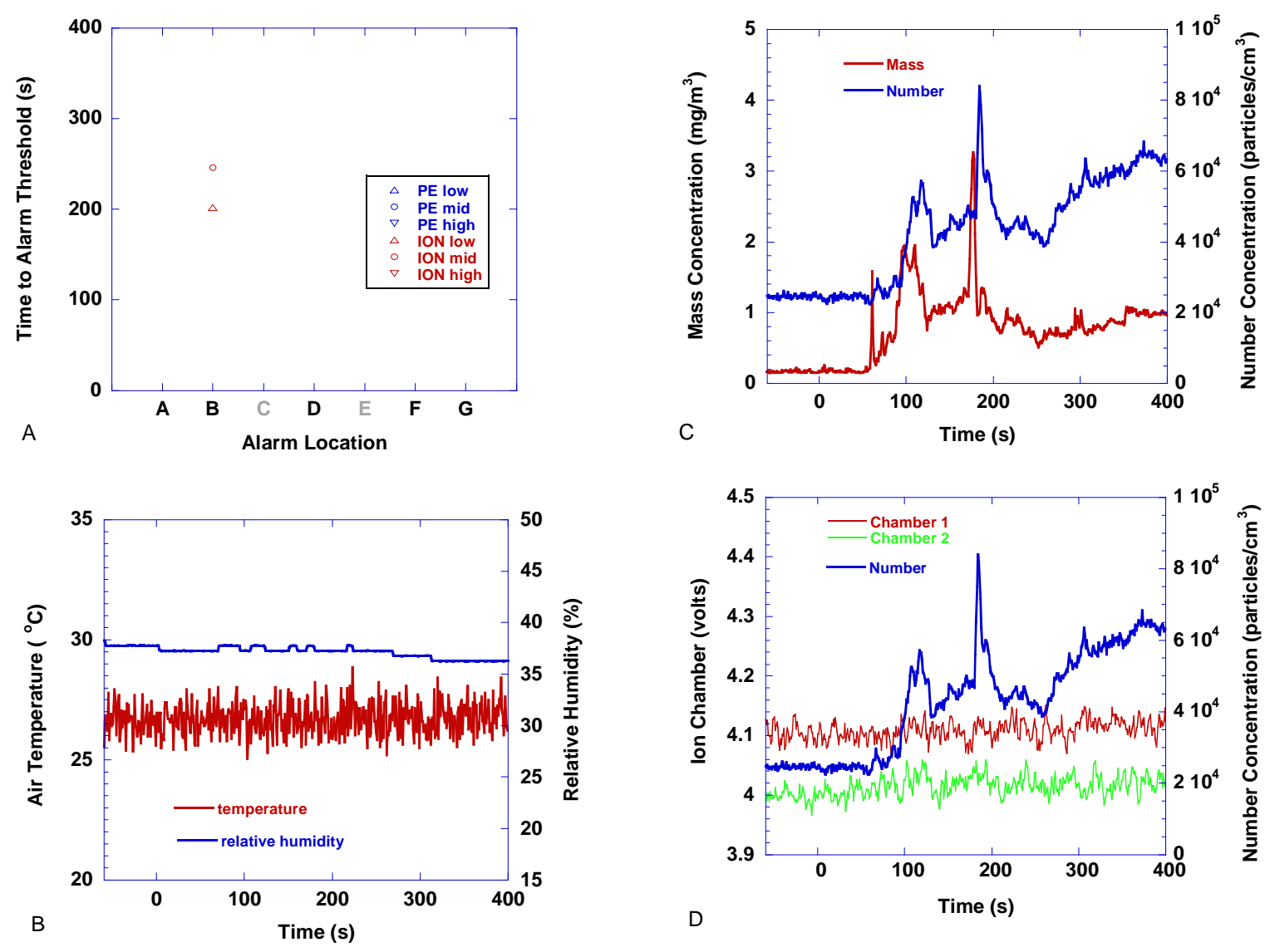

Figure 177. Cigarette smoking - two smokers smoking one cigarette each in kitchen area. Floor fan was off. Cigarettes lit at $\mathrm{t}=0$

Figure 178 shows the results for the case with the floor fan off. All ionization alarms reached threshold levels, and all but one before $300 \mathrm{~s}$. Five out of six photoelectric alarms reached threshold levels, the two closest to the source (location B) reached thresholds sooner. The air temperature rise was about $1{ }^{\circ} \mathrm{C}$, while the relative humidity dropped about $3 \%$. The mass and number concentration both start to rise at $120 \mathrm{~s}$ and reach local peak values before $300 \mathrm{~s}$ of 30 $\mathrm{mg} / \mathrm{m}^{3}$ and $1.2 \times 10^{6}$ particles $/ \mathrm{cm}^{3}$. The wick smoldering rate is highest soon after ignition. After a wick smolders away from its ignition coil smoldering proceeds at a steady rate reaching values of approximately $35 \mathrm{mg} / \mathrm{m}^{3}$ and $1.25 \times 10^{6}$ particles $/ \mathrm{cm}^{3}$. The early peaks in the number and mass concentrations are due the higher smoldering rates of the wicks soon after ignition. After dropping from the peak values, the number and mass concentration continued to climb for the duration of the test. The ionization chamber voltages followed the number concentration results, 
realizing the early peak, then climbing for the remainder of the test. By the end of the test, the voltage change for each chamber was nearly $1 \mathrm{~V}$.
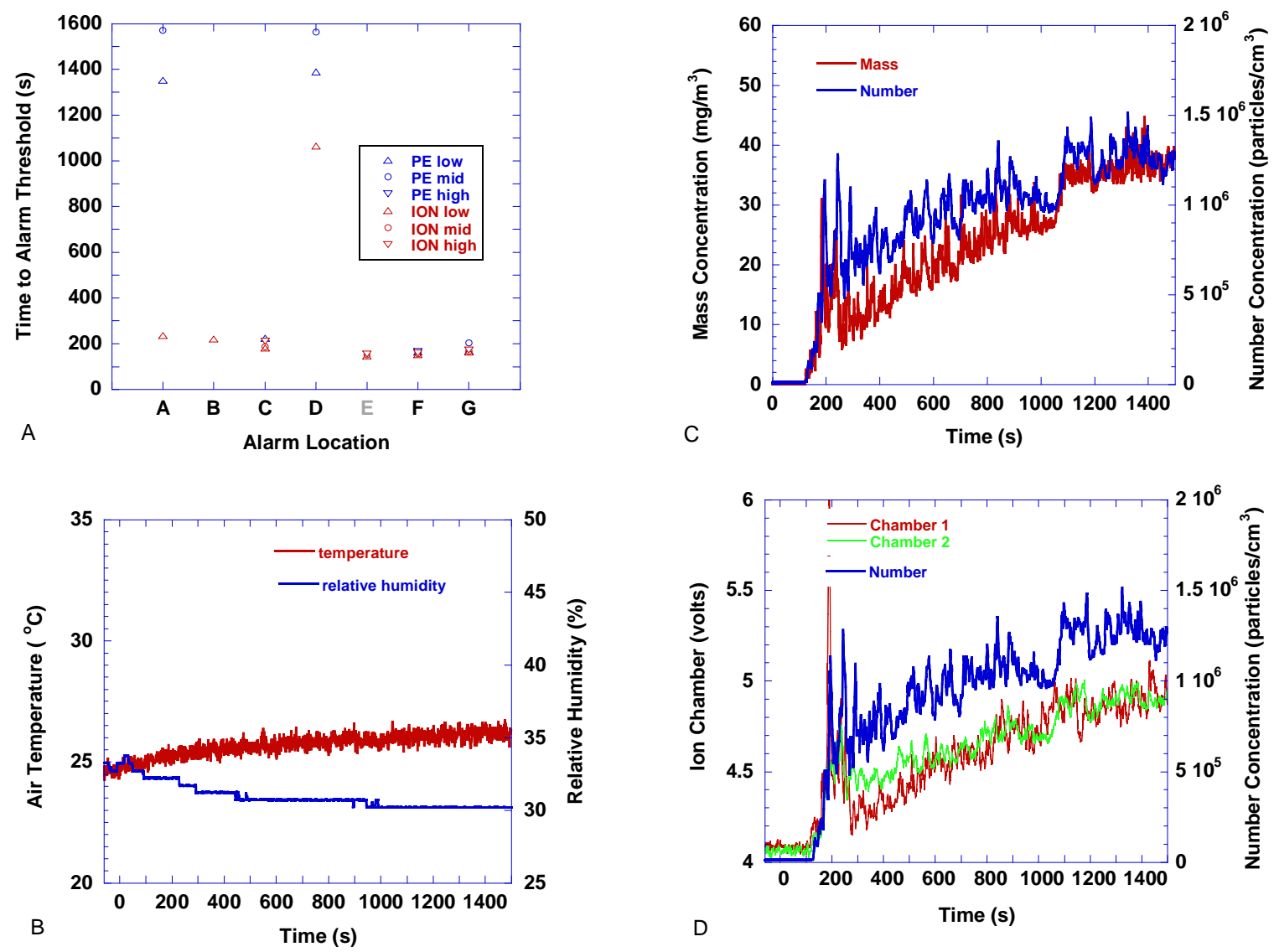

Figure 178. Smoldering cotton wicks - eight sets of four wicks were ignited with a 12 s delay using the staged wick ignition device, located on the living room floor at the chair burn location. Fan was off. Ignition sequence started at $\mathrm{t}=30 \mathrm{~s}$

Figure 179 shows the results for the case with the floor fan on. Again, all ionization alarms reached threshold levels. There is clearly a relationship between the time to reach a threshold and the distance from the source; alarms closer to the source reach threshold levels first. Only three photoelectric alarms reached threshold levels, with the alarm closest to the source $(\mathrm{F})$ reaching it first. The air temperature rose by about $2{ }^{\circ} \mathrm{C}$ and the relative humidity dropped by about $5 \%$ during the test. Contrary to what was observed in the smoldering wick test without forced flow, there were no early peaks in the mass or number concentration. At $100 \mathrm{~s}$ the mass and number concentration start to rise. From $300 \mathrm{~s}$ to the end of the test, the mass concentration increased linearly to $36 \mathrm{mg} / \mathrm{m}^{3}$, while the number concentration increased towards an asymptote 
of approximately $1.1 \times 10^{6}$ particles $/ \mathrm{cm}^{3}$. Initially, the ionization chambers follow the number concentration trend, but from $800 \mathrm{~s}$ on they continue to climb while the number concentration starts to level off. This observation, along with the steady rise in the mass concentration suggests that the mean particle size was increasing during the test.
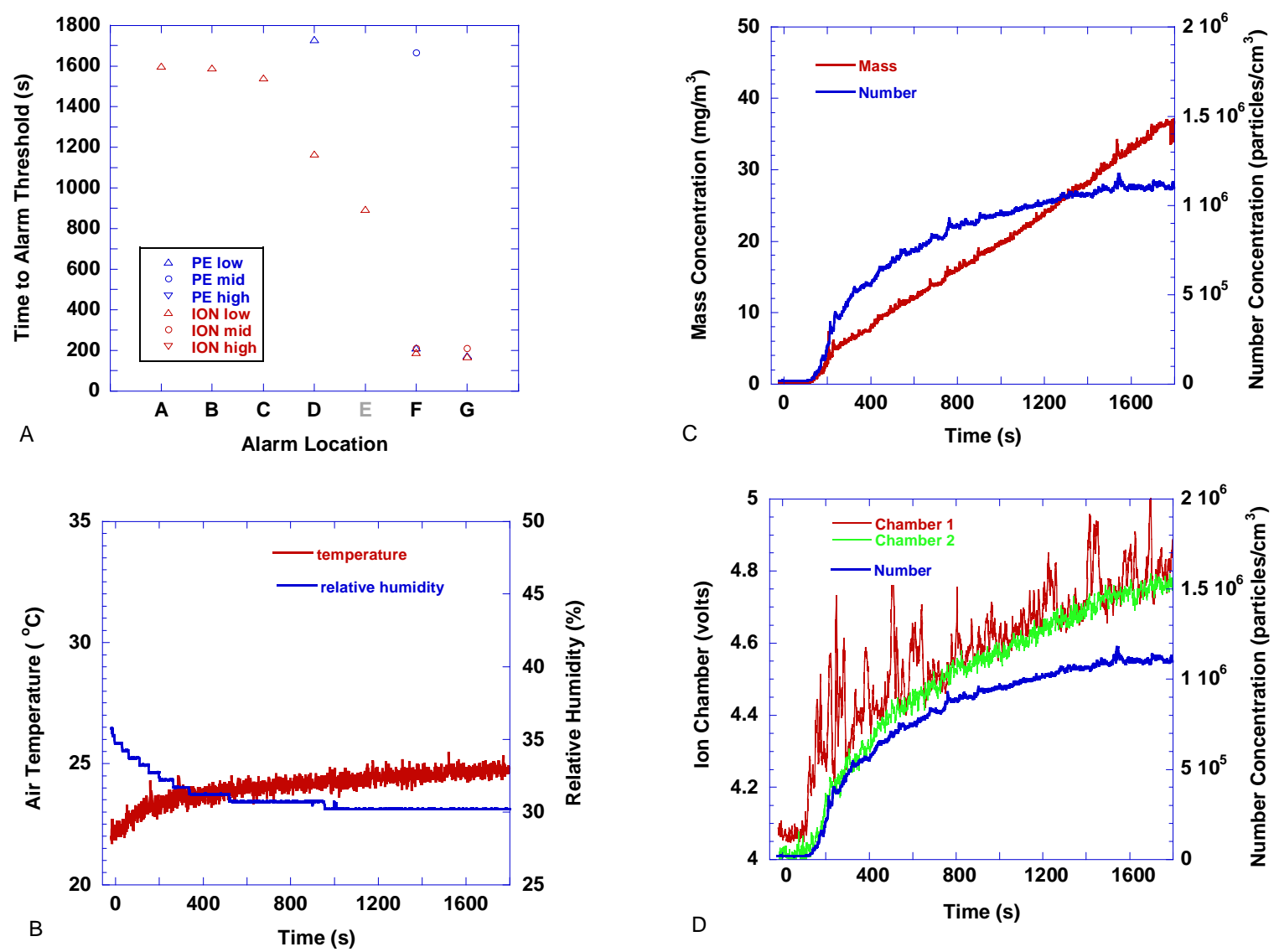

Figure 179. Smoldering cotton wicks -eight sets of four wicks were ignited with a $12 \mathrm{~s}$ delay using the staged wick ignition device, located on the living room floor at the chair burn location.

Fan was on. Ignition sequence started at $\mathrm{t}=30 \mathrm{~s}$

The following generalizations characterize the smoldering cotton scenarios:

- Ionization alarm thresholds were reached earlier and at more locations than photoelectric alarm thresholds.

- The mass and number concentration by the end of the test were nearly the same for both cases. 
- A somewhat counterintuitive observation that in the case with the floor fan on, the time to reach a threshold level for ionization alarms was a strong function of the distance from the source, while in the case with the floor fan off, the time to reach ionization threshold levels occurred nearly simultaneously for six of seven alarms.

\subsubsection{Wood Smolder}

Eight $3.5 \mathrm{~cm} \times 2.0 \mathrm{~cm} \times 1.0 \mathrm{~cm}$ beech wood blocks were placed on a $750 \mathrm{~W}$ electric hot plate that was placed on the chair-burn load cell. Full power was applied to the hot plate. Within about $500 \mathrm{~s}$, the blocks started to pyrolyze. After $20 \mathrm{~min}$, the power to the hot plate was removed. The test was performed with the floor fan off and repeated with the floor fan on.

Figure 180 shows the results for the case with the floor fan off. All six photoelectric alarms reached threshold values, with the ones closer to the source tending to reach thresholds first. Four out of seven ionization alarms reached threshold values, and where three were collocated with photoelectric alarms, the ionization alarms took longer to reach threshold values. Only a very slight increase in air temperature, and decrease in relative humidity was observed. The number concentration started to rise at $550 \mathrm{~s}$, while the mass concentration started to rise appreciably at $700 \mathrm{~s}$. The number concentration peaked at about $1 \times 10^{6}$ particles $/ \mathrm{cm}^{3}$ at $1350 \mathrm{~s}$ while the mass concentration reached a peak of about $65 \mathrm{mg} / \mathrm{m}^{3}$ at $1200 \mathrm{~s}$. The ionization chambers followed the rise in number concentration, and reached voltage change peaks of $0.7 \mathrm{~V}$.

Figure 181 shows the results for the case with the floor fan on. Five out of six photoelectric alarms reached threshold values, the one farthest from the source did not. Four out of seven ionization alarms reached threshold values, again, after photoelectric thresholds were met. Similar to the test above, only a very slight increase in air temperature, and decrease in relative humidity was observed. The number concentration started to rise at $500 \mathrm{~s}$, peaking at $4 \times 10^{5}$ particles $/ \mathrm{cm}^{3}$, while the mass concentration started to rise at $700 \mathrm{~s}$, peaking at $38 \mathrm{mg} / \mathrm{m}^{3}$. Ionization chamber 1 started to rise noticeably at $800 \mathrm{~s}$, and peaking at about 1 volt above the initial voltage at $1250 \mathrm{~s}$. Chamber 2 started to rise at $1000 \mathrm{~s}$ and peaked $0.3 \mathrm{~V}$ above the initial voltage at $1500 \mathrm{~s}$.

The following generalizations characterize the smoldering wood blocks tests:

- Photoelectric alarms reached thresholds earlier and at more locations than ionization alarms.

- The relatively high mass concentration and low number concentration levels suggest a mean particle size larger than the smoldering cotton source. 

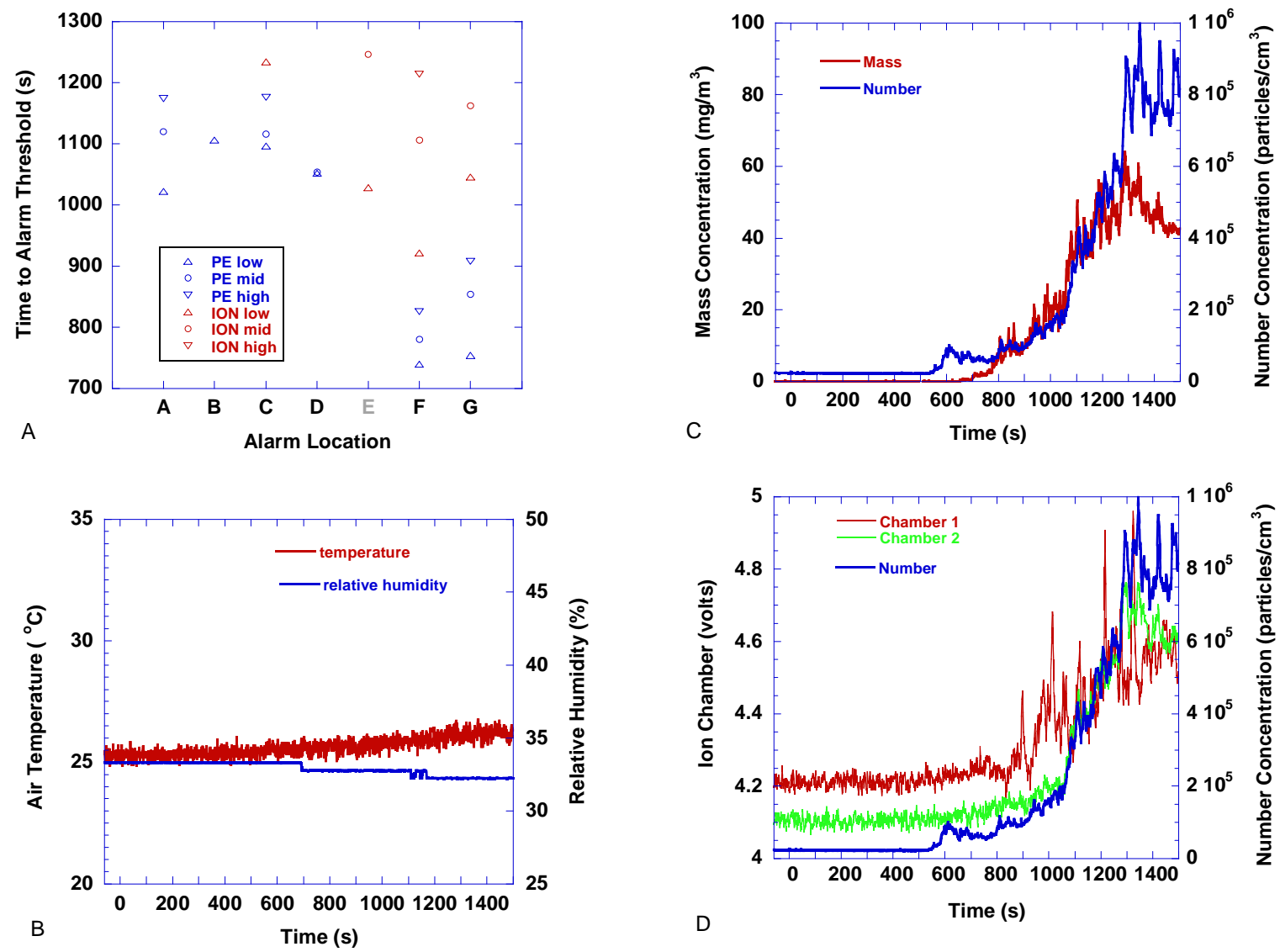

Figure 180. Smoldering wood blocks - eight beech wood blocks on an electric hotplate, located on the living room floor at the chair burn location. Fan was off. Power to hotplate on at $\mathrm{t}=0$ 

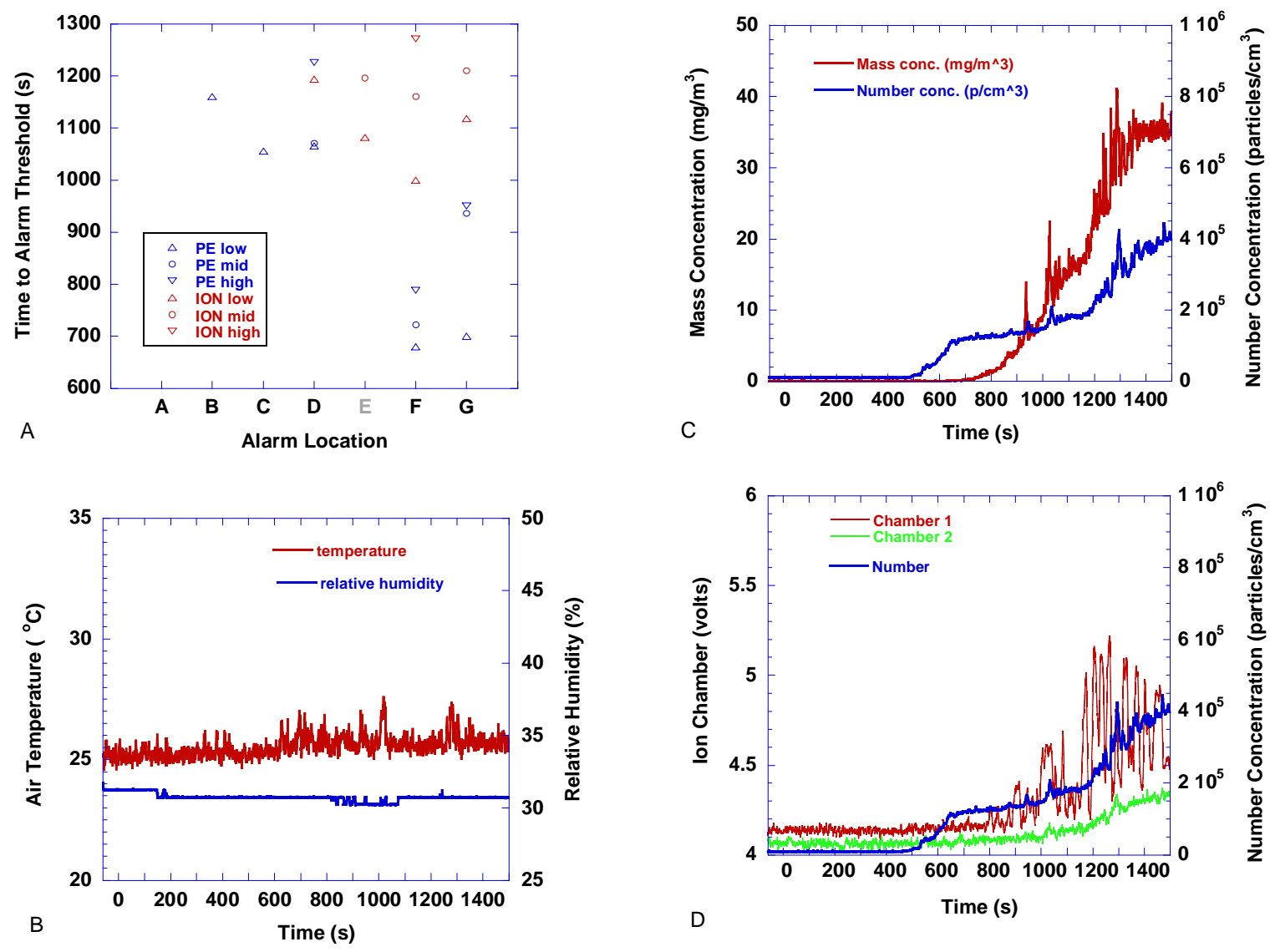

Figure 181. Smoldering wood blocks - eight beech wood blocks on an electric hotplate, located on the living room floor at the chair burn location. Fan was on. Power to hotplate on at $\mathrm{t}=0$

\subsubsection{Polyurethane Foam Smolder}

Blocks of polyurethane foam, (approximately $8 \mathrm{~cm}$ x $8 \mathrm{~cm}$ x $10 \mathrm{~cm}$ ) were removed from a chair cushion of the same type used in the smoldering chair fire scenario in the Home Smoke Alarm tests. A single block was used in each smolder test. A slit was made in the foam at the center of the $10 \mathrm{~cm}$ high block, and a nichrome heating wire (similar to the one used to ignite the chairs for the smoldering fire tests) was inserted. Electric power was applied to the wire to cause the foam block to smolder in the same manner as the fire tests discussed in section 3.3.2.

Smoldering was allowed to proceed for approximately $15 \mathrm{~min}$. The test was repeated three times with the floor fan off and once with the floor fan on. 
Figure 182 shows the results for the first test run with the floor fan off. Several photoelectric alarm thresholds were reached between $200 \mathrm{~s}$ and $400 \mathrm{~s}$. Around $800 \mathrm{~s}$ all alarm thresholds were reached, this corresponded to a transition from smoldering to flaming of the foam block. The air temperature rose slightly until about $800 \mathrm{~s}$ where it rapidly rose by $10{ }^{\circ} \mathrm{C}$ following flaming of the foam. The flaming period was short lived due to the limited amount of material to burn, and the temperature dropped over the next $100 \mathrm{~s}$. The relative humidity dropped by about $5 \%$ during the test. The mass concentration started to rise at $100 \mathrm{~s}$, and by $250 \mathrm{~s}$ reached a local peak of nearly $40 \mathrm{mg} / \mathrm{m}^{3}$. By $500 \mathrm{~s}$ it had dropped down to about $10 \mathrm{mg} / \mathrm{m}^{3}$, then started rising slowly again until $800 \mathrm{~s}$ when it rapidly shot up to a peak greater than $150 \mathrm{mg} / \mathrm{m}^{3}$. The number concentration started to rise at $100 \mathrm{~s}$ and by $300 \mathrm{~s}$ had reached a value of $1 \times 10^{5}$ particles $/ \mathrm{cm}^{3}$, and remained steady until $800 \mathrm{~s}$ when it rapidly increased to a value of $4 \times 10^{6}$ particles $/ \mathrm{cm}^{3}$. The ionization chambers showed an increase of $0.1 \mathrm{~V}$ from $100 \mathrm{~s}$ to $200 \mathrm{~s}$. They remained unchanged until $800 \mathrm{~s}$ when they shot up rapidly by over $2 \mathrm{~V}$.

Figure 183 shows the results for the second test run with the floor fan off. Two photoelectric alarms reached threshold values before $200 \mathrm{~s}$, but no other alarms did. The block of foam initially smoldered vigorously, but as time went on, it apparently stopped. The test was stopped at $800 \mathrm{~s}$. The air temperature rise was on the order of $2{ }^{\circ} \mathrm{C}$, while the relative humidity dropped by about $5 \%$. The mass concentration started to rise at $100 \mathrm{~s}$ and reached a nominally steady value of $6 \mathrm{mg} / \mathrm{m}^{3}$ at $400 \mathrm{~s}$. The number concentration started to rise at about $80 \mathrm{~s}$ and reached a maximum value of $9 \times 10^{4}$ particles $/ \mathrm{m}^{3}$ by the end of the test. During the test, the increases in the ionization chambers voltages were $0.2 \mathrm{~V}$ and $0.05 \mathrm{~V}$ for chamber 1 and 2 respectively. 

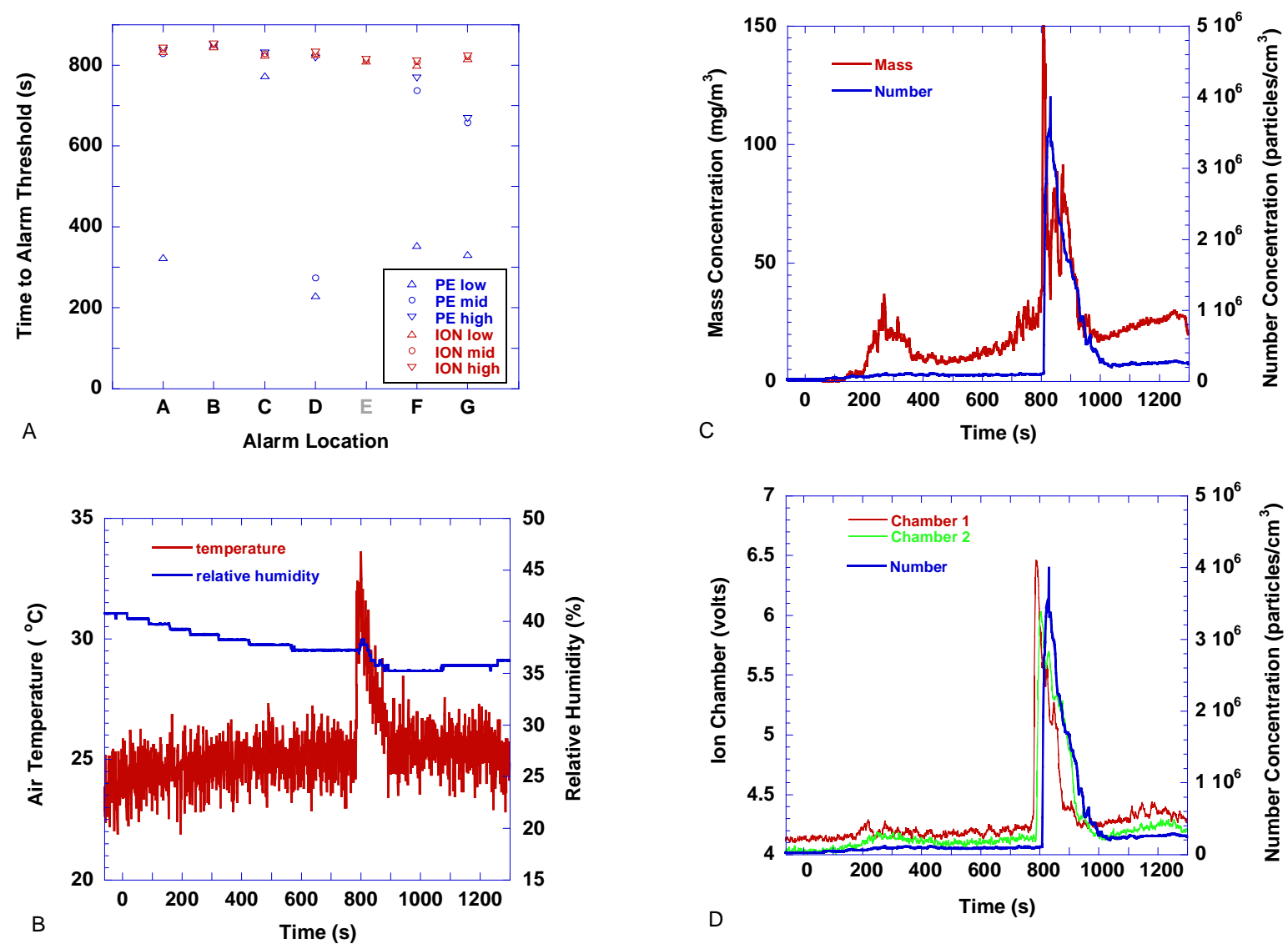

Figure 182. Smoldering foam - A polyurethane foam block, located on the living room floor at the chair burn location and ignited with an electrically powered nichrome wire heater imbedded in the block. Fan was off. Power to igniter on at $\mathrm{t}=0$. Sample flamed at $798 \mathrm{~s}$

Figure 184 shows the results for the third test run with the floor fan off. Three out of six photoelectric alarms reached threshold values, the three closest to the source. Air temperature rise was on the order of a couple of degrees Celsius, while the relative humidity dropped by about $5 \%$ during the test. The mass concentration started to rise steadily at $100 \mathrm{~s}$ and reached a plateau of $13 \mathrm{mg} / \mathrm{m}^{3}$ at $700 \mathrm{~s}$. The number concentration started to increase around $50 \mathrm{~s}$ and reached a plateau of almost $6 \times 10^{4}$ particles $/ \mathrm{cm}^{3}$ by $600 \mathrm{~s}$. The spike in the mass concentration and number concentration just after the $600 \mathrm{~s}$ mark could be due to a transient flame inside the foam block. Flashing flames were observed in lab tests during smoldering of this chair foam. Sometimes the flashing led to sustained flaming, and sometimes not. Almost no change in either ionization chamber voltage was observed. 

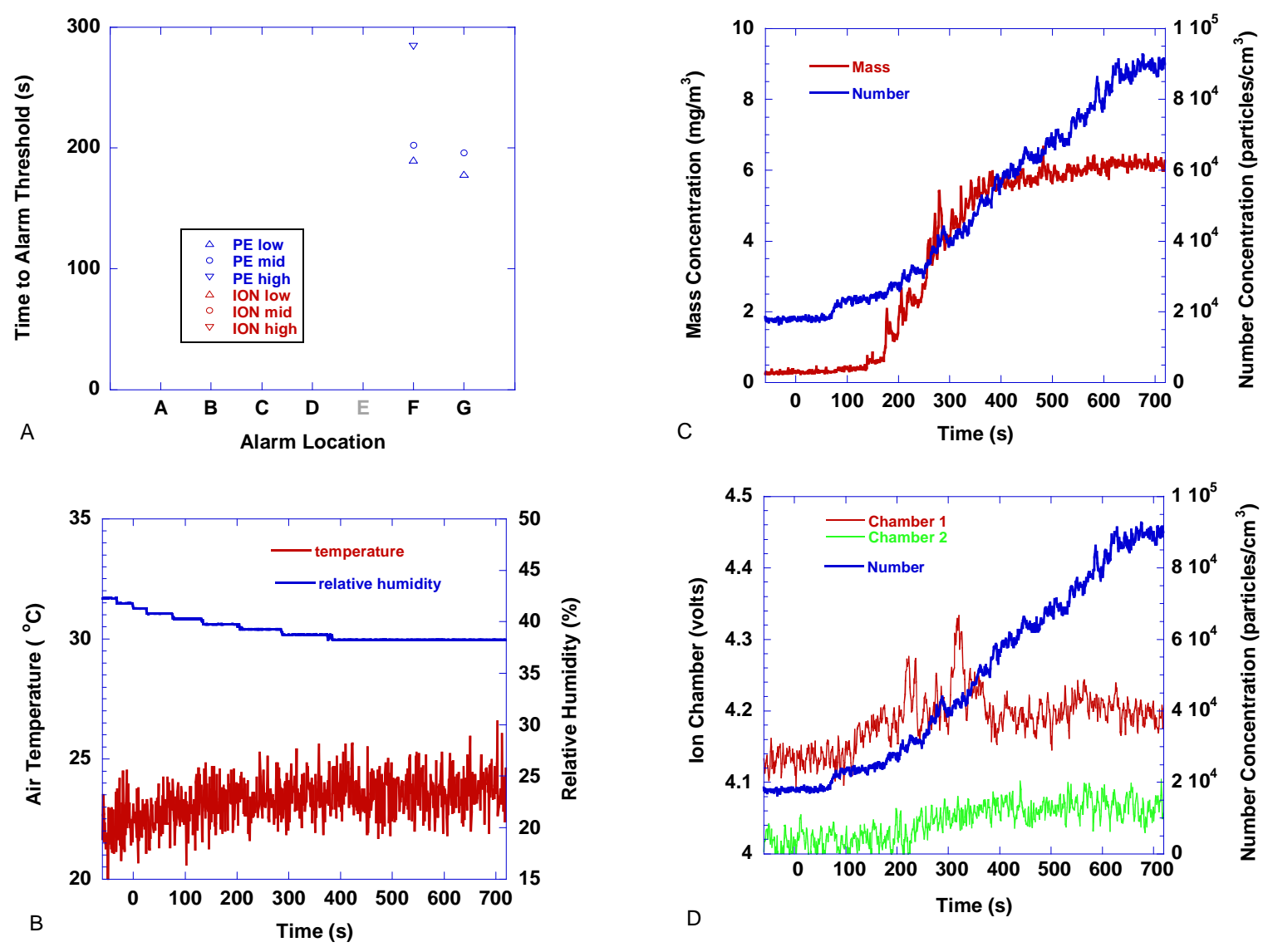

Figure 183. Smoldering foam - a polyurethane foam block, located on the living room floor at the chair burn location and ignited with an electrically powered nichrome wire heater imbedded in the block. Fan was off. Power to igniter on at $\mathrm{t}=0$. Sample stopped smoldering

Figure 185 shows the results for the test run with the floor fan on. The three photoelectric alarms closest to the source reached threshold values, no other alarms did. Same as the last test, the air temperature rise was on the order of a couple of degrees Celsius, while the relative humidity dropped by about $5 \%$. The mass and number concentration both started to rise around $50 \mathrm{~s}$. The mass concentration reached a peak of about $30 \mathrm{mg} / \mathrm{m}^{3}$ at $260 \mathrm{~s}$, while the number concentration reached a peak of $1.8 \times 10^{5}$ particles $/ \mathrm{cm}^{3}$ at $360 \mathrm{~s}$. By $500 \mathrm{~s}$ both mass and number concentration had leveled off to nearly steady values of $5 \mathrm{mg} / \mathrm{m}^{3}$ and $1 \times 10^{5}$ particles $/ \mathrm{cm}^{3}$ respectively. Ionization chambers reached peak values between $200 \mathrm{~s}$ and $300 \mathrm{~s}$, with voltage rises of $0.15 \mathrm{~V}$ and $0.2 \mathrm{~V}$ for chambers 1 and 2 respectively. 

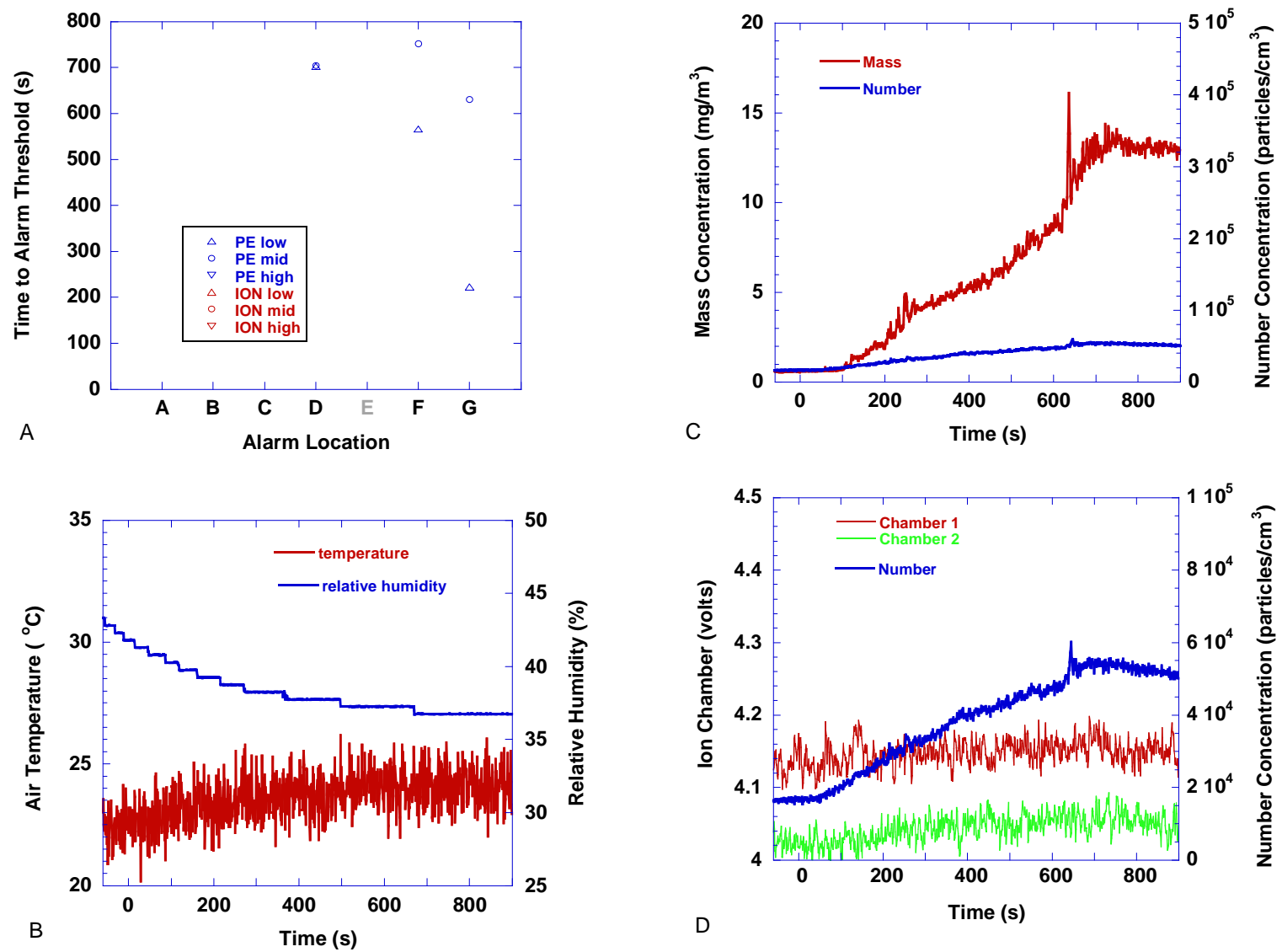

Figure 184. Smoldering foam - a polyurethane foam block, located on the living room floor at the chair burn location and ignited with an electrically powered nichrome wire heater imbedded in the block. Fan was on. Power to igniter on at $\mathrm{t}=0$

The following generalizations characterize the smoldering foam tests:

- This test showed a lot of variation in terms of smoke production.

- The propensity was for photoelectric alarms to reach threshold values during smoldering, and all alarms to reach thresholds after transition to flaming. 

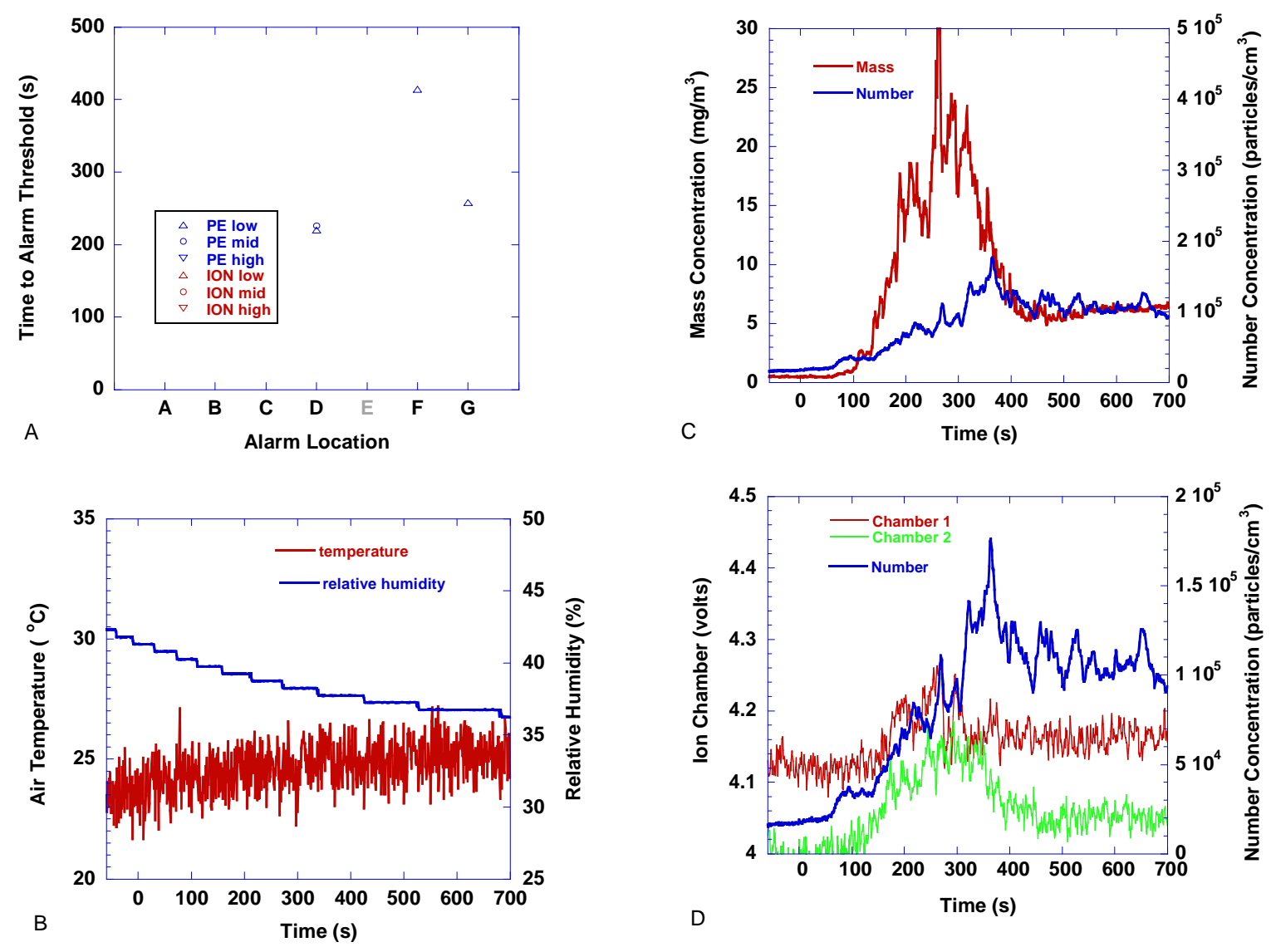

Figure 185. Smoldering foam - a polyurethane foam block, located on the living room floor at the chair burn location and ignited with an electrically powered nichrome wire heater imbedded in the block. Fan was on. Power to igniter on at $\mathrm{t}=0$

The nuisance source test results performed here supported the two main conclusions drawn from the field studies on nuisance alarms, the type of alarm (ionization or photoelectric) and the location of the alarm (relative to the source) affect the frequency of nuisance alarms.

Specifically, it was observed that the properties of the aerosol produced and its concentration affect whether either type of alarm threshold would be met. A relatively high number concentration of small particles is sufficient to produce nuisance alarms in ionization-type detectors, while not causing photoelectric-type alarms to activate. A high mass concentration of large particles can have the exact opposite outcome. In a quiescent environment only disturbed by a cooking heat source, cigarette, candle flame, or smoldering source, a distinct trend of an alarm's location relative to a source, and the propensity to reach an alarm threshold was observed. Forced flow conditions do not always follow that trend due to more rapid mixing, dilution and the directional nature of the flow relative to the source and the alarms. 


\subsection{FE/DE Emulation of Nuisance Sources}

Several preliminary tests were performed in the FE/DE with the intent of reproducing similar environments observed in the manufactured home tests above. The tests included toasting bread, a burning candle, margarine and butter heated in a pan, smoldering cotton and wood blocks. The data collected included the air temperature and relative humidity inside the test section, laser light extinction, MIC current, carbon monoxide and carbon dioxide concentration, barometric pressure, flow velocity, and the output from two modified photo, ion, $\mathrm{CO}$, thermistor units (D1 and D8, which were placed near the rear (location A) and front (location G) doors, respectively in the manufactured home; see figure 141 for locations)

The main objective of these tests was to demonstrate that important features of the selected scenarios could be reproduced in the FE/DE. Reproduction of perfect time histories of smoke and gas concentration, temperature and velocity at any detector location was not the goal. The tests were run at two different fan flows which produce flow velocities on the order of what was observed in the manufactured home test during no forced flow and forced flow tests (see section 6.3.1 for details of flow velocities). The results are presented below with a comparison to observations in the manufactured home tests.

\subsubsection{Cotton Wick Calibration}

Two calibration tests using the staged wick ignition source were performed. These tests were identical to the cotton wick calibration tests in section 5. The results are shown in figures 186 - 188. The electrochemical cell in D1 was not functioning properly and its results are not presented. Notice that the MIC signal fluctuated in clean air conditions as well as during steady smoke levels as shown in figure 187. This was caused by a problem in the picoammeter that was used to monitor the MIC and transmit a scaled voltage signal to the FE/DE data acquisition board. For this test and the FE/DE nuisance tests described below, this noisy MIC signal was present, and thus the MIC signal should be viewed as a relative indication of how a MIC would respond. Figure 186 shows repeated results for the photoelectric sensors compared to the laser light extinction. The sensor from unit D1 rose from an initial value of zero, while the sensor from unit D8 starts out at a value of about 5. An increase in the output for each of these sensors of about five would be the alarm threshold equivalent to $2.5 \%$ obscuration. Figure 187 shows repeated results for the ionization sensors compared to the MIC output. For these sensors, a decrease of 25 from the initial level would be the equivalent of about $1 \% / \mathrm{ft}$ obscuration. Figure 188 shows the results for the $\mathrm{CO}$ sensor in unit $\mathrm{D} 1$ compared to the $\mathrm{CO}$ concentration from the gas analyzer for the repeated tests. The initial jump in the $\mathrm{CO}$ sensor response from zero to 10 was attributed to signal noise; this periodic noise was observed throughout the tests. The carbon monoxide results are presented below, but no further discussion of the results will be made here. 

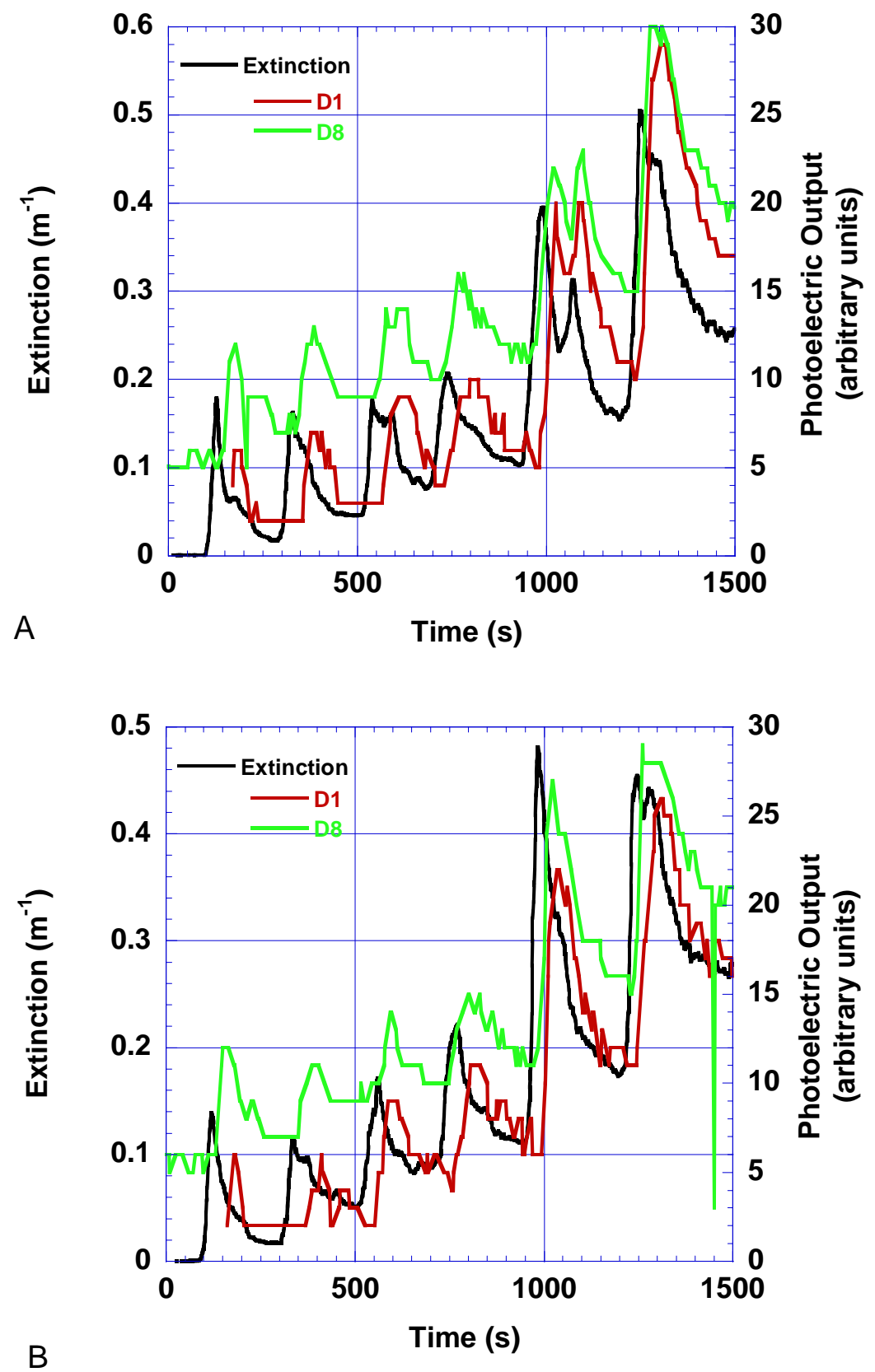

Figure 186. Photoelectric sensor response to cotton smolder smoke calibration test. $A$ and $B$ indicate repeated tests 

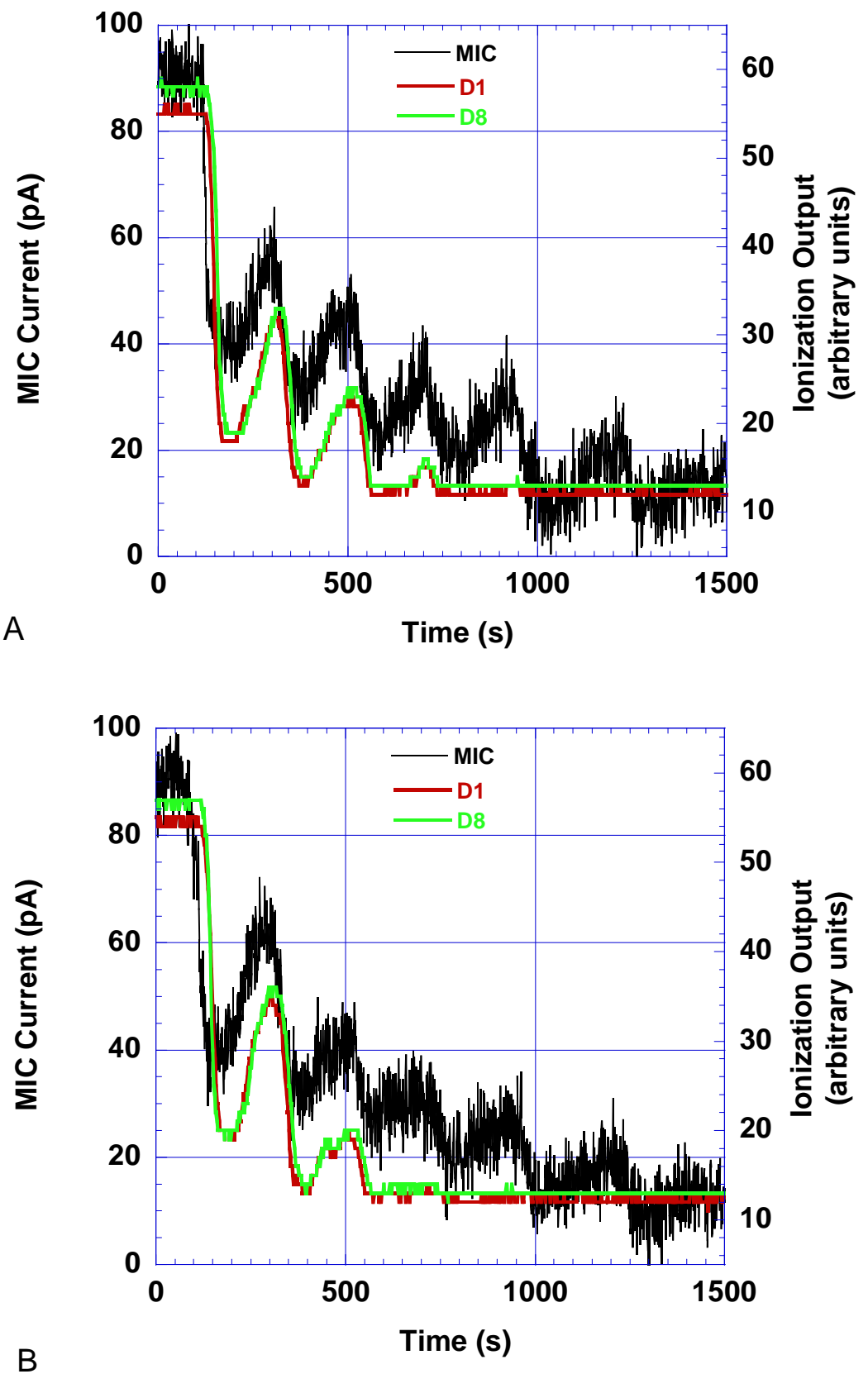

Figure 187. Ionization sensor response to cotton smolder smoke calibration test. $A$ and $B$ indicate repeated tests 

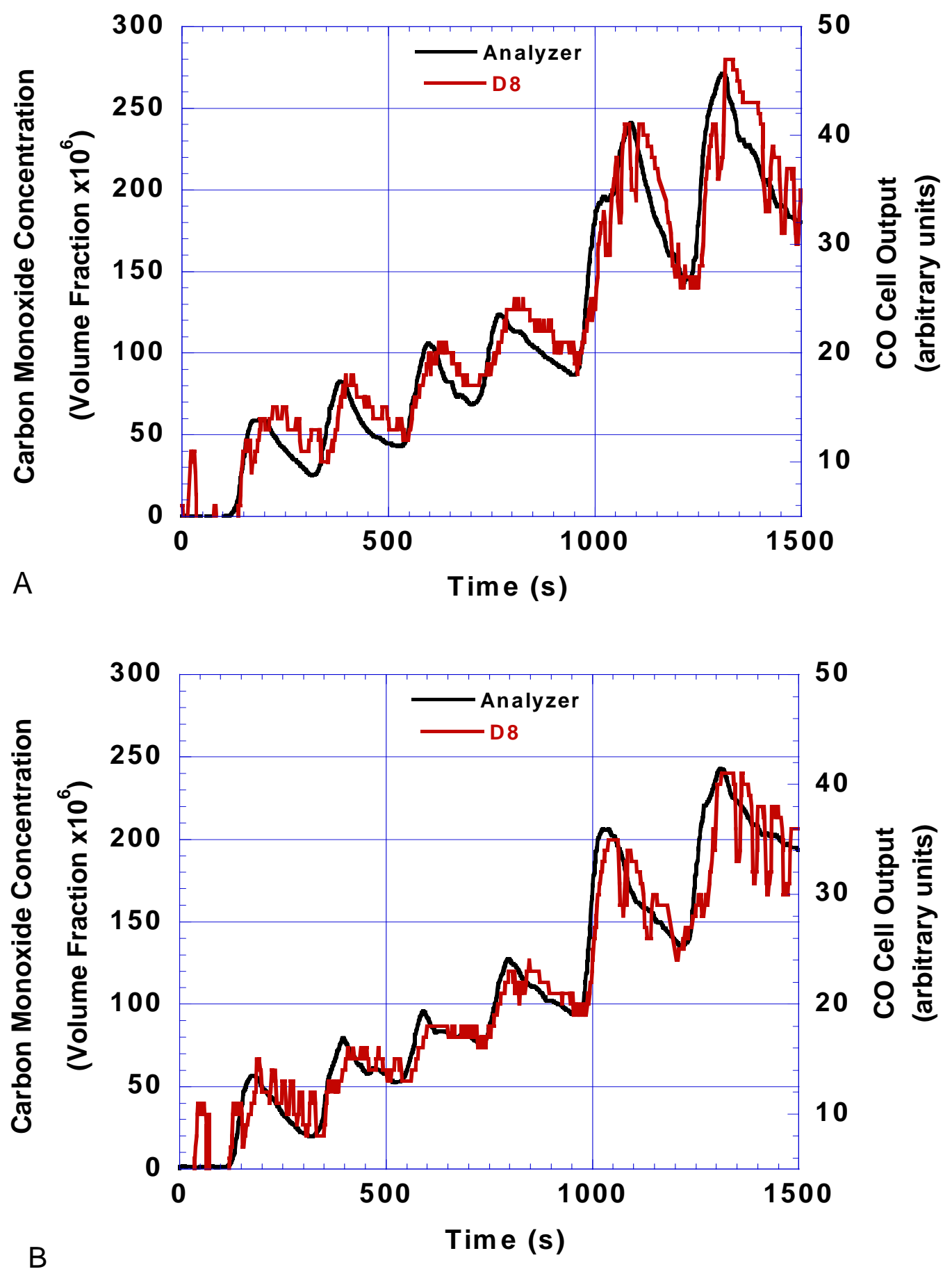

Figure 188. Electrochemical cell CO sensor response to cotton smolder smoke calibration test. A and B indicate repeated tests 


\subsubsection{Cotton Smolder Smoke Fire Scenario}

The cotton smolder smoke scenario employed the same ignition sequence used in the manufactured home tests, but with half of the number of wicks (16). Tests were conducted at fan speeds of $7 \mathrm{~Hz}$ and $12 \mathrm{~Hz}$, and each was repeated. The baffle plate was installed in the duct upstream of the test section to produce turbulent flow characteristics similar to those observed in the manufactured home tests. (The baffle plate was left in during all subsequent tests.)

Figures 189 and 190 show results for repeat runs at a fan speed of $7 \mathrm{~Hz}$. The extinction was observed to peak at about $0.3 \mathrm{~m}^{-1}$ for the first test, and $0.5 \mathrm{~m}^{-1}$ for the second test. This difference is attributed to variation in the time to ignition for the two sets of wicks. At about $500 \mathrm{~s}$ the wicks were approaching a steady burning rate. The photoelectric and ionization sensor alarms reach threshold levels. Figure 191 and 192 show results for repeat runs at a fan speed of $12 \mathrm{~Hz}$. Although the smoke concentration was lower due to the dilution with the higher duct flow, both photoelectric and ionization alarms reach threshold levels. The photoelectric sensor signal rise lags the rise in smoke concentration, and responds slower than the ionization sensor

for all of these tests. This is partially due to smoke entry lag into the photoelectric sensor and an artifact from filtering the photoelectric sensor signals.

Both the FE/DE results and the manufactured home results for sensor units near the source achieve alarm threshold levels for both types of alarms during the ignition phase of the wicks when the peak amount of smoke was produced. The smoke concentration in the upper layer of the manufactured home continued to rise because it was a closed system.

\subsubsection{Wood Smolder Smoke Fire Scenario}

The generation of wood smolder smoke was identical to the method used in the manufactured home. Eight beech wood blocks were placed on the electric hot plate which was located at the bottom of the vertical rise in the duct. At time $=0$ the heater was turned on. Figures 193 to 196 show the results for repeated tests at $7 \mathrm{~Hz}$ and $12 \mathrm{~Hz}$ fan flows.

The photoelectric sensors reach the alarm threshold levels slightly earlier than the ionization sensors. In the manufactured home tests, photoelectric alarm thresholds were reached earlier than the ionization alarm thresholds, but the time differential tended to be larger than what was observed in the FE/DE tests due to the inherently smaller volume of the FE/DE apparatus. 

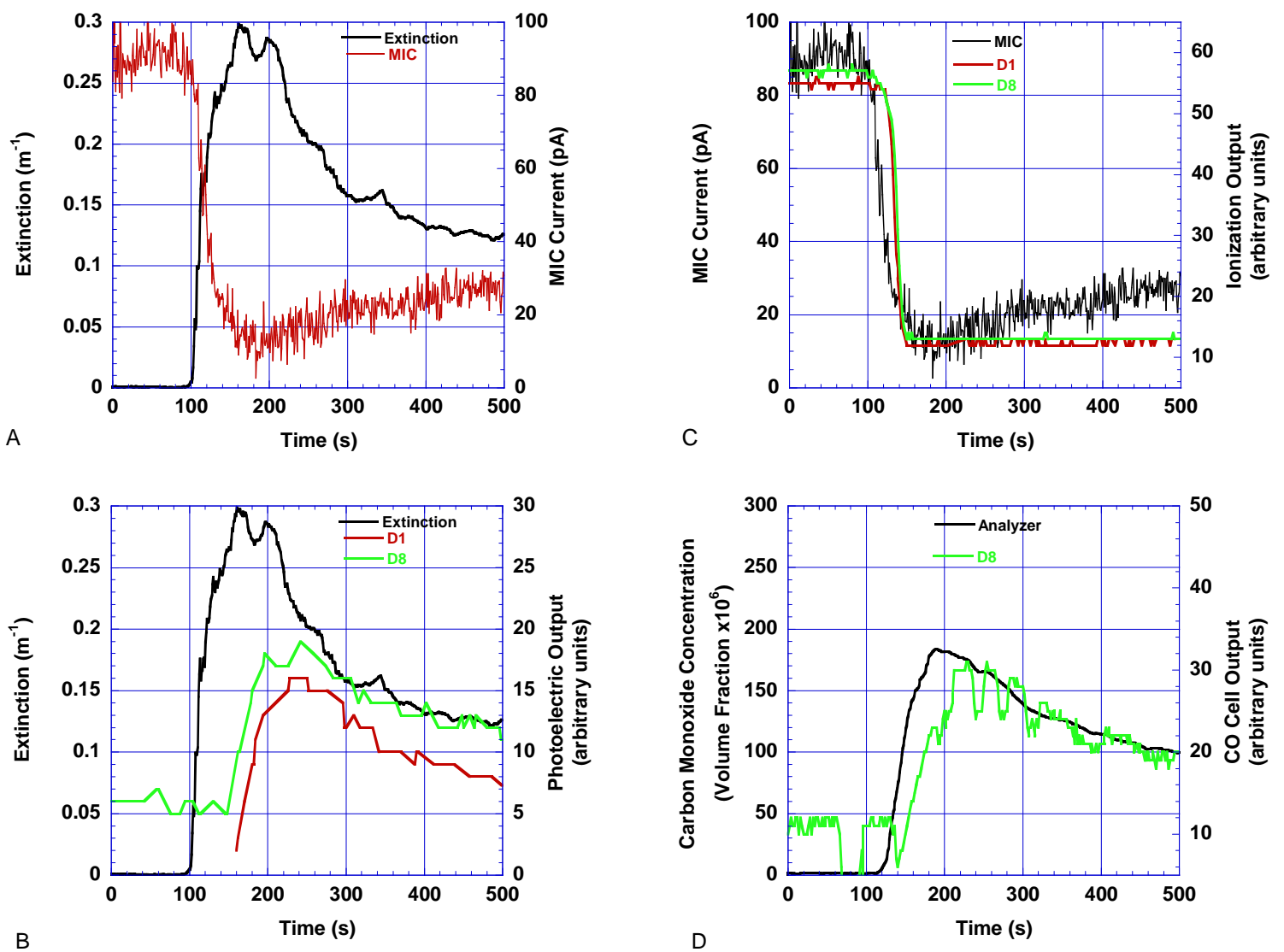

Figure 189. Smoldering cotton fire scenario, fan speed $7 \mathrm{~Hz}$ 

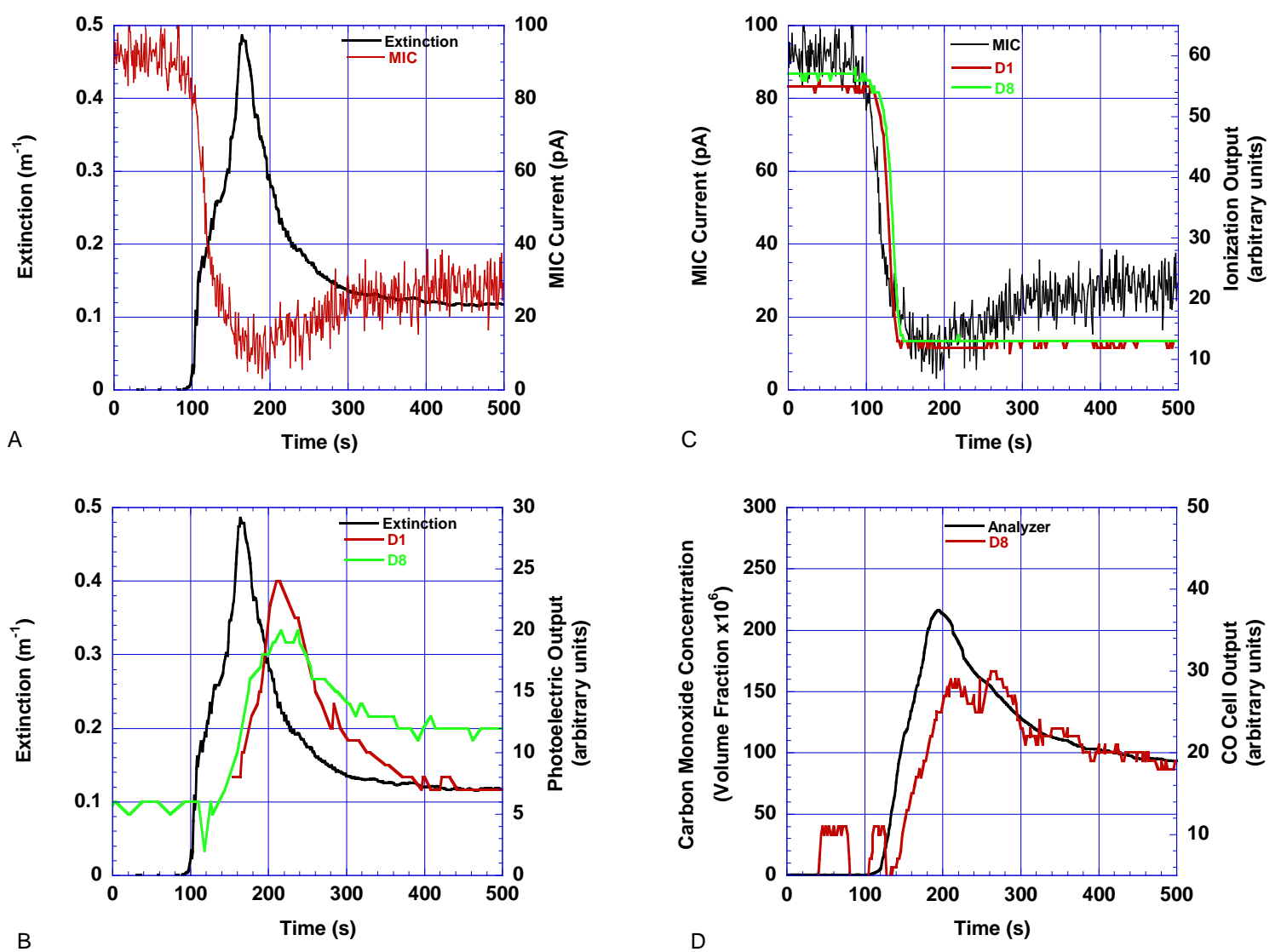

Figure 190. Smoldering cotton fire scenario, fan speed $7 \mathrm{~Hz}$ 

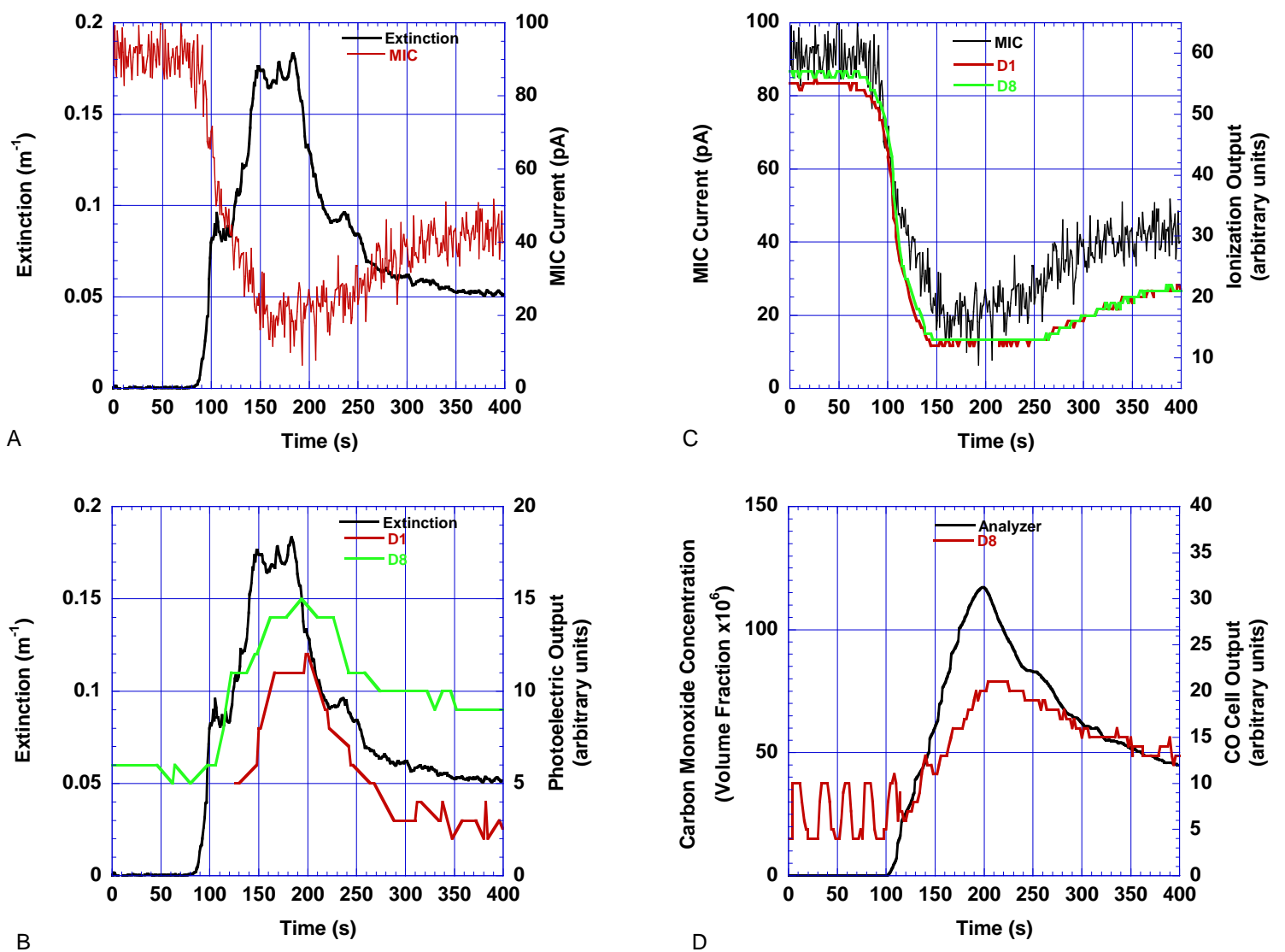

Figure 191. Smoldering cotton fire scenario, fan speed $12 \mathrm{~Hz}$ 

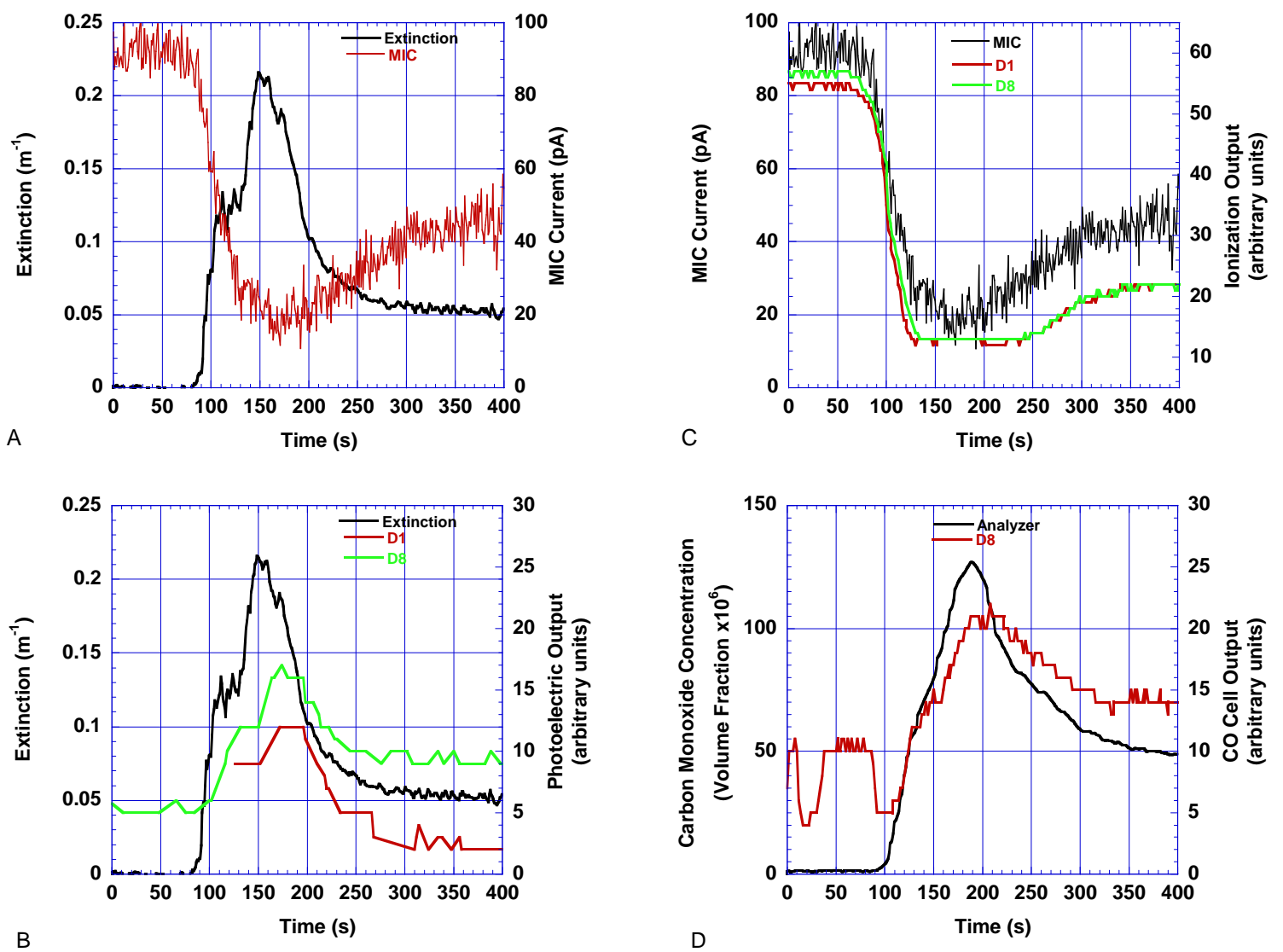

Figure 192. Smoldering cotton fire scenario, fan speed $12 \mathrm{~Hz}$ 

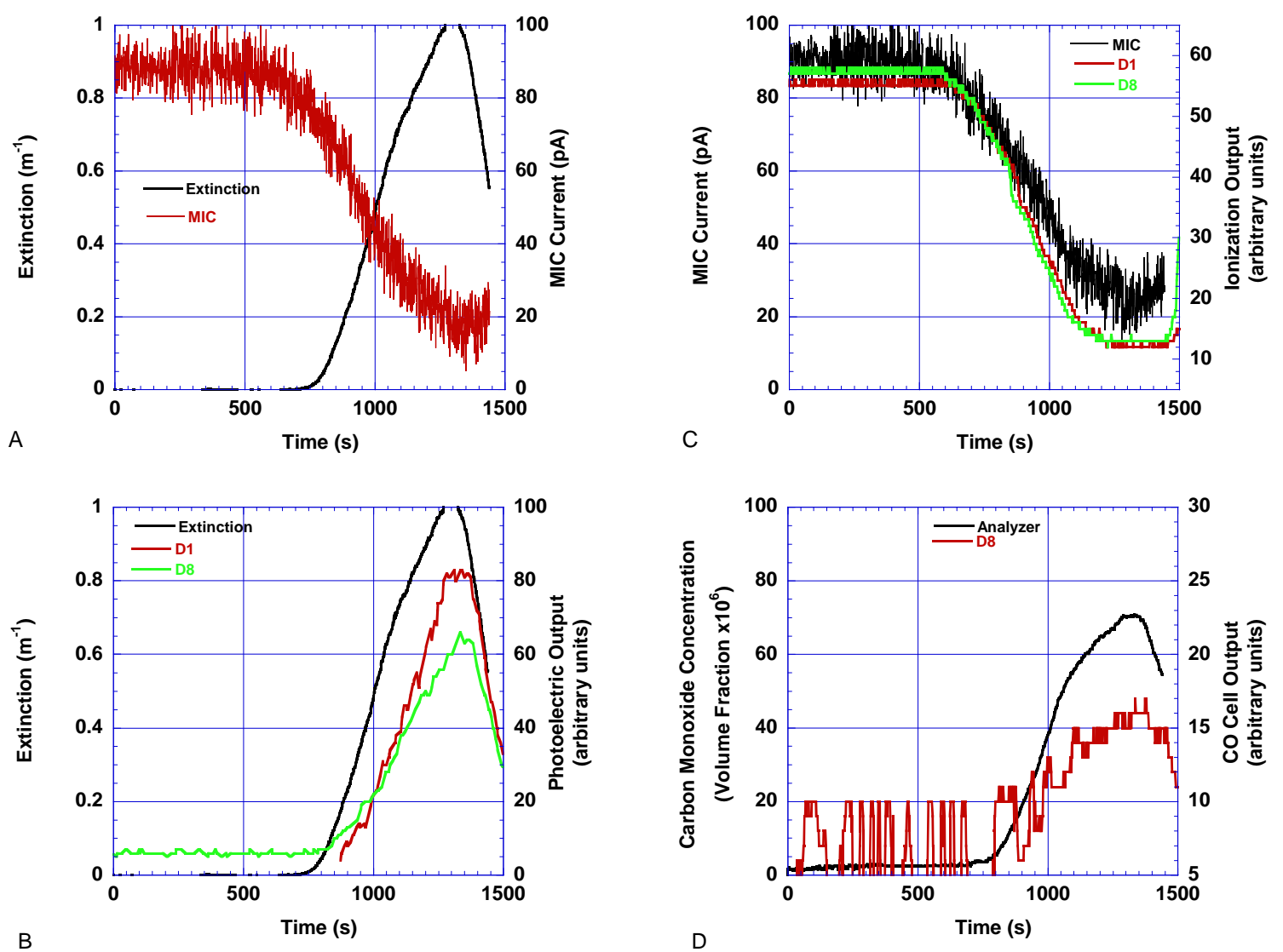

Figure 193. Smoldering wood fire scenario, fan speed $7 \mathrm{~Hz}$ 

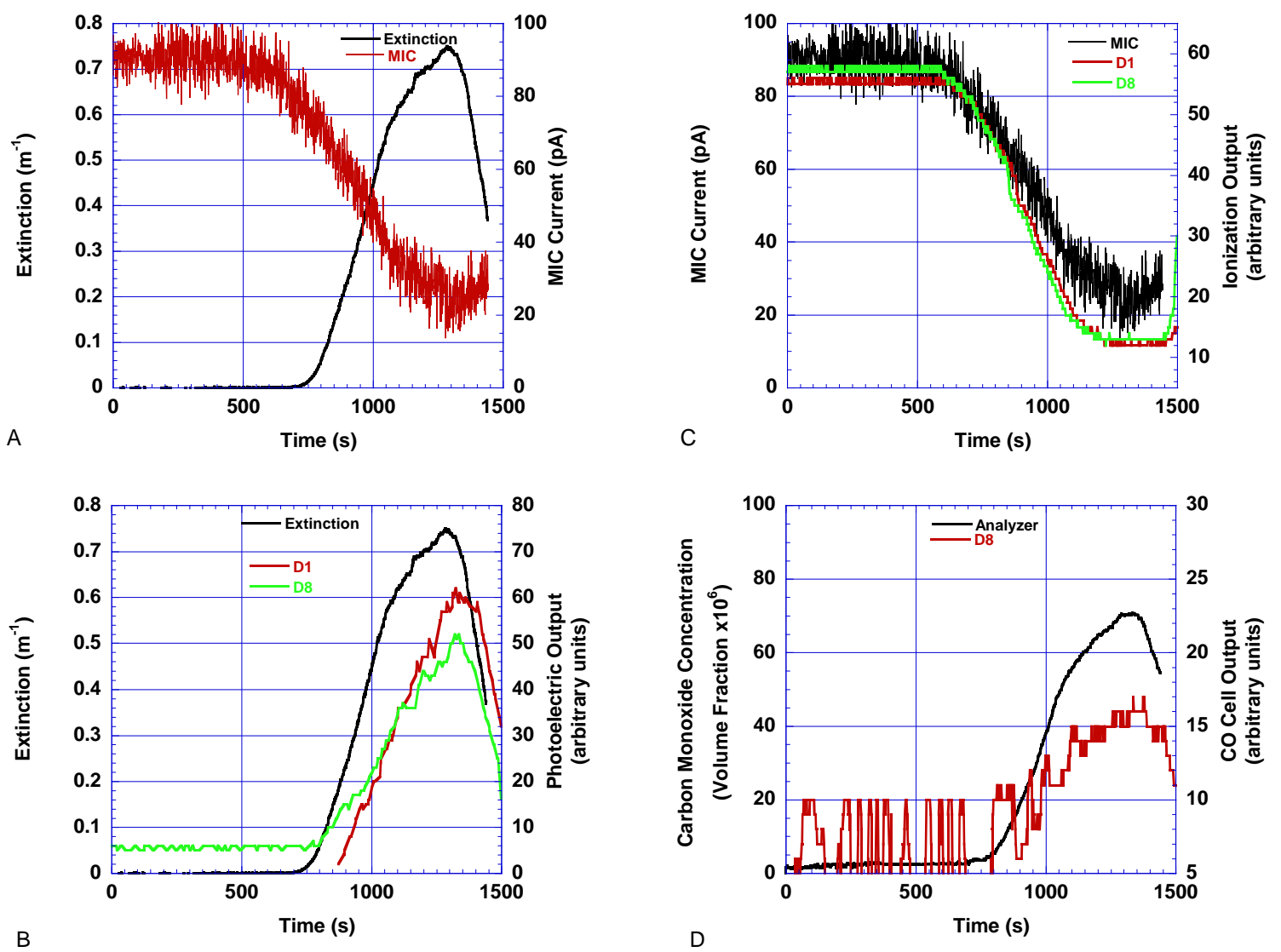

Figure 194. Smoldering wood fire scenario, fan speed $7 \mathrm{~Hz}$ 

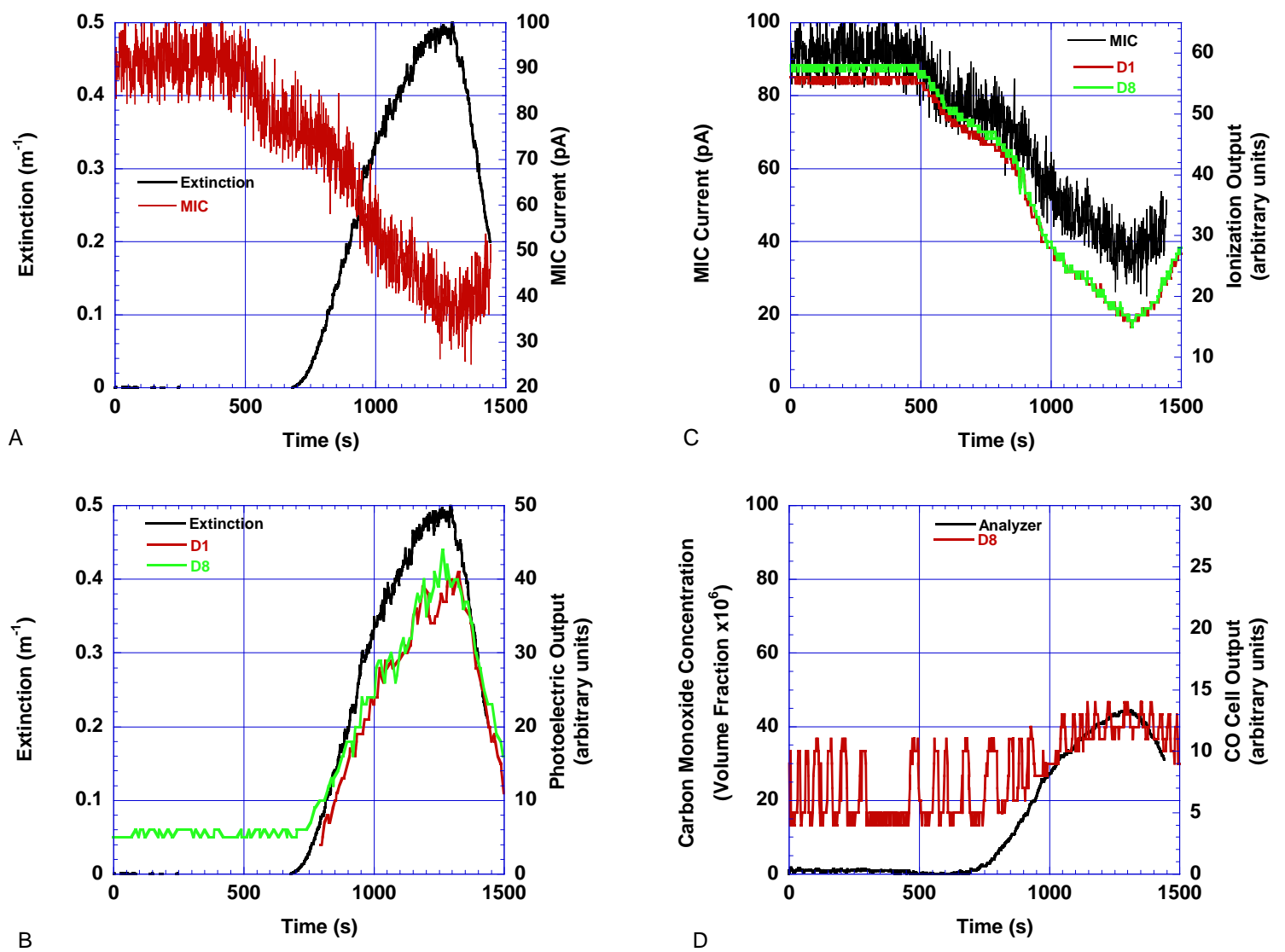

Figure 195. Smoldering wood fire scenario, fan speed $12 \mathrm{~Hz}$ 

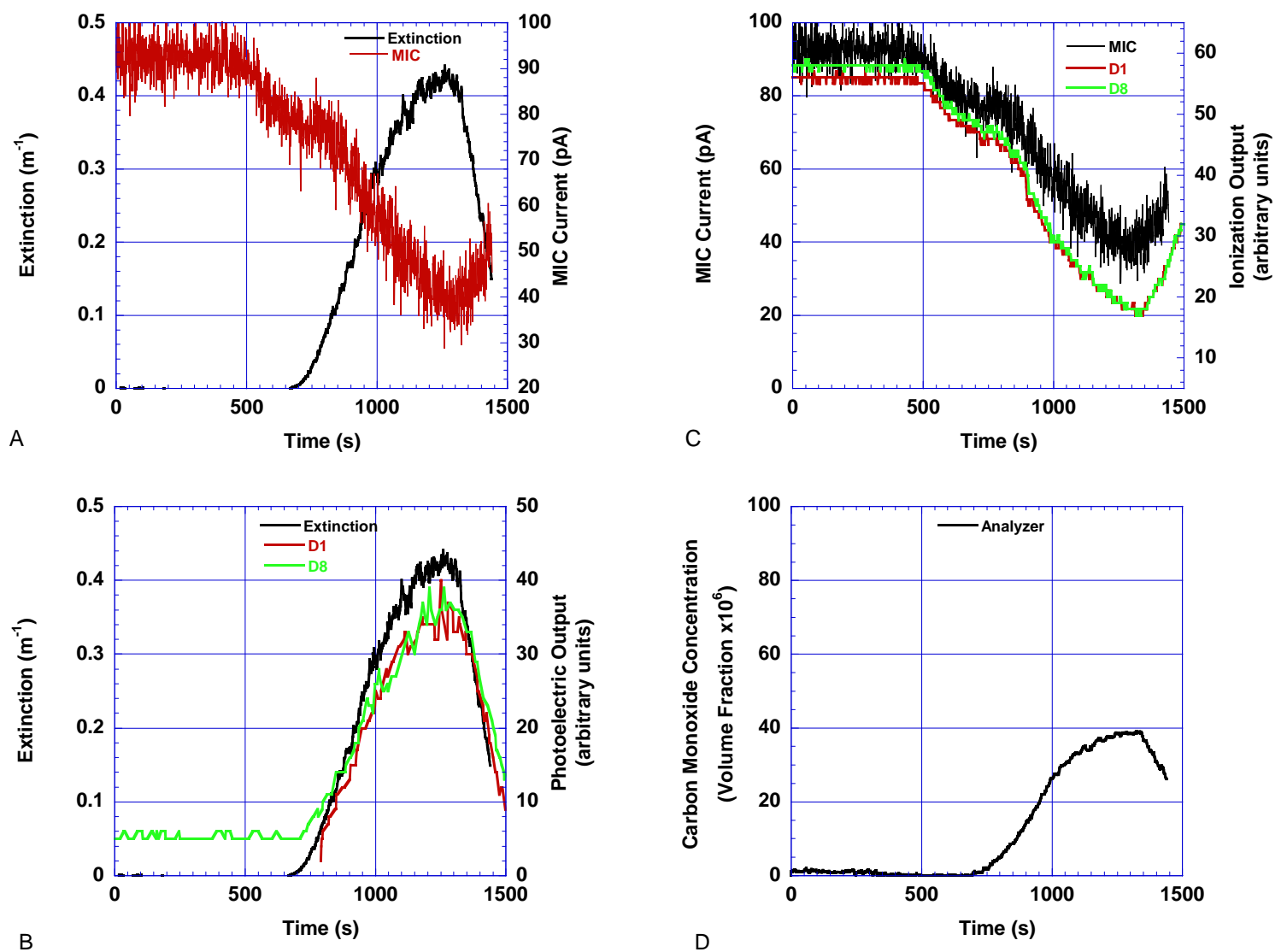

Figure 196. Smoldering wood fire scenario, fan speed $12 \mathrm{~Hz}$ 


\subsubsection{Candle Flame Nuisance Scenario}

A single tea candle, the same brand used in the manufactured home tests, was placed in the FE/DE duct and lit. Figure 197 shows the results. This produced a slight decrease in the MIC output and in the ionization sensors, but not sufficient for an ionization alarm threshold. There was no observable light extinction. These observations mirror the manufactured home results, where no alarm thresholds were met. Only an increase in aerosol number concentration and a slight rise in the ionization chambers were evident.
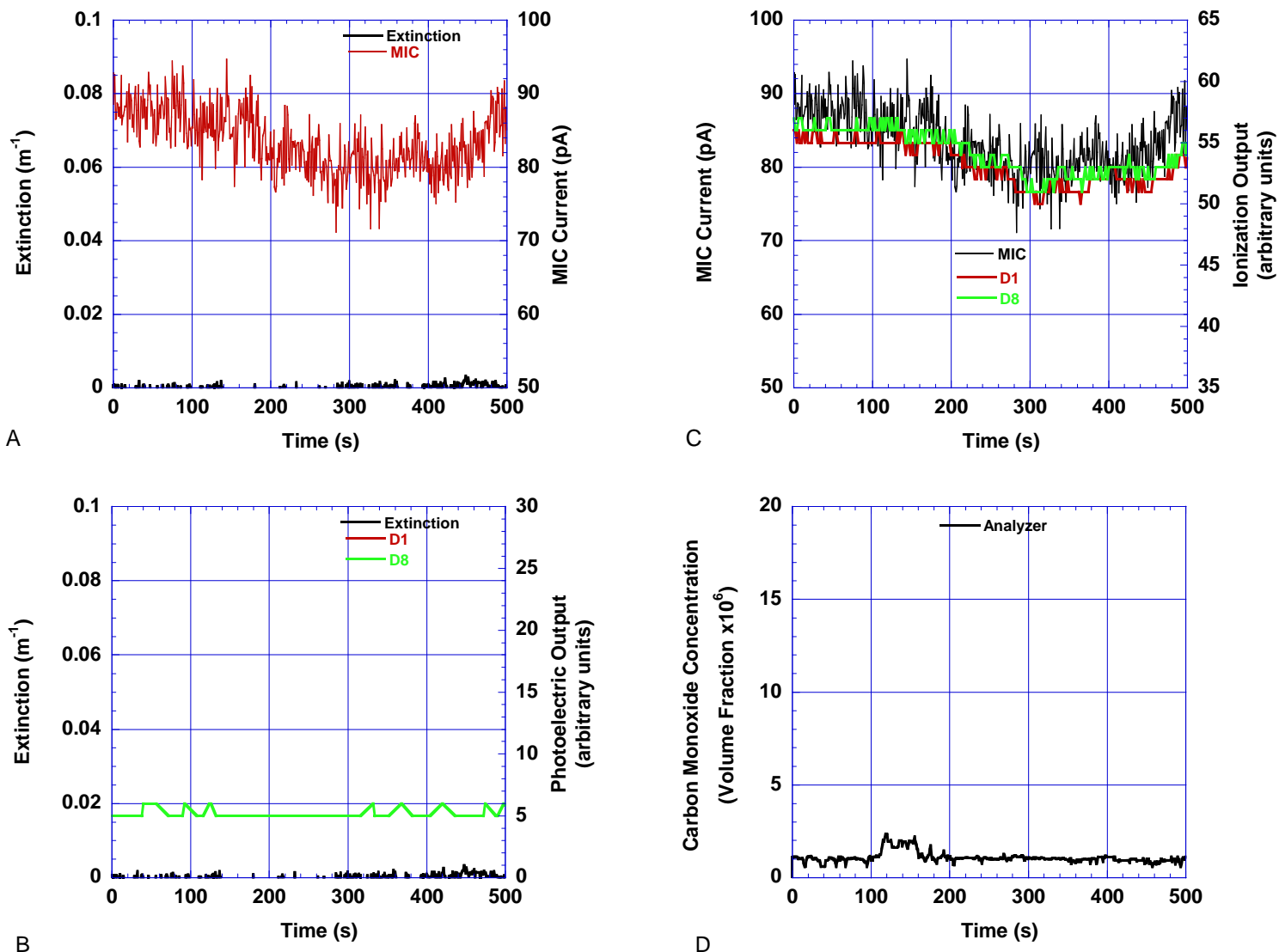

Figure 197. Candle flame, fan speed $7 \mathrm{~Hz}$ 


\subsubsection{Heated Margarine or Butter Nuisance Scenario}

In these tests, a portable, thermostatically controlled, $15 \mathrm{~cm}$ diameter electric hotplate was used to heat either $1 / 2$ tablespoon of margarine or butter placed in a $15 \mathrm{~cm}$ diameter cast-iron frying pan. All tests were performed at a fan flow of $7 \mathrm{~Hz}$.

Figures 198 to 200 show the results. No ionization alarm thresholds were reached during the margarine tests or the butter test. Photoelectric thresholds were reached during one margarine test and the butter test.

The FE/DE tests are somewhat different than the manufactured home tests in that the hot plate used in the FE/DE did not apparently heat up the cast-iron pan to temperatures reached on the cast-iron pan placed on the electric range. The thermostat in the portable hot plate does not let the temperature reach a level where the margarine or butter will smoke heavily.

\subsubsection{Toasting Bread Nuisance Scenario}

The same toaster and brand of bread that was used in the manufactured home tests was used in the FE/DE tests. The only difference was that one slice of bread was used in the FE/DE tests. For these tests, the toaster was turned on at $60 \mathrm{~s}$ and turned off at $300 \mathrm{~s}$. Three tests at a fan speed of $7 \mathrm{~Hz}$ and two tests at $12 \mathrm{~Hz}$ were performed.

The results are shown in figures 201 to 205. Consistent with the manufactured home tests, both ionization and photoelectric alarm thresholds were reached, with the ionization alarms thresholds reached first. The delays between the extinction measurements and the photoelectric sensor signals were evident during all of the tests. 

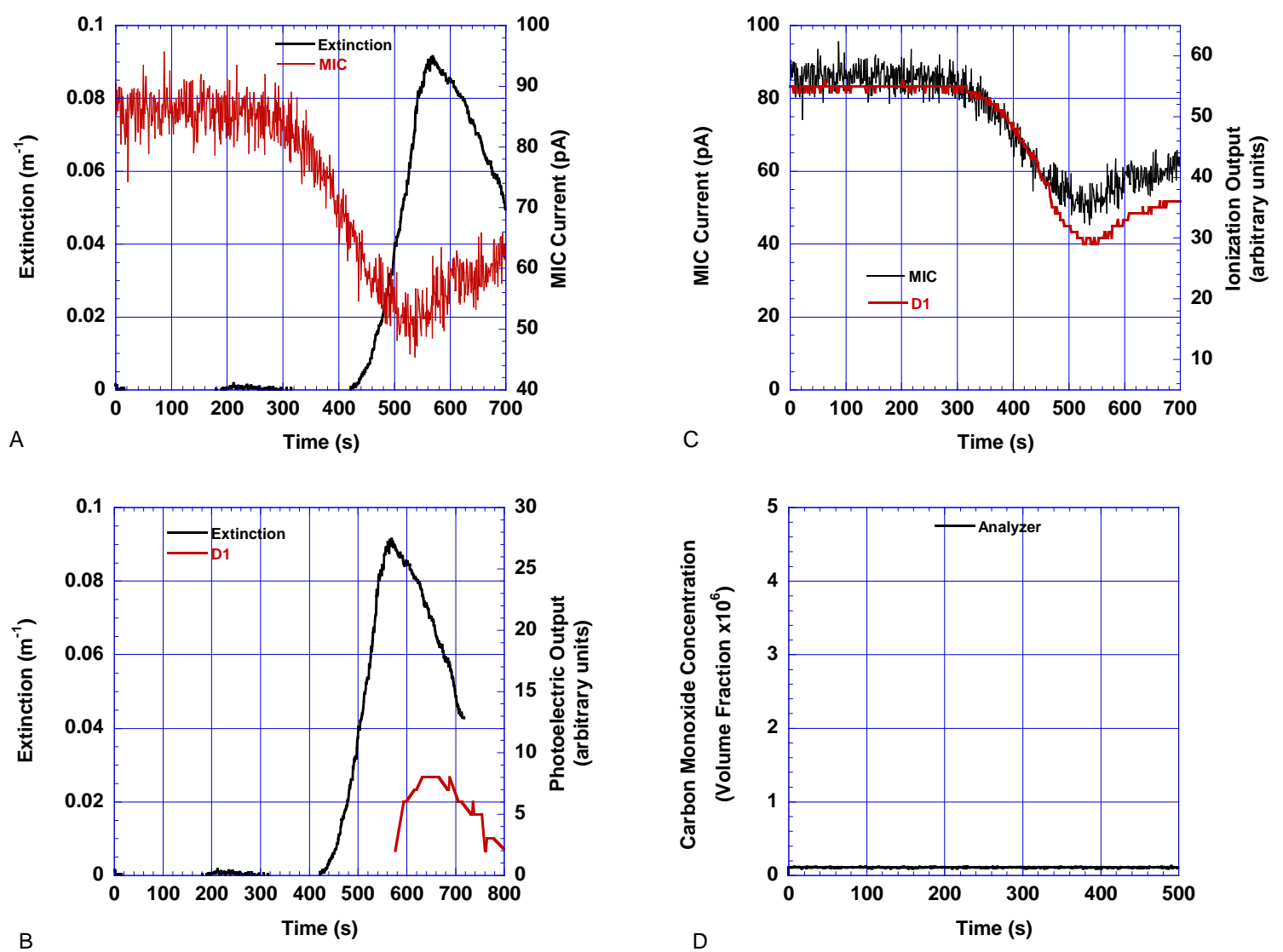

Figure 198. Margarine heated in a pan, fan speed $7 \mathrm{~Hz}$ 

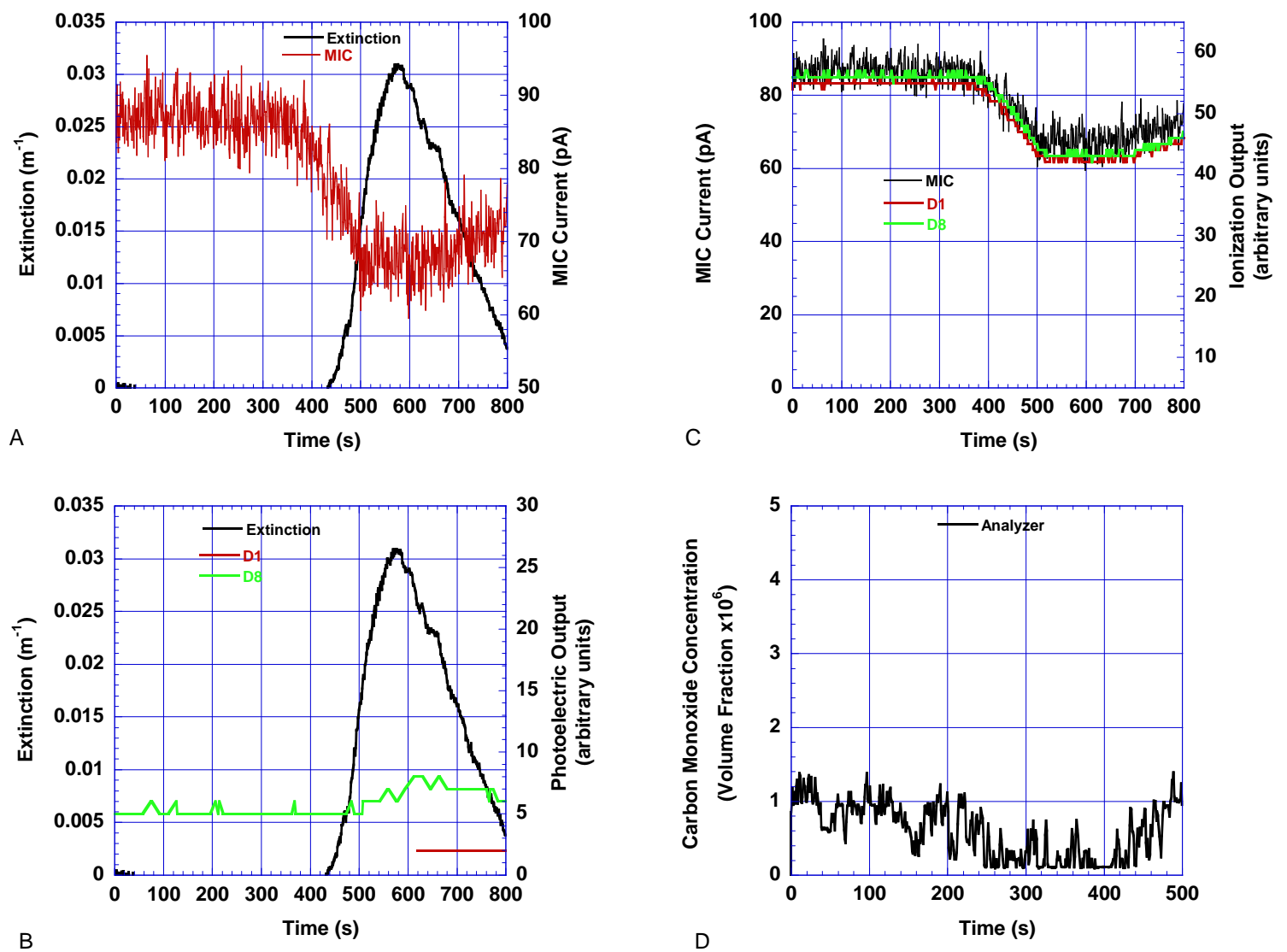

Figure 199. Margarine heated in a pan, fan speed $7 \mathrm{~Hz}$ 

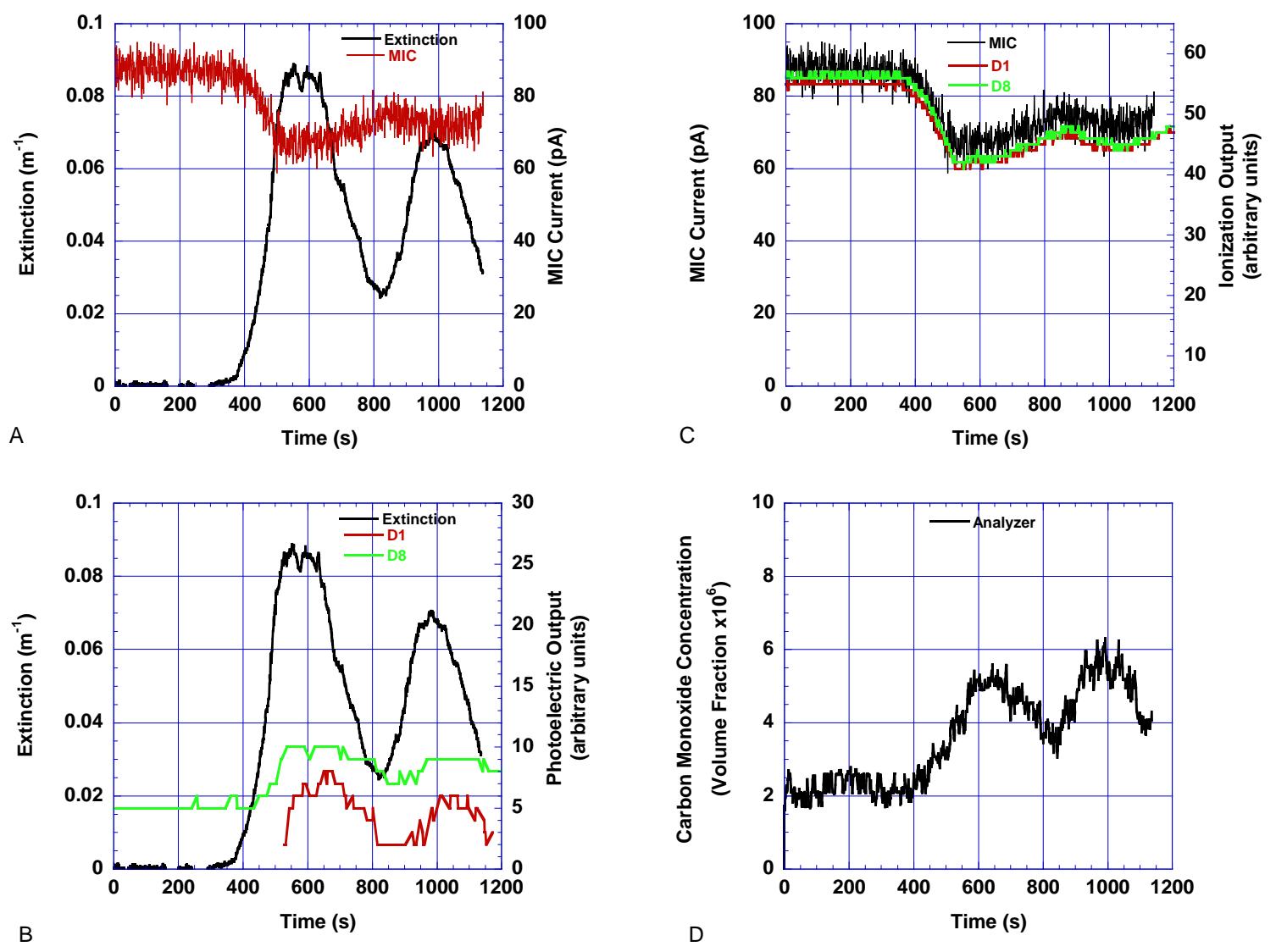

Figure 200. Butter heated in a pan, fan speed $7 \mathrm{~Hz}$ 

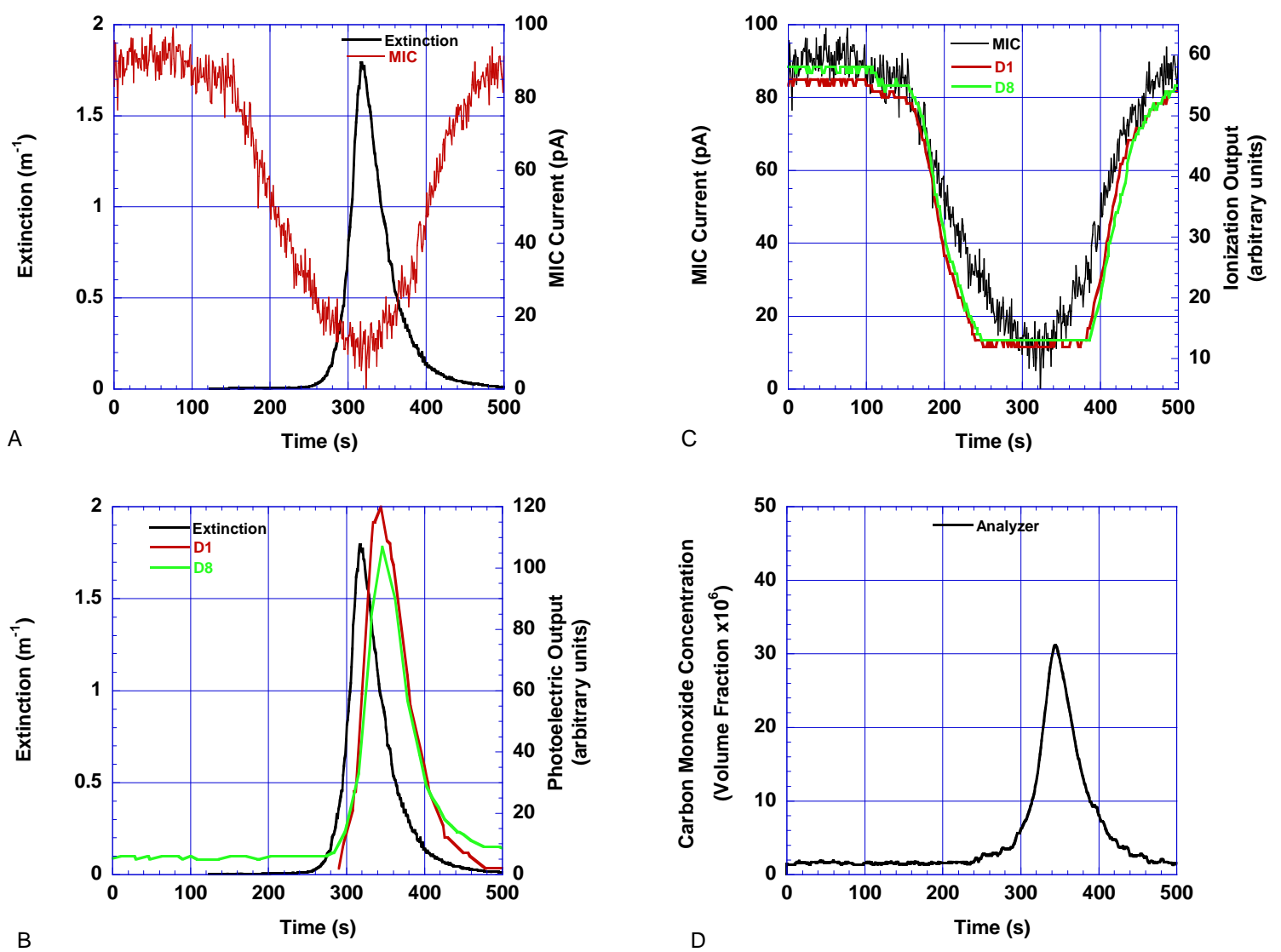

Figure 201. Bread in a toaster, fan speed $7 \mathrm{~Hz}$ 

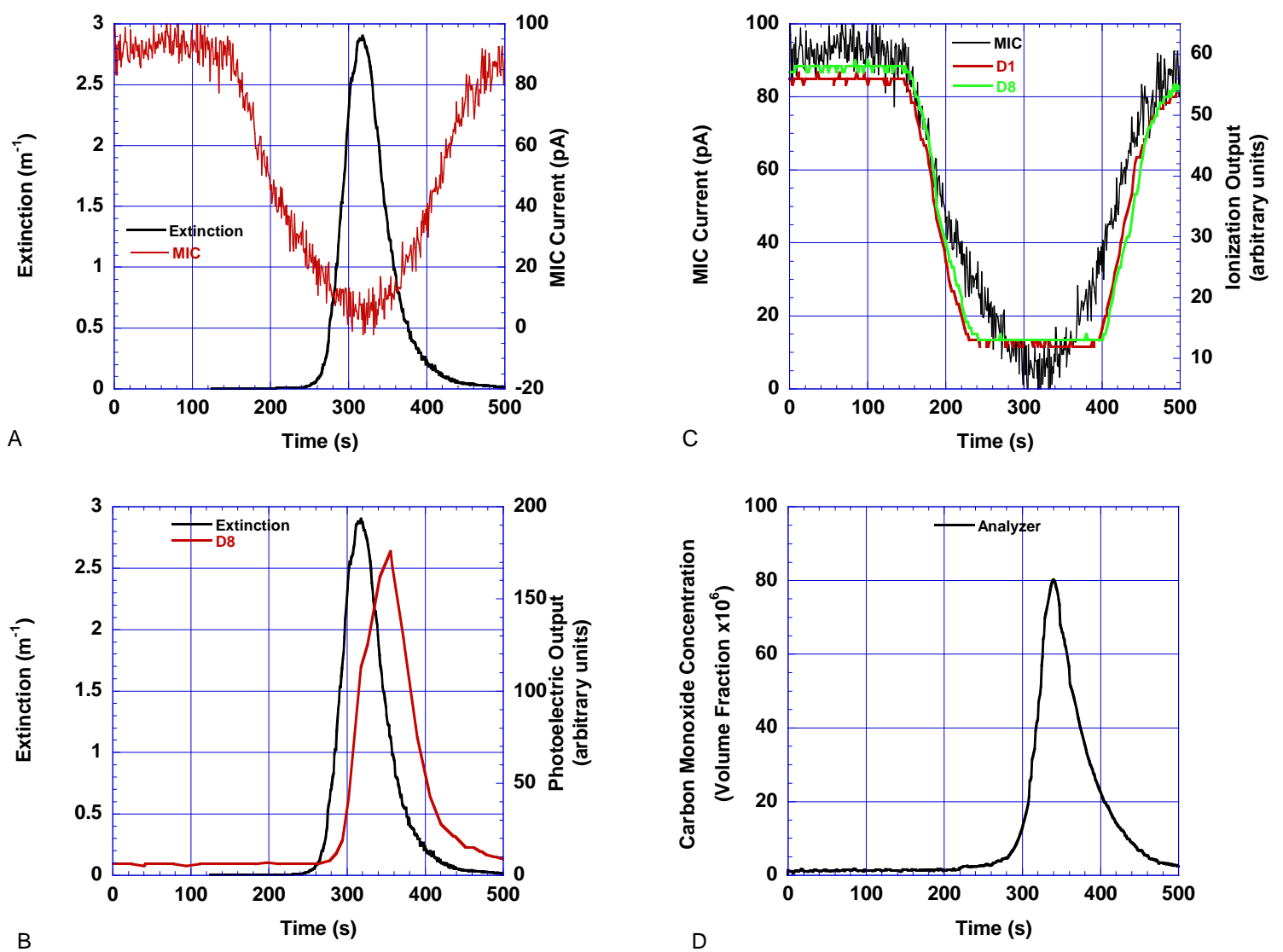

Figure 202. Bread in a toaster, fan speed $7 \mathrm{~Hz}$ 

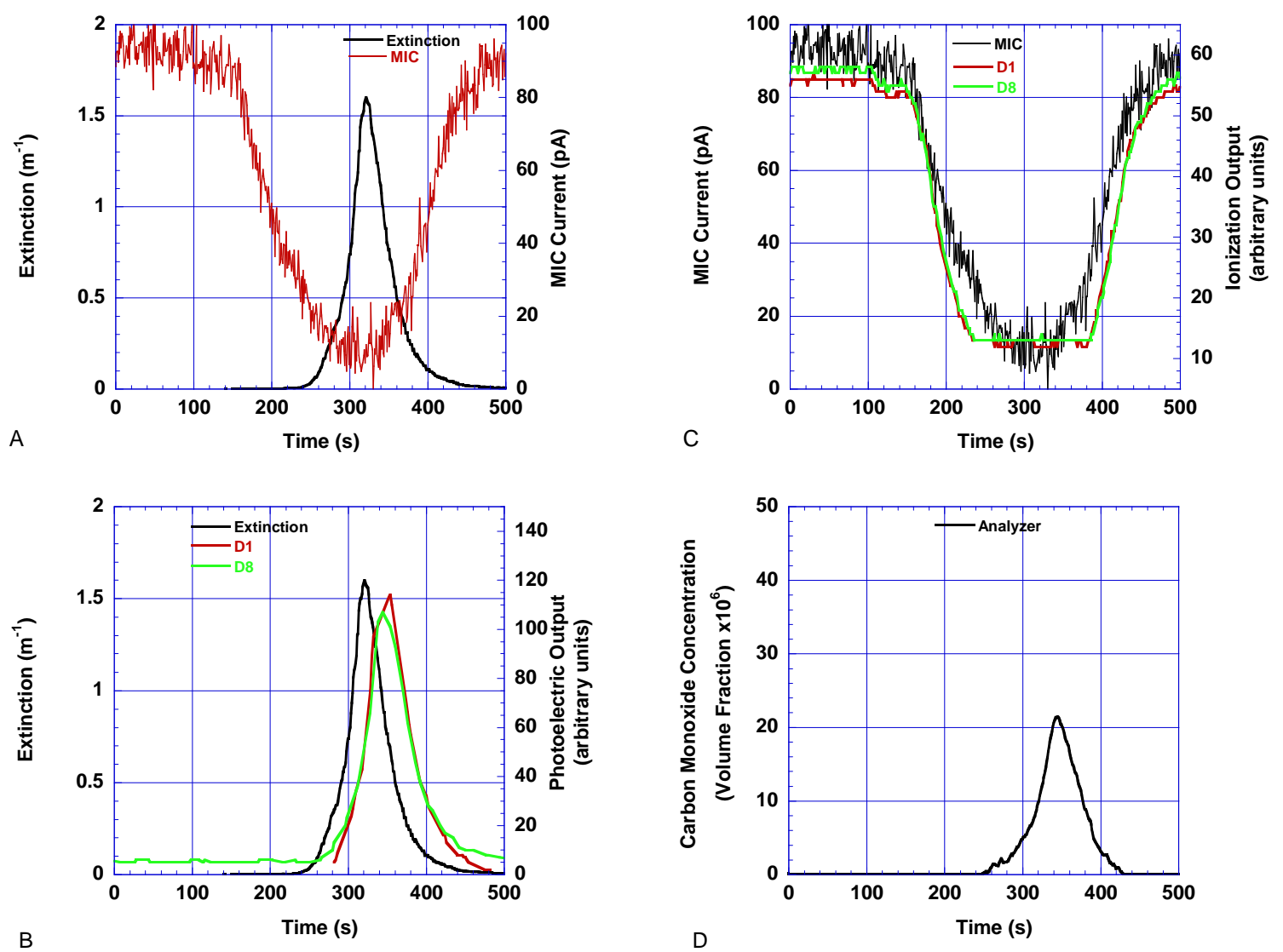

Figure 203. Bread in a toaster, fan speed $7 \mathrm{~Hz}$ 

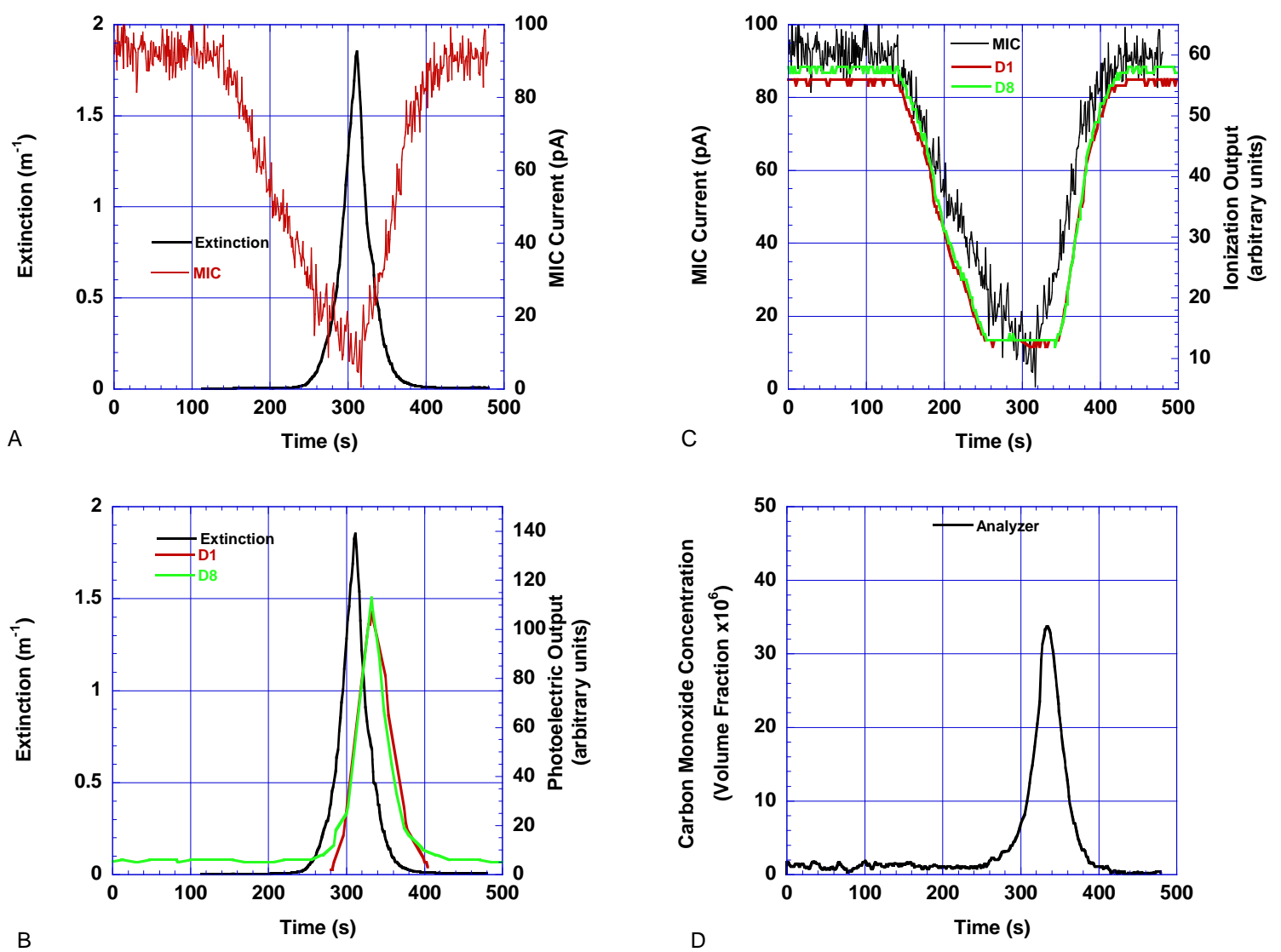

Figure 204. Bread in a toaster, fan speed $12 \mathrm{~Hz}$ 

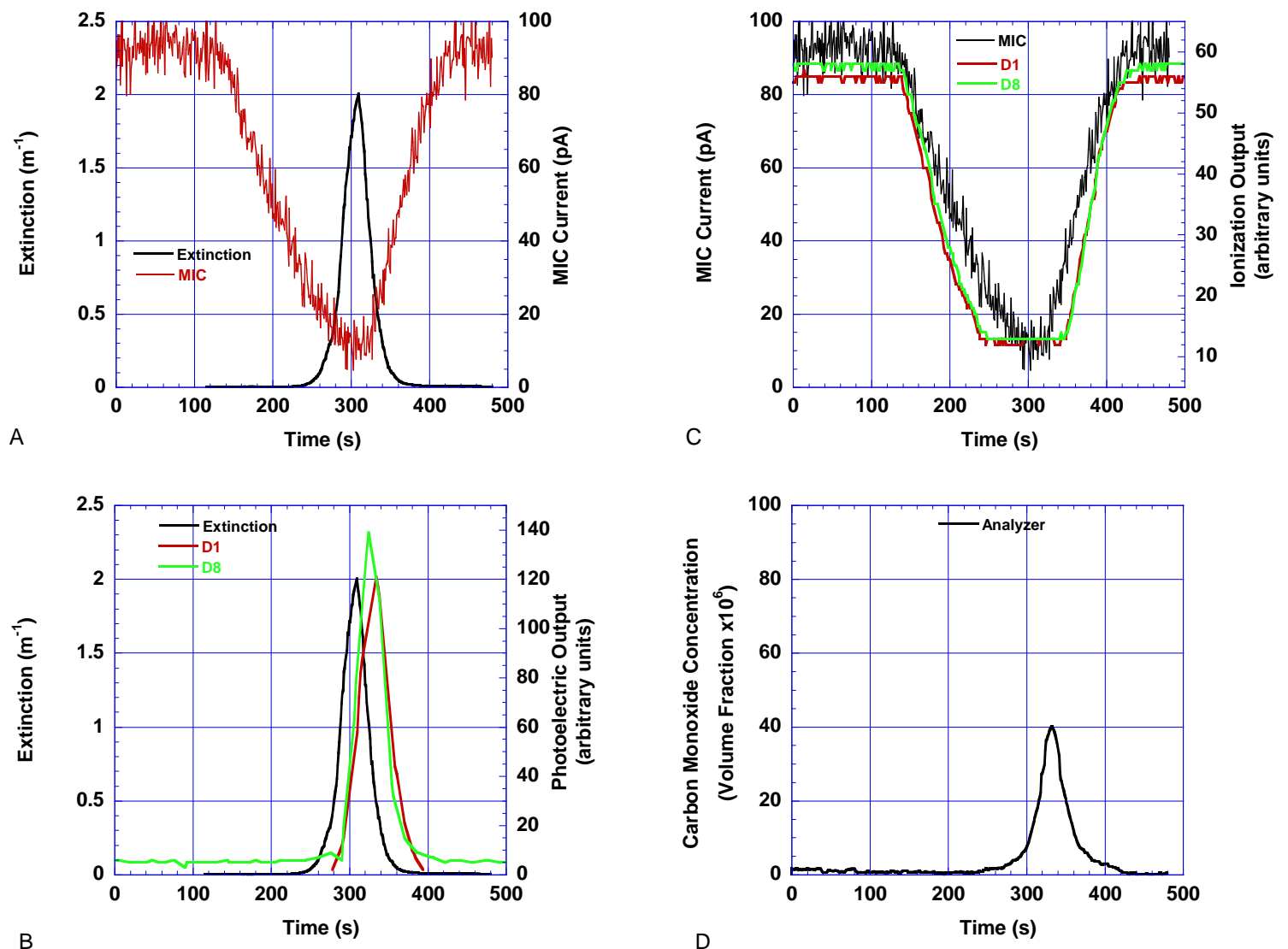

Figure 205. Bread in a toaster, fan speed $12 \mathrm{~Hz}$ 


\section{Discussion}

In 1975, the Indiana Dunes study pioneered the idea of measuring the performance of home smoke alarms by the amount of escape time provided. Escape time is the time between the device alarm and the occurrence of untenable conditions at any point along the primary escape path - generally the corridors and stairs leading from the sleeping rooms to the egress door. While building codes require that sleeping rooms have a secondary exit, usually a window of sufficient size and within $6 \mathrm{~m}(20 \mathrm{ft})$ of grade [42], experience shows that these are rarely used to escape home fires. Thus, calculating the time between alarm activation and the onset of untenable conditions represents the performance metric most appropriate to evaluating the performance of smoke alarms in residences.

\subsection{Smoke Alarm Activation Time}

Smoke alarm requirements in building codes and standards require multiple devices depending on the size and arrangement of the dwelling. Thus, when determining the performance of smoke alarms, the escape time provided by any single device is not (necessarily) appropriate. Rather the escape time provided by the first of several devices at specific locations cited in the codes is the appropriate parameter to report.

For existing homes (and all homes, prior to 1993) smoke alarms are required outside the sleeping rooms and on each additional story of the home. This arrangement will be referred to as "every level" and represents the minimum arrangement allowed by code. In 1993 the National Fire Alarm Code (NFPA 72) was revised to require smoke alarms in every bedroom for new construction in addition to the every level locations, in order to improve audibility in bedrooms where occupants sleep with the door closed and to provide warning to the occupants of bedrooms with closed doors when the fire starts in that bedroom. We will refer to this arrangement as "every level + bedrooms." The greatest escape times would be produced by a smoke alarm in the room of fire origin, but this would be guaranteed only if smoke alarms were required in every room - an arrangement that has never been required in any code. Thus the "every room" result represents the best possible performance that can be compared to "every level" as the minimum performance as a function of number and location.

Alarm times for heat alarms and residential sprinklers are always based on the activation of devices in the room of fire origin. NFPA 72 allows the use of heat alarms on an individual basis (for example, in locations where smoke alarms are not appropriate - in an attic). Similarly, some rooms - e.g., bathrooms, some closets - are not required to have sprinklers in a code-compliant home sprinkler system (NFPA 13D). All fires in these test series were in normally-occupied 
spaces of the structures (living room, bedroom, or kitchen). Placement of heat alarms and residential sprinklers in these spaces is consistent with the appropriate codes.

Tables 23 and 24 shows a summary of alarm activation times for all of the tests. For each alarm technology and fire scenario, the average time to alarm is shown for typical alarm criteria (4.3\%/m for ionization alarms and $6.6 \% / \mathrm{m}$ for photoelectric alarms). For the manufactured home tests, each alarm time value is an average of available replicates. For the two-story home tests, only the flaming chair was replicated; all other data are single-test values.

Tables 23 and 24 also include alarm response times for three different alarm placement analyses described earlier: "Every Level," "Every Level + Bedrooms," and "Every Room." For the "Every Level" placement, alarms in hallways of each structure are included. For the "Every Level + Bedrooms" placement, these alarms are supplemented with alarms included in all the bedrooms of the structure. Finally, the "Every Room" placement included every alarm in all rooms of the structure.

The "Every Level" alarm placement provides a comparison of different smoke alarms and fire scenarios. For flaming fires, the ionization alarms provided the first alarms for all flaming scenarios except the flaming mattress with the door closed in the two-story home (In this scenario, the sprinkler directly above the fire responded first, with alarms outside the room of fire origin following later since flow out of the room was limited to leakage around the door). Average time to first alarm for the all flaming fires range from $30 \mathrm{~s}$ to $3602 \mathrm{~s}$, depending on the specific fire scenario, dwelling geometry, and alarm type. For smoldering fires, the photoelectric alarms provide shorter alarm times compared to other technologies. Average time to first alarm range from $1366 \mathrm{~s}$ to $4829 \mathrm{~s}$, depending on the fire scenario, dwelling geometry, and alarm type. In all but one smoldering test, photoelectric alarms provided the first alarm (for the smoldering chair in the two-story home, the aspirated alarm uses photoelectric alarm technology and for the smoldering mattress in the two-story home, the photoelectric alarm in the dual alarm responds first). For the cooking oil tests, an ionization alarm responded first in the manufactured home while the photoelectric aspirated alarm responded first in the two-story home test.

Providing alarms in the bedrooms in addition to the "Every Level" alarm placement (the "Every Level + Bedroom" alarm placement) reduces the time to alarm for every fire scenario and most alarm technologies, providing an additional $3 \mathrm{~s}$ to $794 \mathrm{~s}$ of available egress time, depending on the specific fire scenario, dwelling geometry, and alarm type. Not surprisingly, alarm times for the bedroom fire scenarios were most affected by the additional alarms since alarms were now included for the room of fire origin. For all but one fire scenario, the same alarm technology was the first to respond in the "Every Level + Bedroom" alarm placement compared to the "Every Level" alarm placement. For this exception, one of the Dual Ion/Photo alarms in the bedroom in one test responded earlier than other alarms and moved the average alarm time downward. Other alarms responded shortly after this alarm. 
In the "Every Room" alarm placement, all alarms in the structures were included in the calculations. For the photoelectric and ionization alarms, no additional reduction in alarm time was noted for the bedroom fire scenarios since the bedroom alarms were already included in the "Every Level + Bedroom" alarm placement. Some additional reduction in alarm times for flaming fire scenarios was expected due to placement of alarms in the room of fire origin for these scenarios.

Alarm placement had the greatest impact on reducing alarm time when alarms in the room of fire origin were included in the scenario. For bedroom fires, the greatest reduction was noted in the "Every Level + Bedrooms" placement scenario. For living room or kitchen fires, the reduction occurred in the "Every Room" placement. Few changes were noted for other alarm locations. In both test geometries, this is likely due to the simple geometries and initial alarm placement. For the manufactured home, the structure is essentially a single large compartment with bedrooms at either end of the structure. Thus, including additional alarms in the main compartment of the structure would be expected to have a minor impact on time to alarm. For the two-story home, there was no soffit between the living room and the stairwell to the upstairs level of the home. Thus, in most fires, alarms in the upstairs hallway of the home, included in the "Every Level" placement, were the first to alarm. Additional alarm locations in other location outside the room of fire origin thus had only a small impact on alarm time. For more complex geometries, the impact of alarm placement can be expected to be more pronounced. 
Table. 23. Average time to alarm (in seconds) for several smoke alarms and fire scenarios in a manufactured home Every Level Installation Criterion

\begin{tabular}{|c|c|c|c|c|}
\hline Flaming & Photo & Ion & $\begin{array}{c}\text { Dual } \\
\text { Ion/Photo }\end{array}$ & Aspirated \\
\hline Living Room & 130 & 73 & 77 & 137 \\
\hline Bedroom & 96 & 61 & 186 & 121 \\
\hline Bedroom (Door Closed) & 619 & 172 & 630 & 643 \\
\hline \multicolumn{5}{|l|}{ Smoldering } \\
\hline Living Room & 4615 & 4829 & 4605 & 4541 \\
\hline Bedroom & 2622 & 3631 & 3471 & 2997 \\
\hline Bedroom (Door Closed) & 3442 & 3428 & 3434 & 3446 \\
\hline \multicolumn{5}{|l|}{ Cooking } \\
\hline Kitchen & 766 & 520 & 912 & 1172 \\
\hline
\end{tabular}

\section{Every Level + Bedrooms Installation Criterion}

\begin{tabular}{|l|c|c|c|c|}
\cline { 2 - 4 } \multicolumn{1}{c|}{ Flaming } & Photo & Ion & $\begin{array}{c}\text { Dual } \\
\text { lon/Photo }\end{array}$ & Aspirated \\
\hline Living Room & 130 & 73 & 77 & 137 \\
\hline Bedroom & 78 & 37 & 186 & 121 \\
\hline Bedroom (Door Closed) & 84 & 34 & 619 & 643 \\
\hline
\end{tabular}

Smoldering
\begin{tabular}{|l|c|c|c|c|}
\hline Living Room & 3856 & 4695 & 4304 & 4541 \\
\hline Bedroom & 2179 & 3618 & 3471 & 2997 \\
\hline Bedroom (Door Closed) & 2648 & 3402 & 3434 & 3446 \\
\hline
\end{tabular}

2648

3434

Cooking

Kitchen

764

520

539

\begin{tabular}{|c|c|c|c|}
\hline \multicolumn{4}{|c|}{ Every Room Installation Criterion } \\
\hline Flaming & Photo & Ion & $\begin{array}{c}\text { Dual } \\
\text { Ion/Photo }\end{array}$ \\
\hline Living Room & 92 & 27 & 77 \\
\hline Bedroom & 78 & 37 & 104 \\
\hline Bedroom (Door Closed) & 84 & 34 & 134 \\
\hline
\end{tabular}

\section{Smoldering}

\begin{tabular}{|l|c|c|c|}
\hline Living Room & 2552 & 4402 & 4304 \\
\hline Bedroom & 2179 & 3618 & 3429 \\
\hline Bedroom (Door Closed) & 2648 & 3402 & 3434 \\
\hline
\end{tabular}

Bedroom (Door Closed)

\section{Cooking}

Kitchen

691

\begin{tabular}{|l|l|}
\hline 487 & 539 \\
\hline
\end{tabular}

Change from Every Level

\begin{tabular}{|c|c|c|}
\hline Photo & Ion & $\begin{array}{c}\text { Dual } \\
\text { Ion/Photo }\end{array}$ \\
\hline-- & -- & -- \\
\hline-18 & -25 & -- \\
\hline-535 & -138 & -11 \\
\hline \multicolumn{3}{|c|}{} \\
\hline-- & -- & -301 \\
\hline-443 & -13 & -- \\
\hline-794 & -26 & -- \\
\hline \multicolumn{2}{|c|}{} \\
\hline-3 & -- & -373 \\
\hline
\end{tabular}

Change from Every Level + Bedrooms

\begin{tabular}{|c|c|c|}
\hline Photo & Ion & $\begin{array}{c}\text { Dual } \\
\text { lon/Photo }\end{array}$ \\
\hline-38 & -45 & -- \\
\hline-- & -- & -83 \\
\hline-- & -- & -485 \\
\hline \multicolumn{3}{|c|}{} \\
\hline-2063 & -427 & -- \\
\hline-- & -- & -42 \\
\hline \multicolumn{2}{|l}{} \\
\hline-73 & -33 & -- \\
\hline
\end{tabular}


Table 24. Average time to alarm (in seconds) for several smoke alarms and fire scenarios in a two-story home

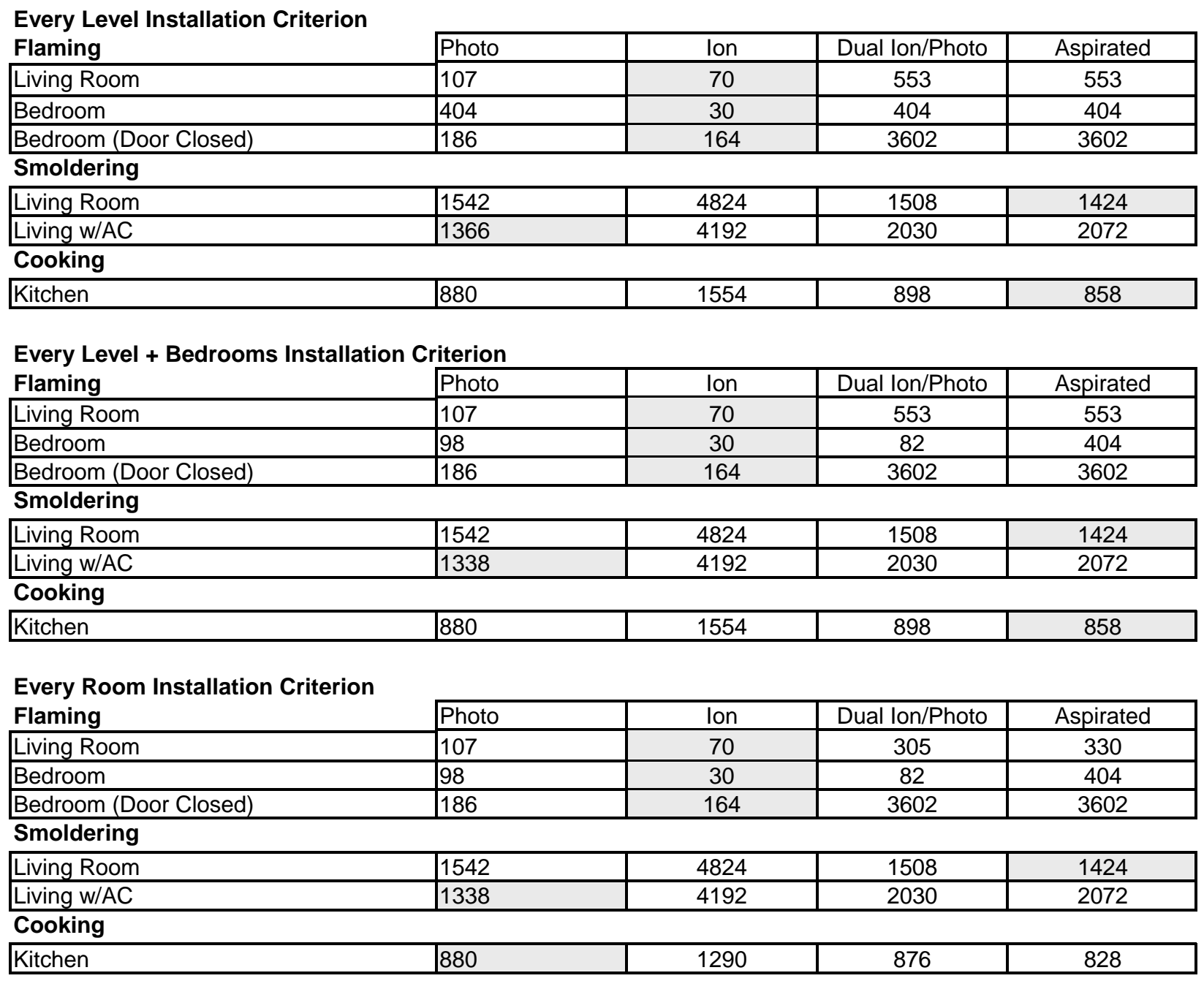




\subsection{Tenability Times}

For this report, the tenability time is when the first tenability limit is exceeded $1.5 \mathrm{~m}(5 \mathrm{ft})$ from the floor at any point along the primary escape path. It should be noted that the conditions at the instrument locations nearest to and in the same room as the fire were excluded since the codes are not designed to protect people intimate with the initial fire. The tolerance of people to fire conditions is a subject of considerable debate worldwide, because it has significant implications for public safety and for product liability. Deciding on appropriate tolerance limits is highly complex because of the broad variability among people and the need for conservatism to protect the more vulnerable portions of any population.

This topic has been the subject of current work by an international committee of experts working as ISO Technical Committee (TC) 92, subcommittee (SC) 3. This group has published a technical standard, ISO TS 13571 [26], that recommends limits of human tolerance to fire products. These limits are also consistent with the recommendations in the SFPE Handbook of Fire Protection Engineering [23]. Limits for elevated temperature and toxic gas species were taken from ISO TS 13571. For smoke obscuration, an optical density of $0.25 \mathrm{~m}^{-1}$ was used as a tenability criterion, a value typically used by the smoke alarm industry.

It should be noted that ISO TS 13571 is considered by most (including its principle authors) to be highly conservative, stating that the "... values are intended to assure with high confidence that even vulnerable people will not be incapacitated, then killed." Thus, any observations based on analyses using these values may look much worse than what would be seen in actual use because tenability is based on the most susceptible of the population. The ISO TS 13571 values represent the best, international consensus so they were used for the primary analysis, but the data has been made available so that others can examine the impact of other limits.

\subsection{Time Needed for Escape}

As with the original Indiana Dunes tests this report does not select a value of time needed for escape since this is highly variable and is a function of the age and condition of the occupants, travel distances, behavior affecting pre-movement times, etc. An independent analysis of the Indiana Dunes data in 1975 [43] chose 3 min as an (arbitrary) reference number. A subsequent study funded by NIST at the University of Massachusetts (Amherst) [44] found average times needed to awaken sleeping occupants, phone the fire department, and evacuate all family members was about $50 \mathrm{~s}$ for families with children and nearly 70 seconds for the elderly.

This section will address escape times for best and worst case fire scenarios occurring in both the manufactured and 2-story homes. The evacuation of occupants in any occupancy consists of a 
series of actions beginning with cues received from the fire situation to eventual movement out of the building or refuge within the space. This escape time analysis assumes that the initial cue is provided by the smoke alarm and that the occupants have received, recognized, and interpreted this and other cues of the fire situation. It is difficult to establish a quantitative result for escape time. Movement time has been monitored and recorded in a number of studies, and especially in residential cases, it is simple to obtain an occupant velocity for unimpeded flow (low density). Premovement times, on the other hand, are more difficult to obtain since these response times are not only dependent on the fire situation, but also on the layout of the house, time of day, placement of items in relation to occupants (such as clothes, phone, personal effects), position of occupants in relation to each other (i.e., are the children in the same room, hiding in a closet in the house), and the role of the person(s) in the house in times of emergency (i.e., will they investigate, gather others).

\subsubsection{Movement Speed}

Movement speeds are calculated using the maximum (unimpeded) exit flow speeds (table 3-14.4 in SFPE Handbook [45]). The population density of the space is low, therefore maximum speeds are considered to be possible. The maximum speed along a horizontal exit route is $1.2 \mathrm{~m} / \mathrm{s}(235 \mathrm{ft} / \mathrm{min})$. The stairs in the two-story home have $18 \mathrm{~cm}$ (7 in) risers and $28 \mathrm{~cm}$ (11 in) treads, which corresponds to a maximum travel speed of $0.95 \mathrm{~m} / \mathrm{s}(187 \mathrm{ft} / \mathrm{min})$. Stair travel speeds only apply to the two-story dwelling.

For elderly occupants, Ando [46] investigated unimpeded horizontal movement times and determined that travel speed for males and females was dependant upon age. A female around the age of 30, to signify a young occupant, would move at a velocity of $1.2 \mathrm{~m} / \mathrm{s}(235 \mathrm{ft} / \mathrm{min})$ and a female at the age of 70 years would move at approximately $0.75 \mathrm{~m} / \mathrm{s}(148 \mathrm{ft} / \mathrm{min})$. The younger occupant's speed agrees with the SFPE handbook's value for unimpeded movement over horizontal components. Proulx [47] performed a study of drilled-evacuation from four apartment buildings and summarized that occupants over 65 years old moved through the stairway at an unimpeded velocity of $0.43 \mathrm{~m} / \mathrm{s}(85 \mathrm{ft} / \mathrm{min})$.

\subsubsection{Premovement Activities}

Bryan [48], Wood [49], and Keating [50] have studied premovement actions of residential occupancies after the recognition phase has been completed. These actions (in no specific order) may be divided among the parental unit or taken on by a single person within the household and are the following: notifying others, getting dressed, calling the fire department, investigating, fighting the fire, and leaving. Wood states that the more serious a resident considers the fire to be, the more likely he/she is to leave the building immediately. Also, in his studies, he found that familiarity with the residence did not affect whether a person attempted to immediately exit the building or first retrieve personal items. In a 3 year study of Seattle residents, Keating found 
that men were more likely to fight the fire, while women were more likely to request information (which could involve notifying others, the fire department, etc). However, since this study occurred more than 20 years ago, it is not clear that this trend would be presently observed.

Pearson and Joost [51]observed that premovement and movement times will be increased for those scenarios which include elderly occupants. They studied response times during the day and night of young adults in good health, elderly in good health, and elderly with arthritic impairments in a residential experimental facility. When comparing total egress times of all scenarios, the elderly subjects in good health took approximately $27 \%$ longer and the arthritic subjects took $44 \%$ longer than the young adult subjects to perform scenario behaviors and movements. Some response activities noted by Pearson and Joost were accompanied by estimated time. Again, these depend on the situation and are only included to give the reader some idea of the time taken to perform the activities shown in table 25 .

Table 25. Sample premovement activity time

\begin{tabular}{|c|c|}
\hline Activity & Response Time (s) for young adults \\
\hline Dressing - putting on robe & 6 to 10 \\
\hline Calling fire department & 15 \\
\hline Obtaining personal items (wallet, keys) & 5 to 10 \\
\hline Awakening loved ones & 5 to 10 per child \\
\hline
\end{tabular}

\subsubsection{Escape Times}

For both the two-story home and the manufactured home, several scenarios representing the shortest escape time, as well as a worst case were calculated. These calculated escape times represent reasonable lower and upper bounds, although shorter and longer escape times may be possible. The best case scenario for the manufactured home is an accidental fire where the occupants are located in the kitchen and begin evacuation immediately upon alarm activation. The worst case scenario includes an arthritic elderly couple asleep at the time of fire ignition. One child is present in each bedroom. It is important to note that there is no feedback between the fire and the occupants accounted for in this calculation. This may extend the escape time significantly.

In the manufactured home, a worst-case evacuation would involve one or both occupants walking from bedroom 2, through the kitchen, through the living room, into bedroom 1, then into

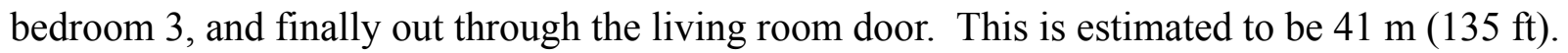


Distances are taken as straight lines (excluding obstacles) and are measured from the furthest corner in any room to the room door. A $41 \mathrm{~m}$ travel distance would take an elderly occupant approximately $55 \mathrm{~s}$ to traverse at $0.75 \mathrm{~m} / \mathrm{s}$. Prior to egress initiation, the occupants are assumed to dress, call the fire department, obtain personal items, and awaken both children. The cumulative response time (as opposed to the movement time) would be $55 \mathrm{~s}$ for a young adult family with 2 children to retrieve. For the elderly response times, multiplying $55 \mathrm{~s}$ by 1.44 gives a value of $80 \mathrm{~s}$. Thus, the total escape time would be approximately $(55 \mathrm{~s}+80 \mathrm{~s})$ or $135 \mathrm{~s}$.

For the 2-story dwelling, movement of an elderly couple from the master bedroom, to bedroom 3 , to bedroom 2, to the stairs, and out the front door totaled $33.7 \mathrm{~m} \mathrm{(110} \mathrm{ft)} \mathrm{on} \mathrm{horizontal}$ surfaces and $5.8 \mathrm{~m}(19 \mathrm{ft})$ on the stairs. The movement time for the elderly arthritic couple with two young children present is $58 \mathrm{~s}$. The premovement time for the two-story home would be identical to that of the manufactured home. Thus, the total worst case escape time for the twostory home is $(58+80)$ or $138 \mathrm{~s}$.

For the best case scenario, movement times in the manufactured home were calculated for the scenarios that would take the shortest amount of time to complete. This includes a young couple making dinner in the kitchen which results in an accidental grease fire. The distance to evacuate from the kitchen fire is approximately $2.7 \mathrm{~m}(9 \mathrm{ft}$.). The kitchen evacuation would take approximately $3 \mathrm{~s}$ to $5 \mathrm{~s}$. Pre-movement time was assumed to be $0 \mathrm{~s}$. In the two-story home, the young couple accidently starts a fire. The distance to evacuate from the kitchen is approximately $11 \mathrm{~m}(36 \mathrm{ft})$. The kitchen evacuation would take approximately $10 \mathrm{~s}$.

Additional scenarios are presented in table 26 . The young family at night scenario is identical to the worst case scenario, except that the young family moves more quickly and takes less time performing premovement tasks. Additionally, the elderly couple in the kitchen would require a somewhat longer time than the young couple in order to evacuate the home from the kitchen. In conclusion, the manufactured home will require $5 \mathrm{~s}$ to $135 \mathrm{~s}$ of escape time in order to reasonably protect the occupants. The two-story home will require approximately $10 \mathrm{~s}$ to $140 \mathrm{~s}$ of escape time. Note that escape times are sensitive to building geometry and occupant behavior assumptions. 
Table 26. Estimates of required escape times for best and worst case scenarios

\begin{tabular}{|c|c|c|c|c|c|c|c|}
\hline \multicolumn{2}{|c|}{ Scenario } & \multicolumn{2}{|c|}{ Premovement Time (s) } & \multicolumn{2}{|c|}{ Movement Time (s) } & \multicolumn{2}{|c|}{ Total Escape Time (s) } \\
\hline & & M. Home & Two-Story & M. Home & Two-Story & M. Home & Two-Story \\
\hline \multirow{2}{*}{ 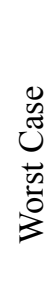 } & $\begin{array}{l}\text { Young } \\
\text { family at } \\
\text { night }\end{array}$ & 55 & 55 & 35 & 35 & 90 & 90 \\
\hline & $\begin{array}{l}\text { Elderly } \\
\text { family at } \\
\text { night }\end{array}$ & 80 & 80 & 55 & 60 & 135 & 140 \\
\hline \multirow{2}{*}{ 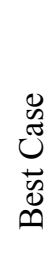 } & $\begin{array}{l}\text { Young } \\
\text { couple in } \\
\text { kitchen }\end{array}$ & $-\mathrm{a}$ & -- & 5 & 10 & 5 & 10 \\
\hline & $\begin{array}{l}\text { Elderly } \\
\text { couple in } \\
\text { kitchen }\end{array}$ & -- & -- & 10 & 15 & 10 & 15 \\
\hline
\end{tabular}

a - best case scenarios neglect any premovement activity; actual escape times are likely to be longer than best case estimates

\subsection{Smoke Alarm Performance}

Table 27 and figures 206 to 208 present the average (for different alarm times of the same type and multiple tests of each scenario) available egress times for ion, photo, aspirated, and $\mathrm{CO}$ alarms as a function of installation requirement (every level, every level + bedrooms, and every room) and fire type (flaming, smoldering, and grease), for the manufactured home tests (tests SDC01 to SDC15 and SDC30 to SDC41). Table 28 includes equivalent results for the two-story home. For heat alarms and sprinklers, only a single location in the room of fire origin was included in the tests. Escape times are calculated as the time to the first alarm activation (for that type of alarm) minus the time to untenable conditions. Negative numbers thus indicate that tenability criteria were exceeded prior to alarm activation. Discussion in this section focuses on the manufactured home tests. Smoke alarm activation times and test tenability times for the two story tests were similar to comparable tests in the manufactured home and are available to facilitate similar analyses for this structure. Appendix B provides detailed data for alarm activation and test tenability.

Increasing alarm coverage from every level to every level plus bedrooms provides room of origin protection for mattress scenarios since the mattress was located in one of the bedrooms. For chair scenarios, this addition would not indicate an increase in protection for the current test series since chairs were never located in bedrooms in these tests. 
On average, all of the alarm types provided positive available egress time for all manufactured home fire scenarios, with average available safe egress times ranging from $36 \mathrm{~s}$ to $2413 \mathrm{~s}$. The alarms did not, however, provide positive available egress time in every test when the alarms were located only on every level but not in bedrooms. (In one manufactured home flaming mattress test with the doorway closed, the photoelectric alarm responded $22 \mathrm{~s}$ after the tenability limit was reached and in one two-story home smoldering chair test, the ionization alarm responded $54 \mathrm{~s}$ after the tenability limit was reached.) Ionization type smoke alarms typically provided the longest egress times for flaming (and grease) fires, and photoelectric smoke alarms typically provided the longest escape times for smoldering fires. Similar trends are seen for the two-story home tests, with ionization alarms providing the longest egress times for flaming fires.

As expected, alarm placement can effect the available safe egress time. For the manufactured home tests, including alarms in bedrooms in addition to those on every level increases average available safe egress time by from $3 \mathrm{~s}$ to nearly $800 \mathrm{~s}$, depending upon fire scenario, alarm type, and alarm placement. Greater increases were noted for photoelectric (up to $794 \mathrm{~s}$ ) or dual photoelectric / ionization alarms (up to $301 \mathrm{~s}$ ) than for the ionization alarms (up to $138 \mathrm{~s}$ ). No increase was seen for the aspirated photoelectric detector. This may be due to the periodic sampling of this detector. Additional increases were seen for the change from every level + bedrooms to every room. For the two-story home tests, the effect was far less pronounced. This was likely due to the interior design of the home. For fires downstairs, the upstairs hallway alarms were typically the first to respond since there was no soffit to impede smoke flow from the first floor into the stairwell to the alarms at the top of the stairs. 
Table. 27. Available egress time (in seconds) for several different alarm technologies and fire scenarios in a manufactured home Every Level Installation Criterion

\begin{tabular}{|c|c|c|c|c|}
\hline Flaming & Photo & Ion & $\begin{array}{c}\text { Dual } \\
\text { Ion/Photo }\end{array}$ & Aspirated \\
\hline Living Room & 89 & 147 & 142 & 82 \\
\hline Bedroom & 58 & 93 & 39 & 45 \\
\hline Bedroom (Door Closed) & 876 & 1323 & 1808 & 852 \\
\hline \multicolumn{5}{|l|}{ Smoldering } \\
\hline Living Room & 351 & 137 & 361 & 425 \\
\hline Bedroom & 1382 & 120 & 362 & 731 \\
\hline Bedroom (Door Closed) & 40 & 54 & 48 & 36 \\
\hline \multicolumn{5}{|l|}{ Cooking } \\
\hline Kitchen & 592 & 838 & 899 & 187 \\
\hline
\end{tabular}

\section{Every Level + Bedrooms Installation Criterion}

\begin{tabular}{|l|c|c|c|c|}
\cline { 2 - 4 } \multicolumn{1}{c|}{ Flaming } & Photo & Ion & $\begin{array}{c}\text { Dual } \\
\text { lon/Photo }\end{array}$ & Aspirated \\
\hline Living Room & 89 & 147 & 142 & 82 \\
\hline Bedroom & 76 & 118 & 39 & 45 \\
\hline Bedroom (Door Closed) & 1411 & 1461 & 1830 & 852 \\
\hline
\end{tabular}

\section{Smoldering}

\begin{tabular}{|c|c|c|c|c|}
\hline Living Room & 351 & 137 & 662 & 425 \\
\hline Bedroom & 1548 & 137 & 362 & 731 \\
\hline Bedroom (Door Closed) & 834 & 80 & 48 & 36 \\
\hline Cooking & & & & \\
\hline
\end{tabular}

\begin{tabular}{|l|l|l|l|l|}
\hline Kitchen & 595 & 838 & 819 & 187 \\
\hline
\end{tabular}

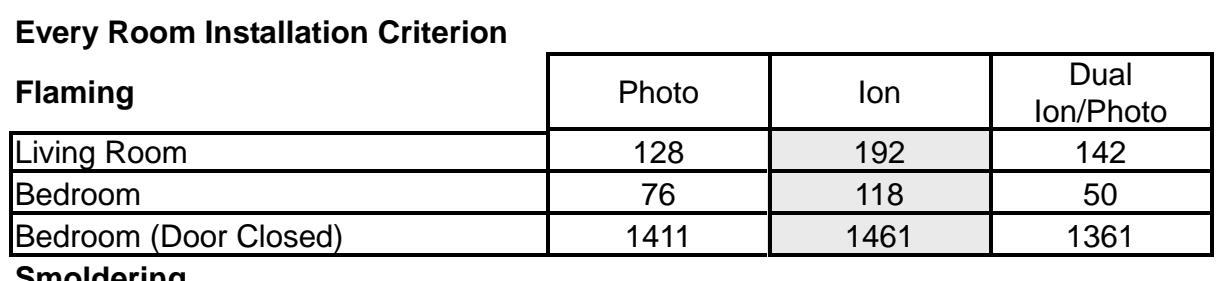

\section{Smoldering}

\begin{tabular}{|l|c|c|c|}
\hline Living Room & 2413 & 563 & 662 \\
\hline Bedroom & 1548 & 109 & 298 \\
\hline Bedroom (Door Closed) & 834 & 80 & 48 \\
\hline
\end{tabular}

\section{Cooking}

\begin{tabular}{|l|l|l|l|}
\hline Kitchen & 667 & 871 & 819 \\
\hline
\end{tabular}

a - blank entry means no change compared to previous installation criterion for this alarm type

Change from Every Level ${ }^{\mathrm{a}}$

\begin{tabular}{|c|c|c|}
\hline Photo & Ion & $\begin{array}{c}\text { Dual } \\
\text { Ion/Photo }\end{array}$ \\
\hline 18 & 25 & \\
\hline 535 & 138 & 22 \\
\hline
\end{tabular}

\begin{tabular}{|l|l|l|}
\hline & & 301 \\
\hline 167 & 17 & \\
\hline 794 & 26 & \\
\hline
\end{tabular}

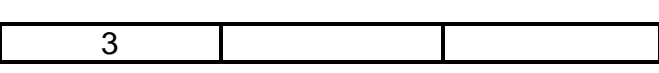

Change from Every Level + Bedrooms ${ }^{\mathrm{a}}$

\begin{tabular}{|c|c|c|}
\hline Photo & Ion & $\begin{array}{c}\text { Dual } \\
\text { Ion/Photo }\end{array}$ \\
\hline 38 & 45 & \\
\hline & & 11 \\
\hline & & \\
\hline
\end{tabular}

\begin{tabular}{|l|l|l|}
\hline 2063 & 427 & \\
\hline & & \\
\hline & & \\
\hline
\end{tabular}

73

\begin{tabular}{|l|l|}
\hline 33 & \\
\hline
\end{tabular}


Table 28. Available egress time (in seconds) for several different alarm technologies and fire scenarios in a two-story home

\begin{tabular}{|c|c|c|c|c|}
\hline \multicolumn{5}{|l|}{ Every Level Installation Criterion ${ }^{a}$} \\
\hline Flaming & Photo & Ion & \begin{tabular}{|c|} 
Dual \\
Ion/Photo \\
\end{tabular} & Aspirated \\
\hline Living Room & 108 & 152 & & \\
\hline Living Room (Replicate) & 134 & 172 & & \\
\hline Living Room (Fully-Furnished) & 144 & 172 & & \\
\hline Bedroom & no alarm & 374 & no alarm ${ }^{b}$ & no alarm \\
\hline Bedroom (Door Closed) & 3416 & 3438 & no alarm & no alarm \\
\hline \multicolumn{5}{|l|}{ Smoldering } \\
\hline Living Room & 3298 & 16 & 3332 & 3416 \\
\hline Living Room (Air Conditioning) & 2773 & -54 & 2108 & 2066 \\
\hline \multicolumn{5}{|l|}{ Cooking } \\
\hline Kitchen & 952 & 278 & 934 & 974 \\
\hline
\end{tabular}

Every Level + Bedrooms Installation Criterion ${ }^{a}$

\begin{tabular}{|c|c|c|c|c|}
\hline Flaming & Photo & Ion & \begin{tabular}{|c|} 
Dual \\
Ion/Photo
\end{tabular} & Aspirated \\
\hline Living Room & 108 & 152 & & \\
\hline Living Room (Replicate) & 134 & 172 & & \\
\hline Living Room (Fully-Furnished) & 144 & 172 & & \\
\hline Bedroom & 306 & 374 & no alarm & no alarm \\
\hline Bedroom (Door Closed) & 3416 & 3438 & no alarm & no alarm \\
\hline \multicolumn{5}{|l|}{ Smoldering } \\
\hline Living Room & 3298 & 16 & 3332 & 3416 \\
\hline Living Room (Air Conditioning) & 2800 & -54 & 2108 & 2066 \\
\hline \multicolumn{5}{|l|}{ Cooking } \\
\hline Kitchen & 952 & 278 & 934 & 974 \\
\hline
\end{tabular}

\section{Every Room Installation Criterion ${ }^{\mathrm{a}}$}

\begin{tabular}{|l|l|c|c|c|}
\cline { 2 - 5 } \multicolumn{1}{c|}{ Flaming } & Photo & Ion & $\begin{array}{c}\text { Dual } \\
\text { Ion/Photo }\end{array}$ & Aspirated \\
\hline Living Room & 108 & 152 & & \\
\hline Living Room (Replicate) & 134 & 172 & & \\
\hline Living Room (Fully-Furnished) & 144 & 172 & & \\
\hline Bedroom & 306 & 374 & no alarm & no alarm \\
\hline Bedroom (Door Closed) & 3416 & 3438 & - & - \\
\hline Smoldering & 3298 & 16 & 3332 & note c \\
\hline Living Room & 2800 & -54 & 2108 & note c \\
\hline Living Room (Air Conditioning) & \multicolumn{5}{|c|}{} \\
\hline Cooking & 952 & 542 & 956 & note c \\
\hline
\end{tabular}

a - blank entry means no alarm of this type was included for this test type

$\mathrm{b}$ - alarm was included, but did not alarm by end of test

c - no additional alarm included of this type 


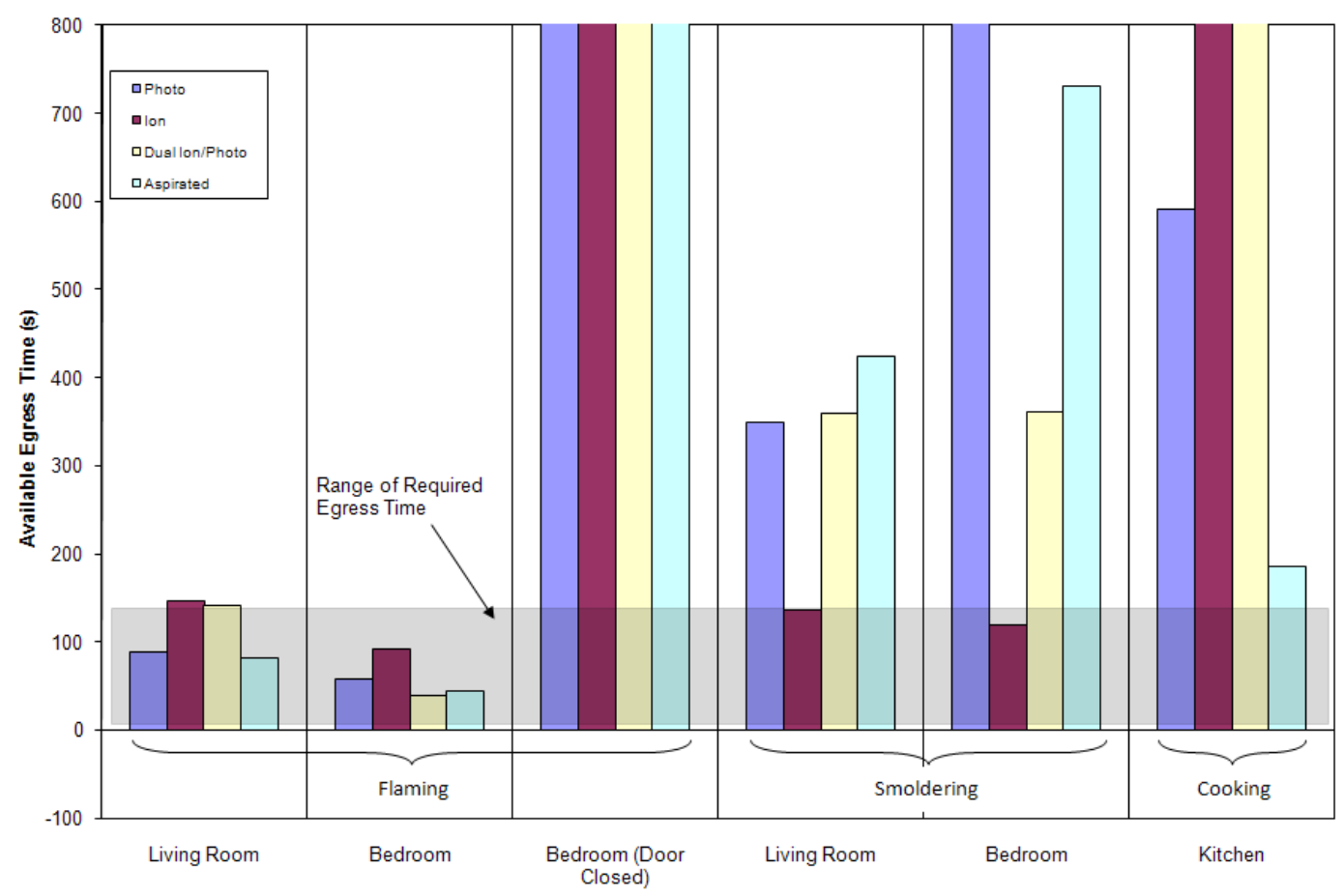

Figure 206. Average available egress time for several different alarm and fire types with alarms on every level for manufactured home tests

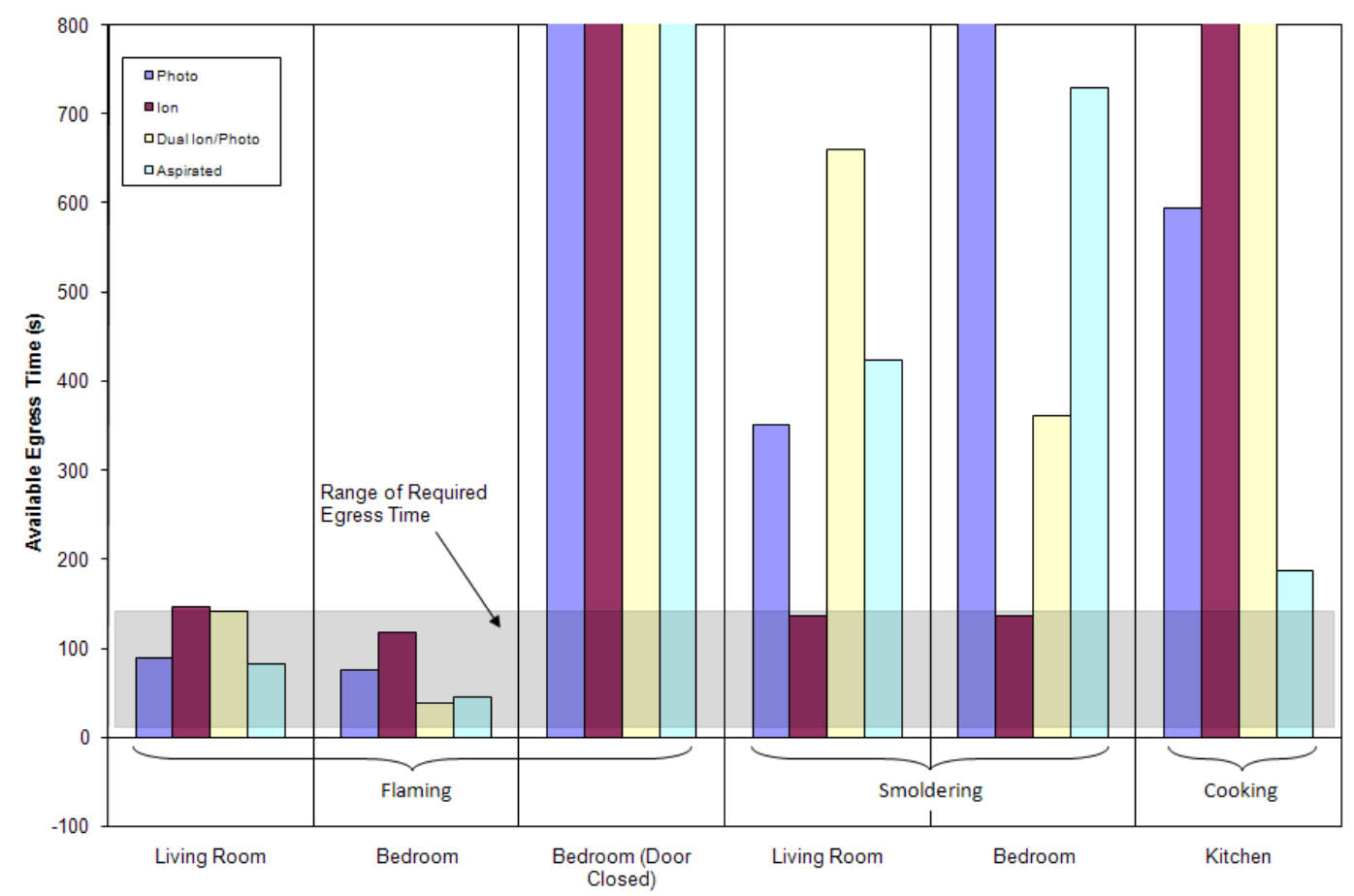

Figure 207. Average available egress time for several different alarm and fire types with alarms on every level + bedrooms for manufactured home tests 


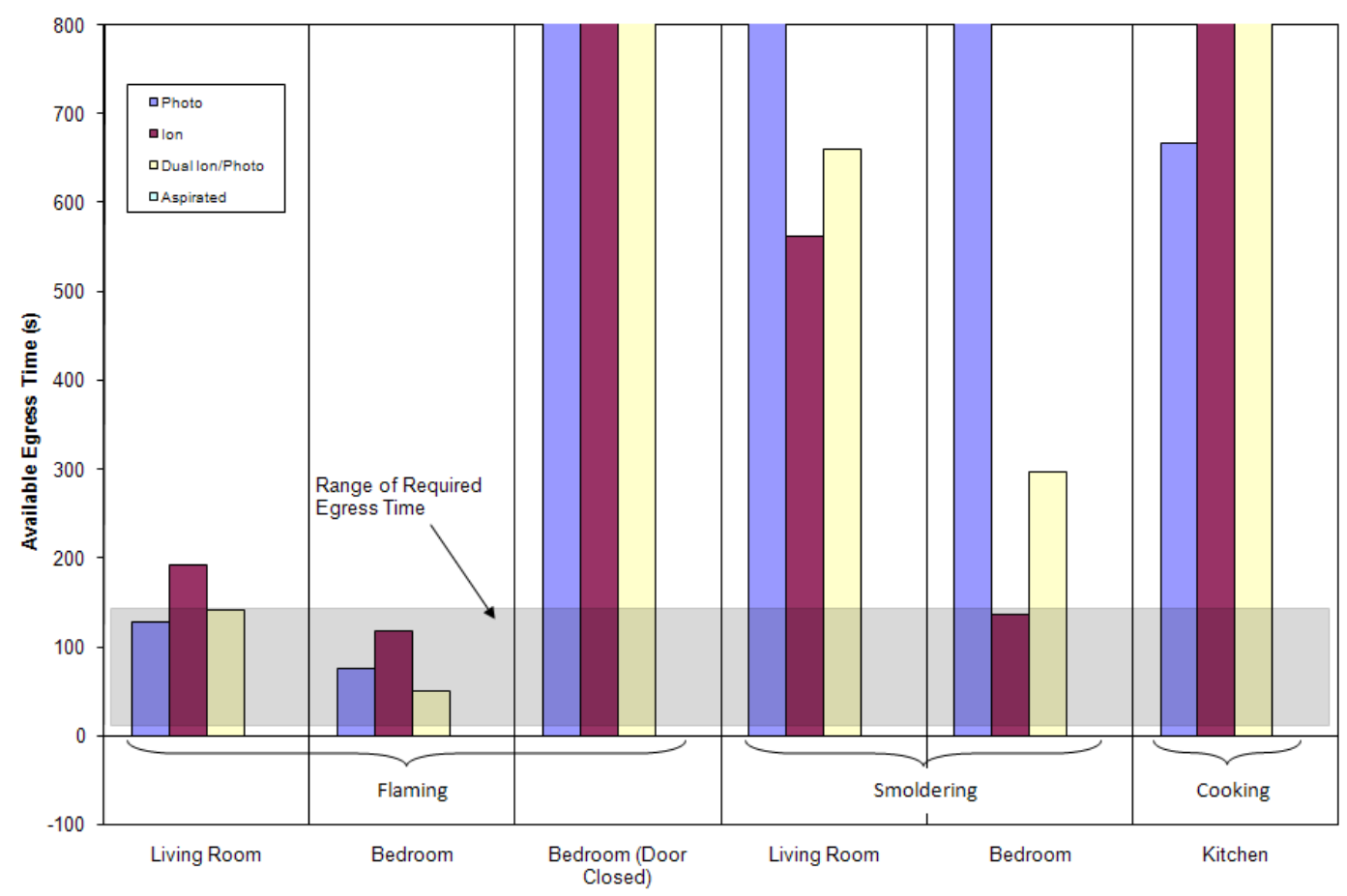

Figure 208. Average available egress time for several different alarm and fire types with alarms in every room for manufactured home tests

It is important to note that there is uncertainty in the estimates of available egress time as noted in section 5.7. For some of the tests, these times were relatively short and in some cases overlapped estimates of the time needed for egress from the buildings. For the most complex egress scenarios (with occupants asleep in all of the bedrooms) with the most sensitive populations (elderly adults with young children requiring egress assistance), the flaming fire scenarios may not provide sufficient time for escape regardless of the alarm technology. Thus, available escape time would be sufficient only if households follow the advice of fire safety educators, including sleeping with doors closed while using interconnected smoke alarms to provide audible alarm in each bedroom, and pre-planning and practicing escape so as to minimize pre-movement and movement times during egress.

\subsection{Other Alarm Technologies}

Additional fire detection technologies were included in the test fires to provide a point of comparison with the smoke alarms and to provide data for future analysis of fire alarms that may include multiple sensor technologies and complex algorithms to better predict accidental fires and thus reduce false alarms. For residential applications in the U.S. and Canada, carbon monoxide alarms, heat alarms, and residential sprinklers are used in concert with smoke alarms. Table 29 shows average alarm times for these technologies for the different fire scenarios in the 
manufactured home. For each alarm technology and fire scenario, the time to alarm is shown for typical alarm criteria (50 ppm for $\mathrm{CO}$ alarms; heat alarms and tell-tale sprinklers were determined from an audible alarm and measured pressure drop, respectively). For the manufactured home tests, each alarm time value is an average of available replicates. Individual calculations for all of the alarms in all of the tests is included as Appendix B.

\subsubsection{Carbon Monoxide} Alarms

The carbon monoxide alarms responded to all fire scenarios, but particularly for scenarios which would be expected to produce significant quantities of carbon monoxide during the combustion process, i.e., smoldering mattress, smoldering chair, and doorclosed flaming chair (which smoldered after the room of origin became oxygen limited). Alarm times ranged from $683 \mathrm{~s}$ to $3548 \mathrm{~s}$, depending upon fire scenario and alarm placement.

\subsubsection{Heat Alarms}

Since nearly all of the tests in this study were limited to the very early stages of fire growth, heat alarms typically responded near the end of the tests and after the transition to flaming for smoldering tests. For flaming fires, alarm times ranged from $163 \mathrm{~s}$ to $1912 \mathrm{~s}$.

Table. 29. Activation time (in seconds) for several different fire detection technologies and fire scenarios in a manufactured home.

\begin{tabular}{|c|c|}
\hline \multicolumn{2}{|c|}{ Every Level Installation Criterion } \\
\hline Flaming & CO Alarm \\
\hline Living Room & 209 \\
\hline Bedroom & 421 \\
\hline Bedroom (Door Closed) & 683 \\
\hline \multicolumn{2}{|l|}{ Smoldering } \\
\hline Living Room & 3581 \\
\hline Bedroom & 3188 \\
\hline Bedroom (Door Closed) & 3458 \\
\hline \multicolumn{2}{|l|}{ Cooking } \\
\hline Kitchen & 1412 \\
\hline
\end{tabular}

\begin{tabular}{|c|c|}
\hline \multicolumn{2}{|c|}{ Every Level + Bedrooms Installation Criteri } \\
\hline Flaming & CO Alarm \\
\hline Living Room & 209 \\
\hline Bedroom & 421 \\
\hline Bedroom (Door Closed) & 683 \\
\hline \multicolumn{2}{|l|}{ Smoldering } \\
\hline Living Room & 3564 \\
\hline Bedroom & 3188 \\
\hline Bedroom (Door Closed) & 3458 \\
\hline \multicolumn{2}{|l|}{ Cooking } \\
\hline Kitchen & 1399 \\
\hline
\end{tabular}

\begin{tabular}{|c|c|c|c|}
\hline \multicolumn{4}{|c|}{ Every Room Installation Criterion (note a) } \\
\hline Flaming & CO Alarm & Heat Alarm & $\begin{array}{c}\text { Tell-tale } \\
\text { Sprinkler }\end{array}$ \\
\hline Living Room & n.a. & 163 & 246 \\
\hline Bedroom & n.a. & 403 & 137 \\
\hline Bedroom (Door Closed) & n.a. & 1912 & 126 \\
\hline \multicolumn{4}{|l|}{ Smoldering } \\
\hline Living Room & n.a. & 5992 & 5914 \\
\hline Bedroom & n.a. & 3798 & 3983 \\
\hline Bedroom (Door Closed) & n.a. & n.a. & 3414 \\
\hline \multicolumn{4}{|l|}{ Cooking } \\
\hline Kitchen & n.a. & 1362 & 1362 \\
\hline
\end{tabular}




\subsubsection{Tell-tale Sprinklers}

The purpose of residential sprinklers is to improve protection against injury, life loss, and property protection. In particular, the design is expected to prevent flashover in the room of fire origin and to improve the chance for occupants to escape or be evacuated [52]. In the tests presented in this report, only the very early stages of a fire were studied, up to the point of incapacitation for occupants. Tell-tale sprinklers were included in the room of fire origin to provide a point of comparison between smoke alarms and sprinkler activation. For flaming fires, response time ranged from $126 \mathrm{~s}$ to $246 \mathrm{~s}$. For smoldering fires, response occurred shortly after the transition to flaming.

\subsection{Comparison with Earlier Tests}

In the original 1975 study [1], over 20 detectors of various generic types and nominal sensitivities were exposed to 40 fires in two actual residences. The fire types included flaming and smoldering chairs and mattresses in the family room, basement, and bedroom, as well as JP-4 in a $20 \mathrm{~cm}$ (8 in) pan in the kitchen. The detector types included ionization, photoelectric, dual gate (combination ionization and resistance bridge), and rate-of-rise heat detectors. Each location included

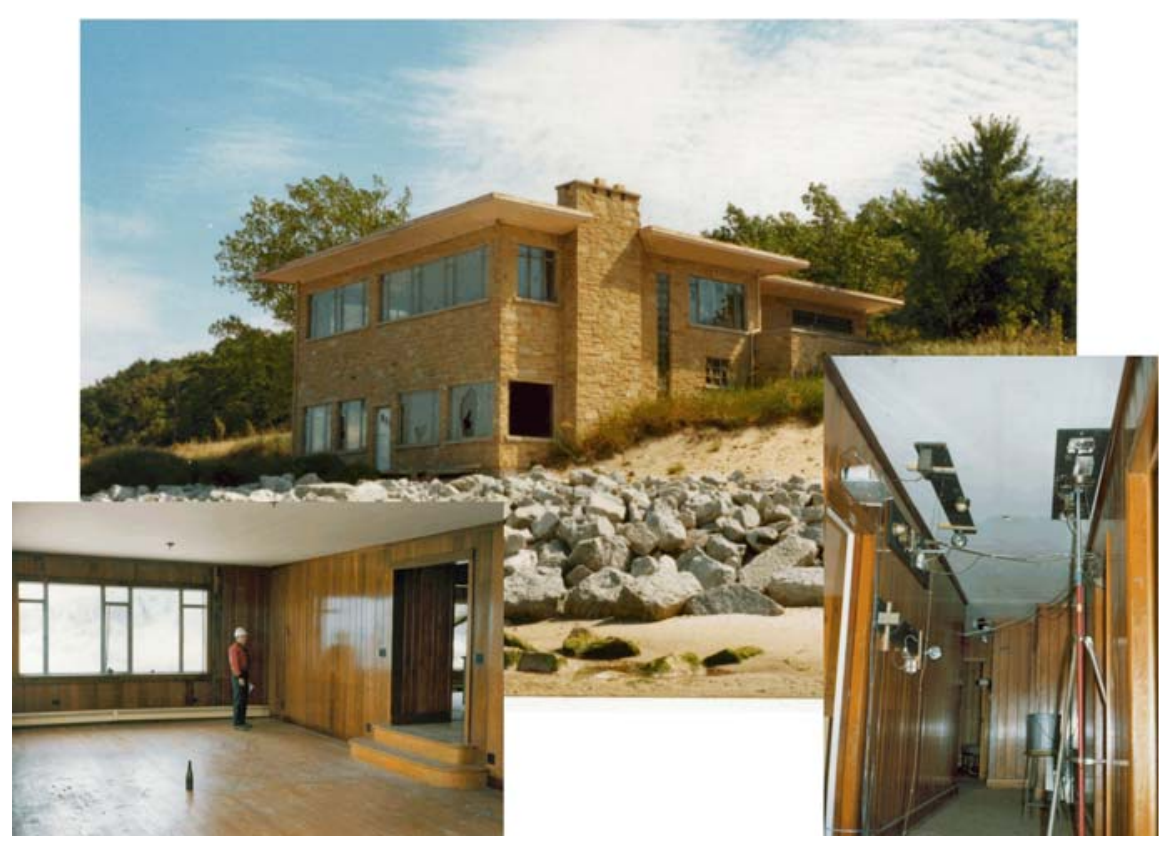

Figure 209. Test structure, sample interior room, and instrumentation in original 1975 Indiana Dunes Experiments both a high sensitivity (one percent per foot obscuration) and a low sensitivity (two percent per foot obscuration) detector.

The two test sites were a two-story brick structure with a basement and a single-story brick residence with basement. Instrumentation for both sites included thermocouple arrays, white light beam obscuration measurements, and equipment to monitor oxygen, carbon monoxide, and carbon dioxide. The instrumentation was placed in the room of origin, representative bedrooms, and along the egress path. 
Escape times in the current study for the ion type alarms for smoldering fires and for both ionization and photoelectric alarms for flaming fires were positive but somewhat short when compared to the performance of similar smoke alarms in the 1975 test series. Table 30 shows a comparison between the two test series.

These differences appear to be not so much because the alarms are activating more slowly, but rather because the tenability limits are being exceeded much faster. For flaming fires, alarm activation was somewhat shorter in the current tests than in the 1975 tests, and time to untenable conditions was dramatically faster in the current tests. This may be attributed partially to the different ISO tenability criteria compared to simpler limits in the 1975 study, and also due to significantly faster fire development observed in the upholstered furniture and mattresses used in these tests. Table 31 shows a comparison of tenability criteria used in the two studies. For the temperature and smoke obscuration criteria, values used in the current study are similar or slightly higher than those used in the 1975 study. For CO concentration, the range for the FED-based model used in the current study includes the value used in the 1975 study, but is also quite a wide range, depending on duration of the fire. For nearly all tests, the temperature or smoke criteria was met prior to the $\mathrm{CO}$ criterion.

A comparison of the fire growth in the two tests can be seen by comparing the temperatures near the ceiling for the tests from the two studies. For this comparison,
Table 30. Comparison of alarm times and times to untenable conditions for 1975 and current studies

\begin{tabular}{|l|l|c|c|}
\cline { 3 - 4 } \multicolumn{2}{c|}{} & 1975 Tests & Current Tests \\
\hline \multirow{2}{*}{ Alarm Times } & Flaming & $146 \pm 93$ & $47 \pm 35$ \\
\cline { 2 - 4 } & Smoldering & $1931 \pm 1103$ & $2042 \pm 876$ \\
\hline \multirow{2}{*}{ Tenability Times } & Flaming & $1036 \pm 374$ & $177 \pm 69$ \\
\cline { 2 - 4 } & Smoldering & $4419 \pm 1790$ & $2148 \pm 1023$ \\
\hline
\end{tabular}

Table 31. Comparison of tenability criteria used in the 1975 and current studies

\begin{tabular}{|l|l|l|}
\cline { 2 - 3 } \multicolumn{1}{c|}{} & 1975 Study & Current Study \\
\hline Temperature & $\mathrm{T} \geq 66{ }^{\circ} \mathrm{C}$ & $\mathrm{T} \geq 88{ }^{\circ} \mathrm{C}^{\mathrm{a}}$ \\
\hline Gas Concentration & $\begin{array}{l}\mathrm{CO} \geq 0.04 \% \\
\text { volume fraction }\end{array}$ & $\begin{array}{l}\mathrm{CO} \geq 0.02-0.3^{\mathrm{b}} \\
\% \text { volume fraction }\end{array}$ \\
\hline $\begin{array}{l}\text { Smoke } \\
\text { Obscuration }\end{array}$ & O.D. $\geq 0.23 \mathrm{~m}^{-1}$ & O.D. $\geq 0.25 \mathrm{~m}^{-1}$ \\
\hline
\end{tabular}

a - value for flaming fires calculated from ISO TS 13571 equation for convected heat

b - range of average values calculated from ISO TS 13571 equation for asphyxiant gases with tenability times for flaming fires and smoldering fires
Table 32. Comparison of fire growth rates in the 1975 and current studies

\begin{tabular}{|l|l|l|}
\cline { 2 - 3 } \multicolumn{1}{c|}{} & \multicolumn{2}{c|}{ Time to $65^{\circ} \mathrm{C}$ near burn room ceiling (s) } \\
\cline { 2 - 3 } \multicolumn{1}{c|}{} & 1975 Study & Current Study \\
\hline Flaming & $969 \pm 527$ & $131 \pm 40$ \\
\hline Smoldering & $4893 \pm 2236$ & $4332 \pm 1893$ \\
\hline
\end{tabular}


the time for the temperature near the ceiling to reach $65{ }^{\circ} \mathrm{C}$ was used as an indication of the rate of fire growth (table 32). Average time for flaming fires was significantly longer in the 1975 tests than in the current study - $969 \mathrm{~s}$ versus $131 \mathrm{~s}$. Average times for smoldering fires in the current test series were comparable to those observed in the 1975 tests. It is important to note, however, that the smoldering fire scenarios are very difficult to reproduce experimentally, and that times in the present study has an uncertainty (based upon one standard deviation) which overlaps the uncertainty in the 1975 study. Therefore, caution should be exhibited in drawing conclusions based upon comparisons of smoldering tenability times between the two studies.

While the 1975 study also used actual upholstered furniture and mattresses, these were purchased from a (charity) resale shop from donated articles. Such items purchased in 1975 would have been new in the early 1960s or even late 1950s and represented materials and constructions of that period. The chairs used in the present study were purchased from a furniture rental store and, while used, were only a year or two old. The mattresses were purchased new. Thus, the materials were certainly significantly different. Fire development in the current tests showed generally similar timing in growth of heat release rate to other recent studies for upholstered furniture [53] and mattresses [54].

In the upholstered furniture study, a set of upholstered chairs was constructed from five different fabric/foam combinations and subjected to a variety of ignition sources suggested by fire statistics. In the study, an effort was made to choose from across the spectrum of typical residential materials although no statistical justification for the particular materials was made. Time to peak heat release rates in excess of those necessary to lead to untenable conditions in structures in the current study ranged from about $150 \mathrm{~s}$ to nearly $1000 \mathrm{~s}$. It is estimated that a comparable value for chairs in the current study is about $300 \mathrm{~s}$. For mattresses, time to incapacitation using similar criteria to the current study ranged from $240 \mathrm{~s}$ to $1150 \mathrm{~s}$, depending upon the mattress. It is important to note that this study included reduced flammability designs not currently on the market. Thus, the materials used in the current study should not be dramatically different than other currently available materials. Still, it is important to note that while both the 1975 study and the current study attempted to use a representative sample of available and important furnishings, each study included only a small fraction of those available in the marketplace.

Conversely, alarms in the current study were, on average, of similar sensitivity compared to the alarms in the 1975 study. In the current study, the sensitivity of the unmodified smoke alarms were discussed in section 2.6. The average sensitivity measured for all alarms tested was $1.54 \% / \mathrm{ft} \pm 0.45 \% / \mathrm{ft}$. In the 1975 study, the average of all alarms tested was $1.91 \% / \mathrm{ft} \pm$ $0.73 \% / \mathrm{ft}$. While the average for the 1975 tests is higher, the uncertainty in the data overlaps. 


\subsection{Nuisance Alarms}

Nuisance alarms in residential settings from typical cooking activities, smoking or candle flames are affected by the properties of the aerosol produced and its concentration, the location of an alarm relative to the source, and the air flow that transports smoke to an alarm. This is not surprising, as the same observations have been made in the fire tests here and other studies. This study provides a detailed set of data that can be used to address several issues involving nuisance alarms and reinforces current suggested practices including the following:

- Clearly, the advice that alarms not be installed close to cooking appliances if at all possible is valid. These results show that homeowners who are able to move the location of an alarm that frequently experiences nuisance alarms would do well to maximize its distance from cooking appliances while keeping it in the area to be protected.

- It was observed that ionization alarms had a propensity to alarm when exposed to nuisance aerosols produced in the early stages of some cooking activities, prior to noticeable smoke production. This phenomenon could be particularly vexing to homeowners who experience such nuisance alarms.

- This data set could be used to assess the efficacy of employing an alarm with its sensitivity set to a level near the least sensitive limit allowable, relative to a more sensitive alarm.

- This study can be used to assess the benefits in terms of reduction of nuisance alarms from a typical ionization alarm with the use of a dual photoelectric/ionization sensor alarm that would maintain better overall sensitivity to a variety of fire sources (flaming and smoldering). Combined with the fire test series data, a basis for the sensitivity level settings on each alarm sensor could be proposed that is tied to test data.

- The so-called "hush" button or any other function that decreases sensitivity through human intervention to silence alarms for a fixed time period requires that a sensitivity adjustment and time period be specified. This data set could be used to assess the effectiveness of a sensitivity adjustment for a specific time period before resetting to the base sensitivity relative to these nuisance scenarios. The data could also be used to compare such a feature to other proposed strategies designed to reduce nuisance alarms.

- Carbon monoxide electrochemical cells at all alarm locations gathered data on the level of carbon monoxide produced during the tests and transported to the alarms. These data in conjunction with the complete fire test series data could be used to verify combined smoke/CO alarm algorithms. 
The FE/DE nuisance source tests captured salient features of some of the manufactured home tests. Clearly more work needs to be done to produce a set of tests and the performance criteria that covers a significant range of residential nuisance sources, and would assure a benefit in terms of nuisance alarm reduction. This work is part of ongoing research with the FE/DE at NIST. 


\section{Summary}

The data developed in this study include measurement of the output of a number of alarm devices for a range of fire scenarios and residences. In addition, temperature, smoke obscuration, and gas concentrations throughout the structures were monitored during this test series. A summary of the major efforts of this study relative to the original 11 goals presented in section 1.3 are discussed below. Specific conclusions are included in section 9 .

\section{Evaluate the performance of current smoke-alarm technology}

Current smoke alarm technology includes two operating principles - the ionization-type sensor and the photoelectric (scattering) sensor. The project included examples of current products containing each of the sensor designs currently being sold in the U.S. market so that the results would be representative of the full range of product performance expected.

Smoke alarms of either the ionization type or the photoelectric type consistently provided positive escape times. In some cases the escape time provided was relatively short and may overlap with a range of estimates of the time necessary for occupant egress.. Consistent with prior findings, ionization type alarms provided somewhat better response to flaming fires than photoelectric alarms, and photoelectric alarms provided (often) considerably faster response to smoldering fires than ionization type alarms.

In many cases, available escape time would be sufficient only if households follow the advice of fire safety educators, including sleeping with doors closed while using interconnected smoke alarms to provide audible alarm in each bedroom, and pre-planning and practicing escape so as to minimize pre-movement and movement times during egress.

\section{Test conditions representative of current fatal residential fires}

To ensure that the room sizes and arrangements, materials of construction, and ventilation conditions were representative of actual dwellings, all tests were conducted in real dwelling units. First, a manufactured home was procured and delivered to the NIST site for use in both fire tests and nuisance alarm tests. The floor plan selected was a three bedroom, two bath arrangement with a master suite at one end and the other bedrooms at the other end. This size and arrangement represents not only manufactured homes but also apartments and condominiums of about $100 \mathrm{~m}^{2}$ (or about $1000 \mathrm{ft}^{2}$ ). A second test site was a three bedroom, twostory, brick home, $139 \mathrm{~m}^{2}\left(1490 \mathrm{ft}^{2}\right)$ in size. 
Careful choice of test scenarios helps insure the representativeness of the study. Important factors included the room of fire origin, ignition source and first item ignited, and the ventilation conditions that affected the fire development and combustion chemistry. A review of recent fire statistics was conducted to select the most important fire scenarios representative of fatal and non-fatal fires in the U. S. In addition to the scenarios themselves, the test matrix considered the need to perform sufficient replicates to allow estimates of experimental uncertainty and repeatability.

\section{Evaluate the efficacy of current requirements for number and location of smoke alarms}

The experimental design provided data on the performance of alarms in various installation arrangements. Groups of alarms were located in the room of fire origin, at least one bedroom, and in a central location on every level. Thus, by considering the alarm times of devices in various locations against tenability times for any test it was possible to determine the escape time provided by various installation arrangements including every room, every level with or without every bedroom, and single detectors. Since both ionization and photoelectric types were included at each location the performance by sensor type is also quantified.

Smoke alarms of either type installed on every level generally provided positive escape times for different fire types and locations. Adding smoke alarms in bedrooms increased the escape time provided, especially for smoldering fires. In addition, occupants of bedrooms sleeping with the door closed would benefit from improved audibility of alarms within the room. This provides data for evaluating whether bedroom smoke alarms should be required in existing homes as they have been in new homes since 1993. It is important to note that the available safe egress times may overlap with the range of estimates of necessary egress time for the residences studied. Some of this is due to conservative tenability criteria based on incapacitation of the most vulnerable occupants that was used for the current study. Use of tenability criteria based on incapacitation or death of healthy individuals would certainly increase the available safe egress time.

Escape times in this study were systematically shorter than those found in a similar study conducted in the 1970s. This is related to some combination of faster fire development times for today's products that provide the main fuel sources for fires, such as upholstered furniture and mattresses, different criteria for time to untenable conditions, and improved understanding of the speed and range of threats to tenability. Still, it is important to note that while both studies attempted to use a representative sample of available and important furnishings, each study included only a small fraction of those available in the marketplace. Since there may be significant differences in the burning behavior between items of furniture, general conclusions are beyond the scope of this study and may warrant additional research. 


\section{Develop standard nuisance alarm sources to be included in the test program}

Smoke alarms are susceptible to alarming when exposed to non-fire aerosols. In residential settings, this typically involves cooking activities or transient, high humidity conditions (i.e., "shower steam"). The approach taken was to define a set of nuisance scenarios, replicate the events that cause nuisance alarms, and quantify the important variables. Translating the results to a set of nuisance source conditions reproducible in a suitable test-bed (i.e., a test room or the fire emulator/detector evaluator) would allow for more comprehensive detector performance testing. Preliminary tests were performed in the FE/DE with this in mind. Programming realistic and reproducible fire and nuisance conditions in the $\mathrm{FE} / \mathrm{DE}$ is an ongoing research project at NIST.

Experiments conducted with common nuisance sources produced data that should be useful in the development of new performance requirements for conditions that should not activate smoke alarms. Since the data include analog signal levels and duration for each of the sensor types they should be useful in evaluating a range of approaches to nuisance alarm reduction from reducing alarm threshold for a specified time ("hush" feature) to decision algorithms and multi-sensor arrays.

\section{Examine other fire detection technologies in combination with smoke alarms}

The program included mechanically aspirated (system-type) photoelectric smoke detectors, residential $\mathrm{CO}$ alarms, heat alarms, and tell-tale residential sprinklers in the tests along with currently available residential smoke alarms. Activation times for these devices will facilitate future analysis and development of fire detection devices using inputs from multiple sensors.

\section{Obtain data on the potential for improvements in performance by new technologies}

Simple alarm times for currently available smoke alarms are not sufficient to identify potential performance improvements so analog sensor data were recorded for both the fire performance and nuisance alarm testing. Additional instrumentation was selected and located to supplement the analog sensor data and allow better quantification of results. Examples included thermocouple measurements of temperatures and temperature gradients, smoke and gas species concentrations in several locations, and convective flow velocities in the ceiling layer at the level of the sensors.

\section{Include fuel items that incorporate materials and constructions representative of current residential furnishings}

Fuel (combustible) items were selected as generically appropriate to the room of origin and fire scenario identified from NFIRS analysis and typical of materials and constructions in common 
use today. Specifically, upholstered chairs were used for living room fires and mattresses for bedroom fires that were purchased from local, retail sources. No modifications were made and no accelerants were used to enhance or retard the burning behavior of the items. Cooking oil used in kitchen fires was also a normal commercial product that was not modified in any way.

\section{Fully characterize test detectors and alarms in a consistent manner to facilitate comparisons}

All detectors (smoke, heat, and CO) were characterized in NIST's FE/DE apparatus under similar conditions using an appropriate source. This allowed direct calibration of all devices in a comparable way and a means to verify the performance of sensors in combination or utilizing algorithms that may be suggested by the results of this study.

\section{Utilize fire models to extend the applicability of the test arrangements and maximize the test instrumentation}

Modeling, using NIST's CFAST and FDS models, was performed prior to testing to evaluate the impacts of the selected fuels and fire scenarios on the instrumentation and test structures and to assist in identifying the most appropriate instrument locations.

\section{Make All of the Data Collected as Widely Accessible as Possible}

Clearly the best method of (global) dissemination of the test data was to employ the Internet. Thus, a public web site was established (http://smokealarm.nist.gov/) on which the detailed results of tests were published as NIST Reports of Tests. This publication vehicle exists as a means to quickly disseminate test data that is factual in nature - no observations or conclusions were included. Thus the web site contains floor plans of the test sites showing instrument locations, details of the fuels and test conditions, and test data in electronic form.

\section{Provide Opportunities to Enhance Public Fire Safety Education}

CPSC, USFA, NFPA, and others have significant efforts in public fire safety education. The information obtained in these tests will provide a basis for improving public fire safety messages and will quantify the protection provided by residential smoke alarms. The project data could be used to show the reduction in protection resulting from one or more devices being nonfunctional as a motivation for testing and maintenance by the homeowner. Video recording of all of the tests from multiple locations were made. These recordings will be made available as part of the information on the public web site for use in public safety announcements and educational materials. 
Finally, a press day was held that resulted in stories carried on all the major news networks and on more than 75 local outlets from Maine to Hawaii. 


\section{Conclusions}

1. The data developed in this study include measurement of temperature and smoke obscuration in addition to gas concentrations for a range of fire scenarios and residences. Measurement of the response of smoke alarms, CO alarms, heat alarms, and tell-tale sprinklers are also included. These data could be of significant value in developing appropriate algorithms for alarms that may include one or more sensor types.

2. Smoke alarms of either the ionization type or the photoelectric type consistently provided time for occupants to escape from most residential fires.

a In many cases, available escape time would be sufficient only if households follow the advice of fire safety educators, including sleeping with doors closed while using interconnected smoke alarms to provide audible alarm in each bedroom, and pre-planning and practicing escape so as to reduce pre-movement and movement times.

b. Smoke alarms may not provide protection for people directly exposed to the initial fire development (so-called "intimate with ignition").

c. Consistent with prior findings, ionization type alarms provided somewhat better response to flaming fires than photoelectric alarms, and photoelectric alarms provided (often) considerably faster response to smoldering fires than ionization type alarms.

d. Smoke alarms of either type installed on every level generally provided positive escape times for different fire types and locations. Adding smoke alarms in bedrooms increased the escape time provided, especially for smoldering fires. It is important to note that the available safe egress times may overlap with the range of estimates of necessary egress time for the residences studied. Some of this is due to conservative tenability criteria based on incapacitation of the most vulnerable occupants that was used for the current study.

e. Escape times in this study were systematically shorter than those found in a similar study conducted in the 1970's. This is related to some combination of different criteria for time to untenable conditions, improved understanding of the speed and range of threats to tenability, and faster fire development times for today's products that provide the main fuel sources for fires, such as upholstered 
furniture and mattresses. It is important to note that while both the 1975 study and the current study attempted to use a representative sample of available and important furnishings, each study included only a small fraction of those available in the marketplace. Still, this study is consistent with other recent studies of furniture and mattresses even though there may be significant differences in the burning behavior between items of furniture.

f. A mechanically aspirated (system-type) photoelectric smoke detector included in the study consistently responded after the other photoelectric smoke alarms, even for smoldering fires where convective flow rates are low and smoke entry might be an issue. Since only one such alarm was included in the study, more general conclusions cannot be drawn.

g. Residential sprinklers activated well after the smoke alarms and after the heat alarms in all of the scenarios. While these sprinklers have an outstanding record of saving lives and property, the later activation time implies that residential sprinkler installations should always include smoke alarms (as currently required in NFPA 13D and 13R) to provide greater escape times for those capable of escaping.

4. Experiments conducted with common nuisance sources produced data that should be useful in the development of new performance requirements for conditions that should not activate smoke alarms. Since the data includes analog signal levels and duration for each of the sensor types they should be useful in evaluating a range of approaches to nuisance alarm reduction from reducing alarm threshold for a specified time ("hush" feature) to decision algorithms and multi-sensor arrays. 


\section{References}

[1] Bukowski, R. W., Waterman, T. E., and Christian, W. J. "Detector Sensitivity and Siting Requirements for Dwellings: A Report of the NBS 'Indiana Dunes Tests."' NFPA No. SPP-43. Nat. Fire Prot. Assn., Quincy, MA, 1975.

[2] McGuire, J. H. and Ruscoe, B. E., The Value of a Fire Detector in the Home, Fire Study No. 9 of the Division of Building Research, National Research Council of Canada, Ottawa, 1962

[3] Hall, J. R., Jr., U. S. Experience with Smoke Detectors and Other Fire Detectors, Who Has Them? How Well Do They Work? When Don't They Work? National Fire Protection Association, 1996.

[4] U.S. Consumer Product Safety Commission; U.S. Fire Administration; National Fire Protection Association; Congressional Fire Services Institute. , "National Smoke Detector Project," U.S. Consumer Product Safety Commission, Washington, DC, 86 p. 1994.

[5] Cleary, T. G., "Fire Emulator/Detector Evaluator: Design, Operation, and Performance. International Conference on Automatic Fire Detection "AUBE '01", 12th. Proceedings. National Institute of Standards and Technology. March 25-28, 2001, Gaithersburg, MD, Beall, K.; Grosshandler, W. L.; Luck, H., Editors, National Institute of Standards and Technology, Gaithersburg, MD NIST SP 965; February 2001. 312-323 pp, 2001.

[6] Smith, Charles, "Smoke Detector Operability Survey: Report on Findings", U.S. Consumer Product Safety Commission, October, 1994

[7] Bukowski, R. W., "Investigation of the Effects of Heating and Air Conditioning on the Performance of Smoke Detectors in Mobile Home. Final Report.'National Bureau of Standards, Gaithersburg, MD, NBSIR 79-1915; 179 p. 1979.

[8] Milke, J. A.; and McAvoy, T. J., “Analysis of Signature Patterns for Discriminating Fire Detection With Multiple Sensors. Fire Technology, Vol. 31, No. 2, 120-136, May 1995.

[9] Gottuk, D.; Rose-Pehrsson, S.; Shaffer, R.; and Williams, F. "Early Warning Fire Detection via Probabilistic Neural Networks and Multi-Sensor Arrays." Proceedings of Research and Practice: Bridging the Gap. Fire Suppression and Detection Research Application Symposium. Proceedings. Fire Protection Research Foundation. February 23-25, 2000, Orlando, FL, 365-369 
[10] "Saving Lives, Saving Money, Automatic Sprinkler A 10 Year Study, A detailed history of the effects of the automatic sprinkler code in Scottsdale, AZ," available from the Home Fire Sprinkler Coalition, http://www.homefiresprinkler.org, 2003.

[11] Peacock, R. D., Averill, J. A., Bukowski, R. D., and Reneke, P. A., "Home Smoke Alarm Project: Manufactured Home Tests," Natl. Inst. Stand. Technol., Report of Test FR 4016, 2001.

[12] Averill, J. A., Peacock, R. D., Bukowski, R. W., and Reneke, P. A., "Home Smoke Alarm Project: Two-Story Home Tests," Natl. Inst. Stand. Technol., Report of Test FR 4017, 2002.

[13] Babrauskas, V., Peacock, R. D., Janssens, M., and Bathos, N. E., "Standardization and Presentation of Fire Data: The FDMS," Fire and Materials, Vol. 15, pp. 85-92, 1991.

[14] "Standard for Safety Single and Multiple Station Smoke Alarms, UL 217," Underwriters Laboratories Inc., Northbrook, IL.

[15] Cleary, T. G., "Home Smoke Alarm Project: Alarm Response Calibration,” Natl. Inst. Stand. Technol., Report of Test FR 4019, 2003.

[16] Helsper, C., Fissan, H., Muggli, J., and Scheidweiler, A., "Verification of Ionization Chamber Theory," Fire Technology, Vol. 19, No,. 1, pp. 14-21, 1983.

[17] Hosemann, J., P., "Uber Verfahren zur bestimmung der Korngrossenvertteiling hockkonzentrierter Polydispersionen von dielektrischen Miepartileln," Ph.D. thesis, Aachen, 1970.

[18] Smith, L., U. S. Consumer Product Safety Commission, private communication, June 1999.

[19] "NFPA 101, Life Safety Code, 2003 Edition," Volume 6 of the National Fire Codes, Natl. Fire Protection Association, Quincy, MA (2003).

[20] "SFPE Engineering Guide to Performance-Based Fire Protection Analysis and Design of Buildings,” NFPA No. SFPE-00, Natl. Fire Protection Association, Quincy, MA (2000).

[21] Peacock, R. D. and Babrauskas, V. "Analysis of Large-scale Fire Test Data.” Fire Safety Journal 17, pp. $387-414,1991$.

[22] Peacock, R. D., Bukowski, R. W., Reneke, P. A., Averill, J. D., and Markos, S. H., "Development of a Fire Hazard Assessment Method to Evaluate the Fire Safety of Passenger Trains." Proceedings of the Fire and Materials 2001 Conference, January 22-24, San Francisco, Interscience Communications, London, pp 67-78. 
[23] The SFPE Handbook of Fire Protection Engineering, 2nd Ed. P. J. DiNenno, Editor. Nat. Fire Prot. Assoc., Quincy, MA, 1995.

[24] Peacock, R. D., Averill, J. A., Bukowski, R. W., and Reneke, P. A., "Home Smoke Alarm Project, Manufactured Home Tests,” NIST Fire Research Report of Test FR4016, Natl. Inst. Stand. Technol., http://smokealarm.nist.gov/Series1.htm, June 2002.

[25] Averill, J. A., Peacock, R. D., Bukowski, R. W., and Reneke, P. A., "Home Smoke Alarm Project, Two-Story Home Tests," NIST Fire Research Report of Test FR4017, Natl. Inst. Stand. Technol., http://smokealarm.nist.gov/Series2.htm, June 2002.

[26] Life threat from fire - Guidance on the estimation for time available for escape using fire data, Proposed Technical Specification, ISO/TS 13571, International Organization for Standardization. 2002.

[27] "NFPA 72, National Fire Alarm Code, 1999 Edition," Volume 5 of the National Fire Codes, Natl. Fire Protection Association, Quincy, MA (2002).

[28] "NFPA 101, Life Safety Code, 2000 Edition," Volume 6 of the National Fire Codes, Natl. Fire Protection Association, Quincy, MA (2002).

[29] Mulholland, G. W., and Liu, B. H., "Response of Smoke Detectors to Monodisperse Aerosols," Journal of Research of the National Bureau of Standards, Vol. 85, No. 3, pp. 223-238, May-June, 1980.

[30] Litton, C. D., “A Mathematical Model for Ionization-type Smoke Detectors and the Reduced Source Approximation,” Fire Technology, Vol. 13, pp. 226-273, 1977.

[31] Mulholland, G. W., and Croarkin, C., "Specific Extinction Coefficient of Flame Generated Smoke,” Fire and Materials, Vol. 24, pp. 227-230, 2000.

[32] Cleary, T.G., "Measurements of the Size Distribution and Aerodynamic Properties of Soot," MS Thesis, University of Maryland, 1989.

[33] Cleary, T.G., Weinert, D.W., and Mulholland, G.W., "Moment Method for Obtaining Particle Size Measures of Test Smokes", Natl. Inst. Stand. Technol., NISTIR 7050, 2003.

[34] Shapiro, Julie, (Smoke Detector Operability Survey: Engineering Laboratory Analysis", U.S. Consumer Product Safety Commission, October, 1994.

[35] Gottuk, D.T., Peatross, M.J., Roby, R.J., and Beyler, C.L., “Advanced Fire Detection Using Multi-signature Alarm Algorithms", Proceedings of the 11th Int. Conf. on Automatic Fire Detection (AUBE '99), March 16-18, 1999, Duisburg, Germany, Ed. Luck, H., pp. 237-246, 1999. 
[36] Ishii, H., Ono, T., Yamauchi, Y., Ohtani, S., "Fire Detection System by Multi-layered Neural Network with Delay Circuit", Proceedings of the 4th International Symposium on Fire Safety Science, July 13-17, 1994, Ottawa, Canada, Ed. T. Kashiwagi, pp. 761-772, 1994.

[37] Cleary, T., and Ono, T., "Enhanced Residential Fire Detection by Combining Smoke and Carbon Monoxide Sensors", Proceedings of the 12th Int. Conf. on Automatic Fire Detection (AUBE '01), March 26-38, 2001, Gaithersburg, MD, Eds., Beall, K., Grosshandler, W., and Luck, H., pp. 346-357, 2001.

[38] Meacham, B.J., "The Use of Artificial Intelligence Techniques for Signal Discrimination in Fire Detection Systems", J. of Fire Prot., Engr., Vol. 6, No. 3, pp 125-136, 1994.

[39] UL 268: Standard for Smoke Detectors for Fire Protective Signaling Systems, 4th ed., Underwriters Laboratories Inc., Northbrook, IL, 1996.

[40] EN 54: Components of Automatic Fire Detection Systems, Part 9, Fire Sensitivity Test, European Committee for Standardization, Brussels, 1982.

[41] Cleary, T. G., "Home Smoke Alarm Project: Alarm Response to Nuisance Sources," Natl. Inst. Stand. Technol., Report of Test FR 4020, 2003.

[42] see, for example, International Building Code 2000, International Code Council, Falls Church, VA, 2000.

[43] Wilson, R. "Computer Analysis of Data on Fire Detectors Available for Purchase in Massachusetts." Data From the National Bureau of Standards Indiana Dunes Tests Record,.Firepro, Inc., Wellesley Hills, MA, National Bureau of Standards, Gaithersburg, MD, 11 p. January 28, 1976.

[44] Nober, E. H.; Peirce, H.; Well, A. D.; Johnson, C. C.; Clifton, C. "Waking Effectiveness of Household Smoke and Fire Detection Devices. Fire Journal, Vol. 75, No. 4, 86-91,130, July 1981.

[45] Nelson, Harold E. and Mowrer, F. W. "Emergency Movement." In The SFPE Handbook of Fire Protection Engineering, $3^{\text {rd }}$ Edition. National Fire Protection Association, Quincy, MA (2002).

[46] Ando, K., Ota, H., and Oki, T. "Forecasting The Flow of People.” Railway Research Review (45) pp. 8-14, 1988.

[47] Proulx, G. "Evacuation Time and Movement in Apartment Buildings." National Research Council of Canada, Ottawa, Ontario, Fire Safety Journal, Vol. 24, No. 3 , 229-246, 1995. 
[48] Bryan, J. L. "Project People - A Study of Human Response in Fire Incidents." Maryland University, College Park. Alena Enterprises of Canada. Fire Retardents: Proceedings of 1977 International Symposium on Flammability and Fire Retardants. May 19-20, 1977, Washington, DC, Technomic Publishing Co., Inc., Westport, CT, Bhatnagar, V.M., Editor, 103-127 pp, 1979.

[49] Wood, P. G. "Survey of Behavior in Fires." Surrey Univ., Guildford, England, Fires and Human Behavior, John Wiley and Sons, New York, Canter, D., Editor(s), 83-95 p., 1980.

[50] Keating, J. P. and E. L. Loftus. "Post Fire Interviews: Development and Field Validation of the Behavioral Sequence Interview Technique." Final Report. Natl Bur. Stand. (U. S.), NBS GCR 84-477, October 1984.

[51] Pearson, R. G., and Joost, M. G. "Egress Behavior Response Time of Handicapped and Elderly Subjects to Simulated Residential Fire Situations." Natl Bur. Stand. (U. S.), NBS GCR 83-429, 1983.

[52] NFPA 13R: Installation of Sprinkler Systems in One- and Two-Family Dwellings and Manufactured Homes, 1999 Edition, 2002 National Fire Codes. National Fire Protection Association, Quincy, MA, 2002.

[53] Cleary, T. G., Ohlemiller, T. J, and Villa, K. M., "The Influence of Ignition Source on the Flaming Fire Hazard of Upholstered Furniture.” Natl. Inst. Stand. Technol., NISTIR 4847, 1992.

[54] Ohlemiller, T. J, and Gann, R. G.., "Estimating Reduced Fire Risk Resulting From an Improved Mattress Flammability Standard.” Natl. Inst. Stand. Technol., Tech. Note 1446, 2002. 


\section{Appendix A}

\section{Alarm Activation and Time to Untenable Conditions During Tests of Residential Smoke Alarms Included in the Study}




\section{Calculated Alarm Times}


For each alarm included in each test of the program, data are included that identify the alarm, its inclusion within several alarm placement scenarios, calibration data for the alarm, and estimated alarm times for different assumed alarm thresholds. Columns in the data include

Key - the alarm type as "Alarm" for an analog-modified alarm, "Dalarm" for an unmodified alarm, "Sprinkler" for a tell-tale residential sprinkler, or COalarm for a carbon monoxide alarm.

Position - the room where the alarm was located

Level, level + br, all - identifies whether the alarm was included in an alarm placement scenario of "Every Level", "Every Level + Bedrooms", or "Every Room" as described in chapter 7 of the report.

dual - the alarm was part of a dual sensor alarm

name - specific alarm identification as detailed in section 2.5

label - a short alarm identification included in test data spreadsheets for this alarm

units - recording units for the measurement as recorded by the data acquisition system during the test, $\mathrm{V}$ or $\mathrm{mV}$

$\mathbf{m 0 ,} \mathbf{m} \mathbf{1}$ - calibration constants for the alarm as detailed in table 1.

ave - average steady-state clear-air alarm output calculated as a average value prior to ignition, $\mathrm{V}$ or $\mathrm{mV}$ consistent with "units" column

low, mid, high - estimated alarm times for low, medium and high alarm threshold values, s

last data column (unlabelled) - end of test measured from ignition, $\mathrm{s}$ 


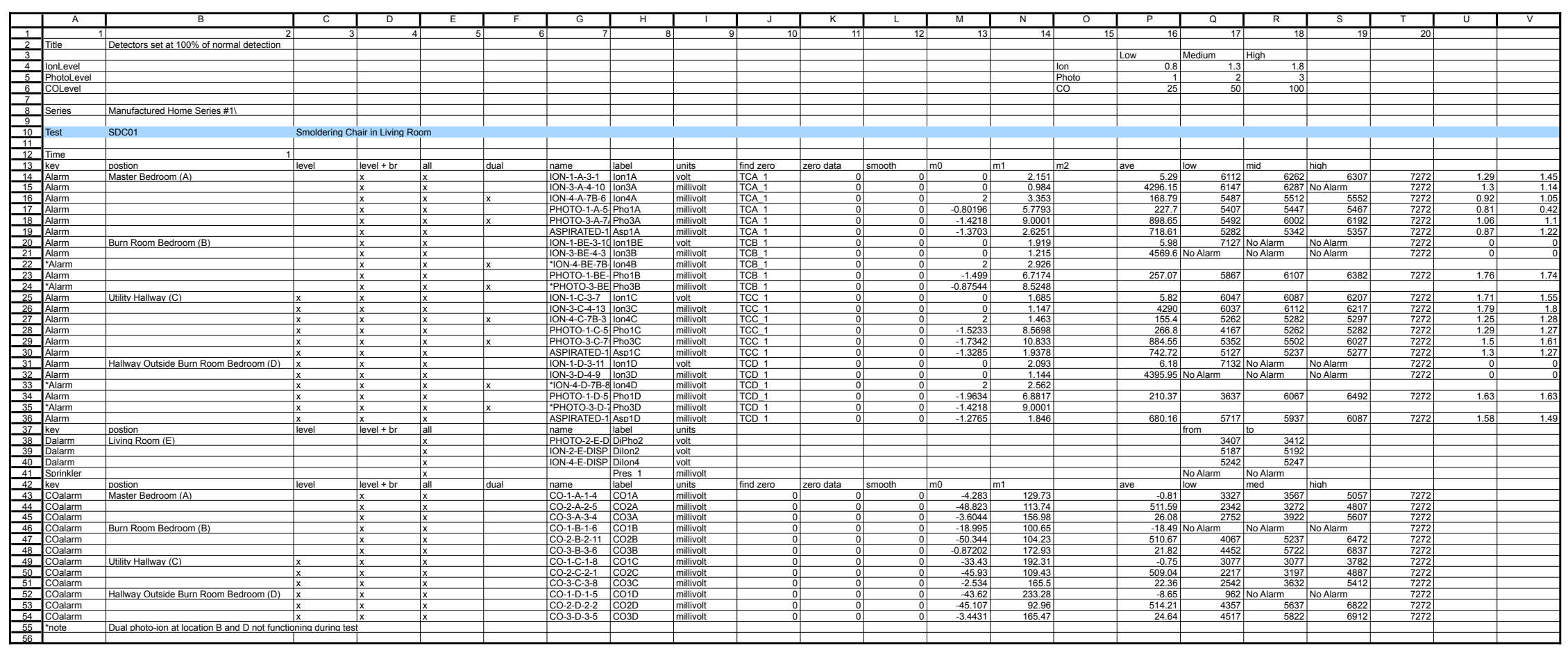




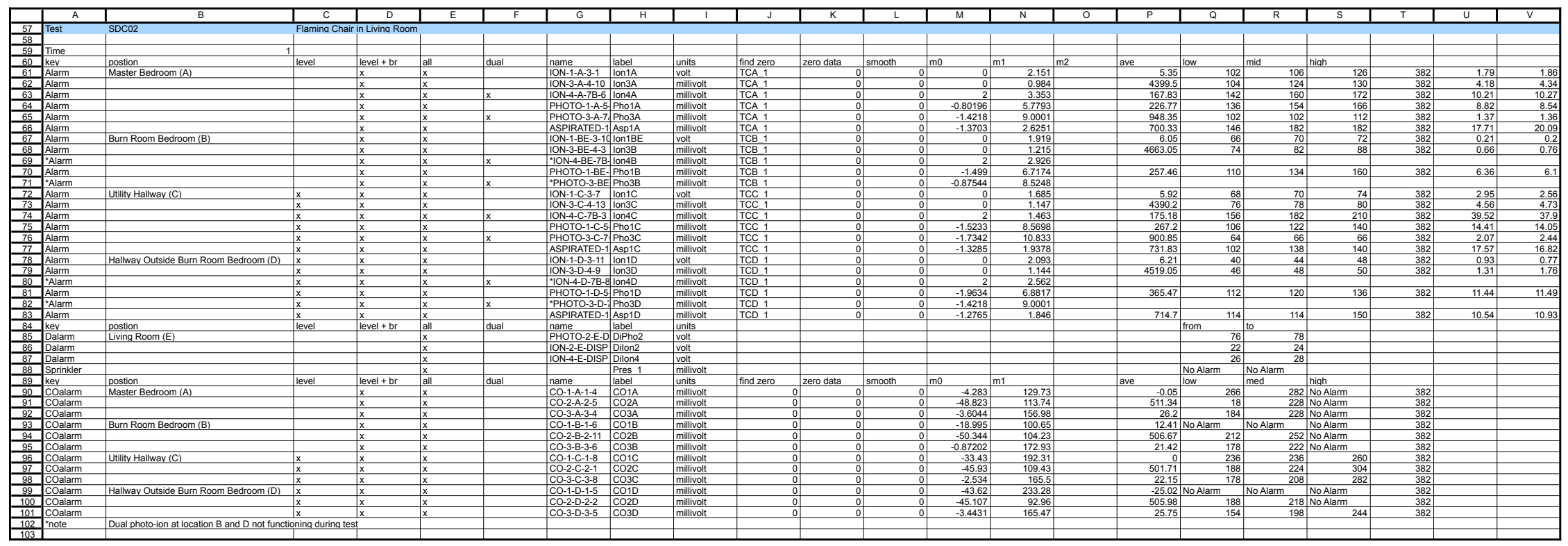




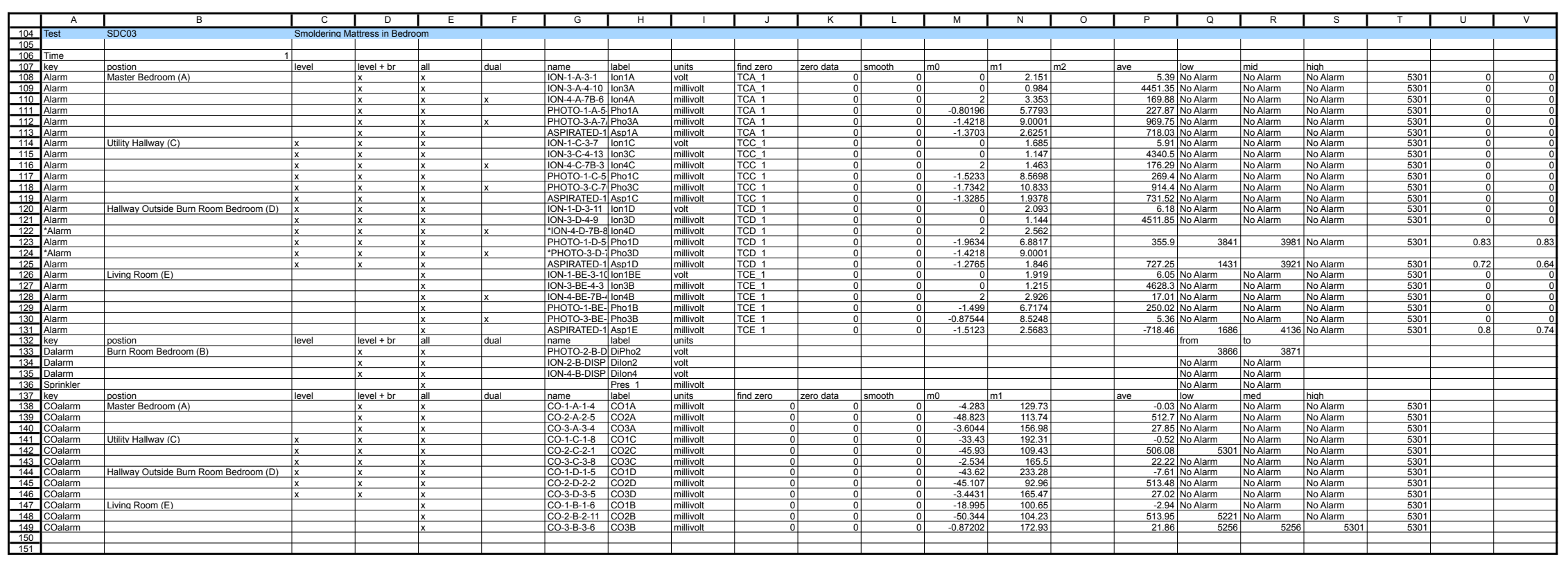




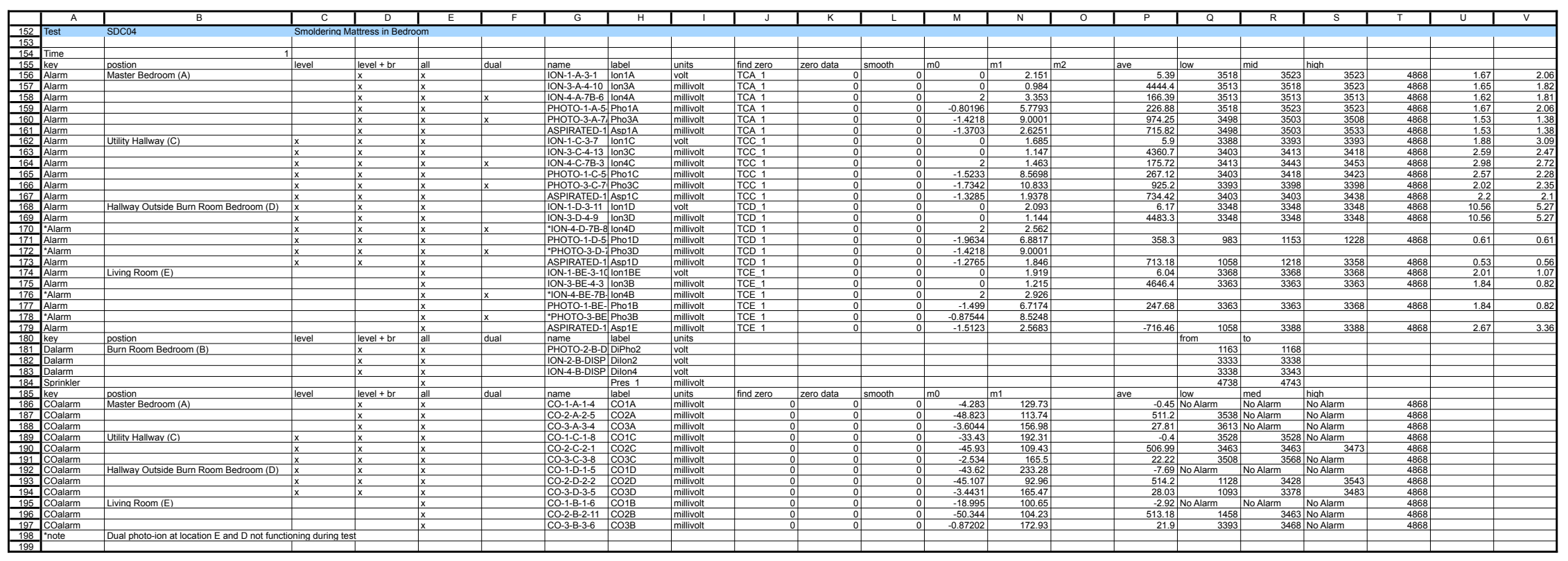




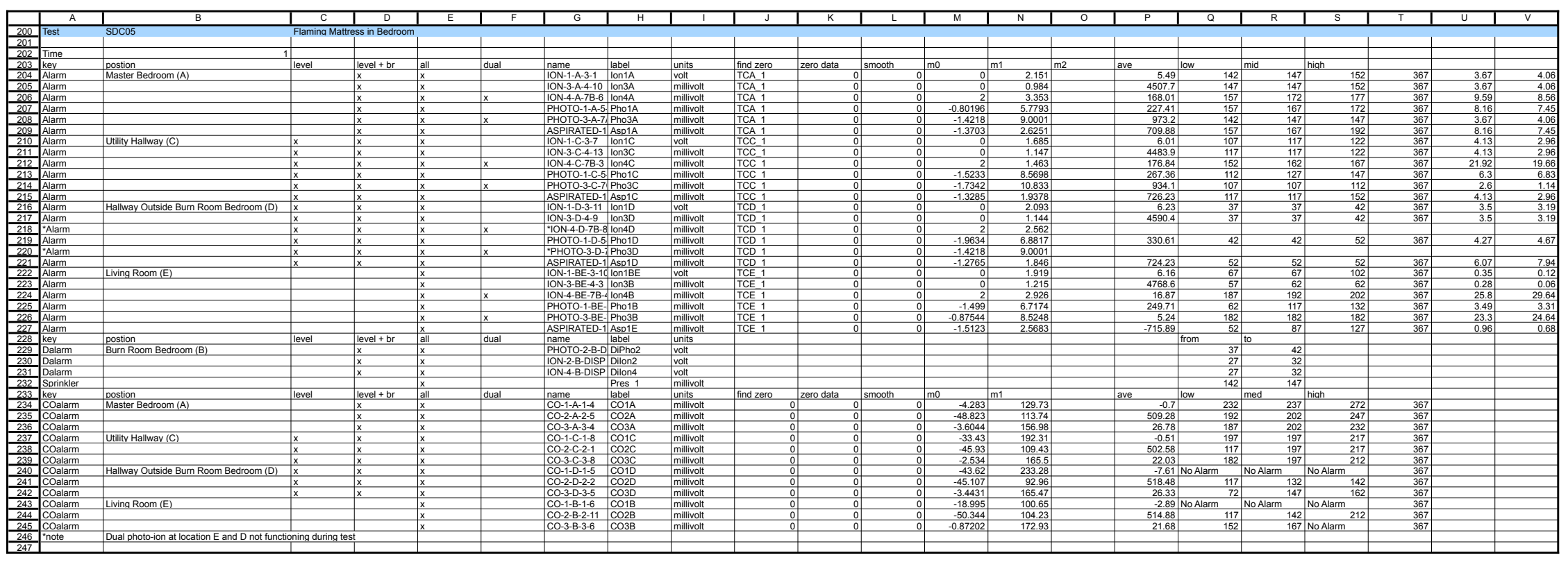




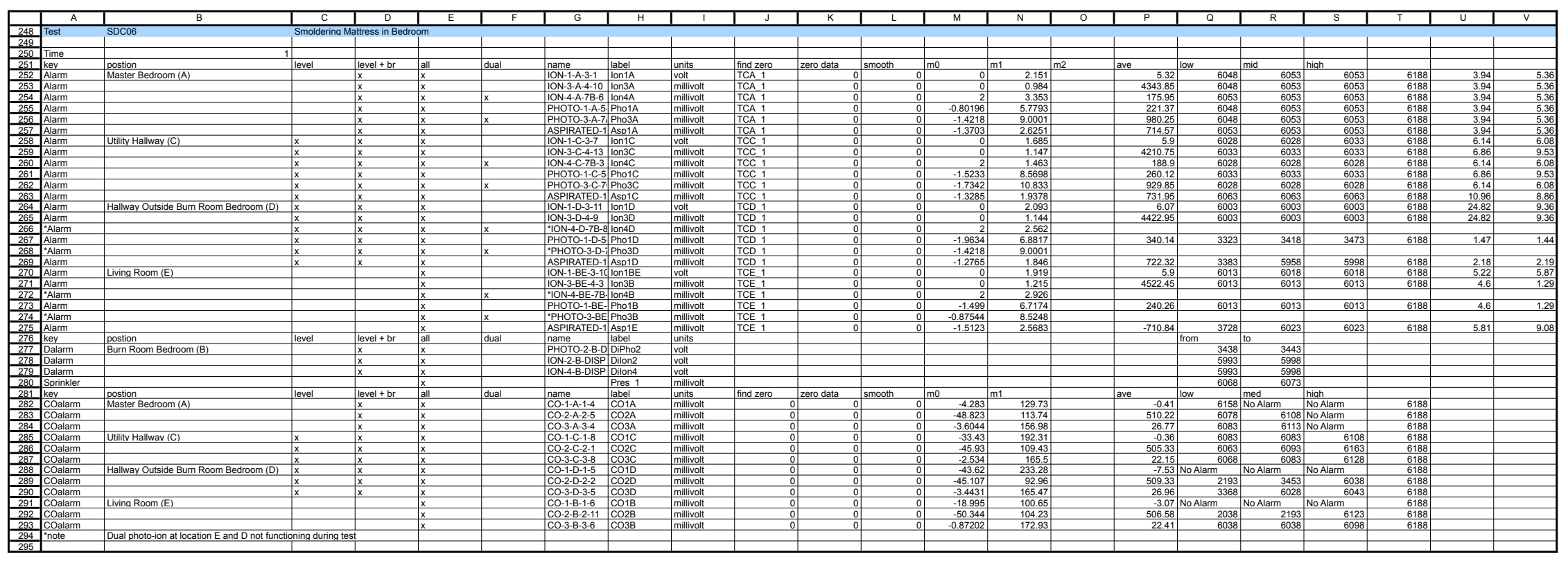




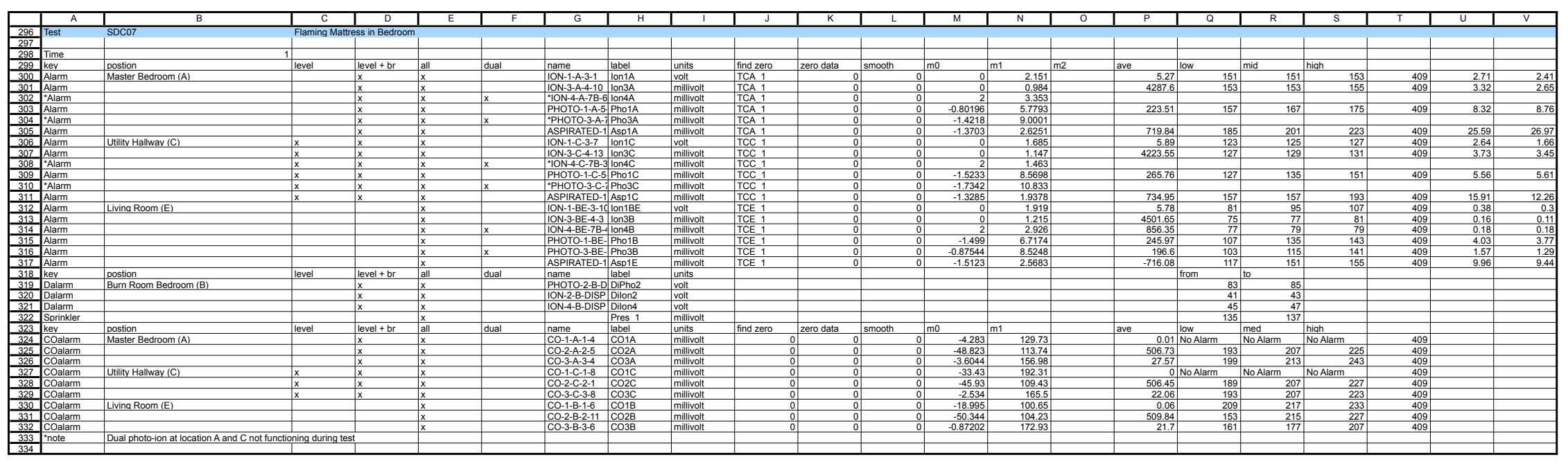




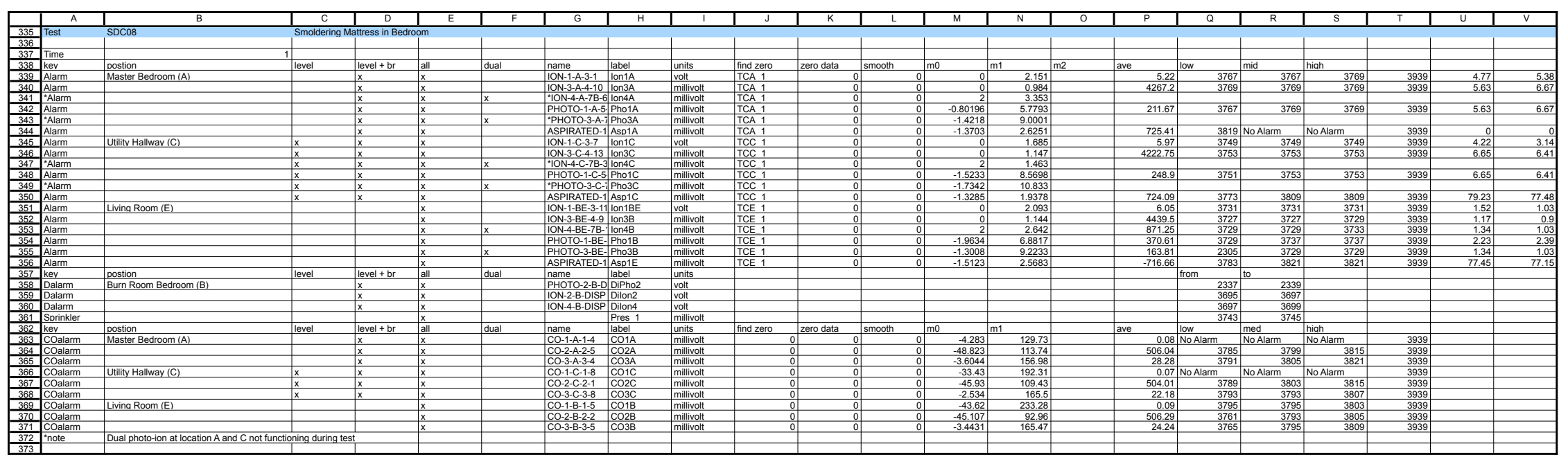




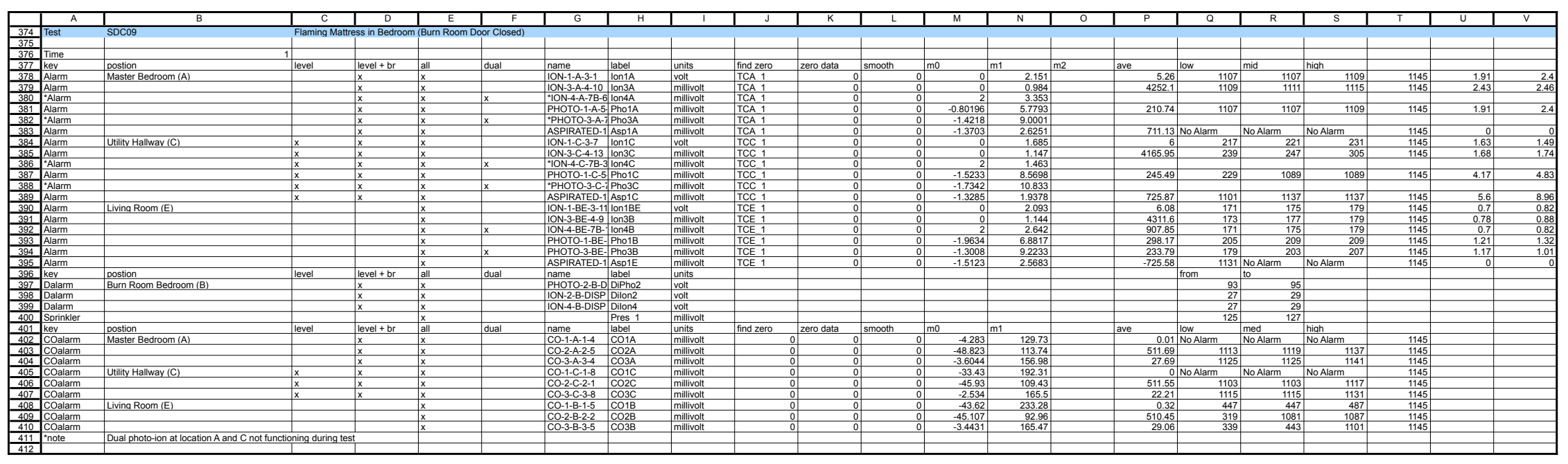




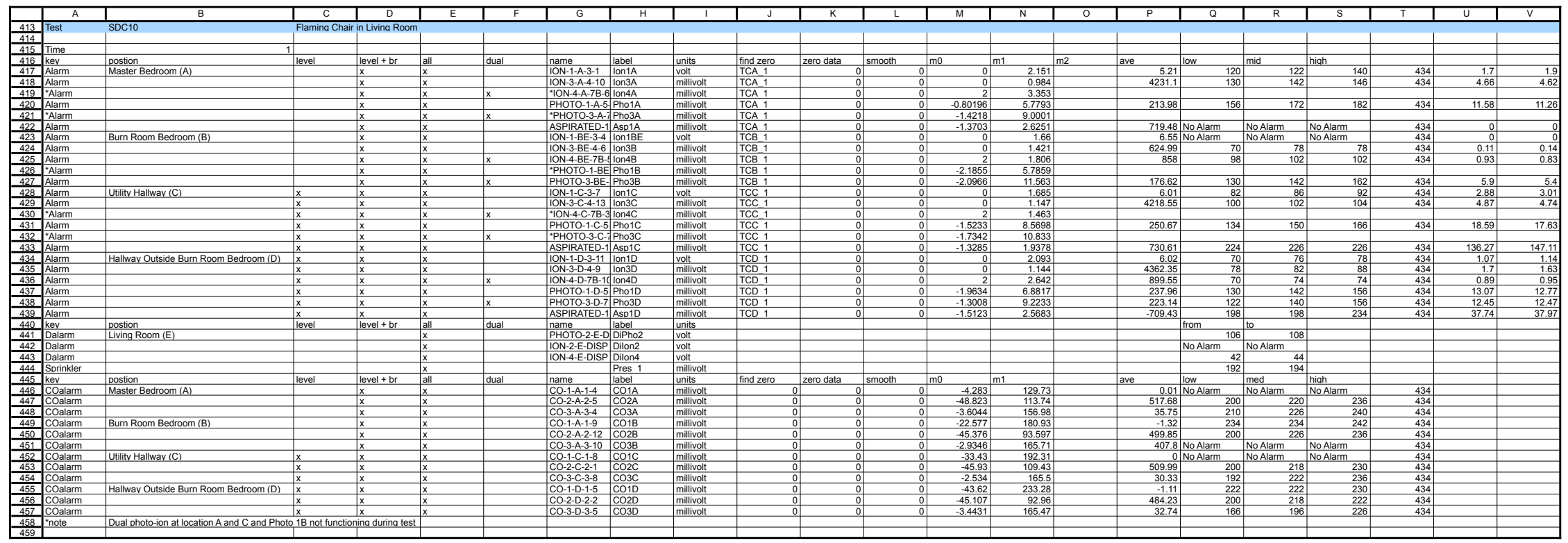




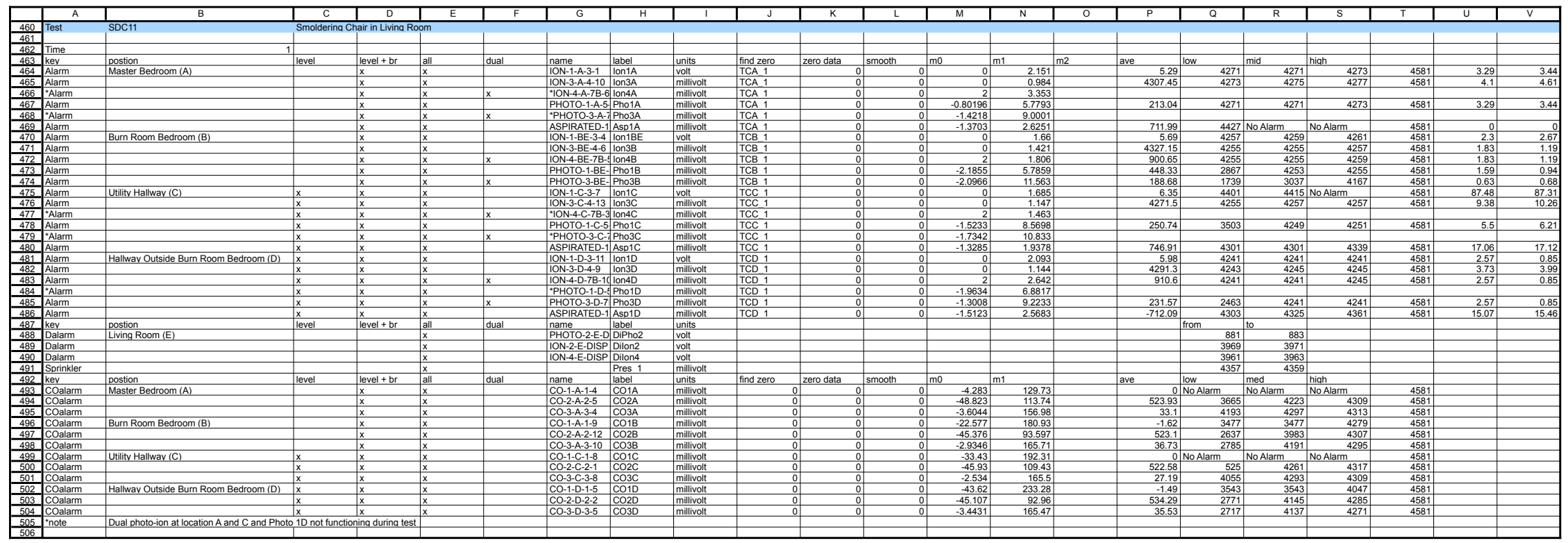




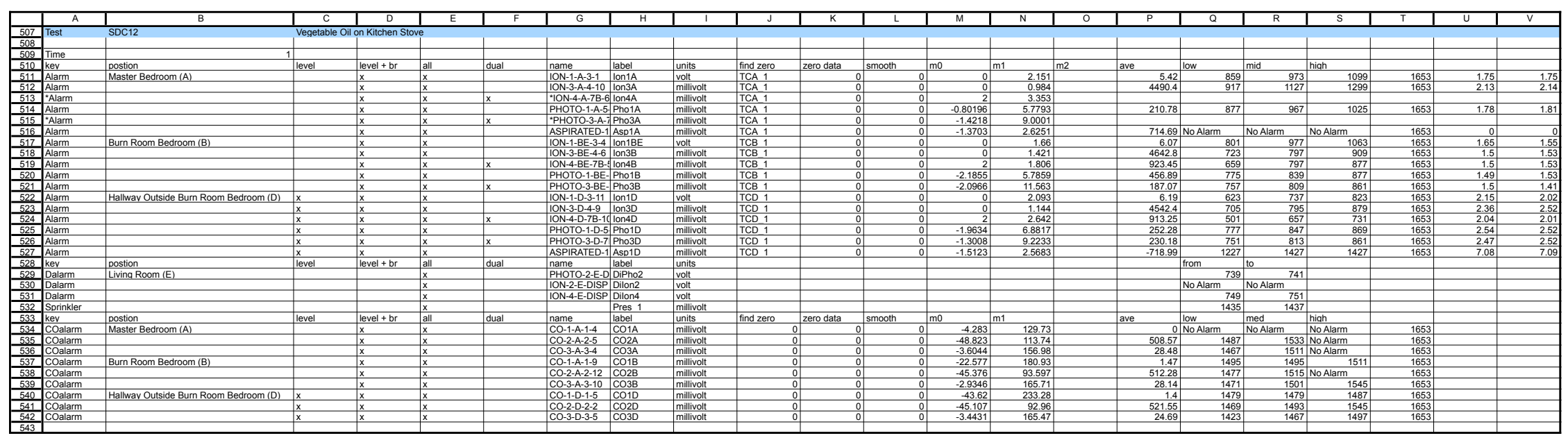




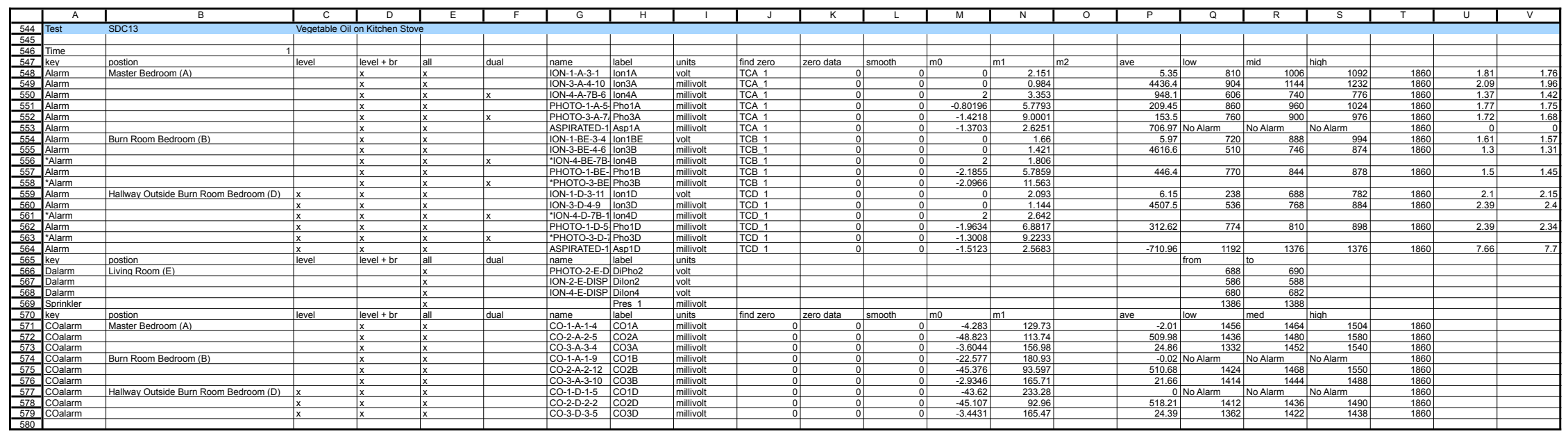




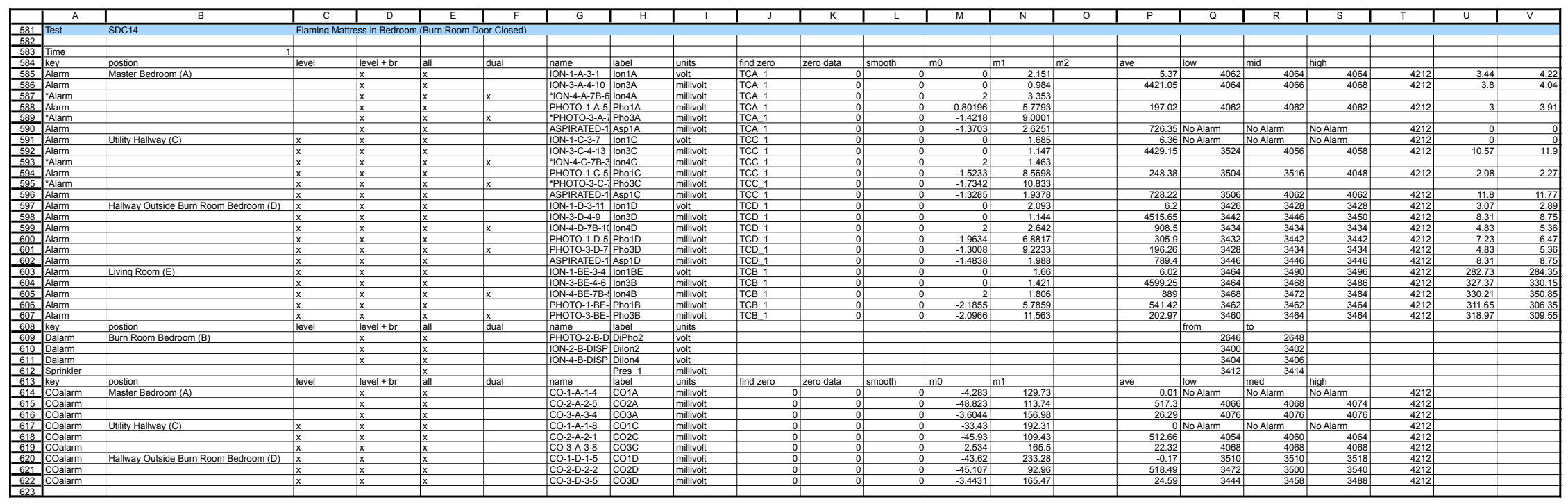




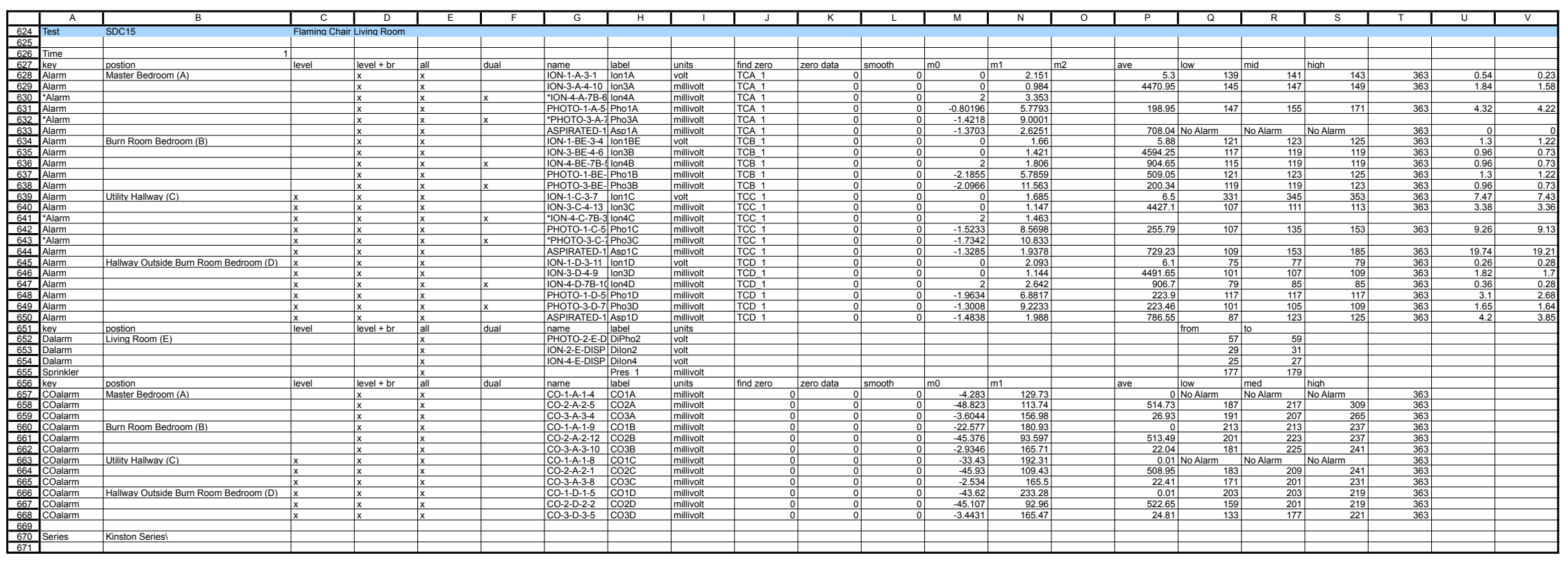




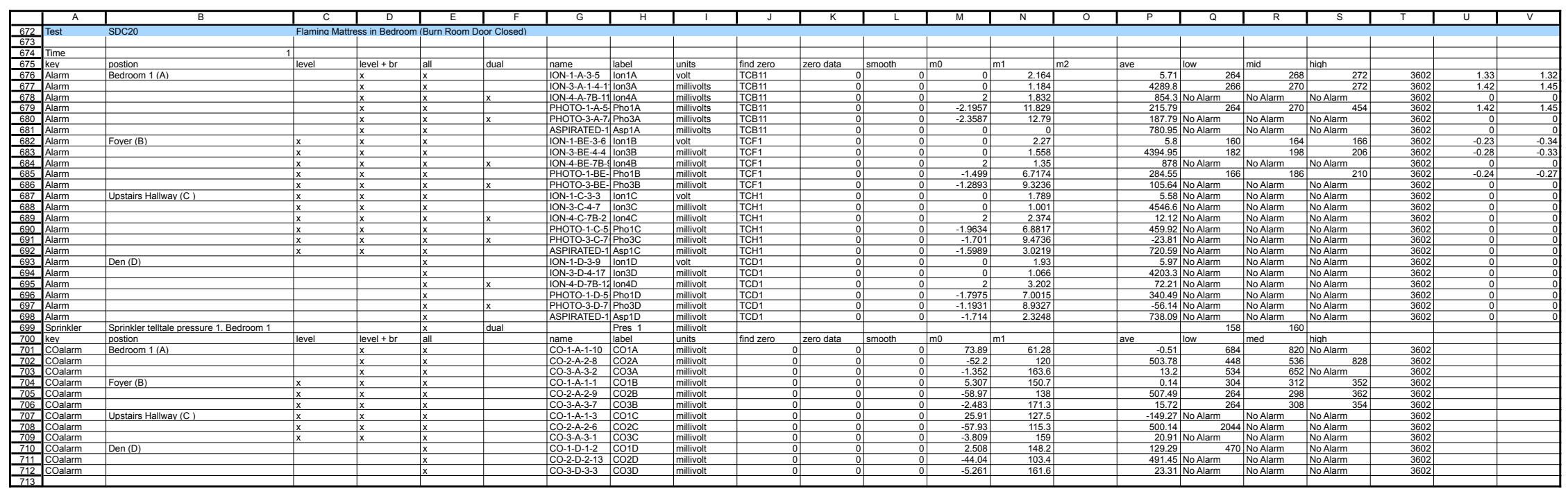




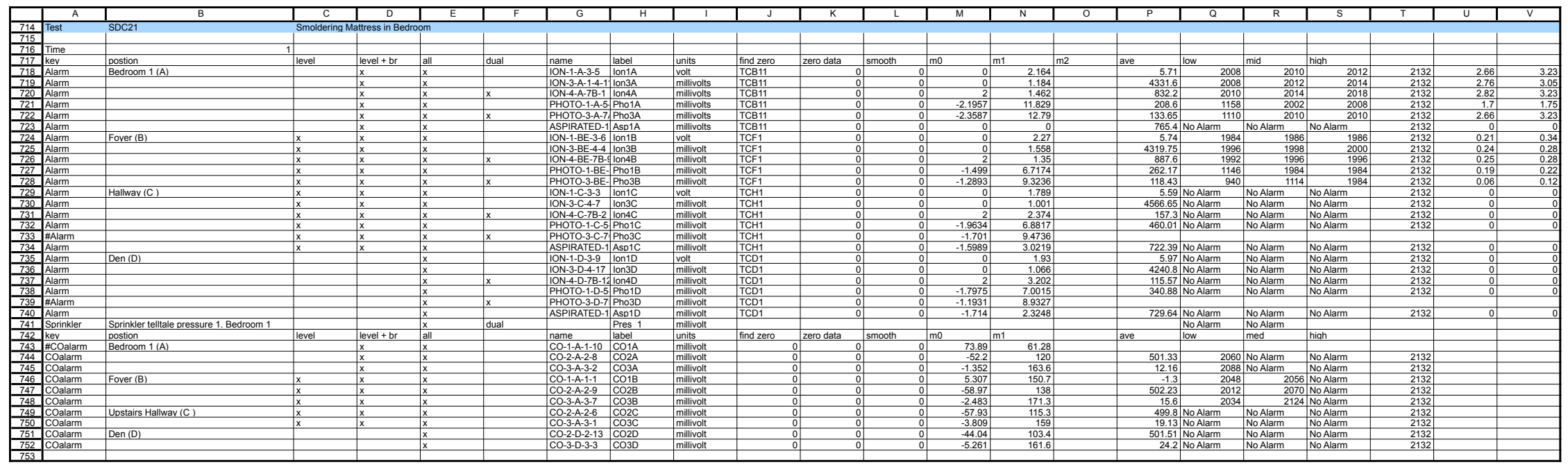




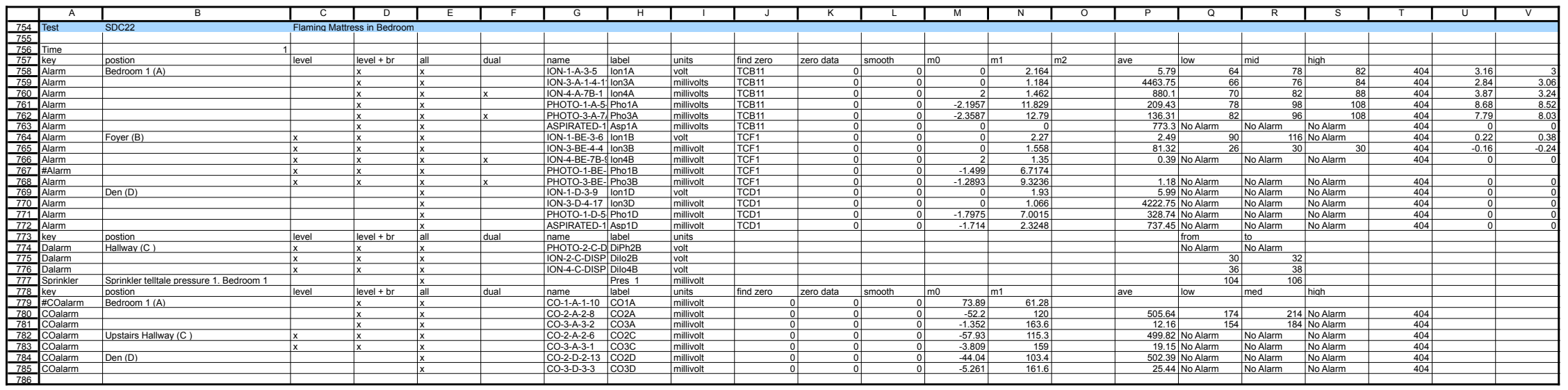




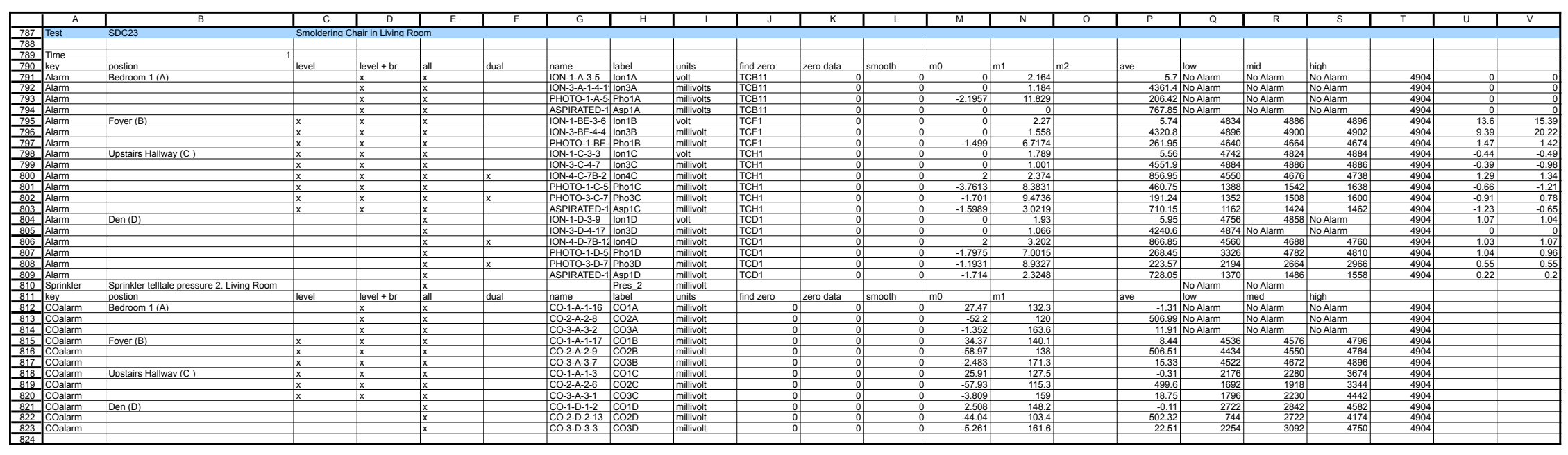




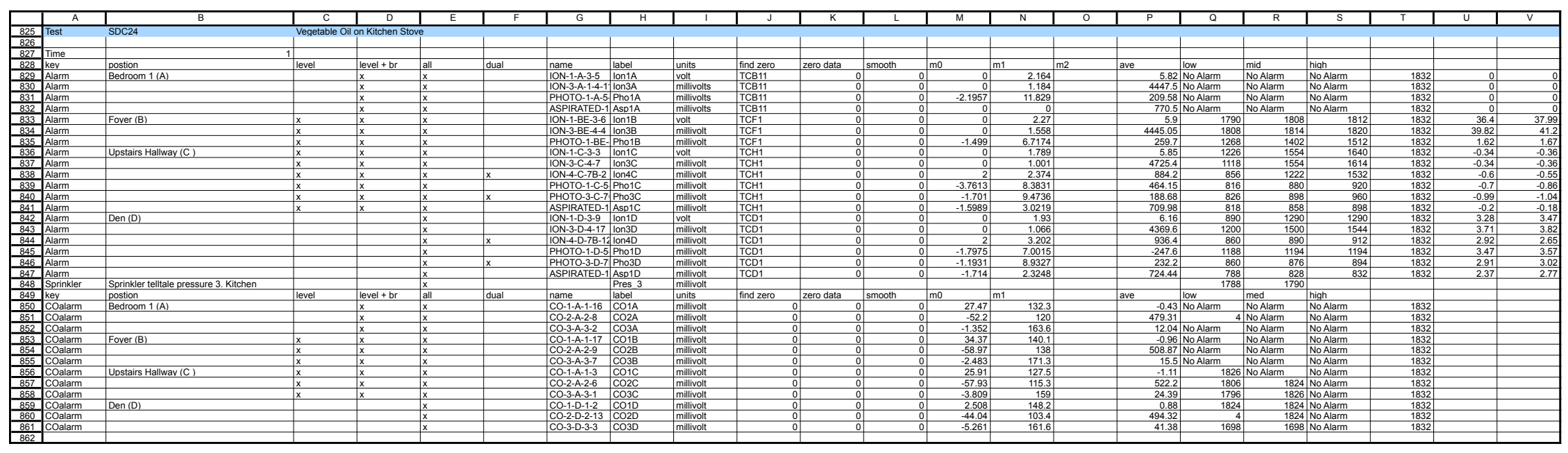




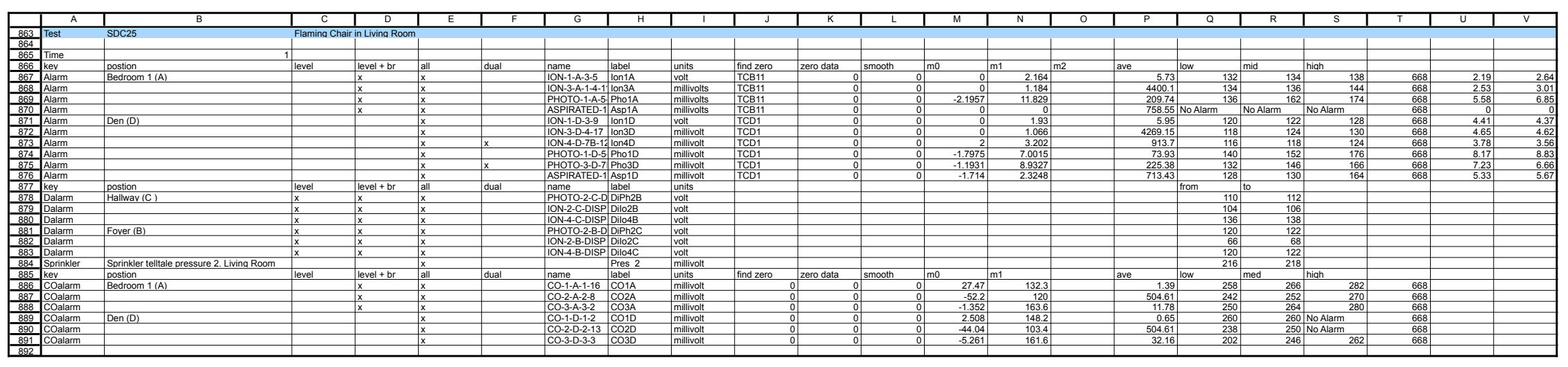




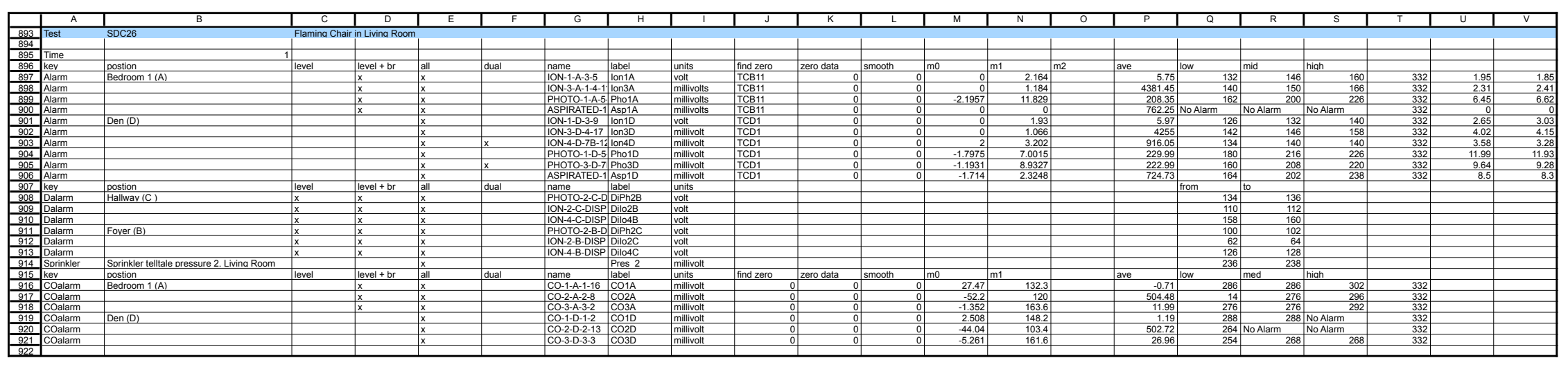




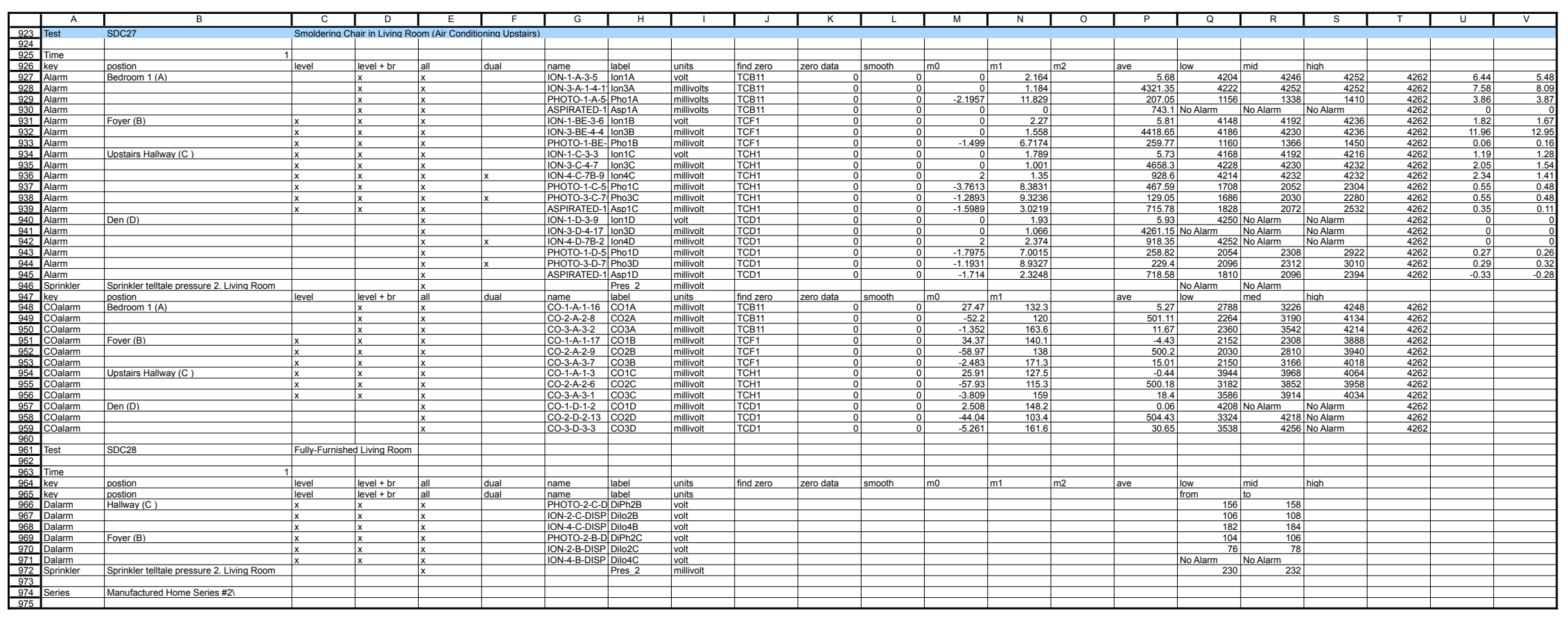




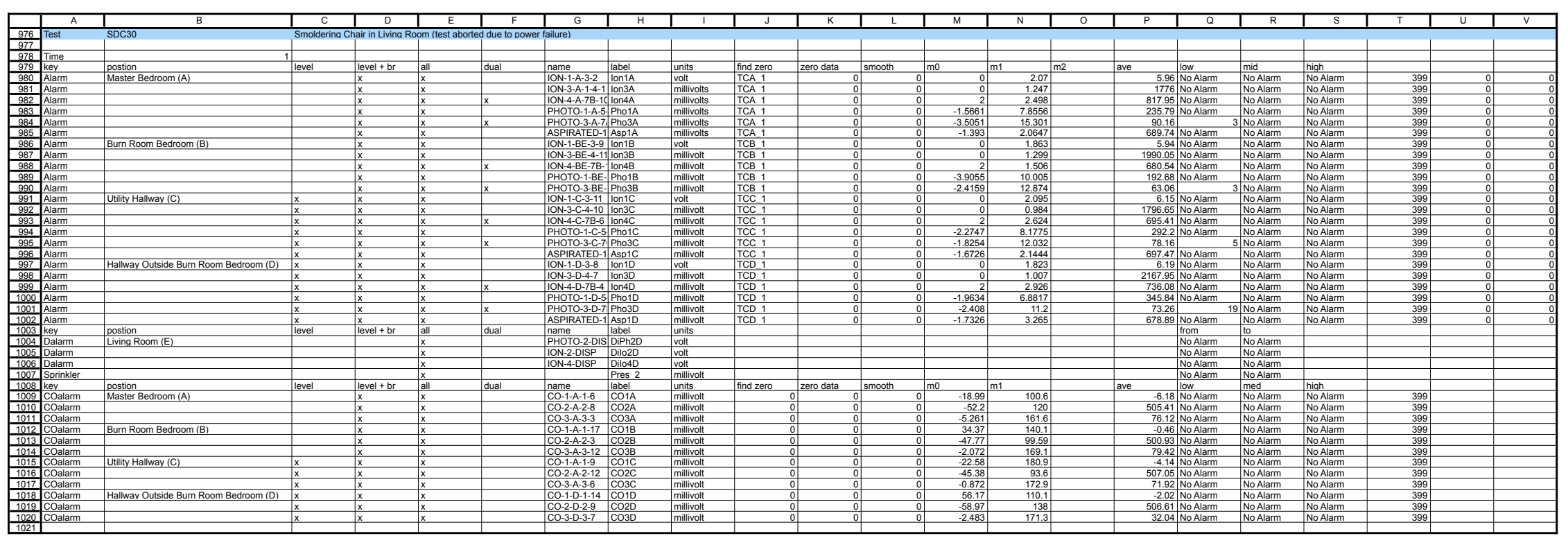




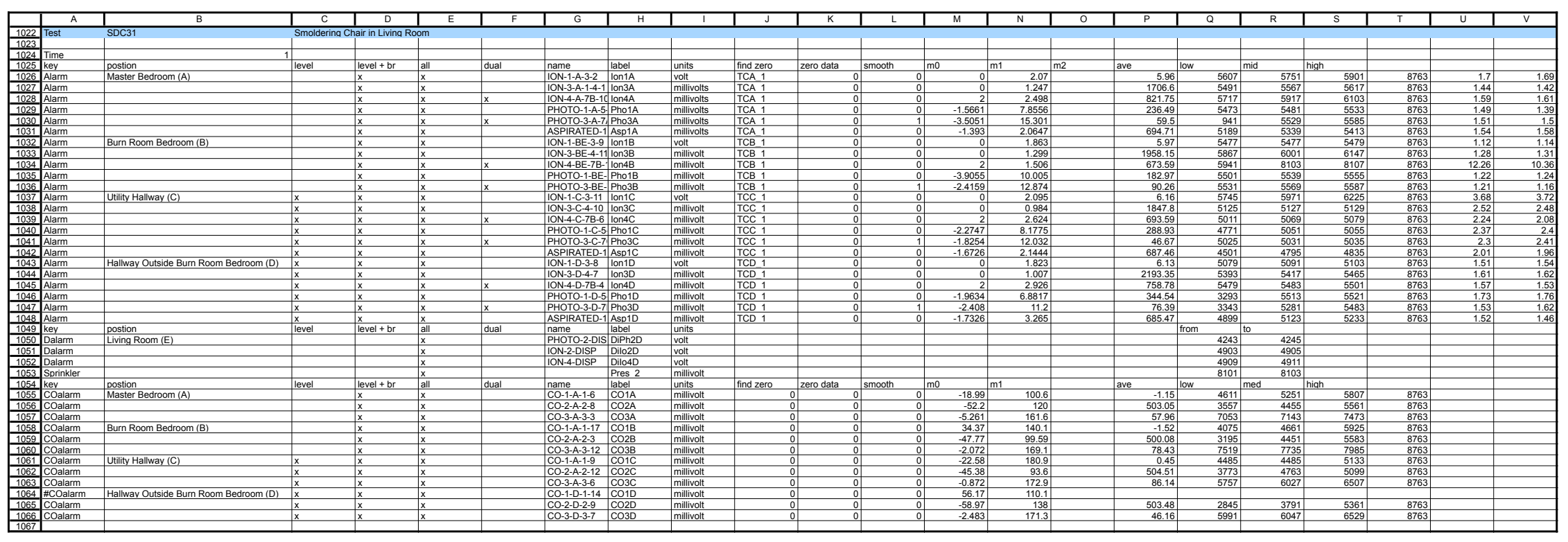




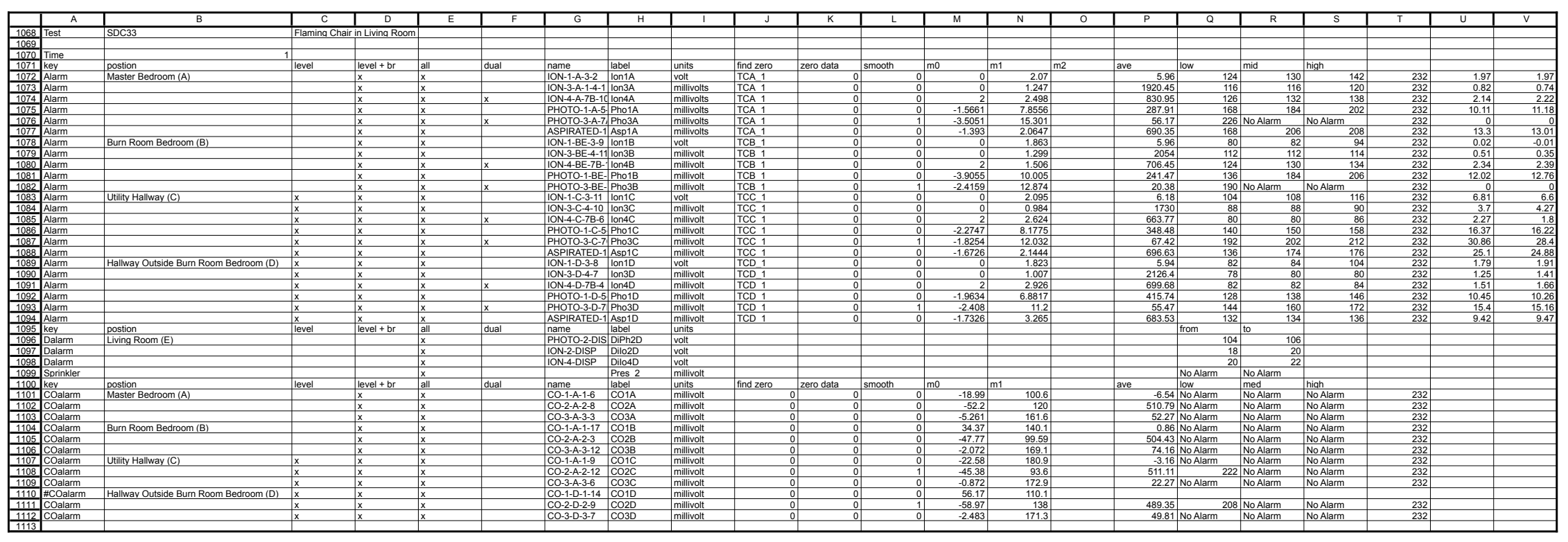




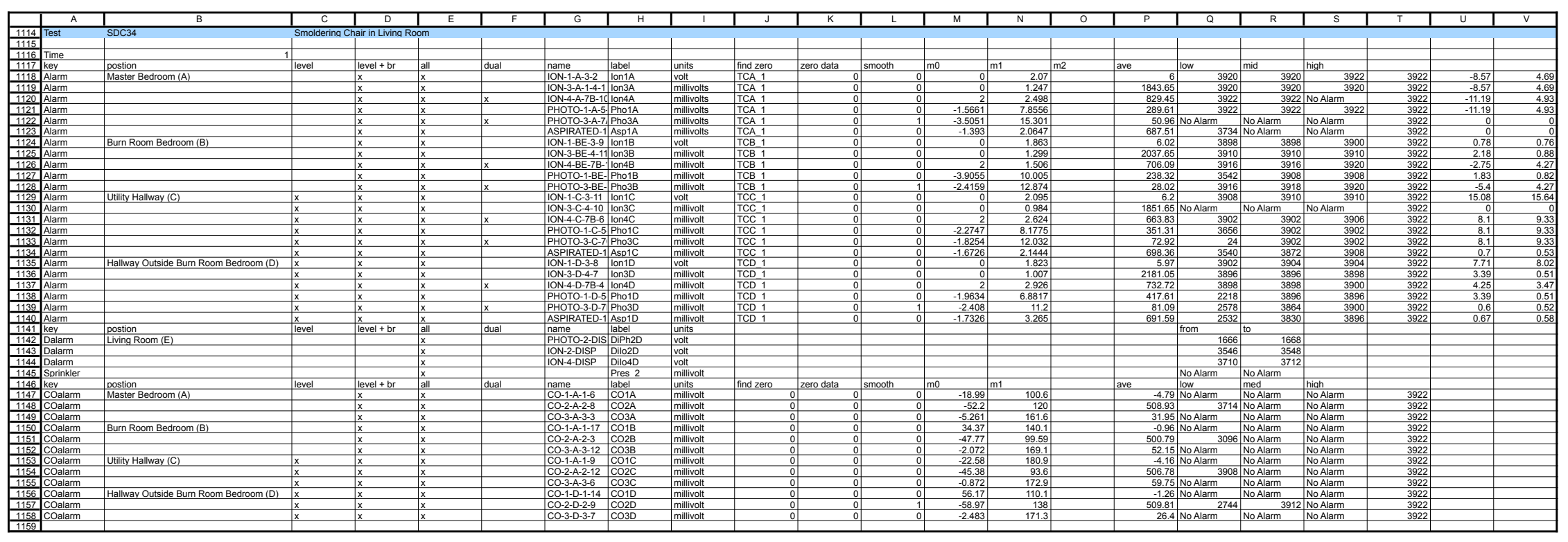




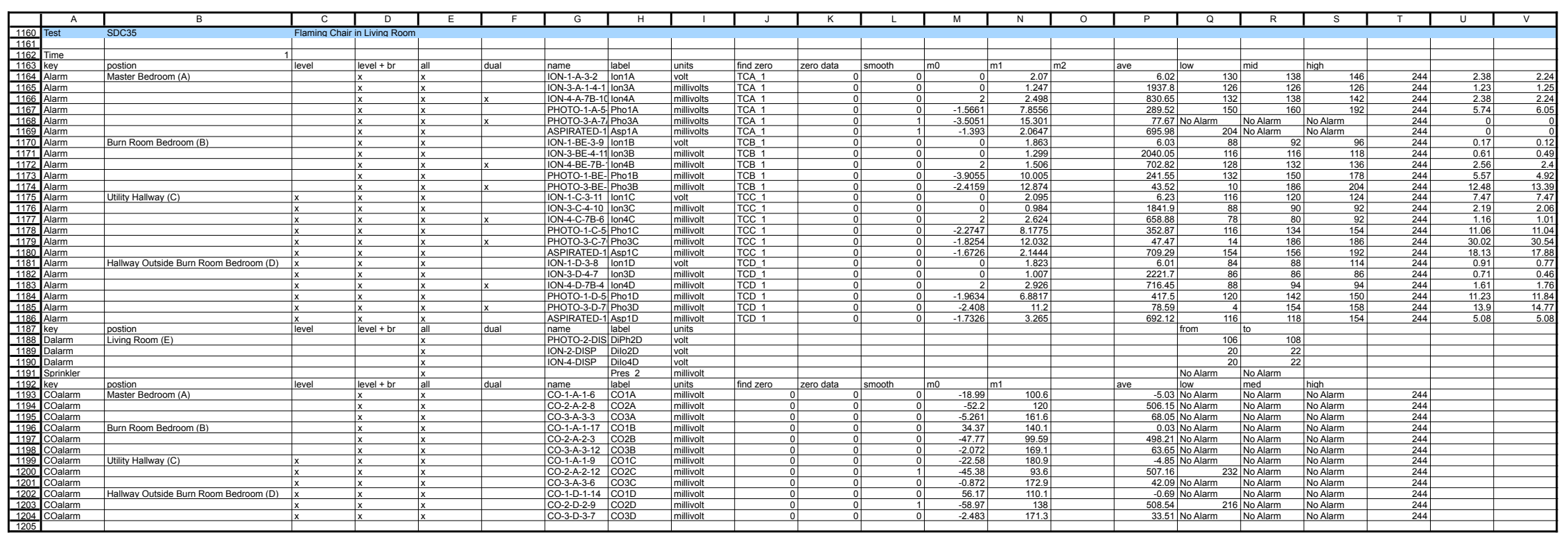




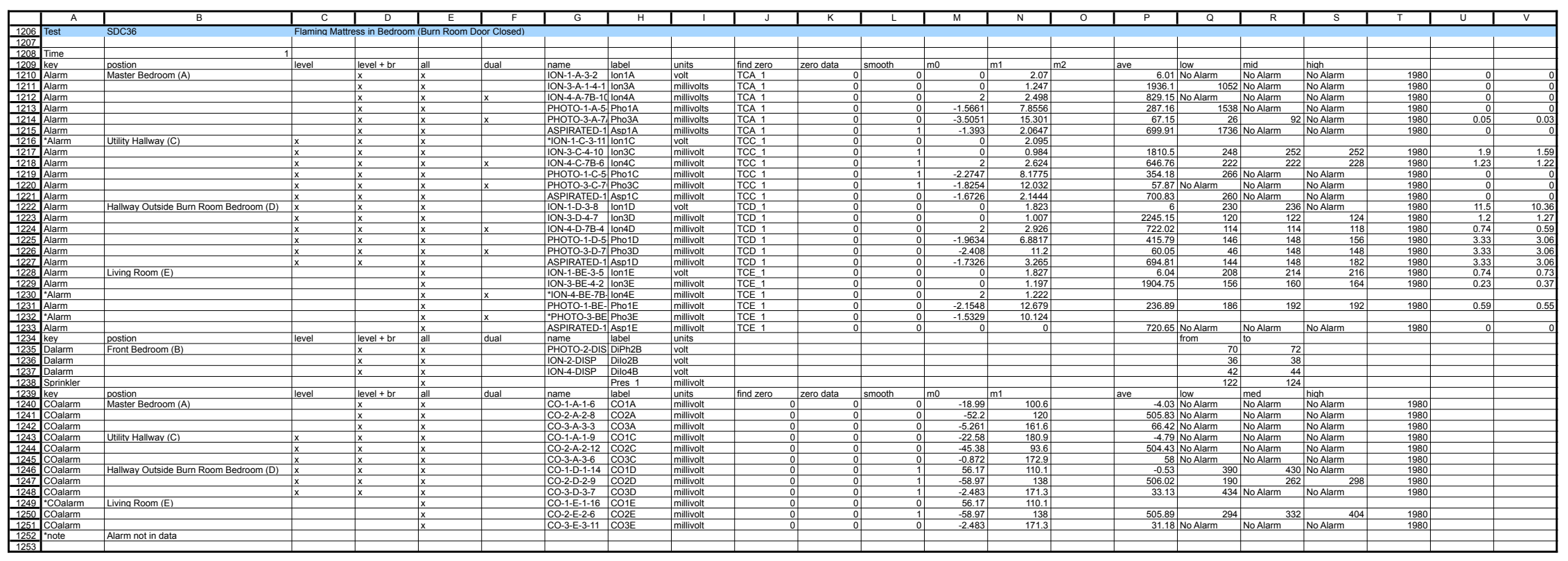




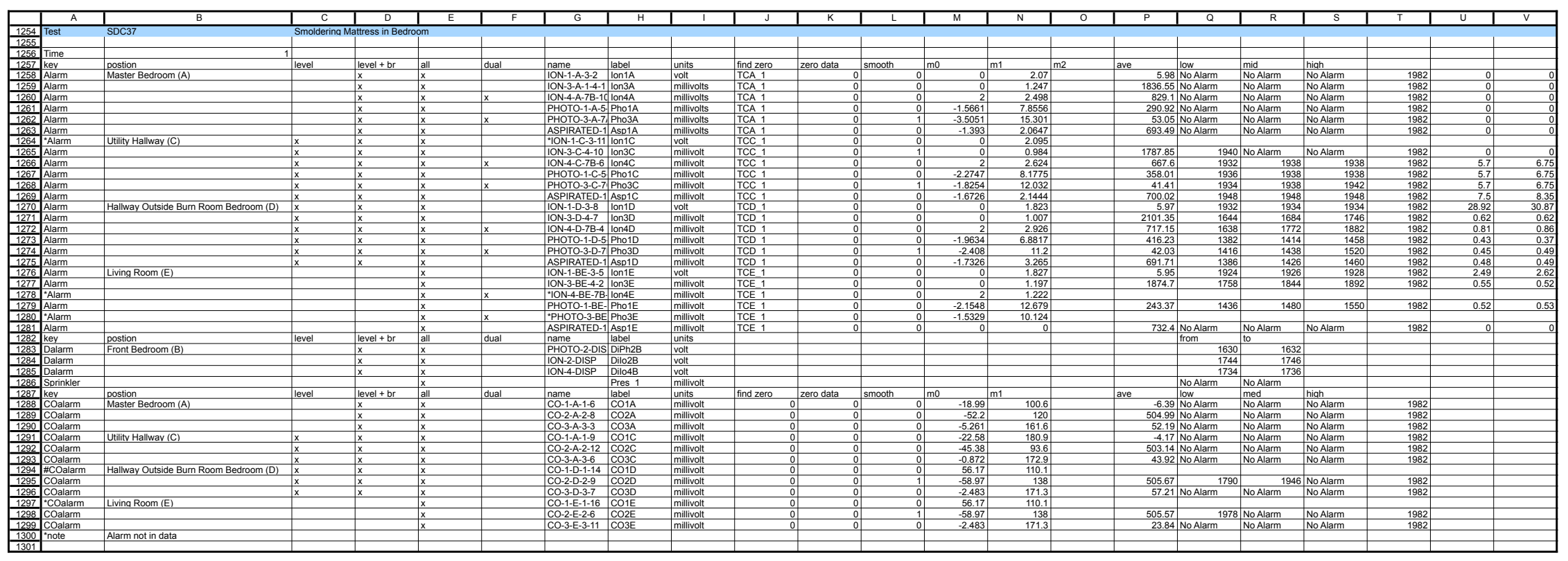




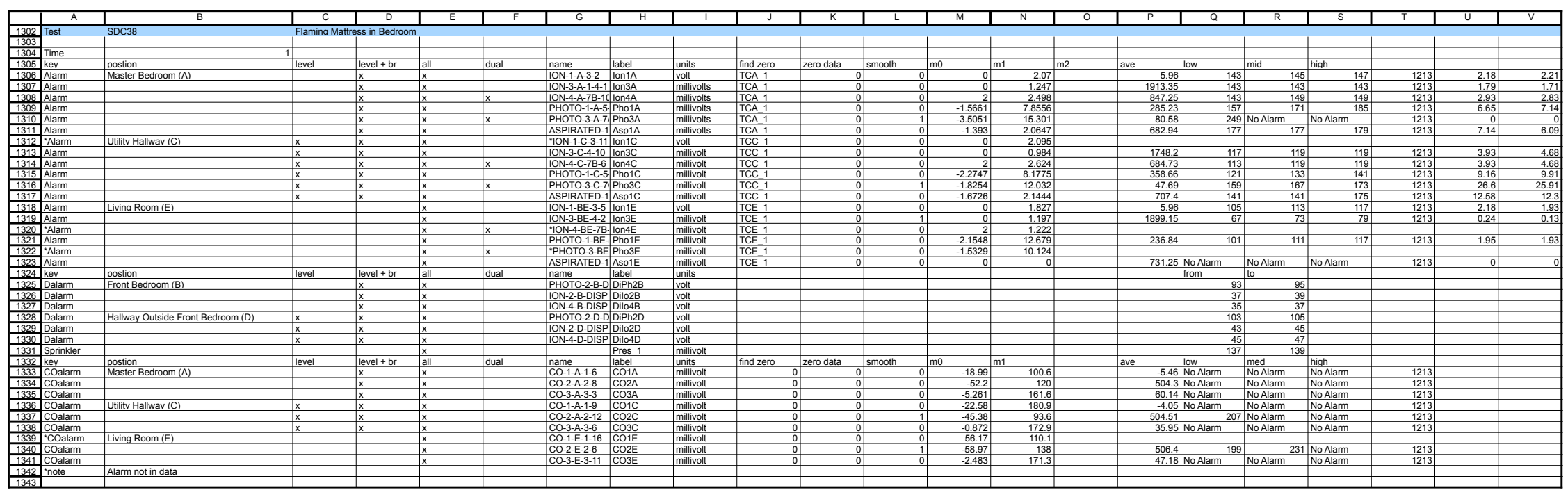




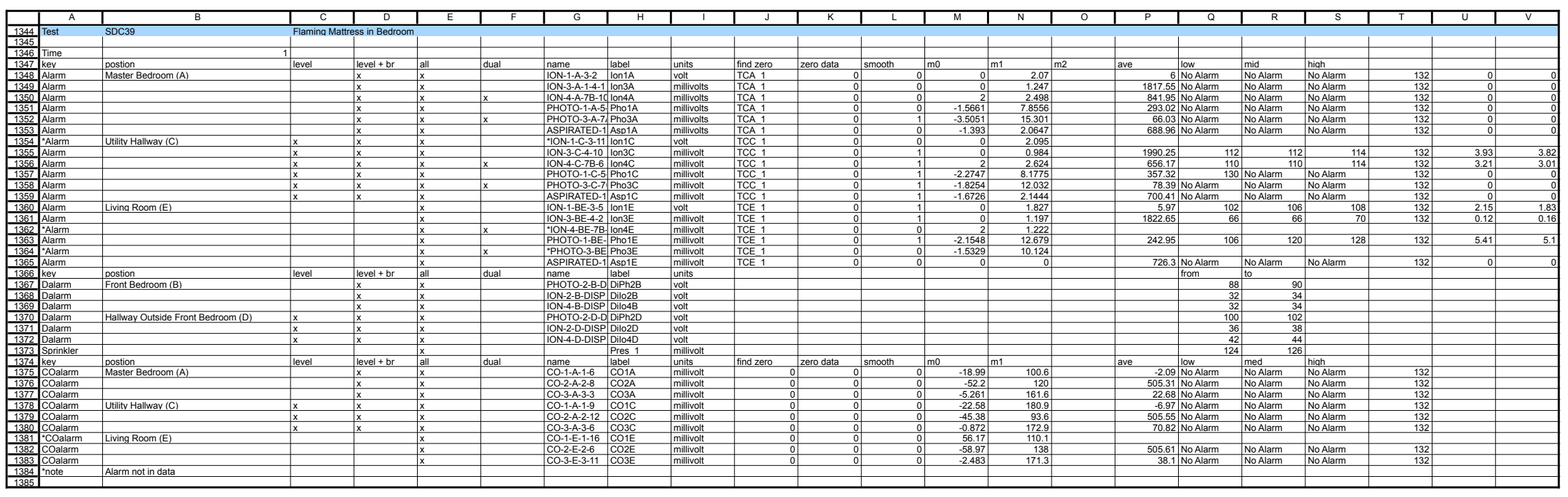




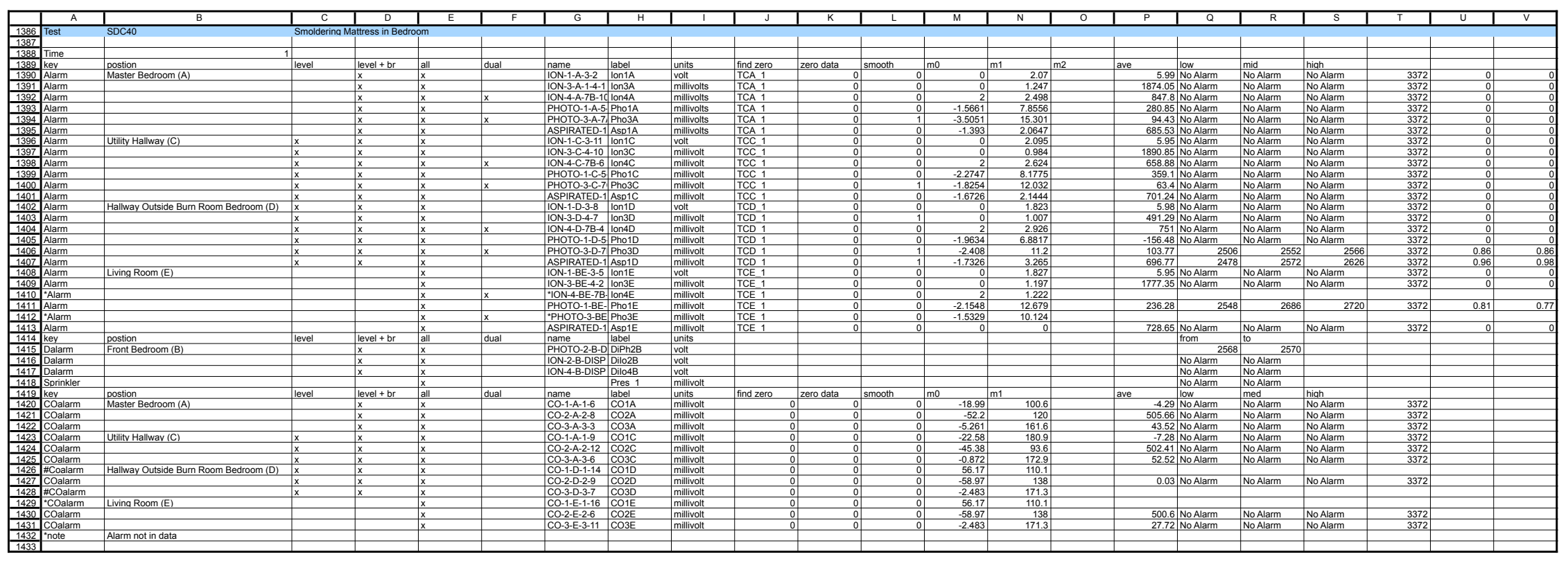




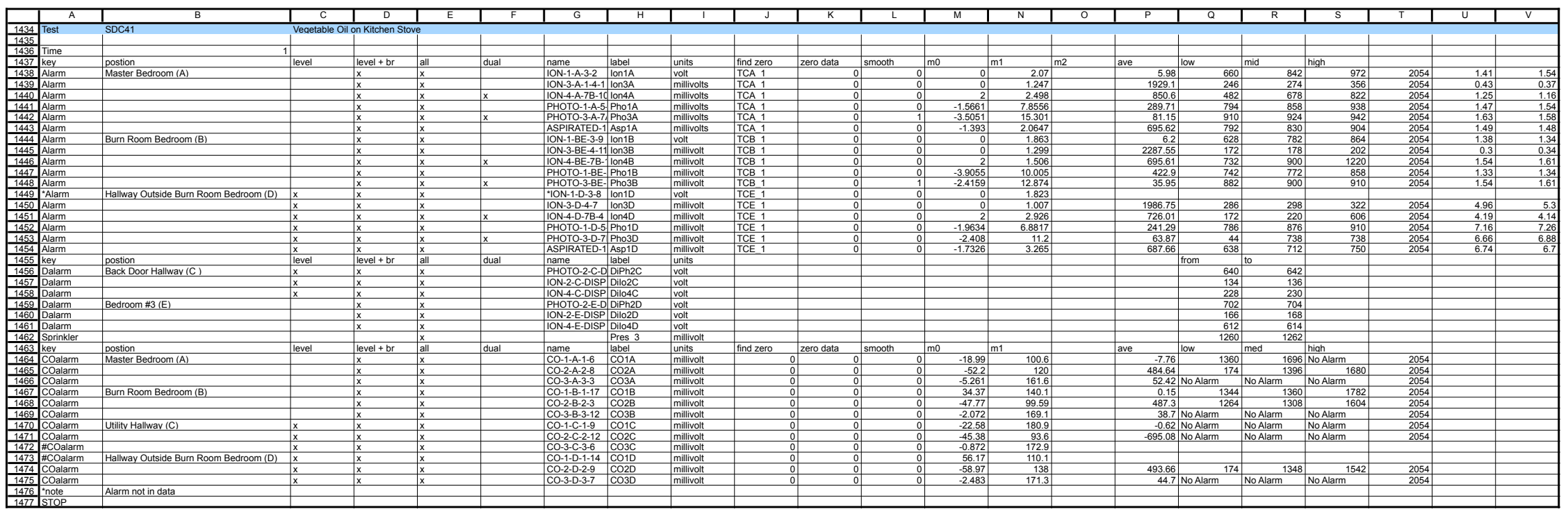




\section{Calculated Times to Untenable Conditions}




\begin{tabular}{|c|c|c|c|c|c|c|c|c|c|c|c|c|c|c|c|c|}
\hline & $\bar{A}$ & $B$ & $\mathrm{C}$ & $\mathrm{D}$ & $\bar{E}$ & $\mathrm{~F}$ & $\bar{G}$ & $\mathrm{H}$ & $\mathrm{T}$ & $\mathrm{J}$ & $\mathrm{K}$ & $\mathrm{L}$ & $\bar{M}$ & $\bar{N}$ & 0 & $\bar{P}$ \\
\hline 1 & & & 3 & 4 & 5 & 6 & 7 & 8 & 9 & 10 & 11 & 12 & 13 & 14 & & \\
\hline 2 & & & & & & & & & & & & & & & & \\
\hline 3 & Series & anufac & & & & & & & & & & & & & & \\
\hline 4 & & & & & & & & & & & & & & & & \\
\hline $\begin{array}{l}5 \\
6\end{array}$ & Test & SDC01 & Smoldering $\mathrm{Cr}$ & air in Living & Room & & & & & & & & & & & \\
\hline 7 & Time & & & & & & & & & & & & & & & \\
\hline 8 & & & & & & & & & & & & & & & & \\
\hline 9 & ISO Gas FED $=0.3$ & & & & & & & & & & & & & & & \\
\hline 10 & & & $\mathrm{CO} 2$ & CO2 Offset & $\mathrm{t}$ CO2 Base & $\mathrm{CO}$ & CO Offset & $\mathrm{HCN}$ & HCN Offset & ASET & Final Value & Minimum & & Outside Roc & om of Origin & \\
\hline 11 & Master Bedroom (A) & FEDG & GASA_3 & \begin{tabular}{|l|}
0.070961 \\
\end{tabular} & 0.06 & GASA_1 & \begin{tabular}{|l|}
0.008282 \\
\end{tabular} & ins & tion & & 0.238 & 4447 & (ISO Gas) & 6277 & (Smoke) & \\
\hline 12 & Utility Hallway (C) & FEDG & GASC 3 & 0.066902 & 0.06 & GASC 1 & 0.000505 & - & - & & 0.256 & & & & & \\
\hline 13 & Front Door Hallway $(\mathrm{F})$ & FEDG & GASF_3 & 0.064811 & 0.06 & GASF_1 & 0 & - & - & & 0.065 & & & & & \\
\hline 14 & Burn Room (BEK) & FEDG & GASB_3 & 1.0265 & 0.06 & GASB_1 & 0.076412 & - & - & 4447 & 0.300 & & & & & \\
\hline$\frac{14}{15}$ & 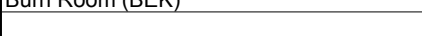 & & & & & & 0.010412 & - & - & & & & & & & \\
\hline 16 & NIST Gas FED $=0.3$ & & & & & & & & & & & & & & & \\
\hline 17 & & & $\mathrm{CO} 2$ & CO2 Offset & $\begin{array}{l}\mathrm{t} \text { CO2 Base } \\
\end{array}$ & $\mathrm{CO}$ & CO Offset & $\mathrm{HCN}$ & HCN Offset & ASET & Final Value & & & & & \\
\hline 18 & Master Bedroom (A) & FEDGN & GASA_3 & \begin{tabular}{|l|}
0.070961 \\
\end{tabular} & 0.06 & GASA_1 & \begin{tabular}{|l|}
0.008282 \\
\end{tabular} & 然 & 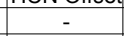 & & 0.000 & & & & & \\
\hline 19 & Utility Hallway (C) & FEDGN & GASC_3 & 0.066902 & 0.06 & GASC_1 & 0.000505 & - & - & & 0.000 & & & & & \\
\hline 20 & Front Door Hallway $(\mathrm{F})$ & FEDGN & GASF_3 & 0.064811 & 0.06 & GASF_1 & 0 & - & - & & 0.000 & & & & & \\
\hline 21 & Burn Room (BEK) & FEDGN & GASB_3 & 1.0265 & 0.06 & GASB_1 & 0.076412 & - & - & & 0.000 & & & & & \\
\hline 22 & & & & & & & & & & & & & & & & \\
\hline$\frac{24}{23}$ & ISO Convected Heat $=0.3$ & & & & & & & & & & & & & & & \\
\hline 24 & & & Temperature & & & & & & & ASET & Final Value & & & & & \\
\hline 25 & Master Bedroom (A) & FEDH & TCA_4 & & & & & & & & 0.145 & & & & & \\
\hline 26 & Burn Room Bedroom (B) & FEDH & TCB_4 & & & & & & & & 0.177 & & & & & \\
\hline$\frac{20}{27}$ & Utility Hallway (C) & FEDH & TCC_ 4 & & & & & & & & 0.155 & & & & & \\
\hline 28 & Hallway Outside Burn Room Bedroom (D) & FEDH & TCD_4 & & & & & & & & 0.162 & & & & & \\
\hline 29 & Living Room (E) & FEDH & TCE 4 & & & & & & & & 0.172 & & & & & \\
\hline 30 & Front Door Hallway (F) & FEDH & TCF_4 & & & & & & & & 0.171 & & & & & \\
\hline 31 & Closed Bedroom (G) & FEDH & TCG & & & & & & & & 0.114 & & & & & \\
\hline 32 & & & & & & & & & & & & & & & & \\
\hline 33 & Smoke Obscuration $=0.25 \mathrm{~m}-1$ & & & & & & & & & & & & & & & \\
\hline 34 & & & OD & & & & & & & ASET & Final Value & & & & & \\
\hline$\frac{34}{35}$ & Master Bedroom (A) & Smoke & SMA 4 & & & & & & & 6277 & 0.255 & & & & & \\
\hline 36 & Burn Room Bedroom (B) & Smoke & SMB 4 & & & & & & & & 0.127 & & & & & \\
\hline 37 & Utility Hallway (C) & Smoke & SMC_4 & & & & & & & 5397 & 0.250 & & & & & \\
\hline 38 & Hallway Outside Burn Room Bedroom (D) & Smoke & SMD 4 & & & & & & & & 0.078 & & & & & \\
\hline 39 & Living Room (E) & Smoke & SME 4 & & & & & & & 6172 & 0.267 & & & & & \\
\hline 40 & Front Door Hallway (F) & Smoke & SMF 4 & & & & & & & 6012 & 0.257 & & & & & \\
\hline 41 & Closed Bedroom (G) & Smoke & $S M G$ & & & & & & & & 0.000 & & & & & \\
\hline 42 & & & & & & & & & & & & & & & & \\
\hline
\end{tabular}




\begin{tabular}{|c|c|c|c|c|c|c|c|c|c|c|c|c|c|c|c|c|}
\hline & $\mathrm{A}$ & $\mathrm{B}$ & $\mathrm{C}$ & $\mathrm{D}$ & $\mathrm{E}$ & $\mathrm{F}$ & $\mathrm{G}$ & $\mathrm{H}$ & $\mathrm{T}$ & $\mathrm{J}$ & $\mathrm{K}$ & $\mathrm{L}$ & $M$ & $\mathrm{~N}$ & 0 & $P$ \\
\hline 43 & Test & SDC02 & Flaming Chair & in Living Roc & & & & & & & & & & & & \\
\hline 44 & & & & & & & & & & & & & & & & \\
\hline 45 & Time & te & & & & & & & & & & & & & & \\
\hline 46 & & & & & & & & & & & & & & & & \\
\hline 47 & ISO Gas FED = 0.3 & & & & & & & & & & & & & & & \\
\hline 48 & & & $\mathrm{CO} 2$ & CO2 Offset & CO2 Base & $\mathrm{CO}$ & CO Offset & $\mathrm{HCN}$ & HCN Offset & ASET & Final Value & Minimum & & Outside Ro & om of Origin & \\
\hline 49 & Master Bedroom (A) & FEDG & GASA_3 & \begin{tabular}{|l|}
0.061349 \\
\end{tabular} & 0.06 & GASA_1 & 0 & - & & & 0.003 & 178 & (Smoke) & 196 & (Smoke) & \\
\hline 50 & Utility Hallway (C) & FEDG & GASC_3 & 0.066478 & 0.06 & GASC_1 & 0.00011 & - & & & 0.007 & & & & & \\
\hline 51 & Front Door Hallway $(F)$ & FEDG & GASF_3 & 0.058458 & 0.06 & GASF_1 & 0.06285 & - & & & 0.011 & & & & & \\
\hline 52 & Burn Room (BEK) & FEDG & GASB_3 & 1.0182 & 0.06 & GASB_1 & 0.000561 & - & & & 0.029 & & & & & \\
\hline 53 & & & & & & & & & & & & & & & & \\
\hline 54 & NIST Gas FED $=0.3$ & & & & & & & & & & & & & & & \\
\hline 55 & & & $\mathrm{CO} 2$ & CO2 Offset & CO2 Base & $\mathrm{CO}$ & CO Offset & $\mathrm{HCN}$ & HCN Offset & ASET & Final Value & & & & & \\
\hline 56 & Master Bedroom (A) & FEDGN & GASA_3 & \begin{tabular}{|r|}
0.061349 \\
\end{tabular} & 0.06 & GASA_1 & 0 & . & & & 0.000 & & & & & \\
\hline 57 & Utility Hallway (C) & FEDGN & GASC_3 & 0.066478 & 0.06 & GASC_1 & 0.00011 & - & & & 0.000 & & & & & \\
\hline 58 & Front Door Hallway $(F)$ & FEDGN & GASF_3 & 0.058458 & 0.06 & GASF_1 & 0.06285 & - & & & 0.000 & & & & & \\
\hline 59 & Burn Room (BEK) & FEDGN & GASB_3 & 1.0182 & 0.06 & GASB_1 & 0.000561 & - & & & 0.000 & & & & & \\
\hline 60 & & & & & & & & & & & & & & & & \\
\hline 61 & ISO Convected Heat $=0.3$ & & & & & & & & & & & & & & & \\
\hline 62 & & & Temperature & & & & & & & ASET & Final Value & & & & & \\
\hline 63 & Master Bedroom (A) & FEDH & TCA_4 & & & & & & & & 0.012 & & & & & \\
\hline 64 & Burn Room Bedroom (B) & FEDH & TCB 4 & & & & & & & & 0.014 & & & & & \\
\hline 65 & Utility Hallway (C) & FEDH & TCC_4 4 & & & & & & & & 0.021 & & & & & \\
\hline 66 & Hallway Outside Burn Room Bedroom (D) & FEDH & TCD_4 & & & & & & & & 0.022 & & & & & \\
\hline 67 & Living Room (E) & FEDH & TCE_4 & & & & & & & & 0.041 & & & & & \\
\hline 68 & Front Door Hallway (F) & FEDH & TCF 4 4 & & & & & & & & 0.040 & & & & & \\
\hline 69 & Closed Bedroom (G) & FEDH & TCG & & & & & & & & 0.003 & & & & & \\
\hline 70 & & & & & & & & & & & & & & & & \\
\hline 71 & Smoke Obscuration $=0.25 \mathrm{~m}-1$ & & & & & & & & & & & & & & & \\
\hline 72 & & & OD & & & & & & & ASET & Final Value & & & & & \\
\hline 73 & Master Bedroom (A) & Smoke & SMA 4 & & & & & & & 196 & 0.280 & & & & & \\
\hline 74 & Burn Room Bedroom (B) & Smoke & SMB_4 & & & & & & & 220 & 0.254 & & & & & \\
\hline 75 & Utility Hallway (C) & Smoke & SMC_4 4 & & & & & & & 186 & 0.255 & & & & & \\
\hline 76 & Hallway Outside Burn Room Bedroom (D) & Smoke & SMD_4 4 & & & & & & & 178 & 0.277 & & & & & \\
\hline 77 & Living Room (E) & & & & & & & & & & & & & & & \\
\hline 78 & Front Door Hallway (F) & Smoke & SMF_4 & & & & & & & 230 & 0.261 & & & & & \\
\hline 79 & Closed Bedroom (G) & Smoke & $S M \bar{G}$ & & & & & & & & 0.007 & & & & & \\
\hline 80 & & & & & & & & & & & & & & & & \\
\hline
\end{tabular}




\begin{tabular}{|c|c|c|c|c|c|c|c|c|c|c|c|c|c|c|c|c|}
\hline & $\mathrm{A}$ & $\mathrm{B}$ & $\mathrm{C}$ & $\mathrm{D}$ & $\mathrm{E}$ & $\mathrm{F}$ & $\bar{G}$ & $\mathrm{H}$ & $\mathrm{T}$ & $\mathrm{J}$ & $\mathrm{K}$ & $\mathrm{L}$ & $M$ & $\mathrm{~N}$ & $\mathrm{O}$ & $\mathrm{P}$ \\
\hline$\frac{81}{82}$ & Test & SDC04 & Smoldering $\mathrm{M}$ & attress in $\mathrm{Bec}$ & droom & & & & & & & & & & & \\
\hline$\frac{82}{83}$ & Time & 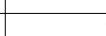 & & & & & & & & & & & & & & \\
\hline 84 & & & & & & & & & & & & & & & & \\
\hline 85 & ISO Gas FED = 0.3 & & & & & & & & & & & & & & & \\
\hline 86 & & & $\mathrm{CO} 2$ & CO2 Offset & CO2 Base & $\mathrm{CO}$ & CO Offset & $\mathrm{HCN}$ & HCN Offset & ASET & Final Value & Minimum & & Outside Ro & om of Origin & \\
\hline 87 & Master Bedroom (A) & & GASA_3 & \begin{tabular}{|l|}
0.032135 \\
\end{tabular} & 0.04 & GASA_1 & 0.005538 & - & - & & & 3383 & (Smoke) & 3443 & (Smoke) & \\
\hline 88 & Utility Hallway (C) & FEDG & GASC_3 & 0.043681 & 0.04 & GASC_1 & $2.55 \mathrm{E}-05$ & - & - & & 0.073 & & & & & \\
\hline 89 & Front Door Hallway $(F)$ & FEDG & GASF_3 & 0.30282 & 0.04 & GASF_1 & 0 & - & - & & 0.000 & & & & & \\
\hline 90 & Burn Room (BEK) & FEDG & GASB_3 & 0.27086 & 0.04 & GASB_1 & 0 & - & - & & 0.032 & & & & & \\
\hline 91 & & & & & & & & & & & & & & & & \\
\hline 92 & NIST Gas FED $=0.3$ & & & & & & & & & & & & & & & \\
\hline 93 & & & $\mathrm{CO} 2$ & CO2 Offset & CO2 Base & $\mathrm{CO}$ & CO Offset & $\mathrm{HCN}$ & HCN Offset & ASET & Final Value & & & & & \\
\hline 94 & Master Bedroom (A) & & GASA_3 & \begin{tabular}{|r|} 
\\
0.032135 \\
\end{tabular} & 0.04 & GASA_1 & 0.005538 & (10) & 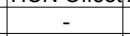 & & & & & & & \\
\hline 95 & Utility Hallway (C) & FEDGN & GASC_3 & 0.043681 & 0.04 & GASC_1 & $2.55 \mathrm{E}-05$ & - & - & & 0.066 & & & & & \\
\hline 96 & Front Door Hallway $(\mathrm{F})$ & FEDGN & GASF_3 & 0.30282 & 0.04 & GASF_1 & 0 & - & - & & 0.000 & & & & & \\
\hline 97 & Burn Room (BEK) & FEDGN & GASB_3 & 0.27086 & 0.04 & GASB_1 & 0 & - & - & & 0.000 & & & & & \\
\hline 98 & & & & & & & & & & & & & & & & \\
\hline 99 & ISO Convected Heat $=0.3$ & & & & & & & & & & & & & & & \\
\hline 100 & & & Temperature & & & & & & & ASET & Final Value & & & & & \\
\hline 101 & Master Bedroom (A) & FEDH & TCA_4 & & & & & & & & 0.090 & & & & & \\
\hline 102 & Burn Room Bedroom (B) & FEDH & TCB 4 & & & & & & & & 0.099 & & & & & \\
\hline 103 & Utility Hallway (C) & FEDH & $\mathrm{TCC}_{4} 4$ & & & & & & & & 0.078 & & & & & \\
\hline 104 & Hallway Outside Burn Room Bedroom (D) & FEDH & TCD_4 & & & & & & & & 0.077 & & & & & \\
\hline 105 & Living Room (E) & FEDH & TCE_4 & & & & & & & & 0.079 & & & & & \\
\hline 106 & Front Door Hallway $(\mathrm{F})$ & FEDH & TCF_4 & & & & & & & & 0.077 & & & & & \\
\hline 107 & Closed Bedroom (G) & FEDH & TCG & & & & & & & & 0.052 & & & & & \\
\hline 108 & & & & & & & & & & & & & & & & \\
\hline 109 & Smoke Obscuration $=0.25 \mathrm{~m}-1$ & & & & & & & & & & & & & & & \\
\hline 110 & & & OD & & & & & & & ASET & Final Value & & & & & \\
\hline 111 & Master Bedroom (A) & Smoke & SMA 4 & & & & & & & 3588 & 0.252 & & & & & \\
\hline 112 & Burn Room Bedroom (B) & Smoke & SMB_4 & & & & & & & 3383 & 0.251 & & & & & \\
\hline 113 & Utility Hallway (C) & Smoke & SMC_4 & & & & & & & 4013 & 3.356 & & & & & \\
\hline 114 & Hallway Outside Burn Room Bedroom (D) & Smoke & SMD_4 & & & & & & & 3443 & 0.273 & & & & & \\
\hline 115 & Living Room (E) & Smoke & SME 4 & & & & & & & 3518 & 0.252 & & & & & \\
\hline 116 & Front Door Hallway $(\mathrm{F})$ & Smoke & SMF_4 & & & & & & & 4053 & 2.384 & & & & & \\
\hline 117 & Closed Bedroom (G) & Smoke & $S M \bar{G}$ & & & & & & & 4008 & 3.482 & & & & & \\
\hline 118 & & & & & & & & & & & & & & & & \\
\hline
\end{tabular}




\begin{tabular}{|c|c|c|c|c|c|c|c|c|c|c|c|c|c|c|c|c|}
\hline & $\mathrm{A}$ & $\mathrm{B}$ & $\mathrm{C}$ & $\mathrm{D}$ & $\mathrm{E}$ & $\mathrm{F}$ & $\mathrm{G}$ & $\mathrm{H}$ & $\mathrm{T}$ & $\mathrm{J}$ & $\mathrm{K}$ & $\mathrm{L}$ & $M$ & $\mathrm{~N}$ & 0 & $\mathrm{P}$ \\
\hline 119 & Test & SDC05 & Flaming Mattre & ss in Bedroc & & & & & & & & & & & & \\
\hline 120 & & & & & & & & & & & & & & & & \\
\hline 121 & Time & te & & & & & & & & & & & & & & \\
\hline 122 & & & & & & & & & & & & & & & & \\
\hline 123 & ISO Gas FED = 0.3 & & & & & & & & & & & & & & & \\
\hline 124 & & & $\mathrm{CO} 2$ & CO2 Offset & CO2 Base & $\mathrm{CO}$ & CO Offset & $\mathrm{HCN}$ & HCN Offset & ASET & Final Value & Minimum & & Outside Ro & om of Origin & \\
\hline 125 & Master Bedroom (A) & FEDG & GASA_3 & 0.043402 & 0.04 & GASA_1 & $\begin{array}{l}0.007707 \\
\end{array}$ & - & - & & 0.012 & 137 & (Smoke) & 162 & (Smoke) & \\
\hline 126 & Utility Hallway (C) & FEDG & GASC_3 & 0.044686 & 0.04 & GASC_1 & 0.001675 & - & - & & 0.021 & & & & & \\
\hline 127 & Front Door Hallway $(F)$ & FEDG & GASF_3 & 0.041174 & 0.04 & GASF_1 & 0.000427 & - & - & & 0.028 & & & & & \\
\hline 128 & Burn Room (BEK) & FEDG & GASB_3 & 1.0002 & 0.04 & GASB_1 & 0.00146 & - & - & & 0.070 & & & & & \\
\hline 129 & & & & & & & & & & & & & & & & \\
\hline 130 & NIST Gas FED $=0.3$ & & & & & & & & & & & & & & & \\
\hline 131 & & & $\mathrm{CO} 2$ & CO2 Offset & CO2 Base & $\mathrm{CO}$ & CO Offset & $\mathrm{HCN}$ & HCN Offset & ASET & Final Value & & & & & \\
\hline 132 & Master Bedroom (A) & FEDGN & GASA_3 & 0.043402 & 0.04 & GASA_1 & 0.007707 & 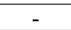 & 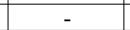 & & 0.000 & & & & & \\
\hline 133 & Utility Hallway (C) & FEDGN & GASC_3 & 0.044686 & 0.04 & GASC_1 & 0.001675 & - & - & & 0.000 & & & & & \\
\hline 134 & Front Door Hallway $(\mathrm{F})$ & FEDGN & GASF_3 & 0.041174 & 0.04 & GASF_1 & 0.000427 & - & - & & 0.000 & & & & & \\
\hline 135 & Burn Room (BEK) & FEDGN & GASB_3 & 1.0002 & 0.04 & GASB_1 & 0.00146 & - & - & & 0.000 & & & & & \\
\hline 136 & & & & & & & & & & & & & & & & \\
\hline 137 & ISO Convected Heat $=0.3$ & & & & & & & & & & & & & & & \\
\hline 138 & & & Temperature & & & & & & & ASET & Final Value & & & & & \\
\hline 139 & Master Bedroom (A) & FEDH & TCA_4 & & & & & & & & 0.009 & & & & & \\
\hline 140 & Burn Room Bedroom (B) & FEDH & TCB 4 & & & & & & & & 0.273 & & & & & \\
\hline 141 & Utility Hallway (C) & FEDH & TCC 4 & & & & & & & & 0.016 & & & & & \\
\hline 142 & Hallway Outside Burn Room Bedroom (D) & FEDH & TCD_4 & & & & & & & & 0.042 & & & & & \\
\hline 143 & Living Room (E) & FEDH & TCE_4 & & & & & & & & 0.020 & & & & & \\
\hline 144 & Front Door Hallway (F) & FEDH & TCF 4 4 & & & & & & & & 0.022 & & & & & \\
\hline 145 & Closed Bedroom (G) & FEDH & TCG & & & & & & & & 0.004 & & & & & \\
\hline 146 & & & & & & & & & & & & & & & & \\
\hline 147 & Smoke Obscuration $=0.25 \mathrm{~m}-1$ & & & & & & & & & & & & & & & \\
\hline 148 & & & OD & & & & & & & ASET & Final Value & & & & & \\
\hline 149 & Master Bedroom (A) & Smoke & SMA 4 & & & & & & & 197 & 0.276 & & & & & \\
\hline 150 & Burn Room Bedroom (B) & Smoke & SMB_4 & & & & & & & 137 & 0.329 & & & & & \\
\hline 151 & Utility Hallway (C) & Smoke & SMC_4 & & & & & & & 182 & 0.341 & & & & & \\
\hline 152 & Hallway Outside Burn Room Bedroom (D) & Smoke & SMD_4 & & & & & & & 162 & 0.403 & & & & & \\
\hline 153 & Living Room (E) & Smoke & SME 4 & & & & & & & 177 & 0.260 & & & & & \\
\hline 154 & Front Door Hallway $(\mathrm{F})$ & Smoke & SMF_4 & & & & & & & 217 & 0.291 & & & & & \\
\hline 155 & Closed Bedroom (G) & Smoke & SMG & & & & & & & & 0.006 & & & & & \\
\hline 156 & & & & & & & & & & & & & & & & \\
\hline
\end{tabular}




\begin{tabular}{|c|c|c|c|c|c|c|c|c|c|c|c|c|c|c|c|c|}
\hline & $\mathrm{A}$ & $\mathrm{B}$ & $\mathrm{C}$ & $\mathrm{D}$ & $\mathrm{E}$ & $\mathrm{F}$ & $\mathrm{G}$ & $\mathrm{H}$ & $\mathrm{T}$ & $\mathrm{J}$ & $\mathrm{K}$ & $\mathrm{L}$ & $M$ & $\mathrm{~N}$ & 0 & $\mathrm{P}$ \\
\hline 157 & Test & SDC06 & Smoldering Ma & attress in Bec & droom & & & & & & & & & & & \\
\hline 158 & & & & & & & & & & & & & & & & \\
\hline 159 & Time & te & & & & & & & & & & & & & & \\
\hline 160 & & & & & & & & & & & & & & & & \\
\hline 161 & ISO Gas FED = 0.3 & & & & & & & & & & & & & & & \\
\hline 162 & & & $\mathrm{CO} 2$ & CO2 Offset & CO2 Base & $\mathrm{CO}$ & CO Offset & $\mathrm{HCN}$ & HCN Offset & ASET & Final Value & Minimum & & Outside Ro & om of Origin & \\
\hline 163 & Master Bedroom (A) & & GASA_3 & \begin{tabular}{|l|}
0.044175 \\
\end{tabular} & 0.04 & GASA_1 & 0.009128 & . & & & & 6008 & (Smoke) & 6068 & (Smoke) & \\
\hline 164 & Utility Hallway (C) & FEDG & GASC_3 & 0.047696 & 0.04 & GASC_1 & 0.000423 & - & & & 0.060 & & & & & \\
\hline 165 & Front Door Hallway $(F)$ & FEDG & GASF_3 & 0.039582 & 0.04 & GASF_1 & 0 & - & & & 0.001 & & & & & \\
\hline 166 & Burn Room (BEK) & FEDG & GASB_3 & 0.99975 & 0.04 & GASB_1 & 0.000419 & - & & & 0.021 & & & & & \\
\hline 167 & & & & & & & & & & & & & & & & \\
\hline 168 & NIST Gas FED $=0.3$ & & & & & & & & & & & & & & & \\
\hline 169 & & & $\mathrm{CO} 2$ & CO2 Offset & CO2 Base & $\mathrm{CO}$ & CO Offset & $\mathrm{HCN}$ & HCN Offset & ASET & Final Value & & & & & \\
\hline 170 & Master Bedroom (A) & & GASA_3 & 0.044175 & 0.04 & GASA_1 & 0.009128 & 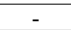 & & & 0.000 & & & & & \\
\hline 171 & Utility Hallway (C) & FEDGN & GASC_3 & 0.047696 & 0.04 & GASC_1 & 0.000423 & - & & & 0.000 & & & & & \\
\hline 172 & Front Door Hallway $(\mathrm{F})$ & FEDGN & GASF_3 & 0.039582 & 0.04 & GASF_1 & 0 & - & & & 0.000 & & & & & \\
\hline 173 & Burn Room (BEK) & FEDGN & GASB_3 & 0.99975 & 0.04 & GASB_1 & 0.000419 & 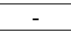 & & & 0.000 & & & & & \\
\hline 174 & & & & & & & & & & & & & & & & \\
\hline 175 & ISO Convected Heat $=0.3$ & & & & & & & & & & & & & & & \\
\hline 176 & & & Temperature & & & & & & & ASET & Final Value & & & & & \\
\hline 177 & Master Bedroom (A) & FEDH & TCA_4 & & & & & & & & 0.106 & & & & & \\
\hline 178 & Burn Room Bedroom (B) & FEDH & TCB 4 & & & & & & & & 0.122 & & & & & \\
\hline 179 & Utility Hallway (C) & FEDH & TCC 4 & & & & & & & & 0.095 & & & & & \\
\hline 180 & Hallway Outside Burn Room Bedroom (D) & FEDH & TCD_4 & & & & & & & & 0.090 & & & & & \\
\hline 181 & Living Room (E) & FEDH & TCE_4 & & & & & & & & 0.094 & & & & & \\
\hline 182 & Front Door Hallway (F) & FEDH & TCF 4 4 & & & & & & & & 0.092 & & & & & \\
\hline 183 & Closed Bedroom (G) & FEDH & TCG & & & & & & & & 0.058 & & & & & \\
\hline 184 & & & & & & & & & & & & & & & & \\
\hline 185 & Smoke Obscuration $=0.25 \mathrm{~m}-1$ & & & & & & & & & & & & & & & \\
\hline 186 & & & OD & & & & & & & ASET & Final Value & & & & & \\
\hline 187 & Master Bedroom (A) & Smoke & SMA 4 & & & & & & & 6098 & 0.279 & & & & & \\
\hline 188 & Burn Room Bedroom (B) & Smoke & SMB_4 & & & & & & & 6008 & 0.408 & & & & & \\
\hline 189 & Utility Hallway (C) & Smoke & SMC_4 & & & & & & & 6078 & 0.293 & & & & & \\
\hline 190 & Hallway Outside Burn Room Bedroom (D) & & SMD_4 & & & & & & & & & & & & & \\
\hline 191 & Living Room (E) & Smoke & SME 4 & & & & & & & 6068 & 0.261 & & & & & \\
\hline 192 & Front Door Hallway $(\mathrm{F})$ & Smoke & SMF_4 & & & & & & & & 0.069 & & & & & \\
\hline 193 & Closed Bedroom (G) & Smoke & SMG & & & & & & & & 0.017 & & & & & \\
\hline 194 & & & & & & & & & & & & & & & & \\
\hline
\end{tabular}




\begin{tabular}{|c|c|c|c|c|c|c|c|c|c|c|c|c|c|c|c|c|}
\hline & $\bar{A}$ & $\mathrm{~B}$ & $\mathrm{C}$ & $\mathrm{D}$ & $\mathrm{E}$ & $F$ & $\bar{G}$ & $\mathrm{H}$ & $T$ & $\mathrm{~J}$ & $\mathrm{~K}$ & $\mathrm{~L}$ & $\bar{M}$ & $\bar{N}$ & $\mathrm{O}$ & $\bar{P}$ \\
\hline 195 & Test & SDC07 & Flaming Mattre & ss in Bedroc & & & & & & & & & & & & \\
\hline 196 & & & & & & & & & & & & & & & & \\
\hline 197 & Time & & & & & & & & & & & & & & & \\
\hline 198 & tinc & & & & & & & & & & & & & & & \\
\hline 199 & ISO Gas FED = 0.3 & & & & & & & & & & & & & & & \\
\hline 200 & & & $\mathrm{CO} 2$ & CO2 Offset & CO2 Base & $\mathrm{CO}$ & CO Offset & $\mathrm{HCN}$ & HCN Offset & tASET & Final Value & Minimum & & Outside Ro & oom of Origin & \\
\hline 201 & Master Bedroom (A) & FEDG & GASA_3 & \begin{tabular}{|l|}
0.053758 \\
\end{tabular} & 0.05 & GASA_1 & \begin{tabular}{|l|}
0.024354 \\
\end{tabular} & 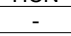 & - & & 0.083 & 145 & (Smoke) & 163 & (Smoke) & \\
\hline 202 & Utility Hallway (C) & FEDG & GASC_3 & 0.050332 & 0.05 & GASC_1 & 0.003521 & - & - & & 0.111 & & & & & \\
\hline 203 & Front Door Hallway (F) & FEDG & GASF_3 & 0.27477 & 0.05 & GASF_1 & 0 & - & - & 289 & 0.313 & & & & & \\
\hline 204 & Burn Room (BEK) & FEDG & GASB_3 & 6.2016 & 0.05 & GASB_1 & 0 & - & - & 229 & 0.362 & & & & & \\
\hline 205 & & & & & & & & & & & & & & & & \\
\hline 206 & NIST Gas FED $=0.3$ & & & & & & & & & & & & & & & \\
\hline 207 & & & $\mathrm{CO} 2$ & CO2 Offset & CO2 Base & $\mathrm{CO}$ & CO Offset & $\mathrm{HCN}$ & HCN Offset & tASET & Final Value & & & & & \\
\hline 208 & Master Bedroom (A) & FEDGN & GASA_3 & \begin{tabular}{|l|}
0.053758 \\
\end{tabular} & 0.05 & GASA_1 & \begin{tabular}{|l|}
0.024354 \\
\end{tabular} & - & - & & 0.000 & & & & & \\
\hline 209 & Utility Hallway (C) & FEDGN & GASC_3 & 0.050332 & 0.05 & GASC_1 & 0.003521 & - & 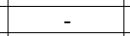 & & 0.000 & & & & & \\
\hline 210 & Front Door Hallway (F) & FEDGN & GASF_3 & 0.27477 & 0.05 & GASF_1 & 0 & 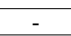 & - & & 0.026 & & & & & \\
\hline 211 & Burn Room (BEK) & FEDGN & GASB_3 & 6.2016 & 0.05 & GASB_1 & 0 & - & - & 391 & 0.300 & & & & & \\
\hline 212 & & & & & & & & & & & & & & & & \\
\hline 213 & ISO Convected Heat $=0.3$ & & & & & & & & & & & & & & & \\
\hline 214 & & & Temperature & & & & & & & ASET & Final Value & & & & & \\
\hline 215 & Master Bedroom (A) & FEDH & TCA_4 & & & & & & & & 0.084 & & & & & \\
\hline 216 & Burn Room Bedroom (B) & FEDH & TCB 4 & & & & & & & 193 & 0.315 & & & & & \\
\hline 217 & Utility Hallway (C) & FEDH & TCC_4 & & & & & & & & 0.217 & & & & & \\
\hline 218 & Hallway Outside Burn Room Bedroom (D) & FEDH & TCD 4 & & & & & & & 217 & 0.351 & & & & & \\
\hline 219 & Living Room (E) & FEDH & TCE_ 4 & & & & & & & 387 & 0.300 & & & & & \\
\hline 220 & Front Door Hallway $(F)$ & FEDH & TCF_4 & & & & & & & 283 & 0.305 & & & & & \\
\hline 221 & Closed Bedroom (G) & FEDH & TCG & & & & & & & & 0.005 & & & & & \\
\hline 222 & & & & & & & & & & & & & & & & \\
\hline 223 & Smoke Obscuration $=0.25 \mathrm{~m}-1$ & & & & & & & & & & & & & & & \\
\hline 224 & & & OD & & & & & & & ASET & Final Value & & & & & \\
\hline 225 & Master Bedroom (A) & Smoke & SMA 4 & & & & & & & 191 & 0.256 & & & & & \\
\hline 226 & Burn Room Bedroom (B) & Smoke & SMB 4 & & & & & & & 145 & 0.253 & & & & & \\
\hline 227 & \begin{tabular}{|l} 
Utility Hallway (C) \\
\end{tabular} & Smoke & SMC 4 & & & & & & & 187 & 0.263 & & & & & \\
\hline 228 & Hallway Outside Burn Room Bedroom (D) & Smoke & SMD_4 & & & & & & & 163 & 0.271 & & & & & \\
\hline 229 & Living Room (E) & Smoke & SME_4 & & & & & & & 181 & 0.265 & & & & & \\
\hline 230 & Front Door Hallway (F) & Smoke & SMF 4 & & & & & & & 199 & 0.379 & & & & & \\
\hline 231 & Closed Bedroom (G) & Smoke & SMG & & & & & & & 219 & 3.599 & & & & & \\
\hline 232 & & & & & & & & & & & & & & & & \\
\hline
\end{tabular}




\begin{tabular}{|c|c|c|c|c|c|c|c|c|c|c|c|c|c|c|c|c|}
\hline & $\mathrm{A}$ & $\mathrm{B}$ & $\mathrm{C}$ & $\mathrm{D}$ & $\mathrm{E}$ & $\mathrm{F}$ & $\mathrm{G}$ & $\mathrm{H}$ & $T$ & $\mathrm{~J}$ & $\mathrm{~K}$ & $\mathrm{~L}$ & $\mathrm{M}$ & $\mathrm{N}$ & 0 & $\mathrm{P}$ \\
\hline 233 & Test & SDC08 & Smoldering Ma & attress in $\mathrm{Bec}$ & droom & & & & & & & & & & & \\
\hline$\frac{234}{235}$ & Time & & & & & & & & & & & & & & & \\
\hline$\frac{235}{236}$ & Time & 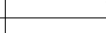 & & & & & & & & & & & & & & \\
\hline 237 & ISO Gas FED $=0.3$ & & & & & & & & & & & & & & & \\
\hline 238 & & & $\mathrm{CO} 2$ & CO2 Offset & CO2 Base & $\mathrm{CO}$ & co Offset & $\mathrm{HCN}$ & HCN Offse & ASET & Final Value & Minimum & & Outside $R$ & oom of Oriain & \\
\hline 239 & Master Bedroom (A) & FEDG & GASA_3 & \begin{tabular}{|l|}
0.046098 \\
\end{tabular} & 0.04 & GASA_1 & 0.020241 & - & - & & 0.164 & 3771 & (Smoke) & 3771 & 1 (Smoke) & \\
\hline 240 & Utility Hallway (C) & FEDG & GASC 3 & 0.047659 & 0.04 & GASC 1 & 0.001858 & - & - & & 0.064 & & & & & \\
\hline 241 & Front Door Hallway $(\mathrm{F})$ & FEDG & GASF_3 & 0.30594 & 0.04 & GASF_1 & 0 & - & - & 3891 & 0.305 & & & & & \\
\hline 242 & Burn Room (BEK) & FEDG & GASB_3 & 5.5062 & 0.04 & GASB_1 & 0 & - & - & 3847 & 0.306 & & & & & \\
\hline 243 & & & & & & & & & & & & & & & & \\
\hline 244 & NIST Gas FED $=0.3$ & & & & & & & & & & & & & & & \\
\hline 245 & & & $\mathrm{CO} 2$ & CO2 Offset & CO2 Base & $\mathrm{CO}$ & CO Offset & $\mathrm{HCN}$ & HCN Offse & ASET & Final Value & & & & & \\
\hline 246 & Master Bedroom (A) & FEDGN & GASA_3 & \begin{tabular}{|r|}
0.046098 \\
\end{tabular} & 0.04 & GASA_1 & 0.020241 & - & - & & 0.000 & & & & & \\
\hline 247 & Utility Hallway (C) & FEDGN & GASC_3 & 0.047659 & 0.04 & GASC_1 & 0.001858 & - & - & & 0.000 & & & & & \\
\hline 248 & Front Door Hallway (F) & FEDGN & GASF_3 & 0.30594 & 0.04 & GASF_1 & 4 & - & - & & 0.240 & & & & & \\
\hline 249 & Burn Room (BEK) & FEDGN & GASB_3 & 5.5062 & 0.04 & GASB_1 & 0 & - & - & & 0.149 & & & & & \\
\hline 250 & & & & & & & & & & & & & & & & \\
\hline 251 & ISO Convected Heat $=0.3$ & & & & & & & & & & & & & & & \\
\hline 252 & & & Temperature & & & & & & & ASET & Final Value & & & & & \\
\hline 253 & Master Bedroom (A) & FEDH & TCA 4 & & & & & & & & 0.104 & & & & & \\
\hline 254 & Burn Room Bedroom (B) & FEDH & TCB_4 & & & & & & & 3783 & 0.301 & & & & & \\
\hline 255 & Utility Hallway (C) & FEDH & TCC_4 & & & & & & & & 0.148 & & & & & \\
\hline 256 & Hallway Outside Burn Room Bedroom (D) & FEDH & TCD_4 & & & & & & & 3807 & 0.319 & & & & & \\
\hline 257 & Living Room (E) & FEDH & TCE_4 & & & & & & & & 0.172 & & & & & \\
\hline 258 & Front Door Hallway (F) & FEDH & TCF_4 4 & & & & & & & & 0.206 & & & & & \\
\hline 259 & Closed Bedroom (G) & FEDH & TCG & & & & & & & & 0.046 & & & & & \\
\hline 260 & & & & & & & & & & & & & & & & \\
\hline 261 & Smoke Obscuration $=0.25 \mathrm{~m}-1$ & & & & & & & & & & & & & & & \\
\hline 262 & & & OD & & & & & & & ASET & Final Value & & & & & \\
\hline 263 & Master Bedroom (A) & Smoke & SMA_4 & & & & & & & 3783 & 0.284 & & & & & \\
\hline 264 & Utility Hallway (C) & Smoke & SMC 4 & & & & & & & 3771 & 0.263 & & & & & \\
\hline 265 & Hallway Outside Burn Room Bedroom (D) & Smoke & SMD_4 & & & & & & & 3801 & 0.279 & & & & & \\
\hline 266 & Living Room (E) & Smoke & SME_4 & & & & & & & 3779 & 0.259 & & & & & \\
\hline 267 & Front Door Hallway (F) & Smoke & SMF_4 & & & & & & & 3785 & \begin{tabular}{l|l}
0.266 \\
\end{tabular} & & & & & \\
\hline 268 & & & & & & & & & & & & & & & & \\
\hline
\end{tabular}




\begin{tabular}{|c|c|c|c|c|c|c|c|c|c|c|c|c|c|c|c|c|}
\hline & A & $B$ & $\mathrm{C}$ & $\mathrm{D}$ & $\mathrm{E}$ & $\mathrm{F}$ & $\mathrm{G}$ & $\mathrm{H}$ & 1 & $\mathrm{~J}$ & $\mathrm{~K}$ & $\mathrm{~L}$ & $M$ & $\mathrm{~N}$ & 0 & $\mathrm{P}$ \\
\hline 269 & Test & SDC09 & Flaming Mattre & ss in Bedroc & om (Burn Ro & om Door $\mathrm{Cl}$ & osed) & & & & & & & & & \\
\hline 270 & & & & & & & & & & & & & & & & \\
\hline 271 & Time & & & & & & & & & & & & & & & \\
\hline 272 & & & & & & & & & & & & & & & & \\
\hline 273 & ISO Gas FED = 0.3 & & & & & & & & & & & & & & & \\
\hline 274 & & & $\mathrm{CO} 2$ & CO2 Offset & CO2 Base & $\mathrm{CO}$ & co Offset & $\mathrm{HCN}$ & HCN Offset & ASET & Final Value & Minimum & & Outside Ro & om of Origin & \\
\hline 275 & Master Bedroom (A) & FEDG & GASA_3 & \begin{tabular}{|l|}
0.045254 \\
\end{tabular} & 0.05 & GASA_1 & 0.02389 & - & - & & 0.058 & 163 & (ISO Heat) & 1067 & (Smoke) & \\
\hline 276 & Utility Hallway (C) & FEDG & GASC_3 & 0.046558 & 0.05 & GASC_1 & 0.002098 & - & - & & 0.014 & & & & & \\
\hline 277 & Front Door Hallway (F) & FEDG & GASF_3 & 0.25434 & 0.05 & GASF_1 & 0 & - & - & & 0.010 & & & & & \\
\hline 278 & Burn Room (BEK) & FEDG & GASB_3 & 0.31543 & 0.05 & GASB_1 & $6.16 \mathrm{E}-05$ & - & - & 187 & 0.319 & & & & & \\
\hline 279 & & & & & & & & & & & & & & & & \\
\hline 280 & NIST Gas FED $=0.3$ & & & & & & & & & & & & & & & \\
\hline 281 & & & $\mathrm{CO} 2$ & CO2 Offset & CO2 Base & $\mathrm{CO}$ & co Offset & $\mathrm{HCN}$ & HCN Offset & ASET & Final Value & & & & & \\
\hline 282 & Master Bedroom (A) & FEDGN & GASA_3 & 0.045254 & 0.05 & GASA_1 & 0.02389 & - & - & & 0.000 & & & & & \\
\hline 283 & Utility Hallway (C) & FEDGN & GASC_3 & 0.046558 & 0.05 & GASC_1 & 0.002098 & - & - & & 0.000 & & & & & \\
\hline 284 & Front Door Hallway $(F)$ & FEDGN & GASF_3 & 0.25434 & 0.05 & GASF_1 & 0 & - & - & & 0.000 & & & & & \\
\hline 285 & Burn Room (BEK) & FEDGN & GASB_3 & 0.31543 & 0.05 & GASB_1 & $6.16 \mathrm{E}-05$ & - & - & & 0.021 & & & & & \\
\hline 286 & & & & & & & & & & & & & & & & \\
\hline 287 & ISO Convected Heat $=0.3$ & & & & & & & & & & & & & & & \\
\hline 288 & & & Temperature & & & & & & & ASET & Final Value & & & & & \\
\hline 289 & Master Bedroom (A) & FEDH & TCA 4 & & & & & & & & 0.030 & & & & & \\
\hline 290 & Burn Room Bedroom (B) & FEDH & TCB_4 & & & & & & & 163 & 0.304 & & & & & \\
\hline 291 & Utility Hallway (C) & FEDH & TCC_4 & & & & & & & & 0.028 & & & & & \\
\hline 292 & Hallway Outside Burn Room Bedroom (D) & FEDH & TCD_4 & & & & & & & & 0.032 & & & & & \\
\hline 293 & Living Room (E) & FEDH & TCE_4 & & & & & & & & 0.029 & & & & & \\
\hline 294 & Front Door Hallway $(\mathrm{F})$ & FEDH & TCF_4 & & & & & & & & 0.029 & & & & & \\
\hline 295 & Closed Bedroom (G) & FEDH & TCG & & & & & & & & 0.021 & & & & & \\
\hline 296 & & & & & & & & & & & & & & & & \\
\hline 297 & Smoke Obscuration $=0.25 \mathrm{~m}-1$ & & & & & & & & & & & & & & & \\
\hline 298 & 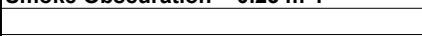 & & $\mathrm{OD}$ & & & & & & & ASET & Final Value & & & & & \\
\hline 299 & Master Bedroom (A) & Smoke & SMA_4 & & & & & & & & 0.189 & & & & & \\
\hline 300 & Utility Hallway (C) & Smoke & SMC_4 & & & & & & & 1113 & 0.307 & & & & & \\
\hline 301 & Hallway Outside Burn Room Bedroom (D) & Smoke & SMD_4 & & & & & & & 1067 & 0.438 & & & & & \\
\hline 302 & Living Room (E) & Smoke & SME_4 4 & & & & & & & 1111 & 0.255 & & & & & \\
\hline 303 & Front Door Hallway $(\mathrm{F})$ & Smoke & SMF_4 4 & & & & & & & 1073 & 0.818 & & & & & \\
\hline 304 & Closed Bedroom (G) & Smoke & SMG & & & & & & & & 0.004 & & & & & \\
\hline 305 & & & & & & & & & & & & & & & & \\
\hline
\end{tabular}




\begin{tabular}{|c|c|c|c|c|c|c|c|c|c|c|c|c|c|c|c|c|}
\hline & A & $\mathrm{B}$ & $\mathrm{C}$ & $\mathrm{D}$ & $\mathrm{E}$ & $\mathrm{F}$ & $\mathrm{G}$ & $\mathrm{H}$ & $I$ & $\mathrm{~J}$ & $\mathrm{~K}$ & $\mathrm{~L}$ & $M$ & $\mathrm{~N}$ & 0 & $P$ \\
\hline$\frac{306}{307}$ & Test & SDC10 & Flaming Chair & in Living Roor & & & & & & & & & & & & \\
\hline 307 & & & & & & & & & & & & & & & & \\
\hline 308 & Time & & & & & & & & & & & & & & & \\
\hline 309 & & & & & & & & & & & & & & & & \\
\hline 310 & ISO Gas FED = 0.3 & & & & & & & & & & & & & & & \\
\hline 311 & & & $\mathrm{CO} 2$ & CO2 Offset & CO2 Base & $\mathrm{CO}$ & co Offset & $\mathrm{HCN}$ & HCN Offset & ASET & Final Value & Minimum & & Outside Ro & om of Origin & \\
\hline 312 & Master Bedroom (A) & FEDG & GASA_3 & $\begin{array}{ll}0.061769 \\
\end{array}$ & 0.06 & GASA_1 & 0.025371 & - & - & & 0.016 & 160 & (Smoke) & 212 & (Smoke) & \\
\hline 313 & Utility Hallway (C) & FEDG & GASC_3 & 0.0611 & 0.06 & GASC_1 & 0.002442 & - & - & & 0.037 & & & & & \\
\hline 314 & Front Door Hallway (F) & FEDG & GASF_3 & 0.42583 & 0.06 & GASF_1 & 0 & - & - & 278 & 0.308 & & & & & \\
\hline 315 & Burn Room (BEK) & FEDG & GASB_3 & \begin{tabular}{|l|l|}
5.5129 \\
\end{tabular} & 0.06 & GASB_1 & 0 & - & - & 258 & 0.312 & & & & & \\
\hline 316 & & & & & & & & & & & & & & & & \\
\hline 317 & NIST Gas FED $=0.3$ & & & & & & & & & & & & & & & \\
\hline 318 & & & $\mathrm{CO} 2$ & CO2 Offset & CO2 Base & $\mathrm{CO}$ & co Offset & $\mathrm{HCN}$ & HCN Offset & ASET & Final Value & & & & & \\
\hline 319 & Master Bedroom (A) & FEDGN & GASA_3 & 0.061769 & 0.06 & GASA_1 & 0.025371 & - & - & & 0.000 & & & & & \\
\hline 320 & Utility Hallway (C) & FEDGN & GASC_3 & 0.0611 & 0.06 & GASC_1 & 0.002442 & - & - & & 0.000 & & & & & \\
\hline 321 & Front Door Hallway $(F)$ & FEDGN & GASF_3 & 0.42583 & 0.06 & GASF_1 & 0 & - & - & & 0.014 & & & & & \\
\hline 322 & Burn Room (BEK) & FEDGN & GASB_3 & 5.5129 & 0.06 & GASB_1 & 0 & - & - & & 0.035 & & & & & \\
\hline 323 & & & & & & & & & & & & & & & & \\
\hline 324 & ISO Convected Heat $=0.3$ & & & & & & & & & & & & & & & \\
\hline 325 & & & Temperature & & & & & & & ASET & Final Value & & & & & \\
\hline 326 & Master Bedroom (A) & FEDH & TCA 4 & & & & & & & & 0.073 & & & & & \\
\hline 327 & Burn Room Bedroom (B) & FEDH & TCB_4 & & & & & & & & 0.098 & & & & & \\
\hline 328 & Utility Hallway (C) & FEDH & TCC 4 & & & & & & & & 0.181 & & & & & \\
\hline 329 & Hallway Outside Burn Room Bedroom (D) & FEDH & TCD_4 & & & & & & & & 0.171 & & & & & \\
\hline 330 & Living Room (E) & FDEH & TCE_4 & & & & & & & & & & & & & \\
\hline 331 & Front Door Hallway $(\mathrm{F})$ & FEDH & TCF_4 & & & & & & & 232 & 0.305 & & & & & \\
\hline 332 & Closed Bedroom (G) & FEDH & TCG & & & & & & & & 0.007 & & & & & \\
\hline 333 & & & & & & & & & & & & & & & & \\
\hline 334 & Smoke Obscuration $=0.25 \mathrm{~m}-1$ & & & & & & & & & & & & & & & \\
\hline 335 & 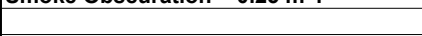 & & $\mathrm{OD}$ & & & & & & & ASET & Final Value & & & & & \\
\hline 336 & Master Bedroom (A) & Smoke & SMA_4 & & & & & & & 212 & 0.277 & & & & & \\
\hline 337 & Burn Room Bedroom (B) & Smoke & SMB 4 & & & & & & & 214 & 0.260 & & & & & \\
\hline 338 & Utility Hallway (C) & Smoke & SMC_4 & & & & & & & 192 & 0.252 & & & & & \\
\hline 339 & Hallway Outside Burn Room Bedroom (D) & Smoke & SMD_4 & & & & & & & 190 & 0.267 & & & & & \\
\hline 340 & Front Door Hallway $(F)$ & Smoke & SMF_4 4 & & & & & & & 160 & 0.265 & & & & & \\
\hline 341 & Closed Bedroom (G) & Smoke & $S M \bar{G}$ & & & & & & & & 0.042 & & & & & \\
\hline 342 & & & & & & & & & & & & & & & & \\
\hline
\end{tabular}




\begin{tabular}{|c|c|c|c|c|c|c|c|c|c|c|c|c|c|c|c|c|}
\hline & $\mathrm{A}$ & $\mathrm{B}$ & $\mathrm{C}$ & $\mathrm{D}$ & $\mathrm{E}$ & $\mathrm{F}$ & $\mathrm{G}$ & $\mathrm{H}$ & $\mathrm{T}$ & $\mathrm{J}$ & $\mathrm{K}$ & $\mathrm{L}$ & $M$ & $\mathrm{~N}$ & 0 & $P$ \\
\hline 343 & Test & SDC11 & Smoldering $\mathrm{Ch}$ & air in Living & Room & & & & & & & & & & & \\
\hline 344 & Juts & & & & & & & & & & & & & & & \\
\hline 345 & Time & te & & & & & & & & & & & & & & \\
\hline 346 & & & & & & & & & & & & & & & & \\
\hline 347 & ISO Gas FED = 0.3 & & & & & & & & & & & & & & & \\
\hline 348 & & & $\mathrm{CO} 2$ & CO2 Offset & CO2 Base & $\mathrm{CO}$ & CO Offset & $\mathrm{HCN}$ & HCN Offset & ASET & Final Value & Minimum & & Outside $R c$ & oom of Origin & \\
\hline 349 & Master Bedroom (A) & FEDG & GASA_3 & \begin{tabular}{|l|}
0.045152 \\
\end{tabular} & 0.05 & GASA_1 & 0.015872 & - & - & & 0.191 & 2901 & (ISO Gas) & 4341 & 1 (Smoke) & \\
\hline 350 & Utility Hallway (C) & FEDG & GASC_3 & 0.046906 & 0.05 & GASC_1 & 0.003377 & - & - & & 0.087 & & & & & \\
\hline 351 & Front Door Hallway $(\mathrm{F})$ & FEDG & GASF_3 & 0.24874 & 0.05 & GASF_1 & 0 & - & - & 4245 & 0.301 & & & & & \\
\hline 352 & Burn Room (BEK) & FEDG & GASB_3 & 5.3871 & 0.05 & GASB_1 & 0.003682 & - & - & 2901 & 0.300 & & & & & \\
\hline 353 & & & & & & & & & & & & & & & & \\
\hline 354 & NIST Gas FED $=0.3$ & & & & & & & & & & & & & & & \\
\hline 355 & & & $\mathrm{CO} 2$ & CO2 Offset & CO2 Base & $\mathrm{CO}$ & CO Offset & $\mathrm{HCN}$ & HCN Offset & ASET & Final Value & & & & & \\
\hline 356 & Master Bedroom (A) & FEDGN & GASA_3 & 0.045152 & 0.05 & GASA_1 & 0.015872 & 然 & - & & 0.000 & & & & & \\
\hline 357 & Utility Hallway (C) & FEDGN & GASC_3 & 0.046906 & 0.05 & GASC_1 & $\begin{array}{l}0.003377 \\
\end{array}$ & - & - & & 0.000 & & & & & \\
\hline 358 & Front Door Hallway $(\mathrm{F})$ & FEDGN & GASF_3 & 0.24874 & 0.05 & GASF_1 & 0 & - & - & & 0.007 & & & & & \\
\hline 359 & Burn Room (BEK) & FEDGN & GASB_3 & 5.3871 & 0.05 & GASB_1 & 0.003682 & - & - & & 0.064 & & & & & \\
\hline 360 & & & & & & & & & & & & & & & & \\
\hline 361 & ISO Convected Heat $=0.3$ & & & & & & & & & & & & & & & \\
\hline 362 & & & Temperature & & & & & & & ASET & Final Value & & & & & \\
\hline 363 & Master Bedroom (A) & FEDH & TCA_4 & & & & & & & & 0.179 & & & & & \\
\hline 364 & Burn Room Bedroom (B) & FEDH & TCB 4 & & & & & & & & 0.187 & & & & & \\
\hline 365 & Utility Hallway (C) & FEDH & TCC 4 & & & & & & & & 0.209 & & & & & \\
\hline 366 & Hallway Outside Burn Room Bedroom (D) & FEDH & TCD_4 & & & & & & & & 0.210 & & & & & \\
\hline 367 & Living Room (E) & FEDH & TCE_4 & & & & & & & 4407 & 0.302 & & & & & \\
\hline 368 & Front Door Hallway $(\mathrm{F})$ & FEDH & TCF_4 & & & & & & & & 0.261 & & & & & \\
\hline 369 & Closed Bedroom (G) & FEDH & TCG & & & & & & & & 0.096 & & & & & \\
\hline 370 & & & & & & & & & & & & & & & & \\
\hline 371 & & & & & & & & & & & & & & & & \\
\hline 372 & Smoke Obscuration $=0.25 \mathrm{~m}-1$ & & & & & & & & & & & & & & & \\
\hline 373 & prione onseration - & & $\mathrm{OD}$ & & & & & & & ASET & Final Value & & & & & \\
\hline 374 & Master Bedroom (A) & Smoke & SMA_4 & & & & & & & 4341 & 0.262 & & & & & \\
\hline 375 & Burn Room Bedroom (B) & Smoke & SMB_4 & & & & & & & 4345 & 0.252 & & & & & \\
\hline 376 & Utility Hallway (C) & Smoke & SMC_4 & & & & & & & 4297 & 0.255 & & & & & \\
\hline 377 & Hallway Outside Burn Room Bedroom (D) & Smoke & SMD 4 & & & & & & & 4331 & 0.271 & & & & & \\
\hline 378 & Front Door Hallway $(\mathrm{F})$ & Smoke & SMF 4 & & & & & & & 3993 & 0.253 & & & & & \\
\hline 379 & Closed Bedroom (G) & Smoke & $S M \bar{G}$ & & & & & & & & 0.033 & & & & & \\
\hline 380 & & & & & & & & & & & & & & & & \\
\hline
\end{tabular}




\begin{tabular}{|c|c|c|c|c|c|c|c|c|c|c|c|c|c|c|c|c|}
\hline & $\mathrm{A}$ & $\mathrm{B}$ & $\mathrm{C}$ & $\mathrm{D}$ & $\mathrm{E}$ & $F$ & $\bar{G}$ & $\mathrm{H}$ & $T$ & $\mathrm{~J}$ & $\mathrm{~K}$ & $\mathrm{~L}$ & $\bar{M}$ & $\mathrm{~N}$ & $\mathrm{O}$ & $\mathrm{P}$ \\
\hline$\frac{381}{382}$ & Test & SDC12 & Vegetable Oil c & on Kitchen S & tove & & & & & & & & & & & \\
\hline 382 & & & & & & & & & & & & & & & & \\
\hline 383 & Time & & & & & & & & & & & & & & & \\
\hline 384 & & & & & & & & & & & & & & & & \\
\hline 385 & ISO Gas FED $=0.3$ & & & & & & & & & & & & & & & \\
\hline 386 & & & $\mathrm{CO} 2$ & CO2 Offset & CO2 Base & $\mathrm{CO}$ & CO Offset & $\mathrm{HCN}$ & HCN Offse & ASET & Final Value & Minimum & & Outside $\mathrm{Rc}$ & om of Origin & \\
\hline 387 & Master Bedroom (A) & & GASA_3 & \begin{tabular}{|l|}
0.055647 \\
\end{tabular} & 0.05 & GASA_1 & 0.007483 & - & - & & 0.101 & 1247 & (Smoke) & 1423 & (Smoke) & \\
\hline 388 & Utility Hallway (C) & & GASC_3 & 0.05711 & 0.05 & GASC_1 & 0.036972 & - & - & 1461 & 0.300 & & & & & \\
\hline 389 & Front Door Hallway $(F)$ & FEDG & GASF_3 & 0.57198 & 0.05 & GASF_1 & 0 & - & - & & 0.000 & & & & & \\
\hline 390 & Burn Room (BEK) & FEDG & GASB_3 & 5.4816 & 0.05 & GASB_1 & $2.84 \mathrm{E}-05$ & - & - & & 0.174 & & & & & \\
\hline 391 & & & & & & & & & & & & & & & & \\
\hline 392 & NIST Gas FED $=0.3$ & & & & & & & & & & & & & & & \\
\hline 393 & & & $\mathrm{CO} 2$ & CO2 Offset & CO2 Base & $\mathrm{CO}$ & co Offset & $\mathrm{HCN}$ & HCN Offse & tASET & Final Value & & & & & \\
\hline 394 & Master Bedroom (A) & & GASA_3 & \begin{tabular}{|l|}
0.055647 \\
\end{tabular} & 0.05 & GASA_1 & 0.007483 & - & - & & 0.000 & & & & & \\
\hline 395 & Utility Hallway (C) & & GASC_3 & 0.05711 & 0.05 & GASC_1 & 0.036972 & - & - & & 0.062 & & & & & \\
\hline 396 & Front Door Hallway (F) & FEDGN & GASF_3 & 0.57198 & 0.05 & GASF_1 & 0 & - & - & & 0.000 & & & & & \\
\hline 397 & Burn Room (BEK) & FEDGN & GASB_3 3 & 5.4816 & 0.05 & GASB_1 & $2.84 \mathrm{E}-05$ & - & - & & 0.000 & & & & & \\
\hline 398 & & & & & & & & & & & & & & & & \\
\hline 399 & ISO Convected Heat $=0.3$ & & & & & & & & & & & & & & & \\
\hline 400 & & & Temperature & & & & & & & ASET & Final Value & & & & & \\
\hline 401 & Master Bedroom (A) & FEDH & TCA 4 & & & & & & & & 0.063 & & & & & \\
\hline 402 & Burn Room Bedroom (B) & FEDH & TCB_4 & & & & & & & & 0.062 & & & & & \\
\hline 403 & Utility Hallway (C) & FEDH & TCC_4 & & & & & & & & 0.064 & & & & & \\
\hline 404 & Hallway Outside Burn Room Bedroom (D) & FEDH & TCD_4 & & & & & & & & 0.064 & & & & & \\
\hline 405 & Living Room (E) & FEDH & TCE_4 & & & & & & & & 0.066 & & & & & \\
\hline 406 & Front Door Hallway $(F)$ & FEDH & TCF_4 4 & & & & & & & & 0.068 & & & & & \\
\hline 407 & Closed Bedroom (G) & FEDH & TCG & & & & & & & & 0.036 & & & & & \\
\hline 408 & & & & & & & & & & & & & & & & \\
\hline 409 & Smoke Obscuration $=0.25 \mathrm{~m}-1$ & & & & & & & & & & & & & & & \\
\hline 410 & 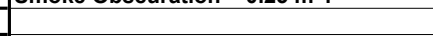 & & $O D$ & & & & & & & ASET & Final Value & & & & & \\
\hline 411 & Master Bedroom (A) & Smoke & SMA_4 & & & & & & & 1423 & 0.257 & & & & & \\
\hline 412 & Burn Room Bedroom (B) & Smoke & SMB_4 & & & & & & & 1453 & 0.266 & & & & & \\
\hline 413 & Utility Hallway (C) & Smoke & SMC_4 & & & & & & & 1315 & 0.257 & & & & & \\
\hline 414 & Hallway Outside Burn Room Bedroom (D) & Smoke & SMD_4 & & & & & & & 1247 & 0.263 & & & & & \\
\hline 415 & Front Door Hallway (F) & Smoke & SMF_4 & & & & & & & 1377 & 0.251 & & & & & \\
\hline 416 & Closed Bedroom (G) & Smoke & SMG & & & & & & & & 0.041 & & & & & \\
\hline 417 & & & & & & & & & & & & & & & & \\
\hline
\end{tabular}




\begin{tabular}{|c|c|c|c|c|c|c|c|c|c|c|c|c|c|c|c|c|}
\hline & A & $\mathrm{B}$ & $\mathrm{C}$ & $\mathrm{D}$ & $\bar{E}$ & $\mathrm{~F}$ & $\bar{G}$ & $\mathrm{H}$ & $T$ & $\mathrm{~J}$ & $\mathrm{~K}$ & $\mathrm{~L}$ & $\mathrm{M}$ & $\bar{N}$ & 0 & $\bar{P}$ \\
\hline 418 & Test & SDC13 & Vegetable Oil c & on Kitchen S & tove & & & & & & & & & & & \\
\hline 419 & & & & & & & & & & & & & & & & \\
\hline 420 & Time & T & & & & & & & & & & & & & & \\
\hline 421 & & & & & & & & & & & & & & & & \\
\hline 422 & ISO Gas FED $=0.3$ & & & & & & & & & & & & & & & \\
\hline 423 & & & $\mathrm{CO} 2$ & CO2 Offset & CO2 Base & $\mathrm{CO}$ & co Offset & $\mathrm{HCN}$ & HCN Offse & ASET & Final Value & Minimum & & Outside $R$ & oom of Origin & \\
\hline 424 & Master Bedroom (A) & FEDG & GASA_3 & 0.05107 & 0.05 & GASA_1 & 0.01502 & - & - & & 0.101 & 1176 & (Smoke) & 1400 & (Smoke) & \\
\hline 425 & Utility Hallway (C) & & GASC 3 & 0.053608 & 0.05 & GASC 1 & 0.003159 & - & - & & 0.037 & & & & & \\
\hline 426 & Front Door Hallway $(F)$ & FEDG & GASF_3 & 0.4629 & 0.05 & GASF_1 & 0 & - & - & & 0.000 & & & & & \\
\hline 427 & Burn Room (BEK) & FEDG & GASB_3 & 6.392 & 0.05 & GASB_1 & 0 & - & - & & 0.095 & & & & & \\
\hline 428 & & & & & & & & & & & & & & & & \\
\hline 429 & NIST Gas FED $=0.3$ & & & & & & & & & & & & & & & \\
\hline 430 & & & $\mathrm{CO} 2$ & CO2 Offset & CO2 Base & $\mathrm{co}$ & co Offset & $\mathrm{HCN}$ & HCN Offse & ASET & Final Value & & & & & \\
\hline 431 & Master Bedroom (A) & FEDGN & GASA_3 & \begin{tabular}{|l|}
0.05107 \\
\end{tabular} & 0.05 & GASA_1 & 0.01502 & - & - & & 0.000 & & & & & \\
\hline 432 & Utility Hallway (C) & & GASC_3 & 0.053608 & 0.05 & GASC_1 & 0.003159 & - & - & & 0.000 & & & & & \\
\hline 433 & Front Door Hallway (F) & FEDGN & GASF_3 & 0.4629 & 0.05 & GASF_1 & 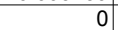 & - & - & & 0.000 & & & & & \\
\hline 434 & Burn Room (BEK) & FEDGN & GASB_3 & 6.392 & 0.05 & GASB_1 & 0 & - & - & & 0.233 & & & & & \\
\hline 435 & & & & & & & & & & & & & & & & \\
\hline 436 & ISO Convected Heat $=0.3$ & & & & & & & & & & & & & & & \\
\hline 437 & & & Temperature & & & & & & & ASET & Final Value & & & & & \\
\hline$\frac{438}{438}$ & Master Bedroom (A) & FEDH & TCA 4 & & & & & & & & 0.057 & & & & & \\
\hline 439 & Burn Room Bedroom (B) & FEDH & TCB_4 & & & & & & & & 0.056 & & & & & \\
\hline 440 & Utility Hallway (C) & FEDH & TCC_4 4 & & & & & & & & 0.059 & & & & & \\
\hline 441 & Hallway Outside Burn Room Bedroom (D) & FEDH & TCD 4 & & & & & & & & 0.059 & & & & & \\
\hline$\frac{442}{442}$ & Living Room (E) & FEDH & TCE 4 & & & & & & & & 0.063 & & & & & \\
\hline 443 & Front Door Hallway $(\mathrm{F})$ & FEDH & TCF_4 & & & & & & & & 0.067 & & & & & \\
\hline 444 & Closed Bedroom (G) & FEDH & TCG & & & & & & & & 0.025 & & & & & \\
\hline 445 & & & & & & & & & & & & & & & & \\
\hline 446 & Smoke Obscuration $=0.25 \mathrm{~m}-1$ & & & & & & & & & & & & & & & \\
\hline 447 & & & $\mathrm{OD}$ & & & & & & & ASET & Final Value & & & & & \\
\hline 448 & Master Bedroom (A) & Smoke & SMA_4 & & & & & & & 1400 & 0.259 & & & & & \\
\hline 449 & Utility Hallway $(C)$ & Smoke & SMC_4 & & & & & & & 1376 & 0.413 & & & & & \\
\hline 450 & Hallway Outside Burn Room Bedroom (D) & Smoke & SMD 4 & & & & & & & 1176 & 0.252 & & & & & \\
\hline 451 & Front Door Hallway (F) & Smoke & SMF_4 & & & & & & & 1350 & 0.251 & & & & & \\
\hline 452 & Closed Bedroom (G) & Smoke & SMG & & & & & & & & 0.036 & & & & & \\
\hline 453 & & & & & & & & & & & & & & & & \\
\hline
\end{tabular}




\begin{tabular}{|c|c|c|c|c|c|c|c|c|c|c|c|c|c|c|c|c|}
\hline & $\mathrm{A}$ & $B$ & $\mathrm{C}$ & $\mathrm{D}$ & $E$ & $\mathrm{~F}$ & $\bar{G}$ & $\mathrm{H}$ & $\mathrm{T}$ & $\mathrm{J}$ & $\mathrm{K}$ & $\bar{L}$ & $\bar{M}$ & $\mathrm{~N}$ & $\mathrm{O}$ & $\mathrm{P}$ \\
\hline 454 & Test & SDC14 & Smoldering Ma & attress in Bedr & droom (Burn & Room Doo & Closed) & & & & & & & & & \\
\hline 455 & & & & & & & & & & & & & & & & \\
\hline 456 & Time & & & & & & & & & & & & & & & \\
\hline 457 & & & & & & & & & & & & & & & & \\
\hline 458 & ISO Gas FED $=0.3$ & & & & & & & & & & & & & & & \\
\hline 459 & & & $\mathrm{CO} 2$ & CO2 Offset & CO2 Base & $\mathrm{CO}$ & CO Offset & $\mathrm{HCN}$ & HCN Offset & ASET & Final Value & Minimum & & Outside Ro & om of Origin & \\
\hline 460 & Master Bedroom (A) & FEDG & GASA_3 & 0.053447 & 0.05 & GASA_1 & 0.008797 & - & - & & 0.168 & 3438 & (ISO Heat) & 3482 & (Smoke) & \\
\hline 461 & Utility Hallway (C) & FEDG & GASC_3 & 0.057068 & 0.05 & GASC_1 & 0.001845 & - & - & & 0.063 & & & & & \\
\hline 462 & Front Door Hallway $(F)$ & FEDG & GASF_3 & 0.55287 & 0.05 & GASF_1 & 0 & - & - & & 0.230 & & & & & \\
\hline 463 & Burn Room (BEK) & FEDG & GASB_3 & $\begin{array}{l}5.5859 \\
\end{array}$ & 0.05 & GASB_1 & 0 & - & - & 3478 & 0.309 & & & & & \\
\hline 464 & & & & & & & & & & & & & & & & \\
\hline 465 & NIST Gas FED $=0.3$ & & & & & & & & & & & & & & & \\
\hline 466 & & & $\mathrm{CO} 2$ & CO2 Offset & CO2 Base & $\mathrm{CO}$ & CO Offset & $\mathrm{HCN}$ & HCN Offset & ASET & Final Value & & & & & \\
\hline 467 & Master Bedroom (A) & FEDGN & GASA_3 & \begin{tabular}{|l|}
0.053447 \\
\end{tabular} & 0.05 & GASA_1 & 0.008797 & & 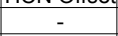 & & 0.000 & & & & & \\
\hline 468 & Utility Hallway (C) & FEDGN & GASC_3 & 0.057068 & 0.05 & GASC_1 & 0.001845 & - & - & & 0.000 & & & & & \\
\hline 469 & Front Door Hallway (F) & FEDGN & GASF_3 & 0.55287 & 0.05 & GASF_1 & 0 & - & - & & 0.130 & & & & & \\
\hline 470 & Burn Room (BEK) & FEDGN & GASB_3 & 5.5859 & 0.05 & GASB_1 & 0 & - & - & 3586 & 0.302 & & & & & \\
\hline 471 & & & & & & & & & & & & & & & & \\
\hline 472 & ISO Convected Heat $=0.3$ & & & & & & & & & & & & & & & \\
\hline 473 & & & Temperature & & & & & & & ASET & Final Value & & & & & \\
\hline 474 & Master Bedroom (A) & FEDH & TCA_4 & & & & & & & & 0.122 & & & & & \\
\hline 475 & Burn Room Bedroom (B) & FEDH & TCB_4 & & & & & & & 3438 & 0.301 & & & & & \\
\hline 476 & Utility Hallway (C) & FEDH & TCC_4 4 & & & & & & & & 0.110 & & & & & \\
\hline 477 & Hallway Outside Burn Room Bedroom (D) & FEDH & TCD_4 & & & & & & & & 0.113 & & & & & \\
\hline 478 & Living Room (E) & FEDH & TCE_4 & & & & & & & & 0.113 & & & & & \\
\hline 479 & Front Door Hallway $(\mathrm{F})$ & FEDH & TCF_4 & & & & & & & & 0.114 & & & & & \\
\hline 480 & Closed Bedroom (G) & FEDH & TCG & & & & & & & & 0.078 & & & & & \\
\hline 481 & & & & & & & & & & & & & & & & \\
\hline 482 & Smoke Obscuration $=0.25 \mathrm{~m}-1$ & & & & & & & & & & & & & & & \\
\hline 483 & & & $\mathrm{OD}$ & & & & & & & ASET & Final Value & & & & & \\
\hline 484 & Master Bedroom (A) & Smoke & SMA_4 & & & & & & & & 0.247 & & & & & \\
\hline 485 & Utility Hallway (C) & Smoke & SMC_4 & & & & & & & 4058 & 0.448 & & & & & \\
\hline 486 & Hallway Outside Burn Room Bedroom (D) & Smoke & SMD_4 & & & & & & & 3482 & 0.257 & & & & & \\
\hline 487 & Front Door Hallway (F) & Smoke & SMF_4 & & & & & & & 4032 & 0.433 & & & & & \\
\hline 488 & & & & & & & & & & & & & & & & \\
\hline
\end{tabular}




\begin{tabular}{|c|c|c|c|c|c|c|c|c|c|c|c|c|c|c|c|c|}
\hline & $\mathrm{A}$ & $\mathrm{B}$ & $\mathrm{C}$ & $\mathrm{D}$ & $E$ & $\mathrm{~F}$ & $G$ & $\mathrm{H}$ & 1 & J & $\mathrm{K}$ & $\mathrm{L}$ & $\mathrm{M}$ & $\mathrm{N}$ & $\mathrm{O}$ & $P$ \\
\hline 489 & Test & SDC15 & Flaming Chair & in Living Roc & & & & & & & & & & & & \\
\hline 490 & & & & & & & & & & & & & & & & \\
\hline 491 & Time & & & & & & & & & & & & & & & \\
\hline 492 & & & & & & & & & & & & & & & & \\
\hline 493 & ISO Gas FED $=0.3$ & & & & & & & & & & & & & & & \\
\hline 494 & & & $\mathrm{CO} 2$ & CO2 Offset & CO2 Base & $\mathrm{CO}$ & CO Offset & $\mathrm{HCN}$ & HCN Offset & ASET & Final Value & Minimum & & Outside Rc & oom of Origin & \\
\hline 495 & Master Bedroom (A) & FEDG & GASA_3 & 0.047309 & 0.05 & GASA_1 & \begin{tabular}{|r|}
0.00717 \\
\end{tabular} & - & 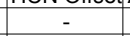 & & 0.014 & 177 & (Smoke) & & 3 (Smoke) & \\
\hline 496 & Utility Hallway (C) & FEDG & GASC 3 & 0.052957 & 0.05 & GASC 1 & 0.001446 & - & - & & 0.013 & & & & & \\
\hline 497 & Front Door Hallway (F) & FEDG & GASF_3 & 0.26104 & 0.05 & GASF_1 & 0 & - & - & & 0.095 & & & & & \\
\hline 498 & Burn Room (BEK) & FEDG & GASB 3 & 0.32699 & 0.05 & GASB_1 & 0.54492 & - & - & & 0.237 & & & & & \\
\hline 4999 & & & & & & & & & & & & & & & & \\
\hline 500 & NIST Gas FED $=0.3$ & & & & & & & & & & & & & & & \\
\hline 501 & & & $\mathrm{CO} 2$ & CO2 Offset & CO2 Base & $\mathrm{CO}$ & CO Offset & $\mathrm{HCN}$ & HCN Offset & ASET & Final Value & & & & & \\
\hline 502 & Master Bedroom (A) & FEDGN & GASA 3 & 0.047309 & 0.05 & GASA_1 & \begin{tabular}{|l|}
0.00717 \\
\end{tabular} & Then & 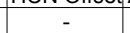 & & 0.000 & & & & & \\
\hline 503 & Utility Hallway (C) & FEDGN & GASC 3 & 0.052957 & 0.05 & GASC 1 & 0.001446 & - & - & & 0.000 & & & & & \\
\hline 504 & Front Door Hallway (F) & FEDGN & GASF_3 & 0.26104 & 0.05 & GASF_1 & 0 & - & - & & 0.000 & & & & & \\
\hline 505 & Burn Room (BEK) & FEDGN & GASB_3 & 0.32699 & 0.05 & GASB_1 & 0.54492 & 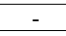 & - & & 0.016 & & & & & \\
\hline 506 & & & & & & & & & & & & & & & & \\
\hline 507 & ISO Convected Heat $=0.3$ & & & & & & & & & & & & & & & \\
\hline 508 & & & Temperature & & & & & & & ASET & Final Value & & & & & \\
\hline 509 & Master Bedroom (A) & FEDH & TCA_4 & & & & & & & & 0.034 & & & & & \\
\hline 510 & Burn Room Bedroom (B) & FEDH & TCB 4 & & & & & & & & 0.049 & & & & & \\
\hline 511 & Utility Hallway (C) & FEDH & TCC 4 & & & & & & & & 0.058 & & & & & \\
\hline 512 & Hallway Outside Burn Room Bedroom (D) & FEDH & TCD_4 & & & & & & & & 0.062 & & & & & \\
\hline 513 & Living Room (E) & FEDH & TCE 4 & & & & & & & & 0.086 & & & & & \\
\hline 514 & Front Door Hallway $(\mathrm{F})$ & FEDH & TCF 4 & & & & & & & & 0.175 & & & & & \\
\hline 515 & Closed Bedroom (G) & FEDH & TCG & & & & & & & & 0.010 & & & & & \\
\hline 516 & & & & & & & & & & & & & & & & \\
\hline 517 & Smoke Obscuration $=0.25 \mathrm{~m}-1$ & & & & & & & & & & & & & & & \\
\hline 518 & & & OD & & & & & & & ASET & Final Value & & & & & \\
\hline 519 & Master Bedroom (A) & Smoke & SMA 4 & & & & & & & 213 & 0.299 & & & & & \\
\hline 520 & Utility Hallway (C) & Smoke & SMC_4 & & & & & & & 187 & 0.273 & & & & & \\
\hline 521 & Hallway Outside Burn Room Bedroom (D) & Smoke & SMD_4 & & & & & & & 189 & 0.266 & & & & & \\
\hline 522 & Front Door Hallway (F) & Smoke & SMF_4 & & & & & & & 177 & 0.293 & & & & & \\
\hline 523 & & & & & & & & & & & & & & & & \\
\hline
\end{tabular}




\begin{tabular}{|c|c|c|c|c|c|c|c|c|c|c|c|c|c|c|c|c|}
\hline & $\mathrm{A}$ & $\mathrm{B}$ & $\mathrm{C}$ & $\mathrm{D}$ & $E$ & $\mathrm{~F}$ & $\mathrm{G}$ & $\mathrm{H}$ & $\mathrm{I}$ & $\mathrm{J}$ & $\mathrm{K}$ & $\mathrm{L}$ & $\mathrm{M}$ & $\mathrm{N}$ & 0 & $P$ \\
\hline 524 & Series & & & & & & & & & & & & & & & \\
\hline 525 & & & & & & & & & & & & & & & & \\
\hline 526 & Test & SDC31 & Smoldering $\mathrm{Ch}$ & air in Living & Room & & & & & & & & & & & \\
\hline 527 & & & & & & & & & & & & & & & & \\
\hline 528 & Time & & & & & & & & & & & & & & & \\
\hline 529 & & & & & & & & & & & & & & & & \\
\hline 530 & ISO Gas FED = 0.3 & & & & & & & & & & & & & & & \\
\hline 531 & & & $\mathrm{CO} 2$ & CO2 Offset & CO2 Base & $\mathrm{CO}$ & CO Offset & $\mathrm{HCN}$ & HCN Offset & ASET & Final Value & Minimum & & Outside R & oom of Origin & \\
\hline 532 & Master Bedroom (A) & FEDG & GASA_3 & \begin{tabular}{|r|}
0.039692 \\
\end{tabular} & 0.04 & GASA_1 & 0.003631 & - & - & 6585 & 0.300 & 3715 & (ISO Gas) & 5321 & 1 (Smoke) & \\
\hline 533 & Utility Hallway (C) & FEDG & GASC_3 & 0.031859 & 0.04 & GASC_1 & 0.003822 & 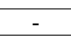 & - & 5339 & 0.300 & & & & & \\
\hline 534 & Front Door Hallway (F) & FEDG & GASF_3 & 0.045835 & 0.04 & GASF 1 & 0.006313 & - & - & 3715 & 0.300 & & & & & \\
\hline 535 & Burn Room (BEK) & FEDG & GASB_3 & 0.020709 & 0.04 & GASB_1 & 0.005459 & - & - & 4905 & 0.300 & & & & & \\
\hline 536 & & & & & & & & & & & & & & & & \\
\hline 537 & NIST Gas FED $=0.3$ & & & & & & & & & & & & & & & \\
\hline 538 & & & $\mathrm{CO} 2$ & CO2 Offset & CO2 Base & $\mathrm{CO}$ & CO Offset & $\mathrm{HCN}$ & HCN Offset & ASET & Final Value & & & & & \\
\hline 539 & Master Bedroom (A) & FEDGN & GASA_3 & 0.039692 & 0.04 & GASA_1 & 0.003631 & - & - & & 0.000 & & & & & \\
\hline 540 & Utility Hallway (C) & FEDGN & GASC_3 & 0.031859 & 0.04 & GASC_1 & 0.003822 & - & - & & 0.000 & & & & & \\
\hline 541 & Front Door Hallway (F) & FEDGN & GASF_3 & 0.045835 & 0.04 & GASF_1 & 0.006313 & - & - & & 0.000 & & & & & \\
\hline 542 & Burn Room (BEK) & FEDGN & GASB 3 & 0.020709 & 0.04 & GASB 1 & 0.005459 & - & - & & 0.000 & & & & & \\
\hline 543 & & & & & & & & & & & & & & & & \\
\hline 544 & ISO Convected Heat $=0.3$ & & & & & & & & & & & & & & & \\
\hline 545 & & & Temperature & & & & & & & ASET & Final Value & & & & & \\
\hline$\frac{345}{546}$ & Master Bedroom (A) & FEDH & TCA 4 & & & & & & & & 0.241 & & & & & \\
\hline 547 & Burn Room Bedroom (B) & FEDH & TCB_4 & & & & & & & & 0.265 & & & & & \\
\hline 548 & Utility Hallway (C) & FEDH & TCC_ 4 & & & & & & & & 0.240 & & & & & \\
\hline 549 & Hallway Outside Burn Room Bedroom (D) & FEDH & TCD 4 & & & & & & & & 0.249 & & & & & \\
\hline 550 & Living Room (E) & FEDH & TCE 4 & & & & & & & & 0.261 & & & & & \\
\hline 551 & Front Door Hallway (F) & FEDH & TCF_4 4 & & & & & & & & 0.257 & & & & & \\
\hline 552 & Closed Bedroom $(\mathrm{G})$ & FEDH & TCG & & & & & & & & 0.180 & & & & & \\
\hline 553 & & & & & & & & & & & & & & & & \\
\hline 554 & Smoke Obscuration $=0.25 \mathrm{~m}-1$ & & & & & & & & & & & & & & & \\
\hline 555 & & & OD & & & & & & & ASET & Final Value & & & & & \\
\hline 556 & Master Bedroom (A) & Smoke & SMA 3 & & & & & & & 5321 & 0.252 & & & & & \\
\hline 557 & Burn Room Bedroom (B) & Smoke & SMB 3 & & & & & & & 5673 & 0.257 & & & & & \\
\hline 558 & Utility Hallway (C) & Smoke & SMC 3 & & & & & & & 5085 & 0.283 & & & & & \\
\hline 559 & Hallway Outside Burn Room Bedroom (D) & Smoke & SMD 3 & & & & & & & 5295 & 0.333 & & & & & \\
\hline 560 & Living Room (E) & Smoke & SME 3 & & & & & & & 5365 & 0.284 & & & & & \\
\hline 561 & Front Door Hallway (F) & Smoke & SMF 3 & & & & & & & 4963 & 0.259 & & & & & \\
\hline 562 & Closed Bedroom (G) & Smoke & SMG & & & & & & & 8249 & 0.255 & & & & & \\
\hline$\frac{502}{563}$ & & & & & & & & & & & & & & & & \\
\hline
\end{tabular}




\begin{tabular}{|c|c|c|c|c|c|c|c|c|c|c|c|c|c|c|c|c|}
\hline & $\mathrm{A}$ & $\mathrm{B}$ & $\mathrm{C}$ & $\mathrm{D}$ & $\mathrm{E}$ & $\mathrm{F}$ & $\mathrm{G}$ & $\mathrm{H}$ & $\mathrm{T}$ & $\mathrm{J}$ & $\mathrm{K}$ & $\mathrm{L}$ & $M$ & $\mathrm{~N}$ & 0 & $P$ \\
\hline$\frac{564}{565}$ & Test & SDC33 & Flaming Chair & in Living Roc & & & & & & & & & & & & \\
\hline & & & & & & & & & & & & & & & & \\
\hline 566 & Time & te & & & & & & & & & & & & & & \\
\hline 567 & & & & & & & & & & & & & & & & \\
\hline 568 & ISO Gas FED = 0.3 & & & & & & & & & & & & & & & \\
\hline 569 & & & $\mathrm{CO} 2$ & CO2 Offset & CO2 Base & $\mathrm{CO}$ & Co Offset & $\mathrm{HCN}$ & HCN Offset & ASET & Final Value & Minimum & & Outside Ro & om of Origin & \\
\hline 570 & Master Bedroom (A) & FEDG & GASA_3 & 0.05066 & 0.04 & GASA_1 & 0.002902 & - & - & & 0.002 & 182 & (Smoke) & 232 & (End of Test & \\
\hline 571 & Utility Hallway (C) & FEDG & GASC_3 & 0.035783 & 0.04 & GASC_1 & 0.000512 & - & - & & 0.001 & & & & & \\
\hline 572 & Front Door Hallway $(\mathrm{F})$ & FEDG & GASF_3 & 0.050041 & 0.04 & GASF_1 & 0.006011 & - & - & & 0.002 & & & & & \\
\hline 573 & Burn Room (BEK) & FEDG & GASB_3 & 0.025553 & 0.04 & GASB_1 & 0.002259 & - & - & & 0.005 & & & & & \\
\hline 574 & & & & & & & & & & & & & & & & \\
\hline 575 & NIST Gas FED $=0.3$ & & & & & & & & & & & & & & & \\
\hline 576 & & & $\mathrm{CO} 2$ & CO2 Offset & CO2 Base & $\mathrm{CO}$ & CO Offset & $\mathrm{HCN}$ & HCN Offset & ASET & Final Value & & & & & \\
\hline 577 & Master Bedroom (A) & FEDGN & GASA_3 & 0.05066 & 0.04 & GASA_1 & 0.002902 & 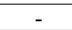 & 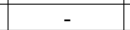 & & 0.000 & & & & & \\
\hline 578 & Utility Hallway (C) & FEDGN & GASC_3 & 0.035783 & 0.04 & GASC_1 & $\begin{array}{l}0.000512 \\
\end{array}$ & - & - & & 0.000 & & & & & \\
\hline 579 & Front Door Hallway $(F)$ & FEDGN & GASF_3 & 0.050041 & 0.04 & GASF_1 & 0.006011 & - & - & & 0.000 & & & & & \\
\hline 580 & Burn Room (BEK) & FEDGN & GASB_3 & 0.025553 & 0.04 & GASB_1 & 0.002259 & 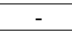 & - & & 0.000 & & & & & \\
\hline 581 & & & & & & & & & & & & & & & & \\
\hline 582 & ISO Convected Heat $=0.3$ & & & & & & & & & & & & & & & \\
\hline 583 & & & Temperature & & & & & & & ASET & Final Value & & & & & \\
\hline 584 & Master Bedroom (A) & FEDH & TCA_4 & & & & & & & & 0.007 & & & & & \\
\hline 585 & Burn Room Bedroom (B) & FEDH & TCB 4 & & & & & & & & 0.008 & & & & & \\
\hline 586 & Utility Hallway (C) & FEDH & TCC 4 & & & & & & & & 0.009 & & & & & \\
\hline 587 & Hallway Outside Burn Room Bedroom (D) & FEDH & TCD_4 & & & & & & & & 0.009 & & & & & \\
\hline 588 & Living Room (E) & FEDH & TCE_4 & & & & & & & & 0.012 & & & & & \\
\hline 589 & Front Door Hallway $(\mathrm{F})$ & FEDH & TCF_4 & & & & & & & & 0.013 & & & & & \\
\hline 590 & Closed Bedroom (G) & FEDH & TCG & & & & & & & & 0.005 & & & & & \\
\hline 591 & & & & & & & & & & & & & & & & \\
\hline 592 & Smoke Obscuration $=0.25 \mathrm{~m}-1$ & & & & & & & & & & & & & & & \\
\hline 593 & & & OD & & & & & & & ASET & Final Value & & & & & \\
\hline 594 & Master Bedroom (A) & Smoke & SMA 3 & & & & & & & & 0.080 & & & & & \\
\hline 595 & Burn Room Bedroom (B) & Smoke & SMB_3 3 & & & & & & & & 0.158 & & & & & \\
\hline 596 & Utility Hallway (C) & Smoke & SMC_3 & & & & & & & & 0.117 & & & & & \\
\hline 597 & Hallway Outside Burn Room Bedroom (D) & Smoke & SMD_3 & & & & & & & & 0.034 & & & & & \\
\hline 598 & Living Room (E) & Smoke & SME_3 & & & & & & & & 0.150 & & & & & \\
\hline 599 & Front Door Hallway $(\mathrm{F})$ & Smoke & SMF_3 3 & & & & & & & 182 & 0.254 & & & & & \\
\hline 600 & Closed Bedroom (G) & Smoke & $S M \bar{G}$ & & & & & & & & 0.007 & & & & & \\
\hline 601 & & & & & & & & & & & & & & & & \\
\hline
\end{tabular}




\begin{tabular}{|c|c|c|c|c|c|c|c|c|c|c|c|c|c|c|c|c|}
\hline & $\mathrm{A}$ & $\mathrm{B}$ & $\mathrm{C}$ & $\mathrm{D}$ & $\mathrm{E}$ & $\mathrm{F}$ & $\mathrm{G}$ & $\mathrm{H}$ & $\mathrm{T}$ & $\mathrm{J}$ & $\mathrm{K}$ & $\mathrm{L}$ & $M$ & $\mathrm{~N}$ & 0 & $\mathrm{P}$ \\
\hline 602 & Test & SDC34 & Smoldering $\mathrm{Ch}$ & air in Living & Room & & & & & & & & & & & \\
\hline 603 & & & & & & & & & & & & & & & & \\
\hline 604 & Time & & & & & & & & & & & & & & & \\
\hline 605 & & & & & & & & & & & & & & & & \\
\hline 606 & ISO Gas FED = 0.3 & & & & & & & & & & & & & & & \\
\hline 607 & & & $\mathrm{CO} 2$ & CO2 Offset & CO2 Base & $\mathrm{CO}$ & Co Offset & $\mathrm{HCN}$ & HCN Offset & ASET & Final Value & Minimum & & Outside Ro & om of Origin & \\
\hline 608 & Master Bedroom (A) & FEDG & GASA_3 & \begin{tabular}{|l|}
0.049596 \\
\end{tabular} & 0.04 & GASA_1 & 0 & - & - & & 0.013 & 3882 & (ISO Gas) & 3922 & (End of Test & \\
\hline 609 & Utility Hallway (C) & FEDG & GASC_3 & 0.030053 & 0.04 & GASC_1 & 0.000136 & - & - & & 0.038 & & & & & \\
\hline 610 & Front Door Hallway $(F)$ & FEDG & GASF_3 & 0.05043 & 0.04 & GASF_1 & 0.001379 & - & - & & 0.094 & & & & & \\
\hline 611 & Burn Room (BEK) & FEDG & GASB_3 & 0.023504 & 0.04 & GASB_1 & 0.000352 & - & - & & 0.258 & & & & & \\
\hline 612 & & & & & & & & & & & & & & & & \\
\hline 613 & NIST Gas FED $=0.3$ & & & & & & & & & & & & & & & \\
\hline 614 & & & $\mathrm{CO} 2$ & CO2 Offset & CO2 Base & $\mathrm{CO}$ & CO Offset & $\mathrm{HCN}$ & HCN Offset & ASET & Final Value & & & & & \\
\hline 615 & Master Bedroom (A) & FEDGN & GASA_3 & \begin{tabular}{|l|}
0.049596 \\
\end{tabular} & 0.04 & GASA_1 & 0 & . & - & & 0.000 & & & & & \\
\hline 616 & Utility Hallway (C) & FEDGN & GASC_3 & 0.030053 & 0.04 & GASC_1 & 0.000136 & - & - & & 0.000 & & & & & \\
\hline 617 & Front Door Hallway $(\mathrm{F})$ & FEDGN & GASF_3 & 0.05043 & 0.04 & GASF_1 & 0.001379 & - & - & & 0.000 & & & & & \\
\hline 618 & Burn Room (BEK) & FEDGN & GASB_3 & 0.023504 & 0.04 & GASB_1 & 0.000352 & 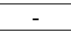 & - & & 0.000 & & & & & \\
\hline 619 & & & & & & & & & & & & & & & & \\
\hline 620 & ISO Convected Heat $=0.3$ & & & & & & & & & & & & & & & \\
\hline 621 & & & Temperature & & & & & & & ASET & Final Value & & & & & \\
\hline 622 & Master Bedroom (A) & FEDH & TCA_4 & & & & & & & & 0.103 & & & & & \\
\hline 623 & Burn Room Bedroom (B) & FEDH & TCB 4 & & & & & & & & 0.110 & & & & & \\
\hline 624 & Utility Hallway (C) & FEDH & $\mathrm{TCC}_{4} 4$ & & & & & & & & 0.096 & & & & & \\
\hline 625 & Hallway Outside Burn Room Bedroom (D) & FEDH & TCD_4 & & & & & & & & 0.097 & & & & & \\
\hline 626 & Living Room (E) & FEDH & TCE_4 & & & & & & & & 0.101 & & & & & \\
\hline 627 & Front Door Hallway $(\mathrm{F})$ & FEDH & TCF_4 & & & & & & & & 0.100 & & & & & \\
\hline 628 & Closed Bedroom (G) & FEDH & TCG & & & & & & & & 0.077 & & & & & \\
\hline 629 & & & & & & & & & & & & & & & & \\
\hline 630 & Smoke Obscuration $=0.25 \mathrm{~m}-1$ & & & & & & & & & & & & & & & \\
\hline 631 & & & OD & & & & & & & ASET & Final Value & & & & & \\
\hline 632 & Master Bedroom (A) & Smoke & SMA 3 & & & & & & & & 0.041 & & & & & \\
\hline 633 & Burn Room Bedroom (B) & Smoke & SMB_3 3 & & & & & & & & 0.013 & & & & & \\
\hline 634 & Utility Hallway (C) & Smoke & SMC_3 & & & & & & & 3906 & 0.257 & & & & & \\
\hline 635 & Hallway Outside Burn Room Bedroom (D) & Smoke & SMD_3 & & & & & & & & 0.185 & & & & & \\
\hline 636 & Living Room (E) & Smoke & SME_3 & & & & & & & & 0.011 & & & & & \\
\hline 637 & Front Door Hallway $(\mathrm{F})$ & Smoke & SMF_3 3 & & & & & & & 3882 & 0.377 & & & & & \\
\hline 638 & Closed Bedroom (G) & Smoke & $S M \bar{G}$ & & & & & & & & 0.012 & & & & & \\
\hline 639 & & & & & & & & & & & & & & & & \\
\hline
\end{tabular}




\begin{tabular}{|c|c|c|c|c|c|c|c|c|c|c|c|c|c|c|c|c|}
\hline & $\mathrm{A}$ & $\mathrm{B}$ & $\mathrm{C}$ & $\mathrm{D}$ & $\mathrm{E}$ & $\mathrm{F}$ & $\mathrm{G}$ & $\mathrm{H}$ & $\mathrm{T}$ & $\mathrm{J}$ & $\mathrm{K}$ & $\mathrm{L}$ & $M$ & $\mathrm{~N}$ & 0 & $\mathrm{P}$ \\
\hline$\frac{640}{641}$ & Test & SDC35 & Flaming Chair & in Living Roc & & & & & & & & & & & & \\
\hline$\frac{641}{642}$ & Time & 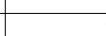 & & & & & & & & & & & & & & \\
\hline 643 & & & & & & & & & & & & & & & & \\
\hline 644 & ISO Gas FED = 0.3 & & & & & & & & & & & & & & & \\
\hline 645 & & & $\mathrm{CO} 2$ & CO2 Offset & CO2 Base & $\mathrm{CO}$ & Co Offset & $\mathrm{HCN}$ & HCN Offset & ASET & Final Value & Minimum & & Outside Roc & om of Origin & \\
\hline 646 & Master Bedroom (A) & FEDG & GASA_3 & \begin{tabular}{|l|}
0.047879 \\
\end{tabular} & 0.04 & GASA_1 & 0.000461 & - & - & & 0.000 & 182 & (Smoke) & 244 & (End of Test & \\
\hline 647 & Utility Hallway (C) & FEDG & GASC_3 & 0.033633 & 0.04 & GASC_1 & 0.00157 & - & - & & 0.001 & & & & & \\
\hline 648 & Front Door Hallway $(F)$ & FEDG & GASF_3 & 0.046268 & 0.04 & GASF_1 & 0 & - & - & & 0.001 & & & & & \\
\hline 649 & Burn Room (BEK) & FEDG & GASB_3 & 0.02238 & 0.04 & GASB_1 & 0.00452 & - & - & & 0.003 & & & & & \\
\hline 650 & & & & & & & & & & & & & & & & \\
\hline 651 & NIST Gas FED $=0.3$ & & & & & & & & & & & & & & & \\
\hline 652 & & & $\mathrm{CO} 2$ & CO2 Offset & CO2 Base & $\mathrm{CO}$ & CO Offset & $\mathrm{HCN}$ & HCN Offset & ASET & Final Value & & & & & \\
\hline 653 & Master Bedroom (A) & FEDGN & GASA_3 & 0.047879 & 0.04 & GASA_1 & 0.000461 & 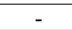 & 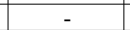 & & 0.000 & & & & & \\
\hline 654 & Utility Hallway (C) & FEDGN & GASC_3 & 0.033633 & 0.04 & GASC_1 & 0.00157 & - & - & & 0.000 & & & & & \\
\hline 655 & Front Door Hallway $(\mathrm{F})$ & FEDGN & GASF_3 & 0.046268 & 0.04 & GASF_1 & 0 & - & - & & 0.000 & & & & & \\
\hline 656 & Burn Room (BEK) & FEDGN & GASB_3 & 0.02238 & 0.04 & GASB_1 & 0.00452 & - & - & & 0.000 & & & & & \\
\hline 657 & & & & & & & & & & & & & & & & \\
\hline 658 & ISO Convected Heat $=0.3$ & & & & & & & & & & & & & & & \\
\hline 659 & & & Temperature & & & & & & & ASET & Final Value & & & & & \\
\hline 660 & Master Bedroom (A) & FEDH & TCA_4 & & & & & & & & 0.007 & & & & & \\
\hline 661 & Burn Room Bedroom (B) & FEDH & TCB 4 & & & & & & & & 0.008 & & & & & \\
\hline 662 & Utility Hallway (C) & FEDH & $\mathrm{TCC}_{4} 4$ & & & & & & & & 0.008 & & & & & \\
\hline 663 & Hallway Outside Burn Room Bedroom (D) & FEDH & TCD_4 & & & & & & & & 0.009 & & & & & \\
\hline 664 & Living Room (E) & FEDH & TCE_4 & & & & & & & & 0.012 & & & & & \\
\hline 665 & Front Door Hallway $(\mathrm{F})$ & FEDH & TCF_4 & & & & & & & & 0.011 & & & & & \\
\hline 666 & Closed Bedroom (G) & FEDH & TCG & & & & & & & & 0.005 & & & & & \\
\hline 667 & & & & & & & & & & & & & & & & \\
\hline 668 & Smoke Obscuration $=0.25 \mathrm{~m}-1$ & & & & & & & & & & & & & & & \\
\hline 669 & & & OD & & & & & & & ASET & Final Value & & & & & \\
\hline 670 & Master Bedroom (A) & Smoke & SMA 3 & & & & & & & & 0.060 & & & & & \\
\hline 671 & Burn Room Bedroom (B) & Smoke & SMB_3 3 & & & & & & & & 0.101 & & & & & \\
\hline 672 & Utility Hallway (C) & Smoke & SMC_3 & & & & & & & & 0.121 & & & & & \\
\hline 673 & Hallway Outside Burn Room Bedroom (D) & Smoke & SMD_3 & & & & & & & & 0.143 & & & & & \\
\hline 674 & Living Room (E) & Smoke & SME_3 & & & & & & & & 0.232 & & & & & \\
\hline 675 & Front Door Hallway $(\mathrm{F})$ & Smoke & SMF_3 3 & & & & & & & 182 & 0.251 & & & & & \\
\hline 676 & Closed Bedroom (G) & Smoke & $S M \bar{G}$ & & & & & & & & 0.014 & & & & & \\
\hline 677 & & & & & & & & & & & & & & & & \\
\hline
\end{tabular}




\begin{tabular}{|c|c|c|c|c|c|c|c|c|c|c|c|c|c|c|c|c|}
\hline & $\mathrm{A}$ & $\mathrm{B}$ & $\mathrm{C}$ & $\mathrm{D}$ & $\mathrm{E}$ & $F$ & $\mathrm{G}$ & $\mathrm{H}$ & $\mathrm{T}$ & $\mathrm{J}$ & $\mathrm{K}$ & $\mathrm{L}$ & $M$ & $\mathrm{~N}$ & 0 & $P$ \\
\hline 678 & Test & SDC36 & Flaming Mattre & ss in Bedroc & om (Burn Ro & om Door Clc & osed) & & & & & & & & & \\
\hline 679 & & & & & & & & & & & & & & & & \\
\hline 680 & Time & te & & & & & & & & & & & & & & \\
\hline 681 & & & & & & & & & & & & & & & & \\
\hline 682 & ISO Gas FED = 0.3 & & & & & & & & & & & & & & & \\
\hline 683 & & & $\mathrm{CO} 2$ & CO2 Offset & CO2 Base & $\mathrm{CO}$ & CO Offset & $\mathrm{HCN}$ & HCN Offset & ASET & Final Value & Minimum & & Outside Ro & om of Origin & \\
\hline 684 & Master Bedroom (A) & FEDG & GASA_3 & 0.043555 & 0.04 & GASA_1 & 0.00039 & 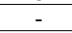 & - & & 0.016 & 128 & (Smoke) & 1922 & (Smoke) & \\
\hline 685 & Utility Hallway (C) & FEDG & GASC_3 & 0.02837 & 0.04 & GASC_1 & 0.000358 & - & - & & 0.031 & & & & & \\
\hline 686 & Front Door Hallway (F) & FEDG & GASF_3 & 0.048868 & 0.04 & GASF_1 & 0.004965 & - & - & & 0.062 & & & & & \\
\hline 687 & Burn Room (BEK) & FEDG & GASB_3 & 0.024205 & 0.04 & GASB_1 & 0.002849 & - & - & 486 & 0.302 & & & & & \\
\hline 688 & & & & & & & & & & & & & & & & \\
\hline 689 & NIST Gas FED $=0.3$ & & & & & & & & & & & & & & & \\
\hline 690 & & & $\mathrm{CO} 2$ & CO2 Offset & CO2 Base & $\mathrm{CO}$ & CO Offset & $\mathrm{HCN}$ & HCN Offset & ASET & Final Value & & & & & \\
\hline 691 & Master Bedroom (A) & FEDGN & GASA_3 & 0.043555 & 0.04 & GASA 1 & 0.00039 & 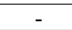 & 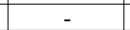 & & 0.000 & & & & & \\
\hline 692 & Utility Hallway (C) & FEDGN & GASC_3 & 0.02837 & 0.04 & GASC_1 & $\begin{array}{l}0.000358 \\
\end{array}$ & - & - & & 0.000 & & & & & \\
\hline 693 & Front Door Hallway $(\mathrm{F})$ & FEDGN & GASF_3 & 0.048868 & 0.04 & GASF_1 & 0.004965 & - & - & & 0.000 & & & & & \\
\hline 694 & Burn Room (BEK) & FEDGN & GASB_3 & 0.024205 & 0.04 & GASB_1 & 0.002849 & 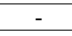 & - & & 0.125 & & & & & \\
\hline 695 & & & & & & & & & & & & & & & & \\
\hline 696 & ISO Convected Heat $=0.3$ & & & & & & & & & & & & & & & \\
\hline 697 & & & Temperature & & & & & & & ASET & Final Value & & & & & \\
\hline 698 & Master Bedroom (A) & FEDH & TCA_4 & & & & & & & & 0.048 & & & & & \\
\hline 699 & Burn Room Bedroom (B) & FEDH & TCB 4 & & & & & & & 158 & 0.310 & & & & & \\
\hline 700 & Utility Hallway (C) & FEDH & $\mathrm{TCC}_{4} 4$ & & & & & & & & 0.044 & & & & & \\
\hline 701 & Hallway Outside Burn Room Bedroom (D) & FEDH & TCD_4 & & & & & & & & 0.048 & & & & & \\
\hline 702 & Living Room (E) & FEDH & TCE_4 & & & & & & & & 0.046 & & & & & \\
\hline 703 & Front Door Hallway $(\mathrm{F})$ & FEDH & TCF_4 & & & & & & & & 0.046 & & & & & \\
\hline 704 & Closed Bedroom (G) & FEDH & TCG & & & & & & & & 0.041 & & & & & \\
\hline 705 & & & & & & & & & & & & & & & & \\
\hline 706 & Smoke Obscuration $=0.25 \mathrm{~m}-1$ & & & & & & & & & & & & & & & \\
\hline 707 & & & $O D$ & & & & & & & ASET & Final Value & & & & & \\
\hline 708 & Master Bedroom (A) & Smoke & SMA 3 & & & & & & & & 0.053 & & & & & \\
\hline 709 & Burn Room Bedroom (B) & Smoke & SMB_3 & & & & & & & 128 & 0.324 & & & & & \\
\hline 710 & Utility Hallway (C) & Smoke & SMC_3 3 & & & & & & & & 0.093 & & & & & \\
\hline 711 & Hallway Outside Burn Room Bedroom (D) & Smoke & SMD_3 & & & & & & & 1922 & 0.251 & & & & & \\
\hline 712 & Living Room (E) & Smoke & SME_3 & & & & & & & & 0.063 & & & & & \\
\hline 713 & Front Door Hallway $(\mathrm{F})$ & Smoke & SMF_3 3 & & & & & & & & 0.177 & & & & & \\
\hline 714 & Closed Bedroom (G) & Smoke & SMG & & & & & & & & 0.102 & & & & & \\
\hline 715 & & & & & & & & & & & & & & & & \\
\hline
\end{tabular}




\begin{tabular}{|c|c|c|c|c|c|c|c|c|c|c|c|c|c|c|c|c|}
\hline & $\mathrm{A}$ & $\mathrm{B}$ & $\mathrm{C}$ & $\mathrm{D}$ & $\mathrm{E}$ & $\mathrm{F}$ & $\mathrm{G}$ & $\mathrm{H}$ & $\mathrm{T}$ & $\mathrm{J}$ & $\mathrm{K}$ & $\mathrm{L}$ & $M$ & $\mathrm{~N}$ & 0 & $\mathrm{P}$ \\
\hline 716 & Test & SDC37 & Smoldering Ma & attress in Bec & droom & & & & & & & & & & & \\
\hline 717 & & & & & & & & & & & & & & & & \\
\hline 718 & Time & & & & & & & & & & & & & & & \\
\hline 719 & & & & & & & & & & & & & & & & \\
\hline 720 & ISO Gas FED = 0.3 & & & & & & & & & & & & & & & \\
\hline 721 & & & $\mathrm{CO} 2$ & CO2 Offset & CO2 Base & $\mathrm{CO}$ & Co Offset & $\mathrm{HCN}$ & HCN Offset & ASET & Final Value & Minimum & & Outside Roc & om of Origin & \\
\hline 722 & Master Bedroom (A) & FEDG & GASA_3 & \begin{tabular}{|l|}
0.048702 \\
\end{tabular} & 0.04 & GASA_1 & 0 & - & - & & 0.000 & 1644 & (Smoke) & 1982 & (End of Test & \\
\hline 723 & Utility Hallway (C) & FEDG & GASC_3 & 0.031533 & 0.04 & GASC_1 & 0.001689 & - & - & & 0.005 & & & & & \\
\hline 724 & Front Door Hallway $(\mathrm{F})$ & FEDG & GASF_3 & 0.052475 & 0.04 & GASF_1 & $6.78 \mathrm{E}-05$ & - & - & & 0.001 & & & & & \\
\hline 725 & Burn Room (BEK) & FEDG & GASB_3 & 0.027423 & 0.04 & GASB_1 & 0.004191 & - & - & & 0.023 & & & & & \\
\hline 726 & & & & & & & & & & & & & & & & \\
\hline 727 & NIST Gas FED $=0.3$ & & & & & & & & & & & & & & & \\
\hline 728 & & & $\mathrm{CO} 2$ & CO2 Offset & CO2 Base & $\mathrm{CO}$ & CO Offset & $\mathrm{HCN}$ & HCN Offset & ASET & Final Value & & & & & \\
\hline 729 & Master Bedroom (A) & FEDGN & GASA_3 & \begin{tabular}{|r|}
0.048702 \\
\end{tabular} & 0.04 & GASA_1 & 0 & . & - & & 0.000 & & & & & \\
\hline 730 & Utility Hallway (C) & FEDGN & GASC_3 & 0.031533 & 0.04 & GASC_1 & $\begin{array}{l}0.001689 \\
\end{array}$ & - & - & & 0.000 & & & & & \\
\hline 731 & Front Door Hallway $(F)$ & FEDGN & GASF_3 & 0.052475 & 0.04 & GASF_1 & $6.78 \mathrm{E}-05$ & - & - & & 0.000 & & & & & \\
\hline 732 & Burn Room (BEK) & FEDGN & GASB_3 & 0.027423 & 0.04 & GASB_1 & 0.004191 & 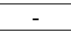 & - & & 0.000 & & & & & \\
\hline 733 & & & & & & & & & & & & & & & & \\
\hline 734 & ISO Convected Heat $=0.3$ & & & & & & & & & & & & & & & \\
\hline 735 & & & Temperature & & & & & & & ASET & Final Value & & & & & \\
\hline 736 & Master Bedroom (A) & FEDH & TCA_4 & & & & & & & & 0.050 & & & & & \\
\hline 737 & Burn Room Bedroom (B) & FEDH & TCB 4 & & & & & & & & 0.059 & & & & & \\
\hline 738 & Utility Hallway (C) & FEDH & $\mathrm{TCC}_{4} 4$ & & & & & & & & 0.047 & & & & & \\
\hline 739 & Hallway Outside Burn Room Bedroom (D) & FEDH & TCD_4 & & & & & & & & 0.048 & & & & & \\
\hline 740 & Living Room (E) & FEDH & TCE_4 & & & & & & & & 0.049 & & & & & \\
\hline 741 & Front Door Hallway $(\mathrm{F})$ & FEDH & TCF_4 & & & & & & & & 0.048 & & & & & \\
\hline 742 & Closed Bedroom (G) & FEDH & TCG & & & & & & & & 0.039 & & & & & \\
\hline 743 & & & & & & & & & & & & & & & & \\
\hline 744 & Smoke Obscuration $=0.25 \mathrm{~m}-1$ & & & & & & & & & & & & & & & \\
\hline 745 & & & OD & & & & & & & ASET & Final Value & & & & & \\
\hline 746 & Master Bedroom (A) & Smoke & SMA 3 & & & & & & & & 0.028 & & & & & \\
\hline 747 & Burn Room Bedroom (B) & Smoke & SMB_3 & & & & & & & 1644 & 0.258 & & & & & \\
\hline 748 & Utility Hallway (C) & Smoke & SMC_3 & & & & & & & & 0.153 & & & & & \\
\hline 749 & Hallway Outside Burn Room Bedroom (D) & Smoke & SMD_3 & & & & & & & & 0.243 & & & & & \\
\hline 750 & Living Room (E) & Smoke & SME_3 & & & & & & & & 0.062 & & & & & \\
\hline 751 & Front Door Hallway (F) & Smoke & SMF_3 & & & & & & & & 0.134 & & & & & \\
\hline 752 & Closed Bedroom (G) & Smoke & $S M \bar{G}$ & & & & & & & & 0.015 & & & & & \\
\hline 753 & & & & & & & & & & & & & & & & \\
\hline
\end{tabular}




\begin{tabular}{|c|c|c|c|c|c|c|c|c|c|c|c|c|c|c|c|c|}
\hline & $\mathrm{A}$ & $\mathrm{B}$ & $\mathrm{C}$ & $\mathrm{D}$ & $\mathrm{E}$ & $\mathrm{F}$ & $\mathrm{G}$ & $\mathrm{H}$ & $\mathrm{T}$ & $\mathrm{J}$ & $\mathrm{K}$ & $\mathrm{L}$ & $M$ & $\mathrm{~N}$ & 0 & $\mathrm{P}$ \\
\hline 754 & Test & SDC38 & Flaming Mattre & ss in Bedroc & & & & & & & & & & & & \\
\hline 755 & & & & & & & & & & & & & & & & \\
\hline 756 & Time & te & & & & & & & & & & & & & & \\
\hline 757 & & & & & & & & & & & & & & & & \\
\hline 758 & ISO Gas FED = 0.3 & & & & & & & & & & & & & & & \\
\hline 759 & & & $\mathrm{CO} 2$ & CO2 Offset & CO2 Base & $\mathrm{CO}$ & CO Offset & $\mathrm{HCN}$ & HCN Offset & ASET & Final Value & Minimum & & Outside Ro & om of Origin & \\
\hline 760 & Master Bedroom (A) & FEDG & GASA_3 & \begin{tabular}{|l|}
0.047578 \\
\end{tabular} & 0.04 & GASA_1 & 0.000177 & . & - & & 0.000 & 143 & (Smoke) & 159 & (Smoke) & \\
\hline 761 & Utility Hallway (C) & FEDG & GASC_3 & 0.031745 & 0.04 & GASC_1 & 0.000136 & - & - & & 0.002 & & & & & \\
\hline 762 & Front Door Hallway $(\mathrm{F})$ & FEDG & GASF_3 & 0.049872 & 0.04 & GASF_1 & 0.003 & - & - & & 0.009 & & & & & \\
\hline 763 & Burn Room (BEK) & FEDG & GASB_3 & 0.022815 & 0.04 & GASB_1 & 0.00448 & - & - & & 0.045 & & & & & \\
\hline 764 & & & & & & & & & & & & & & & & \\
\hline 765 & NIST Gas FED $=0.3$ & & & & & & & & & & & & & & & \\
\hline 766 & & & $\mathrm{CO} 2$ & CO2 Offset & CO2 Base & $\mathrm{CO}$ & CO Offset & $\mathrm{HCN}$ & HCN Offset & ASET & Final Value & & & & & \\
\hline 767 & Master Bedroom (A) & FEDGN & GASA_3 & 0.047578 & 0.04 & GASA_1 & 0.000177 & 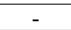 & 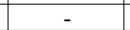 & & 0.000 & & & & & \\
\hline 768 & Utility Hallway (C) & FEDGN & GASC_3 & 0.031745 & 0.04 & GASC_1 & 0.000136 & - & - & & 0.000 & & & & & \\
\hline 769 & Front Door Hallway $(\mathrm{F})$ & FEDGN & GASF_3 & 0.049872 & 0.04 & GASF_1 & $\begin{array}{r}0.003 \\
\end{array}$ & - & - & & 0.000 & & & & & \\
\hline 770 & Burn Room (BEK) & FEDGN & GASB_3 & 0.022815 & 0.04 & GASB_1 & 0.00448 & 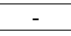 & - & & 0.000 & & & & & \\
\hline 771 & & & & & & & & & & & & & & & & \\
\hline 772 & ISO Convected Heat $=0.3$ & & & & & & & & & & & & & & & \\
\hline 773 & & & Temperature & & & & & & & ASET & Final Value & & & & & \\
\hline 774 & Master Bedroom (A) & FEDH & TCA_4 & & & & & & & & 0.021 & & & & & \\
\hline 775 & Burn Room Bedroom (B) & FEDH & TCB 4 & & & & & & & & 0.087 & & & & & \\
\hline 776 & Utility Hallway (C) & FEDH & TCC 4 & & & & & & & & 0.014 & & & & & \\
\hline 777 & Hallway Outside Burn Room Bedroom (D) & FEDH & TCD_4 & & & & & & & & 0.022 & & & & & \\
\hline 778 & Living Room (E) & FEDH & TCE_4 & & & & & & & & 0.016 & & & & & \\
\hline 779 & Front Door Hallway $(\mathrm{F})$ & FEDH & TCF_4 & & & & & & & & 0.017 & & & & & \\
\hline 780 & Closed Bedroom (G) & FEDH & TCG & & & & & & & & 0.021 & & & & & \\
\hline 781 & & & & & & & & & & & & & & & & \\
\hline 782 & Smoke Obscuration $=0.25 \mathrm{~m}-1$ & & & & & & & & & & & & & & & \\
\hline 783 & & & OD & & & & & & & ASET & Final Value & & & & & \\
\hline 784 & Master Bedroom (A) & Smoke & SMA 3 & & & & & & & & 0.040 & & & & & \\
\hline 785 & Burn Room Bedroom (B) & Smoke & SMB_3 & & & & & & & 143 & 0.262 & & & & & \\
\hline 786 & Utility Hallway (C) & Smoke & SMC_3 3 & & & & & & & & 0.069 & & & & & \\
\hline 787 & Hallway Outside Burn Room Bedroom (D) & Smoke & SMD_3 & & & & & & & 159 & 0.271 & & & & & \\
\hline 788 & Living Room (E) & Smoke & SME_3 & & & & & & & & 0.062 & & & & & \\
\hline 789 & Front Door Hallway $(\mathrm{F})$ & Smoke & SMF_3 3 & & & & & & & 177 & 0.467 & & & & & \\
\hline 790 & Closed Bedroom (G) & Smoke & SMG & & & & & & & & 0.012 & & & & & \\
\hline 791 & & & & & & & & & & & & & & & & \\
\hline
\end{tabular}




\begin{tabular}{|c|c|c|c|c|c|c|c|c|c|c|c|c|c|c|c|c|}
\hline & $\mathrm{A}$ & $\mathrm{B}$ & $\mathrm{C}$ & $\mathrm{D}$ & $\mathrm{E}$ & $\mathrm{F}$ & $\mathrm{G}$ & $\mathrm{H}$ & $\mathrm{T}$ & $\mathrm{J}$ & $\mathrm{K}$ & $\mathrm{L}$ & $M$ & $\mathrm{~N}$ & 0 & $\mathrm{P}$ \\
\hline 792 & Test & SDC39 & Flaming Mattre & ss in Bedroc & & & & & & & & & & & & \\
\hline 793 & & & & & & & & & & & & & & & & \\
\hline 794 & Time & te & & & & & & & & & & & & & & \\
\hline 795 & & & & & & & & & & & & & & & & \\
\hline 796 & ISO Gas FED = 0.3 & & & & & & & & & & & & & & & \\
\hline 797 & & & $\mathrm{CO} 2$ & CO2 Offset & CO2 Base & $\mathrm{CO}$ & Co Offset & $\mathrm{HCN}$ & HCN Offset & ASET & Final Value & Minimum & & Outside Ro & om of Origin & \\
\hline 798 & Master Bedroom (A) & FEDG & GASA_3 & \begin{tabular}{|l|}
0.051722 \\
\end{tabular} & 0.04 & GASA_1 & & - & - & & 0.000 & 120 & (Smoke) & 132 & (End of Test & \\
\hline 799 & Utility Hallway (C) & FEDG & GASC_3 & 0.038548 & 0.04 & GASC_1 & 0.001465 & - & - & & 0.001 & & & & & \\
\hline 800 & Front Door Hallway $(\mathrm{F})$ & FEDG & GASF_3 & 0.056685 & 0.04 & GASF_1 & 0.000271 & - & - & & 0.000 & & & & & \\
\hline 801 & Burn Room (BEK) & FEDG & GASB_3 & 0.026912 & 0.04 & GASB_1 & 0.00198 & - & - & & 0.001 & & & & & \\
\hline 802 & & & & & & & & & & & & & & & & \\
\hline 803 & NIST Gas FED $=0.3$ & & & & & & & & & & & & & & & \\
\hline 804 & & & $\mathrm{CO} 2$ & CO2 Offset & CO2 Base & $\mathrm{CO}$ & CO Offset & $\mathrm{HCN}$ & HCN Offset & ASET & Final Value & & & & & \\
\hline 805 & Master Bedroom (A) & FEDGN & GASA_3 & 0.051722 & 0.04 & GASA_1 & & 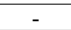 & 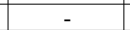 & & 0.000 & & & & & \\
\hline 806 & Utility Hallway (C) & FEDGN & GASC_3 & 0.038548 & 0.04 & GASC_1 & 0.001465 & - & - & & 0.000 & & & & & \\
\hline 807 & Front Door Hallway $(\mathrm{F})$ & FEDGN & GASF_3 & 0.056685 & 0.04 & GASF_1 & 0.000271 & - & - & & 0.000 & & & & & \\
\hline 808 & Burn Room (BEK) & FEDGN & GASB_3 & 0.026912 & 0.04 & GASB_1 & 0.00198 & 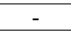 & - & & 0.000 & & & & & \\
\hline 809 & & & & & & & & & & & & & & & & \\
\hline 810 & ISO Convected Heat $=0.3$ & & & & & & & & & & & & & & & \\
\hline 811 & & & Temperature & & & & & & & ASET & Final Value & & & & & \\
\hline 812 & Master Bedroom (A) & FEDH & TCA_4 & & & & & & & & 0.003 & & & & & \\
\hline 813 & Burn Room Bedroom (B) & FEDH & TCB 4 & & & & & & & & 0.027 & & & & & \\
\hline 814 & Utility Hallway (C) & FEDH & TCC 4 & & & & & & & & 0.002 & & & & & \\
\hline 815 & Hallway Outside Burn Room Bedroom (D) & FEDH & TCD_4 & & & & & & & & 0.003 & & & & & \\
\hline 816 & Living Room (E) & FEDH & TCE_4 & & & & & & & & 0.002 & & & & & \\
\hline 817 & Front Door Hallway $(\mathrm{F})$ & FEDH & TCF_4 & & & & & & & & 0.002 & & & & & \\
\hline 818 & Closed Bedroom (G) & FEDH & TCG & & & & & & & & 0.002 & & & & & \\
\hline 819 & & & & & & & & & & & & & & & & \\
\hline 820 & Smoke Obscuration $=0.25 \mathrm{~m}-1$ & & & & & & & & & & & & & & & \\
\hline 821 & & & $O D$ & & & & & & & ASET & Final Value & & & & & \\
\hline 822 & Master Bedroom (A) & Smoke & SMA 3 & & & & & & & & 0.017 & & & & & \\
\hline 823 & Burn Room Bedroom (B) & Smoke & SMB 3 & & & & & & & 120 & 0.266 & & & & & \\
\hline 824 & Utility Hallway (C) & Smoke & SMC_3 3 & & & & & & & & 0.012 & & & & & \\
\hline 825 & Hallway Outside Burn Room Bedroom (D) & Smoke & SMD_3 & & & & & & & & 0.098 & & & & & \\
\hline 826 & Living Room (E) & Smoke & SME_3 & & & & & & & & 0.011 & & & & & \\
\hline 827 & Front Door Hallway $(\mathrm{F})$ & Smoke & SMF_3 3 & & & & & & & & 0.041 & & & & & \\
\hline 828 & Closed Bedroom (G) & Smoke & SMG & & & & & & & & 0.005 & & & & & \\
\hline 829 & & & & & & & & & & & & & & & & \\
\hline
\end{tabular}




\begin{tabular}{|c|c|c|c|c|c|c|c|c|c|c|c|c|c|c|c|c|}
\hline & $\mathrm{A}$ & $\mathrm{B}$ & $\mathrm{C}$ & $\mathrm{D}$ & $\mathrm{E}$ & $\mathrm{F}$ & $\mathrm{G}$ & $\mathrm{H}$ & $\mathrm{T}$ & $\mathrm{J}$ & $\mathrm{K}$ & $\mathrm{L}$ & $M$ & $\mathrm{~N}$ & 0 & $\mathrm{P}$ \\
\hline 830 & Test & SDC40 & Smoldering Ma & attress in Bec & droom & & & & & & & & & & & \\
\hline 831 & & & & & & & & & & & & & & & & \\
\hline 832 & Time & te & & & & & & & & & & & & & & \\
\hline 833 & & & & & & & & & & & & & & & & \\
\hline 834 & ISO Gas FED = 0.3 & & & & & & & & & & & & & & & \\
\hline 835 & & & $\mathrm{CO} 2$ & CO2 Offset & CO2 Base & $\mathrm{CO}$ & Co Offset & $\mathrm{HCN}$ & HCN Offset & ASET & Final Value & Minimum & & Outside Ro & om of Origin & \\
\hline 836 & Master Bedroom (A) & FEDG & GASA_3 & 0.050621 & 0.04 & GASA_1 & & - & - & & 0.000 & 3372 & (End of Tes & 3372 & (End of Test & \\
\hline 837 & Utility Hallway (C) & FEDG & GASC_3 & 0.033271 & 0.04 & GASC_1 & 0.000426 & - & - & & 0.002 & & & & & \\
\hline 838 & Front Door Hallway $(F)$ & FEDG & GASF_3 & 0.057496 & 0.04 & GASF_1 & $8.13 \mathrm{E}-05$ & - & - & & 0.012 & & & & & \\
\hline 839 & Burn Room (BEK) & FEDG & GASB_3 & 0.02539 & 0.04 & GASB_1 & $\begin{array}{l}0.002366 \\
\end{array}$ & - & - & & 0.017 & & & & & \\
\hline 840 & & & & & & & & & & & & & & & & \\
\hline 841 & NIST Gas FED $=0.3$ & & & & & & & & & & & & & & & \\
\hline 842 & & & $\mathrm{CO} 2$ & CO2 Offset & CO2 Base & $\mathrm{CO}$ & CO Offset & $\mathrm{HCN}$ & HCN Offset & ASET & Final Value & & & & & \\
\hline 843 & Master Bedroom (A) & FEDGN & GASA_3 & 0.050621 & 0.04 & GASA_1 & & 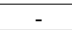 & 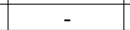 & & 0.000 & & & & & \\
\hline 844 & Utility Hallway (C) & FEDGN & GASC_3 & 0.033271 & 0.04 & GASC_1 & 0.000426 & - & - & & 0.000 & & & & & \\
\hline 845 & Front Door Hallway $(\mathrm{F})$ & FEDGN & GASF_3 & 0.057496 & 0.04 & GASF_1 & $8.13 \mathrm{E}-05$ & - & - & & 0.000 & & & & & \\
\hline 846 & Burn Room (BEK) & FEDGN & GASB_3 & 0.02539 & 0.04 & GASB_1 & 0.002366 & - & - & & 0.000 & & & & & \\
\hline 847 & & & & & & & & & & & & & & & & \\
\hline 848 & ISO Convected Heat $=0.3$ & & & & & & & & & & & & & & & \\
\hline 849 & & & Temperature & & & & & & & ASET & Final Value & & & & & \\
\hline 850 & Master Bedroom (A) & FEDH & TCA_4 & & & & & & & & 0.075 & & & & & \\
\hline 851 & Burn Room Bedroom (B) & FEDH & TCB 4 & & & & & & & & 0.078 & & & & & \\
\hline 852 & Utility Hallway (C) & FEDH & $\mathrm{TCC}_{4} 4$ & & & & & & & & 0.068 & & & & & \\
\hline 853 & Hallway Outside Burn Room Bedroom (D) & FEDH & TCD_4 & & & & & & & & 0.068 & & & & & \\
\hline 854 & Living Room (E) & FEDH & TCE_4 & & & & & & & & 0.070 & & & & & \\
\hline 855 & Front Door Hallway $(\mathrm{F})$ & FEDH & TCF_4 & & & & & & & & 0.069 & & & & & \\
\hline 856 & Closed Bedroom (G) & FEDH & TCG & & & & & & & & 0.053 & & & & & \\
\hline 857 & & & & & & & & & & & & & & & & \\
\hline 858 & Smoke Obscuration $=0.25 \mathrm{~m}-1$ & & & & & & & & & & & & & & & \\
\hline 859 & & & OD & & & & & & & ASET & Final Value & & & & & \\
\hline 860 & Master Bedroom (A) & Smoke & SMA 3 & & & & & & & & 0.062 & & & & & \\
\hline 861 & Burn Room Bedroom (B) & Smoke & SMB_3 3 & & & & & & & & 0.199 & & & & & \\
\hline 862 & Utility Hallway (C) & Smoke & SMC_3 & & & & & & & & 0.182 & & & & & \\
\hline 863 & Hallway Outside Burn Room Bedroom (D) & Smoke & SMD_3 & & & & & & & & 0.005 & & & & & \\
\hline 864 & Living Room (E) & Smoke & SME_3 & & & & & & & & 0.066 & & & & & \\
\hline 865 & Front Door Hallway (F) & Smoke & SMF_3 & & & & & & & & 0.079 & & & & & \\
\hline 866 & Closed Bedroom (G) & Smoke & $S M \bar{G}$ & & & & & & & & 0.010 & & & & & \\
\hline 867 & & & & & & & & & & & & & & & & \\
\hline
\end{tabular}




\begin{tabular}{|c|c|c|c|c|c|c|c|c|c|c|c|c|c|c|c|c|}
\hline & $\mathrm{A}$ & $\mathrm{B}$ & $\mathrm{C}$ & $\mathrm{D}$ & $\mathrm{E}$ & $\mathrm{F}$ & $\mathrm{G}$ & $\mathrm{H}$ & $\mathrm{T}$ & $\mathrm{J}$ & $\mathrm{K}$ & $\mathrm{L}$ & $M$ & $\mathrm{~N}$ & 0 & $\mathrm{P}$ \\
\hline 868 & Test & SDC41 & Vegetable Oil 0 & n Kitchen S & tove & & & & & & & & & & & \\
\hline 869 & & & & & & & & & & & & & & & & \\
\hline 870 & Time & te & & & & & & & & & & & & & & \\
\hline 871 & & & & & & & & & & & & & & & & \\
\hline 872 & ISO Gas FED = 0.3 & & & & & & & & & & & & & & & \\
\hline 873 & & & $\mathrm{CO} 2$ & CO2 Offset & CO2 Base & $\mathrm{CO}$ & CO Offset & $\mathrm{HCN}$ & HCN Offset & ASET & Final Value & Minimum & & Outside Ro & om of Origin & \\
\hline 874 & Master Bedroom (A) & FEDG & GASA_3 & \begin{tabular}{|l|}
0.044481 \\
\end{tabular} & 0.04 & GASA_1 & 0.000481 & . & - & & 0.031 & 1106 & (Smoke) & 1252 & (Smoke) & \\
\hline 875 & Utility Hallway (C) & FEDG & GASC_3 & 0.031651 & 0.04 & GASC_1 & 0.000375 & - & - & & 0.040 & & & & & \\
\hline 876 & Front Door Hallway $(\mathrm{F})$ & FEDG & GASF_3 & 0.044046 & 0.04 & GASF_1 & 0 & - & - & & 0.020 & & & & & \\
\hline 877 & Burn Room (BEK) & FEDG & GASB_3 & 0.023451 & 0.04 & GASB_1 & 0.001923 & - & - & & 0.073 & & & & & \\
\hline 878 & & & & & & & & & & & & & & & & \\
\hline 879 & NIST Gas FED $=0.3$ & & & & & & & & & & & & & & & \\
\hline 880 & & & $\mathrm{CO} 2$ & CO2 Offset & CO2 Base & $\mathrm{CO}$ & CO Offset & $\mathrm{HCN}$ & HCN Offset & ASET & Final Value & & & & & \\
\hline 881 & Master Bedroom (A) & FEDGN & GASA_3 & 0.044481 & 0.04 & GASA_1 & 0.000481 & 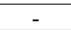 & 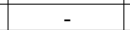 & & 0.000 & & & & & \\
\hline 882 & Utility Hallway (C) & FEDGN & GASC_3 & 0.031651 & 0.04 & GASC_1 & 0.000375 & - & - & & 0.000 & & & & & \\
\hline 883 & Front Door Hallway $(\mathrm{F})$ & FEDGN & GASF_3 & 0.044046 & 0.04 & GASF_1 & 0 & - & - & & 0.000 & & & & & \\
\hline 884 & Burn Room (BEK) & FEDGN & GASB_3 & 0.023451 & 0.04 & GASB_1 & 0.001923 & - & - & & 0.000 & & & & & \\
\hline 885 & & & & & & & & & & & & & & & & \\
\hline 886 & ISO Convected Heat $=0.3$ & & & & & & & & & & & & & & & \\
\hline 887 & & & Temperature & & & & & & & ASET & Final Value & & & & & \\
\hline 888 & Master Bedroom (A) & FEDH & TCA_4 & & & & & & & & 0.080 & & & & & \\
\hline 889 & Burn Room Bedroom (B) & FEDH & TCB 4 & & & & & & & & 0.083 & & & & & \\
\hline 890 & Utility Hallway (C) & FEDH & TCC 4 & & & & & & & & 0.089 & & & & & \\
\hline 891 & Hallway Outside Burn Room Bedroom (D) & FEDH & TCD_4 & & & & & & & & 0.093 & & & & & \\
\hline 892 & Living Room (E) & FEDH & TCE_4 & & & & & & & & 0.095 & & & & & \\
\hline 893 & Front Door Hallway $(\mathrm{F})$ & FEDH & TCF_4 & & & & & & & & 0.094 & & & & & \\
\hline 894 & Closed Bedroom (G) & FEDH & TCG & & & & & & & & 0.037 & & & & & \\
\hline 895 & & & & & & & & & & & & & & & & \\
\hline 896 & Smoke Obscuration $=0.25 \mathrm{~m}-1$ & & & & & & & & & & & & & & & \\
\hline 897 & & & OD & & & & & & & ASET & Final Value & & & & & \\
\hline 898 & Master Bedroom (A) & Smoke & SMA 3 & & & & & & & 1312 & 0.254 & & & & & \\
\hline 899 & Burn Room Bedroom (B) & Smoke & SMB_3 3 & & & & & & & 1252 & 0.255 & & & & & \\
\hline 900 & Utility Hallway (C) & Smoke & SMC_3 & & & & & & & 1178 & 0.418 & & & & & \\
\hline 901 & Hallway Outside Burn Room Bedroom (D) & Smoke & SMD_3 & & & & & & & 1106 & 0.259 & & & & & \\
\hline 902 & Living Room (E) & Smoke & SME_3 & & & & & & & 1386 & 0.271 & & & & & \\
\hline 903 & Front Door Hallway $(\mathrm{F})$ & Smoke & SMF_3 3 & & & & & & & 1168 & 0.257 & & & & & \\
\hline 904 & Closed Bedroom (G) & Smoke & $S M \bar{G}$ & & & & & & & & 0.064 & & & & & \\
\hline 905 & & & & & & & & & & & & & & & & \\
\hline
\end{tabular}




\begin{tabular}{|c|c|c|c|c|c|c|c|c|c|c|c|c|c|c|c|c|}
\hline & A & $\mathrm{B}$ & $\mathrm{C}$ & $\mathrm{D}$ & $E$ & $\mathrm{~F}$ & $\bar{G}$ & $\mathrm{H}$ & $T$ & $\mathrm{~J}$ & $\mathrm{~K}$ & $\mathrm{~L}$ & $\mathrm{M}$ & $\bar{N}$ & $\mathrm{O}$ & $\mathrm{P}$ \\
\hline 906 & Series & nston : & riesl & & & & & & & & & & & & & \\
\hline 907 & & & & & & & & & & & & & & & & \\
\hline 908 & Test & SDC20 & Flaming Mattre & ss in Bedroc & om (Burn Ro & om Door C & osed) & & & & & & & & & \\
\hline 909 & & & & & & & & & & & & & & & & \\
\hline 910 & Time & & & & & & & & & & & & & & & \\
\hline 911 & & & & & & & & & & & & & & & & \\
\hline 912 & ISO Gas FED $=0.3$ & & & & & & & & & & & & & & & \\
\hline 913 & & & $\mathrm{CO} 2$ & CO2 Offset & CO2 Base & $\mathrm{CO}$ & CO Offset & $\mathrm{HCN}$ & HCN Offse & ASET & Final Value & Minimum & & Outside $\mathrm{R}$ & toom of Origin & \\
\hline 914 & Bedroom 1 (B1) & FEDG & GASB3 & \begin{tabular}{|c|}
0.04454 \\
\end{tabular} & 0.05 & GASB1 & 0.004347 & - & - & & 0.033 & 210 & (ISO Heat) & 360 & \begin{tabular}{l|l|l|}
2 (End of Test \\
\end{tabular} & \\
\hline 915 & Upstairs Hallway $(\mathrm{H})$ & FEDG & GASF3 & \begin{tabular}{|l|}
0.056339 \\
\end{tabular} & 0.05 & GASF1 & 0.003391 & - & - & & 0.002 & & & & & \\
\hline 916 & Bedroom 3 Burn Room (B3) & & & & & & & & & & & & & & & \\
\hline 917 & Living Room Burn Room (L) & & & & & & & & & & & & & & & \\
\hline 918 & Foyer $(\mathrm{F})$ & & & & & & & & & & & & & & & \\
\hline 919 & $\operatorname{Den}(\mathrm{D})$ & & & & & & & & & & & & & & & \\
\hline 920 & Kitchen $(\mathrm{K})$ & & & & & & & & & & & & & & & \\
\hline 921 & & & & & & & & & & & & & & & & \\
\hline 922 & NIST Gas FED $=0.3$ & & & & & & & & & & & & & & & \\
\hline 923 & & & $\mathrm{CO} 2$ & CO2 Offset & CO2 Base & $\mathrm{CO}$ & CO Offset & $\mathrm{HCN}$ & HCN Offse & ASET & Final Value & & & & & \\
\hline 924 & Bedroom 1 (B1) & FEDGN & GASB3 & 0.04454 & \begin{tabular}{|r|}
0.05 \\
0.05
\end{tabular} & GASB1 & 0.004347 & & 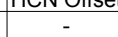 & & 0.000 & & & & & \\
\hline 925 & Upstairs Hallway $(\mathrm{H})$ & FEDGN & GASF3 & \begin{tabular}{|l|}
0.056339 \\
\end{tabular} & 0.05 & GASF1 & 0.003391 & - & - & & 0.000 & & & & & \\
\hline 926 & Bedroom 3 Burn Room (B3) & & & & & & & & & & & & & & & \\
\hline 927 & Living Room Burn Room (L) & & & & & & & & & & & & & & & \\
\hline$\frac{211}{928}$ & $\begin{array}{l}\text { Foyer }(F) \\
\text { Fol }\end{array}$ & & & & & & & & & & & & & & & \\
\hline 929 & Den (D) & & & & & & & & & & & & & & & \\
\hline 930 & Kitchen $(\mathrm{K})$ & & & & & & & & & & & & & & & \\
\hline 931 & & & & & & & & & & & & & & & & \\
\hline 932 & ISO Convected Heat $=0.3$ & & & & & & & & & & & & & & & \\
\hline 933 & & & Temperature & & & & & & & ASET & Final Value & & & & & \\
\hline 934 & Bedroom 1 (B1) & FEDH & TCB14 & & & & & & & & 0.120 & & & & & \\
\hline 935 & Upstairs Hallway $(\mathrm{H})$ & FEDH & $\mathrm{TCH} 4$ & & & & & & & & 0.121 & & & & & \\
\hline 936 & Bedroom 3 Burn Room (B3) & FEDH & TCB34 & & & & & & & 210 & 0.315 & & & & & \\
\hline 937 & Living Room Burn Room (L) & FEDH & TCL4 & & & & & & & & 0.098 & & & & & \\
\hline 938 & Foyer (F) & FEDH & TCF4 & & & & & & & & 0.100 & & & & & \\
\hline 939 & $\operatorname{Den}(\mathrm{D})$ & FEDH & TCD4 & & & & & & & & 0.094 & & & & & \\
\hline 940 & Kitchen $(\mathrm{K})$ & FEDH & TCK4 & & & & & & & & 0.094 & & & & & \\
\hline 941 & & & & & & & & & & & & & & & & \\
\hline 942 & Smoke Obscuration $=0.25 \mathrm{~m}-1$ & & & & & & & & & & & & & & & \\
\hline 943 & & & OD & & & & & & & ASET & Final Value & & & & & \\
\hline 944 & Bedroom 1 (B1) & Smoke & SMB12S & & & & & & & & 0.223 & & & & & \\
\hline 945 & Upstairs Hallway $(\mathrm{H})$ & Smoke & SMH2S & & & & & & & & 0.145 & & & & & \\
\hline 946 & Bedroom 3 Burn Room (B3) & & & & & & & & & & & & & & & \\
\hline 947 & Living Room Burn Room (L) & & & & & & & & & & & & & & & \\
\hline 948 & Foyer (F) & Smoke & SMF2S & & & & & & & & 0.083 & & & & & \\
\hline 949 & $\operatorname{Den}(\mathrm{D})$ & Smoke & SMD2S & & & & & & & & 0.036 & & & & & \\
\hline 950 & Kitchen $(\mathrm{K})$ & & & & & & & & & & & & & & & \\
\hline 951 & & & & & & & & & & & & & & & & \\
\hline
\end{tabular}




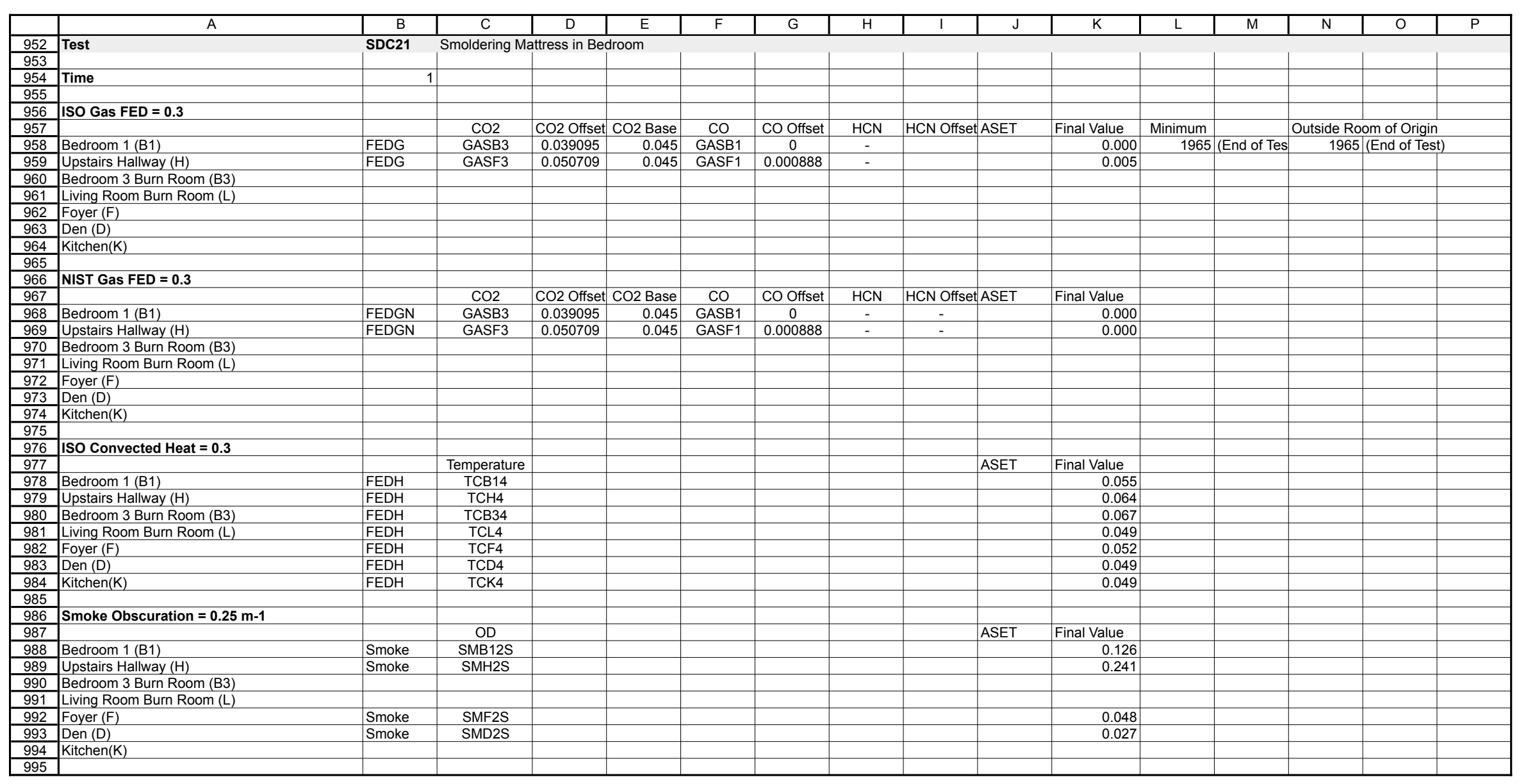




\begin{tabular}{|c|c|c|c|c|c|c|c|c|c|c|c|c|c|c|c|c|}
\hline & $\mathrm{A}$ & $\mathrm{B}$ & C & $\mathrm{D}$ & $\mathrm{E}$ & $\mathrm{F}$ & $\bar{G}$ & $\mathrm{H}$ & $T$ & $\mathrm{~J}$ & $\mathrm{~K}$ & $\mathrm{~L}$ & $\mathrm{M}$ & $\mathrm{N}$ & $\mathrm{O}$ & $\mathrm{P}$ \\
\hline 996 & Test & SDC22 & Flaming Mattre & ss in Bedro & & & & & & & & & & & & \\
\hline 997 & & & & & & & & & & & & & & & & \\
\hline 998 & Time & & & & & & & & & & & & & & & \\
\hline 999 & & & & & & & & & & & & & & & & \\
\hline $\begin{array}{ll}0000 \\
100\end{array}$ & ISO Gas FED = 0.3 & & & & & & & & & & & & & & & \\
\hline 1001 & & & $\mathrm{CO} 2$ & CO2 Offset & CO2 Base & $\mathrm{co}$ & CO Offset & $\mathrm{HCN}$ & HCN Offset & ASET & Final Value & Minimum & & Outside Ro & om of Origin & \\
\hline 1002 & Bedroom 1 (B1) & FEDG & GASB3 & 0.055825 & 0.05 & GASB1 & 0 & & e & & 0.000 & 114 & (Smoke) & 404 & (End of Test & \\
\hline 1003 & Upstairs Hallway $(\mathrm{H})$ & FEDG & GASF3 & \begin{tabular}{|l|}
0.044064 \\
\end{tabular} & 0.05 & GASF1 & \begin{tabular}{|l|}
0.000421 \\
\end{tabular} & - & - & & 0.000 & & & & & \\
\hline 1004 & Bedroom 3 Burn Room (B3) & & & & & & & & & & & & & & & \\
\hline 1005 & Living Room Burn Room (L) & & & & & & & & & & & & & & & \\
\hline 1006 & Foyer $(F)$ & & & & & & & & & & & & & & & \\
\hline 1007 & Den (D) & & & & & & & & & & & & & & & \\
\hline 1008 & Kitchen $(\mathrm{K})$ & & & & & & & & & & & & & & & \\
\hline 1009 & & & & & & & & & & & & & & & & \\
\hline 1010 & NIST Gas FED $=0.3$ & & & & & & & & & & & & & & & \\
\hline 1011 & & & $\mathrm{CO} 2$ & CO2 Offset & CO2 Base & $\mathrm{CO}$ & CO Offset & $\mathrm{HCN}$ & HCN Offset & ASET & Final Value & & & & & \\
\hline 1012 & Bedroom 1 (B1) & FEDGN & GASB3 & 0.055825 & 0.05 & GASB1 & 0 & - & - & & 0.000 & & & & & \\
\hline 1013 & Upstairs Hallway $(\mathrm{H})$ & FEDGN & GASF3 & \begin{tabular}{|l|}
0.044064 \\
\end{tabular} & 0.05 & GASF1 & \begin{tabular}{|l|}
0.000421 \\
\end{tabular} & 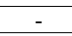 & - & & 0.000 & & & & & \\
\hline 1014 & Bedroom 3 Burn Room (B3) & & & & & & & & & & & & & & & \\
\hline 1015 & Living Room Burn Room (L) & & & & & & & & & & & & & & & \\
\hline 1016 & Foyer $(F)$ & & & & & & & & & & & & & & & \\
\hline 1017 & $\operatorname{Den}(\mathrm{D})$ & & & & & & & & & & & & & & & \\
\hline 1018 & \begin{tabular}{|l|} 
Kitchen $(\mathrm{K})$ \\
\end{tabular} & & & & & & & & & & & & & & & \\
\hline 1019 & & & & & & & & & & & & & & & & \\
\hline 1020 & ISO Convected Heat $=0.3$ & & & & & & & & & & & & & & & \\
\hline 1021 & & & Temperature & & & & & & & ASET & Final Value & & & & & \\
\hline 1022 & Bedroom 1 (B1) & FEDH & TCB14 & & & & & & & & 0.015 & & & & & \\
\hline$\frac{1024}{1023}$ & Upstairs Hallway $(\mathrm{H})$ & \begin{tabular}{|l} 
FEDH \\
\end{tabular} & TCH4 & & & & & & & & $\begin{array}{l}0.013 \\
0.013\end{array}$ & & & & & \\
\hline 1024 & Bedroom 3 Burn Room (B3) & FEDH & TCB34 & & & & & & & & 0.043 & & & & & \\
\hline 1025 & Living Room Burn Room (L) & FEDH & TCL4 & & & & & & & & 0.012 & & & & & \\
\hline 1026 & Foyer (F) & FEDH & TCF4 & & & & & & & & 0.011 & & & & & \\
\hline 1027 & $\operatorname{Den}(\mathrm{D})$ & FEDH & TCD4 & & & & & & & & 0.011 & & & & & \\
\hline 1028 & Kitchen $(\mathrm{K})$ & FEDH & TCK4 & & & & & & & & 0.011 & & & & & \\
\hline 1029 & & & & & & & & & & & & & & & & \\
\hline 1030 & Smoke Obscuration $=0.25 \mathrm{~m}-1$ & & & & & & & & & & & & & & & \\
\hline 1031 & & & $\mathrm{OD}$ & & & & & & & ASET & Final Value & & & & & \\
\hline 1032 & Bedroom 1 (B1) & Smoke & SMB12S & & & & & & & 114 & 0.295 & & & & & \\
\hline 1033 & Upstairs Hallway (H) & Smoke & SMH2S & & & & & & & & 0.012 & & & & & \\
\hline 1034 & Bedroom 3 Burn Room (B3) & & & & & & & & & & & & & & & \\
\hline 1035 & Living Room Burn Room (L) & & & & & & & & & & & & & & & \\
\hline 1036 & Foyer $(F)$ & Smoke & SMF2S & & & & & & & & 0.028 & & & & & \\
\hline 1037 & $\operatorname{Den}(\mathrm{D})$ & Smoke & SMD2S & & & & & & & & 0.040 & & & & & \\
\hline 1038 & Kitchen $(\mathrm{K})$ & & & & & & & & & & & & & & & \\
\hline 1039 & & & & & & & & & & & & & & & & \\
\hline
\end{tabular}




\begin{tabular}{|c|c|c|c|c|c|c|c|c|c|c|c|c|c|c|c|c|}
\hline & A & $\mathrm{B}$ & $\mathrm{C}$ & $\mathrm{D}$ & $E$ & $\mathrm{~F}$ & $G$ & $\mathrm{H}$ & I & $\mathrm{J}$ & $\mathrm{K}$ & $\mathrm{L}$ & $\mathrm{M}$ & $\mathrm{N}$ & 0 & $P$ \\
\hline 1040 & Test & SDC23 & Smoldering $\mathrm{Ch}$ & air in Living & Room & & & & & & & & & & & \\
\hline 1041 & & & & & & & & & & & & & & & & \\
\hline 1042 & Time & 1 & & & & & & & & & & & & & & \\
\hline 1043 & & & & & & & & & & & & & & & & \\
\hline 1044 & ISO Gas FED $=0.3$ & & & & & & & & & & & & & & & \\
\hline 1045 & & & $\mathrm{CO} 2$ & CO2 Offset & CO2 Base & $\mathrm{CO}$ & co Offset & $\mathrm{HCN}$ & HCN Offse & ASET & Final Value & Minimum & & Outside R & om of Origin & \\
\hline 1046 & Bedroom 1 (B1) & FEDG & GASB3 & \begin{tabular}{|l|}
0.044208 \\
\end{tabular} & 0.045 & GASB1 & 0.003846 & - & - & & 0.001 & 4672 & (Smoke) & 4840 & (Smoke) & \\
\hline 1047 & Upstairs Hallway $(\mathrm{H})$ & FEDG & GASF3 & 0.045426 & 0.045 & GASF1 & 0.000436 & - & - & & 0.055 & & & & & \\
\hline 1048 & Bedroom 3 Burn Room (B3) & & & & & & & & & & & & & & & \\
\hline 1049 & Living Room Burn Room (L) & & & & & & & & & & & & & & & \\
\hline 1050 & Foyer (F) & & & & & & & & & & & & & & & \\
\hline 1051 & $\operatorname{Den}(\mathrm{D})$ & & & & & & & & & & & & & & & \\
\hline 1052 & Kitchen $(\mathrm{K})$ & & & & & & & & & & & & & & & \\
\hline 1053 & & & & & & & & & & & & & & & & \\
\hline 1054 & NIST Gas FED $=0.3$ & & & & & & & & & & & & & & & \\
\hline 1055 & & & $\mathrm{CO} 2$ & CO2 Offset & CO2 Base & $\mathrm{CO}$ & co Offset & $\mathrm{HCN}$ & HCN Offse & tASET & Final Value & & & & & \\
\hline 1056 & Bedroom 1 (B1) & FEDGN & GASB3 & \begin{tabular}{|l|}
0.044208 \\
\end{tabular} & 0.045 & GASB1 & 0.003846 & - & - & & 0.000 & & & & & \\
\hline 1057 & Upstairs Hallway $(\mathrm{H})$ & FEDGN & GASF3 & 0.045426 & 0.045 & GASF1 & 0.000436 & - & - & & 0.000 & & & & & \\
\hline$\frac{1058}{1058}$ & Bedroom 3 Burn Room (B3) & & & & & & & & & & & & & & & \\
\hline 1059 & Living Room Burn Room (L) & & & & & & & & & & & & & & & \\
\hline 1060 & Foyer (F) & & & & & & & & & & & & & & & \\
\hline 1061 & $\operatorname{Den}(\mathrm{D})$ & & & & & & & & & & & & & & & \\
\hline 1062 & Kitchen $(\mathrm{K})$ & & & & & & & & & & & & & & & \\
\hline 1063 & & & & & & & & & & & & & & & & \\
\hline 1064 & ISO Convected Heat $=0.3$ & & & & & & & & & & & & & & & \\
\hline 1065 & & & Temperature & & & & & & & ASET & Final Value & & & & & \\
\hline 1066 & Bedroom 1 (B1) & FEDH & TCB14 & & & & & & & & 0.077 & & & & & \\
\hline 1067 & Upstairs Hallway $(\mathrm{H})$ & FEDH & TCH4 & & & & & & & & 0.076 & & & & & \\
\hline 1068 & Bedroom 3 Burn Room (B3) & FEDH & TCB34 & & & & & & & & 0.073 & & & & & \\
\hline 1069 & Living Room Burn Room (L) & FEDH & TCL4 & & & & & & & & 0.074 & & & & & \\
\hline 1070 & Foyer (F) & FEDH & TCF4 & & & & & & & & 0.072 & & & & & \\
\hline 1071 & $\operatorname{Den}(\mathrm{D})$ & FEDH & TCD4 & & & & & & & & 0.065 & & & & & \\
\hline 1072 & Kitchen $(K)$ & FEDH & TCK4 & & & & & & & & 0.066 & & & & & \\
\hline 1073 & & & & & & & & & & & & & & & & \\
\hline 1074 & Smoke Obscuration $=0.25 \mathrm{~m}-1$ & & & & & & & & & & & & & & & \\
\hline 1075 & & & OD & & & & & & & ASET & Final Value & & & & & \\
\hline 1076 & Bedroom 1 (B1) & Smoke & SMB12S & & & & & & & & 0.120 & & & & & \\
\hline 1077 & Upstairs Hallway $(\mathrm{H})$ & Smoke & SMH2S & & & & & & & 4840 & 0.258 & & & & & \\
\hline 1078 & Bedroom 3 Burn Room (B3) & & & & & & & & & & & & & & & \\
\hline 1079 & Living Room Burn Room (L) & & & & & & & & & & & & & & & \\
\hline 1080 & Foyer (F) & Smoke & SMF2S & & & & & & & 4672 & 0.273 & & & & & \\
\hline 1081 & $\operatorname{Den}(\mathrm{D})$ & Smoke & SMD2S & & & & & & & & 0.111 & & & & & \\
\hline 1082 & Kitchen $(K)$ & & & & & & & & & & & & & & & \\
\hline 1083 & & & & & & & & & & & & & & & & \\
\hline
\end{tabular}




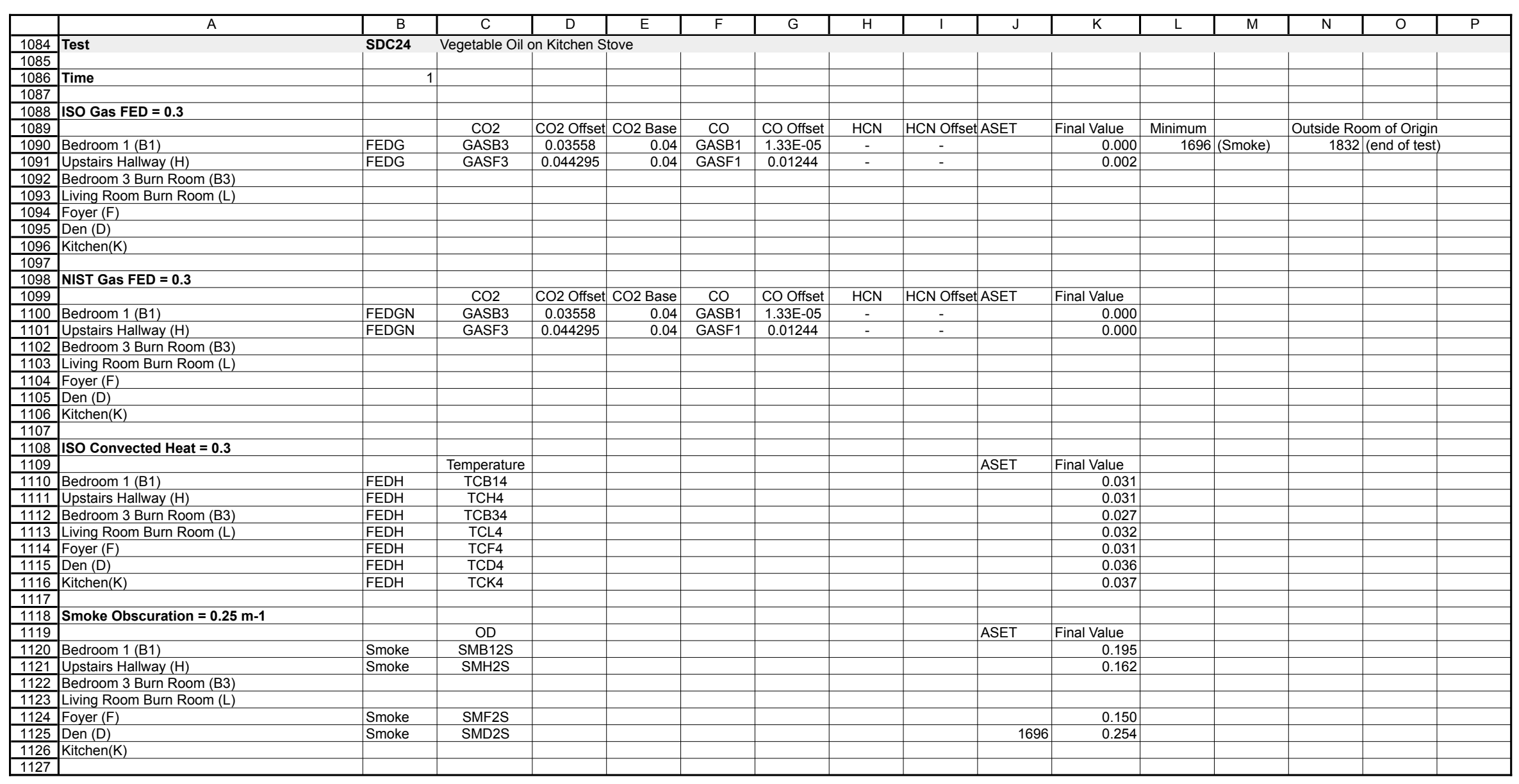




\begin{tabular}{|c|c|c|c|c|c|c|c|c|c|c|c|c|c|c|c|c|}
\hline & $\mathrm{A}$ & $B$ & $\mathrm{C}$ & $\mathrm{D}$ & $E$ & $F$ & $\bar{G}$ & $\mathrm{H}$ & $T$ & $\mathrm{~J}$ & $\mathrm{~K}$ & $\mathrm{~L}$ & $M$ & $\bar{N}$ & $\mathrm{O}$ & $\mathrm{P}$ \\
\hline 1128 & Test & SDC25 & Flaming Chair & in Living Roc & & & & & & & & & & & & \\
\hline 1129 & & & & & & & & & & & & & & & & \\
\hline 1130 & Time & - & & & & & & & & & & & & & & \\
\hline 1131 & & & & & & & & & & & & & & & & \\
\hline 1132 & ISO Gas FED = 0.3 & & & & & & & & & & & & & & & \\
\hline 1133 & & & $\mathrm{CO} 2$ & CO2 Offset & CO2 Base & $\mathrm{CO}$ & CO Offset & $\mathrm{HCN}$ & HCN Offset & ASET & Final Value & Minimum & & Outside Roc & om of Origin & \\
\hline 1134 & Bedroom 1 (B1) & FEDG & GASB3 & 0.042139 & 0.04 & GASB1 & \begin{tabular}{|l|}
0.000502 \\
\end{tabular} & - & & & 0.008 & 202 & (Smoke) & \begin{tabular}{|r|}
220 \\
\end{tabular} & (Smoke) & \\
\hline 1135 & Upstairs Hallway $(\mathrm{H})$ & FEDG & GASF3 & \begin{tabular}{|l|}
0.040747 \\
\end{tabular} & 0.04 & GASF1 & \begin{tabular}{|l|}
0.002588 \\
\end{tabular} & - & & & 0.006 & & & & & \\
\hline 1136 & Bedroom 3 Burn Room (B3) & & & & & & & & & & & & & & & \\
\hline 1137 & Living Room Burn Room (L) & & & & & & & & & & & & & & & \\
\hline 1138 & Foyer (F) & & & & & & & & & & & & & & & \\
\hline 1139 & $\operatorname{Den}(\mathrm{D})$ & & & & & & & & & & & & & & & \\
\hline 1140 & Kitchen $(\mathrm{K})$ & & & & & & & & & & & & & & & \\
\hline 1141 & & & & & & & & & & & & & & & & \\
\hline 1142 & NIST Gas FED $=0.3$ & & & & & & & & & & & & & & & \\
\hline 1143 & & & $\mathrm{CO} 2$ & CO2 Offset & CO2 Base & $\mathrm{CO}$ & CO Offset & $\mathrm{HCN}$ & HCN Offset & ASET & Final Value & & & & & \\
\hline 1144 & Bedroom 1 (B1) & FEDGN & GASB3 & \begin{tabular}{|l|}
0.042139 \\
\end{tabular} & 0.04 & GASB1 & \begin{tabular}{|l|}
0.000502 \\
\end{tabular} & . & & & 0.000 & & & & & \\
\hline 1145 & Upstairs Hallway $(\mathrm{H})$ & FEDGN & GASF3 & \begin{tabular}{|l|}
0.040747 \\
\end{tabular} & 0.04 & GASF1 & \begin{tabular}{|l|}
0.002588 \\
\end{tabular} & - & & & 0.000 & & & & & \\
\hline 1146 & Bedroom 3 Burn Room (B3) & & & & & & & & & & & & & & & \\
\hline 1147 & Living Room Burn Room (L) & & & & & & & & & & & & & & & \\
\hline 1148 & Foyer (F) & & & & & & & & & & & & & & & \\
\hline 1149 & Den (D) & & & & & & & & & & & & & & & \\
\hline 1150 & Kitchen $(K)$ & & & & & & & & & & & & & & & \\
\hline 1151 & & & & & & & & & & & & & & & & \\
\hline 1152 & ISO Convected Heat $=0.3$ & & & & & & & & & & & & & & & \\
\hline 1153 & & & Temperature & & & & & & & ASET & Final Value & & & & & \\
\hline 1154 & Bedroom 1 (B1) & FEDH & TCB14 & & & & & & & & 0.039 & & & & & \\
\hline 1155 & Upstairs Hallway $(\mathrm{H})$ & FEDH & $\mathrm{TCH} 4$ & & & & & & & & 0.249 & & & & & \\
\hline 1156 & Bedroom 3 Burn Room (B3) & FEDH & TCB34 & & & & & & & & 0.038 & & & & & \\
\hline 1157 & Living Room Burn Room (L) & FEDH & TCL4 & & & & & & & & 0.104 & & & & & \\
\hline 1158 & Foyer (F) & FEDH & TCF4 & & & & & & & & 0.054 & & & & & \\
\hline 1159 & Den (D) & FEDH & TCD4 & & & & & & & & 0.007 & & & & & \\
\hline 1160 & Kitchen $(\mathrm{K})$ & FEDH & TCK4 & & & & & & & & 0.011 & & & & & \\
\hline 1161 & & & & & & & & & & & & & & & & \\
\hline 1162 & Smoke Obscuration $=0.25 \mathrm{~m}-1$ & & & & & & & & & & & & & & & \\
\hline 1163 & & & $\mathrm{OD}$ & & & & & & & ASET & Final Value & & & & & \\
\hline 1164 & Bedroom 1 (B1) & Smoke & SMB12S & & & & & & & 250 & 0.281 & & & & & \\
\hline 1165 & Upstairs Hallway $(\mathrm{H})$ & Smoke & SMH2S & & & & & & & 220 & 0.254 & & & & & \\
\hline 1166 & Bedroom 3 Burn Room (B3) & & & & & & & & & & & & & & & \\
\hline 1167 & Living Room Burn Room (L) & & & & & & & & & & & & & & & \\
\hline 1168 & Foyer (F) & Smoke & SMF2S & & & & & & & 202 & 0.255 & & & & & \\
\hline 1169 & $\operatorname{Den}(\mathrm{D})$ & Smoke & SMD2S & & & & & & & 250 & 0.301 & & & & & \\
\hline 1170 & Kitchen $(\mathrm{K})$ & & & & & & & & & & & & & & & \\
\hline 1171 & & & & & & & & & & & & & & & & \\
\hline
\end{tabular}




\begin{tabular}{|c|c|c|c|c|c|c|c|c|c|c|c|c|c|c|c|c|}
\hline & A & $\mathrm{B}$ & $\mathrm{C}$ & $\mathrm{D}$ & $E$ & $\mathrm{~F}$ & $G$ & $\mathrm{H}$ & $\mathrm{I}$ & $\mathrm{J}$ & $\mathrm{K}$ & $\mathrm{L}$ & $\mathrm{M}$ & $\mathrm{N}$ & 0 & $P$ \\
\hline 1172 & Test & SDC26 & Flaming Chair & in Living Roc & & & & & & & & & & & & \\
\hline 1173 & & & & & & & & & & & & & & & & \\
\hline 1174 & Time & 1 & & & & & & & & & & & & & & \\
\hline 1175 & & & & & & & & & & & & & & & & \\
\hline 1176 & ISO Gas FED $=0.3$ & & & & & & & & & & & & & & & \\
\hline 1177 & & & $\mathrm{CO} 2$ & CO2 Offset & CO2 Base & $\mathrm{CO}$ & CO Offset & $\mathrm{HCN}$ & HCN Offse & ASET & Final Value & Minimum & & Outside Ro & om of Origin & \\
\hline 1178 & Bedroom 1 (B1) & FEDG & GASB3 & \begin{tabular}{|l|}
0.035784 \\
\end{tabular} & 0.04 & GASB1 & 0 & - & - & & 0.001 & 226 & (Smoke) & 236 & (Smoke) & \\
\hline 1179 & Upstairs Hallway $(\mathrm{H})$ & FEDG & GASF3 & \begin{tabular}{|l|}
0.043844 \\
\end{tabular} & 0.04 & GASF1 & 0.00226 & - & - & & 0.007 & & & & & \\
\hline 1180 & Bedroom 3 Burn Room (B3) & & & & & & & & & & & & & & & \\
\hline 1181 & Living Room Burn Room (L) & & & & & & & & & & & & & & & \\
\hline 1182 & Foyer (F) & & & & & & & & & & & & & & & \\
\hline 1183 & Den (D) & & & & & & & & & & & & & & & \\
\hline 1184 & Kitchen $(\mathrm{K})$ & & & & & & & & & & & & & & & \\
\hline 1185 & & & & & & & & & & & & & & & & \\
\hline 1186 & NIST Gas FED $=0.3$ & & & & & & & & & & & & & & & \\
\hline 1187 & & & $\mathrm{CO} 2$ & CO2 Offset & CO2 Base & $\mathrm{CO}$ & CO Offset & $\mathrm{HCN}$ & HCN Offse & tASET & Final Value & & & & & \\
\hline 1188 & Bedroom 1 (B1) & FEDGN & GASB3 & \begin{tabular}{|l|}
0.035784 \\
\end{tabular} & 0.04 & GASB1 & 0 & - & - & & 0.000 & & & & & \\
\hline 1189 & Upstairs Hallway $(\mathrm{H})$ & FEDGN & GASF3 & 0.043844 & 0.04 & GASF1 & 0.00226 & - & - & & 0.000 & & & & & \\
\hline 1190 & Bedroom 3 Burn Room (B3) & & & & & & & & & & & & & & & \\
\hline 1191 & Living Room Burn Room (L) & & & & & & & & & & & & & & & \\
\hline 1192 & Foyer (F) & & & & & & & & & & & & & & & \\
\hline 1193 & $\operatorname{Den}(\mathrm{D})$ & & & & & & & & & & & & & & & \\
\hline 1194 & Kitchen $(\mathrm{K})$ & & & & & & & & & & & & & & & \\
\hline 1195 & & & & & & & & & & & & & & & & \\
\hline 1196 & ISO Convected Heat $=0.3$ & & & & & & & & & & & & & & & \\
\hline 1197 & & & Temperature & & & & & & & ASET & Final Value & & & & & \\
\hline 1198 & Bedroom 1 (B1) & FEDH & TCB14 & & & & & & & & 0.018 & & & & & \\
\hline 1199 & Upstairs Hallway $(\mathrm{H})$ & FEDH & $\mathrm{TCH} 4$ & & & & & & & & 0.102 & & & & & \\
\hline 1200 & Bedroom 3 Burn Room (B3) & FEDH & TCB34 & & & & & & & & 0.015 & & & & & \\
\hline 1201 & Living Room Burn Room (L) & FEDH & TCL4 & & & & & & & & 0.028 & & & & & \\
\hline 1202 & Foyer (F) & FEDH & TCF4 & & & & & & & & 0.020 & & & & & \\
\hline 1203 & $\operatorname{Den}(\mathrm{D})$ & FEDH & TCD4 & & & & & & & & 0.005 & & & & & \\
\hline 1204 & Kitchen $(K)$ & FEDH & TCK4 & & & & & & & & 0.007 & & & & & \\
\hline 1205 & & & & & & & & & & & & & & & & \\
\hline 1206 & Smoke Obscuration $=0.25 \mathrm{~m}-1$ & & & & & & & & & & & & & & & \\
\hline 1207 & & & OD & & & & & & & ASET & Final Value & & & & & \\
\hline 1208 & Bedroom 1 (B1) & Smoke & SMB12S & & & & & & & 258 & 0.252 & & & & & \\
\hline 1209 & Upstairs Hallway $(\mathrm{H})$ & Smoke & SMH2S & & & & & & & 236 & 0.260 & & & & & \\
\hline 1210 & Bedroom 3 Burn Room (B3) & & & & & & & & & & & & & & & \\
\hline 1211 & Living Room Burn Room (L) & & & & & & & & & & & & & & & \\
\hline$\frac{1212}{1212}$ & Foyer (F) & Smoke & SMF2S & & & & & & & 226 & 0.264 & & & & & \\
\hline 1213 & $\operatorname{Den}(\mathrm{D})$ & Smoke & SMD2S & & & & & & & 266 & 0.274 & & & & & \\
\hline 1214 & Kitchen $(\mathrm{K})$ & & & & & & & & & & & & & & & \\
\hline 1215 & & & & & & & & & & & & & & & & \\
\hline
\end{tabular}




\begin{tabular}{|c|c|c|c|c|c|c|c|c|c|c|c|c|c|c|c|c|}
\hline & A & $\mathrm{B}$ & $\mathrm{C}$ & $\mathrm{D}$ & $\mathrm{E}$ & $\mathrm{F}$ & $\bar{G}$ & $\mathrm{H}$ & $\mathrm{I}$ & $\mathrm{J}$ & $\mathrm{K}$ & $\mathrm{L}$ & $\mathrm{M}$ & $\mathrm{N}$ & 0 & $P$ \\
\hline 1216 & Test & SDC27 & Smoldering $\mathrm{Ch}$ & air in Living & Room (Air C & onditioning & Upstairs) & & & & & & & & & \\
\hline 1217 & & & & & & & & & & & & & & & & \\
\hline 1218 & Time & 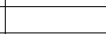 & & & & & & & & & & & & & & \\
\hline 1219 & & & & & & & & & & & & & & & & \\
\hline 1220 & ISO Gas FED = 0.3 & & & & & & & & & & & & & & & \\
\hline 1221 & & & $\mathrm{CO} 2$ & CO2 Offset & CO2 Base & $\mathrm{CO}$ & CO Offset & $\mathrm{HCN}$ & HCN Offset & ASET & Final Value & Minimum & & Outside R & om of Origin & \\
\hline 1222 & Bedroom 1 (B1) & FEDG & GASB3 & 0.056035 & 0.06 & GASB1 & \begin{tabular}{|l|}
0.035784 \\
\end{tabular} & - & & & 0.000 & 4138 & (Smoke) & $413 \varepsilon$ & (Smoke) & \\
\hline 1223 & Upstairs Hallway $(\mathrm{H})$ & FEDG & GASF3 & \begin{tabular}{|l|}
0.083994 \\
\end{tabular} & 0.06 & GASF1 & \begin{tabular}{|l|}
0.043844 \\
\end{tabular} & - & & & 0.000 & & & & & \\
\hline 1224 & Bedroom 3 Burn Room (B3) & & & & & & & & & & & & & & & \\
\hline 1225 & Living Room Burn Room (L) & & & & & & & & & & & & & & & \\
\hline 1226 & Foyer (F) & & & & & & & & & & & & & & & \\
\hline 1227 & Den (D) & & & & & & & & & & & & & & & \\
\hline 1228 & Kitchen $(\mathrm{K})$ & & & & & & & & & & & & & & & \\
\hline 1229 & & & & & & & & & & & & & & & & \\
\hline 1230 & NIST Gas FED $=0.3$ & & & & & & & & & & & & & & & \\
\hline 1231 & & & $\mathrm{CO} 2$ & CO2 Offset & CO2 Base & $\mathrm{CO}$ & CO Offset & $\mathrm{HCN}$ & HCN Offset & ASET & Final Value & & & & & \\
\hline 1232 & Bedroom 1 (B1) & FEDGN & GASB3 & \begin{tabular}{|l|}
0.056035 \\
\end{tabular} & 0.06 & GASB1 & \begin{tabular}{|l|}
0.035784 \\
\end{tabular} & - & & & 0.000 & & & & & \\
\hline 1233 & Upstairs Hallway $(\mathrm{H})$ & FEDGN & GASF3 & 0.083994 & 0.06 & GASF1 & 0.043844 & - & & & 0.000 & & & & & \\
\hline$\frac{1230}{1234}$ & Bedroom 3 Burn Room (B3) & & & & & & & & & & & & & & & \\
\hline 1235 & Living Room Burn Room (L) & & & & & & & & & & & & & & & \\
\hline 1236 & Foyer (F) & & & & & & & & & & & & & & & \\
\hline 1237 & Den (D) & & & & & & & & & & & & & & & \\
\hline 1238 & Kitchen $(K)$ & & & & & & & & & & & & & & & \\
\hline 1239 & & & & & & & & & & & & & & & & \\
\hline$\frac{1250}{1240}$ & ISO Convected Heat $=0.3$ & & & & & & & & & & & & & & & \\
\hline 1241 & & & Temperature & & & & & & & ASET & Final Value & & & & & \\
\hline 1242 & Bedroom 1 (B1) & FEDH & TCB14 & & & & & & & & 0.017 & & & & & \\
\hline 1243 & Upstairs Hallway $(\mathrm{H})$ & FEDH & $\mathrm{TCH} 4$ & & & & & & & & 0.025 & & & & & \\
\hline 1244 & Bedroom 3 Burn Room (B3) & FEDH & TCB34 & & & & & & & & 0.019 & & & & & \\
\hline 1245 & Living Room Burn Room (L) & FEDH & TCL4 & & & & & & & & 0.029 & & & & & \\
\hline 1246 & Foyer (F) & FEDH & TCF4 & & & & & & & & 0.027 & & & & & \\
\hline 1247 & Den (D) & FEDH & TCD4 & & & & & & & & 0.030 & & & & & \\
\hline 1248 & Kitchen $(\mathrm{K})$ & FEDH & TCK4 & & & & & & & & 0.030 & & & & & \\
\hline 1249 & & & & & & & & & & & & & & & & \\
\hline 1250 & Smoke Obscuration $=0.25 \mathrm{~m}-1$ & & & & & & & & & & & & & & & \\
\hline 1251 & & & OD & & & & & & & ASET & Final Value & & & & & \\
\hline$\frac{1252}{1252}$ & Bedroom 1 (B1) & & & & & & & & & & & & & & & \\
\hline 1253 & Upstairs Hallway $(\mathrm{H})$ & Smoke & SMH2S & & & & & & & 4138 & 0.254 & & & & & \\
\hline 1254 & Bedroom 3 Burn Room (B3) & & & & & & & & & & & & & & & \\
\hline 1255 & Living Room Burn Room (L) & & & & & & & & & & & & & & & \\
\hline 1256 & Foyer (F) & Smoke & SMF2S & & & & & & & 4214 & 0.347 & & & & & \\
\hline 1257 & $\operatorname{Den}(\mathrm{D})$ & Smoke & SMD2S & & & & & & & & 0.085 & & & & & \\
\hline 1258 & Kitchen $(\mathrm{K})$ & & & & & & & & & & & & & & & \\
\hline 1259 & & & & & & & & & & & & & & & & \\
\hline
\end{tabular}




\begin{tabular}{|c|c|c|c|c|c|c|c|c|c|c|c|c|c|c|c|c|}
\hline & $\mathrm{A}$ & $\mathrm{B}$ & $\mathrm{C}$ & $\bar{D}$ & $\mathrm{E}$ & $\bar{F}$ & $\bar{G}$ & $\mathrm{H}$ & $T$ & $\mathrm{~J}$ & $\bar{K}$ & $\mathrm{~L}$ & $\bar{M}$ & $\bar{N}$ & $\mathrm{O}$ & $\bar{P}$ \\
\hline 1260 & Test & SDC28 & Fully-Furnishec & d Living Roo & & & & & & & & & & & & \\
\hline 1261 & & & & & & & & & & & & & & & & \\
\hline 1262 & Time & & & & & & & & & & & & & & & \\
\hline 1263 & & & & & & & & & & & & & & & & \\
\hline 1264 & ISO Gas FED $=0.3$ & & & & & & & & & & & & & & & \\
\hline 1265 & & & $\mathrm{CO} 2$ & CO2 Offset & CO2 Base & $\mathrm{co}$ & CO Offset & $\mathrm{HCN}$ & HCN Offse & ASET & Final Value & Minimum & & Outside Ro & om of Origin & \\
\hline 1266 & Bedroom 1 (B1) & FEDG & GASB3 & \begin{tabular}{|l|}
0.0299 \\
\end{tabular} & 0.035 & GASB1 & 0.003877 & - & - & 352 & 0.309 & 236 & (Smoke) & 250 & (Smoke) & \\
\hline 1267 & Upstairs Hallway $(\mathrm{H})$ & FEDG & GASF3 & 0.037252 & 0.035 & GASF1 & 0.008643 & - & - & 326 & 0.316 & & & & & \\
\hline 1268 & Bedroom 3 Burn Room (B3) & & & & & & & & & & & & & & & \\
\hline 1269 & Living Room Burn Room (L) & & & & & & & & & & & & & & & \\
\hline 1270 & Foyer (F) & & & & & & & & & & & & & & & \\
\hline 1271 & Den (D) & & & & & & & & & & & & & & & \\
\hline 1272 & Kitchen $(\mathrm{K})$ & & & & & & & & & & & & & & & \\
\hline 1273 & & & & & & & & & & & & & & & & \\
\hline 1274 & NIST Gas FED $=0.3$ & & & & & & & & & & & & & & & \\
\hline 1275 & & & $\mathrm{CO} 2$ & CO2 Offset & CO2 Base & $\mathrm{CO}$ & CO Offset & $\mathrm{HCN}$ & HCN Offse & ASET & Final Value & & & & & \\
\hline 1276 & Bedroom 1 (B1) & FEDGN & GASB3 & 0.0299 & 0.035 & GASB1 & 0.003877 & 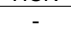 & - & & 0.092 & & & & & \\
\hline 1277 & Upstairs Hallway $(\mathrm{H})$ & FEDGN & GASF3 & \begin{tabular}{|l|}
0.037252 \\
\end{tabular} & 0.035 & GASF1 & 0.008643 & - & - & & 0.091 & & & & & \\
\hline 1278 & Bedroom 3 Burn Room (B3) & & & & & & & & & & & & & & & \\
\hline 1279 & Living Room Burn Room (L) & & & & & & & & & & & & & & & \\
\hline 1280 & Foyer (F) & & & & & & & & & & & & & & & \\
\hline 1281 & Den (D) & & & & & & & & & & & & & & & \\
\hline 1282 & Kitchen $(\mathrm{K})$ & & & & & & & & & & & & & & & \\
\hline 1283 & & & & & & & & & & & & & & & & \\
\hline 1284 & ISO Convected Heat $=0.3$ & & & & & & & & & & & & & & & \\
\hline 1285 & & & Temperature & & & & & & & ASET & Final Value & & & & & \\
\hline 1286 & Bedroom 1 (B1) & FEDH & TCB14 & & & & & & & 308 & 0.319 & & & & & \\
\hline 1287 & Upstairs Hallway $(\mathrm{H})$ & FEDH & $\mathrm{TCH} 4$ & & & & & & & 274 & 0.348 & & & & & \\
\hline$\frac{128}{1288}$ & Bedroom 3 Burn Room (B3) & FEDH & TCB34 & & & & & & & & 0 & & & & & \\
\hline 1289 & Living Room Burn Room (L) & FEDH & TCL4 & & & & & & & 262 & 0.397 & & & & & \\
\hline 1290 & Foyer $(F)$ & FEDH & TCF4 & & & & & & & 266 & 0.364 & & & & & \\
\hline 1291 & $\operatorname{Den}(\mathrm{D})$ & FEDH & TCD4 & & & & & & & 298 & 0.364 & & & & & \\
\hline 1292 & Kitchen $(\mathrm{K})$ & FEDH & TCK4 & & & & & & & 298 & 0.312 & & & & & \\
\hline 1293 & & & & & & & & & & & & & & & & \\
\hline 1294 & Smoke Obscuration $=0.25 \mathrm{~m}-1$ & & & & & & & & & & & & & & & \\
\hline 1295 & & & OD & & & & & & & ASET & Final Value & & & & & \\
\hline 1296 & Bedroom 1 (B1) & Smoke & SMB12S & & & & & & & 270 & 0.341 & & & & & \\
\hline 1297 & Upstairs Hallway $(\mathrm{H})$ & Smoke & SMH2S & & & & & & & 250 & 0.266 & & & & & \\
\hline 1298 & Bedroom 3 Burn Room (B3) & & & & & & & & & & & & & & & \\
\hline 1299 & Living Room Burn Room (L) & & & & & & & & & & & & & & & \\
\hline$\frac{1300}{1300}$ & Foyer (F) & Smoke & SMF2S & & & & & & & 236 & 0.270 & & & & & \\
\hline 1301 & $\operatorname{Den}(\mathrm{D})$ & Smoke & SMD2S & & & & & & & 268 & 0.345 & & & & & \\
\hline 1302 & Kitchen $(\mathrm{K})$ & & & & & & & & & & & & & & & \\
\hline
\end{tabular}




\section{Appendix B}

\section{FTIR Gas Measurement in Home Smoke Alarm Tests}

This appendix, reprinted with permission from the Institute for Research in Construction, National Research Council of Canada, uses the International System of Units (metric units) but not in full accordance with NIST style. The information is printed in its original format to preserve its integrity. 


\title{
FTIR Gas Measurement in Home Smoke Alarm Tests
}

Research Report 107

Date of Issue: September 2002

\author{
Authors: Joseph Z. Su \\ Malgosia Kanabus-Kaminska
}

Published by

Institute for Research in Construction

National Research Council Canada

Ottawa, Canada

K1A 0R6 


\title{
FTIR GAS MEASUREMENT IN HOME SMOKE ALARM TESTS
}

\author{
By \\ Joseph Z. Su and Malgosia Kanabus-Kaminska \\ Fire Risk Management Program \\ Institute for Research in Construction \\ National Research Council of Canada
}

\begin{abstract}
The National Research Council of Canada performed Fourier Transform Infrared (FTIR) gas measurements during fire detection experiments (Tests 1-14) in a manufactured home, as part of a joint study with several U.S. organisations and federal agencies to evaluate current requirements and technology of residential smoke detectors. The objectives were to identify toxic species (such as $\mathrm{HCl}, \mathrm{HCN}, \mathrm{NO}_{x}, \mathrm{HBr}$ and $\mathrm{HF}$ ) produced from test fires and to quantify their concentrations for use in determination of the onset of untenable conditions.

Fire scenarios included flaming and smouldering fires of a mattress in a bedroom, upholstered chair in the living area and cooking oil fires. The size, growth rate and duration of the test fires were closely controlled to create small, slow smouldering fires (1 to 2 hours) and short flaming fires (3 minutes or less). While these test fires provided the greatest challenge for smoke detectors to detect the fires early before becoming fully developed ones, the experiments were terminated well before reaching conditions that would produce a significant amount of toxic species.

FTIR spectra collected during all these tests show spectral features from $\mathrm{CO}, \mathrm{CO}_{2}$ and water vapour. FTIR spectra collected during the chair and mattress smouldering and cooking oil fires also show absorption characteristic of volatile hydrocarbon compounds. There was no apparent absorption from chemical species such as $\mathrm{HCl}, \mathrm{NO}_{x}, \mathrm{HBr}$ and $\mathrm{HF}$ in the FTIR spectra. These chemical species were below the minimum detection limit of the FTIR spectrometer in this full-scale experimental set-up. HCN was only detected in Test 14 with an under-ventilated condition and its maximum concentration was 60 ppm. Carbon monoxide and carbon dioxide were primary gas products produced in these fire detection tests.
\end{abstract}




\section{TABLE OF CONTENTS}

1.0 INTRODUCTION

2.0 EXPERIMENTS

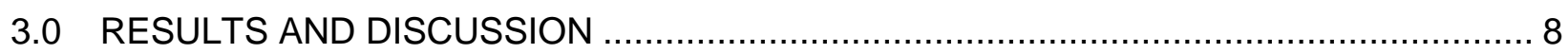

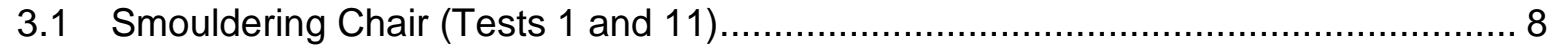

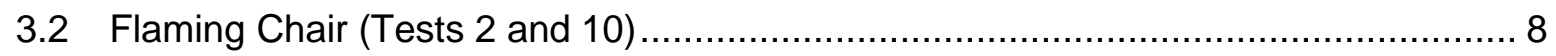

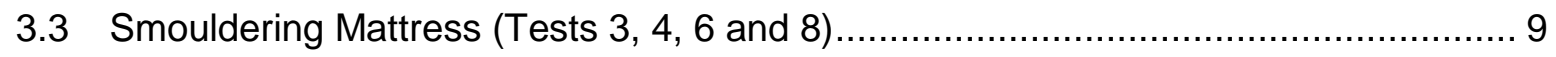

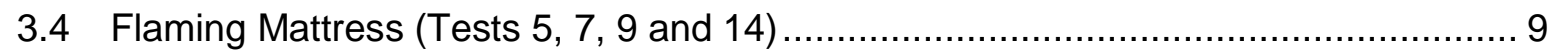

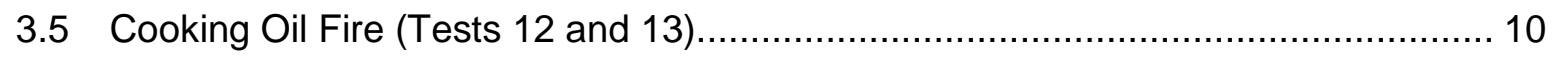

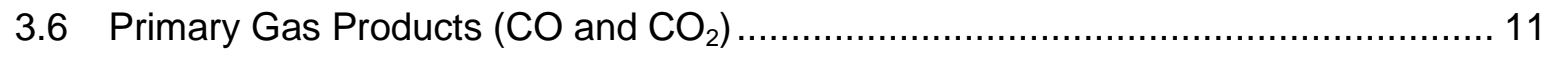

3.7 Secondary Gas Products ............................................................................... 11

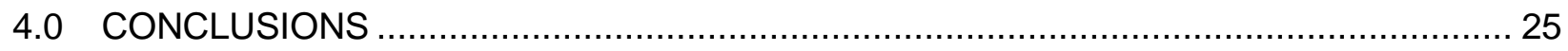

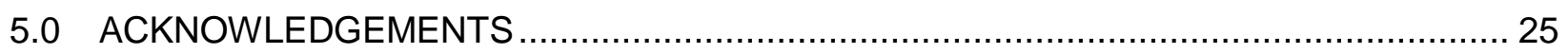

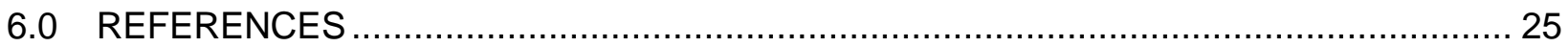

\section{LIST OF FIGURES}

Figure 1. Manufactured home and FTIR measurement system .......................................... 4

Figure 2. Plan view of a manufactured home, fire locations and

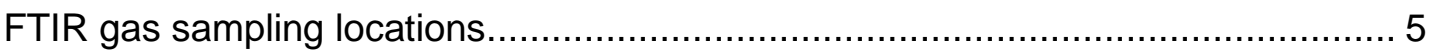

Figure 3. Upholstered chairs before and after smouldering and flaming fire tests ................... 6

Figure 4. Twin-size mattresses before and after smouldering and flaming fire tests .............. 7

Figure 5. 4-min average of FTIR spectra for fire location after first smoke alarm (Test 1) ..... 12

Figure 6. 4-min average of FTIR spectra for fire location (Test 1) ..................................... 12

Figure 7. 4-min average of FTIR spectra for the master bedroom (Test 1) .......................... 12

Figure 8. 4-min average of FTIR spectra for the master bedroom (Test 1) ......................... 13

Figure 9. 2-min average of spectra from fire location before water discharge in Test 2........ 13

Figure 10. 1-min average of spectra at peak smoke obscuration in the master bedroom

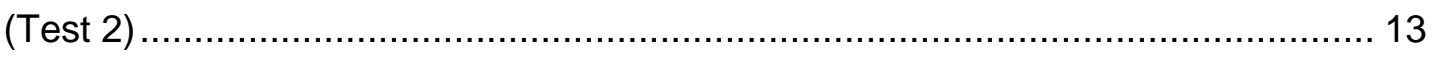

Figure 11. Average of spectra for fire location in the last 4 minutes of mattress smouldering (Test 4)

Figure 12. Average spectrum for the fire room during peak smoke obscuration in Test 6 with mattress smouldering ........................................................................ 14

Figure 13. 1-min average of spectra for the fire room after first smoke alarm in Test 8 with mattress smouldering ........................................................................... 14

Figure 14. Average of spectra for the fire room during the 50-s flaming in Test 4 .................. 15

Figure 15. Average of spectra for the fire room during the 60-s flaming in Test 6 .................. 15 
Figure 16. Average of spectra for the fire room during the 100 -s flaming in Test 8 .............. 15

Figure 17. 1-min average of spectra for the fire room during suppression in Test $4 \ldots \ldots \ldots \ldots \ldots . . . .16$

Figure 18. 4-min average of spectra for the master bedroom during/after suppression

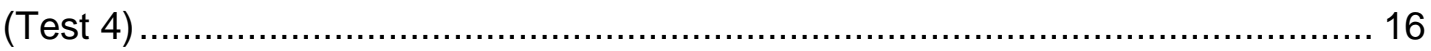

Figure 19. 2-min average of the spectra for the fire room during mattress flaming (Test 5).... 16

Figure 20. 3-min average of the spectra for the fire room during mattress flaming (Test 7) .... 17

Figure 21. 2-min average of the spectra for the fire room during mattress flaming (Test 9).... 17

Figure 22. 2-min average of the spectra for the fire room after fire extinguishment (Test 9)... 17

Figure 23. Average of the spectra for the fire room during the last $30 \mathrm{~s}$ of mattress smouldering (Test 14) ............................................................. 18

Figure 24. 30-s average of the spectra for the fire room after mattress flaming (Test 14) ....... 18

Figure 25. 90-s average of the spectra for the fire room after water spray (Test 14) ............. 18

Figure 26. 60-s average of the spectra for the fire room after all doors opened (Test 14) ....... 19

Figure 27. Average of spectra for last 2 minutes of cooking-oil heating (Test 13) ................ 20

Figure 28. Average of spectra for last 2 minutes of cooking oil flaming (Test 13)................. 20

Figure 29. 2-min average of spectra after cooking-oil fire extinguishment (Test 13) .............. 20

Figure 30. Carbon dioxide concentration in master bedroom from FTIR and NDIR

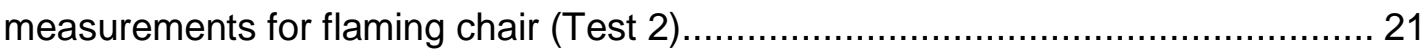

Figure 31. Carbon dioxide concentration in master bedroom from FTIR and NDIR measurements for smouldering chair (Test 11).....

Figure 32. Carbon dioxide concentration in fire room from FTIR and NDIR measurements for flaming mattress (Test 5)

Figure 33. Carbon dioxide concentration in fire room from FTIR and NDIR measurements for smouldering mattress (Test 6) 22

Figure 34. Carbon dioxide concentration in master bedroom from FTIR and NDIR measurements for cooking oil fire (Test 12)

Figure 35. Carbon monoxide concentration in fire room from FTIR and NDIR measurements for flaming mattress (Test 5)

Figure 36. Carbon monoxide concentration in fire room from FTIR and NDIR measurements for smouldering mattress (Test 6)

Figure 37. Carbon monoxide concentration in master bedroom from FTIR and NDIR measurements for flaming chair (Test 10) 


\title{
FTIR GAS MEASUREMENT IN HOME SMOKE ALARM TESTS
}

\author{
by \\ Joseph Z. Su and Malgosia Kanabus-Kaminska \\ Fire Risk Management Program \\ Institute for Research in Construction \\ National Research Council of Canada
}

\section{$1.0 \quad$ INTRODUCTION}

Smoke alarms are important and cost-effective fire protection devices in residential dwellings, providing fire detection and evacuation warnings for occupants in case of fires. Between 1985 and 1995, Canada's death rate in fires declined by more than 40 per cent, much of this decline is attributed to the use of residential smoke alarms and the enforcement of relevant codes and standards. This is also the case in the United States where the fire death rate has declined by approximately 50\% since 1975 .

In spite of this, the fire protection community has an ongoing task to maximize the benefit of current smoke detector technologies and develop new technologies in order to improve residential fire safety. As a contributor, the National Research Council of Canada (NRC) joined forces with several U.S. organizations and federal agencies to evaluate the current state of residential smoke detector requirements (known as the Dunes II Tests). This experimental program, commissioned by the U.S. Fire Administration and U.S. Consumer Product Safety Commission and executed by the National Institute of Standards and Technology (NIST), are another systematic evaluation of residential smoke detector technologies, 25 years after the first Indiana Dunes Tests [1].

The research involved 2 series of full-scale fire detection experiments. Series 1 experiments were conducted in a manufactured home at a NIST test site [2]. Series 2 experiments were conducted in a two-storey single house in Kinston, North Carolina [3]. The research program began in October 2000 and is completed in September 2002. The research aimed to determine the response of smoke, heat, and carbon monoxide detectors to modern residential fires and the available egress time before the onset of untenable conditions. General information on the research program is on the web site http://smokealarm.nist.gov/.

Inhalation of toxic smoke generated by fires has been the major cause of fire fatalities. NRC's role in this important testing program has been to provide expertise in performing Fourier Transform Infrared (FTIR) measurement and analysis of smoke and toxic gases (such as $\mathrm{HCl}$, $\mathrm{HCN}, \mathrm{NO}_{x}, \mathrm{HBr}$ and $\mathrm{HF}$ ) during fire detection experiments and to participate in the project steering committee. The objectives were to identify and quantify the concentrations of harmful species produced from test fires and to provide data for use in determination of the onset of untenable conditions.

NRC participated in, and performed the FTIR gas measurement during Series 1 experiments in the manufactured home at the NIST site (Tests 1-14 in May and June 2001). The results of the FTIR measurement and data analysis for toxic gases are documented in this report. 


\subsection{EXPERIMENTS}

Experimental details of detector placement, instrumentation for various measurements (temperature, heat release rate, mass loss rate, smoke properties, response of various detectors, etc.) and test conditions are described in the NIST report [2]. The following sections only address matters that directly relate to the FTIR gas measurement.

Figure 1 shows a manufactured home and FTIR measurement system that were used in full-scale fire detection experiments. This was a 900-square-foot one-storey house with 3 bedrooms, a bathroom, a kitchen, and a living area. Figure 2 shows a plan view of the test house, fire locations, and FTIR measurement points.

Fire scenarios included flaming and smouldering fires of a twin-size mattress in the end bedroom and an upholstered chair in the living area. Figures 3-4 shows chair and mattress fuel packages and ignition sources used in the experiments. The flaming fire was started using an electric match that was inserted into a fold of the upholstered chair or mattress. The smouldering fire was started using an electric nichrome-wire loop (3.8-cm or 10-cm diameter) that was inserted into the polyurethane foam of the chair or mattress. An autotransformer was used to regulate the electric current to the smouldering loop in order to control the heat. Fire scenarios also included cooking oil fires, ignited by heating corn oil $(500 \mathrm{~mL})$ on a gas burner. These fuel packages were representative of common household items that were often first ignited in real fire incidents. In each experiment, the electric power to the ignition source or the gas burner was turned on 3 minutes after the start of the test.

NRC performed FTIR gas measurements during 14 full-scale fire detection experiments (8 experiments with fire origin in the end bedroom, 4 experiments with fire origin in the living area and 2 experiments with fire origin in the kitchen). Four gas sampling ports were located along the centre line of the house as shown in Figure 2. These included a ceiling sampling port ( $2.4 \mathrm{~m}$ above floor) in each of the 3 rooms of fire origin, and a target sampling port in the remote master bedroom at $1.5 \mathrm{~m}$ above floor ( $0.6 \mathrm{~m}$ from the end wall).

Each experiment involved a ceiling sampling port in the room of fire origin and the target sampling port for the gas measurement. Gas samples were drawn by pumps from the ceiling sampling port in the room of fire origin and the target sampling port in the master bedroom through two gas sampling lines to 2 FTIR spectrometers, respectively. Pyrex cylindrical gas cells (one with $10-\mathrm{cm}$ pathlength and $110-\mathrm{mL}$ volume, the other with $100-\mathrm{cm}$ pathlength and 120 -mL volume) with $\mathrm{KBr}$ optics were used for the FTIR spectrometers. The gas samples flowed through the gas cells at a flow rate of $9 \mathrm{~L} / \mathrm{min}$. To prevent the condensation of sample gases, the sampling lines and gas cells were heated (the temperature setting was $93^{\circ} \mathrm{C}$ for the line to the fire room and $121^{\circ} \mathrm{C}$ for the line to the master bedroom). The infrared light that emitted from the spectrometer passed through the gas cell. The FTIR spectrometers scanned the gas samples over a wide frequency range of 400 to $4500 \mathrm{~cm}^{-1}$ at a $1-\mathrm{cm}^{-1}$ resolution.

Chemical compounds that give rise to a permanent or oscillating dipole moment can absorb the infrared light at unique characteristic frequencies, which serve as their "fingerprints". Species, such as $\mathrm{HCl}, \mathrm{HCN}, \mathrm{NO}_{x}, \mathrm{HBr}$ and $\mathrm{HF}$, absorb the infrared light and leave "fingerprints" at their characteristic frequencies in the infrared spectrum. The magnitude of the absorption of each species at its characteristic frequencies is a function of its concentration. With calibration of the FTIR spectrometers using standard gases at known concentrations, the concentration of each species produced during fire experiments can be quantified. 
Table 1 shows a matrix of the FTIR measurement during the fire experiments. The bathroom window was partially open $(33 \mathrm{~cm} \times 20 \mathrm{~cm}$ opening) to make up air in the house. All exterior doors and other windows were closed during each experiment. The master bedroom door was always open. The doors of the smallest bedroom and the bathroom were always closed. The end bedroom door was open unless otherwise specified.

TABLE 1

FTIR Measurement during Tests in Manufactured Home (May and June, 2001)

\begin{tabular}{|c|c|c|c|c|c|}
\hline & Test 1 & Test 2 & Test 3 & Test 4 & Test 5 \\
\hline Fire Origin & Living Area & Living Area & End Bedroom & End Bedroom & End Bedroom \\
\hline Fuel & $\begin{array}{c}\text { Upholstered } \\
\text { Chair }\end{array}$ & $\begin{array}{c}\text { Upholstered } \\
\text { Chair }\end{array}$ & Mattress & Mattress & Mattress \\
\hline Burning Mode & Smouldering & Flaming & Smouldering & Smouldering & Flaming \\
\hline $\begin{array}{c}\text { Burst to } \\
\text { Flame at }\end{array}$ & $2 \mathrm{~h} 8 \mathrm{~min}$ & $3 \mathrm{~min}$ & no flame & $58 \mathrm{~min}$ & $31 / 2 \mathrm{~min}$ \\
\hline $\begin{array}{c}\text { FTIR Gas } \\
\text { Sampling }\end{array}$ & $2 \mathrm{~h} 11 \mathrm{~min}$ & $15 \mathrm{~min}$ & $1 \mathrm{~h} 33 \mathrm{~min}$ & $1 \mathrm{~h} 10 \mathrm{~min}$ & $14 \mathrm{~min}$ \\
\hline
\end{tabular}

\begin{tabular}{|c|c|c|c|c|c|}
\hline & Test 6 & Test 7 & Test 8 & Test 9 & Test 10 \\
\hline Fire Origin & End Bedroom & End Bedroom & End Bedroom & $\begin{array}{l}\text { End Bedroom } \\
\text { (Door Closed) }\end{array}$ & Living Area \\
\hline Fuel & Mattress & Mattress & Mattress & Mattress & $\begin{array}{c}\text { Upholstered } \\
\text { Chair }\end{array}$ \\
\hline Burning Mode & Smouldering & Flaming & Smouldering & Flaming & Flaming \\
\hline $\begin{array}{l}\text { Burst to } \\
\text { Flame at }\end{array}$ & $1 \mathrm{~h} 42 \mathrm{~min}$ & $3 / 2 \min$ & $1 \mathrm{~h} 4 \mathrm{~min}$ & $3 \mathrm{~min}$ & $\begin{array}{c}71 / 2 \text { min } \\
\left(2^{\text {nd }} \text { ignition }\right)\end{array}$ \\
\hline $\begin{array}{l}\text { FTIR Gas } \\
\text { Sampling }\end{array}$ & 1 h 52 min & $15 \mathrm{~min}$ & $1 \mathrm{~h} 11 \mathrm{~min}$ & $25 \mathrm{~min}$ & $15 \mathrm{~min}$ \\
\hline
\end{tabular}

\begin{tabular}{|c|c|c|c|c|l|}
\hline & Test 11 & Test 12 & Test 13 & Test 14 & \\
\hline Fire Origin & Living Area & Kitchen & Kitchen & $\begin{array}{c}\text { End Bedroom } \\
\text { (Door Closed) }\end{array}$ & \\
\hline Fuel & $\begin{array}{c}\text { Upholstered } \\
\text { Chair }\end{array}$ & Cooking Oil & Cooking Oil & Mattress & \\
\hline Burning Mode & Smouldering & Flaming & Flaming & $\begin{array}{c}\text { Smouldering } / \\
\text { Flaming }\end{array}$ & \\
\hline $\begin{array}{c}\text { Burst to } \\
\text { Flame at }\end{array}$ & $1 \mathrm{~h} 13 \mathrm{~min}$ & $26 \mathrm{~min}$ & $25 \mathrm{~min}$ & $1 \mathrm{~h}$ & \\
\hline $\begin{array}{c}\text { FTIR Gas } \\
\text { Sampling }\end{array}$ & $1 \mathrm{~h} 20 \mathrm{~min}$ & $33 \mathrm{~min}$ & $36 \mathrm{~min}$ & $1 \mathrm{~h} 14 \mathrm{~min}$ & \\
\hline
\end{tabular}

Note: the electric power to the ignition source or the gas burner was turned on 3 min after start of a test. 


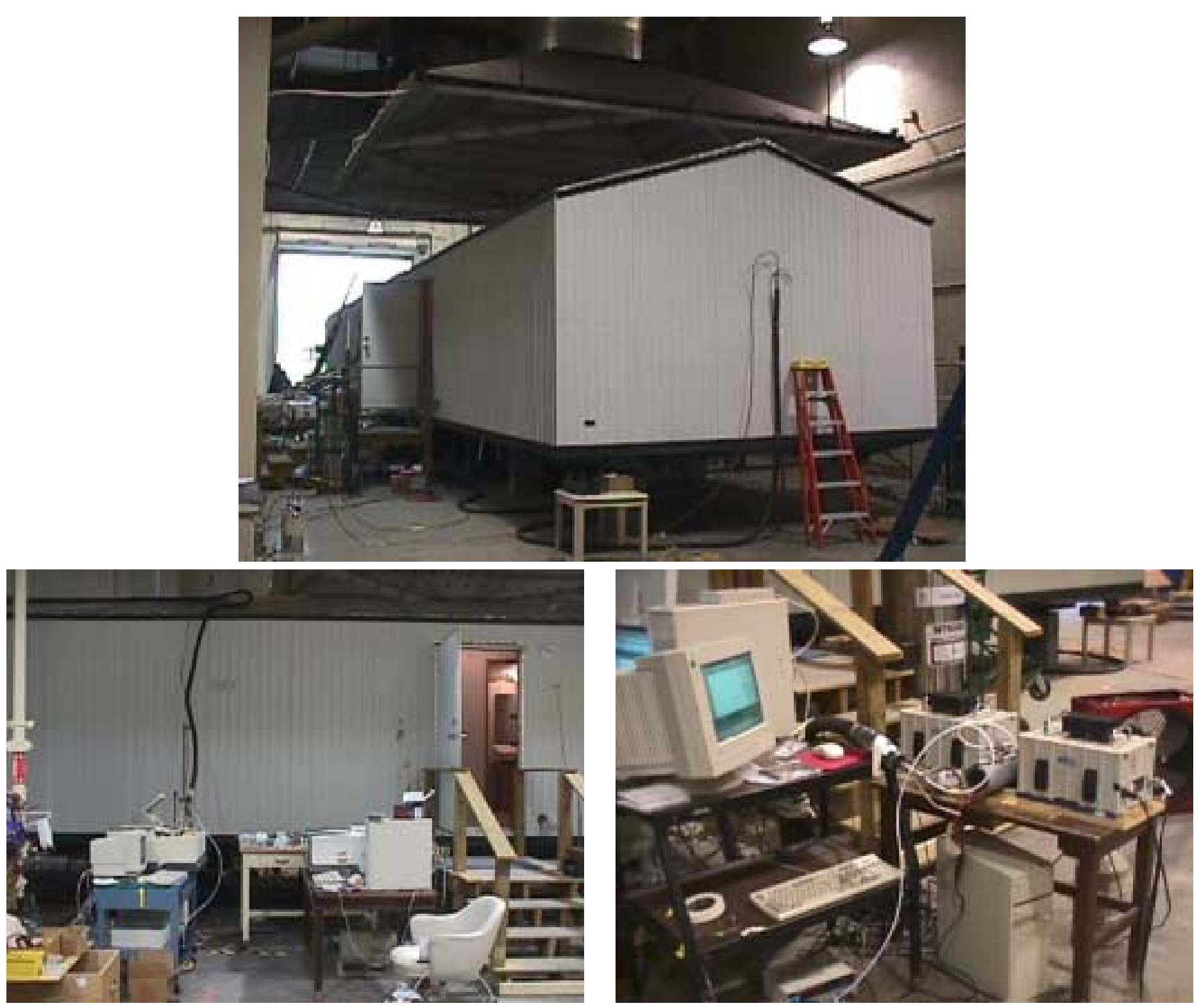

Figure 1. Manufactured home and FTIR measurement system 


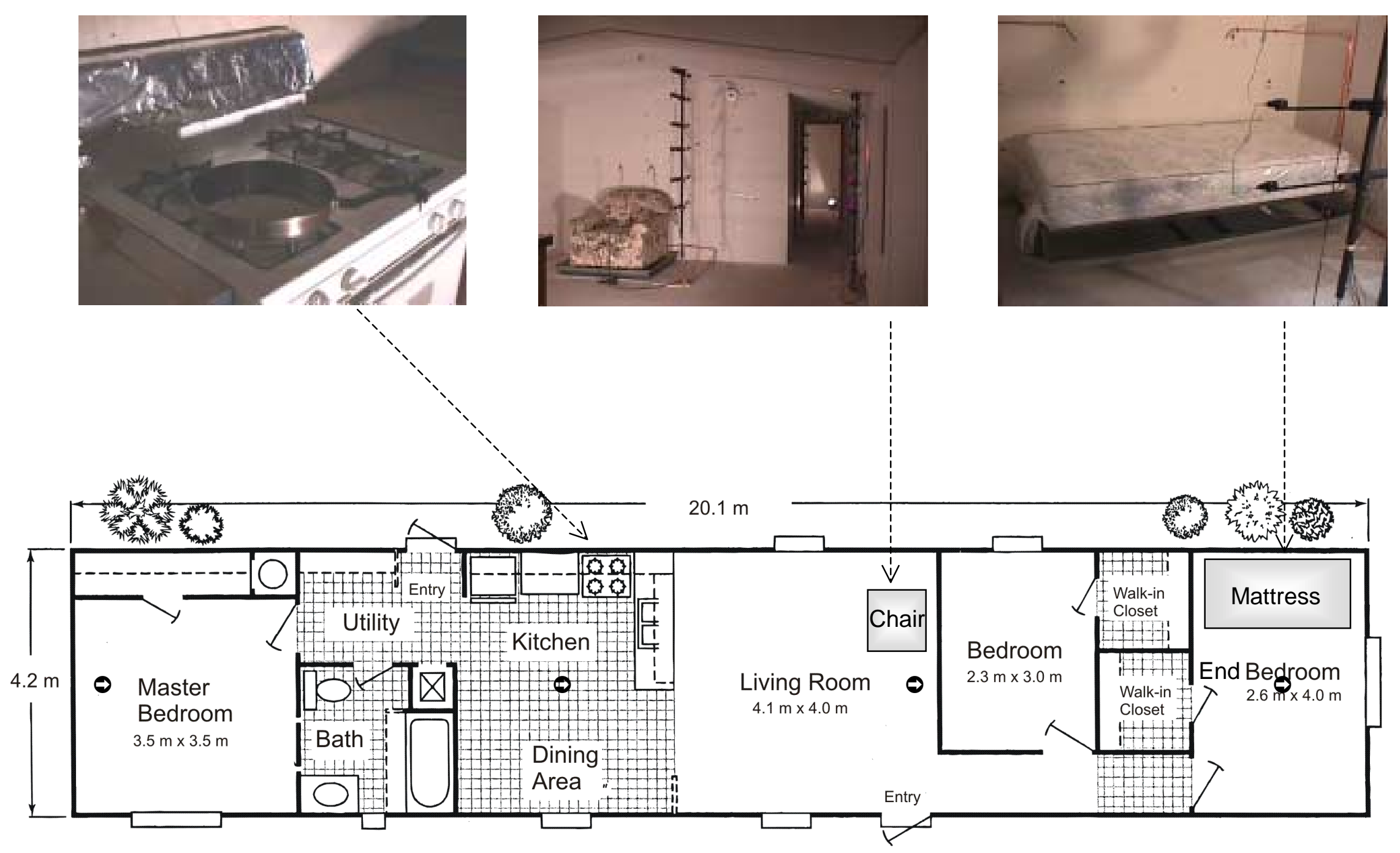

Figure 2. Plan view of a manufactured home (floor plan courtesy of NIST), fire locations (chair, mattress, kitchen stove) and FTIR gas sampling locations $(\boldsymbol{\theta})$ 

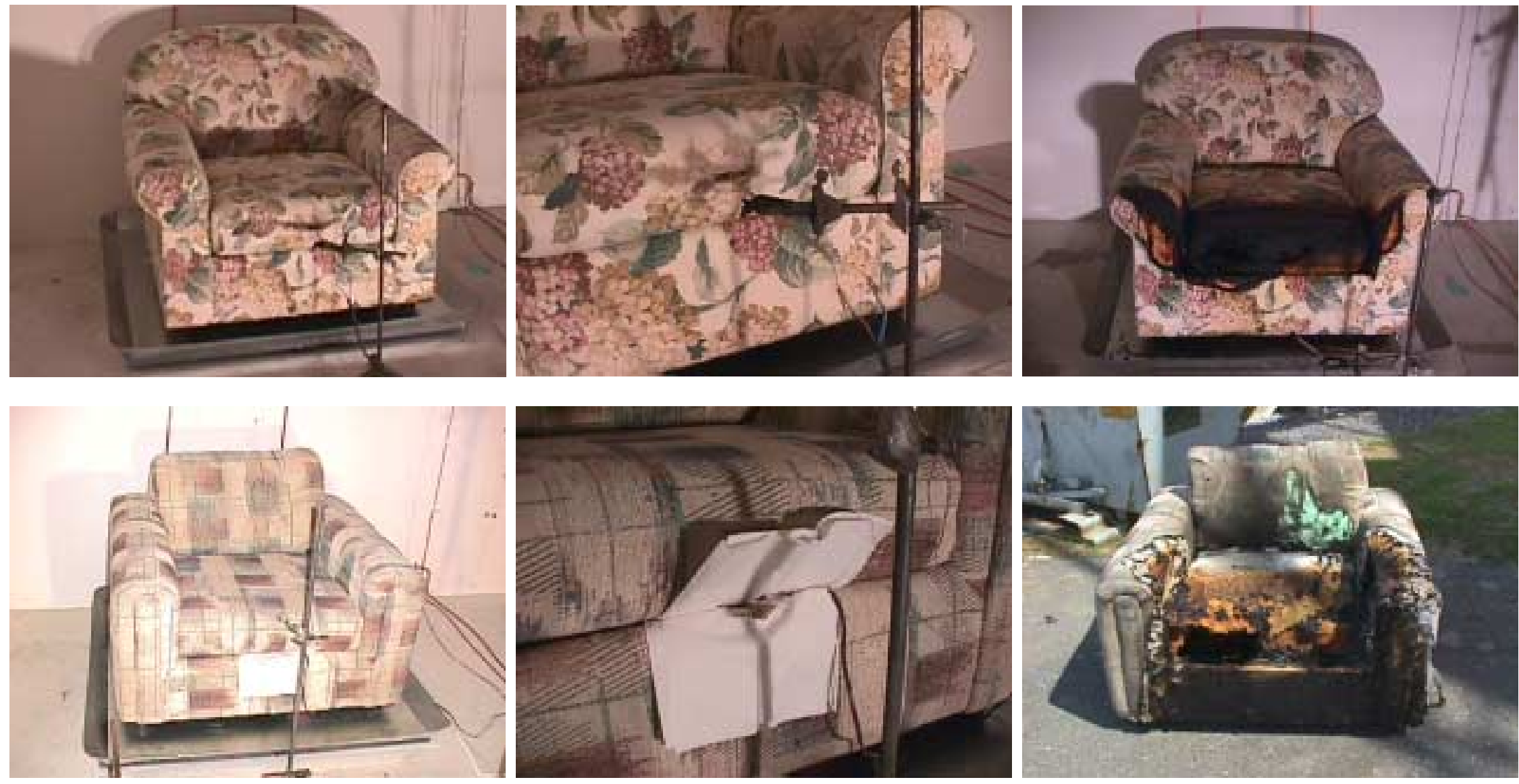

Figure 3. Upholstered chairs before and after smoldering ( $1^{\text {st }}$ row $)$ and flaming $\left(2^{\text {nd }}\right.$ row $)$ fire tests 

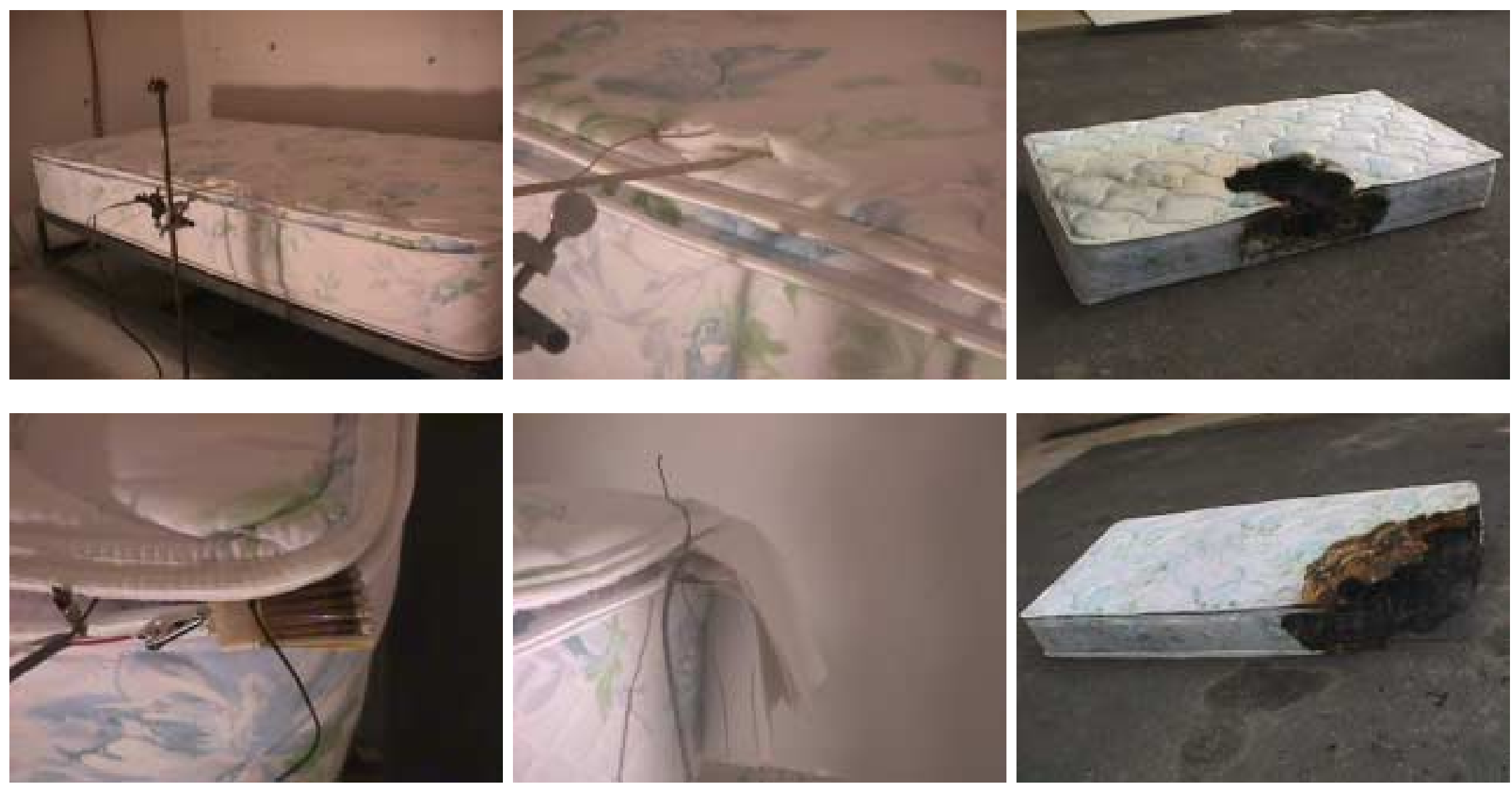

Figure 4. Twin-size mattresses before and after smoldering ( $1^{\text {st }}$ row $)$ and flaming $\left(2^{\text {nd }}\right.$ row $)$ fire tests 


\subsection{RESULTS AND DISCUSSION}

A total of 50,000 frames of FTIR spectra (approximately 2 gigabytes) was collected during Tests 1-14 and analyzed in various ways to determine gas components in smoke. Final analysis results are presented as follows along with representative spectroscopic information.

\subsection{Smouldering Chair (Tests 1 and 11)}

In Tests 1 and 11, an upholstered chair was used as the fuel package to create a smouldering fire in the living area. The smouldering fire was controlled to develop very slowly. The ceiling temperature rise at the living area was $3^{\circ} \mathrm{C}$ or less during the smouldering period.

Figure 5 shows an FTIR spectrum after the first smoke alarm was actuated ( 57 min after energizing the ignitor) during Test 1, which is a 4-min average of the spectra from 56 to $60 \mathrm{~min}$ for the fire location. Figure 6 shows another average spectrum from the fire location and Figure 7 shows an average spectrum for the master bedroom for the following 4 minutes.

In Test 1 , most of other detectors were actuated at least 87 min after energizing the ignitor. Figure 8 shows a 4-min average spectrum for the master bedroom (around $104 \mathrm{~min}$ ) when the smoke obscuration, concentrations of $\mathrm{CO}_{2}$ and $\mathrm{CO}$ and analog outputs of various detectors in the master bedroom were at peak values.

These spectra are representative of all spectra collected in Tests 1 and 11 during the smouldering chair fire, showing absorption from $\mathrm{CO}, \mathrm{CO}_{2}$ and water vapour as well as an absorption band around $3000 \mathrm{~cm}^{-1}$. This absorption band around $3000 \mathrm{~cm}^{-1}$ is characteristic of $\mathrm{C}-\mathrm{H}$ stretching of volatile hydrocarbon compounds.

\subsection{Flaming Chair (Tests 2 and 10)}

In Tests 2 and 10, an upholstered chair was used as the fuel package to create a flaming fire in the living area. The first smoke alarm was actuated $24 \mathrm{~s}$ and $44 \mathrm{~s}$ after the ignition in Test 2 and Test 10, respectively; activation of many other detectors followed. Approximately 3 minutes after flaming, experimental conditions met the criteria for terminating the test (set by NIST) and water was discharged on the fire. The tests were ended with fire suppression and ventilation. Before the fire suppression, the ceiling temperature of the living area reached $125^{\circ} \mathrm{C}$ in Test 2 and $500^{\circ} \mathrm{C}$ in Test 10.

Figure 9 shows a 2-min average of the spectra from the fire location before the water discharge in Test 2. Figure 10 shows a 1-min average of the spectra from the master bedroom when the smoke obscuration and $\mathrm{CO}_{2}$ concentration in the master bedroom were at peak values in the same test.

These spectra are representative of all spectra collected in Tests 2 and 10 during the flaming chair fire. The spectra show absorption only from $\mathrm{CO}, \mathrm{CO}_{2}$ and water vapour. 


\subsection{Smouldering Mattress (Tests 3, 4, 6 and 8)}

In Tests 3, 4, 6 and 8, a twin-size mattress was used as the fuel package to create a smouldering fire in the end bedroom. During the smouldering period (90, 55, 99 and 61 min long, respectively), the change of temperatures in the house was very small in these tests; the maximum temperature rise was approximately $2^{\circ} \mathrm{C}$ in the room of fire origin.

Figures 11-13 are a few of representative spectra during mattress smouldering. Figure 11 shows an FTIR spectrum in Test 4, which is an average of the spectra from the fire location during the last 4 minutes (51 to 55 min from energizing the ignitor) of smouldering. Figure 12 is an average of the spectra from the fire room during a peak period of smoke obscuration when the first smoke alarm actuated (3443 s after energizing the ignitor) in Test 6. Figure 13 is a 1-min average of the spectra from the fire room when the first smoke alarm actuated (1950 s after energizing the ignitor) in Test 8 . These spectra show absorption from water vapour, $\mathrm{CO}, \mathrm{CO}_{2}$ and an absorption band around $3000 \mathrm{~cm}^{-1}$ due to $\mathrm{C}-\mathrm{H}$ stretching of volatile hydrocarbon compounds.

The mattress smouldering changed to flaming 55, 99 and 61 minutes after energizing the ignitor in Tests 4, 6 and 8, respectively. Figure 14 shows an average of the spectra from the fire room during the 50 -second-long flaming in Test 4 . Figure 15 shows an average of the spectra from the fire room during the 60 -second-long flaming in Test 6 . Figure 16 shows an average of the spectra from the fire room during the 100-second-long flaming in Test 8 . These spectra also show $\mathrm{CO}$ and $\mathrm{CO}_{2}$ absorption as well as absorption from water vapour and $\mathrm{C}-\mathrm{H}$ stretching of volatile hydrocarbon compounds.

Once the mattress started flaming, the ceiling temperature in the fire room rose quickly. The transition from smouldering to flaming was the criterion for terminating the tests (set by NIST). Water was discharged to suppress the fire (flaming only lasted for approximately 50-100 $\mathrm{s}$ in these mattress smouldering tests). Figure 17 shows a 1-min average of the spectra from the fire room during the fire suppression in Test 4. Figure 18 shows a 4-min average of the spectra from the master bedroom during and after the fire suppression in Test 4 when the smoke obscuration and analog output of various detectors were at peak values in the master bedroom.

The spectra shown in Figures 11-18 are representative of all spectra collected in Tests 3, 4, 6 and 8. Other than $\mathrm{CO}, \mathrm{CO}_{2}$, water and $\mathrm{C}-\mathrm{H}$ stretching of volatile hydrocarbon compounds, there was no distinct absorption from any other chemical species in the FTIR spectra.

\subsection{Flaming Mattress (Tests 5, 7, 9 and 14)}

In Tests 5, 7, 9 and 14, a twin-size mattress was used as the fuel package to create a flaming fire in the end bedroom. Note that the fire-room door was closed in Tests 9 and 14.

In Tests 5 and 7 , the first smoke alarm actuated $32 \mathrm{~s}$ and $43 \mathrm{~s}$ after the ignition, respectively. Activation of many other detectors followed. Approximately 3 minutes after ignition, experimental conditions met the criteria for terminating the tests (set by NIST) and water was discharged to the fire. The test was ended with fire suppression and ventilation. Before the fire suppression, the ceiling temperature of the fire room reached $180^{\circ} \mathrm{C}$ in Test 5 
and $500^{\circ} \mathrm{C}$ in Test 7 . Figure 19 shows a 2-min average of the spectra from the fire location during the flaming period in Test 5 . Figure 20 shows a 3-min average of the spectra from the fire room during the flaming period in Test 7 . The spectra show absorption pattern only from $\mathrm{CO}, \mathrm{CO}_{2}$ and water vapour.

Test 9 was similar to Tests 5 and 7 . But, the fire room door was closed in Test 9. The first smoke alarm actuated $30 \mathrm{~s}$ after the ignition. Experimental conditions met the criteria for terminating the test approximately 3 minutes after ignition; water was discharged to extinguish the fire. Before the fire suppression, the ceiling temperature of the fire room reached $340^{\circ} \mathrm{C}$. Figure 21 shows a 2-min average of the spectra from the fire room during mattress flaming. Figure 22 shows a 2-min average of the spectra from the fire room after fire extinguishment. The spectrum shows absorption only from $\mathrm{CO}_{2}, \mathrm{CO}$ and water vapour.

Test 14 was similar to Test 9 with the fire-room door being closed. However, the mattress smouldered for $3400 \mathrm{~s}$. The first smoke alarm in the fire room was actuated $2648 \mathrm{~s}$ after energizing the ignitor. Figure 23 shows a 30-s average of the spectra from the fire room during the last 30 seconds of mattress smouldering. The mattress fire became a flaming one approximately $3400 \mathrm{~s}$ after energizing the ignitor. Figure 24 shows a 30 -s average of the spectra from the fire room during flaming. Approximately 1 minute after flaming, experimental conditions met the criteria for terminating the test and water was discharged to extinguish the fire (the ceiling temperature of the fire room reached $380^{\circ} \mathrm{C}$ before the fire suppression). Figure 25 shows a 1.5-min average of the spectra from the fire room after water spray. The fire room door was kept closed for another 9 minutes. Then, the doors of the fire room, front and back entrances were opened to allow smoke moving from the fire room to other parts of the house and outside. Figure 26 shows a 1-min average of the spectra from the fire room after all the doors were opened.

Figures 19-26 are representative of all spectra collected in Tests 5, 7, 9 and 14 during the flaming mattress fire. These spectra show absorption mainly from $\mathrm{CO}, \mathrm{CO}_{2}$ and water vapour. Prior to mattress flaming in Test 14, the spectra indicate weak absorption band around $3000 \mathrm{~cm}^{-1}$ due to $\mathrm{C}-\mathrm{H}$ stretching of volatile hydrocarbon compounds released during pyrolysis of the mattress. Since this test was conducted with an under-ventilated condition, the spectra did show absorption from HCN. The HCN concentration was at a maximum of $60 \mathrm{ppm}$, which was determined from the 1-min average spectra immediately after spraying water on the fire.

\subsection{Cooking Oil Fire (Tests 12 and 13)}

In Tests 12 and 13, corn oil (500 mL) in a cooking pan was heated on a gas burner until flaming. The propane stove was turned on 3 minutes after the start of the test. Smoke started to be produced (smouldering mode). The first smoke alarm actuated 9 to 10 minutes after heating the oil in these two tests. The cooking oil reached $382-387^{\circ} \mathrm{C}$ and auto-ignited $22-23$ minutes after being heated in these two tests. In Test 12, the cooking oil flamed for 2.5 minutes and was manually extinguished. In Test 13, the cooking oil flamed for 5 minutes and then selfextinguished. Before the fire extinguishment, the ceiling temperature in the living area was $121^{\circ} \mathrm{C}$ in Test 12 and $118^{\circ} \mathrm{C}$ in Test 13 ; the ceiling temperature in the master bedroom was $59^{\circ} \mathrm{C}$ in Test 12 and $55^{\circ} \mathrm{C}$ in Test 13.

Figures 27-29 show 2-min average of the spectra from the kitchen area at different stages of Test 13. Figure 27 shows a 2 -min average of the spectra from the kitchen area during the last 2 minutes of heating (before flaming). Figure 28 shows a 2-min average of the spectra 
from the kitchen area during the last 2 minutes of flaming. Figure 29 shows a 2-min average of the spectra from the kitchen area after fire self-extinguishment. The spectra show absorption from $\mathrm{CO}, \mathrm{CO}_{2}$, water vapour and an absorption band around $3000 \mathrm{~cm}^{-1}$ due to $\mathrm{C}-\mathrm{H}$ stretching of volatile hydrocarbon compounds. These spectral features are representative of all spectra collected in Tests 12 and 13 with the cooking oil fire.

\subsection{Primary Gas Products $\left(\mathrm{CO}\right.$ and $\left.\mathrm{CO}_{2}\right)$}

$\mathrm{CO}$ and $\mathrm{CO}_{2}$ are primary gas products produced in the fire detection tests. The FTIR spectrometer was calibrated on site for $\mathrm{CO}_{2}$ at concentrations of $1089 \mathrm{ppm}, 5123 \mathrm{ppm}$ and $2.01 \%$ and for $\mathrm{CO}$ at concentrations of $216 \mathrm{ppm}$ and $1031 \mathrm{ppm}$. The transport time of the gas sample in the sampling lines was $10 \pm 2 \mathrm{~s}$, which was determined by aspirating the $2.01 \% \mathrm{CO}_{2}$ calibration gas through the sampling lines.

Figures 30-37 show typical $\mathrm{CO}$ and $\mathrm{CO}_{2}$ concentrations from the FTIR analysis. Results from the FTIR measurement are consistent with those measured by NIST using the nondispersive infrared (NDIR) gas analyzers. For smouldering fires, most of the concentration rise happened after transition to flaming, similar to other parameters measured by NIST.

\subsection{Secondary Gas Products}

The main objectives of the FTIR measurement were to identify secondary gas products from the test fires. In addition to $\mathrm{CO}$ and $\mathrm{CO}_{2}$, the FTIR measurement showed absorption around $3000 \mathrm{~cm}^{-1}$, characteristic of $\mathrm{C}-\mathrm{H}$ stretching of volatile hydrocarbon compounds from the chair and mattress smouldering as well as cooking oil fires. There was no apparent absorption from chemical species such as $\mathrm{HCl}, \mathrm{NO}_{x}, \mathrm{HBr}$ and $\mathrm{HF}$ in the FTIR spectra. In other words, these chemical species were below the minimum detection limit (MDL) of the FTIR spectrometer in this full-scale experimental set-up. HCN was only detected in Test 14 with an underventilated condition and its maximum concentration was $60 \mathrm{ppm}$.

The MDL depended not only on the quality of FTIR spectrometer's infrared source/detector and optical stability but also on operation parameters such as gas cell, number of scans and spectral resolution, as well as gas sampling systems used in the experiments. The MDL under this full-scale experimental set-up and operation conditions was estimated to be 30 ppm for $\mathrm{HCN}$ and $\mathrm{HF}, 50$ ppm for $\mathrm{HCl}$ and $\mathrm{NO}_{2}$, and $100 \mathrm{ppm}$ for $\mathrm{NO}$ and $\mathrm{HBr}$, based on previous calibration and data from similar gas sampling tests at NRC as well as library data from the FTIR spectrometer manufacturer. 


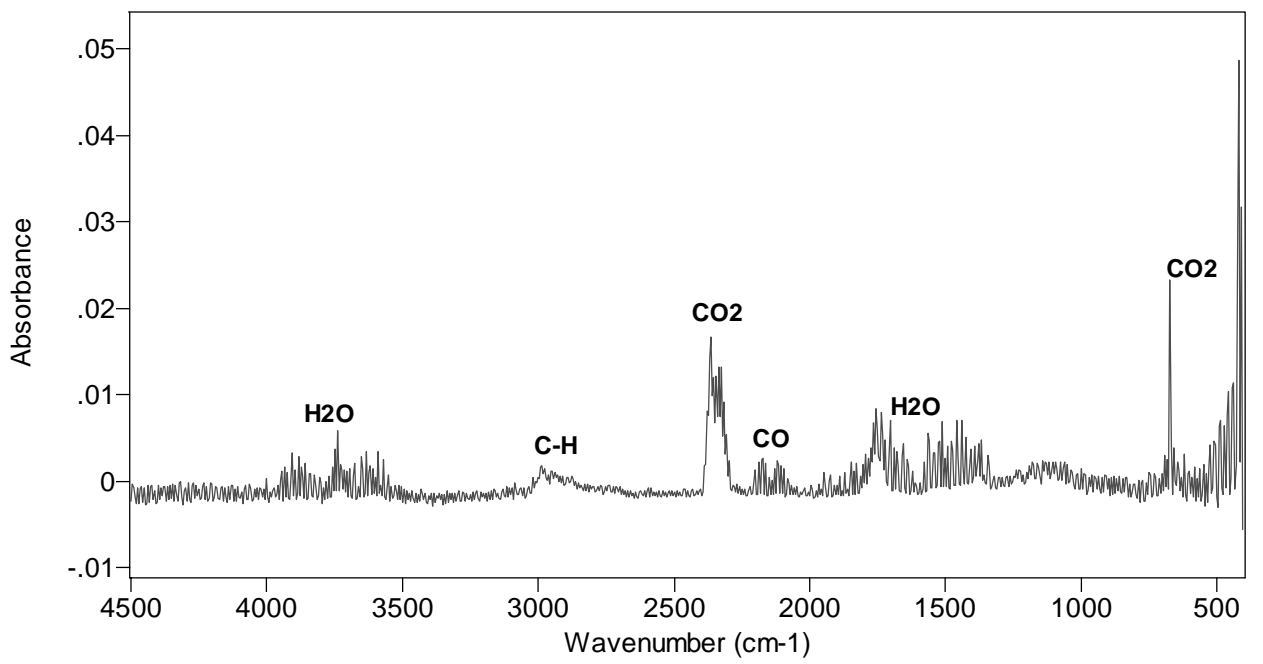

Figure 5. 4-min average of FTIR spectra for fire location after first smoke alarm (Test 1)

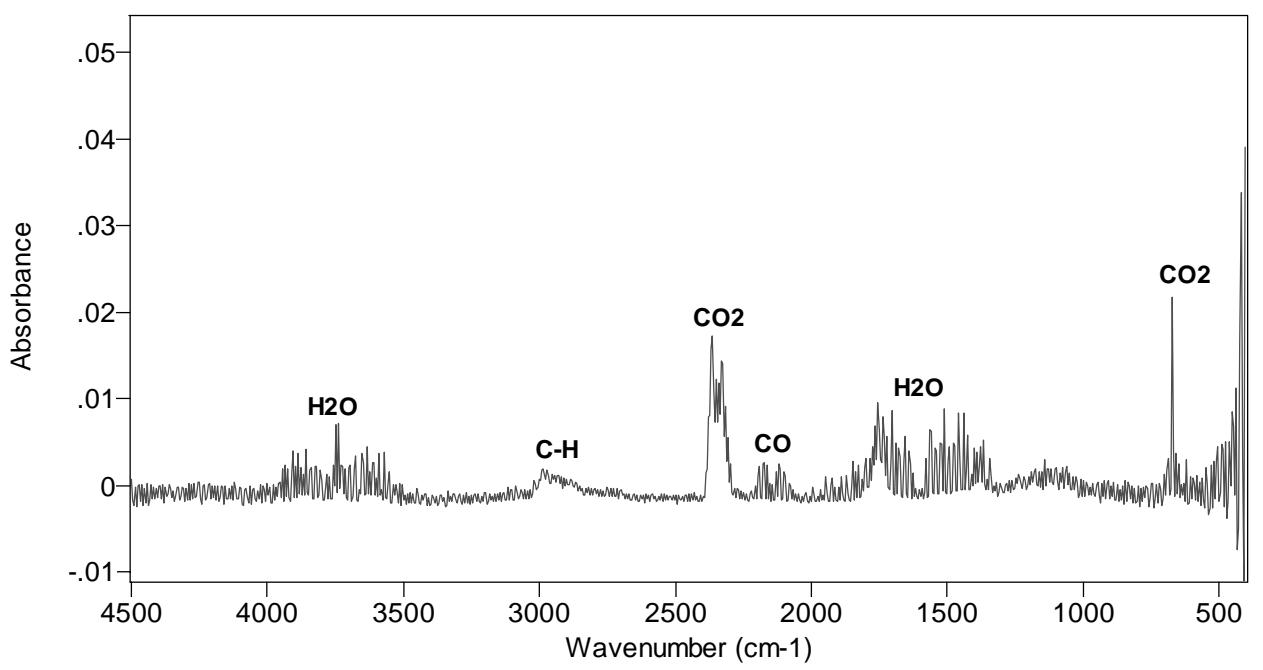

Figure 6. 4-min average of FTIR spectra (at $60 \mathrm{~min}$ ) for fire location (Test 1)

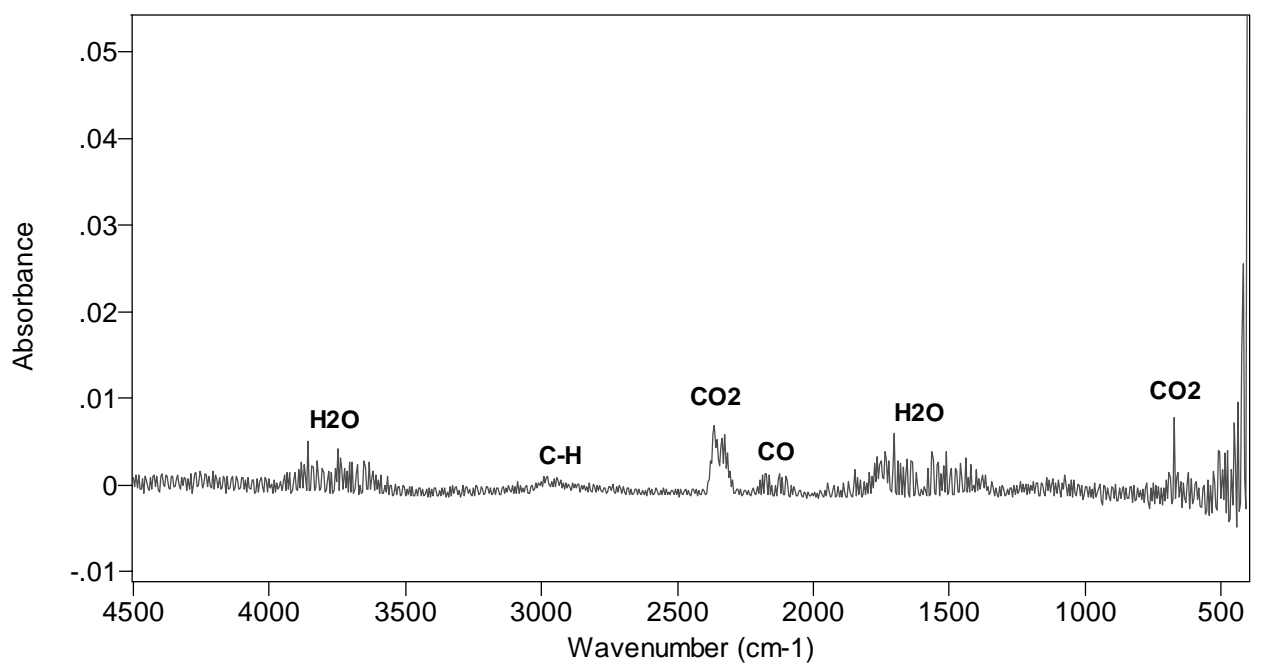

Figure 7. 4-min average of FTIR spectra (at $60 \mathrm{~min}$ ) for master bedroom (Test 1) 


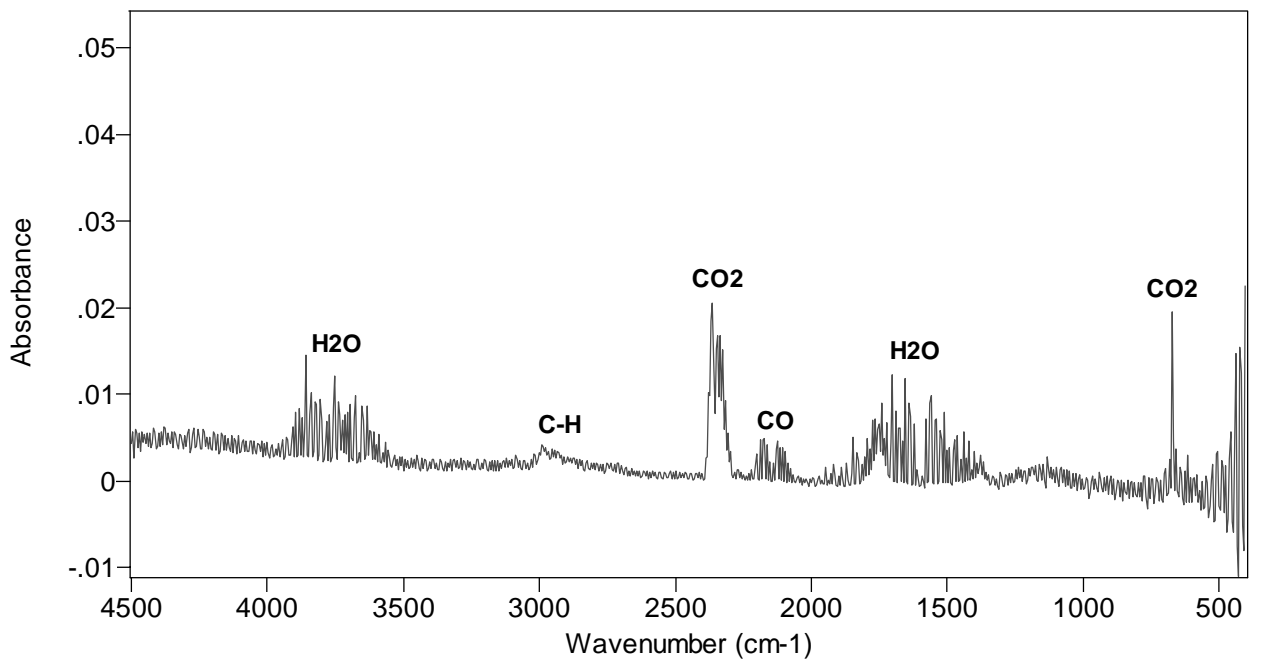

Figure 8. 4-min average of FTIR spectra (at $104 \mathrm{~min}$ ) for master bedroom (Test 1)

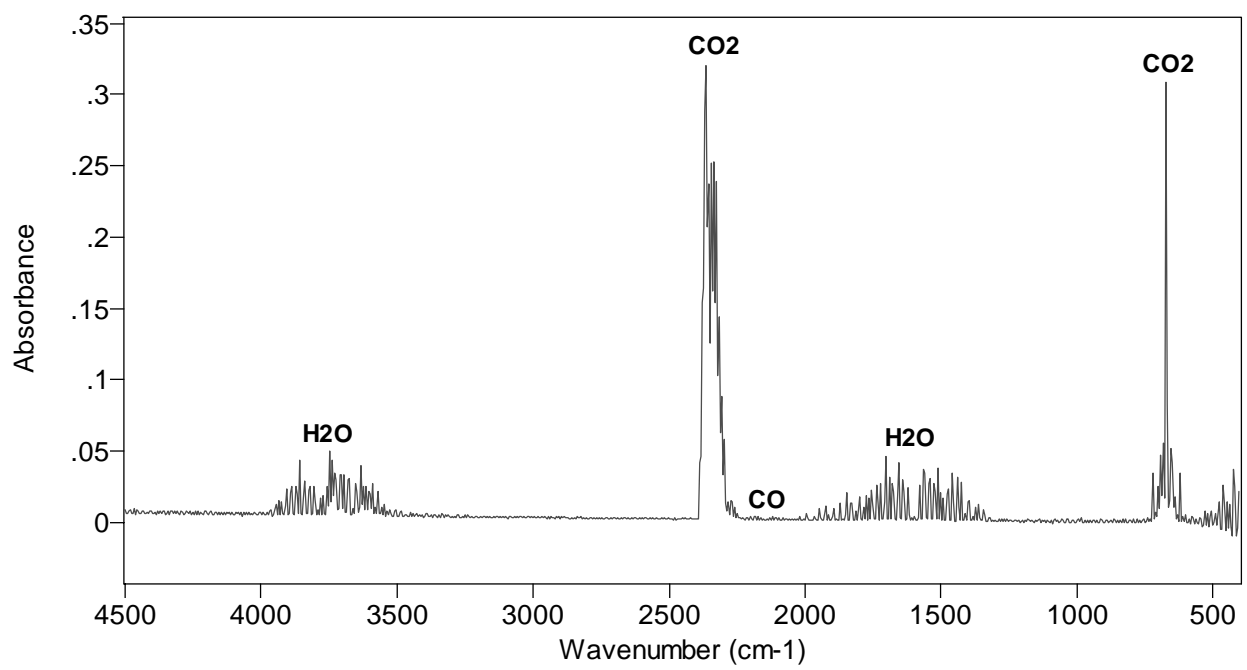

Figure 9. 2-min average of spectra from fire location before water discharge in Test 2

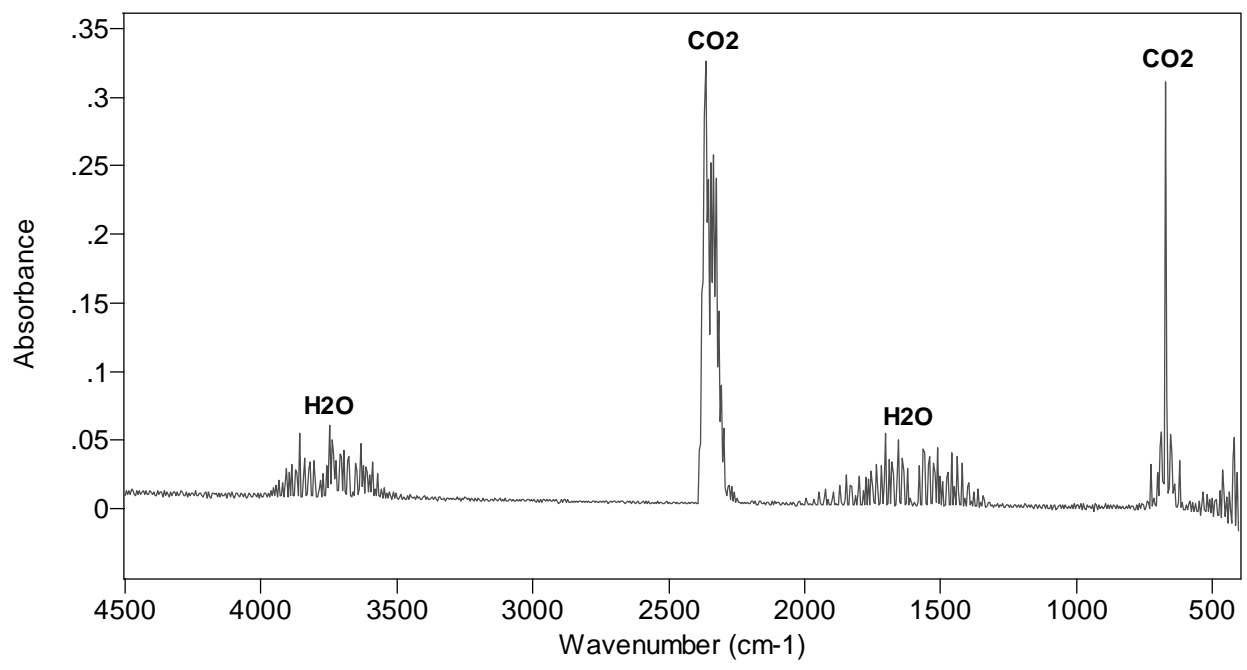

Figure 10. 1-min average of spectra at peak smoke obscuration in master bedroom(Test 2) 


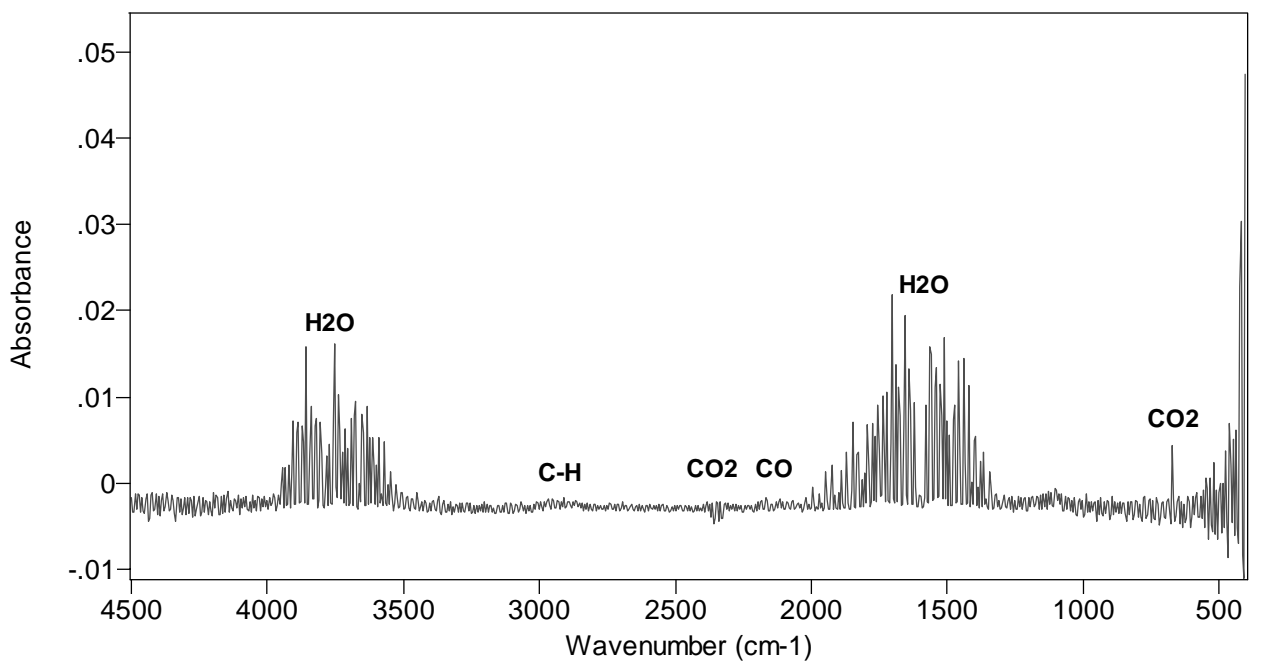

Figure 11. Average of spectra for fire location in last 4 minutes of mattress smouldering (Test 4)

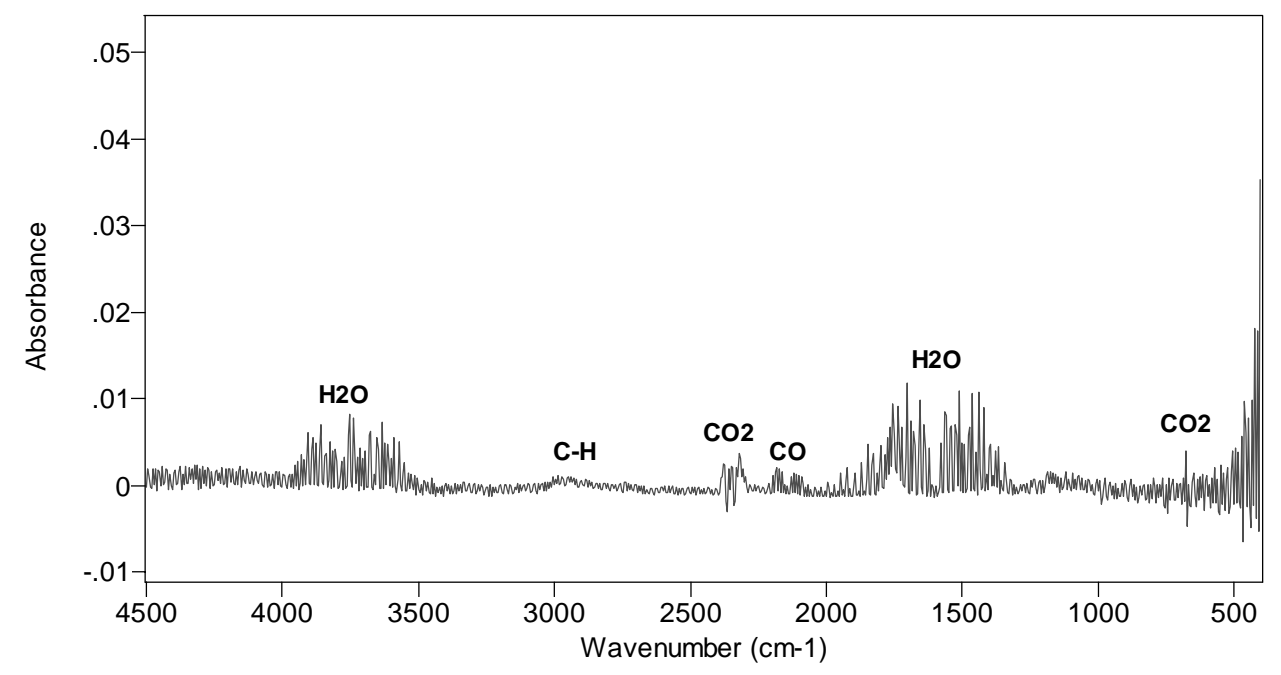

Figure 12. Average spectrum for fire room during peak smoke obscuration in Test 6 with mattress smouldering

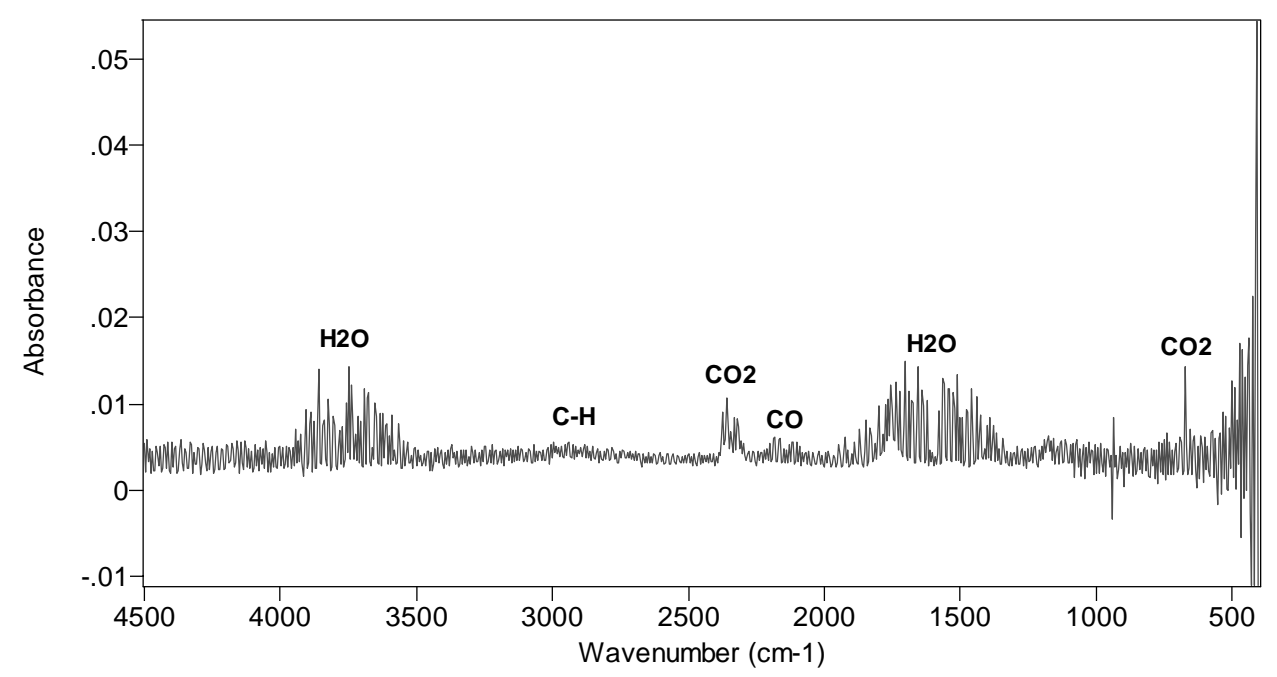

Figure 13. 1-min average of spectra for fire room after first smoke alarm in Test 8 with mattress smouldering 


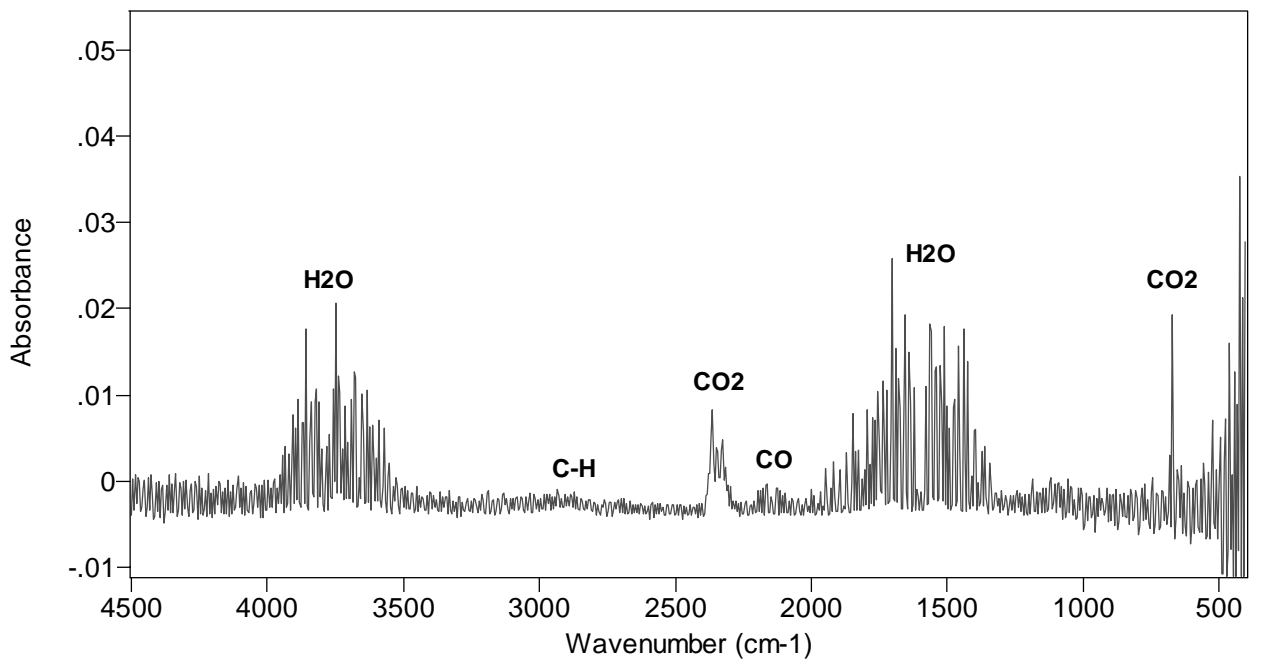

Figure 14. Average of spectra for fire room during the 50-s flaming in Test 4

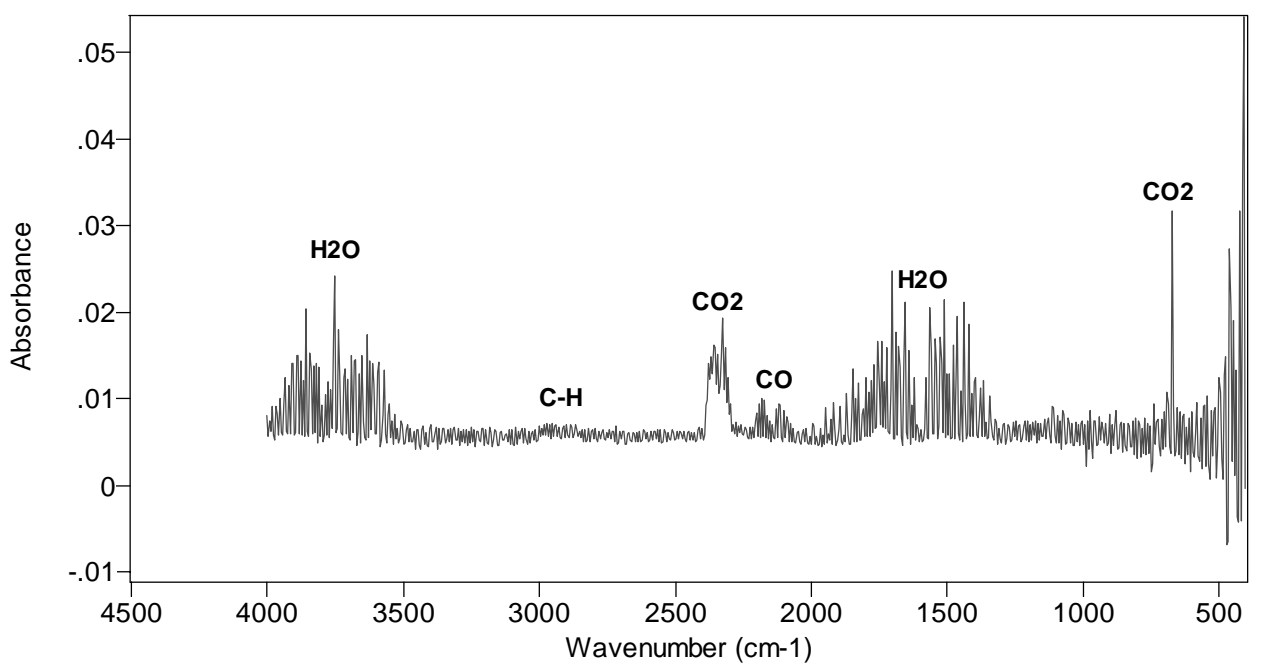

Figure 15. Average of spectra for fire room during the 60-s flaming in Test 6

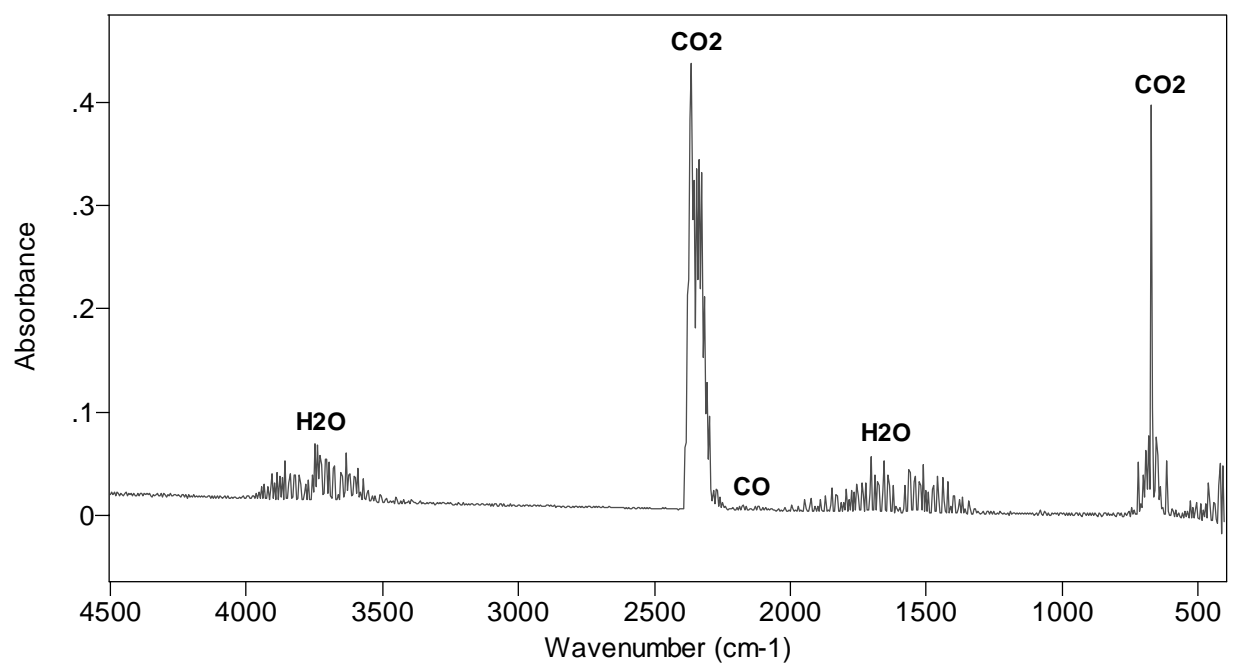

Figure 16. Average of spectra for fire room during the 100-s flaming in Test 8 


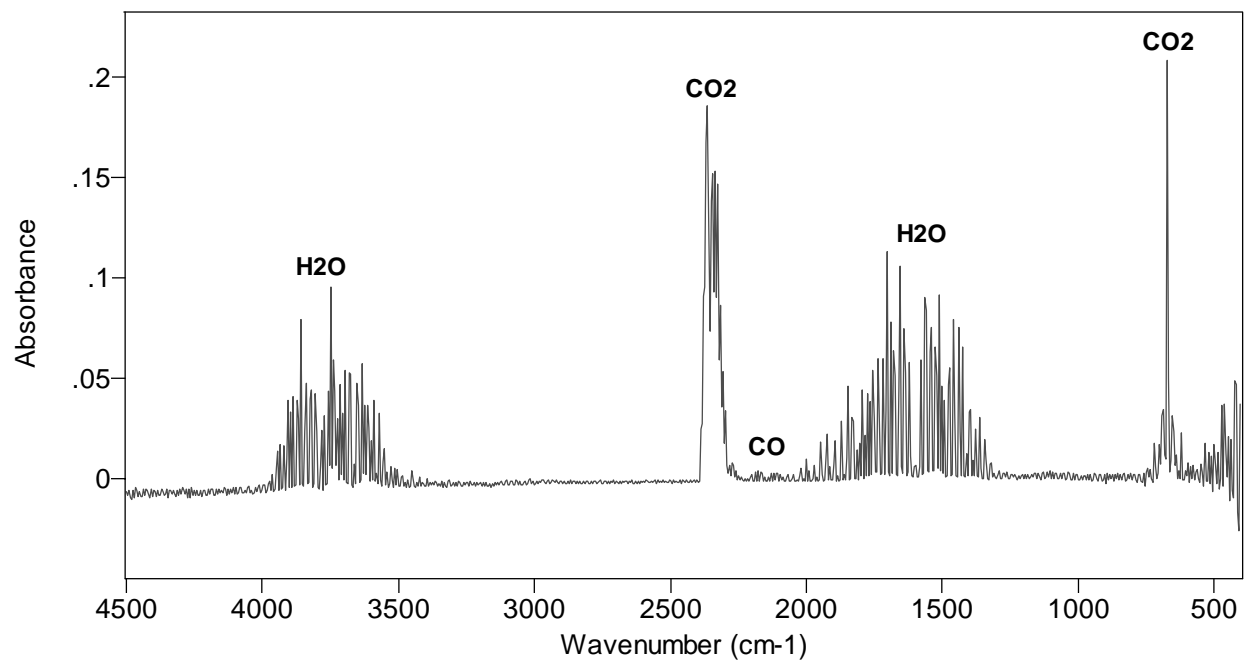

Figure 17. 1-min average of spectra for fire room during suppression in Test 4

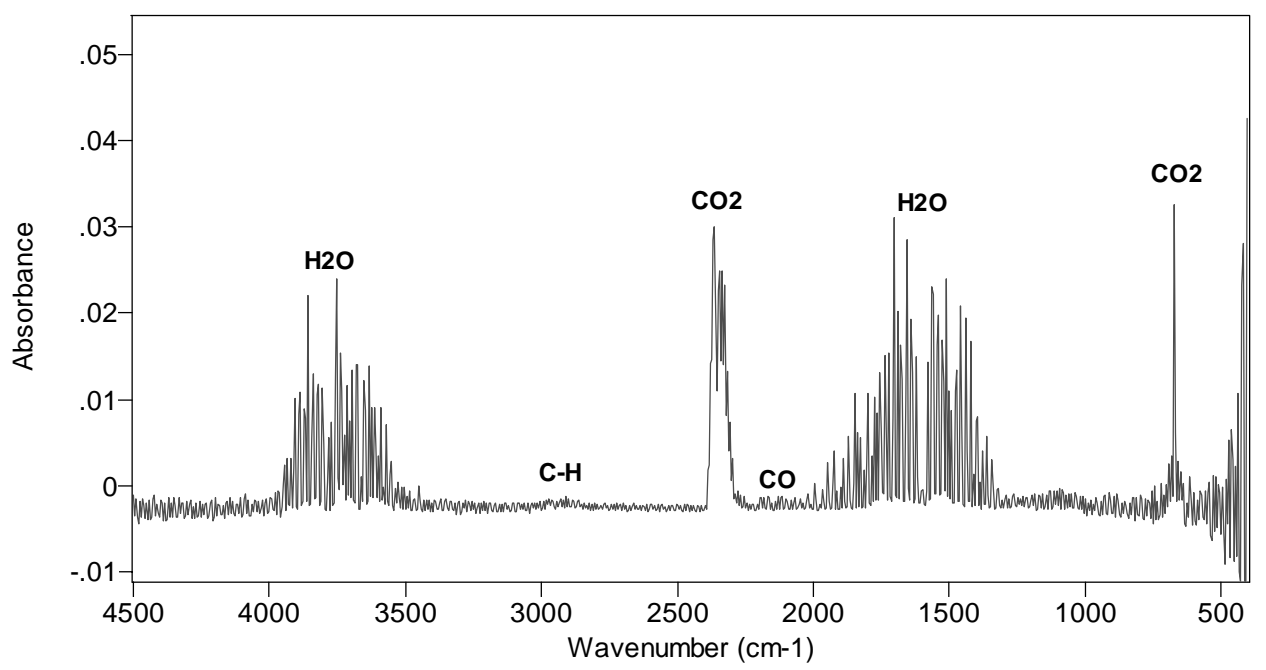

Figure 18. 4-min average of spectra for master bedroom during/after suppression(Test 4)

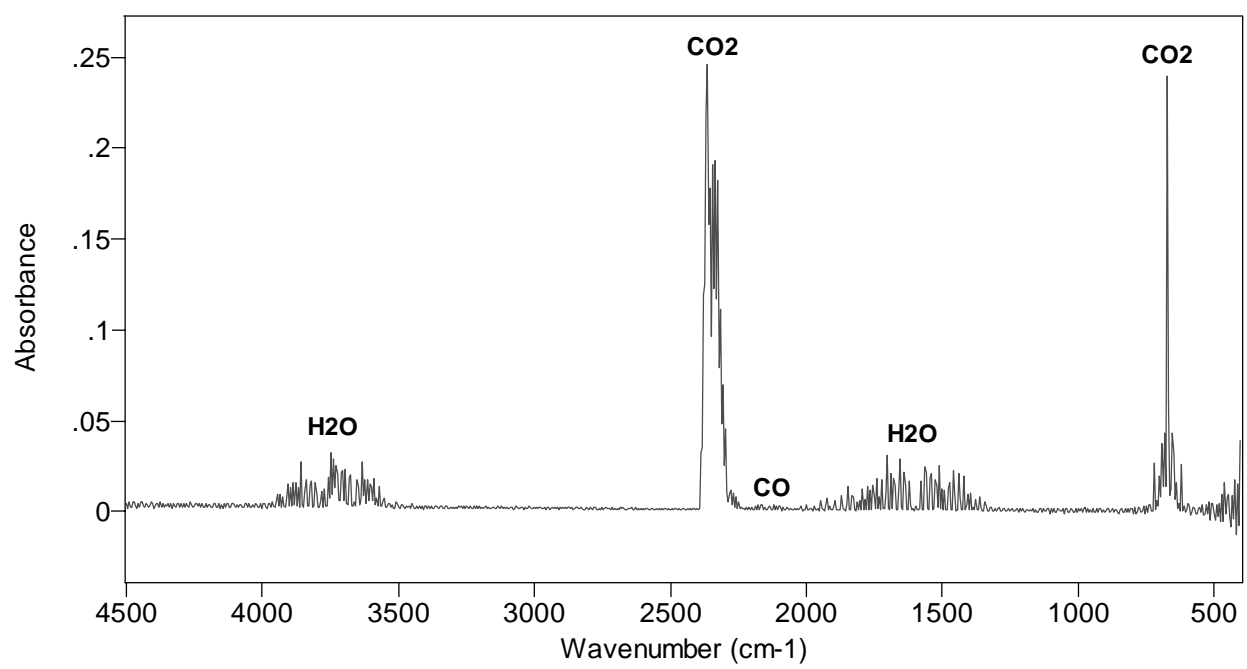

Figure 19. 2-min average of spectra for fire room during mattress flaming (Test 5) 


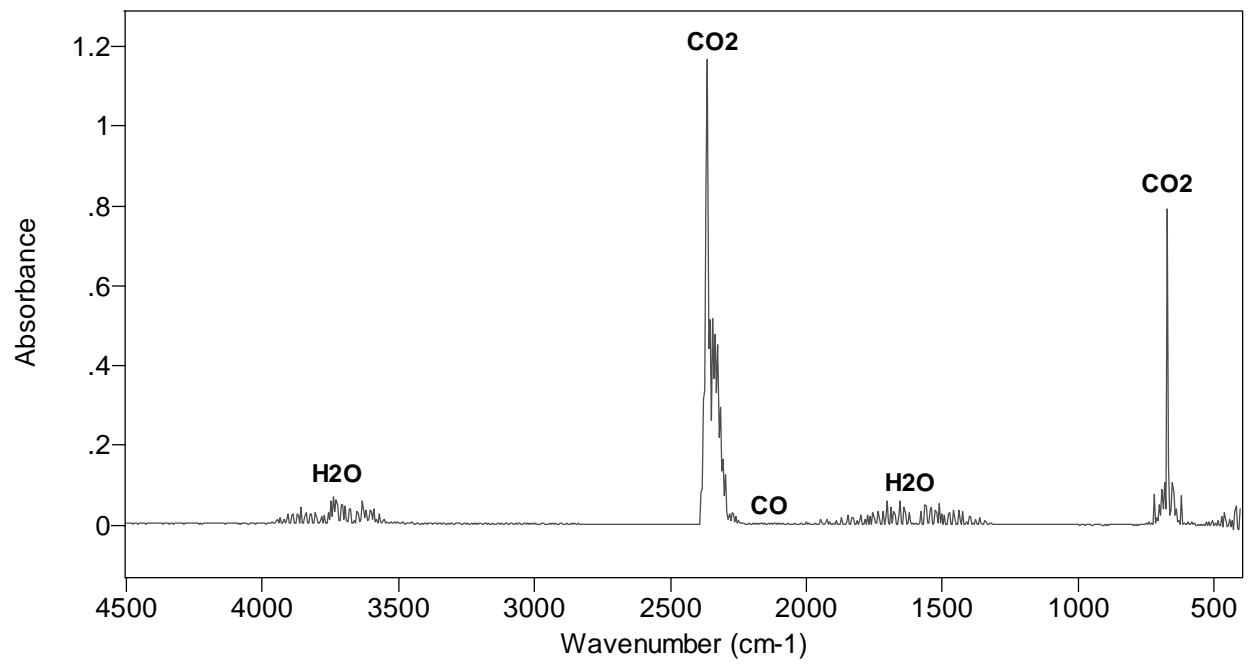

Figure 20. 3-min average of spectra for fire room during mattress flaming (Test 7)

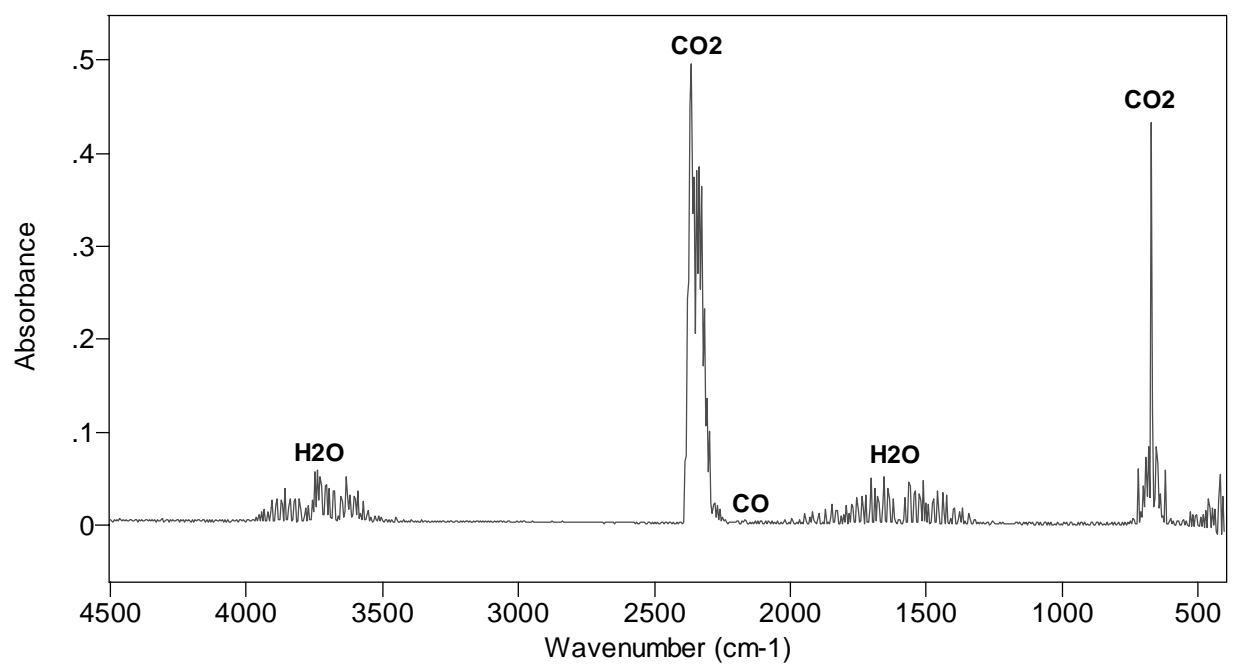

Figure 21. 2-min average of spectra for fire room during mattress flaming (Test 9 )

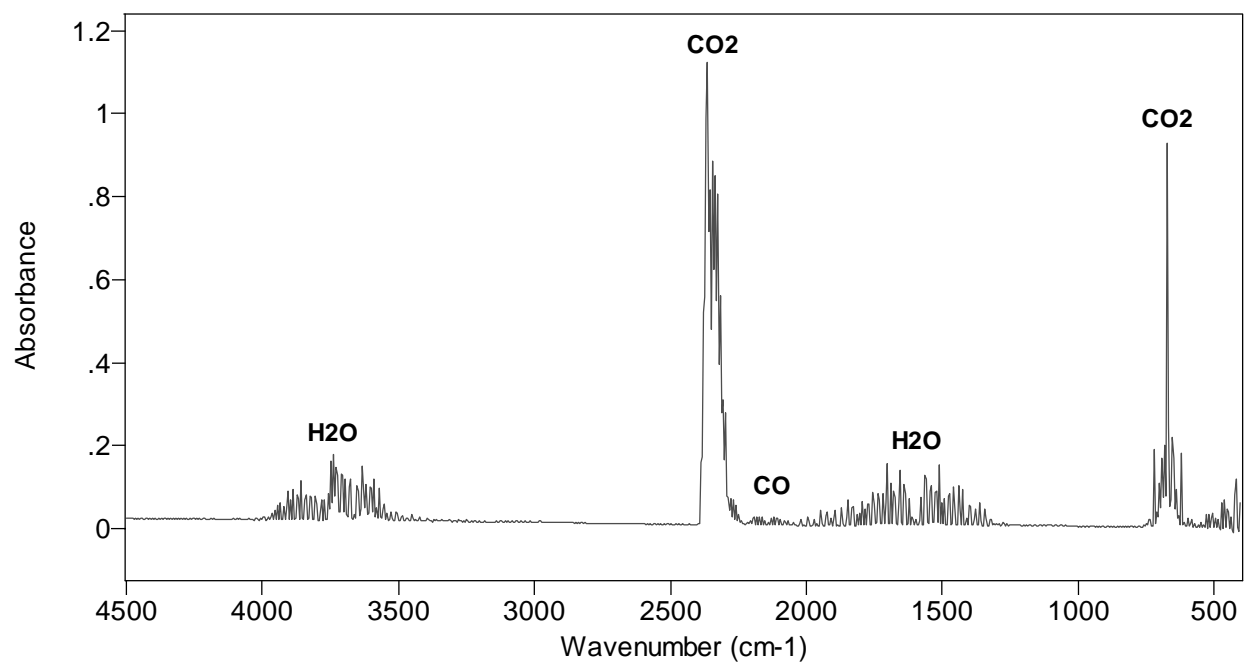

Figure 22. 2-min average of spectra for fire room after fire extinguishment (Test 9) 


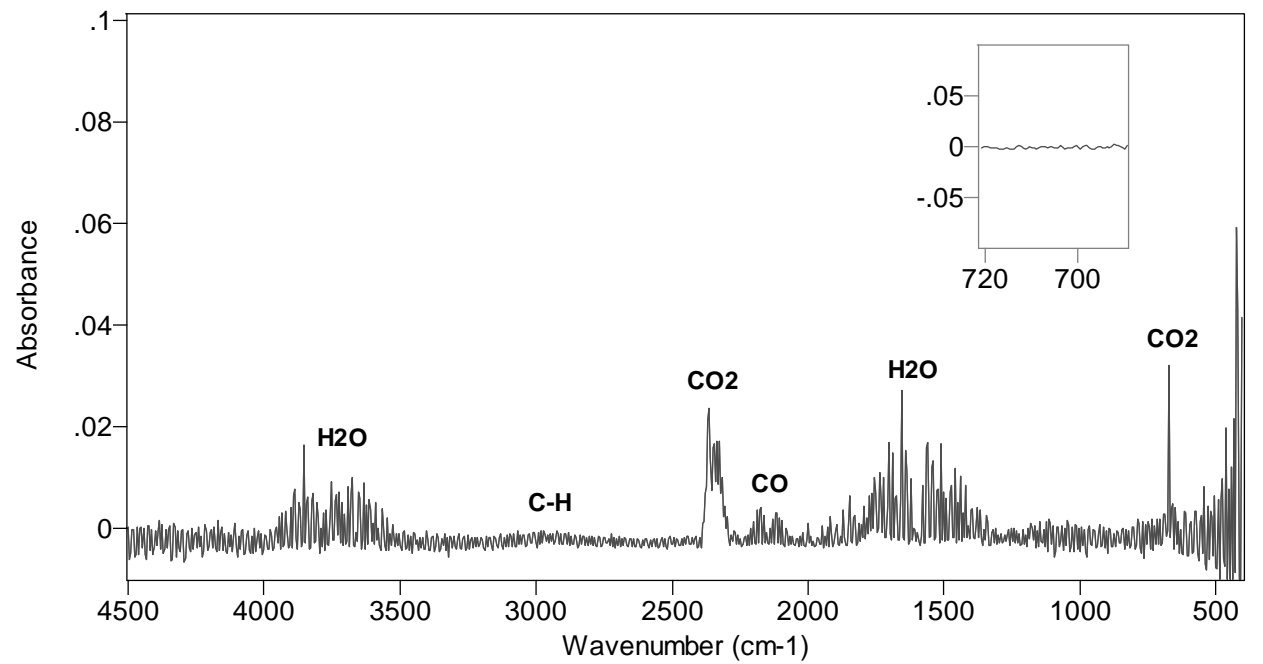

Figure 23. Average of spectra for fire room during the last $30 \mathrm{~s}$ of mattress smouldering (Test 14)

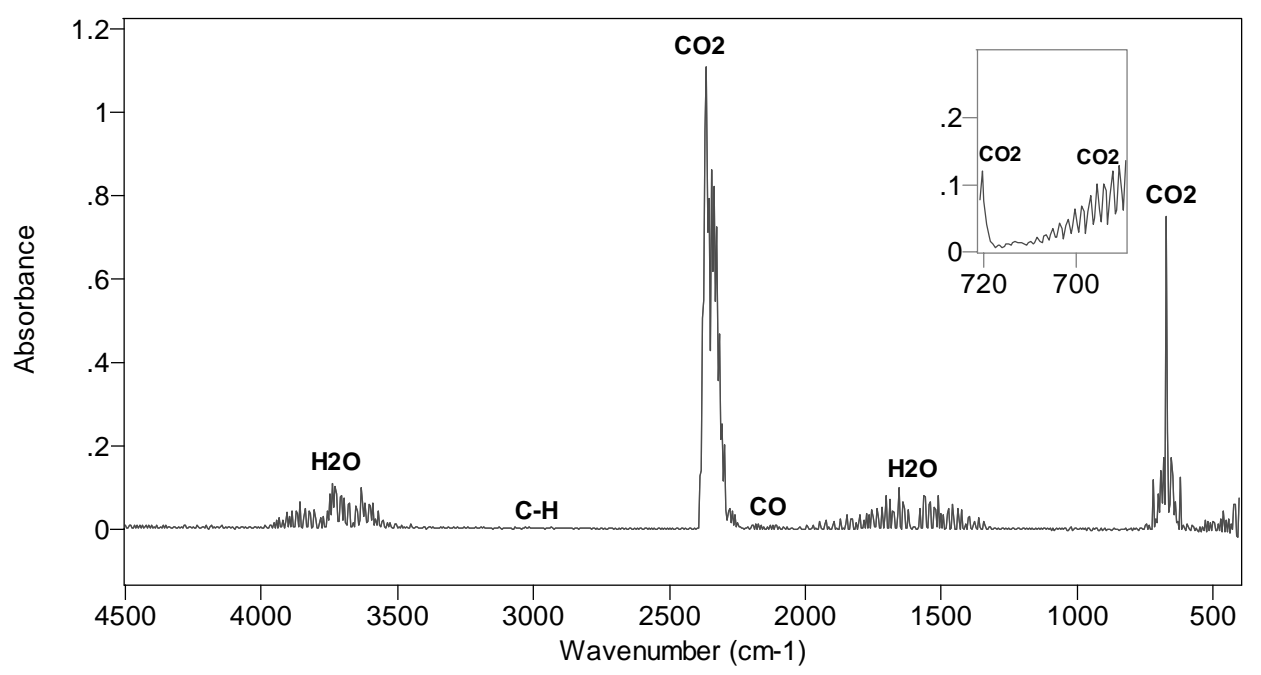

Figure 24. 30-s average of spectra for fire room after mattress flaming (Test 14)

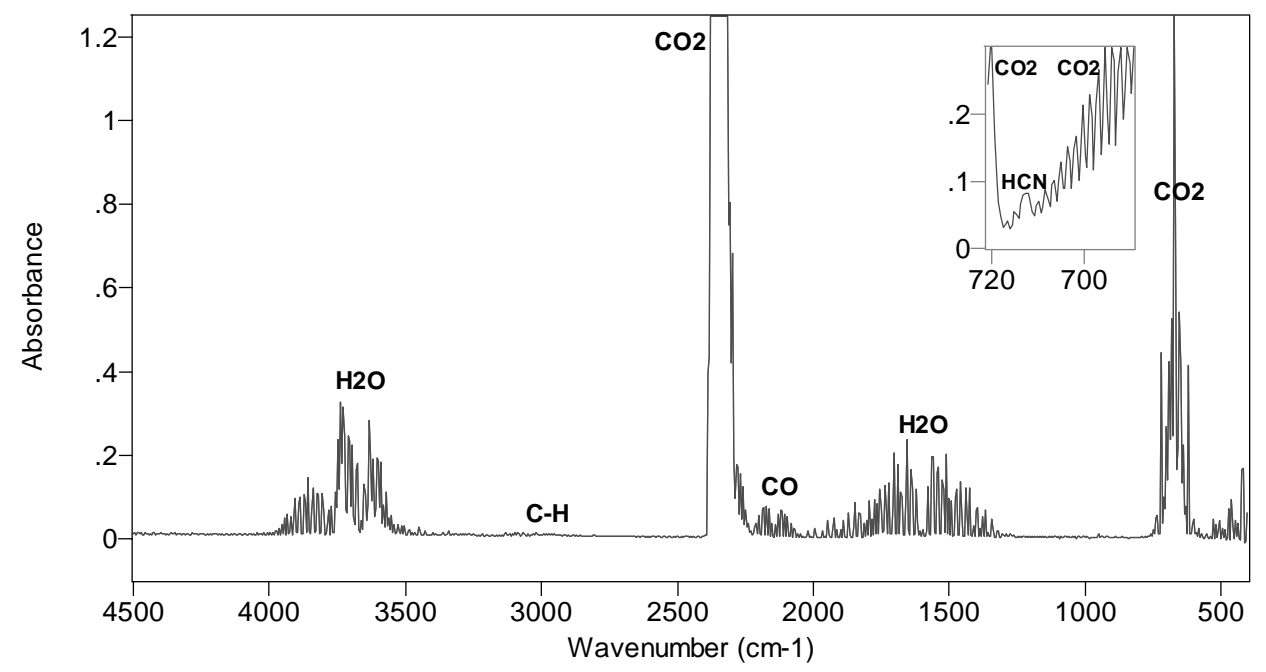

Figure 25. 90-s average of spectra for fire room after water spray (Test 14) 


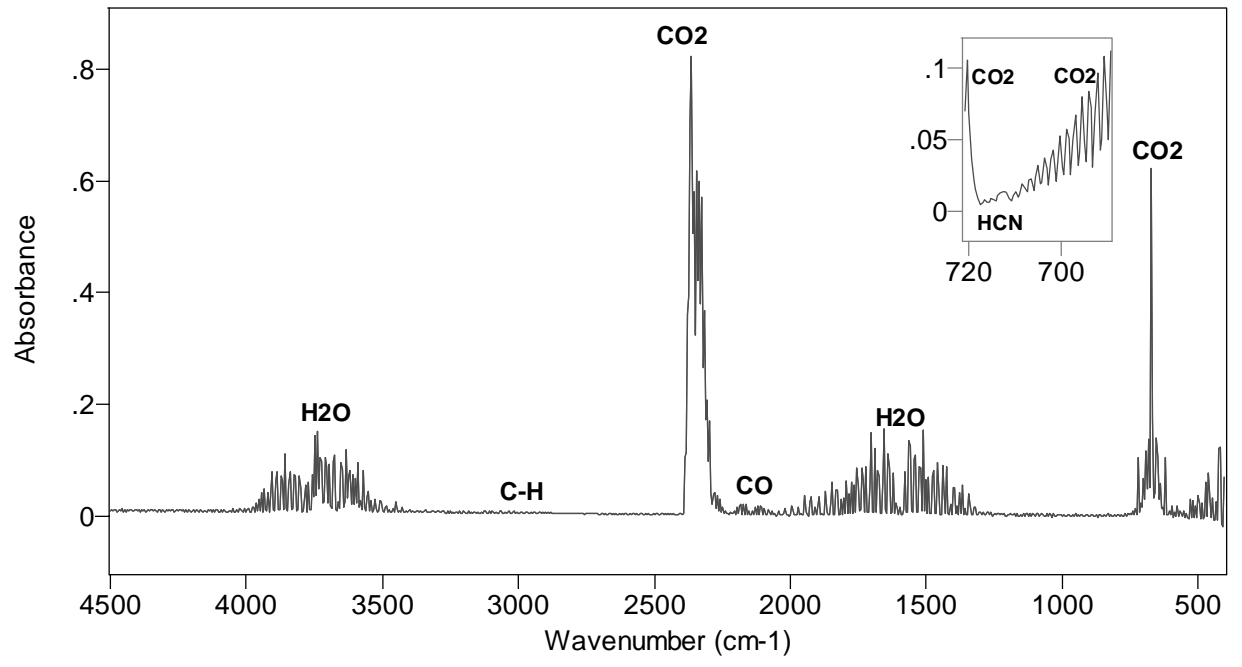

Figure 26. 60-s average of spectra for fire room after all doors opened (Test 14) 


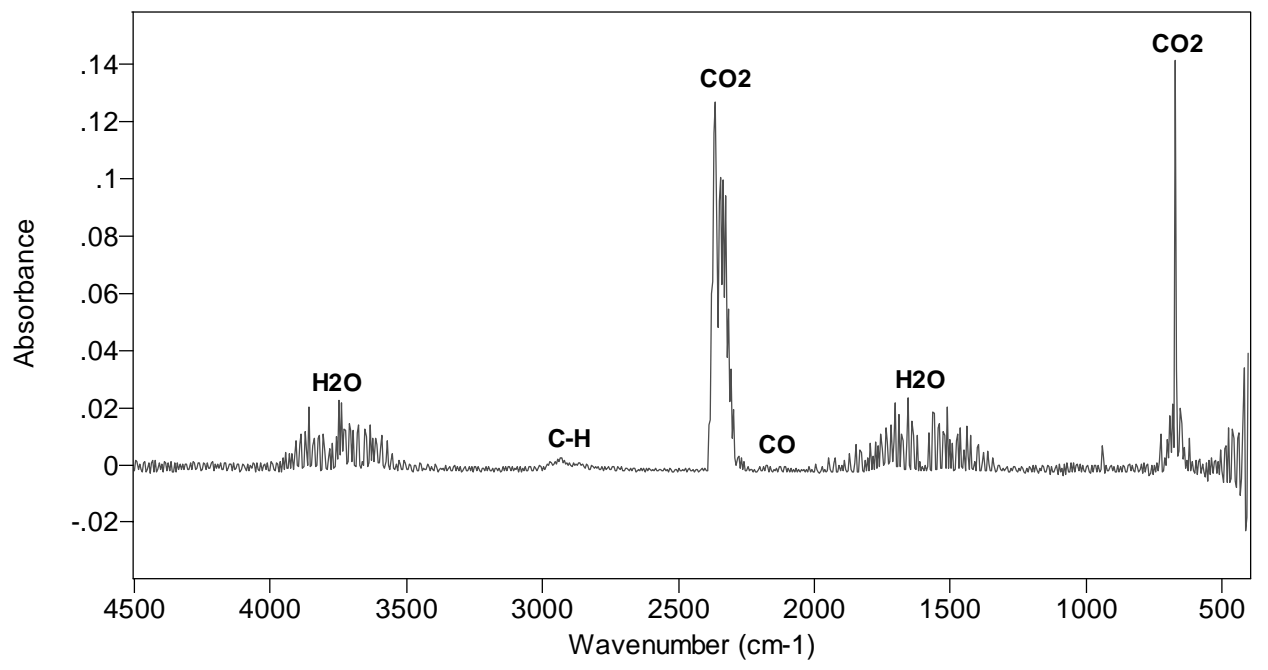

Figure 27. Average of spectra for last 2 minutes of cooking-oil heating (Test 13)

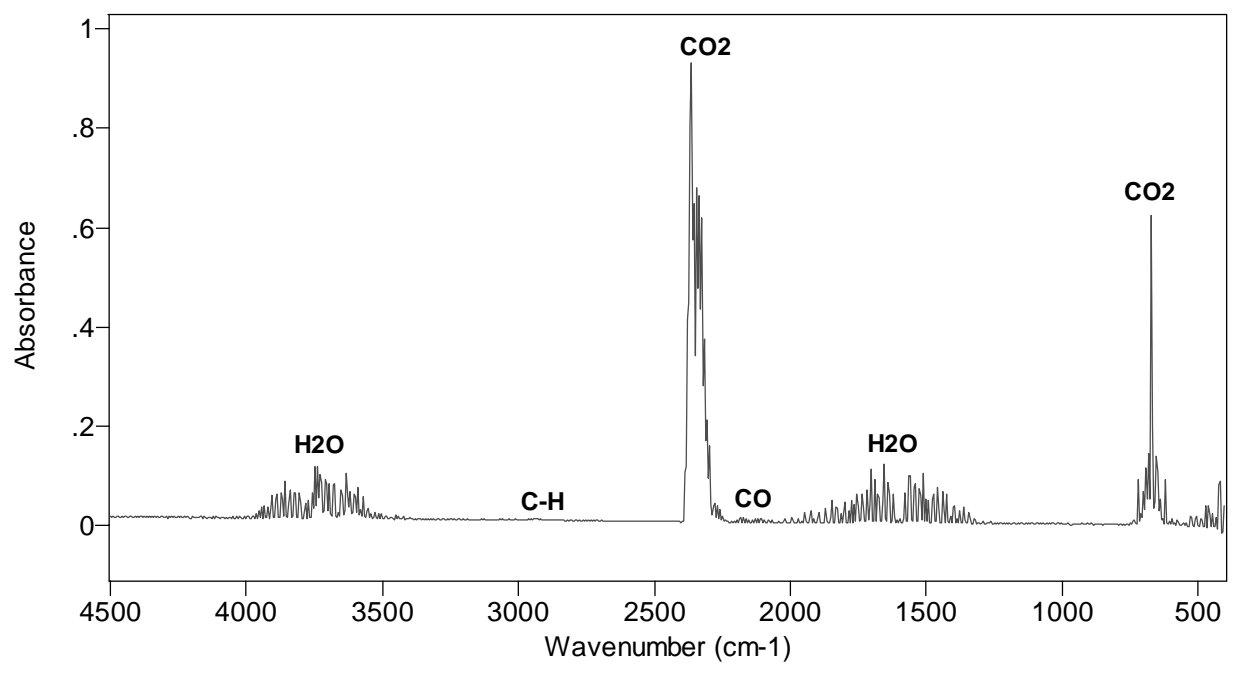

Figure 28. Average of spectra for last 2 minutes of cooking-oil flaming (Test 13)

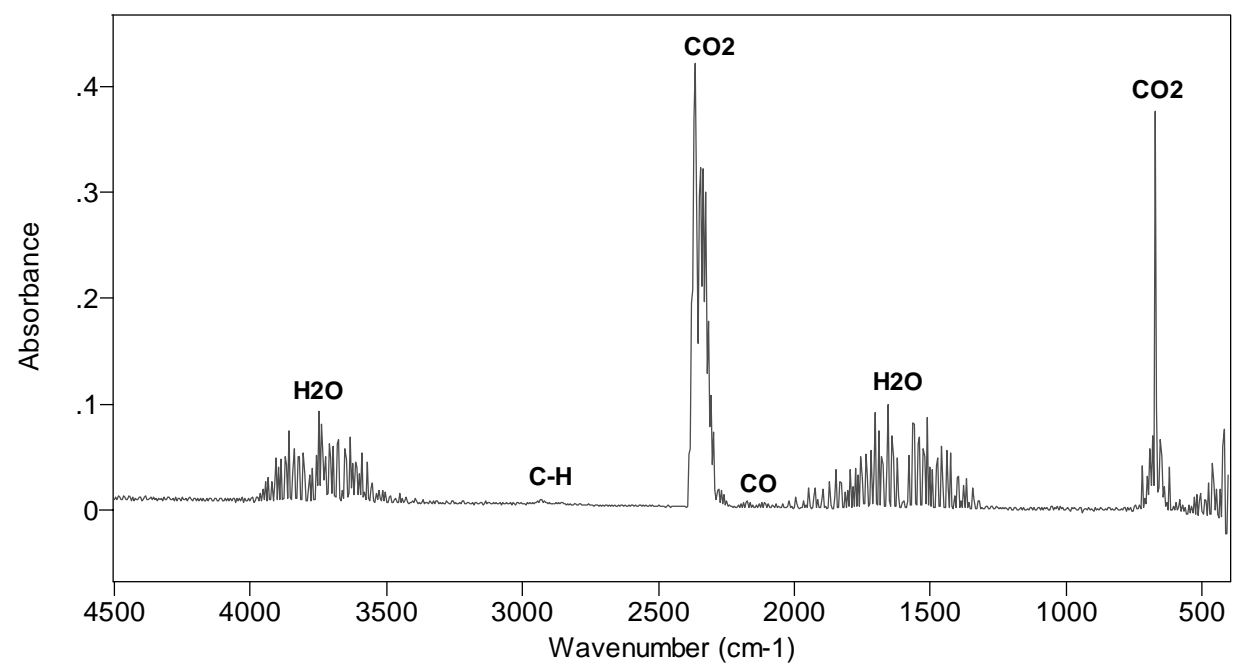

Figure 29. 2-min average of spectra after cooking-oil fire extinguishment (Test 13) 


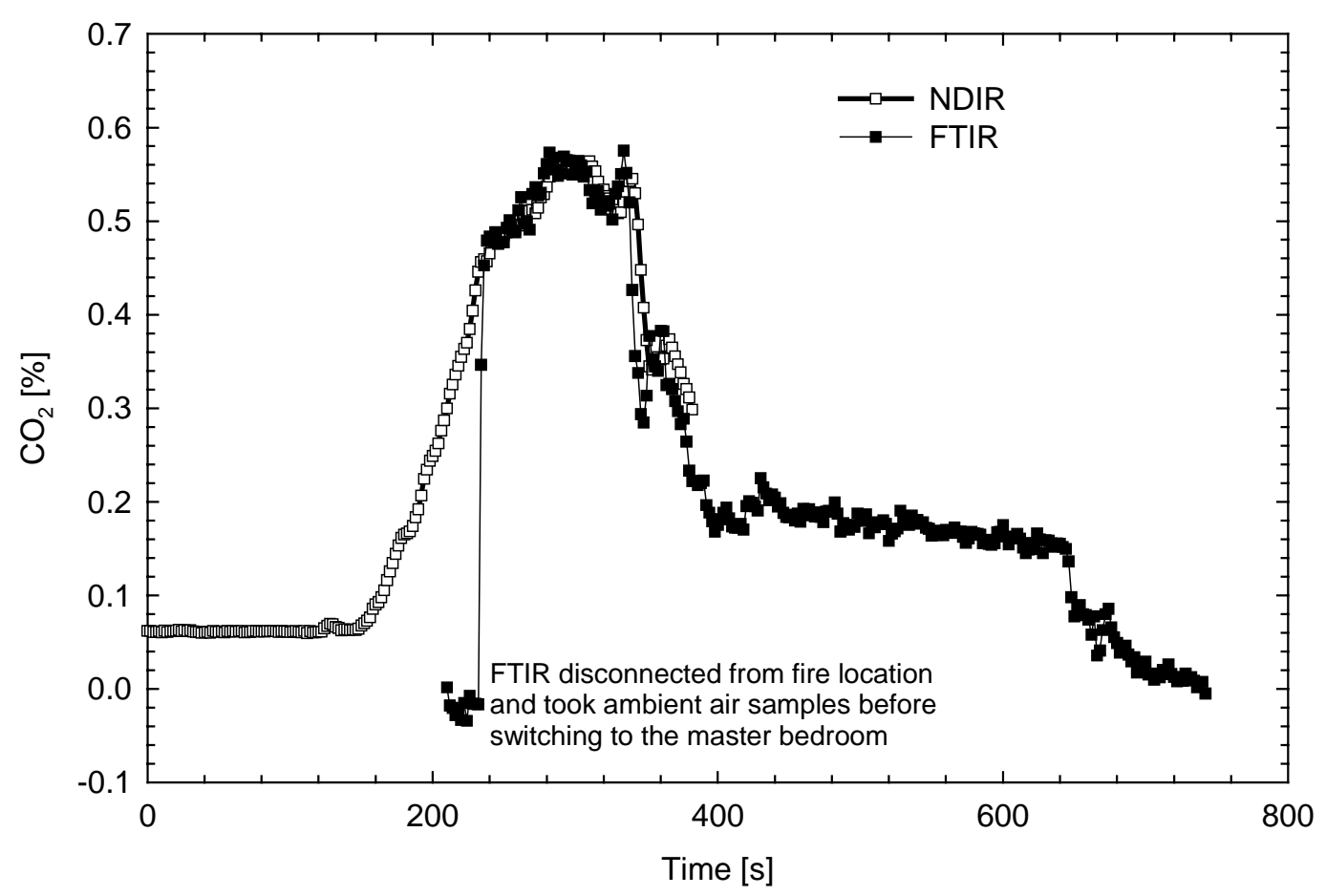

Figure 30. Carbon dioxide concentration in master bedroom from FTIR and NDIR measurements for flaming chair (Test 2)

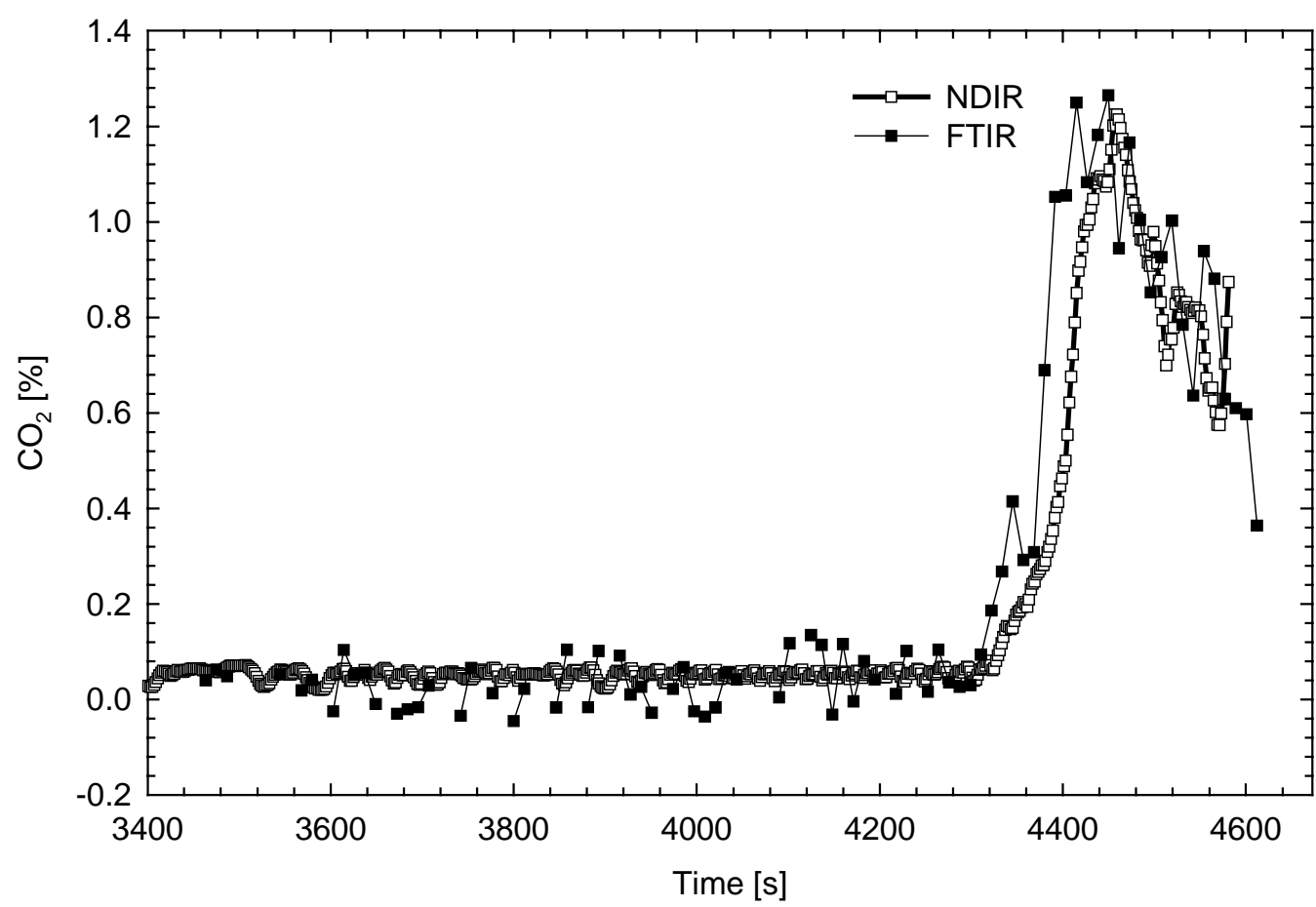

Figure 31. Carbon dioxide concentration in master bedroom from FTIR and NDIR measurements for smouldering chair (Test 11) 


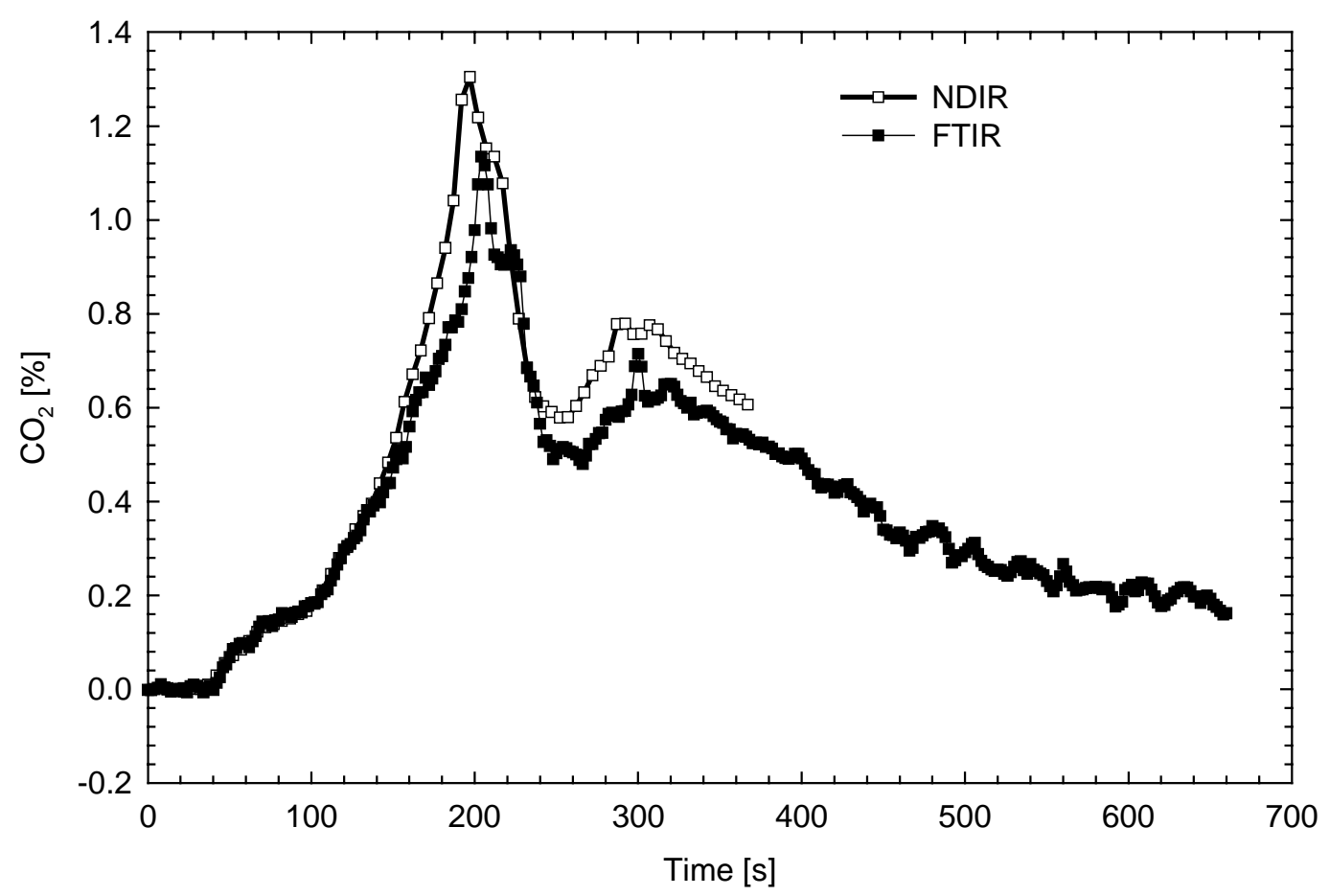

Figure 32. Carbon dioxide concentration in fire room from FTIR and NDIR measurements for flaming mattress (Test 5)

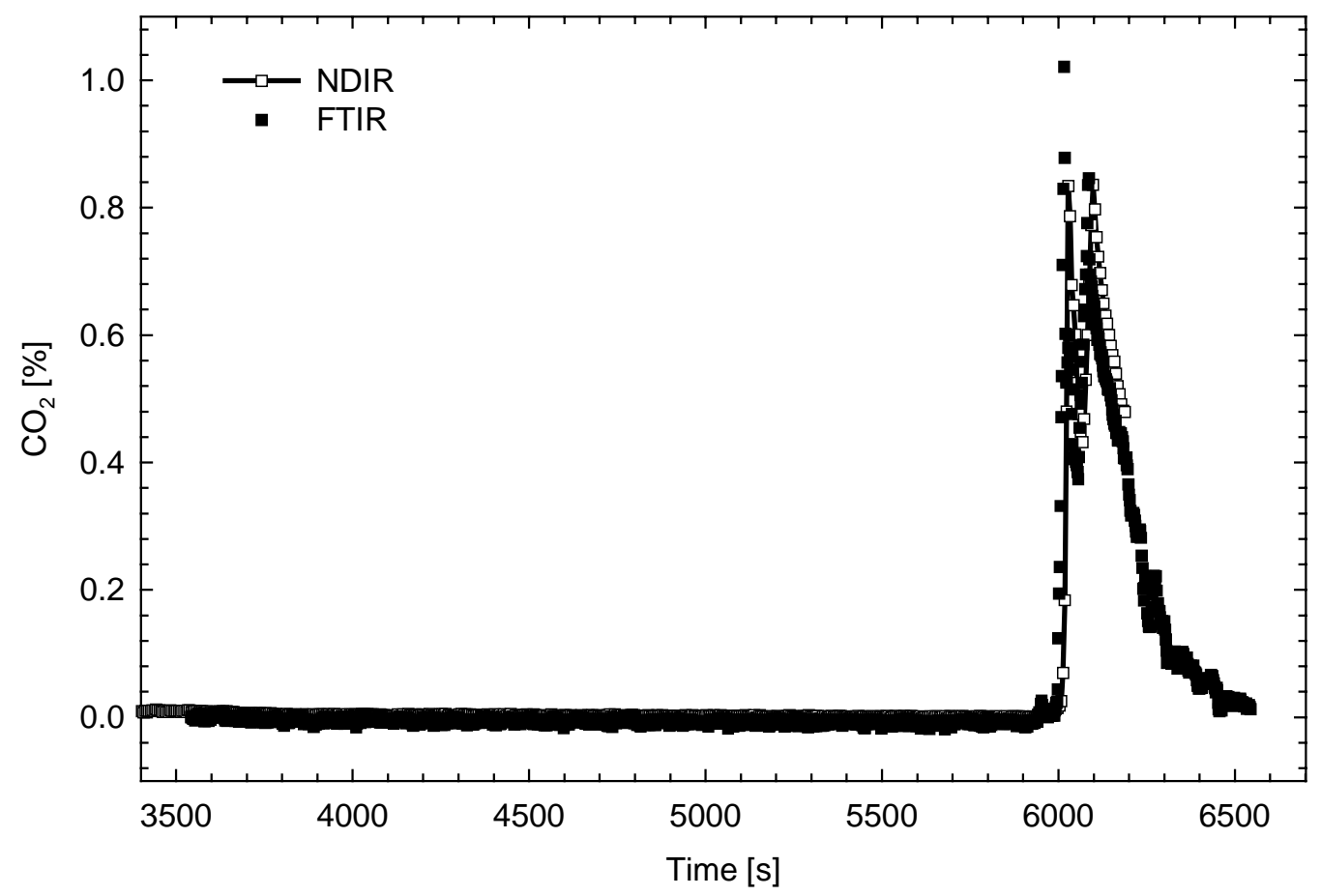

Figure 33. Carbon dioxide concentration in fire room from FTIR and NDIR measurements for smouldering mattress (Test 6) 


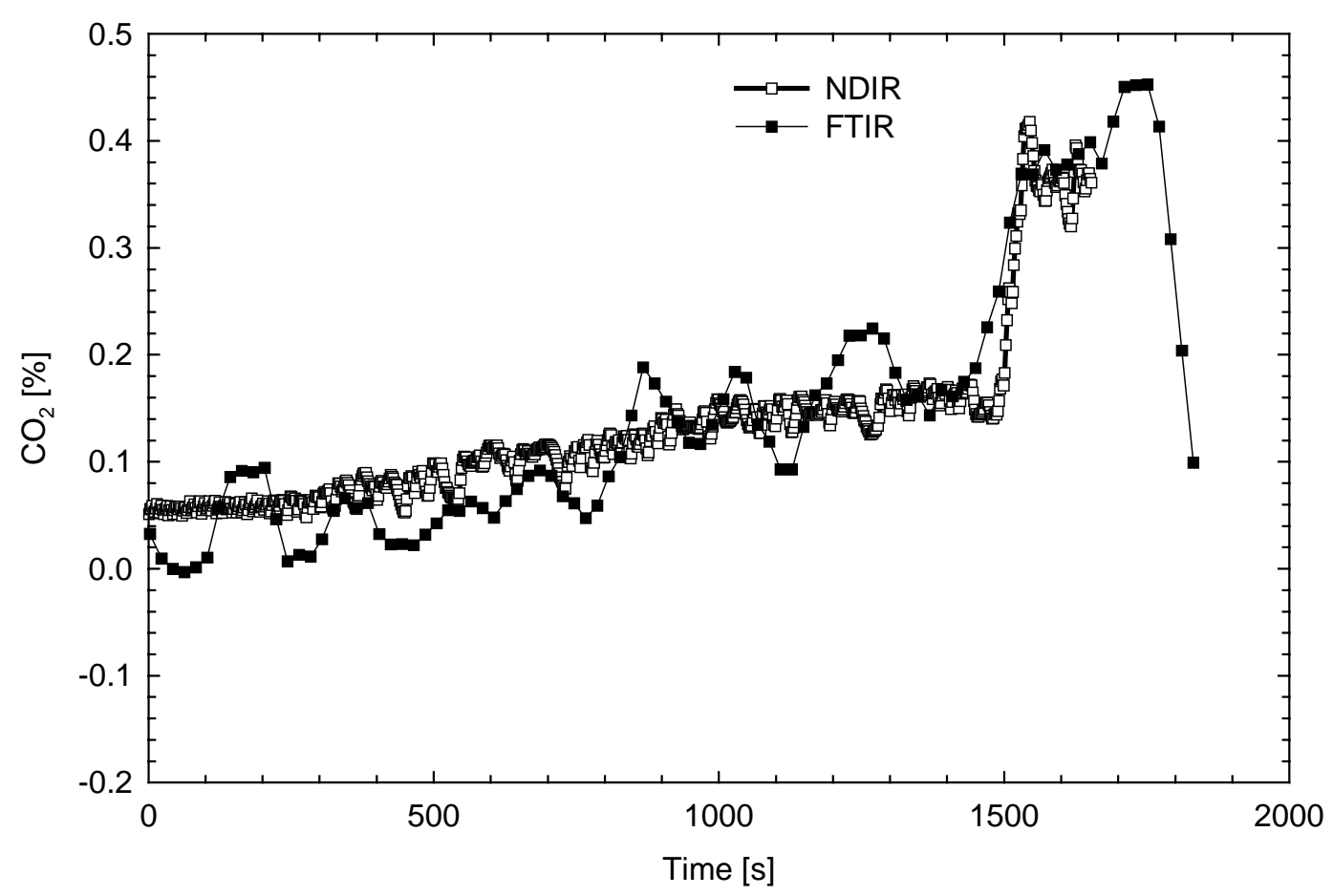

Figure 34. Carbon dioxide concentration in master bedroom from FTIR and NDIR measurements for cooking oil fire (Test 12)

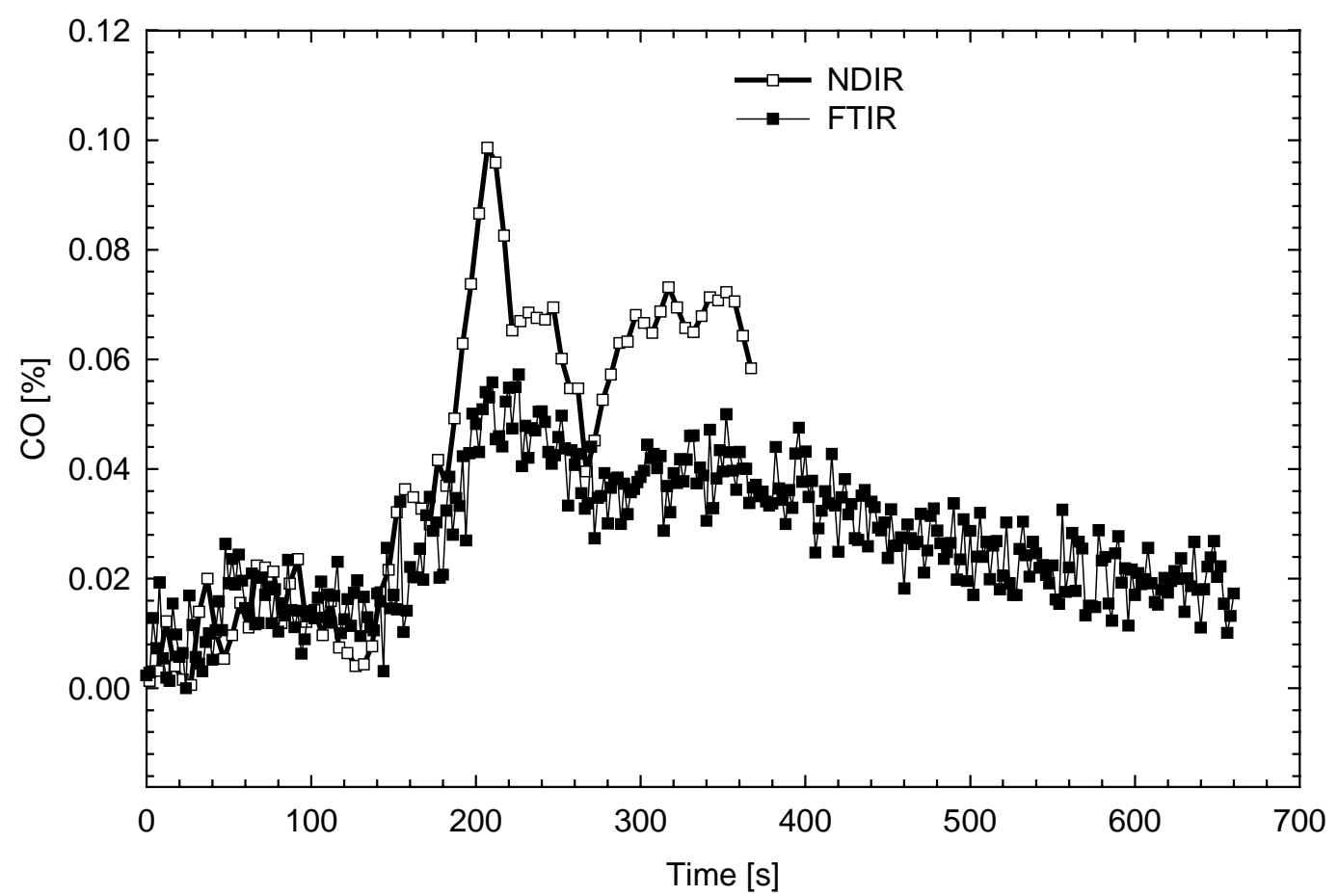

Figure 35. Carbon monoxide concentration in fire room from FTIR and NDIR measurements for flaming mattress (Test 5) 


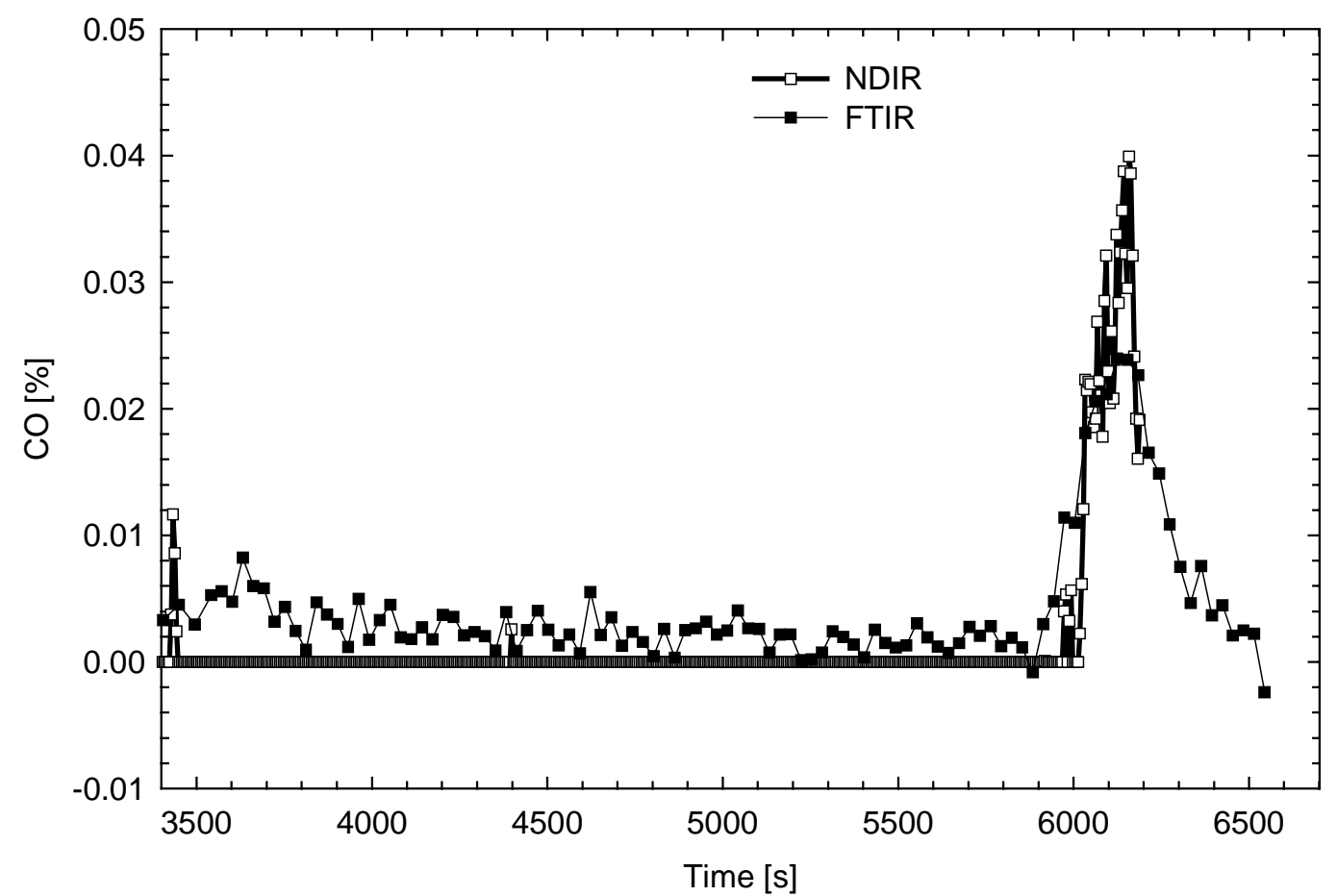

Figure 36. Carbon monoxide concentration in fire room from FTIR and NDIR measurements for smouldering mattress (Test 6)

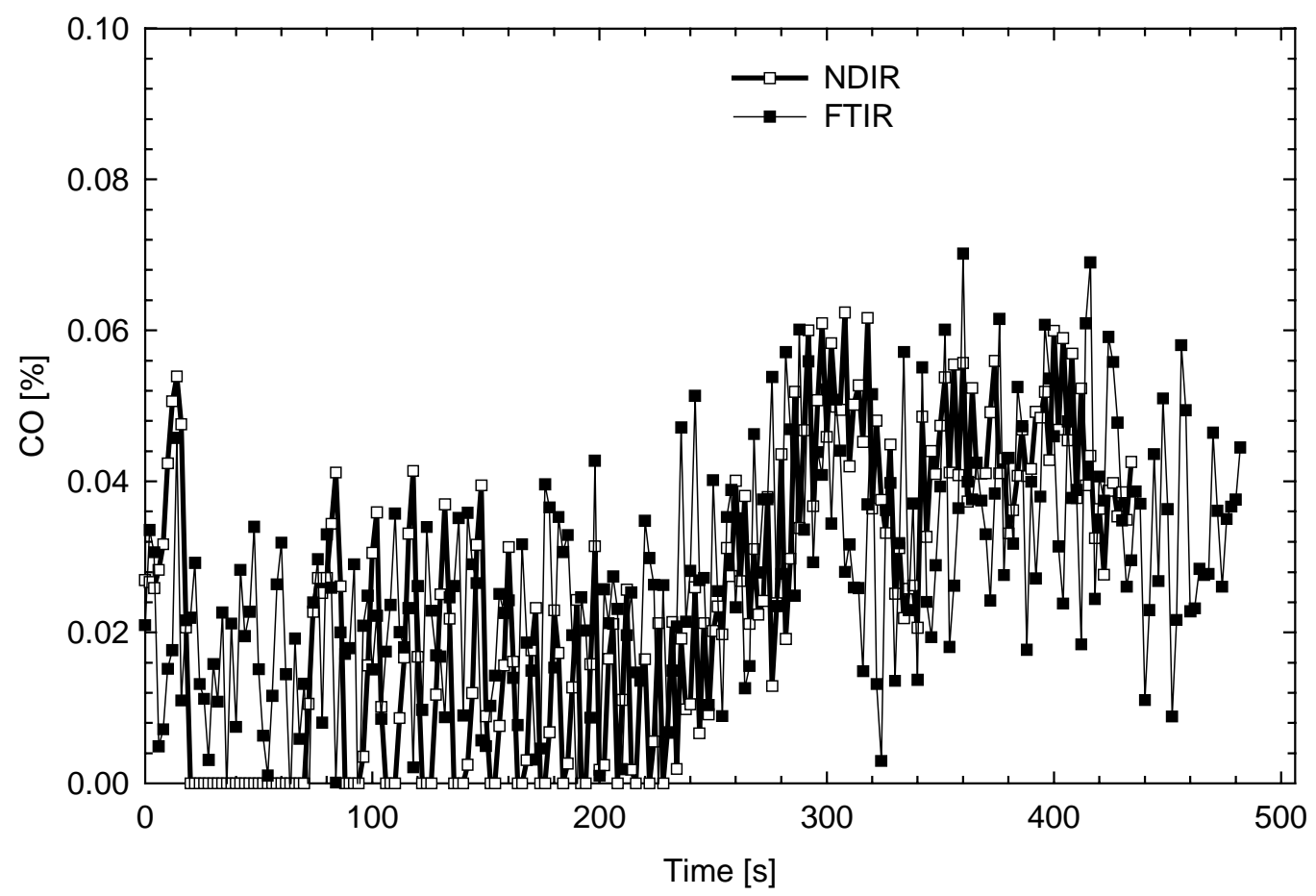

Figure 37. Carbon monoxide concentration in master bedroom from FTIR and NDIR measurements for flaming chair (Test 10) 


\subsection{CONCLUSIONS}

The small, slow smouldering fires and short flaming fires provided the greatest challenge for the smoke detectors to detect the fires early before becoming fully developed ones. However, the experiments were terminated well before reaching conditions that would produce a significant amount of chemical species, such as $\mathrm{HCl}, \mathrm{HCN}, \mathrm{NO}_{x}, \mathrm{HBr}$ and $\mathrm{HF}$, above the minimum detection limit of the FTIR spectrometer in this full-scale experimental set-up. Carbon monoxide and carbon dioxide were primary gas products produced in Tests 1-14. There was no apparent absorption from chemical species such as $\mathrm{HCl}, \mathrm{NO}_{\mathrm{x}}, \mathrm{HBr}$ and $\mathrm{HF}$ in the FTIR spectra. FTIR spectra collected during the chair and mattress smouldering and cooking oil fires show absorption in region characteristic of volatile hydrocarbon compounds. The FTIR measurement only detected absorption from $\mathrm{HCN}$ (60 ppm at maximum) during Test 14 with an under-ventilated condition.

\subsection{ACKNOWLEDGEMENTS}

NRC acknowledges financial contributions from the Canadian Fire Alarm Association and Health Canada. NRC also acknowledges supports from NIST, in particular, Mr. Richard W. Bukowski, Mr. Nelson P. Bryner, Dr. Marc R. Nyden and the testing crew for logistic and experimental support.

\subsection{REFERENCES}

1. Bukowski, R. W., Waterman, T. E., and Christian, W. J., "Detector Sensitivity and Siting Requirements for Dwellings: A Report of the NBS 'Indiana Dunes Tests."' NFPA No. SPP. 43. Nat. Fire Prot. Assn., Quincy, MA, 1975.

2. Peacock, R.D., Averill, J.D., Bukowski, R.W., and Reneke, P.A., "Home Smoke Alarm Project, Manufactured Home Tests," NIST Report of Test FR 4016, Fire Research Division, Building and Fire Research Laboratory, National Institute of Standards and Technology, Gaithersburg, MD, June, 2002.

3. Averill, J.D., Peacock, R.D., Bukowski, R.W., and Reneke, P.A., "Home Smoke Alarm Project, Two-Story Home Tests," NIST Report of Test FR 4017, Fire Research Division, Building and Fire Research Laboratory, National Institute of Standards and Technology, Gaithersburg, MD, June, 2002. 